\title{
$7 x^{3}+2$
}

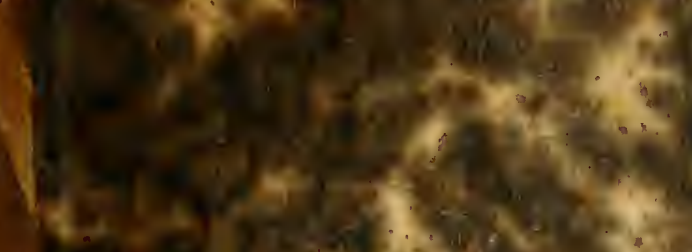 \\ 10.}

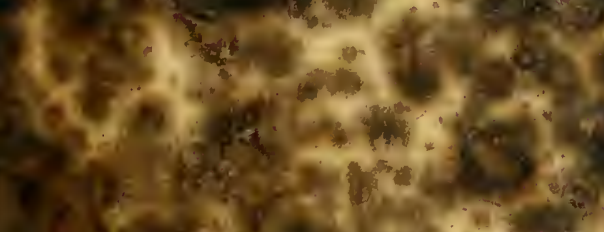

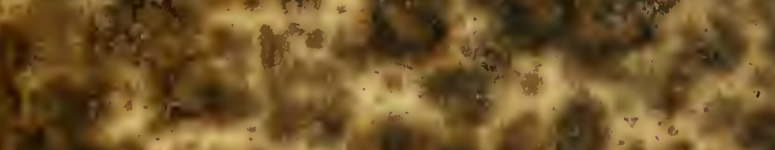
Q

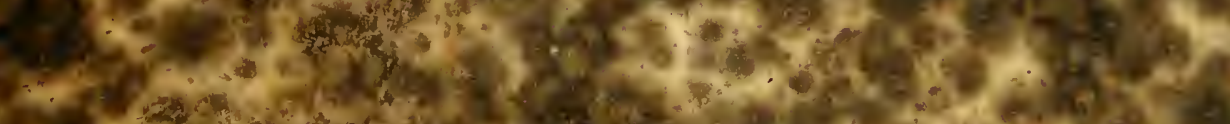

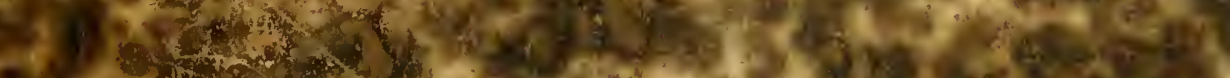

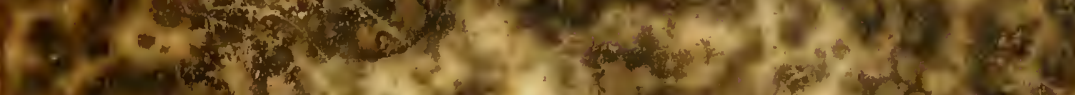

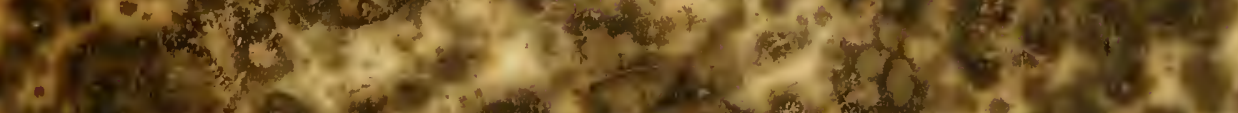

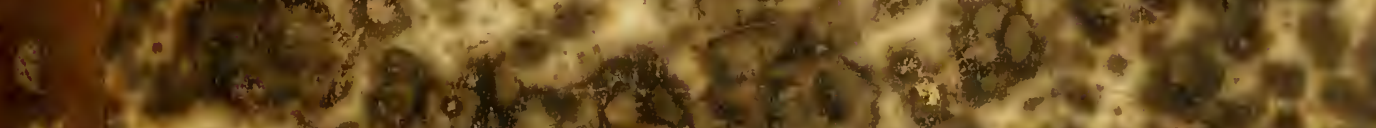

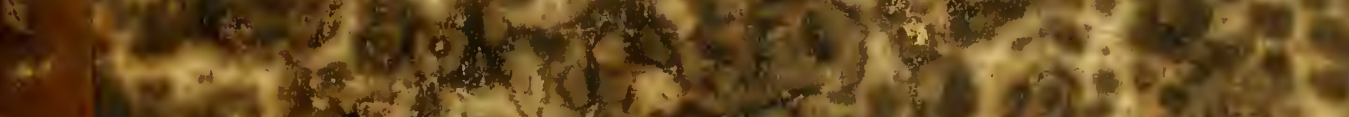

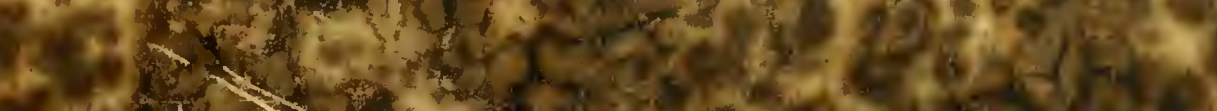

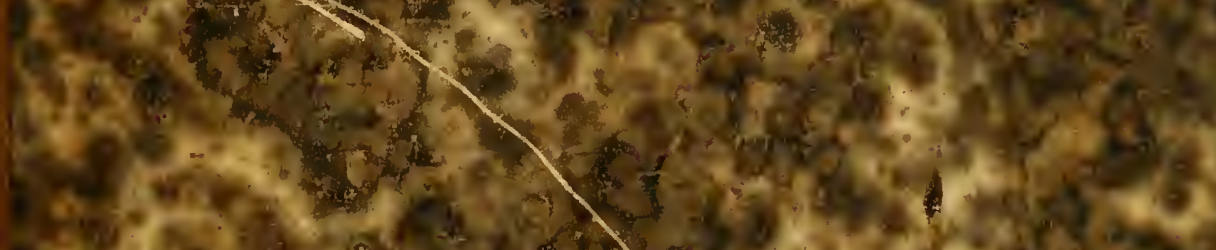

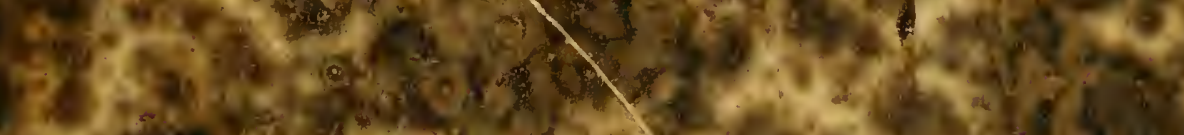

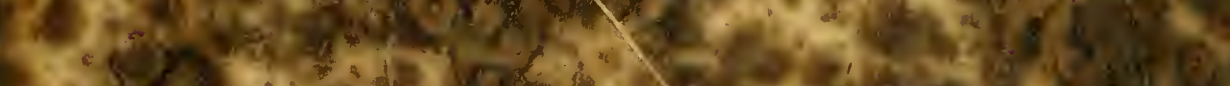

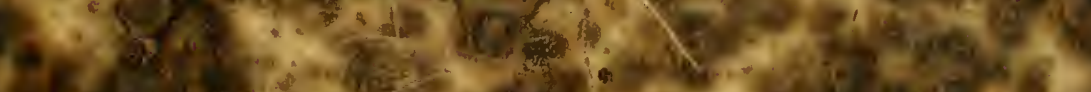

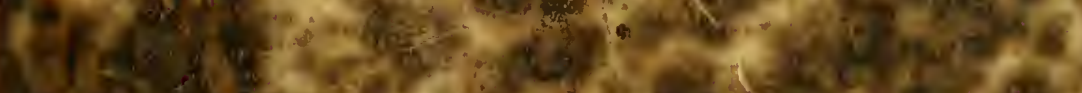

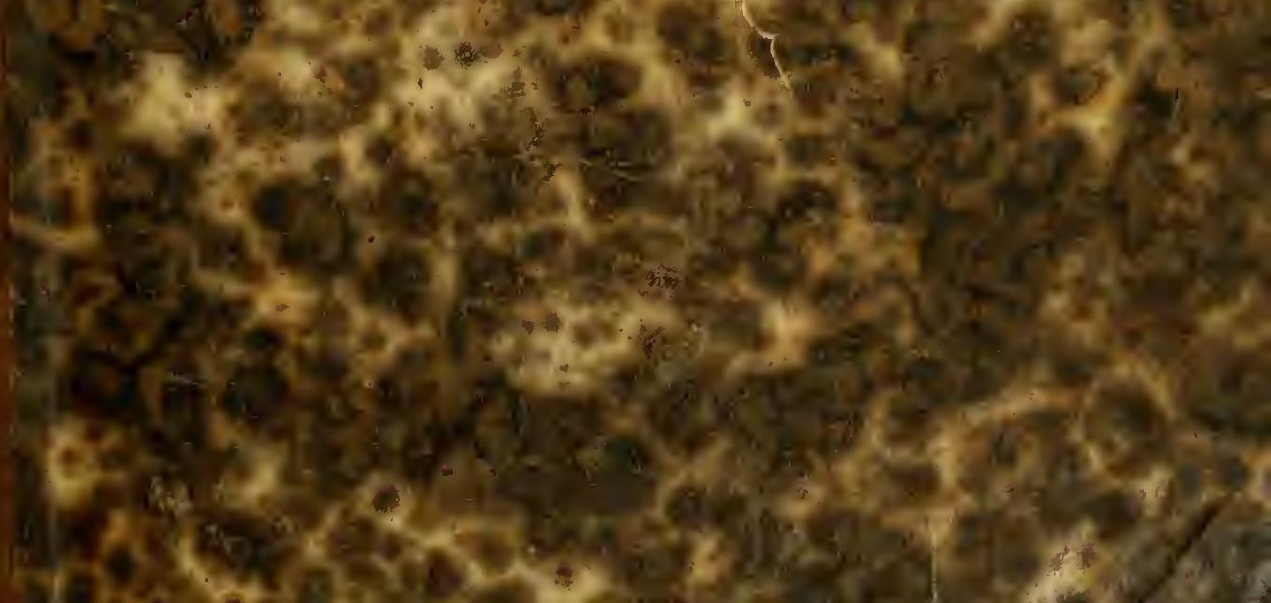


- wor un

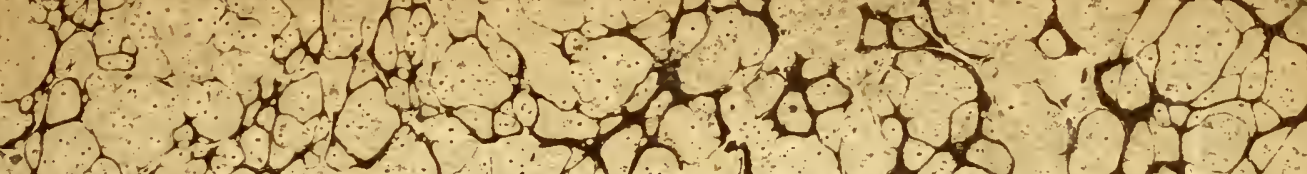
$5 \times 3,-2$, o 20

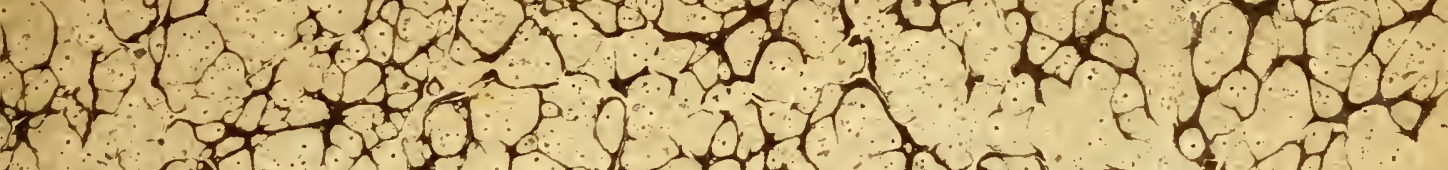
(4) 1,0101, rot ox o (y) 14,2

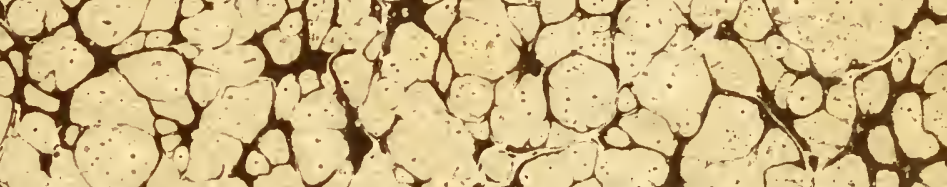
* $\times 2 \times 19$

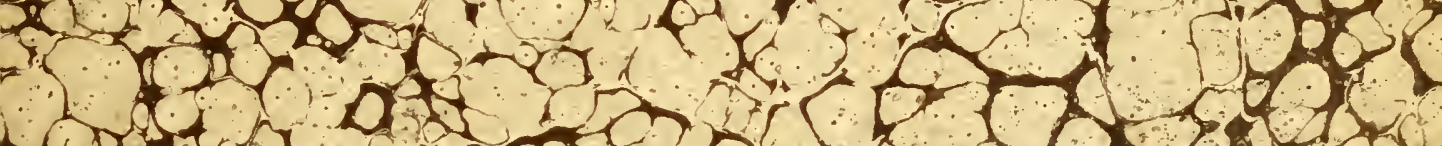

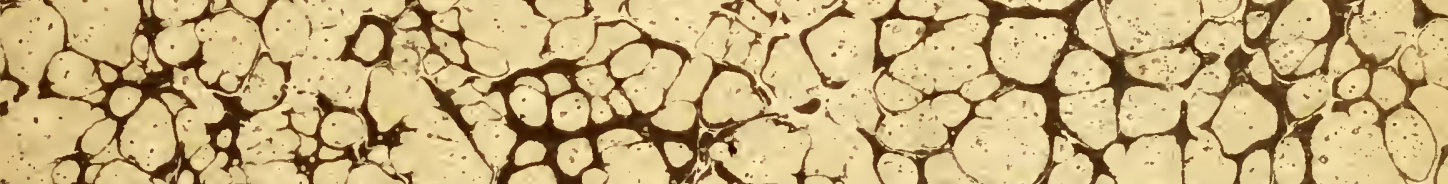

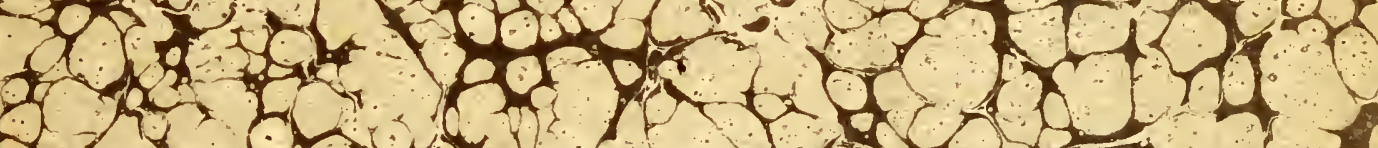

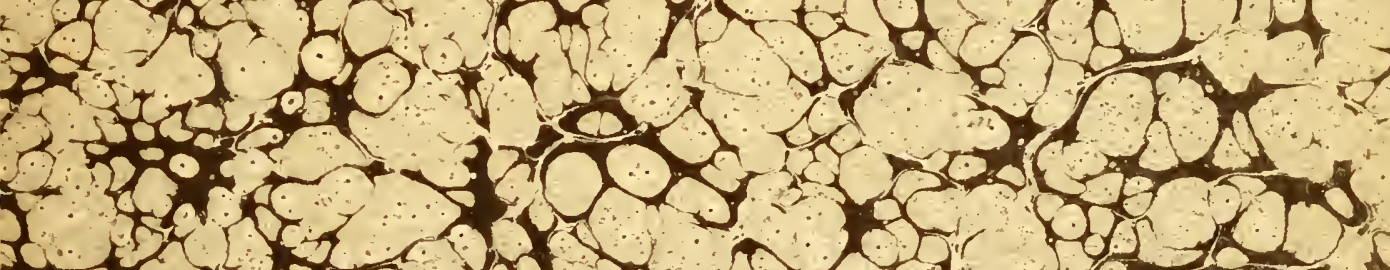
1. 2700

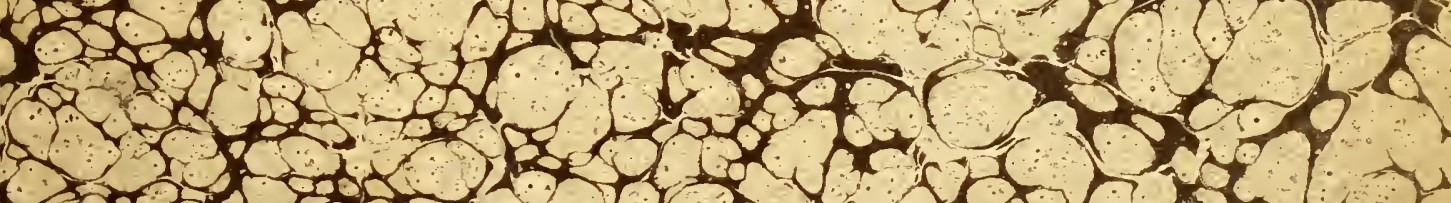

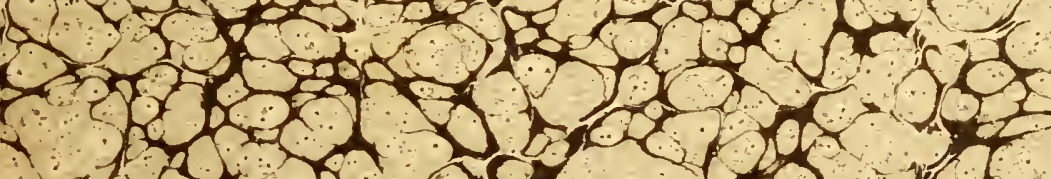

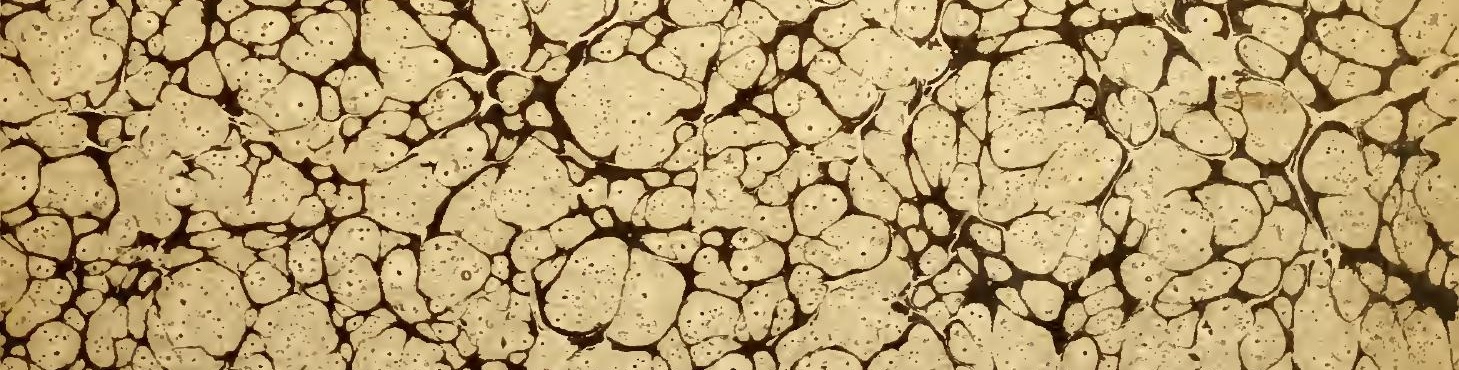

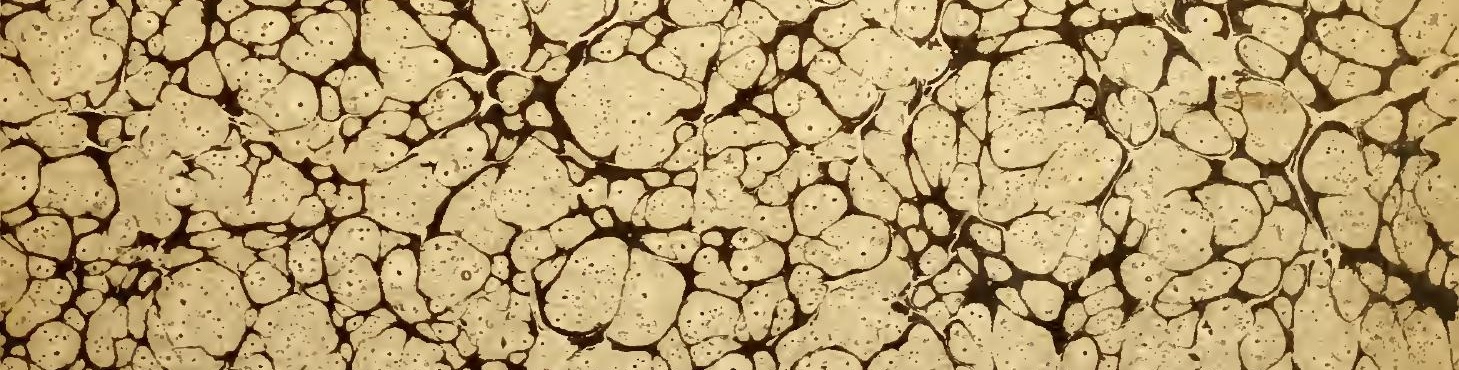

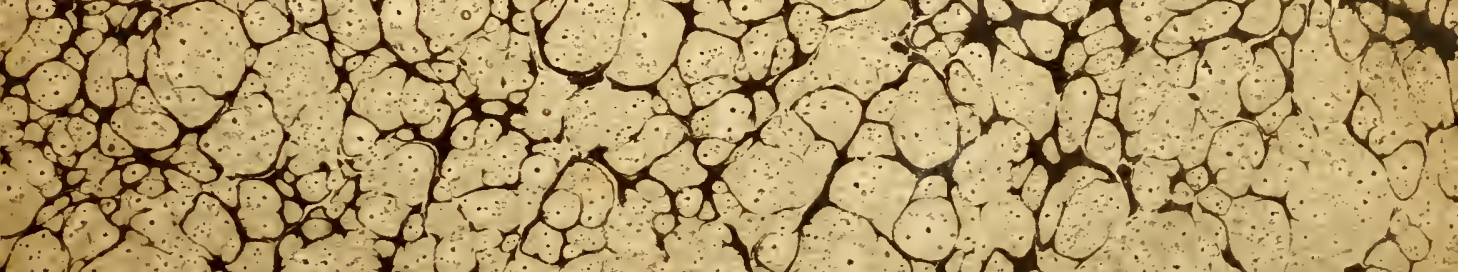

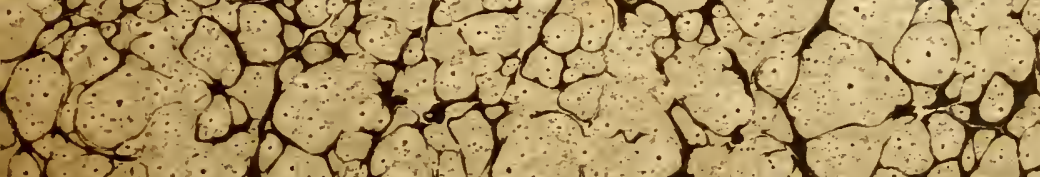



$2 \quad 15167^{3}$

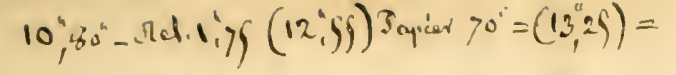





$$
10,
$$




\section{INDEX FUNGORUM,}

SISTENS

\section{ICONES ET SPECININA SICCA NUPERIS TEUPORIBUS EDITA; ADJECTIS SYNONYMIS}

\section{HERMANN HOFFMANN, \\ BOTANICES PROF, P, O. GISSENSI.}

INDICIS MYCOLOGICI EDITIO AUCTA.

\section{LIPSIAE,}

SUMPTIBUS A. FÖRSTNERI, BIBLIOPOLAE.

(A. FELIX.)

1863. 


$$
\begin{aligned}
& 4^{2}+603 \\
& \cdot \ln 3 \\
& \log 3
\end{aligned}
$$




\section{EIN LEI'T U N G.}

Da seit dem grossen, fiir seine Zeit abschliessenden Systema mycologicum von E. FrIEs (1S21-1S29 kein neneres systematisch geordnetes Hauptwerk, welches die ganze Pilzkunde unfasste, veröffentlicht worden ist, so erscheint es als ein dringendes liedürfniss, bis zur Publication eines solchen die grosse Masse der Pilze wenigstens vorlïufig alphabetiseh zu ordnen und auf diese Weise zugänglich zu machen. Frues' Epicrisis 1536-1835) unfasst nur die bis dahin erschienenen $\mathrm{Hy}_{\text {- }}$ menonyceten; desselben Verfassers Summa vegetab. Scandinaviae (1849) bezieht sich in der Aufählung der Species ausschliesslich auf Sehweden und giebt, wie die vorige Schrift, fast keine Citate von Herbarien, welche ein so wichtiges Hülfsmittel fiir diese Studien geworden sind. Dasselbe gilt von 'S'Trennz' Nomenclator (1862) und in noch höherem Grade von Fisies' Monographia Hymenomycetùm. I. (1557). BERKELEY Outlines of brit. fungology (1569) und Ribennorst's Deutsctulands Fry-

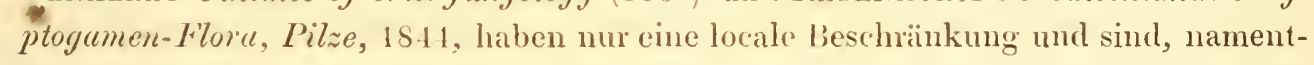
lich ersteres, bezïglich der Citate unvollständig. In Uebrigen aber sind wir auf Monographien beschränkt, welche nur einen kleinen Theil der Mykologie umfassen und ron sehr ungleichen Werthe sind. - Vorliegender lndex sehliesst sieh demnach der '/eitfolge der P'ublicationen nach dem Systema mycologicum von F'ries an, wobei ich jedoch ansdrücklich bemerke, dass die ebengenannten anderen Werke, namentlich die Epicrisis, R.вixinorst u. s. w, als im Besitze eines jeden Mykologen befindlich vorausgesetzt, 1 . h. die dort enthaltenen Citate und Synonyme der Regel nach hier nicht noch einmal abgedruckt sind. Dieser Index ist bestimmt, diese Werke zu ergänzen, nicht sie zu ersetzen.

Ich habe eine Zeit lang geschwankt, ob ich nicht all e seit Fries und (für Deutschland) R.ıBexiorst publicirten neuen species aufinehmen sollte, auch die sehr grosse Zahl derjenigen, welche nur durch Diagnosen, nicht aber durch Abbildungen oder Specimina sicca bekannt gemacht worden sind. In der That ist eine Uebersicht dieser über die Maassen zerstreuten Literatur ein dringendes und nicht länger abzuweisendes Desiderat, und schon als Vorarbeit für ein zuküuftiges $S y$ stema mycologicum ganz unentbehrlich. A ber das vorliegende Werk wäre dadurch noch manches Jahr hinausgeschoben worden, und so möge es denn auch in dieser anspruchsloseren Form nach Kräften nützen und fördern. Es würde alsdann aueh statt der ganz äusserlichen und zufälligen alphabetischen Anordnung die systematische geboten gewesen sein; zu einer solehen Arbeit aber fiihle ich mich, in Betracht der jetzigen Bewegung in der Mykologie, keineswegs berufen. Ueberdiess hätte cliess wieder ein alphabetisches und Synonymenregister bedingt, womit eine, 
bedeutende Vergrösserung des Linfanges rerbuuden war. Wo soll man dam auch cin knde finden! lilosse systematische Anffiihnung der Namen (mit den Citaten) würle Wenigen genügrt hiben; zu den nenen Arten allein die 1)iagnosen hinzufiigen, wäre eine störende Halbheit; sie iibcrall geben, führt natürlich zu einem vollständigen systemu. worïher ich nich schon ansgesprochen habe. Es geht eben hier, wie üherall, das Erreichbare bleiht stets weit hinter den zn Wünschenden zurück, und ohme eine gewise lesignation ist an einen Abschluss nicht zu denken. Ich meines Theils finde, dass die Wissenschaft wic der IIorizont ist; je weiter man vorangeht, desto mehr sicht nan dic Endlosigkeit, man lernt sich endlich beschränken, man muss einem Andem z̈berlasien, was man nicht selbst thm will oder kann. Fnd ein soldher Wohlthäter wird anch fïr dic systentatische Pilzkunde eimmal wieder geboren werden. Fïrs Erste glanbe ich, dass Wohlwollende und billig Denkende mir fïr meinc nühsane und trockene Arbeit Dank wissen werden. So möge denn ein Sammehwerk in jenen sinne Solchcn überlassen bleiben, welche, nit genügender Musse ausgerïstet, in den grossen literarischen Centralpuncten leben und dort die vollständigen und reichen Ḧ̈̈lfsnittel erschöpfend ausnutzen können, deren viele mir nur im Fluge zu durchblättern vergömnt war. Ich glaube, rlass einem solchen Gelehrten rieser mein Index als eine branchbare Hülfe sich erweisen werde, und rechne einstweilen daranf, dass derselbe bis dahin allen Denjenigen, welche sich mit dem bestimnen von Pilzen beschäftigen, dienlich sein kam. - Lm indess auch lem system atischen Bedürfniss wenigstens cinigermassen in die IIände zu arbeiten, so weit diess bei einem Register dieser Art möglich ist, habe ich übcrall, wo es anging, auf FuIEs' Summa veg. Sc. verwiesen, als dem derzeit vollständigsten, ja einzigen Werke, welches das ganze System der P'llze gleichmässig durchgearbeitet, wenigstens bezüglich der Genera, darlegt; ein Werk, das ohnediess kein Mykologe entbehren kamn. Zugleich wurchen hierduch eine grosse Anzahl werthroller synonymischer Aufklärungen gewomnen, so dass ich die Mühe nicht für eine vergeudete halte, um so melır, als das diesem Buche beigegebene Register nur die Genera enthält, und man danach sehr Vieles, negen der veränderten Nomenclatur, kaum auffinden kamn. Ich habe nur noch hinzuzufügen, dass ich mich auf's Emsigste bemüht habe, dem vorliegenden Werke durch dic Sorgfalt in den Citaten, sowie durch den bedeutend vergrösserten Reichthum an benutzten Quellen - worïber ein Blick anf die folgende Lebersicht der citirten Hauptwerke im Vergleich zu meinem Index mycologicus in der Botanischen 'Leitung' 1560 genügenden Aufschluss giebt - müglichste Brauchbarkeit und Vollständigkeit zu verschaffen.

Der Verfasser. 


\title{
INDEX FUNGORUII.
}

\author{
EXPLICATIO NOMINUM ABBREVIATORUM.
}

Alger. = Exploration scientifique de l'Algérie. 1) $40-1512$. Bot. Mycologie par Montagne ete. 1) $15-1555$. livr. $1-17$.

Ann. Mag. n. h. = Annals and Magazine of natu-

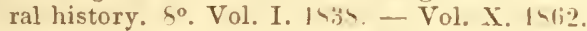

Ann. se. n. $=$ Annales des sciences naturelles, Botanique. Sér. I. XIII. IS24. - XXX 15333. sér. II. $1534 \mathrm{ff}$. Sér. III u. IV bis $156 \mathrm{j}^{2} \mathrm{XII}$

Act. 'Tur. = Mémoires de l'Académie de Turin. $1^{\text {to }}$. Sér. I. $1759 \mathrm{ff}$. bis 1557.

Afz. = Fries, E., Reliquiae Afzelianae; sist. ic. fung. in Guinea coll. Upsala 1>(j0. fol. t. 1-12.

Badluam $=$ C. D. Badham, a treatise of the esculent funguses of England. Loudon, Reeves. 1547. S०.

Bail S. = Bail, System der Pilze, 1 $\$ 5 \varsigma$. taf. ..

Berk. J. = MI. J. Berkeley, Introduction to cryptogamic Botany, $1>57$, \०. Holzschnitte im Text .

Berk. Out. = M. J. Berkeley, Outlines of british fungology. I.ondon. 1560. So. Namen auf den Tafeln ohne Autoren.)

Bisch. = Bischoff, Handbuch der bot. Terminologie, $1 \$ 30 \mathrm{ff}$. $4^{\text {to }}$. Separatabdruck : Kryptogamenkunde mit 30 Tafeln. 1660 meist Copien'.

Bivona $=1$. Stirpium rar. minusque cognitar. in Sicilia sponte proren. desc, ic. $3 \mathrm{Hefte}, \mathrm{Pa}$ normi 1515.

Bonite = Voyage autour du monde exécuté sur la Bonite. 1 $36-153 \%$. Botanique par Gaudichaud. I. $1>14-1516$. Text in so. citirt bis p. 204).

Bon. = Bonorden, Handbuch der Mykologie. 1551 Atlas in $4^{\text {to }}$. Fig. ..

Bon. Con. = Bonorden, Coniomycetes, in Abhandl. d. nat. Ges, in Halle. 1560. Y. 3. u. 1. $1^{\text {to }}$.

Borszezow $=$ Fungi ingrici. Beitr. z. Pflanzenkunde des russ. Reichs. Lief. 10. Petersb. 1 5ว Kais. Akad. der Wissensehaften.

Bot. Zt. = Botanische Zeitung ron Mohl und Schlechtendal. $1 \checkmark 43-1562$.

Brit. f. = Berkeley british fungi. - So $^{\circ}$ (specim. sicc.) fsc. $1(1836)$ - fsc. 4 (1843).

Bull. ac. belg. = Bulletins des séances de la classe des seiences de l'ac. roy. de Belgique. I. (1532) -1560 .

Canar. = Hist. nat. des iles Canaries p. Webb et Berthollet. Paris 1840 . To. III. $2^{\text {me }}$ partie. plt. cell. p. Montagne).

Carol. = Ravenel fungi caroliniani exsiccati. Charleston. J. Russel. $4^{\text {to }}$. fsc. 1. 1552. - 5. 1560.
Casp. Schimm. = Rob. Caspary, über Sehimmelpilze. Monatsber. Berlin. Akad. Mai. 1555. 50.

Chile = C1. Gay, Hist. politica e fisica de Chile. Paris et Chile $1<14-1$. Champ. p. Montagne. sect. Cryptog. no. ..

Coems. Pil. = E. Coemans, Monogr, du genre Pilobolus. Ac, r. Belg., mém. cour. et sav. étr. 1561. XXX. $4^{\text {to }}$.

Comm. it. = Commentario della societa eritto gamologica italiana. 5\%. n. 1 ( 1561$)-4$ (563). Genova, tipi de' sordo-muti.

Cord. Anl. = Corda, Anleitung zum Studium der Mykologie. Prag, 1 $\$ 2$. >o. Taf... Fach... Fig... C'd. Ie. = Corda, Icones fung. tom. IV. taf. 1. 2. V. VI.

de Bary Ir. = de Bary, Brandpilze. 1553. 5o.

Demid. $=$ Demidoff, Voyage clans la Russie méridionale. Paris. 1542. Bot. crypt. par Léveillé. Atlas in Fol.

Dsm. = Desmazières, Plts. erypt. de France. Exsiec. Edit. 2. $1536-1551$. fse. 1-37. - Ed. 3. $15.33-1560$. Lille. $4^{\text {to }}$.

Dub. Hyst. = Duby, Mém. sur la tribu des Hysterinées. Vol. XVI. des mém. de la Soe, d. Phys, et d'hist. nat. de Genève. 1561. $4^{\text {to. }}$.)

Fl. dan. = Flora danica. $1519-1561$. vol. . taf. ..

Flora = Flora, od. regensburger botan. Zeitung. $1<29-1<61$.

Fres. = G. Fresenius, Beiträge zur Mykologie. $4^{\text {to }} .155(1-63$. Frankf, bei Brönner. taf. $1-13$. Fig. ..

Fres. Ent. = G. Fresenius, Ueber die Pilzgattung Entomoplithora. Abhdl. Senckenb. natf. Ges. Frankfurt. H. II. 15.5\%. taf. 9. $4^{\text {tn }}$.

Fries $\mathrm{Sr}$. = E. Fries, Sreriges ätliga och giftiga Sranpar, utgifna af K. Vetensk. Akad. Stockholm, 1560. t. 1-1\%. u. t. 2\%-14.

Fuck. rh. = L. Fuckel Fungi rhenani exsiccati. no. $1-500$. $4^{\text {to }}$. 1663. Östrich i. Pheingau beim Verf.

Fuck. Nass. = L. Fuckel, Enumeratio Fungorum Nassoviae. Ser, 1. 1561. 5o. Jahrb. Ver, f. Naturk. v. Nassau. F. XV. p. 1. Fig. ..

Fuck.s. = L. Fuekel, Auswahl getrockneter Pilze nach dieser Enumeratio geordnet.

Gaudich. voy. = de Freyeinet, Voyage autour du monde. 151 \%-1520. Botanique par Gaudichaud. Planches in fol. 1926. taf. ..

Iledw. = Hedwigia, von Rabenhorst. $1552 \mathrm{ff}$. taf. .. Fig. .. I. II. no. $1-13$. (1563). 
Hoffm. Ic. = II. Hoffmann, Icones analyticae fungorum. (iiessen. $4^{t 0}$. Heft $1-31<t i 1-1<t i 3$.

Hook. I.ond. J. $=11$. J. Hooker, I,ondon Journal of Botany. I. 1\$12 bis VI. 1417.

Honk. J. $=$ Hooker's Journ, and Kiew-Garden Miscellany, I-IX. 1 1 $5 \bar{i}$.

IIrz. = Ilarzer, Naturgetr. Abb. der - Pilze. 1>12. fol. taf.

IIuss. = II' T. J. Inssey, Illustrations of british mycology. $f^{10}$. Iondon. I. 15 17 ; II. 1555.

Ital. = Erbario crittogamico italiano, ed. de Notaris, resati .. no. 1-gun incomplet.

Jungh. pr. = Junghuhn, Praemissa in floram eryptog. Javae insulae. fsc. 1. tab. 15. Extr. des 'Transactions de la soe. des sciences ct des arts

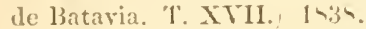

kl. = lilotzschii herb. viv. mycolog. Centuria IXX; cont. cura Riabenhnrst. no. .. $1^{\text {to }}$.

lil. II. = dto., editio nova 2 ; Centuria I-VIII. $145.5 \mathrm{ff}$. $120 . . .4^{\text {to }}$.

Kiops Fl. bat. = Kops, Flora batava, Amsterdam. rol. I. 1 $\backslash 4 \overline{7}-\mathrm{XI}$. 14.53. lith. Habitusbilder rihne Analysen.

Krypt. Bad. = Iirrptogamen Ijarlens, von Jack, Leiner u. Stitzenberger. Exsiccata. Constanz ap. Leiner, " $\%$. Citata ex Indice . no. 1-600.

Kühn, Krr. = J. Kühn, Krankheiten der Culturgewächse. Berlin. 1 45 s. S०.

Letell. = I cones fungorum; Figures des champignons serv, de suppl. aux planches de Bulliard. $4^{\text {to }}$ taf. 603-711). seconde édit. $1 \checkmark 39 \mathrm{ff}$. Paris, Meilhac.

Lib. = MI. A. Libert, Plantae eryptogamicae quas in Arduenna collegit. fase. 1. $\langle 430-4153 \pi$. - Leodii, Des. $4^{\circ}$.

Lindl. $\mathbf{~ . ~ K . ~ = ~ J . ~ L i n d l e y , ~ V e g e t a b l e ~ K i n g d o m . ~}$ London. 1553. (3. edit. . - o.

Linn. = Linnaea, ed. Schlechtendal, rol. 3 1529 $-31 \quad 1562$

Linn. Journ. = Journal of the proceedings of the Linnean Soc. Botany. vol. 114.56 , ff.

Linn. Trans. $=$ Transaction of the Iinnean Society. London. $1^{\text {to }}$. I $1791-$ XXXII 1459 .

Loud. = Loudon's Encyclopedia of plants. 14.5.5. >o. London. Abb. im Text, kleine ('opien, meist naeh Sowerby.

Micr. J. = Quarterly Journal of mieroscopical science. London. 50 . I 1554 - VIII I Lfill. Ser. II. rol. I 1561 .

Nat. hist. Rer. $=$ London natural history review. S०. $1 \backslash 6,1$

Norweg. = J. Chr. Sommerfelt, Plantarum crypt. Norwegiae Centuria I 1526 - II 1530 . ('hristiania. $4^{\circ}$.

Not. Sfer. = de Notaris, Sferiacei italici, Genora 15.63. Centuria 1. fig. $1-1000$.

Nov. Act. = Nova Acta Leopoldina. $f^{\text {to }}$. ab 1429 . rol. XI bis XXVIII $14 ; 1$
Pay. b. cr. = J. Paycr, Botanique cryptogamique. Paris. 14.51). 5०. Copien.

Thöb. Gift. = P. I'höbus, Deutschlands kryptog. Giftgewächse. 1536 . $4^{\text {to }}$.

P'urton. = An appendix to the midland flora Fngland, 2 parts. Jondon. 1521. vol. III. part. 1. 2. $8^{\circ}$.

Regè G. F. = E. Regel, (tartenflora. $1551-1862$.

R. f. = Rabenhorst, Fungi europaei. [Ser. II.] Centuria I $1559-$ TI 1563.

Robin. = Ch. Robin, Hist. n. d. végét, parasites. $1553.3 \%$

Roques $=$ Roques, Histoire des champignons. Paris. 1511. Atlas. gr. "”.

Sagra Cuba $=$ Ramon de la Sagra, Hist. pliys. Ile de C'nba. Bot.: Crypt. plts cellulaires par C. Montagne. Paris $1 \checkmark 3>-1512$. Abb. in Fol. Text in ${ }^{\circ}$.

Schacht Kart. = II. Schacht, Bericht über die Kartoffelpflanze u. ihre Krankheiten. Berlin, Wiegandt. $1456 \%$. $f^{\text {to }}$.

Schnzl. = A. Schnizlein, Iconogr. famil, plantar. $4^{\text {to }}$.

Schweiz. = Schweizerische liryptogamen, ges. u. herausg. von Wartmann und schenk. $1562 \mathrm{ff}$. no. $1-106$ 'citata ex Indiee. S.t. Gallen, typ. scheitlin. $>^{\circ}$.

Staude $=$ Fr. Staude, Die Schwämme Mitteldeutschlands, insbes. Coburgs. Coburg 155\%. Fol. 52 Fig. Meist Copien.

Sturm h. = Sturm, Deutschlands Flora. III. Pilze. Heft 1 1 $13 \mid-36 \quad 1562$.

S. = E. Fries, Summa vegetabilium Scandinaviae. 1449. $>$.

Tul. F. h. = L. R. et Ch. Tulasne, Fungi hypogaei. 1<51. Paris. fol.

Tul. Sel. = Iidem, selecta fungorum earpologia. I. 1561 . fol.

Ung. Ex. = Unger, Exantheme der Pflanzen. $15.33 .<0$

Tent. $=\dot{A}$. Fenturi, Studi micologici. Brescia 1542. Kil.-Fol. - Habitusbilder ron Agaricis etc.

Tent. mi. = Tenturi, miceti del' agro Bresciano.

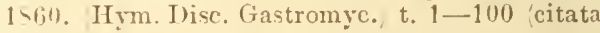
ex indice.

Vitt. $=(\therefore$. Vittadini, Monographia tuberacearum. Iediolani, typ. Rusconi. 1531. $4^{\text {lo }} .5$ Taf.

Viv. = Dom. Viviani, i funghi d'Italia. Genora 1-34. fol. t. $1-60$.

Voges. = Mougeot, Nestler, Schimper: Stirpes rogeso-rhenanae. Bruyerii Tog. $4^{\circ}$. 1410-1s(ill. fse. $1-15$.

West. = Westendorp u. Wallays, Herbier eryptogamique belge. fsc. 1 | $5.511-24$ 15.5. Courtrai, Jaspin. $4^{\circ}$. citata ex indice operis, . 




\section{INDEX FUNGORUM,}

Acalyptospora nervisequa. - Castagno pl. Marseill. 14t5. t. 11.

Acantlomyces aculeata. - Zeitschr. f. wissensch. Zoologie. 15.55. IX, t. 16. f. 6 ff. Lebert, Achitonium. - [S. 451.]

- acicola. - [S. $4 \$$ ].

Achlya cf. Saprolegnia. - S. 491.]

Achorion Sclıönleinii Remak, ef. Mycoderma Favi Gruby. - Robin. v. p. t. 3. f. 6. 11. 13. t. 13. f. 3 .

Achroomyces, - Bot. Zt. 1553. t. 3. f. 21-23. (Ricss.)

- tumidus. - Bon. t. 11. f. 231.

Acinula. - [S. 47.]

- candicans Fr. [S. 47\%.] ef. Sclerotium vestitum Ns.

- Clarus Fr. - ef. Selerot. Clav.

- profusa Fr. - [S. $47 \%$.]

A cionium. - [S. $151 * * * 452$.]

A c la di u m conspersum. - [S. 492.] - Bon. t. 1. f. 101. - cf. Bisch. f. 3759 .

- curvatum. - Bon. t. 4. f. 95.

- heterosporum Wllr. - Fuck. rh. 122.

-_.- roseum. - Letell. 642. 4. (Aspergill.).

A e un osporium Cd. - S. 490.]

- botryoideum. - Cd. Anl. t. 13. f. 17: 7-9.

A cremonium. - [S. 494.]

- album Bon. - Bot. Zt. 1553, t. 7. f. 10.

Sturm. h. 25 - t. 9. Preuss.'

- alternatum Lk. - Bisch. f. 30 s1.

erectum. - Bon. t. t. f. 103.

-.. fuscum Schmidt. - Loud. f. 16569. - Cd.

Anl. t. B. f. $5: 3$. - Pay. b. cr. p. 72. f. 321 .

- spicatum. - Bon. t. 4. f. 104.

- Vaccinii. - Fuck. rh. 159.

- verticillatum Lk. - Bisch. f. $3 S_{02}$. - Will-

komm, Wunder d. Mikr. 1556. p. 91. f. e. Kl. $1.759^{\mathrm{b}}$.

Acrocylindrium copulatum.- Bon.t.6. f. 14 .

- elegans. - Bot. Zt. 1853. t. 7. f. 11. Bon.)

granulosum. - Bon. t. S. f. 172.

- minimum. - Bot. Zt. 1\$53. t. 7. f. 13. (Bon.)

Acrop h y tum tuberculatum. - Zeitschr. f. wiss.

Zool. 1555. IX. t. 17. f. 13-17. Lebert).

Acroscyphus. - [S. 399.]

Acrospeira mirabilis B. B. - Berk. I. p. 305. f. 69 . a. - R. f. 62 .

Acrospermum. - [S. 400.37\%.]

- compressum Td. - [S. 400.] Loud. f. $16306 .-$

K1. 73S. - Cd. Anl. t. F. f. $54: 21-22$. -

Bisch. f. 3406 . - Dsm. \$26. - Kl. II. 35. -

Fuck. s. 503. - Tul. Sel. I. 135.) - Brit.

f. 270. - Purton. I. t. 19. f. 3. - Lib. 32.
Acrosperm um cornutum Fr. - S. 400.] zu Agar. tuberosus Bull. (ef. Hedw. I. no. 14. [IV.] u. 15 ; Tul. Sel. I. 105 .

- conicum. - s. 401 .

-_ foliicolum. - Carol. II. 65.

-_- fructigenum. - cf. Monilia f. gracile. - Cd. Anl. t. F. f. 54:23.

graminum Lib. - S. 4110.] Dsm. 472. -

K1. 927. 1553. - Kl. II. T66. - Bail S. t. 11.Lib. 33. - Wstd. 1253.

- - monilioides? - Ung. Fx. t. 3. f. 20. - ef.

Monilia graminis.

— pyramidatum Fr. - Bisch. f. 3396.

- Kobergéanum. - Dsm. III. 51.

- sclerotioides Fr. = Typhula, Bail : Hedw. I. no. 14. 15 ; Bail Syst, 71.

Acrosphaeria. - [S. 35$]^{* *}$. $]$

- annulipes. - Cd. Anl. t. F. f. $56: 13-16$. collabens. - Cd. Anl. t. F. f. $56: 17$.

Acrosporium ef. Oidium.

- appendiculatum. - Flora 1529. t. 1. f. 2.

Unger .

- bulbigerum Ns. (ef. Torula).

Cerasi. - Verh. d. Ver. z. Beförd. d. Garten-

baues in Preussen. 1551. t. 1. B. (Braun, Casp., de $B$.

- monilioides. Ns. - Wstd. 299. - Loud.

f. 16576 . - Flora 1529. t. 1. f. 1.3. (Unger). -

$\mathrm{Zu}$ Erysiphe graminis, Tul. Sel. I. 212.

- tenue. - Bon. t. 4. f. 91.

Acrostalagmus. - [S. $490^{\text {****. }}$.

cinnabarinus. Cd. [S. 491.] $=$ Verticill. la. teritium Harting. - Lindl, v. K. p. 44. f. 28. Willkomm Wund. d. Mikr. 1S56. p. 92. f. a-e.

Fuck. s. 152. - André, ökon. Neuigk. 1847. t. 4. f. $17-20$ (Cord.). - : Bot. Zt. 1854 . t. 8 . A. d. Hoffm. - Kl. 1276. - R. f. \$4. Fuck. rh. 155. cylindrosporus, - Kl. 1350. geniculatus $\mathrm{Pr}$. - Kl. 12 is. herbarum Pr. - Kl. 1277. murinus Ces. - Kl. II. i4. olivaceus. - Cd. Anl. t. B. f. 17: 4-6.

- persistens. - Pay. b. cr. p. 74. f. 33\%.

Acrothamnium. - [S. 521.496**.]

violaceum. [S. 337 : 10.] - Letell. 635, 4. -

K1. $14 \$ 2$.

Acrotheca Gei F. - Fuck. s. 335.

Acrothecium. - [S. 492.]

multisporum. - Sturm.h. 29-.t.43. (Preuss.)

simplex B. B. - Ann. Mg. n. h. 1861. VII.

t. 16. f. 16 . p. 352 .

Actidium. [S. 401.] - Bail S.t. 18. 
Actidium Haenkei. - Cd. Anl. t. F. f. 59: $20-21$.

- hysterioides. [S. 401.] - Cd. Anl. t. F. f. $59: 15-19$.

- Fr. - Bisch. f. 3552 .

Actigea. - [S. 111.]

A ctinobotrys 'Tulasnei $=$ I'cronospora ganglioniformis. - Bot. Kt. 16.j(i. t. 5. f. 22 . - K1. II. 321 .

Actinocladium. - s. 164 . curtum. - S. flis. rhodosporum Ehrb. - Bisch. f. 3736 .

Actinocnemis ef. Montagnites.

Actinodermium. - [S. $443^{* *}$.

Actinonema. - S. 124. caulincola. - Wstd. 150.

— Crataegi Fr. - [S. 421.] - Voges. 564 . Fuck. s. 359.

— Padi DC. - S. 424. Robergei. - S. $424^{*}$.] - Wstd. 150. Rosae. [S. 424.] - Fuck. s. 360. ef. Asteroma radiosum.

— Uvariae I.év. - [S. $124^{*}$.

A c tin ospira cliartarum. - Cd. J. VI. 23.

A c tinotheciu m caricicola.-Kl.1976. - Hedw. I. t. 11. f. 3 .

Actinothyrium. [S. 420.]

Cytisi Fuck. - Bot. Zt. 1561. t. 10. 2. p. 249.

Praminis Kz. - [S. 420.] - Dsm. 1023. Wstd. 222. - Bisch. f. 35\$1. - Loud. f. 16itss. - Bail S. t. 15. - Pay. b. cr. p. 78. f. 369 . K1. 556. - Kl. 11. 54. - Voges. 65\%. - Iib. 165. - Ital. 853 .

Acurtis. [S. 337.]

A ecidiol um exanthematicum. - Ung. Ex. t. 3.

f. $17-19 .-$ Bisch. f. 3559 .

A ecid ium. [S. 510.]

abietinum A. S. - Bisch. f. 3552.

Abietis Fr. - [S. 510.]

Actaeae Op. - R. f. 155

Adoxae Op. - Wstd. 666. - Kl. 780. -

Kl. II. 371 .

albescens Grev. - Dsm. 830.

Allii ursini P. - Kl. $57 \mathrm{~S}$.

Alliorum P. - [S. 511.]

Amelanchieris D. - Dsm. 87\%.

Anemones P. - Wstd. 662. - FI. dan. XIII.

2217,1

Aquilegiae P. - Wstd. 744.

Ari. - Kl. 1175. (Cent. XIX.).

- virginici. - Carol. IV. 96.

Ariae D. - Dsm. 578 .

Aristolochiae. - Ital. 794.

aroidatum. - Carol. IV. 95.

Asparagi Lsch. - Kl. 1179.

Asperifoliacearum P. - [S.511.]. Wstd. 834. Asperifolii P. - Wstd. 85; 834. - Kl. II.

258. - Fuck. s. 13. - Fl. dan. XIII. 2219.1.-

Dsm. 665. - Ital. 896. - Fuck. rh. 274.

- aurantiacum. - R. f. 592. - Bon. Con.

p.208. t. 3. f. 35 (huc Caeoma Erythron. Lk. u.a.).

- australe. - Hook. Lond. J. II. 1843. t. 22.

f. 5. (Berk.).

Behenis DC. - Brit. f. 340 . - R. f. $486 .-$

Ital. 549 (? 349).

Berberidis Gmel. - [S. 511.] - Dsm. 136. -

Voges. 86. - Wstd. 22\%.

- - P. : Krypt. Bad. 415. - Bisch. f. $3 \$ 55$.

- Loud. f. 16676 . - de Bary Br. t. 5. f. 6. 7. cf. elongatum v. - Landw. Ann. d, mecklenb. patr. Ver, 1862, no, 2. (Kühn).
Aecidium Berheridis fructigenum Iasch. - Kl. $11 \%$.

bifrons. - Ung. Ex. t. 3. f. 17. t. 4. f. 21.

Galiorum. - K̈l. 1490.

Ranunculac. - Wstd. 744.

Leguminos. - Wstcl. 743.

Bunii DC. - Dsm. 1063. - Kl. 1496.

Isupleuri Kz. - Kl. 690. - Fuck. rh. 269.

Calystegiae Cast. - Dsm. 1371.

cancellatum P. - Voges. 154. - Wstd. 123.

- Bisch. f. $354 \%$ - Brit. f. 55 . - Brit. f. 263

(Dothidea collic.!

- candidum I3on. - R. f. 155. - Bon. Con.

t. 3. f. 36. p. 211 (= Acc. Phaseolor. Wilr.).

- IVstd. 125 .

- Caprifoliacearum DC. ef. Aec. Ionicerae.

[S. 511.$]$

- Cathartici Schum. - Fl. dan. XIII. 2218. 3.

Cichoracearum DC. - Wstd. \$33. - Kl. II.

91. - Fuck. s. 12. - Dsm. 1370; III. 12. -

Ital. $59 \varsigma$. - Fuck. rh. 272.

- v. Crepidis. - Kl. 696.

Cimicifugarum. - Carol. I. 94.

Circaeae. - Brit. f. 319. 342. - Kl. II.

372. - Ital. 659 .

- Clematidis DC. - Wstd. 664. - Dsm. 1005.

- Kl. 996. - Ital. 419.

columellatum Schum. - Fl. dan. XIII. 2219.

2. - Norweg. 176.

- columnare A. S. - Bisch. f. 3941. - Kl.

1593. - Ital. 46. 96.

- Compositarum. - [S. 510.] - Kk1. 1594. -

Carol. V. 97. - Wstd. 836. (Bellidis). - Krypt.

Bad. 344. - Fuck. rh. $270,271$.

- Doronici. - Kl. II. 691

— Mrt. f. Hieracii Schum. - K1. 756.

—- Hieracii f. Crepidis. - Kl. II. 369.

-_ Lapsanae. - K1. II. 370.

_-_. Rumicis. - Wstd. 325.

- Tussilaginis. - Brit. f. 322. - Fuck.

s. $11 . \mathrm{b}$.

Convallariae Schum. - [S. 511.] - Kl. 888.

a. b. c (Cent. XIV.). - Wstd. 835 .

f. Majanthemi et Paridis. - Kl. II. 88

Convolvulacearum. - Kl. 1492.-Ital. 47.97.

cornutum P. (cf. Roestelia c.). - [S.510.] -

Bisch. f. 3542 . - Fuck. s. 22. a. b. - Loud.

f. 16678 (unter 16673). - Dsm. 1117. - De-

Cand. Org. vég. 152 7 . II. t. 60. f. 5. (Hedwig f.).

- Voges. 183. - Wstd. 556. 742 (v. Sorbi).

1156 (Mespili).

- f. Amelanchieris. - Kl. 1993.

_ - . Mali cf. Roestelia penicillata.

f. Oxyacanthae ef. Aec. laceratum.

corruseans Fr. - [S. 510.]

- crassum P. - Voges, 89. - Brit. f. 110.

Ung. Ex, t. 4. f. 22. - Dsm. 832. - Biscl. f. 3562 , u. 3546 .

- Schum. - Fl. dan. XIII. 2215. 2. -

de Bary Br. t. 6. f. 1. - Carol. I. 92. - Letell. 620. 1. - Lib. 90. - Wstd. 557. 1155: Pedicularis. - Ital. 450 .

- f. Frangulae. - Kl. II. 192.

- Cruciferarum DC. - [S. 511.]

Cydoniae. - Dsm. 13 \%3.

elatinum A. S. - Voges, 255. - Bisch.

f. 3553 .

__ elegans. - Carol. II. \$s.

elongatum. - Bot. Zt. 1959. t. 11. f. 3

Keimung. Hoffm.). 


$$
1 .
$$



A ecidium elongatum f. Berberidis: Fuck. s. 14. b. - Fuck. rh. 275.

— f. Rhamni P. - Kl. 695. — Fuck. s,

14. a. - Fuck. rh. $27 \%$.

Epilobii DC. - R. f. 4 ऽ . - Dsm. III. 11. -

Brit. f. 317. - Wstd. 559. - Ital. 597.

Ervi W. - West. 1065.

Gmel: Dsm. 1004.

hirsuti. - Kl. 1459.

Euphorbiae. -- Dsm. 1004.

- P.: Biscli. f. 3550. - Fuck. s. 17. -

de Jary Br. t. 5. f. 1-5. - Kil. II. 292. -

J3rit. f. 229. - Wstd. 337. - Fuck. rh. 279.

Cyparissiae. - Voges. Si.

- - sylvaticae DC. - Ann. sc. n. 1517.

VII. t. †. f. $29-31 ; 15.5$. II. t. 9. f. $21-33$

"Tulsn..

- Euphorbiarum. - [S. 511.? - I)sm. III.

5.51. - Wstd. $\$ 3 \%$. - Krypt. Bad. 16. - Ital. 218.

- exanthematicum. - Bon. t. 1. f. 20.

- Falcariac 1'. - Wstd. 716. - Bisch. f. 3551.

- Fuck. s. 10. -- Sehntz1. t. 12. f. 7-10. -

K1. $11 i i ;=$ Aec. grandidentatum Bon.). -

Fuck, rh. 2tis.

—_ Fediac. - Ital. 597.

- olitoriae, - K1. 1792.

Foeniculi Cast. - Dsm. 10111.

Frangulacearum Schw. - [S. 511.$]$

Frangulae Schum, - F1. dan. XiII. 2:14.

2. - Kil. 276.783 .

- fuscum Sow, - Wstd. 541.

Galii 1'. - lil.691. - Fuck. s.15. - Fuck.

rh. $2(t)$.

- Galiorum P. - [S. 511.]

— Geranii DC. - [S. 511.] — Kil. 695. - lil.

II. 376 .

Glaucis, - Tijdschr. v. n. Gesch. 1S15. XII.

t. 6. f. I Doz. Mllk.

- grandidentatum Bon. Con. 209. t. 3. f. 37 .

(= Aec. 'Tussilag. Cd.).

- Grossulariae DC - [S. 511.] - Wstd. 745.

- Ism. 1006. - Loud. f. 16673. - Bon. t. 3. f. $6 i$.

- P. : Kil. 691. - de Bary Br. t. 5. f. \&.

t. 7. f. 1. - lil. II. 191.

- fructigenum. - Kl. 1794.

Hieracii Schum. - Fl. dan. XIII. 2215. 3.

Holböllii Horn. - Fl. dan. XIII. 2220.

hydnoideum. - Carol. IV. 91.

irregulare DC. - Wstd. 665.

Labiatarum DC. [S. 511.] - R. f. 593 (Salviae).

- laceratum DC. - Brit. f. 111. -- Carol. V.

96. - Bisch. f. 3\$15. - Fuck. s. 23. - Loud. f. $1667 \%$.

- Sow: Wstd. 556 .

— Crataegi Ariae DC. - Wstd. 1064.

dium).

Oxyacanthae. - Dsm. 137 Centri-

- Lampsanae. - Wstd. 747, 555.

— Leguminosarum Lk. - [S. 511.] - Fuck.

s. 15. b. - Dsm. 1372. - Fuck. rh. 266. v. Orobi tuberosi. - Kl. 1059.

- - verni. - Krypt. Bad. 416. Leontodontis. - Dsm. 666. - Wstd. StS.

leucospermum. - [S. j11.] - Voges. 155. -

ki. 1495. - Fuck. s. S. - Ki. II. 25\% - Brit.

f. 226. - Wstd. 662. - Krypt. Bad. 145.

- Ligustri. - Sturm. h. 34. t 10 (Strauss).

lineare. - Wstd. 91, 331 .

Lonicerae S-1. - Ital. 4S, 95. - Kl. 577.
A ecidium luminatum. - Carol. I. 91 (nitens Schw..

- Lycopsidis Dsv. - Wstd. $\$ 34$.

Lysimachiae S - 1. - R. f. 391.

Magellanicum. - Crypt. Bot. antarct. voy.

Erebus. 1 $\ 51$. t. 163, 2.

- Majanthae. - Wstd. \$35.

- Meleagris Dub. - Dsm. 16s.

- Mercurialis Schum. - Fl. dan. XIII. 2216. 2.

- Mespili. - Dsm. 1118. - Wstd. 1156.

Myrtilli schum. - F1. dan. XIII. 221!. 3.

nitens Schw. - cf. luminatum.

Nymphoidis DC. - Bisch. 3954.

Onagrariarum. - [S.511.]

Orchidearum D. - Dsm. 663. - K1.1690. -

IIstd. 560 .

- Orobi. - Wstd. 743.

-_- Oxyacanthae. - Wstd. 556.

- Paconiae. - Wstd. $4 \$ 4$.

- Parnassiae Grav. - Dsm. 665. - de Bary

Bir. t. 6. f. 1 .

- Rbh.: Kl. 1065.

- Pedicularis Libz. - Dsm. 1002. - Kl. 1193.

- Wstd. 1155.

- penicillatum A. S. - Wstd, 556.

_- I'ericlymeni Schum. — Letell. 620. 3 (Xy-

lost . - Fl. dan. XIII. 221s. 1. - Kl. II. 246. -

Wstd. 532 . - Fuck. rh. 276.

P'ersonatarum s. 1. - [S. 511 . ?

Phaseolorum Wllr. (candidum Bon.). - Kil.

s 5 i. - Kil. II. 373. - R. f. 1Ss. - Wstd. 1:1.

- Fuck. rh. 267.

_- Pini P. - ef. Periderm. [S.510.] - Bisch. 3949. - Loud. f. 16662 - Voges. 156. - Carol. I. 93. - Wstd. 660 .

- v. Ephedrae. - Carol. III. 95.

Plantaginis. - Ital. 217.

poculiforme Wllr. - elongatum Lk.

- Berberidis. - Wstd. 227.557: Rhamnor.

Polygonorum P. - [S. 511.]

Populi Schun. - [S. 512.

Prenanthis P. - Iil. 1178.

- Primulacearum DC. - [S. 511.] - cf. Aec.

Lysimachiae S-l.

- Primulae DC. - Brit. f. 341. - Dsm. 661.

pulchellum Schrad. - Wstd. 559.

- punctatum $l^{\prime}$. - [S. 511.] - Fl. dan. XIII.

2217. 2. - Fuck. rh. 262.

- quadrifidum DC. - Brit. f. 227. - Dsm.

169. - Letell. 620.4. - Wstd. 661.

- Ranunculacearum P. - [S. 511.] - Kl.

1494. - Wstd. S4. - Ital. 660. - Fuck. rh. $263-265$.

— - Aquilegiae. - K1. II. 791.

- Clematidis (petiolorum). - R. f. 254.

- Krypt. Bad. 114. - Wstd. 66t.

- Ficariae. - Dsm. 831. - Krypt. Bad.

323. - Kl. II. S9. - Fuck. s. 9.

- Lycoctoni. - R. f. 96.

Lycoctoni. - R. f. 96.
Ranunculi. - Krypt. Bad. 322.

- Thalictri. - Wstd. 485. - Fuck. s. 9. c.

Ranunculi. - Fl. 891. - Fuck. s. 9. a. -

Schum.: Fl: dan. XIII. 2216. 1.

Aquilegiae et 'Thalictri). - Kl. 7S5.

resinaecola Rud. - [S. 511.]

Rhamni P. - Dsm. 1007. - Iil. 290. -

Letell. 620. 2. - Wstd. 55S.

Thei. - Wstd. $32 \mathrm{~S}$.

rubellatum Lk. -- Rumicis S-1. - Kl. 7S2.

- Kl. II. 790 . 
Aecidium rubcllum DC. - [S. 511.] - Dsm. 6;67. - Loud. I6050. - Wstd. 325. - Ital. 300 Tumic.

- Rumicis, - Voges. 2s6. - Purton. I. t. 26. - Wstd. 324. - Ital. 300.

i.5.

Scillac. - Fuck, rh. 242.

Scrophulariae. - Lib. 192. - Wstd. 115\%.

Serophularinearum Lasch. - Fil. 461. 751. -

Kl. II. 3it. - ef. Uredo Ser.

Sedi ('es. - Iil. I115i.

Senceionis D. - Ism. 135.

sii Falcariae. - Wstd. ift.

Silenacearum. - S. 511 .

Sonchi. - Wstd. 1160. - KI. II. 2\9.

Statices. - Wstd. 1:65.

Synantherearum S-1. - S. \$10.

Thalictri. - of. Ranunc. - S. 511. -

Loud. 1665 ?.

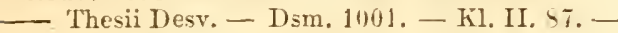
Brit. f. $31 \%$.

- Tragopogi P. - [S. 511.] - Voges. 365. -

Fl. dan. XIII. 2216. 3. - Krypt. Bad. 413. -

Wstd. 433 .

__ Trifolii repentis. - Ital. 216. - Kl. J659 u. 1931.

C'ast. : de Bary Br. t. 6. f. 2.

- Tussilaginis Cd. 'grandident. Bon.) - Loud. f. 16679 . - Cd. Anl. t. C. f. 26: 1-2. - Pay. b. cr. p. 70. f. 312 u. 314 . - Bon. t. 1. f. 25 . Kl. II. 799. - Voges. 390. 55. - Norweg. 175. - Wstd. 663. - Ital. 655.

- Umbelliferarum. - [S. 511.] - K]. II. 375.

_- urceolorum Schum. - Wstd. 750.

- Urticae DC. - Dsm. 131. - Wstd. S5.

- Schum.: Fl, dan. IIII. 2217. 3. -

R. f. 390 . - de Bary Br. t. 6. f. 3.t. T. f. 2. Fuck. s. 19. - Voges. 359. - Brit. f. 112. Fuck. rh. 251 .

is!.

_- Valerianacearum DC. - [S. 511.] - K1. 559.

- Fuck. rh. 273.

- Valerianae. - Kl. 1792.

255 .

erbasci. - Ital. 5 15. - K1. 1191. - R. f.

Viciae. - Wstd. 743.

Violac. - Brit. f. 228. - Fl, dan. XIII.

2215. 1. - Kil. II. 291. - Wstd. 225. - Fuck. rh. 275 .

- Violarum. - [S. 511.] - Wstd. 229. - Kl.

890. - Dsm. III. 555.

- Xylostei. - cf. Periclymeni.

- zonale. - Dsm. III. 13.

Aegerita. - [S. 47\%.] - cf. Tuburcinia.

caesia Fr. - Fuck. s. 221.

— candida P. - [S. 472 u. 47T.] - Bisch. f.

3653. - Fuck. s. 223. - Ann. Mg. n. h. 1559.

III. t. 9. f. 7 (Berk. et Br.). - kil. 1131. -

Kl. II. 241. - Fuck. rh. 163.

- crustacea. - Wstd. 300 .

- perpusilla. - Ann. sc. n. 1540. XIV. t. 1.

_- plagiospora. - Bon. t. 11. f. 221 .

- punctiformis. - [S. 497.3.]

A erophyton. - [S. $465.459 *$.]

- principis Eschw. - Bisch. f. 3791.

A thalium. - [S. 449.]

- Havum Grer. of. Aeth, vaporar. - Cd. Anl.

t. C. f. 31: 5. 6. - Schntzl. t. 15. f. 57. 59.

- melaenum Cher. - zu Licea cylindrica? -

(=Dermodium fallax Nis. f. 103 ; Wilr. n. 2114.).
Aethalium septicum P. - [S. 419.] - Bisch. f. 3631 .

Fr.: Zeitschr. f. wiss. Zool. 15.99. X. t. T. de Bary. - Fuck. S. 29s. a. b. - Keimung: Terhandl. zool. bot. Ges. Wien 1559. t. 1. f. II. Bail!.

- - L. v. flavum. - Kl. 1027.

_- v. cinnamom. - Dsin. 154.

- - v. raporariorum; cf. Aeth. rapor.

vaporariorum Bull. - [S. 419**.] - R.f. S1.

Agaricus. - [S. 2 72.$]$ - Typi subgenerum: cf.

Hoffm. Ic. an. fg. ; ef. Amanita. Entwickelung:

Pay. bot. cr. p.9. f. 47-19. - Bolbitius. Coprinus. - Cortinarius. - Gomphidius. Hygrophorus. - Lactarius. - Lentinus. - Lenzites. - Marasmius. - Montagnites. - Nyctalis. - Panus. -- Paxillus. - Pholiota Bail S. t. 36, - Russula. - Schizophyllun. - Stylobates. - Trogia. - Xerotus.

- Fructific. : Ann. sc, n. 1537. VIII. t. S-11. Léveillé).

abstrusus Fr. - [S. 292.]

acerbus Bull. - Vent. mi. 53. f. 6.7. -

Ital. 534. Trichol. - Vent. t. 5. f. 6s. 69.

- acerosus Fr. - [S. 25.5.]

- acervatus Fr. - - $\$$. 250.]

- acicola. - [S. 2s2.] - Linn. 1530. V. t. 6. f. 3 Jungh. .

- acris (Lact.) Bolt. - Loud. f. 15791. Schum. : Fl. dan. XIII. 226i, 2.

— Bolt. : Bisch. f. 3411. - Ann. sc. n.

1537. VIII. t. S. f. $i$ fruct. Lév. . - [S. 30\%.]

- actinorhizus. - [\$. 273. 36.] - Ann. sc. n.

1536. V. t. 12.

- acuminatus Schff. - [S. 297.]

- acutesquamosus. [S. 273.] - Huss. II. 5. -

Vent. mi. 55. f. 3. 4 .

acutus Schum. = mutabil. v. denudat. Fl. dan. - P.: [S. 306.]

_ adiposus B. - [S. 259.] - Fl. dan. XII. 207 . 1. - Hrz. t. 79. - Berk. Out. t. s. f. 2.

- Brit. f. 16.

_— adnatus Schum. - FI. dan. XII. 211\$. 2.

- Adonis. - $\$ .251$.

_-_ adscendens schum. - [S. 27S.]

- adustus Russ. - [S. 310.] - Loud. f.

15791. - Huss. I. 73.

- aeruginosus Curt. (Psall.) [S. 295.] - Loud.

f. $16019 .-$ Fops Fl. bat. XI. S15. 2. - Hrz.

t. 42. - Bail S. t. 35. - Bot. Zt. 1556. t. 5. f. 1. Pollinar. Hoffm.) - Dsm. III. 34 . IIuss. I. 35.

- aestivus Schum. = praecox Fl. dan.

—. aestuans Fr. - [S. 275.] - Letell. 699.

Aethiops Scop. - [S. 257.]

aetites. - [S. 25:.]

affricatus Fr. [S. 2\$3.]

Agardhii. - [S. 291.]

agathosmus. - [S. 307 .]

aggregatus Schff. - [\$. 27S.

aimatochelis. - Huss. I. 19; cf. hacmatochelis.

albellus Roq. t. 16. f. 1-3. - [S. 276.]

albescens. - Viv. 9.

albidus. - Wstd. 995. - Act. 'Tur. 1503.

XII. t. 11. Balbis.

- albo-brunneus Trich.). - [S. 2it.] - Loud.

f. 15754 . - Bot. Zt. 1556. t. 5. f. 3. Pollinar. Hoffin. est butyraceus, t. Fr. - Vir. 32. Vent. mi. i. f. $3-5$; 93. f. 1. 2. - Ital. 327.

_ albo-crenatus. - Linn. is30. V. t. 6. f. 4 ¡Jungh. . 


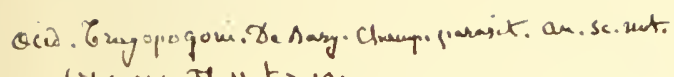
4. Sar. $x x \cdot 31 \cdot 11 \cdot t \cdot 7-10$. 
| 
Agaricus albo-cyaneus Dsm. - [S. 29.5.]

albo-fimbriatus. - [S. 275.]

albo-niger Russ. - Kr. : semicreınus.

albo-nitens Fr. [S, 561.]

albo-violaceus. - S. 302 .

- albuininosus. - Ilook. Lond. J. 1-17. VI.

t. 20 . f. 5 .

- albus Schff, Trich./.-[S. 2;6.] - Loud.

f. 157 1. - Kl. S05. - Berk. Out. t. 4. f. 6. P. ef. Ag. Columbetta.

alcalinus Fr. - [S. 2\$2.] - K1. 609.

aleuriatus Fr. - is. 293.]

algericus. - Alger. t. 31.1.

algidus Fr. - [S. 25.5.]

alliaceus Bull., Schun. = porreus Fl. dan. -

Muller, Pflanzenstaat. 2\%l. ; Spor. Jasid. 'Copic

nach Link . Purton. II. t. 11.

__ alneus I. = Schizophyllum comn.

alnicola Fr. - [S. 2!91.] - Kil. 21!.

alternatus Schum. - Fl, dan. XI. 196iJ. 1.

alumnus. - [S. 250.]

alutaceus Russ.) - S.311.] - Roq. t. 10.

f. 3. - Berk. Out, t. 13. f. S. - IIrz. t. 5!). -

Carol. 1I. 6. - Letell. 643 'var.). -- Staude

t. S. . . 5 .

- C'litopilus - Mail S. t. 37.

amabilis Ilook. - Lond. J. I-12. t. 1 I.

'Berk.'.

amadelphus. - [\$. 313.

- amarellus Lasch. - Kil. j0s.

amarus; cf. fascicularis. - S. 27\%.] - Vent.

mi. 30. f. 1. 2 .

- ambustus. S. 2\1.

- amethysteus. - Vent. mi. 25. f. I-6. -

Roq. t. 15. f. 3. - Staude t. 6. f. 2.

- amianthinus. - S. 273.31.]

_ amictus. - S. $2 \varsigma 2$.

- ammophilus, - Alger. t. 31. s.

- amplus P. - \$. 277.?

amurcus. - S. 301.]

- anapachus. - Letell. 613.

anatinus. - S. $25 \%$

Anatolii Lév. - Demirl. Russ. t. 1. f. 2.

- androsaceus Maras. L. - [S. 31\%] - Kil. II. 610.

Fr.: Dsin. 1250. - Bot.Zt. 1560. t. 14.

f. 13 Hoffm.! - R. f. 104. - Dsm. III. 317.-

Voges. 677. - Brit. f. 131. - Wstd. 1252. -

Vent. mi. 42. f. 2.

anfractus. - [S. 300.]

- anginaceus. - Linn. 1\$30. V. t. 6. f. 1.

(Jungh.).

- angulosus. - [S. 305.]

- angustissimus. - [S. 279.]

- angustus P. - [S. 257.]

anisatus P. - Roq. t. 15. f. 4. - Hrz. t. $5 \varsigma$.

- anomalus. [S. 303.] - Kl. 1506. - Berk.

Out. t. 12. f. 4 .

- v. incurrua. - Letell. 634 .

anthracophilus Lasch. - Kl. 110 S.

apalus. - [S. 293.]

apicreus Flamm. - Bail. S. t. 36.

apiculatus. - [S. 259.]

appendiculatus B. [S. 296.] - Dsm. 251 .

- Kl. 1703. - Berk. Out. t. 11. f. 3. 4.

- applanatus P. - [S. 294, 27S.] - Kl. Cent. VIII.

applicatus B. - [S. 2S5.? - Bisch. f. 3291. -

Lib. 19.

applicatus v. quercicola Lasch. - Kl. 1103.

aquatilis. - [S. 291.]
Agaricus aquilus. - [S. 297.]

aquosus. -- [S. 290.]

- arachuostreptus. - J.etell. 617.

- arbustivus. - [S. 307.]

- archyropus. - [S. 313. 10.]

- arcuatus. - [S. 276 .]

- arenarius. - Kl. 1310.

_ _ argematus. - [S. 309.]

- argentatus. - [S. 302.] - Ital. 535 Cortin. .

- argutus. - [S. 302.]

— argyraceus. - S. 274: 44;275.]

- aridus Fr. - [S. 272.17.$]$

- armeniacus Schff. - S. 301. 46; 302. 71 ;

305. - Loud. f. 15970. - Bot. Kt. 1456. t. 5.

f. 7 Pollinar. Hoffin. .

armillatus. - [\$. 304.302*].

_- aromaticus. - Roq. t. 16. f. 4. 5.

- arquatus. - [S. 310.

- Arrhenii. - [\$. 24?.

29211.

- arvensis Schff. - [S. 291.] - Kil. 317. -

3erk. Out. t. 10. f. 1. - Huss. I. 76. 77. -

Vent. mi. 4.4 .

_ arvinaceus. - [S. 301.]

— asemus collyb. - S. 279.] - Iil. 13112.

- Fr.: R. f. 4 .

- Schff: Fries Sv, t. 1.

- asper Amanita - [S. 272. 16.] - Loud.

f. $15739 .-$ Vent. mi. 1i3. f. 2. 3.

- asperellus I.eptonia - [S. 25i.] - Kl.

1504. - Ann. Mg. n. h. I\$35. I. t. 1. f. 21.

p. 101\%. Spor. Berk.!.

- aspideus. - [S. 369.?

- - aspratus. - C'arol. V. 4.

__ astragalinus. - [\$. 291.]

_ atomatus. - [S. 297 .

- atramentarius Fr. Coprin.). - Kl. 219.

- I.k. : Loud. f. 16041. - Bot. Zt. Is.j\$.

t. 5. f. 4. Anat. Bonord. - Badhain t. 10. 1.

- Huss. II. 14. - Vent, mi. 56. f. 1. 2.

- atratus. - [S. 2S1.]

- atrides. - [S. 2\5.]

- atripes. - [S. 2SH.]

- atro-albus Bolt. - [S. 2S2.] - Loud. f.

15551 .

atro-cinereus. - S. 275.]

atro-coeruleus. - [S. 255.] - Dsm. 1S03. -

Bot. Zt. 1S5 S. t. 6. f. 5. a. b. Anat. Bonord.).

- atro-cyaneus. - [S. 2S1.]

_ atro-marginatus. - [S. 251.]

- atro-rufus Schff.; cf. montanus P. - Kil.

1710. [S. 296.]

- atro-sanguineus. - Act. Tur. 1505. XIV.

t. 1. Cumino .

atro-tomentosus B. Panus, - Bisch.f. 3246.

- Hrz. t. 55. - Fuck. s. 1075.

- atro-virens P. - Kl. 307. (= A. brevipes

Bull. var. ; Ag. humilis P.).

- attenuatus. - Letell. 632.

— augustus. - [S. 294.] - Fries Sv. t. 39.

- aulaxinus. - Ann. se. n. 1S13. XX. t. 15.

f. 3 (Montg.).

- aurantiacus Roq. ; cf. caesareus.

P. : Loud. f. 15795 - [S. 309.]

aurantio-marginatus. - [S. 251.]

aurantius. - S. 274. 41.]

- auratus Russ.). - [S.311.] - Vent. mi.

62. f. 1. 2 .

- aureus Bull. - [S. $259 ; 255$.$] - Fl. dan.$

x. 1799. 
Ag aricus aureus Sow.: Loud. f. 15975. - Roq. t. 15 . f. 2 .

- Mat. : Kl. 213. - v. truncicola Iaselı: lil. 702. - (Ifygroph.) [S. 307.] - (Pholiot.

Huss. I. $11 ;-$ Viv. 2. Auricula. - [S. 277.] auripes Secr. = hyomalis Osb.

- aurivellus B. - [S. 259.] - Fl. dan. XII. 2074 . Kl. 19.

tus Fr.

- Aurora Batsch. - Wstd. 1390.

austcrus. - [S. 291.]

arenaceus. - S. 251.

azureus. - [S. 303.]

azymus. - [S. 291.]

Babingtonii Blox. Nolan.) - Ann. Mg. n. h.

15.51. XIII. t. 15. f. 1. p. 400. (B. B.'.

- badipes. - [s. 292.] - Bot. Zt. 155s. t. 5. f. 5. Bonord. Anat.).

balaninus. - [S. 2\$1.] - Mag. Zool. Bot.

1537. 1. t. 15. f. 2. p. j09. (Berk.) - Ann. Mg.

n. h. 1535. I. t. 4. f. 21. (Fruct. Berk.)

balaustinus. - [S. 305 .

balteatus. - S. 300 .

Batschianus, - [S. 256.]

Belliae. - [S. 2S4.] - Ann. Mg. n. l. 1811.

VI. t. 10. Berk. .

bellus. - [S. 279.]

betulinus, - Wstd. 145 .

biarmillatus. - Staude t. 10. f. $6 \sim$ S.

bifidus Bull.; cf. Russula furcata.

biformis. - [S. 304.]

bifrons. - [S. 296.]

birrus. - S. 289 .

bivelus. - [S. $303.302^{*}$.] ; cf. fulgens. -

Letell. 6s9.

— blandus Pollin. - Berk. J. p. 241. f. 60. b.

blattarius, - [S. 289.]

blennius. - [s. 309.] - Brit. f. 3. - Kl.

616. - Fl. dan. XI. 1961. 2. - Nov. Aet. 1 \$ 12 .

XIX. II. t. 56. f. 6-15 (Basid. Spor. Phöb./ -

Lact. Dsm. III. 346.

— bolaris. - [S. 302.] - Berk. Out. t. 19. f. 1. Boltoni P. - Loud. f. 16035.

bombycinus, - [S. 255.] - Vent. mi. 16.

f. 3. 4. - Berk. Out. t. 7. f. 1.

- Bongardi. - [S. 290.]

- borealis. - [S. 276.]

- bovinus. - [S. 304.]

- breripes Trichol.j - [S. 276.] - Bail S. t. 35 .

ef. Ag. atrorirens $\mathrm{P}$.

brevis. - [S. 561 .

- brumalis. - [S. 275.$]$

— brunneo-fulrus. - [S. 30 4.

- brunneo-villosus. - Linn. 1S30. V.t. 6. f.

(Jungh.).

- brunneus. - [S. 304.]

-_ bubalinus Schum. = impatiens Fl. dan.

bufonius. - [S. 2;5.]

— bulbiger A. S. - [S. 273. 11.] - Dietr.

Flor. bor. t. 373 .

- bulbosus. - [S. 304.] - Roq. t. 23. f. 1. 2. cf. Ag. phalloides).

—- Sow.: Loud. f. 1595\%. - Vent. mi. $\$ 9$.

f. 7. Cortinar.).

bullaceus Bull. - [S. 296.] - Bisch. f. 3421.

Bulliardi. - [S. 302 .

butyraceus Bull. - [S. 279.] - Fl. dan.

XIII. 2266.1 ; cf. albo-brunneus.
Agaric us byssisedus. - [S. 285**, 294.]

- cacabus. - [S. 27S.]

_ caelatus. - [S. 276.$]$

- caesareus. - Amanit.) Badham. t. 15. 1. -

Roq. t. 22.

—- Schff. : Bisch. f. 331 S. - Hrz. t. 80 .

- Scop.: Vent. t. 1. f. 3. t. - Cd. Anl.

t. II. f. $77:$ 16. 17. - Viv. 30. - Vent. mi. 1.

f. $1.2 ; 7$. f. 1.2 ; ค. f. 2 ; 15 . f. 1. 2 .

_ caesariatus. - [S. 291.]

— caespitosus Bolt. - Loud. f. 15911.

- calamistratus. - [S. 290.]

— ealathus. - [S. 2 \% 5.$]$

- calceatus, - S. 295 .

- caligatus. - Viv. 35.

- caliginosus. - Linn. 1530 . V. t. 6. f. 13.

Jungh.

callisteus. - [S. 302.]

callosus Fr. - [S. 296.] - Loud. f. $1602 \varsigma$.

calochrous. - [S. 300.] - Brit. f. 15. -

Berk. Out. t. 12. f. 3. - Letell. (5)1.

- calopus (Maras.). - [S. 313.] - Kl. II. 109.

- Lib. $31 \mathrm{~S}$.

_ camarophyllus Fr. (Clit.). - Loud. f. 15936.

camelinus Lasch. - Kil. 507.

- camerinus. - [S. 292.

- Campanella B. Omplal. - [S. 2S1.] - Kl.

401. - R. f. 105. - Carol. II. 2.

- campanulatus. - [S. 293. 297.] - Koops Fl.

bat. XI. \$20. 2. - Galera) Bail S. t. 36.

- - L.: Kl. 1109.

- campester L. - [S. 294.] - Huss. I. 90. -

Viv. $43-45$. - Vent. t. 5. f. $35-41$. - Hrz. t. 9. - Roq. t. 14. - Bisch. f. 3466 . - Loud.

f. 16013. - Fries Siv. t. 5. - Kiops Fl. bat. X. 735. - Berg, Charakteristik. 1560. t. 2. f. 12. - Schntzl. t. 16. f. 23. 21 (Spor. Basid.). Staude t. 6. f. 1. - Vent. mi. 2. f. 1. 2 u. S. f. $5-$ S. - t. 29. t. 43 . f. $5 ; 57 ; 69$ - Bot. Zt. 1556. t. 5. f. 4. 25-25. (Spor. Entwicklg. Hoffm.). - Kl. II. 102. - Berk. Out. t. 10. f. 2.3. - Iil. 105; Cent. VI. suppl. : var. ; 703. - Dsm. 817. - Pringsh. Jhrb. II. t. 32. f. 44. (Keimung. Hoffm.) - Letell. 659. - Badham. t. 4. 5. (cf. exquis.).

$\square-$ v. edulis Bull. - Hrz. t. 60.
- v. pratincola, - Kl. 1709.
- v. brevipes, longipes, torosus : Viv. 13.
v. radicatus, albus: Vir. 44.
v. fulvaster, costatus: Viv. 15.

f. 126. - cf. subdulcis.

_ camurus. - [S. 303.]

- canaliculatus Schum. - Fl, dan. XI. 1S44. 2. cancrinus. - [S. 257.]

candelaris. - [S. 305.]

candicans (Clitoc.). - [S. 277.] - Kl. 1303. - P. : Fl. dan. XII. 2021. 1.

candidus. - [S. 313.] - (Maras.)

Candollianus P. - [S. 296.] - Kl. 405.

- Fr.: Biscb. f. 3432 . - Ann. se. n.

1537. VIII. t. 9. f. 15. (Fructif. Léveillé). canescens. - Lctell. 655. (mollis Sow.). caninus. - [S. 303 ; ib. no. 100.]

canobrumneus. - [S. 296.]

- cantharellus L. ; cf. Canthar. cibar. - Wstd. 340 .

- capcratus. - [S. 299.] - Fl. dan. X. 1675. -

(Cortin.) Bot. Zt. 15js. t. 5. f. 13. (Anat. Bonord.).

- caperatus v. albus. - Letell. 664 (pudic.). 



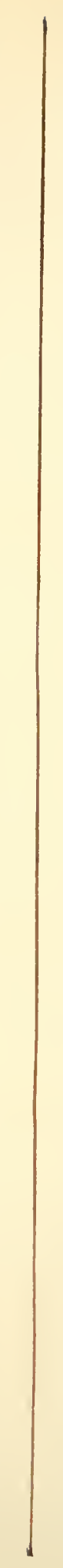


Agaricus capillaris Schum. - [S. 2S3.] - Fl. dan. X. t. 1670. 1. u. XII. 2142.1. - Fuck. s. 1114. - Carol. III. 1.

— capnoides. - S. 295.]

- caprinus Scop. - [S. 307.]

- caput Medusae. - [S. 295.]

- carbonarius Fr. - [S. 291.] - Kil. 303.

carcharias. - [S. 2-3. 34.

- cáriosus (Aman.). - Bot. Zt. 15.j5. t. 6 .

f. I. g. Anat. Bonord.

carneo-albus. - Carol. I. 2.

carneo-tomentosus. - Bot. Zt. 1550. 1. 5.

f. 29. 31. Entwicklg. Hoffm., - cf. Panus torulosus.

- carneo-virens. - Linn. V. 1530. t. 6. f. 2. 'Jungh.'.

— carncus. - [S. 276.] - Brit. f. \&.

- carnosus. - Loud. f. 15749.

- carpophilus. - [S. 293.

_- cartilagineus. - [S. 564.]

- caryophyllatus. - Viv. 3.

- cascus. - [S. 296.

- castaneo-rufus. - cf. subdulcis.

- castaneus Bull. - [S. 305.] - Bisch. f. 3465. catinus. - [S. 2is.]

caudatus. - [S. 29\%.] - Kirypt. Bad. 15\%.

caudicinus. - cf. mutabilis.

caulicinalis Sow. - Huss. I. 65. - Loud.

f. $1590 \%$.

causticus. - [S. 301*.

- cauticinalis (Collyb.) Bull. - [S. 250*.] -

R. f. $10 \%$.

- cauticinus $\mathrm{Sw}$ - - [S. 313: 26.]

- Ceciliae. - J3erk. Out. t. 3. f. 5.

centrifugus. - [S. 300.]

- centunculus. - [S. 292.]

- cepaeoides. - Kl. 1705 .

- cepaestipes (Lepiota' Fr. - [S. 273.] - Fl.

dan. X. 1795. - Lindl. v. Ii. p. 29. f. 5.

- Sow.: Loud. f. 16011. - Sturm. h.

31 -. t. 1. (Sz1.). - Kl. 1904. - Ital. 437.

ceraceus Sow. - Loud. f. $15 \$ 10$. Kl.

1102. - (Hygroph.) [S. 30S.]

- ceraseus. - Fl. dan. X. 1677. 2.

cerasinus, - [S. $30 \% *$. ]

cerealis (Trichol.). - Kl. 1301.

cerinus P. - [S. 276.] R. f. 402 .

- b. flavus. - Kl. 1903.

cernuus. - [S. 296.]

cerodes. - [S. 292.]

cerussatus Fr. - [S. 27\%.] - Fl. dan. X.

1796.

- cervinus. - [S. 256, 275.] - Hoffm. : Bisch.

f. 3268 .

- Schff.: Kl. 211.

Cesatii (Crepidot.) - Kl. 1506.

cetratus. - [S. 25S.]

chalybeus P. - [S. 257.] - Loud. f. 15946.

- Ann. IIg. n. h. 1S3S. I. t. 4.f. 24. (Spor.

Berk.). - (Lepton.) Bail S. t. 37.

- chamaeleontinus (Russ.). - [S. 311.]

chelidonius. - [S. 25.3.]

chioneus. - [S. 294*.] - cf. septicus.

chloranthus. - [S. 281.]

chlorophanus (Hygroph.) Fr. - [S. 308.] -

Hoffm. Ic. t. 5 .

- chloroticus. - Jinn. 1830. V. t. 7. f. 1. (Jungh.).

- chordalis Fr. - [S. 313.] - R. f. 103. -

kl. 1210. (Maras.).

Christinae. - [S. 292.

chrysenteron. - [S. 276.]
A garicus chrysites. - [S. 275. 77.]

- chrysodon. - [S. 30\%.]

chrysoleucus. - [S. 253.]

—- chrysophaeus. - Berk. Out. t. 7. f. 5.

- chrysophyllus. - [S. 2S3.]

- chrysorhoeus (Lact. . - [S. 310.]

— cidaris. - [S. 292.]

- cilicioides. - [S.30S.] - (I act. . - Kil. 60j.

- cimicarius P. - Kl. 1205.

- cimmerius. - [S. 2\$2.]

- cinerascens. - Viv. 20. - Vent. mi. 37.

f. $1-3$.

cinereo-violaceus. - [\$. 302.]

cinereus Fr. (Coprin.). - Loud. f. 16044.

Roq. t. 21. f. 2.3.

- cingulatus Ahnf. - [S. 274. 45.] - Linn.

1\$30. V. t. 10. Fries!.

- cinnabarinus. - [S. 302.]

- cinnamomeus Schum. = lateritius Fl, dan. -

Cortin. v. rubicundus. - Kl. II. 403. - (Dermocybe) R. f. 201. - S. 303.] - Letell. 652. Huss. I. 30.

—_ cinu.) v. semisanguineus. - Letell. $61 \$$.

- circellatus (Lactar.) - [S. 309.]

- circinatus Sclium. = rubescens Fl. dan. -

SS. 290 : $555 ; 254$.

cirrhatus Schum. - [S. 250.] - Kl. 1111. -

'Tul. Sel. I. 112. )

$60 \%$.

- citrinus. - Vent. mi. 13. f. 1. 2. - Roq.

t. 23 . f. 3. 4 .

cladophyllus Myceu.). - Ann. sc. n. 1543.

XIX. t. i. f. 1. Lév.!

- clandestinus. - [S. 25S.]

claricolor. - [S. 300.]

- clavicularis. - S. 2S3.]

clavipes (Trichol.). - [S. 277.] - Bot. Zt.

IS55. t. 6. f. 7. Anat. Bonord.).

clavularis. - [S. 2\$3.]

clavus Batsch. - [S. 2\$3 : dilat., 290. 250.]

$-\longrightarrow$ = Fibula Fl. dan.

_- Bull. : Loud. f. 15870.

cliduchus. - S. 300.$]$

- clivensis B. B. (Psilocybe). - Ann. Mg. n.

h. 1 S61. VII. t. 14. f. 3. p. 376 .

- clivularum. - Letell. 676.

— clusilis. - S. 2SI.]

- clypeatus Bolt. = semiputris Fl. dan. - Berk.

Out. t. 7.f. 6. - [S, 2S7.]

- clypeolarius Bull. - [S. 273.] - Fl. dan. X.

1732. 1. - (Lepiot.) Loud. f. 15742. - Bisch.

f. $32 \%$ - Kl. II. 406. - Carol. V. 2. - Vent.

mi. 51. f. $3-5$.

- Cnista. - [S. 276.]

_ coalescens. - Viv. 16.

_- coccineus Sow. = strobilinus Fl, dan. - $\{\mathrm{Hy}$ groph.) Kops Fl. bat. XI. S10. 1.

- P.: Loud. f. 15\$13. - Nov. Act. " $\$ \$ 42$.

XIX. II. t. 56. f. 16-21 (Phöb.).

Schff.: Kl. 315. - Hygr. [S. 30S.] -

Dsin. 1624. - Huss. I. 61. - R. f. 505. -

Krypt. Bad. 60.

cochleatus P. - Loud. f. 15916. - (Lentin.).

f. 4 . Fr.: Bisch. f. 3252 . - Berk. Out.t. 19.

P.: K1. 706.

coelestinus. - [S. 2S8.]

coerulescens. - [S. 300.] -- Vent. mi. 53.

f. 10. - Alger. t. 30.1. (Lact.).

- coeruleus (Russ.). - Vent. t. 7. f.61.62. -

Staude t. 9.f. 4. (Russ.). - Coffeae. - Viv. 11. 
Agaricus coffeatus. - [S. 277.]

- cuhaerens. - S. 242.

collariatus, - [S. 2\2.

collinitus Sow. - $\therefore .301 .1$ - Vir. 45. -

Ioud. f. 15!)\}. - IIr\%. t. 29. - Cortinar.

kil. I $111, \ldots$ Ital, Is

(ollinus. - S. 241).

colossus, - S. 275

colubrimus 1'. = clypeolarius Fl. dan.

- columbetta. - S. $2 \%$. - I.etell. 62\%. /al-

bus $\mathrm{l}$. .

_ colus. - \$. 3015.

— colyubadinus. - \$. 303.

comatus. -- Badliam t. 10.2. - Kops Fl. bat.

VIII. 591. - Coprin. I.k. : Loud. f. 16039.

- Kil. 21

Miill. : ] Irz. t. 21.

- - v. ovatus. - Nov. Act. 1842. XIX. II.

t. iti. f. lit. Iamell. : T'höb.'

comitialis. - S. $27 \%$.

comosus. - S. 2\??.

- compressus With. - Lond. f. 15551.

- comptulus 13. 13. - 5. 294. 275.] - Ann.

Ig. n. h. I-fil. VII. t. 14. f. 4. p. 376 .

Concha IIoffin. v. hercyn. subterr. t. 17. f. 3. concinmus Bolt. = stipatus Fl, dan.

confluens 1. Collyb. .- [S. 250.] - Kil. 12.

- kl. II. 2112 . - Brit. f. 124.

_ confragosus. - S. 259. ?

- congener Schum. = clegans Fl. dan.

- conglobatus Vitt. - Vent. t. 4. f. 2S. 29.

congregatus Sow. Coprin. - - Sturm. h.

19 -. t. 2. Corda.

Fr. : Loud. f. $160+2$.

conicus Hygroph. - - \$. 305.] - Kops Fl.

bat. XI. $\$ 10.2$. - ib. $\$ 45$.

_- Scop. : Hrz. t. 2s. B.

_- Schff. : Loud. f. 15\$11. - Vent. mi.

-3. f. 3. 4 .

conicus Schff. Panaeol. - Kl. 407.

- Schff.: v. nigrescens Fr.: Kl. 507. -

Vent. mi. 90. f. $1-4$.

— conigenus Fr. Coll. $)$ - S. 250.] - Fuck.

s. 1126 .

HH. : Bot. Zt. 1556. t. 5. f. 13. = = tenacellus Fr. . Keimung'; - Pringsh. Jhrb. II. t. 32. f. 45.

_ conissans Flammula,. - [S. 291.] - Kl.

1706.

- comnatus Schum. - Fl. dan. XI. 1905.

- conocephalus (Coprinar.]. - [S. 293.] - Kl. 1901 .

- conopilus, - [S. 296.] - Hoffm. Ic. t. 7. f. 2.

(Pratell.).

- conquisitus. - Alger. t. 30. 5. Omphal.).

- consobrinus (Russ. - - [S. 311.]

conspersus. - [S. 293.]

- constrictus. - [S. 274. 46.]

- contiguus Bull. - Vent. mi. 99. f. 3-5.

- - controversus. - S. 309.] - (Lact.) Nov. Act.

1 42. XIX. 11. t. 56. f. 2-5. (Spor. Phöb.).

- I.: Vent. t. 6. f. 49.50 - (Gal.)

Loud. f. $15755 .-$ Viv. 56. - Vent. mi. 77. -

Fries Sv. t. 29.

- coprinoides Cd. - Bisch. f. 3303.

- coprophilus (Psiloc.). - [S. 296.] - Bail

S. t. 35 .

- coracinus. - [S. 2S0.]

coriaceus 13. - cf. Irpex canescens.

Bull. : = Lenzites betulina. - Wstd. 145.

coriipellis. - [S. 254.]

comucopiae. - of. dimidiatus, - Viv. 7 .
Agaricus cornueopioides. - Purton. 1. t. 12.

- corrosus. - 'S. 300 .]

corrugis. - S. 296.]

corruscaus. - S. 301.?

-n corticalis Bull. $=\Lambda \mathrm{g}$. Myc.' hyemalis Osb. corticatus. - [S. 2 †.

corticola P. - s. 253.] - Sturm. h. 31 -.

t. 2 Szl. - ? 13ull.: I,oud. f. 15599.

- Myc.) Fr. : Kl. Jos.
- v. b., Fr. $=$ Ag. hyemalis Osb.

- coryphacus. - [S. 274. 54.] - Vent. mi. 36.

f. 1.2 .

__ ('ossus. - \$. 307.' - Brit. f. 2.

- costatus. - S. $25 \%$.

- cothurnatus. - S. 295.

- cotoneus. - S. 303.

- craspedius. - [S. 294.]

- crassus. - [S. 300 .

- craterellus. - Alger. t. 31.5.

_ craticius. - [S. 302.564.$]$

_ cremor. - S. 309.] - Lact.

- crenulatus Schum. - [S. 281.] - Fl. dan.

XIII. 2265. 1.

- cretaceus. - SS. 294.] - Berk. Out. t. 10.

f. 5. - Fries Sv. t. 39.

- crinitus Schwz. = Lentinus Lecomtei Fr.

crispus 'Turp. - Canthar. Dutroch., Tul.

sel. I. 100.

- cristatus (Lep.). - [S. 273.] - Brit. f. 1. -

Huss. 1. 45. - Loud. f. 15743 .

- Bolt. : Hrz. t. 44. B. - Berk. Out. t. 3.

f. 7. - Kl. 502 .

crobolus, - [S. 293.]

- crocatus. - [S. 252.]

- croceo-coeruleus. - [S. 301.]

_ croceo-lamellatus. - Letell. 665. mollis :).

- croceo-sanguineus. - Chile. n. 7. f. 4.

croceus, - [S. 303.]

cruentus. - [S. 282.]

crustuliniformis. - Vent. mi. 13. f. 6.7.-

Staude. 1557 . t. 2. f. 4. - Berk. t. 9. f. 1. -

Vent. mi. S9. f. 5. 6. - Staude t. 4. f. 2.

- cryptarum. - Letell. 611.

- crystallinus. - [S. 301.]

— cucullatus. - Fl. dan. X. 1675. 2.

- Cucumis P. - [S. 292.] — Loud. f. 15969.

- cudon. - [S. 256.]

- cumatilis. - [S. 301.$]$

-_ cuneifolius. - [S. 275.]

- cupressiformis. - Hook. J. VIII. t. 5. f. 3.

_ curtipes Schum. = blennius Fl. dan. [S. 27\%.]

- curtus. - [S. 300.]

- curvipes. - [S. 259.]

- cyanellus. - [S. 257.]

[ cyanescens Russ... - [S.311*.] - Sturm.

h. 33 .t. 1. (Strauss.).

- cyaneus. - [S. 300. 31; 302. 78.]

- cyarites, - [S. 302.]

- cyanopus. - [S. 300.]

- cyanoxanthus (Russ.). - [S. 311.]

cyathiformis Bull. - [S. 278.] - Loud. f.

15912. - Hrz. t. 65. - Kl. 21\%. - Kl. II. 201.

- Hoffm. Ic. t. 3. - Huss. II. 1. - Lib. 118. -

Schweiz. 25.

— v. aithopus Fr. - Bisch. f. 3271 .

Cyathula. - [S. 309.] - Lact.

cyclophilus Lasch. - Kl. 309.

cyphellaeformis. - [S. 2S5.] - Mag. Zool.

Bot. 1837. I. t. 15. f. 3. p. 511. (Berk.).

- cypriacus. - [S. 305.]

danascenus. - [S. 305.] 



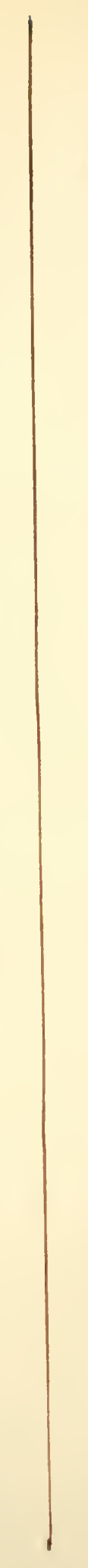


Agaricus dealbatus Fr. (Clit.). - [S. 27i.] Loud. f. 15525.

- Sow. : K1. 1304. - Huss. I. 39 ; II. 36. debilis. - [S. 2\$2.]

decastes. - [S. $27 \%$. $]$

decipiens. - cf. Irpex fusco-violaceus. - [S.

305.] - Letell. 694. - Hoffm. Ic. t. 9. f. 1.

(Cortinar. .

- decolorans. - [S. 301.] - Russ. [S. 311.

- decoloratus. - [S. 301.]

decoratus. - [S. 2\$9.?

decorus. - [S. 244.]

decumbens. - [\$. 30.3.

decussatus. - [S. 291.]

deglubens. - S. 290.

dehiscens. - Viv. 49.

rlelibutus. - [S. 302.]

delicatus. - [S. 273. 40.]

- deliciosus L. Lact.). - [S. 309.] - Loud.

f. 15797 . - Vent. t. 6. f. 55. 56. t. 13. f. 122 .

123. - Hrz. t. 10. - K1. 6. - Fries Sv. t. 6. -

K1. II. 110. - Badham, t. 6. 2. - Huss. I. 67.

- Viv. 13. - Vent. mi. 72, f. 5—; s0. f. 1.

2. - Staude t. 7. f. $1-3$.

- delicus Russ.). - [S. 310.] - Vent. t. 9.

f. 79.50 .

- deliquescens $\mathrm{Kl}$. - cf. Coprinus extinctor. -

(Psathyrella. Bail S. t. 35. - Hrz.t. 37 fuscsc.)

- demissus. - [S. 279.] - Hoffn. Ic. t. 15.

f. 1. Clitoc.

dentatus. - [S. 311.4.] - F1. dan. X. 1735. 2.

- denticulatus Schum. - FI. dan. XIII. 2269. 1.

- depallens Russ.). - [S. 311.] — K1. 1711.

- depexus. - [S. 303.]

- depluens. - [S. 294.] - Hoffm. Ic. t. 15. f. 2.

- depressus. - [S. 306.$]$

- descissus. - [S. 291.]

- destrictus. - [S. 291.

- detonsus. - [S. 306.]

- diabolicus. - [S. 303.]

diatretus. - [S. 279.]

dibaphus, - [S. 300 .

dichrous. - [S. 257.]

difformis. - [S. 279.]

diffractus. - [S. 290.]

- digitaliformis Bull. - Sturm. h. 19. t. 3.

(Cd.). - Coprin.) Bail S. t. 35.

dilatatus. - [S. 243.]

- dilutus. - [S. 305. 302, 72.]

- dimidiatus. - Letell. 695 Cornucopiae P.,

ostreatus Fr.).

- discoideus. - [S. $30 \%$.]

-_ discopus, - Anm. sc. n. 1S41. XVI. t. 14.f.4.

(Lév.).

- dispar. - [S. 250.] - Ann. sc. n. 1534. II.

t. 4. f. 3. Mont.).

dispersus. - [S. 295.]

- disseminatus P. - [S. 297.] - Dsm. 1620.

- Krypt. Bad. 340. - Fl. dan. XI. 1849. -

Loud. f. 16035. - (Psathrll.) P.: K1. 402, 403.

- Ki. II. $40 \%$. - R. f. 110 . - Ital. $22 \mathrm{~s}$.

- dissiliens. - [S. 2S2.]

- distans (Hygr.). - Berk. Out. t. 13. f. 1.

- distortus. - [S. 279.]

- ditopus. - [S. 279.]

- dolabratus. - [S. 305.]

- domesticus. - Huss. II. 19. - Kl. 1704.

- dothiophorus. - [S. 564.]

- dryinus P. - [S. 2St.] - Huss. II. 29. -

(Pleurot.) Huss. II. 33. - Loud. f. 15917.

- dryophilus Bull. - [S. 2SO.] -Fl. dan. XII.

2019. 1 u. 2. - R. f. 204 . - Krypt. Bad. 440.

Hoffmann, Index Fungorum.
Agaricus dryophilus Sow.: Loud. f. $15 \$ 60$. Berk. Out. t. 5. f. 7. - Badham t. 5. 2. - Huss. I. 39 .

dulcamarus A. S. (Hebel.). - R. f. 404.

Dunalii (Lentin). - Berk. Out. t. 15. f. 2.

duracinus. - [S. 305.]

durus. - [S. 25s.]

dycmogalus. - Ann. sc. n. 1837. VIII. t. 10.

f. 25. (Fructif. Léveillé).

eburneus Bull. [S.307.] K1. 209; Cent. VII.

Suppl. - Kl. II. 402. - Fl. dan. XI. 1907. 2. -

Hrz. t. 62. - Loud. f. 15750.

Berk. Out. Dsm. 1218. - Bail S. t. 34. -

Berk. Out. t. 15. f. 1. - Viv. 17.

echinatus. - [S. 294. 295.]

echinipes. - [S. 293.]

- echinulatus. - Hook. Lond. J. 1842. I.

t. 14. (Berk.).

ectypus. - [S. 279.

edulis. - cf. Ag. campester.

effocatellus. - Viv, 18.

elasticus. - K1. II. 405.

elatior. - [S. 301.] - Act. Turin. 1805.

XIV.t. 1. (Cumino).

elatus. - S. 290.]

elegans Fr. - [S. 281.] - Fl. dan. XII.

2024. 2. - Fuck. s. 1120. (Myc.).

elegantior. - [S. 301.]

elephantinus Russ.).- [S. 310.]

elixus. - [S.277.] - Lind1. v. K. p. 33.f. 1.

(Basid.).

- elodes Fr. (Cortin.). - [S. 2S6.] - K1. 1112.

elotus. - S. 300.]

elytroides. - [S. 564.$]$

embolus. - [S. 294.]

- emeticus Russ.). - [S. 311.] - Staude t. 8.

f. 1-3. - Loud. f. 15779. - Roq. t. 11. -

K1. 902, - Huss. I. 41.

- emollitus. - [S. 301 *.]

- emunctus. - [S. 302.]

- enchymosus. - [S. 292.]

ephebeus. - [S. 2S6.]

- ephemerus Fr. - Carol. V. 11. (Coprin.). -

Fl. dan. XI. 1960. 1. - (Copr.) P.: Loud. f.

$1604 \%$ - Nov. Act. 1842 . XIX. II. t. 56. f. $72-$

7S. Spor. Phöb.).

- epibryus. - [S. 294.]

—- Epichysium. - [S. 564. 253.]

- epiphloeus. - [S. 564.]

- epiphyllus. - [S. 314.] K1. II. 611. - Fl.

dan. X. 1676.1.

- Pers.: Loud. f. 15878. - (Maras.)

Bot. Zt. 1S60. t. 14. f. 12. (Hoffm.) - Kl. II.

611. - Krypt. Bad. 43S. - Dsm. 913. - Brit.

f. 11 . W Wtd. 556 .

epipoleus. - [S. 302.]

epipterygius Scop. - [S. 2S3.] - (Myc.) Fl.

dan. XII. 2075. 2. u. XII. 2139. 2. - Bot. Zt.

1560. t. 14. f. 9. (Hoffm.). - R. f. 401. -

Loud. f. $15596 .-$ Kl. 903 ; Cent. XV., Suppl.

- K1. II. 607. - Dsm. III. 665.

- epixanthus. - [S. 295.]

- epixylon. - Bull. ac. belg. 1\$39. VI.1. p. 34.

t. 4. (Histologie. Ch. Morren).

- equestris (Trichol.). - [S. 27!. 53.] - Loud.

f. 15762 . Berk. Out. t. 4. f. 2.

- erebius. - [S. 285.]

- ericaeus P. - [S. 296.] - Loud. f. 16026.

- ericetorum. - [S. 278.] - Fi. dan. X. 16:2.1.

- Loud. f. 15906.

- ericeus Bull. = virgineus $\mathbf{F l}$. dan.

Erinaceus. - [S. 293.] 
Agaricus eriocephalus Bull. - Kl. 205.

- ermineus. - [S. 273.]

- erosus. - [S. 2S1.]

— erubescens. - [S. 307.] - Letell. 055 .

- erugatus. - [S. 305.]

Eryngii DC. - Letell. 693. - Vent. t. 4.

f. 26.27 . - Fent. mi. 65 . f. $1-3$.

erythrinus. - [S. 305.]

- erythrocephalus. - Ann. se. n. 1\$41. XVI.

t. 14. f. 3. (Lév.).

erythropus. - [S. 313.] Maras.

- escharoides Fr. Naucor. . - [S. 293.] - Kl.

304.

- esculentus Wulf. - [S. 2S0.] - Bisch. f.

3270. - Loud. f. 15567. - Collyb.) K1. 204.

- euchrous. - [S. $25 \%$.]

- eumorphus. - [S. $303 *$.]

— euosmus. - Huss. I. 75. Pleurot.).

— evernius Fr. - [S. 304.] - Loud. f. 159.55.

- excelsus. - [S. 272.14.] - Berk. Out. t. 3.

f. 3. - Letell. 640. - Tent. mi. 35. f. 1-3. excissus. - [S. 276.]

- excisus. - [S. 252.]

- excoriatus Schff. - [S. 273.] - Vent. t. 3.

f. 24. 25. - Kops Fl. bat. X. 725. 2. - Loud.

f. 15741. - Cd. Anl. t. H. f. $77: 15.19 .-$

Létell. 610. - Viv. 49. - Vent. mi. 19. f. 6. 7:

- exilis. - [S. 255.]

- expallens. - [S. 275.$]$

— exquisitus. - Badham. t. 6. 1. zu Ag. campester.).

- exsculptus. - [S. 250.]

- exsertus. - Viv. 46.

- exsuccus. - Viv. 56. - Vent. mi. 100.

f. $3-5$.

fabaceus. - Carol. III. 3.

fagetorum. - [S. 252.]

fagineus Schum. = A. Schumacheri Fl. dan.

fallax (Russ.). - [S. 310, must., 311.] -

K1. 1603 .

fasciatus. - [S. 306.]

- fascicularis. - [S. 295.] - Huss. II. 15. -

Fl. dan. XII. 2075. - Loud. f. 16023. - Bisch.

f. 3471. - Viv. 47. - Staude t. 5. f. 5. - Ital.

227. - Kops Fl. bat. XI. S24. 2. - Roq. t. 15.

f. 1. - Berk. t. 11. f. 1. - Hrz. t. 33. - Bot.

Zt. 1856 . t. 5. f. 19. g. Pollinar. Hoffm., -

K1. 21 . Kl. II. 3.

fascinans. - [S. 309.] - (Lact.)

fastibilis. - S. 290.] - Dsm. 1\$01. -

Staude. 1552. t. 4. f. 3. - Loud. f. 1595 ว. -

(Hebel.) Bail S. t. 36. - Bot. Zt. 1555. t. 5.

f. 6. (Anat. Bonord.). - Kl. 409. 713.

f. 4 .

Astigiatus. - S. 290.] - Berk. Out. t. 8.

- fatuus. - [S. 297 .

favillaris. $-[S .2 ; \%$. $]$

felleus Fr. (Russ.). - [S. 311.] - Kl. 705.

401 .

fertilis (Entolom.). - Bail S. t. 3\%. Kl.

- y. intybaceus. - Ann. Mg. n. h. 1535.

I. t. 4. f. 22. 23. (Fructif. Berk.).

festivus. - [S. 292.]

fibrillosus. - [S. 297 .]

fibrosus. - S. $290 * * *$.

Fíbula Bull. - S. 294.] - Fl, dan, XIII,

2265. 1. - Loud. f. 15903 . - Dsm. III. 667. -

(et Swartzii) Brit. f. 13.

ficoides. - S. 305. 21.]

- filopes Bull. - [S. 252.] - F1. dan. XII.

2022. 2. - Bot. Zt. 18566. t. 5. f. 6. (Pollinar.

Hoffm.). - Mycena) R. f. 3 .
Agaricus fimbriatus Bolt. (Clit.). - [S. 294.] Loud. f. 15529 .

fimetarius. - Hoffm. Ic. t. 9. f. 2. (Coprin.). fimicola Fr. (Panaeol.) - - S. 297.\} - Kl. 410.

- P.: R. f. 10 . firmus. - [5. 290.]

- flabelliformis Bolt. -- Loud. f. 15923. -

Lentin. Fuck. s. 1034 .

flabellus. - S. 304.

flaccidus sow. Clitc. . - [S. 27s. 283.

$306 *$.$] - Loud. f. 15516$ ! u. 15974. - Kl.

210.

- Schff. : Loud. f. 15956. - Brit. f. 4.

Hammans. - S. 259.]

flavicans. - S. 299.

- flavidus Schff. Flamm. - - [S. 291.] - Kl.

503.

flavipes Schum. = epipterygius Fl. dan.

flaro-albus. - S. 2S1.

- flaro-brunneus 'Trichol.'. - [S. 274.] - Kl.

1602. - Letell. 707 .

flavo-virens A. S. - Hrz. t. 22. - Kl, 3.

Harus Lasch. - Kl. 1903.

flexipes P. (Telamon.). - [S. 304.] - Kl.

1107.

Hexuosus. - [S. 309.] (Lact.). - Hrz. t. 43.

- Huss. I. 59. - Ann. Mg. n. h. 1535. I. t. 4.

f. 17. Fructif. Berk.

- Schum. = rhodopolius Fl. dan.

floccifer B. B. Flamm.) - Ann. Mg. n. h.

1561. VII. t. $14: 1$. p. 374 .

floccipes. - S. 250 .

- floridulus. - S. 250.

- flosculosus. - Hook. Lond. J. I. 1942. t. 14.

(Berk.) - Crypt. Bot. antarct. voy. Erebus.

1554. t. 162. 2. Coprin.).

- fluxilis. - S. 2>5.]

- focalis. - S. 273. 42 .

- foeniculaceus. - S. 313.]

foenisecii. - [S. 296.] - Huss. I. 39. -

Berk. Out. t. 11, f, 5 .

F1. dan. XII. 2139.1. - Bisch. f. 3263.

- foetens Fr. Russ. - - [S. 311.] - K1. 1513.

- Loud. f. 157 -2. - Hrz. t. 24. - Cd. Anl.

t. H. f. $77: 20-24$. - Vir. 41. - Vent. mi.

45. f. 4. 5 ; 92 : f. 4. - Fries Sv. t. 40 .

- foetidus, - [S. 313.] Maras. . - Loud. f.

15576. Collyb.).

formosus. - [S. $25 \%$.]

- fornicatus. - S. 305.

fracidus. - S. 274. 52.

- fragilis Russ. . - S. 311.] - Stu.h.14.t.53.

- fragrans Sow. - [S. 279.] - Loud. f. 15910.

- Clit. Hoffm. Ic. t. 3. - Bail s. t. 39. -

Dsm. 161\%. - Letell. 655.

— Friesii (Lepiota. - Ki. 1501.

- frumentaceus. - S. 275.]

- frustulentus. - [S. 294.]

- fucatus Fr. Trichol.). - [S. 274: 5S.] -

Kil. 1105 .

_- fulgens. - [S. 301.] - Letell. 646. (bive-

lus $\beta$ Fr.i:

- fuliginosus: - [S.310:] (Lact.) - Roq. t. 20.

f. $2 .-$ Hrz. t. 19 .

- fulmineus. - [S. 301.]

fulvescens, - [S. 305.]

- fulvo-albicans. - Roq. t. 21. f. 1.

fulrus $S$ chum. = raginatus Fl. dan.

fumosus P. - [S. $27 \%$. - Letell. 669. -

Hrz. t. 23. - (Clitoc.) Kl.:302. 


Agaricus furcatus Fr. _- (Russ.) Bail S. t. 33. - S.311.' - Fl. dan.XI.1909. 1. - Roq.t. 12. f. 2. - Phöb. Gitt. t. t. f. 1.2. - Vir.6u. (c. var. heterophylla).

— furfuraceu; P. - [S. 293.] - Loud. f. 15996.

__ furnaceus. - Letell. 653.

furrus. [S. 27 , ]

fuicellus. - Kl. 1905.

- fuscescens Schff. Hrz. t. 37. (sec. Rbh.).

Linn. 1-311. V. t. 1i. f. 9. (Jungh.).

- fuscipes v. rubiginoso-maculatus Fr. - Fl. dan. XIII, 2:ti.3. ‥

— fusco-purpureus P. - [S. 313.] Maras.!. -

Bi.ch. f. 3202. - Loud. f. 15864 - (Clitoc.)

Kl. 5119. - Brit. f. 12i, 124.

fuscus Schum. I stiparus Fl. dan.

fusipes Bull. - - 2 . 2iy. - Berk, Out. t. 5. f. i. - Loud. f. 154 i. - Fl. dan. t. $160 \overline{\text {. }}$ (Fr. Ep.). - Hoffin. Ic. t. 4. - cum Sclerotio: Ann. sc. n. 1 th 3. XX. t. i.. f. 1. (Lév.). - Bot. Zt. 1 .59. t. 11. f. 32 . Keimg. Hoffm.). - Huss. II. 49 ,

— fusus. - [S. 291.]

galericulatus (Myc.) Scop. - [S. 252*.] Vent. mi. 57. f. 3. - t. 11. - Bot. Zt. $1 \leq 611$. t. 14. f. 10. Keimg. Hoffm.' - Bisch. f. 3242 . - Vent. t. S. f. T6. - Loud. f. 15553. - Kl. $610 .-$ R. f. ว. - Dsm. III. 343 .

- gallinaceus. - S. 27 .

- galopus. - 'S. 2-3.] - Berk. Out. t. 6. f. 2. - gambosus. - ${ }^{2}$. 2 6.] - Berk. Out. t. 4.

f. 5. - Fries Sr. Ł. 9. Pomonae Lz. Krbh. . gausapatus. - 275.

Gavanus (Amanit.). - Chile n. 7. f. 9. gentilis. - [S. 304.] - Brit. f. 135. geophyllus Sow. - [S. 291.295**.] - Loud.

f. 15993 . - Nor. Act. 1S42. NIX. II. t. 56.

f. $4^{6}-60$. Anat. Phöb. - Bot. Zt. 1556. t. 5 .

f. \. (Pollinar. Hoffm.! - Kl. 615. - Dsm.

III. 4.55. 459.

—_ Georgii Sow. - (Clusii Huss. I, \$3. - Trichol. grareols.).

- Sow. : Loud. f. 16014.

geotropus. - S. 275.]

germanus, - S. 305 .

gibberosus. - [S. 294.]

gibbus.

v. infundibuliformis Fr. - K1. 10.

- giganteus Clit. - - S. 27S.] - Loud. f.

15514. - Huss. I. 79.

- minor. - Letell. $6 \$ 2$.

gilvo-brunneus. - Linn. $1 \$ 30$. V. t. 6. f. 12.

(Jungh.).

— gilvus P. (Clit.) - - S. 275.) - Letell. 670.

- Loud. f. 15515 . - Hrz. t. 75.

— glandicolor. - - S. 304.]

- glaucophyllus, - Kl. 1503.

- glaucopus Schff. (Cortin.). - S. 300.] -

Huss. II. 30. - K1. 712. - Loud, f. 15963.

- glebarum. - Crypt. Bot. antarct. roy. Ere-

bus. 1554 . t. 162.3 .

- gliodermus. - S. 563.]

- globosus Schum. - Fl, dan. XII. 2145. 1.

- globulifer Gr. = ? racemosus P. (Tul. Sel.).

- gloiocephalus. - Letell. 645.

- glutinosus. - cf. Gomphidius. - [S. 2\$9*.]

- glyciosmus Lact.) - [S. 310.] - Kl. 1512.

- gomphus. - Letell. 603 (rutilus).

- Gordoni B. B. - Ann. Mg. n. h. 1561. VII.

t. 15 . f. 7 . p. $37 \%$.

- gossypinus. - [S. 297.]
Agaricus gracilentus. - [S. 273.]

- gracilis, - [S. 297.] - Bot. Zt. 1859. t. 5.

f. 2. (Anat. Bonord.) - (Gomphid.) Berk. Out.

t. 12. f. $i$.

gracillimus. - [S. 294.] - Alger. t. 31. 2.

grallipes. - [S. 301.$]$

- graminicola Ns. - [S. 2S0*.] - Bisch.

f. 3ํ.j!

- graminum Maras.). - Berk, Out. t. 14. f. 8.

- Lib. 119.

- grammocephalus. - Berk. Out. t. 1. f. 1.

Fructif.i.

- grammopodius. - [S. 276.] - Huss. II. 41.

- granulosus. - [S. $273.7-$ Huss. I. $15 .-$

FI. dan. X. 1677. 1. u. X. 1795. - Fuck. s.

112. - Loud. f. $15745 .-$ Hrz. t. 44. A. -

Bisch. f. 3+61. - Hoffm. Ic. t. 13. f. 1. (Lepiot.). (cf. ochraceus Hrz.)

- graveolens. - $\left[\right.$ S. $276: 104 ; 275^{* *} ; 276$ :

100 .

griseo-cyaneus. - [S. 257.

gríseo-pallidus. - [S. 254.]

grisen-rubellus. - [S. 2SS.

- griseus Fr. (Omphal.). - [S. 284.] - Kl.

1207 .

- grossus. - cum Sclerotio: Ann. sc. n. 1813.

XX. t. 6. f. 2. (Léveille).

- grumatus. - [S. 279.]

- gypseus, - [S. 2S1.]

gyrans. - [S. 279.]

_ gyroflexus. - [S. 296.]

- haematites. - S. 294.]

_ haematocephalus Mt. (Maras.). - Sagra Cuba

t. 17. f. 4 .

- haematochelis. - Huss. I. 19. - Roq. t. 17. f. 2 .

haematophyllus. - Brit. f. 121. - Mag.

Zool, Bot. 1537. I. t. 15. f. 1. p. 507. (Berk.).

- haematopus. - [S. 252.]

- hariolorum. - [S. 2S0.]

- haustellaris. - [S. 294.

- hebes. - [S. 296.

- helomorphus. - [S. 291.]

- helvelloides. - [S. 304.]

- helrolus. - S. 304 .

- helvus Lact.). - [S. 309.] - Kil. II. 109.

- hemitrichus. - [S. 304.$]$

- herpeticus: - [S. 301.] - Roq. t. 20. f. 3.

- heteroclitus Fr. - [S. 2S9.] - Klotzsch in

Dietr. Flor. boruss, t. 356 . - Hoffm. Ic. t. 14.

f. 2. (Derm. Phol.).

heterophyllus Fr. (Russ.). - [S. 311.] -

Badham t. 10.3.- Berk. t. 13. f. 5. - Huss.

I. 54. - cf. furcatus.

- hiascens. - [S. 297.]

- hiemalis. - cf. hyem.

— hilaris. - [S. 292.]

— hinnuleus. - [S. 304.]

—_ hircinus. - [S. 302.$]$

- hirneolus Fr. (Clit.). - [S.277.] - R.f. 109.

- hirtipes Schum. - [S. 259.] - Fl. dan. X.

1730. 2.

— ? hirtus Wllr. - Kl. 1909.

- hispidulus. - [S. 2S6; 273.].

- hiulcus. - S. 291.]

- holosericeus. - [S. 273.]

[ Hookeri. - [S. 290**.] - Hook. J. 1852.

IV. t. 6 .

$\longrightarrow$ hordus. - [S. 275.]

- horizontalis Bull. - [S. 292.]

$-\beta$. crenulatus. - Verh. zool. bot. Ges.

Wien. 1560 . X. p. 809 . (Schulzer). 
Agaricus Hornemanni. - [S. 295.]

hortensis. - Vir. 11.

Hudsoni P. - S. 311.] - Dsm. 165. -

Loud. f. I.s-i9. - (Maras.) Kl. 1+0:3.

humilis P. - cf. atrovirens P. - [S. 276.]

humorus. - [S. ….]

hybridus Sow. - [S. 291.] — Loud. f. 15972.

hydrochrous. - [S. 251 *.]

hydrogrammus Schum. = phyllophilus $\mathrm{Fl}$.

dan. - Omph.) Bail S. t. 35. - [S. 233.] -

Vent. mi. 96. f. i.

hydrojonides (Hypor.). - Comment. soc. cr.

it. 1-til. 2. t. 3. f. 3. p. 61. (Cesati).

hydrophilus Bull. = stipatus Fl. dan. -

[S. 296.]

hydrophorus. - [S. 29i.]

hyemalis Osbeck. - cf. Ag. corticalis. -

[S. 253.] Bull. t. 514.

Lasch.: Kl. 306.

hygrophytus. - S. 296 .

hymeninus. - Alger. t. 31.4.

hypnophilus. - Letell. 706. 2. (perpusill.).

hypnorum Schrk. - [S. 294.] - Loud. f.

160u1. - Bot. Zt. 1556. t. 5. f. 21. e. (Pollin.

Hoffm.).

- hypotheius Fr. - [S. 307.] - Fl. dan. XI.

1914. 2. - Loud. f. 15752 . - (Limac.) Kl. 603. hypsipus. - S. 295.]

hysginus. - [S. 309.] - (Lact.) - Vent.

mi. 97. - f. S. 9.

- hystrix. - [S. 290.

janthinus. - [S. 2S2.]

- ichoratus. - [S. 310.] (Lact.)

- jecorinus. - [S. 309.] (Lact.)

Jerdoni B. B. Naucor.). - Anu. Mg. n. h.

1561. VII. t. 14. f. 2. p. 375.

iliopodius. - [S. 304.]

illibatus. - [S. 302.]

illinitus. - [S. 273. 39.] — Loud. f. 15744.

- Hoffm. Ic. t. 13. f. 2. (Lepiot.).

illuminus. - [S. 305.$]$

- imbricatus (Trichol.). - [S. 275.] - Kl. II.

404. - Berk. Out. t. 4. f. 3.

imbutus. - S. 305.]

impatiens Fr. - [S. 297.] - Fl. dan. XI.

1960. 2.

impennis. - 'S. 304.]

- imperialis. - [\$. 274.47.]

- impudiers. - [\$. 313.] (Maras.).

- inamoellus. - [S. 276.]

- incanus. - S. 257.]

- incariitus Relh. = roseus Fl. dan.

incilis. - S. 27S.]

- incisus. - S. 304.$]$

inclinatus. -- [S. 2S2.]

- incomtus. - S. 279.]

- inconspicuus. - Hook. Lond. J. 1842. I. t. 14.

(Berk.).

- incrustatus Schum. = caperatus Fl. dan.

- incurvus Schum. = urbus F1. dan. - [S. 303.]

- Indigo (Lactar.). - Carol. II. 4.

- inflexus Maras.). - Chile n. T. f. 5.

- infractus. - [S. 300.]

- Infula. - [S. 258.]

- infundibuliformis. - [S. 27\%.] - Berk. Out.

t. 5. f. 2. - Carol. V. 3. - Viv. 5. - Tent. mi. S5. f. 1. 2.

- ingratus Schum. - [S. 250.] - Fl. dan. XIII.

2266.2

- inodermus. - [S. 563.]

inodorus Schum. = plexipes Fl. dan.

inolens. - S. 2S1.]
Agaricus inopus Fr. - [S. 291.] - Loud. f. $1.39-7$.

inormatus Sow. (Clit.). - Loud. f. 15\$28. -

Bot. Zt. I 55-. t. 5. f. 4. (Anat. Bonord.).

- inquilinus. - S. $29 ?$ ?.]

— insidiosus. - Letell. 631.

insititius (Maras.). - [S.314.] - Berk. Out.

t. is. f. ti.

insul-us. - [S. 3109.] (Lact.). - Berk. Out.

t. Iis. f. 2 .

integer L. (Russ.). - [S. 310.12;311.] -

Hrz. t. ti3. - Plıöb. Gift. t. $3 ; 4: 1.2$. -

(Russ.) Bot. Zt. 1 j5. t. 5. f. 10. (Anat. Bonord.j.

- v. sanguinea B. - Hrz. t. 26.

__ v. virescens Schff. - Hrz. t. 54 .

- integrellus P. (Omphal.). - [S. 284.] -

Kl. $111 \cdots$.

- intentus. - S. 301.]

- inunctus. - [S. 295.]

- inversus. .. [S. 27 ○.]

- involucratus. - Alger, t. 3 t. 7.

involutus Panus, Ruthea, Paxill.) B. -

Kops F1. bat. X. 754 . - Loud. f. 16003 .

Ann. Mg. n. h. 1539. I. t. A. f. 27.25. (Fructif.

Berk.). - Berk. Out. t. 12. f. 5. - Cd. Ic. Y.

t. 10. f. 91. (Fruct.) - Kl. 20.

- iocephalus, - Carol. V. 1.

_ ionides. - [S. 276.$]$

- ionipterus (Clitpil.). - Comment. soc. cr. it.

1S61. 2. t. 3. f. 1. p. 60. (Cesati).

- ionus. - Krbh. t. TI. f. 5-9.

— Iris. - [S. 251.? Berk. Out. t. 6. f. 3.

- irregularis. - S. 305.]

- irriguus. - $[$ S. 30s.]

- irroratus Schum. = phalloides Fl. dan.

- irrufatus. - [S. $27 \%$.

— isabellinus. - S. 305.

— jubarinus. - [S. 305.$]$

- jubatus. - [S. 257.]

— juglandinus. - [S. $2 \$ 8$.

- juncicola. - S. 2S3.? - Bot. Zt. 1S58. t. 5

f. 11. (Anat. Bonord.).

- junonius. - S. 2\$9.?

- Karlii Rbh. Maras.). - R. f. 506.

— Kretschmari. - S. $295 * *$.]

- laccatus. - (litoc. - S. 279.] - Kops

Fl, bat. XI. 524. 1. - Berk. Out. t. 5. f. 3. Scop. : Loud. f. 155 t. - R. f. 305 . Schff.: Hrz. t. 45. B. - Nov. Act.

15 2. XIX. II. t. 56. f. 22-25. (Pollin. Phöb.).

- Bot. Zt. 1 566. t. 5. f. 11 . (Pollin. Hoffm.). -

Kil. 11. - Kl. II. 105. - Huss. I. 47.

— - v. perpusillus sicop. - R. f. 503.

laceratus. - S. 305: 36.1

lacerus Fr. - [S. 290.] - Fl. dan. XI. 1546.

1. - Kl. 315. - Hoffn. Ic. t. 12. f. 1. Dermin.).

- Lacmus Schum. = pratensis Fl. dan. -

[S. 30S: 27 .]

[acrymabundus Prat. Hyphol.) Sow. - [S.

296.] - Loud. f. 16021. - Bail S. t. 35. - ef.

Ag. velutinus.

—— Bull. : Iil. 214. - Hoffm. Ic. t. 15. f. 3. lactescens Sow. = subdulcis Fl. dan.

lacteus P. - [S. 2S1.] - (Russ.).- [S. 310.]

- ? Vent. mi. 81. f. 1.2. - Fl. dan. XI. 14.45.

1. u. XII. 2111. 1. - Mycen.) Bull.: K1. 607.

— Bull.: Wstd. 556.

- v. pityus. - Kil. 1705.

— lactifluus. - Kops Fl. bat. XI. 874.

- laetus Hygroph.). - Ki. II. 612.- [S. 30S.] 

1 
Agaricus laevigatus Schum. = epipterygius $\mathrm{Fl}$. dar. - [S. 242.]

lamellatus Poll. - W'std. 12:3.

lampropus Fr.

- v. fuscescens. - Kil. 1104.

lanatus Schum. = peronatus Fl. dan.

Sow. - [S. 312.]

lancipes. - [S. 2; $; 9$.

languidus. - [S. 313.] (Maras.).

laniger. - S. 303. 302*.]

lanuginosus kull. - [S. 290.] - Loud. f.

15991. - (Inoc.) KI. II. 104.

Lappula. - S. 2 $>$ i.

laqueatus. - [S. 274. 50.]

largus. - [S. 300).]

Laschii. - S. 5ti3.

lascivus Fr. (Trichol.). - [S. 276.] - Kl. 12119

lateritius Schff. - (Hyphol.) [S. 293.] -

Loud. f. Ifill2:2.

Fr.: Fl, dan. XI. 1516. 2. - Nov.

Act. 1942. XIX. II. t. 56. f. 61-63. Fruetif.

Phöb.) - HIoffm. Ic, t. 15. f. 13: 17. Is veli evolut.). - Huss. I. 60.

\section{Gajera. Fr. - Kl. 215}

- latus. - [S. 3010. 301:51.

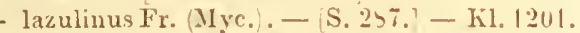
Leightonii. - S. 2\5*.] - Ann. MIg. n. h.

15 4t. XIII. t. 9. f. I. p. 3 t3 l3erk.

lenticularis. - [S. 272. 19.]

lentus. - S. 291.

- leoninus Schff. - S. 256. - Bisch. f. 3120.

- Berk. Out. t. T. f. 4.

- lepideus Fr. - Loud. f. 15915.

- lepidus Russ.). - [S. 310.] - Iluss. II. 32.

Lerehei. - S. 273.41.

- leucocephalus. - S. 276.]

- leucopliacus. - [S. 307.]

- leucophyllus. - S. 564 .

- lemcopus Bull. - [S. 564.] - Bisch. f. 3460 .

- leucosporus, - [S. $273^{* * *}$.

- leucoxanthus. - S. 275.

- Leveilleanus. - 'Tijdschr. v, mat. Geschied.

1st5. XII. t. 5. 'Doz. Molk.).

- libertatis. - S. 296. ?

- licinipes. - [S. 304.]

- ligatus. - [\$. 30\%.]

- lignatilis Fr. - [S. 2) . ] - Fl. dan. X. 1797. - Bull. - cf. Ag. apicreus.

- limacinus. - \$\$. 307.]

limbatus Lasch. Iycen.). - K1. 1203. -

R. f. 502 .

- limonius. - [S. 304.$]$

- limosus. - [s. 2s5.]

- limpidus. - [S. 255.]

- limulatus. - [S. 292.]

- Linckii. - [S. 257.]

- Lindgreenii, - [S. 304.]

- lineatus. - [S. 2\$1.]

- Linnaei Russ.). - [S. 310.]

- liquidus. - [S. 302.]

- Liquiritiae. - [S. 292.]

- lituus. - [S. 253.]

livido-ochraceus. - [S. $303^{* *}$.]

- lividus. - [S. 2S6.]

- livor. - [S. 305.]

- lixivius. - [S. 276.]

- lobatus Sow, invers, v. Fr.). - Huss. II. 43.

longicaudus Schum. = tenacellus Fl. dan. -

Berk. Out. t. 9. f. 2. - [S. 290.]

- longinquus. - Crypt. Bot. antaret. voy. Ere-

bus. 1554. t. 163. ว.
Agaricus longipes Bull. - [S. 279.] - Bisch. f. 3423 . - Vent. mi. 50. f. 5 ; 5 i. f. 3.4 ; il. Si.

- loricatus. - [S. 275.]

- In ripes. - [S. 2;9.]

-_ Loveianus. - Berk. Out. t. T. f. 2.

- lubricus. - [s. 291.]

lucifer. - S. 249.]

lucifugus. - [\$. 291.] - Nov. Act. $1 \$ 12$.

XIX. II. t. 56. f. $39-47$ - (Fructif. Phöb.).

- ludius. - [S. 2911.]

- lugubris. - [S. 24\%.]

- lupinus. - [S. 291.]

— luridus (Trichol.). - [S. 275.] - Loud. f. 15 itil.

15790. (Lactar.). - [S. 309. 17.] - Loud. luscinus. - [S. 277.]

- lustratus. - [S. 300.]

- Iuteo-albus. - [S. 2S1.] - Bot. Zt. $185 \mathrm{~s}$.

t. 6. f. 3. (Anat. Bonord.).

- lutcolus. - Wstd. 310.

- luteo-nitens. - [S. 295.]

- luteus (Russ.) - [S. 311.]

- lutincola Iasch. - Kl. 1707.

__ lyratus. - [S. 301.]

- imacilentus. - [S. 250.]

- macrocephalus. - Ann. Mg. n. h. 1839. I.

t. f. f. 11)-12. Fructif. Berk.

- macropus P. (Cortin.). - [S. 303.] - Kl. 1901. - Kil. II. 1117.

A. S. = caperatus Fl. dan.

_ macrosporus Mtg. = Lentinus Lecomtei Fr.

- maculatus Schum. = magnificus Fl. dan. -

[S. 27\%.]

- A. S. : Huss. II. 20.

- madidus. - [S. 246 .]

_ magnificus Fr. - [S. 272. 11.] - Fl. dan.

XII. 2140.

— maialis Fr. - [\$. 25\%.] - Loud. f. 15947.

- malachius. - [S. 302.] - Kl. 1909.

- malicorius. - S. 303. ?

- mammosus. - [S. 25S.] - IIuss. II. 42.

Bertuch Bilclerb. XXXI. 2. - S. 309.] (Lact.).

_- Mappa. - [\$. 272. 4.] - Vent. t. 13. f. 124.

125. - IIrz. t. 5. - Bot. Zt. 155s. t. 6. f. 1. f.

Anat. Bonord.).

- Mariae KI. - Linn. 1\$32. VII. t. S.

- maritimus Fr. Obs. = lacerus Fl. dan. -

[S. 290 .

- mastiger B. B. Psathyra - Ann. Mg. n.h.

1561. VII. t. 14. f. 6. p. 377 .

— mastoideus Fr. - [S. 273.] - Fl. dan. XII.

2144. - Berk. in Mag. Zool. Bot. 1537. I. t. 2.

f. $1 .-$ iv. 19.

— mastrucatus Fr. - [S.255.] - Loud. f. 15929.

_- maurus Fr. - [S. 2S3.]

- maximus. - [S. 27S.]

- medius. - [S. 2S5.] - Fl. dan, X, 1676. 2.

_ medullatus. - [S. 273. 37.]

- melaleucus. - [S. 276.]

- melanops. - Bull, ac. belg. Ser. 2. To. 11.

p. 660 . f. 5. (Wstd.).

- melantinus, - [S. 273*.]

- melinoides. - [S. 292.] - Dsm. 1619. -

Kl. 1504. - Berk. Out. t. 9. f. 3.

melizeus. - [S. 307.]

- melleus Vahl. (Armill.). - [S. 274. 45.] -

Loud. f. 15747 . - Vent. t. 3. f. 20. 21. - t. 13.

f. 120. 121. - Kops Fl. bat. X. $775 .--$ Bisch.

f. 3326 . - Hrz, t. 9. - Berk. Out. t. 4. f. 1. -

Staude t.9. f. 4-5. - Ital. 5\$3. - Krypt. Bad. 
2.19. - Bail S. t. 39. - Bot. Zt. 1 559 . t. 13. f. 211-25. '2te Fructif. ? de Bars'. - Kl, 2. Dim. 1 447 - Kl. II. 2. - Carol. II. 1. - Badham t. 16. 3. - Viv. 51. - Vent. mi. 26. 51. Fries. Sv. t. 36.

Agaricus melleus Tahl. v. fuscescens. - Kl.

II. $6 \mu$.

_- mephiticus. - [S. 291.]

- merdarius. - [S. 295.]

- mesodactylius. - Ann. Mg. n, h. 1545. II.

t. 4. f. 1. (Beik.)

- inesomorp aus Bull. - Bisch. f. 3261.

- mesophaeus (Derm.) P. - [S. 2S9.] - Hoffm.

Ic. t. $b$.

- mesotrephus (Hygr.). - Ann. Mg. 11. h.

$155+$. XIII. t. 15. f. 2. p. 102 . (Berk. et Br.).

— metachrous. - S. 275.

metapodius, - [\$. 30k.

metatus H. H. - Bot. Zt. 1\$56. t. 5. f. 12.

11. 15-1\% = vulgaris Fr.). - Kl. II. 301. (item . - Fr.: [S. 2-2.]

- micaceus (Coprin.). - Huss. I. 5.5. - Kops

Fl. bat. X. $320,3 .-$ Ann. Mg. n. h. I-35. I. t. t. f. I - . Basid. Berk. - Ann. sc. n. I :37. VIII. t. 5. f. 1-j. (Fructif. Lév. - Pringsh. Jhrb. II. t. 32, f, 41 . 'Keimg. Hoffm. - Nov. Act. 1542. XIX. II. t. 56. f. 65-71. iSpor. Phöb. . - Bot. Zt. 155ti. t. 5. f. 2.5. Pollinar. Hoffm. . - Bot. Zt. 1659. t. 11. f. 33. Keimg. Hoffin. - K1. 305. - Viv. 2t. - Vent. mi. 29. f. 4. 5. - Ital. 329. - Stu. h. 14. t. 49.

- Michelíanus. - S. 250.]

- miculatus. - [S. 275.]

- miltinus. - [S. 303 .

milvinus. - S. 306 .

- miniatus Hygrophor. :- [S. 305.] - Act.

Tur. 1s05. XIT.t. J. Cuminol.

- Fr.: Hrz. t. 4. a.

minutulus Schff. = disseminatus Fl. dan. mitis P. Pleurot. . - [S. 24.5. - Kl. 70\%.-

Berk. Out. t. 6. f. 9. - Kl. II. 602. - Brit. f. 137 .

- mitissimus Lactar. - SS. 310.7 - Hoffm.

Ic. t. 2. - Bot. Zt. 1656. t. 5. f. 21). d. Basid.

$\mathrm{Hoffm}$.

- mniophilus, - [S. 294 .

mollis Schff. - [S. 294.] - Brit. f. 1与. -

Lond. f. 1600 \%. - Berk. Out. t. 9. f. 6. - Crepid. Kl. 704. - Carol. III. 2.

- Sow. cf. Ag. canescens, - Huss. I. 74. molochinus. - S. 2 s0.

molyoides. - \$. 313.

- momentaneus. - Anm. Mg. n. h. 1535. I:

t. 4. f. 9. Basid. Berk. .

- montanus Psiloc. - K K1. 15110 u. 1i10.

morosus. - Limn. 1s30. V. t. 6. f.6. Jungh. . mortuosus. - S. 279.

- Mouceron. - Tent. mi. 11. f. 1-1. - Fl. dan. X. 1672. 2. (cf. Prunulus.

— Bull.: Vent. t. 4. f. 34. 35.

- mucidus Armill. . - [S. 2i4. 5].] - Loud. f. 15746 .

- Schrad. cf. Ag. splendens Hrz. mucifluus. - [S. 311].]

Mucor B. - Bisch. f. 3254.

mucosus Fr. - Vent. mi. \i. f. 7. .

multicolor. - 'S. 299.]

- multifidus Ns. - Schizophyll. comm. Fr.

- multiformis. - [S. 300.

multiplex Fr. - 'S. 2-1.]

mundatus.

- var.: Rbh. R. f. 13.
Agaricus mundulus Lasch. (Clitpil.). - Kl. 612.

- - v. nigrescens. - Kl. bll.

muralis Sow. - [S. 25t.] - Loud. f. 15905.

- Omphal.j. R. f. Ji)!

murcidus. - [S. 296.]

muricatus. - [S. 249.]

muricinus. - [S. 311\%.]

murinaceus. - [S. 275.80.]-Loud. f. 15852.

[Hygrop'. - [S. 2:5 **. 315.]

—_ murinus. - [S. 2 -1.] - Loud. f. i 5913.

- muscarius ef. Amanita, - [S. 27\%. 7.] -

Kops Fl. bat. VII. 49.5. - Bisch. f. 5. 6. 3156

— S. 5. - Loud. f. 15736 - Vent. t. 1. f. 5. 6.

- Roq. t. 1-. 19.211. f. 1. - Hrz.t. 1.-Hoffm.

Ic. t. 1. - Phöb. Gift. t. 2. - Berg Charakterist. 1560 . t. 1. f. 11. - Bail S. t. 3- - Bot. Zt. -59 , t. 11, f. 17. Schwingfaden, Hoffm.). Bot. Zt. 1-5 t. t. h. f. 1. (Anat. Bonord.). Kl. I. - Müller Pflanzenstaat ㄱ:3. (Copie). Fries Sv. t. 1. - Husi. I. . - Viv. 2!. - - Vent. mi. 2. f. 3. 4; t. 3. u. 4. - Krypt. Bad. 250. Stu. h. 1 t. t. jl.

- muscigenus Schum. - [S. 250.] - Fl. dan.

XII. 212.1. 1.

- Musseron cf. Ag. Mouceron.

- mussivus, - T5. 2\$4.

mustelinus Schum. = candicans Fl. dan. -

- Rusi.j S. 311\%.]

[- musteus. - [S. 309.]

_- mutabilis Schff. - Pholiot.) [S. 2\$9.] -

Fent. t. 9. f. -3. 4 . - Berk. Out. t. 5. f. 3.-

- Kops Fl. bat. XI. \$15. 1. - Bisch. f. 3276.

- Loud. f. 15!191. - Kl. 614. - Badham t. 16.

f. 4. 5 caudicinus . - Huss. II. 2\%. - Staude t. 5. f. $1-3$.

$\longrightarrow$ v. denudatus Fr. - Fl. dan. XII. 2076. 1.

- mutilus. - S. 255 .

- mycenoides. - [S. 259.] — Kl: 1309. Pho-

liot. .

- mycenopsis Fr. - [S. 294.] - Hoffm. Ic. t. 6.

- Mromyces. - Trichol. . - Letell. 663. -

Fl. dan. X. 1729. - Loud. f. 15765. - Schntzl.

Ic. Nachträge t. 2. f. 1. Hymenium'.

-_ myosurus. - [S. 250.]

- mytillinus. - [S. 303.]

[_ nanus. - S. 2S6.]

- napus. - S. 300.

— naucinis. - S. 273.$]$ - Vent. mi. 59. f. 1.2.

- nauseosus Russ... - [S. 311 .]

- nebularis B. - [S. 2 † 7 ] - Dsm. 1616. -

Fl. dan. X. 1734. - Ann. Mg. 11. h. 1834. I.

t. 4. f. 15. Fructif. Berk., - Roq. t. 15. f. 5.

- Bot. Zt. 1556 . t. 5. f. 19. d-f. Basid. Hoffm.)

- Clitoe.. K1. 501. - Brit. f. 5. - Badham

t. 9. 1.-Huss. II. 9. - Viv. 33.

- necator Bull. - $\$ .3119 .37$,$] - Lact.j - Fl.$

dan. XI. 1913. - Roq. t. 13. f. 3. 4.- Phöb.

Gift. t. 6. f. 1-14. - Hrz. t. 70 .

- Neesii Plut. : - Bail S. t. 37.

_ B Barla: Nor. Aet. 1sfit. XXVII. t. 16. nefrens - [S, 2צ४.]

- nictitans. - [S.274.] - Trichol.' Huss. II.

46.

nidorosus. - โS. $2 \succ \bar{i}$.

nidulans P. Pleurot.) - [S. 2\5.] - Kl. 316.

- niger. - Carol. I. 1.

- nigrescens Lasch. - Clitop.' Kl. 611. -

R. f. 13.

- nigricans Russ. . - [S. 310.] - Vent. mi.

49. f. $1-1$.

— nigripes Schrad. — Wstd. 12S2.

- nitellinus. - [S. 279.] 


A garicus nitidus (Russ.) - - [S. 311.] - Loud. f. 157 \%. - Berk. Out. t. 13. f. 7. - (Aman.)

[S. 272. 15.] - Cortin. [S. 302.]

- nivalis. - Loud. f. 15735.

niveo-flavescens, - Viv. 52.

niveus Coprin.) - Fl. dan. X. 16:1. -

(Hygr.) [S. 30..] - Russ.) [S. 311. 26.] Jacq. : Wstd. 1253.

- Scop: Vent. mi. 95. f. 2.

nolitangere. - [S. 297.

- nubilus. - [S. 279 .

— nuccus Bolt. — [S. 292.]. - Loud. f. 16002. nucisedus. - [S. 296.

- nudipes. - [S. 290.

_ nudus Bull. - [S.2 26.] - Fl. dan. XI.1911. 1. - Berk. Out. t. 4. f. 7. - Hrz. t. 31. - Bot.

Zt. 1556. t. 5. f. 20, a. (Pollin. Hffm.).-Dsm.

III. 664. - Hoffm. Ic. t. 11. f. 1. Trichol.).

- obbatus. - [S. 27 .

- obliquus, - Kl. 1508:

- obolus. - [S. 279.

- obrusseus. - [S. 30S.] - Hỵgroph.

Kl. II. 203.

- obscuratus. - [S. 310.] Lact.

- obscurus Schum. = udus Fl. das.

— obsoletus. [S. 279.]. - Clituc.). - R. f. 2.

- obturatus. - [S. 295.] - Letell. 700.

- obtusatus, - S. 297.

- obtusus. - [S. 306.] - R. f. j01. Cortin. ocellatus. - [S. 2So.]

ochraceus Bull. - IIrz, t. 1. b. cf. granula-

tus.. - (Russ.) [S. 311.]

ochreatus Holms. - [S. 295. cf. $439 \%$.] -

Bisch. f. $32>6$.

_ ochrolencus. - [S. 303.] - Russ.) [S. 311.]

- Vent. mi. 53. f. 5. 6.

odorus Bull. Clit. - [S. 277.] - Loud.

f. 15523 . - Hrz. t. 55. - cf. Ag. anisatus. -

Brit. f. 6 .

- ocdematopus Scop. - Vent. mi. 45. f. 1. 2.

- Vent. t. 6. f. 4\%. 15. - Clit.) Schff. : Loud.

f. 15532 .

offuciatus. - S. 2 - 3.

- olearius DC. - Viv. 50. - Vent. mi. 15.

f. 1. 2. - Ann. sc. n. 1515. IX. t. 20. f. $1-10$

(Tulsn.).

- olivaceus (Russ.). - Bot. Zt. 1555.t. 6. f. 2.

(Anat, Bon.)

- olivaceo-albus Fr. - [S. 30T.] - Fl, dan.

XI. 1914. 1. Loud, f. 15751.

olivascens. - "S. 301.]

olorinus. - [S. $27 \%$.

1205 .

- onyehius. - [S. $2 i 6$.

- opacus With. Clit.). - Loud. f. 15534: -

(Maras.) Carol. III. 6:

opicus. - $[$ S. 276.]

- opimus. - - [S. 302.]

- opiparus - [S. 27\%.]

- Opuntiae. - Alger. t. 32, 1.

- orbiformis. - [S. $2-\delta$.

- Orcella Bull, - Vent. t. 4. f. $32,33 .-\mathrm{Cd}$.

Ic. V. t. 10, f. 93 (Fructif.) - [S. 257.] -

Badham t. 13. - Huss. I. 7s. - Vent. mi. 8.

f. $3.4 ; 42$. - Ital. 455 .

- Oreades Bolt. (Maras.). - [S: 313.] ? Bail S.

t. 33. - Bot. Zt. 1 \$60. t. 14. f. 16. (Hoffm:).

- Kops FI. bat. XI. $\$ 30$ - Berk. Qut. t. 14.

f. 5. - Loud. f. $15 \$ 62$ Hrz. t. $17 \mathrm{~b}:-$ R.

f. 301 . - Pringsh. Jahrb. II. t. 32 , f. 47 (Keimg.
Hoffm.). - Staude t. 9. f. 1. - Ital. 4S7. - Kl. 14. 711: var. - Badham t. 8. 3. - Huss. I. 35. - Vir. 24. - Vent. mi. 73. f. 4. 5. -

Fries Sr. t. 31 .

A garicus Oreades Bolt. v. alnetorum Lasch. Kl. $\mathrm{S} 01$.

- oreinus. - [S. 276.$]$

- orellanus. - [S. 3113.]

orichalceus. - [S. 301.]

- ornatus. - [S. 254.]

- ostreatus Jeq. - - [S. 255.] - Kops F1. bat.

X. 780 . - Ann. Mg. n. h. 1S38. I. t. 5. f. 34 .

35. Fructif. Berk.). - Vent. t. 4. f. 30.31. -

Loud. f. 15920. - Bot. Zt. 1556. t. 5. f. 21. f.

Pollin. Hoffm. . - Kl. 308. - Kl. II. 106. -

Brit. f. 135. - ef. dimidiat. Letell. 695 . -

Badham t. 11. - Huss. II. 19. - Viv. 42. -

Vent. mi. 20. 3s.

- ovatus Coprin.). - Kops Fl. bat. XI. S75.

cf. Ag. comatus'.

- ovinus. - [S. 30S.] - Huss. II. 50.

- ovoideus Bull. - caesareus Scop. - Vent.

t. 1. f. 1.2. - Viv. 34. - Vent. mi. 25. f. $1-3$;

59. f. 3 .

- oxyosmus. - [S. 294.]

- oxystomus. - Ann. sc. n. 1536. V. t. 13.

MItg.

- ozes. - [s. 250.]

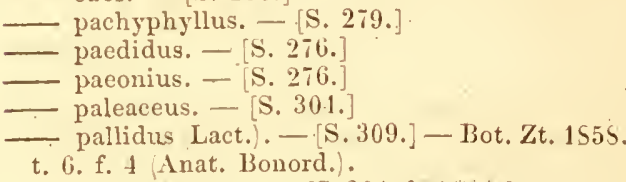

— palmatus Bull. - [S. 294.254***.] - Vent.

t. S. f. 70. 71. - Loud. f. 15925. - Tent. mi.

1. f. 3.4 .

- Palomet. - Letell. 657.

- paludosus. - [S. 294.

_ panaeolus. - [S. 276.] — cf. Paxillus.

—_ pannosus. - [S. 293.]

— pannucius. - [S. 30S.] (Lact.)

—_ Pansa. - [S, 300.]

- pantherinus DC. (Aman.). - [S. 2 72. 12.]

- Fl. dan. XI. 1911. 2. - Vent. t. 1. f. 7. S.

- Bisch. f. 3162. - Loud. f. 15737. - K1. 601.

- ?cf. fuliginos. et herpetic. Roq. - Letell.

639. - cf. verrucosus. - Viv. 26. - Vent. mi.

t. 5 ; t. 6. f. $1.2 ; 9$. f. $1 ; 67 ; 65 ; \mathrm{s} 0 ; 94 ; 94$.

- Staude t. 10. f. 3. 4. - Ital. $2 \$ 9$.

_- panuoides Paxill.). - Berk. Out. t. 12. f. 6.

- papilionaceus Bull. - [S. 297.] - Bail S. t. 3 .

- Kl. 107. - Pringsh. Jahrb. II. t. 32.

f. 42. (Keimg. Hoffm.).

papillatus Hoffm. = lacteus $\mathbf{F l}$. dan.

papulosus. - [S. 301.]

parabolicus. - [S. 292.]

- paragaudis. - [S. 304.]

- parasiticus Bull. - Brit. f. 130. - Loud.

f. $155 \% 2$.

parilis. - [S. 275.]

parkensis. - [S. 2SS.]

- paropsis. - [S, 278.$]$

parvannulatus. - [S. 273.]

- parvulus (Volvar.) : -Kl. : 1401. - Lév. :

[S. 2S6.] - Carol. I. 3.

—- pascuus (Nolan.) - [S. 2SS.] - Kl. 1702.

- Dsm. 1802.

_ pateriformis. - [S, 305.]

patulus. - [S. 2 - 6 . $]$

- pauperculus. - [S. 2\$1.] 
Agaricus pausiacus. - [S. 279.]

pavonius. - [S. 302.]

pectinaceus Bull. - (Russ.) [S. 311.] -

Bisch. f. 34t6. - (Russ.) Ann. se. n. 1837 . VIII.

t. 10. f. 24. (Fructif. Lév.).

pediades. - [S. 293.]

- pelianthinus. - [S. 281.] - Berk. Out. t. 6.

f. 1 .

pelicanthinus Fr. - Loud. f. 15819.

pelliculosus Fr. MI $\left.{ }^{\circ} \mathrm{C}.\right)-[\mathrm{S} .253$.

pellitus. - [S. $2>6$.

pellucidus Bull. - Loud. f. 15 S9s.

peltatus. - [S. 252.

penetrans. - [S. 291.

penicillatus. - [S. 302.]

pennarius. - [S. 30\%.]

- pennatus Psath. - - [S. 297.] - Kil. 1710.

- perbreris.-Hoffm. Ic.t. 14.f. 1. Derm. In.

percomis. - [S. 300.]

perforans Hoffm. Collyb.). - Kl. S06. -

Fuek, s. 1035. - [S. 314.] IIaras.) - Kl. II. 40๔.

R. f. 102. - Schireiz. 24. - Krypt. Bad. 155. pergamenus Swartz. - [S. 309.] - Fl. dan.

XIII. 2265. 2. - Lact. . Cd. Ic. V. t. 10. f.65.

Fructif.

- peronatus Bolt. - Maras. [S. 313.] - Fl. dan. XII. 2019. 2. - Bisch. f. 3261. - Loud.

f. $15 \$ 61$. K 1 . 13. - Berk. Out. t. 14. f. 4. - Brit. f. 125. 126. - Huss. I. 69.

— - r. tomentellus Fr. - Fl. dan. XII.

2018. 1.

perpusillus Fr. - [S. 2S5.] cf. Ag. laceatus. -

Fuck.s.1106. - Letell. 706.2. hypnophilusP., persicinus. - [S. 276.]

- personatus (Trichol.). - [S. 276. 306**.] -

Huss. II. 40. - Loud. f. 157i2. - K1. 209. -

Berk. Out. t. 5. f. 1. - Badham t. 5. 1.

- Persoonii, - [S. 272.18.] - (Lact. [S. 309.

$22 ; 310.49$.

- pes Caprae. - [S. 276.]

- pessundatus. - [S. 275.]

- petaloides Bull. - [S, 2S5.] — Vent. t. 9.

f. 81. \$2. - Vent. mi. t. 54. f. $7-9$.

spathulatus Fr. - Bisch. f. 3245 .

petasatus Fr. - [S. 256.] - Ann. Mg. n. h,

1S44. XIII. t. 9. f. 2. p. 343 . Berk...

— petasiformis, - Cd. Anl. t. H. f. 77: 25-32.

petiginosus. - [S. 290.]

- pezizoides. - [S. 291.] - Act. Tur. $1 \subseteq 05$.

XIV. t. 1. Cumino:

— phaeosporus - S. 295.]

- phalaenarum. - S. 297.

- phaleratus. - [S. 2\$9.]

- phalligerus. - Chile, no. 7. f. 2.

_ phalloides Aman. Fr. - [S. 272. 3.] - Fl.

dan. XII. 2143. 2145. - Ann. Mg. n. h. 1535.

I. t. 4. f. 4. Fructif. Berk.) - Kops Fl, bat.

X. 735; XI. 829.1. - Phöb. Gift. t. 1. - Berk.

Out. t. 3. f. 1. - Bisch. f. 3243. - Loud.

f. 15732. - ? Roq. t. 23. f. 1. 2. - Hrz. t. 5.

(MIappa!. - Fries Sv. t. 2. - K1. 202. - Kl.

II. 603. - : cf. Ag. citrinus. - Viv. 14. 15. -

Staude t. 10. f. 1. 2. - Stu, h. 14. t. 55.

Phiala Schum. - Fl. dan. X. 1730. 1.

philonotis. - [S. 253.]

phlebophorus Dit. - [S. 256.] - Bisch.

f. 3253 . - Loud. f. 15944 . - Stu. h. 1. t. 15. phlyctophorus. - Alger. t. 30. f. 2.

- phoeniceus. - [S. 273.41.]

Phoenix. - [S. 296.]

plolideus Fr. - [S.302.] - Bisch. 'f.3275. -

Inol. Kl. 1902.
A g a ric us phonospermus Bull. - cf. Ag. fertilis. phyllophilus P. - Letell. 605. - Fl. dan.

XI. 1517.

physaloides. - [S. 296.]

- picaceus (Coprin.) Fr. - Loud, f. 16040.

- picinus. - [S. 310.] Lact.).

- picreus P. Dermoc.) - [S. 292]. - K1. 508. pictus. - S. 284.]

pileolarius $\mathrm{Bull} .=$ nebularis $\mathrm{Fl}$. dan. $-(\mathrm{Cli}-$

toc. Huss. I. 66.

— pilipes Sor. - Loud. f. 15995.

— pilulifer Coprin. - Canar. t. 4. f. 6.

_ piluliformis. - [S, 273.]

— pinguis Fr. - [S. 273. 3ৎ.]

— pinsitus. - [S. 2ৎ5.] - Dsm. 1803.

- Piopparello. - Viv. 6. - Vent. mi.40. f. 2;

48. f. 4. 5.

- piperatus Lact. - - [S. 309.] - Kops Fl. bat. XI. S25.1. - Loud. f. 15810 . - Roq. t. 13. f. 1. 2. - Hrz.t. 39. - Pay, bot. cr. p. 6. f. 18. - Ann. sc. n. 1937. VIII. t. 9. f. 17. Fruetif. Lér. - Kl. 901. - Berk. Out. t. 13. f. 3. Dsm. III. 669. - Brit. f. 61. -- Carol. II. 5. Vent. mi. 10. f. 7. - Ital. 439. - Fries Sv. t. 27 .

— pisciodorus Entol. . - Kil. 1509. - Comment. soc. crit. it. 1861.2. t. 3.f.2.p.59. (Ces.). - pithyophilus. - [S. 27i.] - (Maras.) Carol. II. 10.

- pithyreus. - [S. 293.]

- pithyus. - [S. 2S1.]

- placidus. - [S. 28\%.]

- plancus. - [S. 313.]

- planus Schum, - Fl. dan. X. 1733.

_ platyphyllus P. Collyb. . _ [N. 2 2 9.46.1***.]

- Kl. 714

— platypus Ns. - Bisch. f. 3259.

- pleopodius Bull. - Bisch. f. $3+19$. - No-

lan. Bail S. t. 37.

- plexipes Fr. - [S. 2\$1.] - Fl. dan. XII. 2023. 2.

plicatilis Copr. Fr. - Carol. V. 10. Loud. f. 16046 . - Bot. Zt. 1859. t. 11. f. 30.

KKeimg. Hoffm. .

- plicosus Mycen.,. - [S. 2S2.] - Til. 1502.

— Ki. II. 105.

_ plumbeus Bull. (Lact.). - [S. 309.] - Loud.

f. 1550 i. - Cd. Ic. V. t. 10. f. 94 . Fruct.). -

Kil. 101.

— plumbosus. - [S. 305. 301, 57.]

—_ plumiger. - [S. 304.]

— plumosus Bolt. - [S. 290.] - Loud. f. 15990.

- pluteus B. - Loud. f. 15943. - Kl. 904. -

Stu. h. 2. t. 25.

- plutonius. - Canar. t. 5. f. 2. Coprin.).

- pluvius. - [S. 302.]

- politus P. - [S. 2S5.] - Kl.613. - Eccil.)

R. f. 111.

- polyadelphus Mycenaria). - [S. 284.] -

Kil. 1307.

- polycephalus. - [S. 296.]

- polygrammus DC. - [S. 2S2.] - Loud.

f. 15554 . - Bot. Zt. 1556. t. 5. f. 20. b. Pol-

lin. Hoffm.t. - Kl. 605. - Brit. f. 12.

— polystictus. - [S. 273*.]

— pometi. - [S. 2S5.]

Pomonae : ef. Ag. aromatic. Roq. u. gambo-

sus. - Staude t. 9. f. 2. 3.

popinalis. - [S. 25i.]

- populiformis. - Honk. J. VIII. t. 5. f. 2.

- porphyrius Fr. - [S. 2i2.6.] - Fl. dan. XI.

1955. 2. - Bisch. f. 32S5. - Loud. f. 15733. 


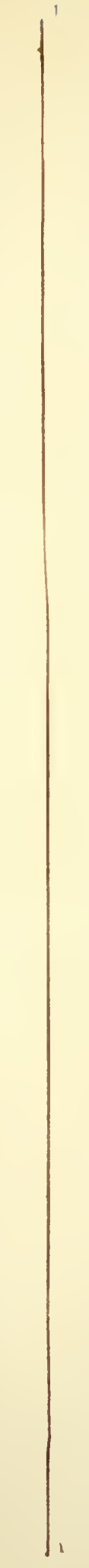



Agaricus porphyropus. - [S. 301.]

- porreus Fr. - MIaras.! [S. 313.] - Fl. dan.

XII. 2020. 2. - Loud. f. $15 \$ 63$. - Iíl. 15.

- Pers. Schum. = prasiosmus Fl. dan. -

Brit. f. 7 .

- porrigens P. - [S. 255.] - Kl. 216.

- porriginosus. - -S. 293.

- portentosus. - [S. 2\%1.56.]

— praecox I'. - S. 255.] - Letell. 605. - Fl. dan. XII. 2uiti. 2. - Berk. Out. t. ?. f, 1. Kl. 106. - Tiv. 12. - v. appendiculatus. - Loud. f. 1601 1:.

- prasinus Trichol. . - Vent. mi. 66. f. 1-3.

- Loud. f. 1.759. - Cortin. S. 301.

prasiosmus Fr. - [S. 313.] - Fl. dan. XII.

2024. 1. - R. f. 302 .

— pratensis. - [S. 294.] - Dsm. 1621. - Fl.

dan. X. 1731. 1. - Hygroph. Hoffm. Ic. t. 5.

Fl. dan. X. 1735. 1. = H. Jacmus Fr. Ep. 219.

- Loud. f. 1543\%. - Hygroph. [S. 3ris.

Fries sv. 30. - Brit. f. 123. - Ituss. I. 40.

- pretiosus Vent. - Vent. t. 5. f. 12-44.

— pristoides. - S. 251 .

_ privignus. [\$. 305.]

- proboseideus. - S. 291.]

- proceris Scop. - S. 273.7 - Badham t. 2.

- Vent. t. 3. f. 22. 23. - Kops Fl. bat. X.

i25. 1. - Fuck. s. 1145 . - Roq. t. 17. f. 3. 4.

- Hrz.t. 16. - Fries Sy. t. 3. - Bisch. f. $325 t$.

- Lep. Loud. f. 15710. - Bail s. t. 35.

Bot. Zt. 145f, t. 5. f. 111. Pollin. Hoffm.)

Kl. 21)1. - Huss. I. 59. - Viv. 5. - Vent. mi.

17. f. 1 ; 1 ᄂ. f. 3 ; 43. f. $1-1$.

- proletarius. - [S. 2๑5.]

- proliferus. - [S. 2>2.]

- prolixus. - [S. 279.]

- prominens. - Viv. 12.

- pronus. - \$. 297.

- protractus. - S. 2 51.$]$

- protuberans. - [S. 564.]

- pruinosus (litoc.) : Kl. II. 205. - Viv. 21. prunuloides. - [S. 256. $290 \cdots+$.

Pranulus P. - [S. 257.] - Badham t. 1. -

Hrz. t. 1:. - Berk. Out. t. 7. f. 7. - Loud.

f. $15936 .-=$ Ag. Mouceron Fl. dan. - Huss.

II. 47. - Viv. 3. 4: v. albellus. - Tent. mi. 64. f. 4 .

— psammocephalus. - [S. 304.] - Huss. I. 72.

- pseudo-unctuosus. - Wstd. 340 .

- psittacinus Schff. - [S. 308.] - Htss. I. 19.

Hrz. t. 17. a. - Bisch. f. 3414. - Vent, mi.

54. f. $1-1 ; 100$. f. $3-5$.

- pterigenus. - [S. 2\$3.] - Brit. f. 134. -

Berk. Out. t. 6. f. 7 .

— ptychophyllus. - Sturm h. 19- t. 5. Cd.

- pubescens Schum. = speciosus Fl. dan.

Wstd. 12S3. - Staude t. 6. f. 4. Lact.

- pudens. - [S. 279.] - Ann. Mg. n. h. 1539.

I. t. 4. f. 20. (Fructif. Berk.) - longipes

Huss. I. So. pudicus. - [S. 2S9.] - cf. Ag. caperatus.

__ Bull. : Huss. II. 31.

pudorinus. - [S. 307.]

puellaris Russ.). - [S. 311.]

pulchellus Russ.) - - Borez. fg. ingr. t. 2.

pullus $\mathrm{P} .=\mathrm{Ag}$. Schumacheri Fl. dan.

_- Schff.: - [S. 279.]

pulmonarius. - [S. 2S5.]

pulverulentus Bull. = fascicularis Fl. dan, - Scop.: [S. 2i6.]

$\mathrm{H}$ off $\mathrm{m}$ a $\mathrm{n}$, Index Fungorum.
A garicus pumilus (Mycen.). - [S. 2S9.] - Bail S. t. 3S. punctatus. - [S. 259.] - Cort.) [S. 304.]

- puniceus Fr. - Hygroph.) [S. 305.] - Loud.

f. 15442 .

- purpurascens. - $\left[\mathrm{S} .307^{* *}.\right]-($ Cortin.) [S. 300.]

- purus P. Iycen. . - [S. 2\$L.] - Hrz.t. 3S.

- Loud. f. 15591. - Kl. 206. - Bot. Zt. 1556.

t. 5. f. 9. Pollin. Hoffm. - R. f. 6. - Dsm.

III. 274. - Huss. II. 49 .

- pusillus F2. - Brit. f. 139. - Kl. 709. -

Ann. Mg. n. h. 1534. I. t. 5. f. 29. Fruet. Berk.).

- Batsch: Ag. virgineus var. Dsm.).

pusiolus. - [S. 292.]

pustulatus Schff. = rubescens Fl. dan. -

P.: [S. 30\%.]

- putidus. - [S. $27 \%$.

- putillus. - [S. 313.] (Maras.) - R. f. 106.

- pygmaeus. - [S. 293.]

- pyriodorus. - [S. 290.]

f pyrogalus Lact.). - [S. 309.] - Roq. t. 13.

f. 5 .

13ull. : Loud. f. 15S05. - Dsm. III. 669.

- pyxidatus Bull. Omphal.) - [S. 283.] -

Bot. Zt. I460.t. 14. f. 7 Hoffm.) - Kl. 310 .

- Berk. Out. t. 6. f. 8. - Bisch. f. 3299 . IIuss. II. 24.

- - v. pusilla. - Kl. s0\%.

— quadricolor. - [s. 304.]

- quercinus. - Wstd. 145.

- quictus Lact. . - [S. 309.] - Kl. (3).

- quinquepartitus. - [S. 274.]

- quisquiliaris. - Hook. Lond. J. 1942. I. t.

11. 'Berk.'.

- racemosus. - [S. 2\$0.] — Loud, f. 15969.

Bisch. f. 3272 .

- - P.: zu tuberos. Rbh.

- rachodes. - Dsm. IHI. 276. - Huss. II. 35.

- Berk. Out. t. 3. f. 6.

- radians. - Amn. sc. n. 1525. XIII.t.10.f. 1.

Desm...-Dsm. 31.

radicatus. - [S. 279.] - Huss. I. 36. -

Vent. ז. 9. f. \5. \$6. - Berk. Out. t. 5. f. 4.-

Collyb. Bail S. t. 35 .

- radicosus. - [S. 259.$]$

- radiosus Pall. - cf. Montagnites.

- ramealis Bull. - [S.313.] Maras.) - Kl. II.

204. - Brit. f. 10. - W'std. 995. - Kl. 802.

Fuck. s. 1125.

- rameutaceus Bull. - [S. 271. 44.] - Dsm.

1246.

rancidus. - [S, 250.] - Hoffn. He, t. 12.

f. 2. Collyb.'

rapaceus. - [S. 300.$]$

— raphanoides Mich. - Kl. 705. - P. : [S. 303.]

- rasilis. - [S. 2i6.]

- Ravenelii, - cf. Lentinus.

- ravidus. - [S. 293.] - (Russ.) [S. 311.]

- reclinis. - [S. 2S4.]

- recutitus. - [S. $2 \div 2.5$.

- redimitus. - [S. 302.]

- reductus. - [S. 2S1.564.]

- Reedii Cortin.). - Huss. II. 45.

- regalis. - [S. 272. 7.]

- relicinus. - [S. 290.]

- renidens. - [S. 305.]

- repandus. - [S. 286. 290.] - Vent. mi. 75.

f. $1-3$.

resimus. - [S. 309.] (Lact.).

resplendens. - [S. 274.]

resutus. - [S. 297.] 
Agaricus reticulatus P. - [S. 293.] - Bisch. f. 3323. - Berk. Out. t. 9. f. 5. - (Cortin.) [S. 303$.

-.. retostus. - [S. 2S1.]

- rliacodes. - $[$ S. 273. 22.]

- rhodocylix. - [S. 245.]

_ rhodopolius. - [S. 297.] - F].dan. X. 1736.

- I.oud. f. 15935.

- Rieckei. - S. 272. S.

- rigens, - [S. 305.] - Alger. t. 31, (i. rigidus. - - $\$$. 304.$]$

- rimosis Bull. - [S. 291.] — Loud, f. 15992.

- Berk. Out. t. \$. f. 5. - Nov. Act. 1512.

XIX. II. t. 56. f. 33-3 - Phöb.) - Ann. Mg.

n. h. 153 . I. t. 4. f. 26 . Fruct, Berk.). -

Staude t. 5. f. 1. - Ital. 32s.

- rimulincola. - K1. 1511. - R. f. 202.

- ringens Fr. - Kl. 503.

- rivulosus. - [S. 27T.]

robustus A.S. - [S. 563.] - Kil. 1204.

Armill. Kl. II. 4.

- romaleus. - I, Ietell. 64t.

- roridus Fr. Mycen. - - [S. 2\3.] - R. f. \.

rosaceus Schuin. = strobilinus Fl. dan. -

- Russ. [S. 310.$]$

rosellus Fr. Mycen.. - - [S. 2\1. u. 273: 29.]

- Fl. dau. XII. 2025. 2. - Krypt. Bad. 439.

- Kl. 1505. - Kl. II. 206. - R.f. 112 . -

Dsm. III. 460 .

_ roseo-albus. - [S. 256.$]$

- roseus $\mathbf{P}$. Schum. = rosellus $\mathbf{F l}$. dan.

- F1. dan. X. 1673.1.

_ Scop.: [S. 273. 25.]

Rotula Scop. Maras. . - [\$. 314.] - Loud.

f. 15574 - Fuek, s. 1037 . - Kl. 220. - Berk.

Out. t. 14. f. 7. - K1. SU1. - Bot. Zt. 1560.

t. 11. f. 11. Hoffin.) - Dsm. III. 345. - Brit. f. 152. - Wstd. 1252.

- ruber Lam. Russ.). - [S. 310.] - Vent.

t. 7. f. 63. 61. - Ki. II. 613. - Vent. mi. 14. f. $1 ; 93$. f. 3. 4. - Loud. f. 15751 .

- DC.: Bisch. f. 3436. - Ann. sc. n.

1\$37. TIII. t. 10. f. 23. Fructif. Lév.).

- rubescens $\mathrm{Fr}_{\mathrm{r}}$ - [S. 272. 10.

- P.: [S. $\left.307^{\star *}.\right]$ - Kops Fl. bat. X.

765. - Fl. dan. XII. 2140 . Tent. t. 2. f. 9.

10. - Aman.) Loud. f. 157.35. - ? cf. fulro-al-

bicans Roq. - Badham t. 12. 1. (Amanita.-

Huss. I. 23. - Viv. 22. 23. - Vent. mi. 31.

f. $3 ; 78$. f. 2. - Staude t. 10. f. 5 .

- var, - Fl. dan. XII. 2147.

Rubi. - [S. 294*.] - Berk. Out. t. 9. f. т. rubidus Berk. - [S. 294.] - Mag. Zool.

Bot. 1537. I. t. 2. f. 2. p. 43 Berk.).

- rubiginosus Schum. = fuscipes rar. Fl. dan.

- P.: [S. 294.] - Wstd. 1254.

- rubricosus. - [S. 305.]

— rubro-marginatus $\mathrm{Fr}$. - [S. 2S1.] - Kl. 504 .

- rudis. $-[$ S. 273. 26.]

- rufo-carneus. - [S. 255*.

- rufo-olivaceus. - [S. 301.]

_ rufus Scop. - [S. 309.] - Huss. I. 15. -

Fi. 7. - (Lact.) Fr. : Fries Sr. t. 11.

- rugatus Fl. dan. - Wstd. 556.

- rugosus. - [S. 2S2.]

- Russula Schff. - Letell. 616. - Vent. t. 3.

f. $17-19$. - Vent. mi. 72 . f. $1-3 ; 91$. f. 1 .

russus. - [S. 300.$]$

- rusticus. - [S. 253.]

f. 11 . rutaceus Lasch. (Lactar.). - K1.606. - R.

— rutilans Schff. - [S. 275.] - Letell. 651. -
Tent. mi. 96.f. 1.-Fl. dan. XI. 1910. 2. - Hrz. t. 31. - Trichol. Loud. f. 15763. - Bot. Zt.

is54. t. 5. f. 9. (Anat. Bonord.). - Kl. 4. 22.

Igaricus rutilus. - Letell. 603 (Gomphus). -

Hluss, II. 24.

- sagatus. - [S. 294.]

saginus. - [S, 300.]

Sininsonii Iév. - Demid. Russ. t. 1. f. 3.

salebrosus. - Ann. Mg. n. h. 1642. IX. t. 9.

f. I. Berk.).

salicinus Schum. = aurivellus Fl. dan. -

P. : [\$. 256.]

salignus P. - [S. 245.] - Letell. 657. -

liops F1. bat. X. 725. 3. - Fuck, s. 1105. -

Bot. Zt. 1455. t. 6. f. 5. d. (Anat. Bonord.). -

Pleurot. Kl. 1505. - Carol. II. 3.

salor. - [S. 302.]

- sambucinus. - [S. 291.]

sandicinus. - [S. 279.]

sanguineus (Russul.). - [S. 311.] - Roq.

t. 12. f. 1 .

- Cortin.). - [S. 303.] - Bail S. t. 31.

Bisch. f. 3161 .

- sanguinolentus Mycen.). - [S. 252.] - Kl.

1306.

- saniosus. - [S. 306.$]$

___ sapidus. - Roq. t. 10. f. 4.

-_ sapineus. - - [S. 291.]

- saponaceus Fr. - [S. 275.] - Kil. 104. -

Trichol. Kil. 1909.

_- sarcitus. - [S. 2S5.]

__ sarcocephalus. - [S. 296.]

- sardellus. - [S. $290^{* *}$. $]$

sardonius (Russ.). - [S. 311.]

- saturninus. - [S. 305.]

saxatilis. - [S. $313 *$.]

scabellus. - S. $250 *$. 291.]

scaber. - [S. 290.] - Loud. f. 15959. -

Nov. Act. 1542. XIX. II. t. 56. f. $26-32$.

Fructif. Phöb.).

- scabiosus. - [S. $\left.2 \varsigma_{6 .}\right]$
- scabrosus. - [S. 2\5.]

scalaris. - [S. 294.]

scalpturatus. - [S. 275.]

scambus. - [S. 291.]

scandens. - [S. 305 .

scaurus, - [S. 301.]

sceptrum. - Coprinus) Linn. 1530. V. t. 6.

f. 10. Jungh.).

- Schumacheri Fr. - [S. 276.] - Fl. dan.

XIII. 2267. 1.

- sciophanus Fr. - [S. 30S.] - F1. dan. XI.

1545. 2.

— scleropus. - Gaudich. voy. 1\$26. t. 2.

scobinaceus, - [S. 295.

scobinellus. - [S. 272. 20.]

scolecinus. - [S. 292.]

- scorodonius Fr. - (Maras.) [S. 313.] - Hrz.

t. 24. A. - Bot. Zt. 1560. t. 14. f. 14. (Hoffni.).

Loud. f. 15565. - Bisch. f. 3315. 3417. - Kl.

16. - Kl. II. 609. - R. f. 105. - Fries Sv. 32. scorpioides. - [S. 293.]

scorteus. - [S. 313.] (Maras.).

- scrobiculatus Scop. - (Lactar.) [S. 305.] -

Ki. 203.

- scutulatus. - [S. 304.]

- scyphiformis Fr. (Omphal.). - [s. 254.] -

R. f. 303.

- scyphoides (Omphal.). - [S. 253.] - Kl.

1604. - R. f. 304 . - Carol. IV. 1.

- sebaceus. - [S. 300.]

segestrinus. - [S. 293.] 



$$
\mid
$$


Igaricus sejunctus (Trichol.). - [S. 271.55.] Loud. f. 15765 .

- Sementino. - Viv. 19.

- semieremus Russ. . - [S. 310.

- semiglobatus B. - [S. 295.] - Loud. f. 16016 .

- IIuss. I. 39

- - semilanceatus. - [S. 296.]

_ seminudus. - [S. 273. 36.]

semiorbicularis. - [S. 293.] - Berk. Ont.

t. 9, f. 2. - ? Ann, sc. n. 1437. VIII. t. ?. f. 19.

Lév. .

- semiovatus Sow. - Joud. f. 16032. - Inn.

Mg. n. h. 1439. I. t. 5. f. 30. Fructif. Berk.

- - Scluum. = semiputris Fl. dan. - Lindl.

v. K. p. 33, f, 2. Fructif.

semiputris Bull. - Fl. dan. XI. 1959. 1, 2.

- semitalis. - -S. 279.

- semirestitus B. B. - $\mathrm{Mnn}$. Mg, n, h. 1:b1.

VII. t. 11, f. 5. p. 37\%.

—_ separatus. - [S. 297.] - Kops Fl. bat. XI.

\$20. 1. - Berk. Out. t. 11. f. 7. - Kil. f0(i.

— septicus Pleurot.). - S. 25.5.] - K1. 13115.

- Ietell. ioti. I chioneus'.

- sepuleralis. - II ook. Lond. J. 1-12. I. t. 15.

33erk.

- sericellus Fr. - S. 2y.] - Jisch. f. 325!1.

- Iil. 12116.

_ sericeus - [S. 2S:.] - Kl. 1401. - ILoffin

Ic. t. 11. f. 2. Wintolom.

- serifluus Lactar. - Berk. Out. t. 13. f. I.

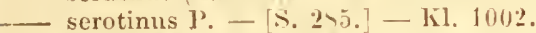

- serrarius. - - \$. 301 .

- serrulatus. - S. 257.

- - sertatus. - Viv. 11.

— sessilis Bull. - Wstd. 1253.

- setipes. - [S. 254 .

— sideroides Nitucor. . - [S. 292.] - Bail S

t. 36 .

_- silaceus. - [S. 293.]

- simiputris. - ef. semiputris.

- sinapizans. - S. 290.

sindonicus. - S. 291 .

sinopieus. S. 2 i . $]$

- sinuatus schum. = phalloides v. Fl. dan.

- [S. 2s6.]

__ sinuosus. - S. 259 .

[ siparius. - S. 293.]

- sistratus. - [S. 273.

- sitaneus. - Bot. Zt. 1555. t. 5. f. 12 Anat.

Bonord.)

- Smithii. [S. 310.49.$]$

sociatus Schum. = ephemerus Fl. dan.

solitarius. - [S. 272. 13.] - Vent. mi. 60.

f. 1.2 .

sordidus - [S. 276.] - Fl. dan. XI. 1S43. 2.

- Triclıol.) Kl. 1503.

- spadiceo-griseus Schff. - [S. 29i.] = stipa-

tus Fl. dan. - Pringsl. Jhrb. II. t. 32 . f. 43 .

(Keimg. Hoffm.).

- spadiceus. - [S. 296.] - (Cortin.) [S. 300.]

- (Hygroph.) [S. 30S.] - Schff: Carol. V. 6.

- sparteus. - [S. 293.]

spathulatus. - [S. 295.$]$

speciosus. - [S. 255.] - Fl. dan. X. 1737.

- Berk. Out. t. 7. f. 3. - (Volvar.) Bail S. t.

37. - Kl. 1601.

spectabilis. - [S. 259.

Speculum. - [S. 2ST.]

speireus. - [S. 2S2.]

sphagnicola. - [S. 2\$3. $313^{* *}$.]

Sphagnorum. - Carol. V. 5.
Agaricus sphaleromorphus Schum. = squamo-

sus v., Fl. dail. - [S. 2SS.]

_ sphinctrinus. - [S. 297.]

- spiculiferus. - Hook. Lond. J. 1St2. I. t.

14. Berk.).

spilomeus Fr. - [S. 303.] - Loud. f. 15960.

— - spinipes Sow. - Loud. f. 15901.

- spintriger. - [\$. 295.]

— spiralis Coprin.). - Canar. t. 1. f. 5.

spissus. - [S. 272.9.$]$

splachnoides. - [S. 314.] Maras.) - Fl.

dan. X. 167 S. 1.

- splendens P. - IIrz. t. 35.

spodoleueus. - [S. 255.] - R. f. 205.

Maras.).

- spodophyllus. - [S. 294.] - Vent. mi. 5i.

f. 5.6 .

- spoliatus. - [S. 290.]

spongiosus Sehum. = butyraceus Fl. dan. -

Lasch: S. 2 4.] - Maras. Carol. III. 7.

- spumosus Flammula! - [S. 291.] - Kl.

II. (jt)1.

squamosus P. Fr. - [S. 295.] - Fl. dan.

XII. 2077. 1, 2. - Berk. Out. t. 10. f. 6.

- Schum. = adiposus Fl. dan.

- Squamula B. = epiphyllus Fl, dan. - Wstd.

$5<6$.

squamulosus P. - [S. 274.] - Kl. 311 .

squarrosus Müll. - [S. 2\$!!.] - Huss. I. S.

- I) $\sin .1122$.

— - Fr.: Kops Fl. bat. X. it3.

_ I': Loud. f. 15974. - IIrz. t. 20. -

Cd. Je. V. t. 10. f. y". Fruct., - Bisch. f. 3!64.

- [il. 301. - Tholiot. Kl. II. 401. - Brit.

f. 17. - Vent. mi. 55. f. 3; 63. f. 5. 6.

stagninus. - [\$. 294.

- stamneus. - [S. 2\2.]

— stellatus Fr. [S. 24.] - Ioud. f. 15902.

— stemmatus. - [S. 305 .

_-Stephensii v. IIudsonii. - Ann. Mg. 11. h.

1454. XIII, t. 15. f. 3. p. 404. Berk. B.).

stereorarius Schun. - [S. 295.] - Fl. dan.

XII. 2141. 2.

- sterilis. - Linn. 1530. V. t. 6. f. S. (Jungh.).

- sticticus. - [S. 292.

- stillatitius. - [S. 302 .

— stiparophyllus. - [S. 275.]

— stipatus. - Bot. Zt. 1556. t. 5. f. 23. (Anat.

Lamell. Hoffm.).

v. caespitosus, - Fl, dan. X, 1673. 2. stipitarius (Collyb.). - Dsm. 1618; - u.

III. 275. - Kl. 1507. - Berk. Out. t. 5. f. 6.

graminealis Brit. f. $132 ; 341$.

- stipularis. - [S. 2\$3.]

— stipulieola. - Kl. 1402 . (Maras.).

- stolonifer. - [S. 250.]

— Storea. - [S. 296.]

— stramineus. - S. 274.43.

strangulatus. - [S. 272.13.]

- striatulus. - [S. 255.] - Dsm. III. 273. -

Carol. IV. 2.

- striatus. - Anu. Mg. n. h. 183S. I. t. 4. f.

13. (Fruetif. Berk.).

stridulus. - [S. 2;9.]

strigiceps. - [S. 291.]

strobiliformis Vitt. - Vent. mi. 45. f. 1-3.

Vent. t. 2. f. 11. 12. - Berk. Out. t. 3. f. 2.

- strobilinus Fr. - [S. 2\$1.] - Fl. dan. XII.

20125. 1:

2- P.: Loud. f. 15559.

strophosus. - [S. 2S9.]

strumosus. - [S. 279.] 
Agaric us stylobates P. - [S. 2\$3.] - Dsm. III. 666. - Fl. dan. XII. 2025. 3. - Bisch. f. 3264. - Berk. Out. t. 6. f. 5. - Sturm h. 2. t. 29

- stypticus (cf. Panus). - Voges. 54ll. Loud. f. $1592 \pi$. - Dsm. 161. - Pringsh. Jhrb. II. t. 32. f. 45. (Keimg. Hoffin.). - Kil. 1s; Cent. VIII. suppl. - Brit. f. 136. - Huss. Il. S. - Lib. 120.

- suavcolens Schum. - [S. 27S.] - Fl. dan.

XI. 1912.1. - K1. 505.

suarissimus Lentil. Fr. - ? Vent. mi. 34.

f. 1.2 .

subalutaceus. - [S. $27 \%$.]

subatratus. - Bot. Zt. 1555. t. 5. f. 1. Anat.

Bonord. .

- subcavus Schum. - [S. 274. 49.] - Fl. dan.

XI. 1443. 1.

- subcernuus Sichum. = squamosus Fl, dan.

- subdulcis Lact.). - [S. 310.] - Fl. dan. X.

1671. - Loud. f. J 5501. - Hrz. t. 53. - Ki]. S.

- Viv. 57. c. v. castanco-rufo et camphorato

$=$ Vent. mi. 55. f. 3.4.

- subferrugineus. - [S. 305.]

sublanatus Sow. - Huss. II. 22. - Loud.

f. 15956 .

- sublateritius. - S. 295.] - Vent. mi. 57.

f. 1. 2 .

subliquescens Schum. - [\$. 296.] - F1. dan.

x. 1732.2 .

- subluteus. - [S. $2 \$ 9$.

— subnotatus. - [S. 303 .

- subpalmatus. - [S. 2\$4.

- subpulverulentus Trichol.,. - Huss. II. 39.

- subpurpurascens. - [S. 300.]

- subradiatus. - [S. 30 S.]

subtilis. [S. 297.]

subtortus. - [S. $300.301,56$.

subulatus Schum. = phyllopliilus Fl. dan.

- subumbonatus. - [S. 310.] Lact.

- succineus. - [S. 250.]

sudorus. - [S. 252.

sudus. - [S. 275.]

suillus. - [S. 302.

sulcatus. - S. $290^{*}$.

sulphureus Bull. - S. 275.] - Fl. dan. XI.

1910. 1. - Troq. t. 16. f. 1j. - Berk. Out. t. 4.

f. 1. - Loud. f. 15s 16 , Kl. 207. - Cortin.

[S. 259*.] - Letell. 671. - Vent. mi. 53.f. \$. 9.

[ superbus. - Linn. 1s30. T. t. 6. f. 11.

Jungh.). - Lasch: K1. 710.

supinus. - [S. 2٪2.

- sylvaticus. - [S. 291.] - ? Vent. mi. Sti.

f. 1.2 .

tabacinus. - [S. 292 .

tabidus. - [S. 310.] (Lact.)

tabularis. - S. 303.$]$

Tagetes I3. II. - Anu. sc. n. 1860. XIV.

t. II. f. 3. p. 142 Mont.,

tageticolor. - Hook. J. VIII. t. 5. f. 1.

talus. - [S. 301\%.]

- tegularis Schum. - [S. 296.] - F1. dan. XI.

1955.1.

- telinolens. - Act. Turin. 11. (1501). t. 2.

'Bellardi .

- temulentus. - [S. 293.]

- tenaccllus P. - [S. 2-0.] - FI. dan. XII.

21121. 2. - cf. Ag. conigenus HH.

— tenax Fr. S. - [\$. 293.] - Jil. II. 605.

- tencllus. - [S. 2\2.]

- tener. - [S. 293.] - Loud. f. 15999. - Ann.

Mg. n. h. 1<34. I. t. 4. f. 25. Fructif. Berk. .

- Il. 312 .
Agaricus tener Schum. = lacteus Fl. dan.

tenerrimus. - [S. 2S3.] - Berk. Out. t. 6. f.6.

'Tentaculum Sow. - Loud. f. 110030.

- tenuipes Aman.). - Alger. t. 30, 4.

tenuis. - [S. 2S2.]

tephroleucus. - [S. 307.]

- terginus. - [\$. 313.] (Maras.).

terreus. [S. $275.257^{*}$.] Kl. 511. - Dsm.

III. 312. - Vent. mi. 39. f. 3. 4.

terrigenus. - [S. 249.]

tessulatus. - [S. 2S4.]

testaccus. - [S. 290.]

thejodes Saut. - Kil. 1106.

thejogalus Bull. - \$. 309.] (Lact.). - Fl.

dan. XI. 1912. 2.

- tigrinus Bull. - Vent. mi. 65. f. 5-7; S4.

f. 5. 1. - Vent. t. 5.f. 15. 46. - Loud.f. 15914.

- kil. 313.

- Tintinnabulum B. = disseminatus Fl. dan.

- S. 292.

- tithymalinus Scop. Lact.). - [S. 310.] -

I.oud. f. 1.5)(J).

- titubans Bull. - Loud, f. 16036. - ef. Bolbitius t.

—_ tofaceus. - [S. 302.]

togularis. - [S. 245.

- tomentosus. - [S. 30s. 310, 11.] (Lact.) -

Linn. 1 3 31. V. t. 6. f. 7. Jungh.) - Krbh.: S. 272.5.$]$

- torminosus 'Lactar. - - [S. 309.] - Kops

Fl. bat. XI. S25. 3. - Vent. mi. 53. f. 3-);

72. f. 4. - Staude t. 6. f. 5. 6. - Vent. t. 6 .

f. 53. 54. - Dsm. 1249. - Hrz.t. 11. - Loud.

f. 15757 . - Phöb. Gift. t. 5. - Bot. Zt. 1556.

t. ว. f. 21. g-k. Pollin. Hoffm. . K1. 5. -

R. f. 1. - Fries Sv. t. 29. cf. Ag. necator Roq.

(1) rnatus. - S. 277.]

torosus. - s. 24s.

torpens. - S. 296.

torquatus. - [S. 314.] (Maras.).

- tertilis. - Roq. t. 16. f. 7. 8. - Loud. f.

$15>17$.

- tortuosus. - [S. 305.]

- trvus. - [S. 304. $302 \%$.]

trachelinus. - is. 253.

traganus Fr. - [S. 302.] - Vent. t. 9. f. 77.

- Vent. mi. 40. f. 3 ; $\$ 5$.

- trechisporus. - [S. 295* *] - Ann. Mg. n.

h. 1534. t. 5. f. 53-56. Fructif. Berk.) -

Berk. Out. t. S. f. 6 ,

tremulus Schff. J'leurot.). - [S. 255.] -

Loud. f. 15933. - Bot. Zt. 1 460 . t. 13. f. 5.

IIoffm.) - ef. Kil. 16115 , Nota.

trepidus. - $[\mathrm{S} .297$.

Tricholoma. - S. 291.]

trichopus. - Gaudich. voy. 1526. t. 1.

tricolor. - S. 254.] - Act. Turin. 1505.

XIV.t. 1. Cumino. - Cortin.) Canar. t. 5 f. 3. tricuspis Scop. = capillaris Fl. dan.

- triformis. - [S. 30 \}.]

- tristis P. - [S. 275.] - Lact.) [S. 309. 34.]

- Ki. $\{507$.

triumphans. - S. 300.$]$

trivialis. - [S. 309.] (Lact.).

trullaeformic. - [S. 2is.]

truncatus. - S. 290.]

Tuba. - [S. 2is.]

'lubae Paul. - Vent. mi. 79. f. 5. 6.

tubaeformis Schwz. = Lentinus friabilis Fr. — Schff. : Ann. sc. n. 1421. I. 23.

tuberculatus. - S. 249: 


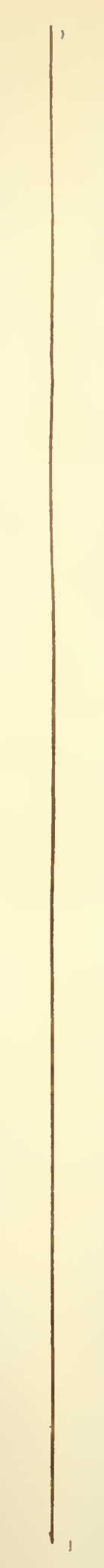



Agaricus tuberosus Fr. - S. 24(1.] - Brit. f. 129. - Fl. dan. XII. 2022. 1. - Fuck. s. 1122. _ _ Bull.: Loud. f. 1566ৎ. - Collyb.' R. f. 9. - Kl. 1202 ; Cent. XV. suppl.

Schum. = dryophilus var., Fl. dan. - tumescens. - Viv. 31.

tumidus. - [S. 275.] - Vent. mi. 95. f. 1.2. - turbidus. - S. $25 \%$.

- turbinatus Bull. - - S. 300.7 - Loud. f. 1596j.) - Schum. = pratensis Fl. dall. - Vent. mi. 37. f. $4-6$; $>$. . f. 4.5.

- turgidus Grev. Clit. . - Lond, f, 15-21. Cortin. S. 302 .

- turmalis. - \$. 300 .

- turpis Lact. - S. 309.] - Kops Fl. bat.

XI. 835 .

- turritus. - S. 276.

- turundus. - [S. 304 .

tylicolor. - S. 241.

- Typhac K. - R. f. 306.

- tyrianthinus. - \$. $27 \%$

udus P. - [S. 296. - F]. dan. XII. 2020. 1.

-Kl. 1(10)3. - Psiloc. Kl. II. 6U1.

ulmarius I3ull. - S. 241. - Loud. f. 15921

Pleurot. Bails. t. 37. - Kl. 1906. - Fries Sv. 37. umbellifer Omphal. .- S. 251. - Kil. 130!!

- Bolt. = epiphyllus Irl. dan. - R. f.

203. - Bot. Zt. 15kill. t. 11. f. 6. Iloffm.

- Bolt. : Wstd. 5-6. - Krypt. Bar. 1lit.

- - ericetor. - Brit. f. 11 pyxid.

- umbilicatus Bull. = candicans Fl. dan. -

S. $2>3$.

- umbratilis. -. .241.

umbrincllus. [S. 2\6.

- umbrinus, - S. 27.7. - Co:tin. S.3111,

$32 ; 301,127.7$ - Lact. S. 309.

umbrosus. - S. 2sij.

uncinatus. - Vent. mi. 73. f. 1. 2.

- undatus. - S. 247.? - Brit. f. 9. - R. f.

403. - \$. 313.? Maras.

- undulatus. - Linn. 1-30. V. t. 6. f. 14.

Jungh. .

— undulosus J. - Dsm. III. 345.
- unguentatus. - [S. 27 - 57.$]$

— inguicularis. - S. 245.

— unguinosus. - [. 304.]

—_ unicolor. - S. 259 .

- uraceus. - [S. 305.$]$

- uranius, - [S. 282.

- urbicus. - S. 304 .

— urbus. - S. 276.] - Fl. dan. XI. 1 444.1.

- urceolatus. - Viv. 11; 27: r. spadic.

- urens Maras. Bull. - S. 312.7 - Kl. II.

103. - perollatus $\mathrm{v}$. tomentos. Fl. dan. Schum. = pergamenus Fl, dan. - Berk.

Out. t. 14. f. 3. - R. f. 101 .

- ustalis. - [S. 274.]

- uvidus. - [S. 309.]

- vaccinus Schff. (Trichol.' - [S. 27.5.]-

Bisch. f. 3279 . - Loud. f. 15761 .

- vaginatus Bull. - [S. 272.1.] - Fl. dan. XII.

2142. 2. - Kops. Fl. bat. X. i5t. - Hrz. t. 14.

- Vent. t. 2. f. 15. 16. - Loud. f. $15734 .-$

Berk. Out. t. 3. f. 4. - Cord. Anl. t. H. f. 77 :

12-15. - Cord. Ic. V. t. 10. f. 97. Fructif. .

- Kl.601. - Huss. II. 34. - Tir. 59. - Vent.

mi. 10. f. $1-4 ; 11$ f. 5.6 ; 5 . f. 1.2 .

Vahlii. - [S. 24s.]

Vaillantii. - [S.313.] Maras.). - Carol. II.9. valesiacus. - Kl. $1>02$.

validus. - [S. 272. S.]

vapidus. - [S. 272. 19.]
Agaricus vaporarius. - [S. 273. 25.] - Bot.

Zt. 1559 . t. fi. f. 6. Anat. Bonord.'. Kirbh.: Fent. mi. 2. f. 1.2.

variabilis $\mathrm{T}$. - - S. 291.] - Huss. I. 50. -

Loud. f. 159:31. - Berk. t. 10. f. 1. - Loud.

f. 16009. - Lib. 221. - Wstd. 12๕3.

tin. [S. 301.?

_ variegatus. - -S. 275.]

— varius Schff. - $[$ S. $3100.301,49$.$] - Loud.$

f. 15964 . - Bot. Zt. 1456. t. 5. f. 24. Anat.

Lamell. Hoffin.).

- vatricosus. - S. 291.]

_ vellereus Fr. Lact. . - [S. 309.] - Loud.

f. 15611. - Phöb. Gift. t. 4. f. $3-12$ - Bail S. t. 33. - Kl. 9. - Brit. f. 122. - Huss. I. 63 .

- velutinus. - \$. 296.] - Kops Fl. bat. X.

769. - Berk. Out. t. 11. f. 2. - Berk. J. p. $2+1$.

f. 60. a. Fructif. - Borcz, fg, ingr. t. 1 .

f. 2. Hygropl.'

_ velutipes Curt. - S. 279.] - Huss. I. 56. -

Iil. 314. - Wstd. 1115\% - Fuck. s. 1124. -

Fl. dan. XIII. 2269. 2. - Kops Fl. bat. X. 799. Loud. f. 1s<5ti. - Iloffm. Ic. t. 4. - Bot.

Zt. 145!!. t. 11. f. 31. Keimg. Hoffm. - Ann.

Mg. n. h. I. 143八. t. 1. f. 16. Fructif. Berk.). -

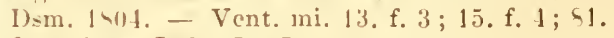

f. $3-5$. Ital. 37.47 .

_ venctus. -

venustus. - Alger. t. 30.3.

verecundus. - [S. 245.]

vermicularis. - is. 275.]

- vernus 13ull. - Vent. t. 2. f. 13.11. - Loud.

f. 15731 .

- verrucosus. - transiens in pantherin. Letell. $1 ; \%$.

— versipellis. - [S. 2\9.]

vervacti. - S. 293.]

- vescus (Russ.). - [S. 311. carn. - rub.] -

Huss. I. $\$ 9$.

- vespertinus. - - [S. 30 ].

- veternosus Russ.]. - [S. 311.] - Vent.

t. 7. f. 59. 60 .

- vibecinus. - [S. 2 - 5.$]$

__ vibratilis. - [S. 302.]

— vietus. Lact. ]. - [S. 309. 15 u. 34.] - Kil.

1907. - Cord. Ic. T. t. 10. f. 96. Fructif.). -

Kützg. Phil. Bot. 1551. t. 9. f. 1. (Anat.).

$\longrightarrow$ Fr. $=$ acris Fl. dan.

- vilis. - [S. 257.]

villosus. - S. 2S9.]

- vinaceus. - [S. $265 *$.]

- vinosus Cd. Pratell. - Sturm. h. 19 -.

t. 1 . Cd.;.

violaceo-ater. - I.etell. 701.

- violaceo-cinereus P. - Kil. 701. - (Cortin.)

Kl. II. 1.

- violaceus. - [S. 302.] - Huss. I. 12. -

Kops Fl. bat. XI. S 44 . - Vent. t. S. f. T4. 75. - Roq. t. 17. f. 1.- Hrz. t. 16. - Loud. f.

15955. - Vent. mi. S3. f. 1. 2 ; 87. f. 1.2.

- violascens. - [S. 305.] Lact.).

_ _ virescens Schff. - Russ.) [S. 310.] - Tent.

mi. 55. f. 1. 2. - Vent. t. 7. f. 57.55 . P.: Bisch. f. 3325 - - (Russ.) Berk.

Out. t. 13. f. 6. - Huss. II. 11. virgatus. - [S. 275.]

- virgineus. - [S. 305.] - Dsm. 1622.1623. --

Fl. dan. XI. $190 \%$. 1.

Wulf.: Tent. t. 5. f. $36.3 \%$. - Hiz. 
t. 15. A. - Loud. f. 15535. - Schntzl. t. 16. f. 25 . - Biseh. f. 3413 . Kl. 604. - Wstd. 61. f. 3 .

A garicus viridifluens Lasch. - Kl. $5 ! 0$. viridis. - [S. 277.] - Roq. t. 12. f. 3.4. -

Vent. mi. -1. f. 1. 2. (Cortin.). virosus. - $[$ S. 272. $]$ - Vent. mi. 9. f. 2 ;

12. f. 2.3 .

- viscidus L. - Bisch. f. 3 469. - Nov. Act. 1ง 42 . XIX. II. t. 56. f. 79-52. Spor.). - ef. Gounphid.

_ - vitellinus (Russ.). - [S. 311.37.]

- viticola. - Carol. V. S. (Maras.).

_ vitilis. - [S. 2\$2.]

- vitreus. - [S. 242.]

- vitrosus. - [S. 295 .

— Vittadinii. - Huss. I. $\ 5$.

vittaeformis. - S. 291.]

volemus. - [S. 310.] Lact.). - Letcll. 621.

- Huss. I. G 7. - Staude t. 7. f. A- . - Ital.

411). - Fries Sr. 36, A.

_ volvaceus Bull. - [S. 255.] - Fl. 'dan. X. 1731.2. - Bisch. f. 3454 . - Vent. t. S. f. 72 . 73. - Nov. Act. 1 32 . XVI. I. t. 6 u. 7. Ns./. - Bisch. f. 3452 .

--1 Amanita virgata $P$. - Letell. 623. -

Viv. 10. - Tent. mi. 49. f. $1-3$; 50. f. 1.2. Ital. 138 .

- vulgaris $\mathbf{P}$. Mycena - - [S. 253.] - R. f. 7.

- Berk. Out. t. 6. f. 4. - Pringsh. Jhrb. II.

t. 32. f. 46. Keimg. Hoffm.) - Bot. Zt. 1560 .

t. 14. f. S. IIoffm. - - cf. metatus Hoffm. -

Brit. f. 133.

vulpinus Sow. — Loud, f. 16006.

_- Webbii. - Canar. t. 4. f. 7.

Wynnei Maras.). - Berk. Out. t. 19. f. 3.

xanthogrammus (Hyporh.). - Commt. soc.

cr. it. 1561. 2. t. 3. f. 1. (es.).

xanthopus. - [S. 250.]

xerampelinus (Russ. - - [S. 310.] - Vent.

mi. 10.f. 5; 13.f. 4.5;39.f. 5. 6 ; 56.f. 3.4; 92 .

- xerophyllon. - Hook. Lond. J. 1St2. I.

t. 7 t. Berk. xylophyllus.

- - viscosus $P$. = blennius Fl. dan. zephirus. - [S. $2>1$.

zinziberatus. - [S. 305.

zizyphinus. - Viv. 21.

- zonarius Bull. - [S. 309.] - Vent. t. 6.

f. 51. 52. - (Lact.) Kops Fl. bat. XI. 525. 2. -

Vent. mi. 91, f. 2.

Agla osp ora. - cf. Sphaeria.

- ocellata. - S. $411 *$.$] - Act. Tur. 1$ S 19.

X. Dec. 5. f. 1. (Notar.). profusa. - [S. 411.] - Ital. 235. - Act.

Tur. 1549. X. Dec. 5. f. 3. Notar.).

Agyrium. - S. 359.342.]

- atrovirens. - [S. 471 .

- caesium. - [S. 360.] - Cd. Anl. t. F. f. 62:

$1-5$.

- chartarun. - Lotos Prag) 1555. t. 1. f. 19.

Peyl.).

flarum. - [S. 360.]

gilvum. - S. 360.

herbarum. - [S. 360.]

nigricans Fr. - [S. 360.471.] - Kl. 149.

nitidum. - Lib. 235.

- pallens. - [S. 360.]

rufum. - [\$. 360.] - Voges. 1096. - Cd.

Anl. t. F. f. 62: 6-9. - Bail S. t. 19. -

? Stictis r. $\mathbf{P}$.

Ailographum. - ef. Aylogr. - [S. $406^{*}$.]
Aleurisma. - [S. 495.] erubescens. - Letell. 635: 4. 5. (zu Sporotrich. sporulos.).

Alphitomorph a. - ef. Erysiphe, Podosphaera, Uncinula. - [5. 195.] - Flora 1538. II. t. 1. Wllr. .

Acerum Wlir. - Wstd. 5.50.

adunca. - Wstd. 80.969 . (Rosac.).

Alni Wllr. - Wstd. 531.

Artemisiae. - Wstd. 111.

Bardanae. - Wstd. 111 .

bicornis Wlly. - Wstd. 550 .

circumfusa. - W'std. $\$ 10$.

cland. v. Aphanes Wlir. - cf. Sphaerotheca

Castagn. - Wstd. 250.

communis. - Wstd. 120.409. $405.554 .73 \overline{7}$.

530. 1359. - Bon. t. 2. f. 60.

Corni Wllx. - Wstd. 552.

depressa. - Wstd. $\$ 11$.

divaricata. - Wstd. 412. 135\%.

ferruginea S. - cf. Sphaerotheca Castagnei.

fuliginea. - Wstd. $2>1$.

fumosa Wllr. - ef. Sphaeroth. Cast. -

Wstd. $2 \succ 1$.

- guttata. - Bon. t. 2. f. 59. - Bot. Zt. 1557.

t. 4. A. 1-12. Bonord.) - Wstd. \$13. (Coryli. - Wstd. 655. (f. Fraxini); 739. (Fagi). horridula v. Cichor. - Wstd. 226.

— Asperif. Wllr. - Wstd. 553.

lamprocarpa v. Balsamin. Wllr. - ef. Sphae-

rotheca Cast. - Wstd. 109.410.

- lenticularis v. Fraxini Wllr. - Wstd. 65.

$7: 39$ : Fagi.

- macularis v. Dryad. IVllr. - ef. Sphaerotheca Cast. - W Wstd. 40 T.

f. Poterii IVIlr. - ef. Sphaerotheca Cast. pannosa W7lr. - cf. Sphaerotlieca pann. penicillata. - Wstd. 112 . 735: Berber. \&31, Alni.

tortilis Wrllr. - Wstd. 552 .

tridactyla Wllr. - ef. Podosphaera Kunzei.

Alph i tom y ces Schrötteri. - Sitz. Ber. Wien.

Ikad. 15ว̌6. Juli. XXI. H. 2. Reisseck.).

Alternaria. - [S. 505.]

- bullata. - [S. 505.] - Wstd. 857.

- chartarum Pr. - Kl. 12\$4. - Sturm, h. 35. t. 1 .

lignicola. - [S. 505.]

tenuis. - [S. 505.] - Cd. Aul. t. B. f. 6 :

6. 7. - Pay. b. cr. p. 69. f. 304 . - Kl. 1772.

Aly sidi u m aequirocum. - Bon. t. 1. f. 15.

- caesium. - Fuck, rh. 73 .

-_ cinereum. - Bon. t. 1. f. 15.

_ epimrees. - Bon. t. 1. f. 14.

- fulvum. - Fuck, rh. 72.

- fuscum. - Bon. t. 1. f. 13.

—- punctatum Bon. - Bot. Zt. 1861. t. S. f. 2. p. 194.

Alyt. - [S. $177^{* *}$.]

Alytosporium. - [S. $\left.496^{* *} .521.\right]$

$\Lambda$ manita. - cf. Agaricus.

- aspera P. - Hrz. t. 61.

- caesarea. - Til. II. 101.

- muscaria. - Schacht, Phys. Bot. 1513. t. 1.

f. 2. 3. 4. Hymen. Zellen . - ej. Lchrb. An.

Phys. I. IS56. p. 166. f. 25. u. p. 74. f. S. Anat.).

- rubescens P. - Hrz. t. 36 .

- umbrina. - Nor. Act. 1 12. XIX. II. t. 5 fi.

f. 1. Spor. Phöb.).

- virgata $P .-$ Nov. Act. 1 \$2. XVI. I. t. 7.

(As.). - cf. Agar. volvaceuts Bull. u. Fl. dan. 


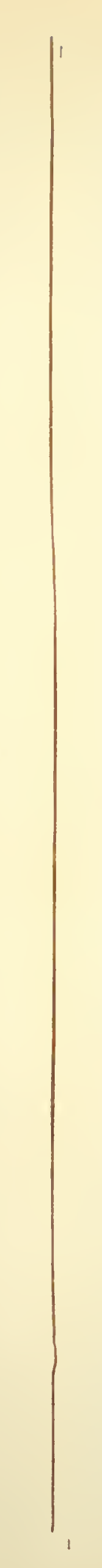


| 
Amblyosporium Botrytis. - Fres. t. 12. f. 17-22. p. 99.

A moe ba. - Act. Turin. 1-25. 29. t. 1. Losana. Myxomycetes p. pte.

A m pelom y ces quisqualis. - Kl. $1669.1669 \mathrm{~b}$. (Cent. XLX.) - Journ. hort. soc. 1554. IX. p. 69. f. 4. - huc Oid. Tuckeri.

Amphiblistrum. - [S. 337. 491.]

Amphichorda. - [S. 464.]

Amphiconium. - [S. 521.]

- petraeum Ns. - Wstd. 699. - Chroolepus aureus.

Amphisphaeria cf. Sphacria.

Amphisporium. - [S. 460.515: 23.]

- versicolor Ns. - Loud, f. 16551 .

Amphitrichum. - [S. 493*.521.]

- olivaceum. - Cd. Anl. t. B. f. 11:1.Kl. 1675.

Amphiura. - [S. $419^{\circ}$.

Angelina. - cf. Ilysterium. - [S. 354.]

Angiopoma. - [S. 415.]

- campanulatum. - Ann. sc. n. 1s41. XVI.

t. 14. Lév.). - ('d. Anl. t. F. f. $55: 5-7$. -

Pay. b. er. p. is. f. 362 u. 36t.

Angioridium. -- s. 451 .

- sinuosum. - Cd. Anl. t. C. f. 2s: 1-1. -

Bisch. f. 3623 . - Lindl. v. K. p. 29. f. 8. -

K1. II. 761 .

Anixia. - [S. 145.]

- fuscescens. - Sphaerosoma f.

- truncigena. - Hoffin. Ic. t. 17. f. 2. Licea sulphurea.

- villosa. - [s. 445 .

A nodotrichu in earneum. - Sturm. h. 25 -

t. 10. (Preuss.).

Anospira. - [S. $455^{*}$.]

Antennaria. - [S. $\left.407^{\circ} .445.\right]$ - cf. Antennularia, Racodium, Torula.

- cellaris. - Wstd. 195.

- cytisophila. - [S. 445.$]$

- elaeophila. - Dsm. III. 100. - Ital, 790.

- laevigata. - Cd. Anl. t. F. f. 53:1-3. -

Pay. b. cr. p. 9S. f. $45 \mathrm{t}$.

- pannosa. - Hook. Lond. J. II. 1543. t. 23.

f. 1. Berk.).

pinophila. - [S. $505 \cdots$. $]$ - Torula fuligi-

nosa Let. - Ital. 791. - Sturm h. 6. t. 10.

— pityophila. - cf. pinophila. - Ital. 791.

Robinsonii. - Hook. Lond. J. II. 1\$43. t. 23.

f. 2. Berk.). - Berk. J. p. 275. f. 63. c. scoriadea M. - cf. Capnodium mucronat.

semiovata. - Ann. IIg. n. h. 1954. XIII.

t. 16. f. 15. p. 469. (Bk. B.).

- Tela. - Kil. 1:355.

Antennataria. - [S. $505^{* *}$.]

Antennatula. - [S. $40 \%^{*}$.]

Antennina. - [S. $407 * 445$.

Antennularia. - cf. Racodium.

- cellaris Rchb. - Bisch. f. 3700 .

- pinophila Rchb. - Bisch. f. $365 s$.

Anthina. - [S. 465.] — ef. Pterula.

aeruginosa. - Fuck. rh. 169.

canofusca. - [S. 465.]

dichotoma Fr. - Bisch. f. 3779 .

filaris. - [S. 465.]

- Hammea P. - [S. 465.] - Brit. f. 206. -

Bisch. f. 3772 . - Berk. Out. t. 21. f. 3.

- flavo-virens. - [S. 465.]

- fulva. - [S. 465.]

ornithopodioides. - [S. 465.]

1 pallida. - Hedw. I. t. 6. f. 1-11. - Kl. 1773.
Anthina purpurea Fr. - Kl, 270.- Hedw. I, t. 6. f. 12-14.

— subulata. - Cd. Anl. t. G. f. il: s. 9.

- umbrina Fr. - Fuck. rh. 16\%. - cf. Isaria.

An trom yces Copridis. - Fries. t. 4. f. $10-45$. A piosporium. - [S. 404.]

— Mali. - Kl. II. 720. — Fuck. s. 493.

- polymorphum. - Cd. Anl. t. F. f. $54: 6$. 7. ef. Ap. Salicis.

- Salicis. - [S. 104.] - Bail S. t. 16.

A poria. - ef. Hysterium. Dub. Hyst. 51.).

- selerotioides, - Dub. Hyst. t. 2. f. 25.

A potemnoum. - [S. 191.]

- maculans. - Bon. t. 2. f. 56 . - Sturm h.

13. t. 39.

A pyrenium.- - S. 470.]

- lignatile Fr. - [S. 470.] - Sturm. 1. 34.

t. S. Strauss

Arachnion. - [S. 443.]

- album. - Carol. IV. 75. - Cd. Anl. t. D.

f. $41: 1-6$.

A reyria. - [S. 457 .

- alutacea Schum. = nutans Fl. dan.

- amomala de $\mathbf{B}$. = serpula Wigd.

- carnea Schum. = fusca Fl. dan.

- cinerea. - S. $45 \%$.] Fl. dan. XI. 1975. i.

- Fr. : Zeitschr. f. wiss. Zool. 1559. X.

t. S. f. 1-5. (de Bary).

- P.: Fuck. s. 2 - 0. - Pringsh. Jhrb. III.

| $\backslash$ il. t. 3. f. 10-12. Wigd.). - Purton. I.

t. 3.) - R. f. 569 - Ann. sc. n. 1s62. XVI.

t. 16. f. $10-12$. Wigd.)

- circinans. - [S. 175 : Lach.

- cylindrica Schum. - Fl. dan. XII. 2090. 3.

$\rightarrow$ decipiens. - Ann. Mg. n. h. 1942. IX. t. 10.

f. 3. (Berk.).

- flava. - ('d. Anl. t. C. f. $31: 3--4$ - - Lindl.

v. K. p. 29 , f. 1.

- flexuosa. - [S. $45 \%$.

- fusca Fr. - [S. 15\%.] - Fl. dan. XII. 2090. 2.

- Fuck. s. 273.

[- globosa. - [\$. 456 : Nass.]

- incarnata $\mathrm{T}$. - [S. 457 . $]$ - Fl. dan. XII. 2090. 1. - Fuck. s. 272. - Bisch. f. 3663. Schntzl. t. 14.f. 34.35.- Kl. \$22. (Cent. XVII.). - Zeitschr. f. wiss. Zool. 1559. X. t. 8. f. 6. (de Bary. - Bot. Zt. 1559. t. 11. f. 16. (Keimg. Hoffm.) - Pringsh. Jhrb. III. 1461. t. 3. f. 8. 9. Fructif. Wigd., - Ann. sc. n. 1\$62. XVI. t. 16. f. S. 9. Wigd.) - Sturm h. 3. t. 14.

- leucocephala. - Wstd. 452.

- nutans. - [S.45\%.] - Fl. dan. XII. 2017.1.

- Kl. 421.

- DC. : Fuck. s. 269. - R. f. 365. (Trichia Bull.) - Pringsh. Jhrb. III. 1561. t. 3. f. $13-15$. Fructif. Wigd.). - Ann. sc. n. 1562. XVI. t. 16.f. 13-15. Wigd.).

ochroleuca Fr. - [S.457.] - Bisch. f. 3692.

- Kl. 1546. - Pringsh. Jhrb. III. 1\61. t. 3.

f. 16. (Capillit. Wigd.). - Ann. sc. n. 1562.

XVI. t. 16. f. 16. Tigd.) - cf. silacea.

- punicea P. - [S. 457.$]$ - Voges. 1456. Wstd. S1. - Cd. Anl. t. C. f. 31: 1.2. - Loud. f. 16523. - Kl. 932. - Fuck. s. 271. - Dsm. 1010. - Il. II. 671. - Schacht, Phys. Bot. 1952. t. XVI. f. 15. Capillit.) - Ztschr. wiss. Zool. 1559. X.t. S.f. T-9. (de Bary). - Pringsh. Jhrb. III. 1S61. t. 3. f. 6. 7. (Fructif. Wigd.).Carol. II. s0. - Jungh. pr. t. 2. f. 10. - Ital. 497. - Ann. sc. n. 1S62. XVI.t. 16. f. 6. ‘. (Wigd.). 2017.2 . 
A reyria ramulosa Wigd. - Pringsh. Jhrb. III. Is61. t. 3. f. 17. Capillit.) - - Ann. sc. 11. 1562. XVI. t. 16. f. 17. Wigd.

- serpula Wigd. - Pringsh. Jhrb. III. Istil. t. 3. f. 18. (Fructif.) - A. anomala de B. Ann. sc. n. 1S62. XVI. t. 16. f. 18. Wigd.

— silacea Ditm. - Sturm 1. 1.t. S. (ochrol.).

- straminea. - S. $45 \%$. 7 .

-_ umbrina Schum. - S. 15\%.] - Fl, dan. XI.

1975.2.

- vermicularis Schum. - ef. sub punicea v.

Fl. dan. S. 457.$]$

A regma. - [S. 507.j - ef. Phragmidium.

- acuminatum. - Wste. 131.

- bulbosum. - Wistd. 157.

mucronatum. - Wstd. 131.

- obtusatum. - Brit. f. 105. - Wstd. $\$ 60$.

— speciosum Fr. - Carol. III. 90. - Berk. J. p. 325. f. 73. a.

Arg y lium. - [S. $43 i^{*}$.]

inquinans Ns. jun. t. 12. = Melanogaster variegatum Bail S. p. 12 ,

Arrhenia Auriscalpium. - S. 312.$]$ cupularis. - cf. Merulius c.

- W. : Sturm.h. 33 - t. 5. Strauss.-

[S. 312.]

- tenella. - S. 312 .

Arthrinium. - [S. 502.] - ef. Camptoum. caricicola. - [S. 502.] - Cd. Anl. t. B.

f. $12: 5 .-$ Bisch. f. 3730 . - Kil. $71 .-\mathrm{Kl}$. 1165. Cryptosp. Caricis (d.) - R. f. $76 .-$ Fuck. rh. 125.

- curvatum Kz. Camptoum . - [S. 502.] -

Iil. 461 ; Cent. XV. suppl. - Fuck. s. $153 .-$

Wistd. 974. - Fuck. rh. 127.

- puccinioides Kz. - S. 502.] - Dsm. 105. -

K1. II. $67 \%$ - Brit. f. 311 . - Fuck. rh. 126.

— scriptum. - Bon. t. 4. f. 92. f. $49-52$. - Fuck. s. 151. - Lib. 394 Sporophl. Gram. Lk., - R. f. 576. - Ital. 241. Fuck. rh. 12s.

Tiliae. - Bon. t. 4. f. 5i.

Arthrobotrys. - [S. 492.]

- oligospora. - Fres. t. 3. f. 1-s.

- recta. - Fl. 1;62.

_- superba. - Cd. Anl. t. B. f. 19:9-11. Bon. t. 9. f. 153. - Pay. b. cr. p. 104. f. $4>1$.

Arthrobotryum atrum. - Ann. Mg. n. h. 1559. III. t. 9. f. 6. Berk. B.,

- Broomii. - R. f. 65.

- stilboideum. - Hedw. 1. t. 4. f. 1. Arthrobotr.).

A rthroderma. - Trichodermati affine.-Micr. Journ. 1 554 . II. t. 9. f. 6-S. p. 240. (Currey.

Arthrographium stilboideum. - Kl. 1521. Hedw. I. t. 1. f.1!

Artotrogus. - [S. 497.] - Myceliosporen von Peronospora Berk.) etc.

- asteroph. - ef. Nyctalis asteroph.

- hydnosporus. - S. 497.] - Journ. hortic. soc. is 16. I. t. 4. f. $2 i-29$. Berk.).

Aschersonia. - [S. 524.]

- taitensis. - Ann. sc. n. 1545. X. t. 6. f. 3. (Nont.).

- turbinata. - Anm. Mg. n. h. 1552. IX. t. S.

f. 1. a - p. 199. (Berk.).

A s chion castaneum Wilr. = Tuber. Cd.

- fuscum Whr. - Kl. 151. (cf. Vittadinion Mont. Z.).

- nigrum. - Kütz. Phil. Bot. 1951. t. 5. f. 1.

(Fructif.).
Ascobolus. - [S. 359.] - Bail. S. t. 20.

aerugineus Fr. = marginatus Fl. dan. -

[S. 355.$]$

_ albidus. - Ann. sc. n. 15.5. X. t. 13. A.

Crouan).

- Brassicae. - Ann. se. n. 155\%. VII. t, 4.

f. C. Crouan'.

carneus. - [S. 355.] - Kl. 1431.

ciliatus Schmidt. - [S. 355.$]$ - Kl. 921. -

Kil. II. 425 .

- cinereus. - Ann. sc. n, 1 55 . X. t. 13. D.

Crouan'.

- coccineus. - Ann. sc. n. 155\%. TII. t. 4.

f. I). Cronan).

conglomeratus. - [S. 35s*.] - Carol. V. 44. coronatus Schum. = Phacidium cor. Fl, dan.

- Wstd. 919.

Crec'hqueraultii. - Ann. se. n. 1559. X.

t. 13. C. Crouan'.

denudatus. - S. 355.]

furfuraceus. - [S. 35ऽ.] - Fl, dan. XI.

1456. 2. - Cd. Anl. t. G. f. 64: 24-29. -

Loud.f. 16279. - Fuck. s. 754. - Dsm. 715. -

Pay. b. cr. p. 5. f. 12 ; p. ๖. f. $24-26$. (Pez.). -

Kil. 522 .

- glaber P. - [A. 355.] - Bisch. f. 3370. -

Fl. dan. XI. 1\$56. I. Kl. 1430.).

- granuliformis. - Ann. sc. n. is 5 s. X.t. 13. F.

('rouan .

- immarginatus. - Ital. 775 .

inquinans. - Wstd. 191.

- insignis. - Ann. sc. n. 1S55. X. t. 13. H.

C'rouan'

- Kerverni. - Ann. se. n. 1555. X. t. 13. B.

(rouan. = glaber $\mathrm{P}$. ex Coems).

- macrosporus. - Ann. sc. n. 1557. VII. t. 4.

f. B. (Crouan' ' = immersus P. ex Cocms.).

- marginatus Schum. - Fl. dan. XI. 1 \$56. III. microscopicus. - Ann. sc. n. 185\%. VII.

t. f. f. F. Crouan .

— miniatus. — Ann. sc. n. 15.55. X. t. 13. I. C'rouan .

- papillatus. - Kl. II. 426. - Fuck. s. 75.5. - Pelletieri. - Ann. sc. n. 155\%. VII. t. 1.

f. A. Crouan, - R. f. 167 .

- pilosus. - [S. 355.$]$

- porphyrosporus Fr. - [S. 355.] - Bisch. f. $33 \pi 1$.

- pulcherrimus. - Ann. sc. n. 1859. X. t. 13.G.

Crouan' - R. f. 355 .

- sexdecimsporus. - Ann. sc. n. 1555. X.

t. 13. E. Crouan :

Solms-Laubachii Rbh. - R. f. 420.

Trifolii Biv. - Brit. f. 69. - Wstd. 399. Dsm. 11 s.

Fr. : ef. Peziza Trif. Lib.

rinosus Berk. - Brit. f. 159 . - R. f. $230 .-$

Berk. Out. t. 2. 3. f. 1.

Ascochyta. - [S. $\left.35 T^{*}.\right]$ - ef. Spilosphaeria, Septoria.

- acerina Lév. - Demid. Russ. t. 5, f. $\boldsymbol{7}$.

Aceris. - Lib. 54.

- Lib.: Fuck. s. 347. - cf. Septor. Ac.

Aegopodii. - Lib. 49. - Wstd. 95. 527.

Aesculi. - Lib. 154. (Depazea aesculicola

Fr. - R. f. 550 .

Anemonis. - Lib. 159.

Aquifolii. - Lib. 3j1. Xyloma u. Phacid.

multivalve;

Atriplicis Lasch. - Kl. 561 .

Calamagrostidis. - cf. Septoria, - Lib. 157.

Cannabis Lasch. - Kl..1059. 


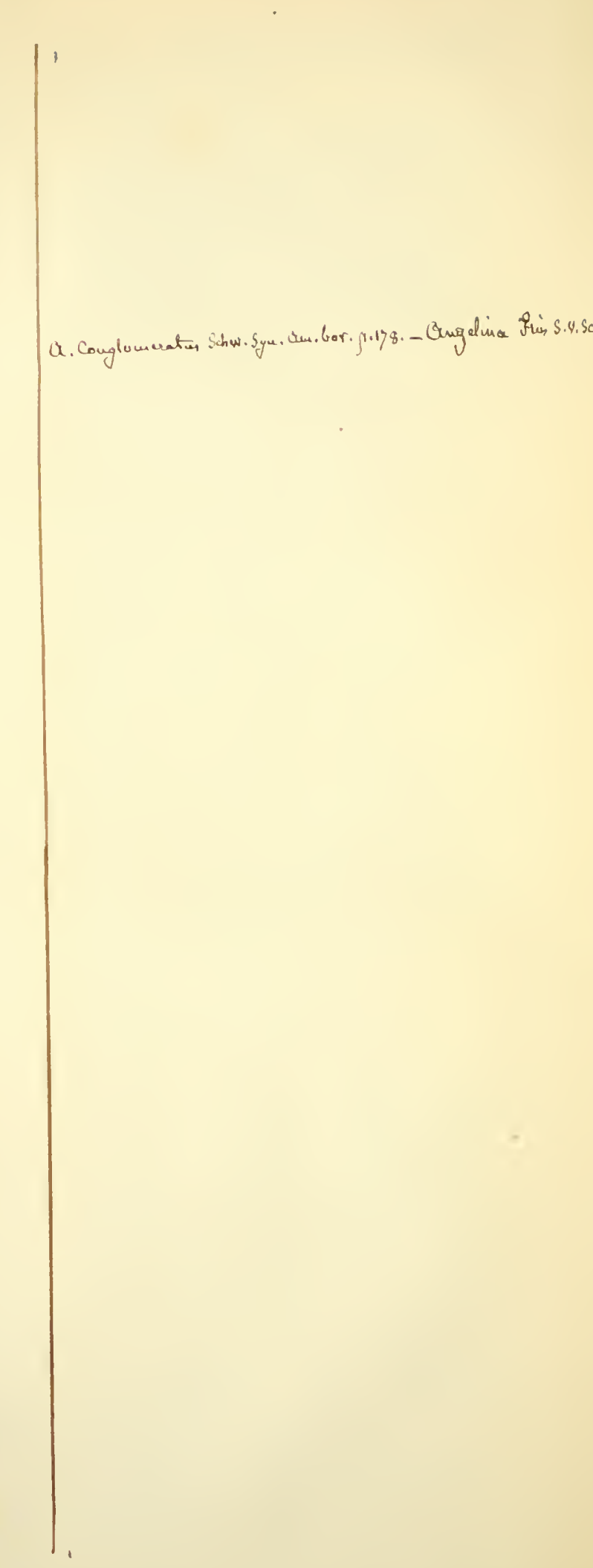




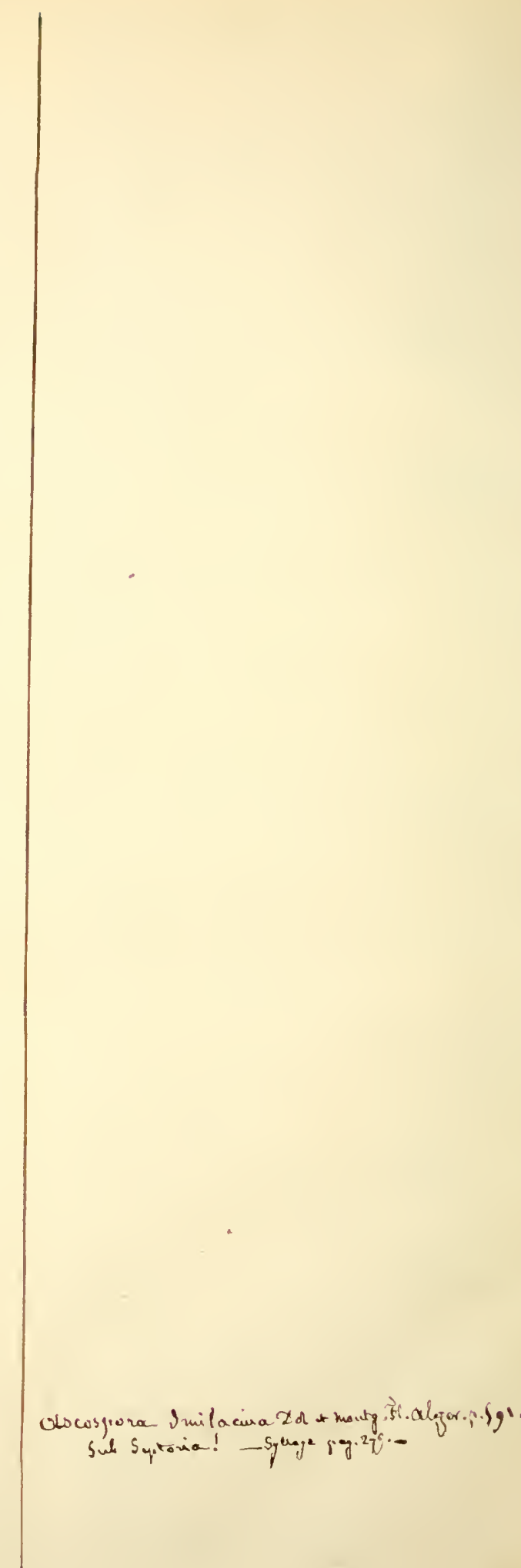


A s c oc hy t a caulium. - Lib. 219. - Wstd. 1123. - Chelidonii. - Lib. 57. - Wstd. 425. - Kil. 679. - Fuck. s. 346. - Kl. II. 552. - Fuck. rh. 451 .

- Convolvuli. - Lib. 56. Sphaera Filum Biv., Phoma Filum Fr. . - Kl. \$59. - Fuck, s. 336.

- Wstd. 295. - Fuck. rh. 476.

- Cormi, - Lib, is.

Crataegi F. - Fuck. s. 345. - Lil). 35\%.

non 152. - Fuck, rh. 473.

- Cytisi. - Lib. 156. - Wstd. 424.

- Dianthi. - I.ib. 155. - Wstd. 293. - Kl.

63. - Wstcl. 616. 293. - Fuck. rh. 190.

Dulcamarae Lasch. - KI. $\$ 55$.

effusa. - I,ib. 35\%.

Epilobii. - Iil. 16.56.

- Rbh.: Fuck. s. 3to. - Fuck. rh. 4\%.

Euphorbiae Lasch. - Kl. $>62$.

Fragariae. - Lib. 155. - Iil. \5!.

— Ibh. : Fuck. s. 349. - Fuck, rlı, 14:3.

Fraxini. - Lib. 4.

Galeopsidis Lasch. - Kil. 11155. - Fuck.

311. - Fuck. rh. 479.

Galii Lasch. - Kl. 1251.

Geranii. - Iil. 1655.

- Graminum Lasch. - Kl. 1155.

Grossulariae. - Lib. 251. - Wstd. 94-1.

Ieraclei. - Lib. 51. - Kil. 1173.

Ilieracii Lasch. - Kil. 1156.

Ilippocastani. - Lib. 151.

Humuli Lasch. - Kl. (j八0.

IIyoseyami Lasch. - Kil. 6 f 1 .

IYperici Lasch. - Kl. 1159. - Fuck. s. 312.

- Fuck. rh. 4 ho.

- Lychnidis. Lasch, - Kil. \57.

Lysimachiae. - I.ib. 253. - K1. 955. - ef.

Septoria Lys. - Wstd. 950.

- Medicaginis. - Fuck. rh. ł৬ל.

Menyanthis Lasch. - I,ib. 251. - Kil. tit.

obducens. - Fuck. rl. 191.

- Orchidis Iasch. - Kl. 9s-.

- Oreoselini Lasch. - Kil. 566.

- Oxyacanthae. - Lib. 152. (Septoria, -

Wstd. 10.

- Padi. - Iib. 153.

- Petroselini. - Lib. 252. - Wstd. 135

- Pisi. - Lib. 59. - Fuek. rh. 457.

- Plantaginis. - Kil. 1742. b.

- Polygoni Tibl. - Kl. 990. - Fuck. s. 339.

- Fuck. rh. $17 \%$.

- quercicola. - Lib. 355. - Wstd. 614.

- quercina. - Lib. $46^{\circ}$.

- Ribis. - Lib. 53. - Wstd. 92. - ef. Septoria $\mathbf{R}^{\prime}$.

Robiniae. - Lib. 35\%. - Ki. 1255. - Wstd.

947. - Fuck. rh. 475.

- Rosarum. - Lib. 50. - Wstd. 93. - Fuck.

s. $33 \mathrm{~s}$. Fuck. rh. 471 ,

Rubi Lasch. - Kl. 1157.

- Ruborum. - Lib. 247. - Wstd. 931.

rubra. - Lib. 352. (Xyloma, Polystigma ${ }^{\wedge}$.

Scabiosae Rbh. - Kl. 1253. - Fuck. s. 319.

- Fuck. rh. 492.

Sedi. - Lib. 249. - Wstd. 943.

Sii. - Wstd. 942: - Kl. 1353.

Silenes Lasch. - Kl. 1256.

stipata. - Lib. 354.

strobilina. - Lib. 150.

Teucrii. - Kl. 1355.

Tiliae Lasch. - Kl. 1160. - Fuck. rh. 492.

Ulmi: - Lib. 52, (Septoria Fr.). - Wstd.

$13 \%$.

Hoffmann, Index Fungörum.
As cocliyta Umbelliferarum Lasch. - Kl. 1159. Y. Chaerophylli. - Kl. 125\%.

Vaccinii. - I.ib. $\$ 7$.

Viburni. - Kil. 1354.

- Rbh.: Fuck, s. 314 . - Fuck, rh, 472.

Viciae. - Lib. 356. - Wistd. 1151.

Virgaureae. - Lib. 55. - Fuck. rh. 195.

Vulnerariae. - Fuck. rl. $4 \$ 9$.

A s com yces bullatus. - Berk. J. p. 25 1. f. 66.c. cf. Ascosporium?

- coerulescens W'std. - Castagn. Mars, t. 10.

- Bull. ac. belg. 1. Ser. XIX. 3. p. 132. f. 4.

__ Mtg. : Dsm. 1305. - Wstd. iti.

Tosquinetii. - Wstcl. 1293. - Bull. ac. belg.

2. Ser. 'Tom. 11. p. 660. f. 4. Wstd.'.

Ascophora. - [S. 45s.]

- arachnoidea. - Regel Gartenflora 1554, t. S7.

f. $1-4$. Reg. .

- Cesatii. - Coemans Monogr. Piloboli. 1561.

p. 65. t. 2. f. E:

- chalybea, - Tijdschr. v. uat. Geschied. $1 \$ 45$.

XII. t. ti. f. 4. Doz. MIk.)

- Choiromyc. - ef. Asc. Rhizopogonis Cd.

- cincrea. - Ḱl. 1361.

- cylindrica: - [S. 524.]

- disciflora $\mathrm{v}$. byssina. - Wstd. 134.

- elcgans. - [S.45s.] - Berk. J. p.2 99. f.67.d.

- Kl. 1363. - Flora. 1457. t. 5. f. 70-52.

Keimg. Bail . - Mucor t. C'oems . - Auerswald bot. Unterhltg. 1 633. p. 34.,. - Fres.t. 12.

f. $13-16$. p. 96 .

— Florac. - Cd. Ic. V. t. 2. f. 2i.

- glauca. - C'd. Ic. V. t. 2. f. 26.

- Mucedo. - s. ł b.] - Cd. Anl. t. C. f. 21:

1-4. - Fuck. s. 146. - Pay. b. er. p. 42.

f. 370. - Bull. ac. belg. 1 5.5 . VIII. p. 770 .

fig. 17. Radix. Coemans,. - Pringsh. Jalırb. II.

t. 30. f. 29. Hoffm.). - Loud. f. 165!7. -

Bisch. f. 3790. - André ükon. Neuigkeiten.

1) 47. t. 1. f. $21-23$. Cd.\}. - Willkomm,

Wund. d. Mikrosk. 1556. p. 90. f. 44. - Il. II.

273. - Fuck, rh. 5\%.

- nucuum. Carol. V. \$9. - Cd. Ic. V. t. 2.

f. 25 .

- perennis. - [S. 4\. 524*

— pulchra Pr. - Kil. 1265.

—_ Rhizopogonis. - Cd. Ic. VI. f. 30.

- stercorea, - Cd. Ic. VI. f. 31.

A seospora. - [S. 425.] - cf. Sphaeria.

Aegopodii Lév. sphaeria $\Lambda$ eg. P. non Pbl.)

- Kl. II. 551. - [S. 425.] - Wstd. 95.

- Asteroma. - [S. 425.] - Fuck. rh. 470.

brunieola Fr. : R.f. 364 . - Fuck, s. 350 . -

IIl. II. 660. - [S. 425.] Fuck. rh. 467 .

- bullatum. - Berk. Out. t. 1. f. 9. b. (Fruct.)

$=$ Ascomyces Berk. Out. p. $36 i$.

- carpinea Fr. - [S. 425.] R. f. 365. (Spluae-

ria c. - Fuck. rh. 466.

deformans. - Berk. Out.t. 1.f. 9. a: (Fruct.).

Mali. - Fuck. rh. 469.

Ostruthii Fr. - [S. 125.] Sphaeria Ös. Cd.

non Fr.): - Kl. II. 550 .

- phomatoides Mt. - Ani. sc. n. 1840. XIV.

t. 19. f. $7: \mathrm{p}: 32 \mathrm{~s}$.

pulverulenta. - Kl. 1754.

pyrenophora. - Bon. t. 3. f. 65.

ribicola. - [S. 425.]

Solidaginis: - [S, 425.] Fuck. s.. 351. -

Fuck. rh. 469 .

Spinaciae. - [S. 425.]

tripunctata. - Bon. t. 3. f. it. 
A scotrich a chartarum. - Amn. Mg. n. h. 153\%.

I. t. 7 . f. s. Berk.).

Aseimotrichum. - [S. 172 * . 50:3**]

- ossium. - Cd. Anl. 1. B. f. 12.: 2, - Sturm

h. 12. t. 22 .

A se roë. - S. 435.]

- actinobola. - Cd. Ic. VI. t. 1. f. 11-14.

- ceylanica. - [s. 431 \%. -.. Hook. Iond. J.

1546. V.t. 19.

- Junghulnii Ś-1.: Schlechtendal de Ascrois genere diss. Is 17 . c. ic.

— pentactina Eudl. - Cd. Ic. VI. t. 4. f. 12. 13. - Cd. Anl. t. E. f. 49: 3. - Pay. b. cr. p. 11 f. f. 562 . - Lindl. r. K. p. 31. f. 23. Bail s. t. 24.

A s e r oё rubra Lab. - Cd. Ic. VI.t. t.f.9-11. -

Hook. Lond. J. I 44. III. p. 191. t.5. A. Berk.

- I3erk. = actinobola Cd. $\mathbf{S}-1$.

viridis I3. H. - $[\mathrm{S}, 434$ †.] Cd. Ic. VI. t. 4 .

f. 19. 20. - Hook. Lond. J. 1s 4. III. t. 5. B.

Aserophallus cruciatus L. M. Cd. Ic. VI.

t. III. f. 49. - Amn. sc. 1. 1545. IV. t. 14.f. 1.

(Mt., - Pay. b. cr. p. 115. f. 55.5.

Aspergillus. - S. 4\$9.]

- spec. bei Pityriasis rersicolor. Vierordt's

Arch. f. physiolog. Heilk. III. H. 3. 1. 49i. Ie.

Gudden?

___ in ovis: Presburg. Terh. Terein f. Naturk.

1557. t. 2. (Kolaczek .

in abdomine Charadrii: Bull. ac. belg. 15!

XV. 1. p. 451. Spring.!

- alternatus. - Amn. Mg. In. h. 1539. I. t. S.

f. 11. Berk.;

- aurantiacus. - Brit. f. 2:2. - Ann. Mg. n.

h. 1S.11. VI, t. 13. f, 22. Berk.).

- candidus. - [S. 4>9.] - Letell. 642. 2.

Monilia,

- elavatus. - Ann. sc. n. 1531. II. t. 3. Dsm,

— Curtisii. - Carol. IV. $\$ 3$.

- dubius. - Berk. J. p. 219, f. 65. b.

- ferrugineus. - Fuck. rh. 15\%.

— flavus. - [S. 159.] — Bon. t. 9. f. 192. - Lk.: Bisch. f. 3722 .

fumigatus. - Fres. t. 10. f. 1-11. p. \$1. glaucus, - [S. 459.]-Cd. Ie. V.t.2.f. 24.-

Fuck. s. 15t. - Cd. Anl. t. C. f. $21:$ i. \$. -

Berk. I. p. 295. f. lis. a. - Pay. b. cr. p. 69. f. 294.

- Bisch. f. 3510 . Willkomm, Wund. d. Mi-

krosk. 1556. p. 91.f.a. - Bot. Zt. 1 5 4.t. 11. de

Bary). - IIicr. Journ. 1457. V. t. 7. f. 1- i.

(Grove). - Kl. 1571. - Schntzl. t. 12. f. 26.27.

Mucor Mucedo:- Brit. f. 20s. - Sturm. h.

11. t. .. - Fres. t. 10. f. 12-16. p. 52 asc.) griseus, - [S, 449.] - Bon. t. 9. f, 154.

incrassatus. - Bull. ac. belg. 1552. XIX.

1. p. 559. Spring.).

laneus Lk. — ? Phymatotrich. lan.

macrosporus. - Bon. t. 9. f. 193.

- maximus Lk. - Letell. 642.1. - Bisch.

f. 3723 .

- mollis. - [S. 4 \$9.]

- nigrescens. - Robin. v. par. t. 5. f. 2.

- ovalispermus Lk. - Cd. Ic. II. f. 7 i.

- penicillatus Grev. - Sturm. h. 11. f. f. -

Loud. f. 16555. - Müller, PHanzenstaat. $1>60$.

265, 7. Copie).

- roseus. - Isetell. 642. 4. (Acladium).

- sulphureus. - Dsm. III. 554.

- virens. - [S. \$\$9.] - Letell. 6+2. 3. Monil.).
Asterin a. - [S. 370 $30^{*} ; 406:$ Limb. ; 56t.]

- Aesculi. - Dsm. I1I. 125.

angulata. - Dsm. III. 124.

Darrinii. - C'rypt. Bot. antarct. voy. Ere-

bus. 1451. t. 164. 2.

- Epilobii. - Dsm. III. 421.

IIederae. - Dsm. III. 7T.

orbicularis, - Carol. 11. 15!.

pelliculosa. - Crypt. Bot. antaret. roy. Fre-

bus. 14;1. t. 161.1.

- Pyracanthae. - I)sm. III. Iㄹㄴ.

- spuria. - Carol. IV. 69.

- stictica. - Crypt. Bot. antaret. roy. Ere-

bus. 14.).1. t. 11il. 4.

L ? umbonata. - Dsin. III. 427.

- vagans. - Dsm. III. 422. 423; 775. - ef.

Asteroma.

Asteroma. - [S. 107. 421.] - cf. Actinonema,

Piggotia, Sphaeria.

- Accris I). - D:m. T13.

- achaenarum. - Dsm. 1691.

- Achilleac. - [S. +22.]

Angelicae. - S. 425.]

- atratum. - S. 2.5.] - Kl.II. 153. — Wstd.

bs 1 .

— Betulae Dsm. - Wstd. 165. - Kl. II. 551.

- - Rob. : Dsm. 746.

- Cacaliae. - Dsm. 1699.

_- Calvadosii. - Dsn. 1649.

- Capreae. - Dsm. 1690.

- Castaneae D. - Dsm. iH.

- Cerasi. - Dsm. III. 11 S.

__ circinans v. Phillyreae. - Dsm. 1658.

- comptum. - Dsm. 1645.

__ Corni D. - Dsm. 711.

- Crataegi Fr. = Actinonema Cr. Fr. Su.

- Berk. : Dsm. 49y. - [S. 124.] - Brit.

f. 12 .

delicatulum Dsm. - Dsm. 1693. III. 770 .

771. - R. f. 363 .

- dendritica Fr. - [S. 425.] - Dsm. 1691. -

Fuck. s. 356 . - Fuck rh. 462.

- elegans. - Dsm. III. 415.

- Epilobii Fr. - [S. 425.] - Fuck. s. 353. -

Fuck. rh. 459.

- Fraxini D. - Dsm. 456.

— fugax. - Dsm. III. $\$ 20$.

__ Fumago. - โS. 425.

_ ? gallicola. — Dsm. III. 772.

- geographica D. - [S. 425.] - Dsm. 495.

- Fr.: Fuck. s. 35\%. - Voges. 1179.

Dothid.) - Wstd. 265. - Fuck. rh. 463. graphoides. - Dsm. III. 116 .

Himantia. - [D. 125.]- Dsm. 742.

- Fr.: Fuck, s. 359. - Fuck. rh. 460.

incomptum. - Dsm. III. 419.

- inflatum. - Dsm. 1656.

Juncaginearum Rbh. - Kl. II. 592.

Labes. - Brit. f. 346 . - Ann. Mg. 12. h.

1-11. VI. t. 11. f. 6. Berk.).

Lonicerae D. - Dsm. 497.

Mali D. - Wstd. 267.-Dsm. 499.

Malorum. - Brit. f. 332 .

Mespili. - Dsm. 1654.

nervisequia. - [S. 425.]

- obscurum. - Dsm. 1692.

Padi. - [S. 124.] - Lib. 60. - Ann. Mg.

n. h. 1541 . VI, t. 11. f. 4. Berk.;.

- Paeoniae W. - Tistd. 1050.

Phyteumae. - Bail S. t. 16.

Polygonati DC. - Toges. 655. - Dsm. iju.

Populi. - Dsm. 1652. - Wstd. 1122. 
Asteriva Séveitl chamying. Exotiquer J'og. Sg.

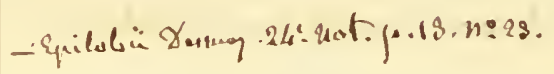




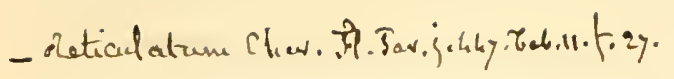


As te rom a Prunellae I'urt. - S. 12.5.] - Dim. 496. - Wstd. 169.

- Pyri. - Dsm. III. 121.

- radiosum Fr. - K1. 34.5. - Actinonema losae Fr. Su. . K1. II. 565.

- reticulatum. - [S. 125.] - Dsm. 1657. ef. Combosira. - Fuck, s. 357. - 1) sm. 739. Brit. f. 11. - Wstd. 636. - I. f. j19. - Fuck. rh. 161.465.

- Robergei D. - Wstd. 150. - D sin. 5011.

- Mosae. - [S. 121. - Ann. Mg. 11. h. 1 11.

VI, t. 11. f. 5. Berk. - I) sm. III. 773. -

Wstd. 111i. - Lirit. f. 311. - Lib. lil.

- Salicis Rob. - Dsin. 715. - Wstd. 924.

- stellare. - 1)sm. 1II. $11 \%$.

- stellatum. - Wstd. 116.

_ subradians. - [S. 125.]

- Ulmi. - Dsm. J699. - Wstd. i20. - Loud. f. 16199 .

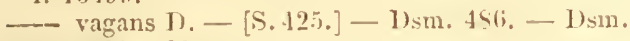
III. 122.423 .

- - v. lilacis. - Dsm. 169.).

- v. Tiliae. - Dsm. 1 (i96.

- v. Frangulac, - Dsm. 1697.

renulosum Fr. - Fuck. s. 35..

Veronicae D. - Dsm. I 41).

- Veronicarum. - Kil. II. T39. - Fuck. rh.

461.

Vineac, - Brit. f. 193.

Virgiliae. - Usm. 1653.

Aylostei. - S. 125.

Asterophora, - S. 441 . $497 \cdots$, of. Nyctat lis et $\Lambda$ sterotrichum. - $\Lambda$ nn, se. $11.1-37$. VIII

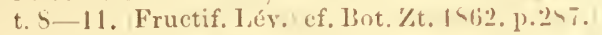
agnicicola. [S. 4! i. 1!]- Fuck. rlı. 154. - agaricoides $\mathrm{Fr}$. - [S. $416 \cdots$. ] - Misch. f. $3691 \%$ - lil. H1. 235. - Brit. f. j99.

- 1ycoperdoides. - S. 416.$]$ - (d. Ic. VI.

f. 5. - Bon. t. 11. f. 22 1. - sturm h. 2. t. 26.

- Fr.: Nyctalidis asterophorne Fr, forma :

de 13 ary ; Bot. Zt. 1559. p. 349.

nauseosa. - liorcz. fg. ingr. 1557. t. (i.

P'ezizac. - C'd. Ic. VI. f. 6. - = Stephanoma strigosum Whlr.: - Fuck. rh. 160.Hoffm. Ic, t. $\rightarrow$ f. 2 .

- physaroides Fr. - Bisch. f. 3691. - Bon. t. 11. f. $225 .-=$ Nyctalidis asterophorae Fr. forma: de Bary, Bot. Zt. 1559. p. 35s. t. 13. f. $1-3$.

- trichioides. - S. 416.$]$

Asterosporium. - $[\mathrm{S}, 50 \mathrm{~S}$.$] - Fres. t. 5$. f. $10-13$.

Hoffmanni. - [S. 504.] - Fuck. s. 132. - - Kz. : Krypt. Bad, 325. - Mlicr. Journ. 15. t. 11. f. $1-9$. Currey. - Kl. $i$ is. Stilb. macr.). - Kil. II. 591 itilbospora ast. Rbh. .Toges. 669. - Wstd. 576. - Fuck, rh. S1. R. f. 5 St.

Asterothecium strigosum W"ll. = Asterophora Pezizae Cd.

Asterotrichum. - [S. 160.454.]

Ditmari. Bon. - cf. Asterophora agaricicola Cd. S. 497.1 !]

Athelia. - [S. 334. $196^{* *}$. 521.] — ef. Thelephora.

- citrina. - Letell. 630. 4. coerulea. - W'std. $76 \%$.

cpiphylla. - zu Cortic. arachnoid. Berk.'. laevis. - Letell. 630. (Himantia Fr.).

_ Typhae. - Ital. 442. Theleph.'.

Atractium. - [S. $469^{* *}$.]

- aurantiacum. - Bon. t. 11. f. 226 .
Asterom a flammeum. - (arol. T. 56 . minus). pallidum. - Bon. t. 10. f. 219.

rigidum Bon. - R. f. $5 \$ 1$. (c. ic.).

Atractobolus. - [S. $439^{\circ}$. cocci sp.; 524.]

— ubiquitarius Tode. - Loud. f. $1631 \%$.

Cord. Anl. t. D. f. 14: 1-7. (ova insector.,

Tul. . - ef. Pez, annularis.

A u erswaldia. - ef. Sphacria lagen.

A ulacographum Dur. - cf. Aylographum

Duh. Hyst. 3i..

A uricularia. ef. 'Thelephora' - - [\$. 333.]

- aurantiaca Schum. = Theleph. hirsuta Fl. dan. - caryoplyllea Sow. - Wstd. 55\%.765.

corrugata Sow. - Wstd. 693.

cucullata. - S. $3333^{*}$.]

- ferruginea Bull. - Wstd. 12๙9.

- Judae Whlb. - Wstd. 760 .

_- Lobata Fr. - [S.333.] (cf. Exidia lob. Som.). lierk. Out. t. Is. f. 1 .

- mesenterica P. - [S. 333.] - Dsm. \$/ৎ. -

[il. 45. - Fuck. S. 915. - IIuss. I1. 6. - Wistd. 6i93. - Ital. 147.

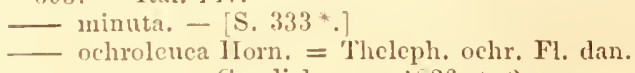

- ornata. - Gaudich, voy. 1-26. t. 2.

_ pallida Schum. = '1'hel. ochroleuca Fl. dan.

- paprina Hull, = Thel, ochroleuca.

— petaluides. - Vent. mi, :17. f. 7.

- phosphorea Sow. - Wstd. ifi.

- pulrerulenta. - Merul, destr. (Tul.). reflexa. - W'std. T66.

variegata.

reticulata, - $-5.325 * \%$

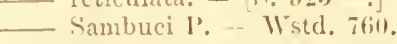

_- tremelloides 13u1l. - Wstd. 1i93.

A y lographum I.ib. $-\left[\right.$. . $\left.406^{*}.\right]=$ Aulaco-

graphum 1)ub. Hyst. 3 .

- Epilobii. - Jilo. 273.

- Festucac. - Lib. 373.

filicinum, - Lih. 275.

- Hederae. - I,ib.272. - Wstd. I is. (K1. II.

52 - Hysterium micrographum Not.) = Ayl. vagum I) $\mathrm{im}$. : Dub. Hyst. 34.

- hieroglyphicum. - Ism. III. 555.

- juncinum. - 1ib. 274. - Dub. Hyst. t. 2.

f. 20 .

Luzulae. - Lib. 372.

maculare 13. B. - Ann. Mg. n. h. 1861. VIr.

t. |6. f. 21 . p. 451 .

- Pinorum D. - Dsm. 294. - R. f. 266. -

1tal. 346.

- ragum D. - Kl. II. 5.5. - Wstd. 179. -

Dsm. 1029. (ef. Hederae) u. III. 559 .

A zosm a helminthospor. - [S. 499.] - Cd. Anl.

t. B. f, 10:2. - Sturm h. 12, t. 1s.

- Punctum. - Dsm. III. 757 .

Azygites. - [S. 4SS.]

Bacillaria. - [S. 3S2**.]

Bactridium. - [S. 474.]

album Bon. - R. f. 473. (cf. Mastigospor. alb. Rs.!.

- candidum. - Cd. Anl. t. B. f. 9: 5. - Pay.

b. cr. p. 73. f. 327 . - Bon. t. 2. f. 47, Mitte. carneum. - Bon. t. 2. f. 47, unten. flavum. - [S. 174.] - Brit. f. 326. - Bon.

t. 2. f. 47 , oben.

- Kz.: R. f. 3 ; 2 .

Helvellae. - Ann. $\mathrm{Ig}$. n. h. 1859. III. t. 9.

f. 3. (Brk. Br.). 
B a dh a mi a. - ef. Physarum. Linn. Trans. IV. 24. (Berk.). - Berk. Out. t. 1. f. 6.

- hyalina. - Linn. Trans. 1S51. XXI. t. 19. f. 3. (Berk.).

nitens. - Linn. Trans. 1551. XXI. t. 19.

f. 1. p. $15 \%$. Berk.).

- pallida. - Linn. Trans. 1551. XXI. t. 19.

f. 2. Berk).

Bal an i um. - [S. $504^{*}$.]

$\mathrm{B}$ als a $\mathrm{mi}$ a. - [S. 43..]

- fragiformis. - Tul. F. hyp. t. 4. f. 3. platyspora. - Tul. F. hyp. t. 15. f. 2.

— vulgaris Vitt. - Vitt. t. 1, 2. - Cd. Ic. V'l. t. 10. f. 99. - Cd. Anl. t. D. f. $46: 12-15$.

Tul. F. hyp. t. 4. f. 4 ; t. 15. f. 1 ; t. 19 . f. 6. Bail S. t. 13. - Kl. 1963. - Kl. II. 246.

Bas idiolum fimbriatum. - Bot.Zt. 1561.p. 173. t. i.f. $30-39$. (Cienk.).

B at tarea. - [S. 410.$]$

- Gaudichaudii. - Ann. sc. n. 1531. II. t. 1. Cd. Anl. t. E: f. 50: 4-6. - Schntzl. t. 11. f. 50.51 .

- phalloides. - Cd. Anl. t. E. f. 50: 1-3. Berk. J. p. 5. f. 5. a. b. Anat. - Hook. J. 1543. II. t. 22. f. 1. (Anat. Berk.). - Loud. f. 1633 5. - Bisch. f. 3463 .

Belonidi um aeruginosum. - Alger. t. 25. -

Bercleasmium Cd. - cf. Sporidesmium concinnum, Cd. Ie. VI. p. 19.

Bertia. - S. 395.] - ef. Sphaeria.

Bilimbios pora Doliolum P. Awd. - R. f. 2ril. $\mathrm{B}$ is pora dicoccun. - Kil. 1655.

- Menzelii. - [S.505.] - Cd. Anl. t. B. f. 6:

1. 2. - Pay. b. cr. p. 69. f. 301 .

- monilioides. - Fuck, rh. 71. - Fres. t. 6.

f. 46-54. - Fuck. s. 136. - Voges. 1350 .

- v. condensata Cd. - Kl. 1077.

Blas totrichum. - [S. 493 *.]

- confervoides. - [S. 493*.] - Pay. b. cr.

p. 73. f. 325 .

- puccinioides. - Sturn. h. 25 - t. 11 . Preuss.

Blennoria. - cf. Trullula. - [S. 475.

- Abietis. - Kl. 1575. - Tharand. Jahrb. 1553. p. 111. c. ic. Stein).

Buxi Fr. - Voges. \2. - Dsm. 239.

Wstd. 751 .

Blox a mia truncata, - Ann. Mg. n. h. 1551.

XIII. p. 46 . t. 16. f. 17 , B. B.) - Berk. In. p. 327 . f. 74. b. - R. f. 165 .

Bolacotrichia grisea. - Ann. Mg. n. h. 1551. VII. p. 97. t. 5. f. 4. (B. B.'

Bolbitius (cf. Agaricus).

- flavidus. - [S. 299.]

- fragilis. - [S. 299.

- luteolus. - [S. 299.]

- mitriformis. - Hook. Lond. J. 1N44. III.

p. 156. t. VI. B.

- pusillus. - Borcz. fg. ingr. t. 1. f. 1.

tener. - Berk. Out. t. 12. f. 2.

titubans Fr. - [S. 299.] - Bisch. f. 3250.-

Bail S. t. 34 .

- vitellinus. - [S. 299.]

Boletus. - Ann. sc. n. 1s37. VIII, t. $\varsigma-11$.

(Fruetif. Lév.). - Rostk. ap. Sturm h. 21-21:

t. $1-15 .-[\mathrm{Su} .315$.

abietinus. - Purton. I. t. 13.

aeneus. - [S. $317,30$.$] - Roq. t. 3. u. I.$

f. 1. : - Rost. apud Sturm t. 37 , = edulis Fr. aestivalis. - [S. $31 \%$.] - Huss. II. 25. -

Fries Sv, t. 13.

- albidus. - Roq. t. \. f. 2.
Boletus alutarius Fr. - [S. 318.] - Krbh.t. 74. f. 8. 9. (ex Berk.).

- Rostk. : [S. 315: 46.]

appendiculatus. - [S. 317.]

artemidorus Lz. - Hrz. t. 13.

- asprellus. - [S. 31S.]

- aurantiacus (ef. scaber). - Roq. t. 9. f. 2. 3.

- Cd. Ic. V. t. 10. f. S2. (Fruct.).

aurantius. - [S. 31 4.45.$]$

auriformis Bolt. - Wstd. 766 .

- badius. - [S. 316.] - Kops F1. bat. XI.

41. - Bail S. t. 31 .

- bovinus L. - [S. 316.] - Huss. I. 34. -

Hrz. t. $76 .-$ Kl. $37 .-$ Cd. Ic. V. t. 10. f. 55.

Fruct.

—_- Rostk. : = mitis. $-[$ S. 316.] - Ital. 332.

- brachyporus. - [S. 316:-17.] - Letell. 60ti.

- Rostk. t. 11. = B. Sistotrema Fr, Su.

- buxeus. - [\$. 317: 31.b.] - Rostk. t. 30.=

13. Obsonium Fr. Su.

calopus. - $[$ S. 316: 19; 317.] - Hrz. t. 6?.

- Vent. mi. 6\%. f. $1-4 ;$ 74. f. 1. 2.

- carnosus. - [S. 316.9 : rut. v.] - Rostk.

t. $14=$ rutilus Fr. Su.

carpineus Sow. = Polypor. adustus Fr.

- castaneus Pull. - [S. 315.] - Huss. II. 17.

Fl. dan. X. 1792 .

- v. badius Fr. - Kl. 108.

cellulosus Fl. dan. = Polyp. vulgaris Fr.

chioneus. - [S. 31ऽ. 45.

- chrysenteron. - [S. 316.] - Roq. t. S. f. :

- Anin. sc. n. 1537. VIII. t. 11. f. 27. Fructió.

Lév:')

- cinnamomeus, - [S. 316. 13. b.]

collinitus. - [S. 316.$]$

- concentricus Schum. = Polypor. adustus Fl. dan.

- concretus. - Alger. t. 32. 3.

- coriaceus. -- Wstd. 97.

- crinitus Spr. : = Polypor. hydnoides Fr.

- crocipodius. - Letell. 666.

_- cyanescens Bull. - [S. 31S.] — Letell. 651.

- Kops Fl. bat. X. 770 . - cf. Bol. sphaeroce-

phalus Barla. - Hrz. t. 71. - Roq. t. S. f. 1. -

Loud. f. 16110.

- dentatus. - [S. 316. 20.] - Rostk. t. $25=$

subtomentos. Fr. Su.

- dictyopus. - [S. 31\%.3\%.] - Rostk. t. 33

$=$ sordarius $\mathrm{Fr}$. Su.

dimidiatus. - Thunb. Fl. jap. t. 39.

dulcis. - Letell. 614.

- edulis Bull. - [S. 317.] - Badham. t. 3. -

Kops Fl. bat. X. 739. - Berk. Out. t. 15. f. fi.

- Vent. t. 10. f. $57-90$ - Bisch. f. 3255 .

3150 . - Roq. t. 4. f. 2 ; t. 5. - Hrz. t. 40.41 .

- Fries Sv. t. 13. - Berg, Charakteristik. 1S60.

t. 2. f. 14. - Kl. 618. - Kl. II. 302. - Ann.

se. n. 1 $>3 \%$. VIII. t. 11. f. 26. (Fructif. Lév.). -

Nov. Act. is t2. XIX. II. t. 57. f. $92-94$. (Spor.

Phöb. . - Rostk. t. $36=$ reticulatus Fr. Su.

? cf. Bol. esculentus Pay.

- Rostk. : [S. 317.32*] - Huss. I. \$1. -

Viv. 25. - Vent. mi. 14. f. $1-3$; t. 22 . -

Staude t. 3. f. 3.

elegans. [S. 316.] - Huss. II. 12.

- elephantinus. - Huss. II. 2.

- eriophorus. - [S. 316: 20.21.] - Rostk

t. $20=$ subtomentosus Fr. Su.

- erythropus P. - [S. 317.] - ef. lurid. -

Bisch. f. 3143 . (? luridi var.). - Phöb. Gift.

t. s. - Hrz. t. 56 . Ann. sc. n. 1s37. VIII.

t. S. f. 9. Fructif. Lév.). 


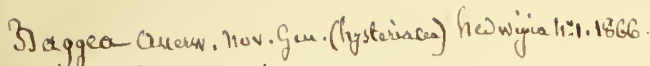
Bull. So. Bot X XII. $.0170 .-$

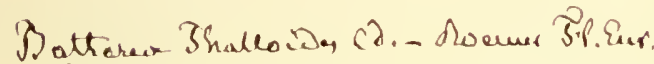
3ace.3.. -

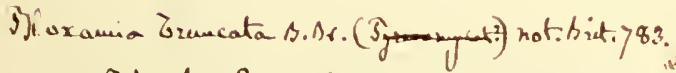

- Tatiagloa Reak +Brim Schain...

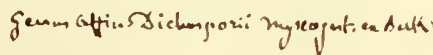



13oletus esculentus P. - Loud. f. 1610s. Pay, b. cr. p. 117 . f, 516.

- felleus Bull. - [S. 31S.] - Kops Fl. bat. $\mathrm{X}, 740.2$.

_- ferrugineus $\mathrm{B} .=$ piperatus $\mathrm{Fl}$. dan. firmus Spr. - Wstd. 1257.

Habelliformis Scop. = Polypor. lucidus Fr.

- Flabellum. - Ioffm. v. hereyn, subt. t. 15. f. 1 .

flavidus. - [\$. 313.3.] - Vent. mi. 97. f. 1-3. - Cd. Ic. V. t. 10. f. \$6. Fructif. . KI. 1113. - Rostk.: [S. 316: 2; zu collinitus,

Fr. 1561.]

- Harus. - [S. 316*.]

- Hoccopus. - cf. strobiloides. - [S. 317.]

- Rostk. : [S.318.41.] versipellis Fr. Su. fuligineus. - [S. $317 ; 318:-41$ u. 45.$]$

fulvidus. - [S. 31 4 .]

- fumosus P. - Wstd. 997.

fuscus. - [S. 316. 20.] - Rostk. t: $12=$ subtomentos. Fr. Su.

- granulatus, - [S. 316.] - Letell. 601. Schntzl. t. 16. f. 26.27. — Vent. mi. 31. f. 1. 2; 69. f. 4. 5 ; 75. f. 1. - Ital. 290. guttatus. - [S. 316.13.]

- holopus. - [S. 315.45.] - Rostk. t. $45=$ scaber v. chionaeus $\mathrm{Fr}$. Su.

- hydnatinus Bosc. - Berl. Mag. II. p. 2. t. 1 . f. 2.3 .

imberbis. - cf. Tramet. gibbosa.

imbricatus. - Wstd. 146.

- impolitus Fr. - [\$. 31\% .] - Kirbh. t. 7 I.

f. 10. 11. ex Berk. ; = sapidus Hrz. - Vent. mi. 99. f. 1.2. - Fries Sv. t. 42.

- incarnatus Schum, = Polypor. abietinus Fl. dan.

infundibuliformis. - Wstd. 429.

- iricleus. - [S. 317.30.] - Rostk.t. $15=$ aeneus Fr. Su.

- lacteus. - Ann. sc.n. 1445. XIX. t. 9. Lév. .

- lanatus. - [S. 316. 20.] - Rost. t. $21=$

subtomentosus Fr. Su.

- laricinus. - [S. 316.] - Huss. I. 25.

- lilaceus. - [S. $316^{* *}$.]

- lividus Bull. - [S.316: 15***.] - Iil. 227.

- lupinus. - [S. $31 \%$.

- Iuridiformis. - [S.317.36.]

- luridus Schff. - [S.317.] - Badham. t. 7, .2.

Huss. II. 26 ; 35 . - Fl. dan. XI. 1962. - Ann. Mg. n. h. 1<36. I. t. 5. f. 39. Fruetif. Berk.).

Phöb. Gift. t. 7; t. 5: 1. 2. — Loud. f. 1.6107. - Kl. $1406 .^{-}$- Berk. Out. t. 15. f, 5. - ? ef. Bol. pemiciosus Roq. - Letell. 67s.

- erythropus Schff. - Letell. 612. ery- $3 .:$ Huss. I. 7. - Viv. 40. - Fries

Sv. t. 12 .

luteus L. - [S. 315.] - Vent. mi. 97. f. 5.

6. - Loud. f. 16103. - Bisch. f. 3459. - Hrz. t: 6. - Kl. II. 615 .

- macroporus. - [S. 316. 13. b.] - Rostk.

t. $13=$ guttatus Fr. Su.

marmoreus. - Roq. t. 6.

medulla panis. - Wstd. $125 \%$.

- mesentericus Schff, = Polyp. giganteus Fl. dan.

Meyeri. - [S. 317.]

mitis. - [S. 316.]

nigrescens. - [S. 315. 45.]

obliquatus Bull. = Polyp. lucidus Fr.

Obsorium. - [S. 31\%:]

olivaceus. - [S. 317.] 13o le tus Ostrea. - Ioffin. v. hercyn, subt. t. 15. f. 2 .

- pachypus. - [S. 317*. 27.] - Letell.611.IIuss. I. 22.

-. pamnosus. - [S. 316. 20.] - Rostk. t. $2 \cdot 2=$ subtomentos. Fr. Su.

- parasiticus. - [S.316.] - Berk. Out. t. 15. f. 1 .

- pascuus P. - Krbh. t. 76. - Sturm. h. 19. t. 1. Cd.)

pelleporus Bull. = Polypor. adustus. Fr.

- perennis. - Wstd. 129.

- perniciosus. - Roq. t. 7. f. $1-3$.

- picrodes. - [S.31\%.26.] - Kl. 1713. -

liostk. t. $24=$ pachypu.s Fr. Su.

- piperatus Bull. - [S. 316.] - Fl. dan. XI.

i4.j0. 2. - Bisch. f. 3129 . - Loud. f. $16105 .-$

lioq. t. 7. f. 4. - Sturm h. 14. t. 60.

- Placenta Schun. = Polypor. zonatus Fl. dan.

- porphyrosporus. -- [S. 317.311*.]

- Preauxii. - Canar. t. 4. f. 1.

prumatus. - [S. 316.$]$

_- pruinosus Fr. - ef. B. rubropruinosus Barla. pubescens Schum. = Polypor. p. Fl. dan.

- pulverulentus Opat. - [S. 316.22.] - Kl.

$226 .=2 u$ radicans Fr. Su. 316 .

- purpurascens. - [S. 316: 1S.] - Rostk.

t. $S=$ pruinatus Fr. Su.

purpureus. - S. 317.] - Fries sv. t. 11.

- radicans. [S. $311 \%$.$] - Cd. Ie. V. t. 111. f. S1.$

Fructif. - Rostk. t. 20 = ? appendicul.

- Wostk. : . S. 317. 23.] ; Fr. Ep. 416.

- regius liz. - [S. 317.] - Vent. t. 10. f. 99. 190.

reticulatus. - $[\mathrm{S} .317$.

Ribis Schum. = Iolypor. R., Fl. dan.

rimosus. - Vent. t. 10. f. 93. 91.

-_ romanus. - Badhan. t. 15, 2.

__ rubeolaris Bull. = luridus Fl. dan.

- rubescens. - [S. 316.$]$

- rubiginosus. - [S. 317.]

rubro-pruinosus. - Nov. Act. 18 til. XXVII.

t. 15. = pruinosus Fr.).

- rufus. - [S. 31S. 11.] - Cd. Ic. V. t. 10.

f. $\$ 3$. Fructif.).

- rutilus. - [S. 316 .

- salicinus Sow. - Wstd. 997.

- sanguifluus. - Vent. mi. 94. f. 3-5.

- sanguineus. - [S. 316.]

- - rhodoxanthus Krbh. = purpureus Fr.

(Rbl..).

- sapidus. - [S. 317. 32.] - Hrz. t. 51.

$=$ impolitus Fr. Su. 317.t.

- Satanas, - Vent. mi. 40. f. 1 ; 78. f. 3.4. Kl. 1712. - ? cf. B. luridus Phöb., marmoreus Roq. - Rostk. t, 31 = luridus Fr. Su. Rostk.: [S. 317.36.$]$

scaber Fr. - [S. 315.] - Badham. t. 7, 1. -

Huss. I. 57. - Kops Fl. bat. X. 740. 1. Ann.

Mg. n. h. is35. I. t. 5. f. 40. 41. (Spor. Berk.).

- Loud. f. 16109. - Vent. t. 10. f. 91.92.-

Cd. Anl. t. H. f. 75 : 5-12. - Hrz. t. 2. -

Fr. Sv. t. 14. - Roq. t. 9. f. 1.2. u. t. 4. f. 1.

- - Bull.: Kl.514. - Vent. mi.31). f. 3.4 ;

$46 ; 47 ; 95$. - Staude t. 3. f. 2.

- aurantiacus Bull. - Hrz. t. 25:

- scobinaceus. - Act. Turin. 1805. XIV. t. 2.

(Cumino).

sericeus. - Krombh. t. 76.

- Sistotrema. - [S. 316.17. u. 16.] - Rostk.

t. $19=$ rubescens $\mathrm{Fr}$. Su.

- sordarius. - - S. 317.$]$ 
Boletus spadiceus. - S. 316.] - Sturm h. 23.

t. 29. hier.).

sphaerocephalus. - Nor. Act. 1560. XXYII.

t. 17. Barla = cyaneus Bull.

- spumeus Sow. - F1. dan. X. 1791.

— squalidus. - [S. 316.]

- squamulosus. - [S. 317.31.] - Rostk. t. 17

= raccinus $\mathrm{Fr} . \mathrm{Su}$.

- Sterbeeckii, - [S. $31 ; \cdots$.$] - Bull. ac. bels.$

1412. IX. 2. t. 2.

- strobilaceus. - [S. 317.] - Vent. mi. 50.

f. 3. 1 .

strobiloides Frbl. . = floccopus Vahl. ? = stro-

bilacens Fr. Ep.

- suberosus 13. = Polypor, adustus Fr.; Fl. dau. - [S. 320.53.

- subtomentosus I. - [S. $316: 20,19$.

Sturm h. 22. t. 20. erio. - Bisch, f. 332 ;. -

Irr. t. 31. - Loud. f. 16106. - K1. 39. -

Huss. 1. 5. - Vir. 27. - Rostk. t. $23=$ chrysent. Fr. Su.

— sulfureus. - S. 316 .

suspectu: lirbh. - cf. impolitus.

- tessellatus. - [S. 317. 26.7 - Rostk.

t. $32=$ olivaceus Fr. Fu.

tuberosus Schum. = luridus Fl, dan.

- unicolor Bull. = Dacdalea un. Fl. dan. -

Wstd. 124.5.

- vaccinus. - [S. 31\%.

— variegatus $S w .-$ [S. $313 ; 13$.$] - Hrz.t. 15$.

- Kl. 35.

- versicolor. - [S. 316.19.] - Wstd. 146. -

Rostk. t. $10=$ chrysenteron Fr. Su.

_ rersipellis. - $\dot{S} .31 \varsigma$.

— vinosus. - Vent, mi. ij. f. 4.5.

- viscidus. - S. $31 \%$.

Bombardia ef. Sphaeria

— fasciculata 'cf. Sphaeria f.) - Kl. 1553. -

[S. 3\$9.] - Krrpt. Bad, 52.

Bostrichonema alpestre Ces. - Ital. 149.

Botryocha et e faginea. - Cd. Ic. VI. t.9. f. 95.

Botr pocladium delectatum Preuss. - Sturm.

h. 36. t. 5 .

Botryosphaeria. - cf. Sphaeria.

Botryosporium. - [S. $490^{* *} .50 \%$.]

- diffusum. - Bon. t. 7 . f. 155. - Sturm

h. $11 . t .5$.

- elegans. - Cd. Anl. t. B. f. 16: 6-9.

hamatum. - Bon. t. 7. f. 157 .

- pulchrum. - Pay. b. cr. p. 101. f. tio. -

Kil. 1360. Cent. XT.

Botrytis. - S. 490. 3!2.] - of. Peromospora, Spicularia, Polyactis, Stachrlidium, Trichosporiun.. - Flora 154i. no. 1. f. 11. Fresen.

- - auf Pollen: C. Mitller, PHanzenwelt.

1557. p. 63. Karsten . - C. Müller, Pflanzenstaat. 1560. p 265. 3. Copie

- acinorum. - Fres. t. 2. f. 15-22.

— acladla. Fres. t. 2, f 23-21.

— aeruginosa Schum. - Fl. dan. XIII. 22\% 3.

- agaricina Lk. Verticill.). - Loud. f. 16579

-- Sturm h. 4. t. 51.

— aggerifera. - Letell. 662. 3. (Bassiana :.

- allochroa Lk. - Bisch. f. 3751.

- arborescens. - Journ. hortic. soc. 1546. I.

t 4. f. 24. Berk.).

- Arenariae. - Journ. hortic. soc. 1\$46. I.

t. 4. f. 22. p. 31. Berk. . - Wstd. 1299.

_- Bassiana Bals. - [S. 490. 3.] - Robin r. par. t. fi. f. 5- - - Berlin, entomol. Ztschr. 1455. t. 2. f. 7; t. 3. f. 12 ; t. 6. f. 29.30 . Lebert.) - Kl. 1052. - Kil. II. 1\% - Journ. hortic. soc. 1416. I. p. 32. 13erk.! - Ital. 792.

Botrytis bicolor Lk. - [S. 490.] - Bisch. f. 3752 . - Jion. t. 6. f. 112.

- brunnenla. - Kil. II. 771.

- cana Ii. - ef. Polyactis c." - Wstd. 435.

S. 490.7

— capitata. - : Letell. 697. 4.

- carnea Schum. - [S. 191.] - Fl. dan, XiII.

$22 \%$. 1. - Fuck. rh. 146 .

- cinerea P. - S. 491.493.] - Fl. dan. XIII

2275. 2. - dazu Polyactis sclerotiophila Kl. cx

('esat. - Kil. i2. - Dism. 225. - Fuck. rh. 114. cinereo-rirens. - Bon. t. 6. f. 14 .

cinnabarina. - S. 491.

- citrina. - Anm. Mg. 12. h. 183ง. I. t. S. f. 12

Berk.

- crustosa Fr. $=$ Peronosp. macrospora Casp.

- Brit. f. 333. - Letell. 697. 1.

- curta. - Brit. f. 209. - Lindl. v. K. p. 33

f. 21. - Ann. Mg. 11. h. 1534. I. t. 5. f. 13

Berk., - Berk. In. p. 245. f. 61. d. - Berk

Out. t. 1. f. 7. b.

— densa. - S. 493.] - Sturm 1. 4. t. 52.

- destructor. - Brit. f. 345. - Ann. Mg. 13

h. 14H1. VI. t. 13. f. 23. Berk.

- didyma. - [N. 492.]

- diffusa A. - Loud. f. 1657 s.

effusa. - Brit. f. 53 ; 335 .

Dsm.: Ann. sc. n. 1537. VIII, t. 1.f. I

Gret. : Dsm. 1091.

elegans, - [S. 490.] - Bon. t. 6. f. 141. -

Schacht, Zuckerrübe. 1563. f. 11. 12.

- elongata. - [S. 491.

- epigaea Lk. - cf. Polyatis ep. - Fuck. rh.

117

- ervthropus c. Sclerotio: Ann. sc. n. 1813.

XX. t. 7. f. 5. Lér. . - Pay. b. cr. p. 6. f. 16 .

- fallax Dsm. = Peronosp. Solani. - Wstd.

1195.

- farinosa Fr. - Brit. f. 335. - Kl. 264. -

= Peronosp. effusa Rbh. - Wstd. 439.

- farinulenta. - Letell. 662. 4.

fulva. - cf. Polyactis.

- furcata. - Fres. t. 2. f. S-11.

- ganglioniformis Bk. - cf. Peronospora. -

S. 493, 3. - Joum. hortic. soc. 1546 . I. t. 4

f. 25. Berk.).

- geminata. - Bot. Zt. 1\47. t. 4. f. 9. = Peronosp. ganglion. ?

_ geophila. - cf. Polyactis geoph.

- gigantea. - Letell. 709. 1.

- grisea Fr. - [S. 491.493.] - Bisch. f. 3731.

- Letell. 709. 4.

- Haplaria. - Cd. Anl. t. B. f. 13:1.

infestans. - Wstd. 1194. - Regel's Garten-

flora. 1654. t. 57. f. 5-10. - Kl. 1679. - cf.

Peronosp. Solani.

- interrupta. - Fres. t. 2. f. 12-14. - ? Iil

II. 771 .

- Jonesii. - Ann. Mg. n. h. 1\$51. XIII. t. 15.

f. 12 . p. 462 . (B. B.).

- isabellina. - K1. 1570.

__ lateritia. - [S. 491.] - Brit. f. 95.

- laxa. - [S. 491.] - Letell. 697. 3.

- leucospora. - [S. 490.]

- macrocarpa. - Ung. Ex. t. 2. f. 13. B. -

ef. Monosporium macr.

[ macrospora. - [S.491** 3.] - Wstd. 129 \%

- Sturm h. 4. t. 50.

- murina Lk. - ef. Sporotrichum murin. -

Sturm h. 3. t. 36 . 
1 

Botrytis nivea. - Ung. Ex. t. 2. f. 14. Schnczl. t. 12. f. 29.

- nutans. - Pay. b. cr. p. (i). f. 24 .

— ochracea. - Letell. Tษ9. 2.

- olivacea. - Letell. 66;2. 2.

- olivaceo-lutea. - Ann. sc. n. 1537. VIIl.

t. 1. f. 2. Dsm.!.

orulifera. - [S. 191.]

pallida. - Carol. V. 91.

- parasitica Cd. (cf, Peronos]. p.). - Cd. Ic.

V. t. 2. f. 15. - Bisch. f. 3743. - Kützing Phi-

los. Bot. 1\$52. t. 19. f. 10. - Berk. In. p. Y2.

f. $23 .-$ Kl. $153 .-$ - Wstd. $\$ 10 .-\mathrm{D} \mathrm{sm} .1093$

Bon. t. 5. f. 124. - Kot. Zt. 1459. t. 11.

f. -. Keimg. Hoffm.! - c.f. nivea. - Dsm. 111 .

159. - Lactucae Brit. f. 331. - Letell 697. 2.

- Journ. hortic. soc. IS I6. I. t. 1. f. 2ti. Berk. plebeja. - Fres. t. 2. f. $1 \ldots i$.

Polyactis Lk. - Wstcl. 146. - Dsm. 64.

- polysperma. - Kintzing Philos. Bot. 14,2.

t. $19 . \mathrm{f} .11$.

- polyspora. - Schntzl. t. 12. f. 24. 25.

Sturm h. 3. t. 35.

pulla. - [S. I91.

Pulvinulus. - lil. 1ל\$1

reptans. - Bon. t. 1j. f. 1 f6.

rhodospora. - Bon. t. (i. f. 1:9).

rufa. - [S. 191.]

- simplex monilis A. S. - zu Erysiphe Grami-

nis Iul. Sel. I. 212.

- Solani cf. infestans; - Peromosp. S.) - lil.

1579.

- sonchicola. - Kl. 1775 .

- spongiosa ? - ef. Scorias sp.

- tenera. - [s. 191.]

- tenuissima. - [s. 193. 2.]

- terrestris. - Amn. Mg. n. h. 1s 11. VI. t. 1.1.

f. 2.1. Berk.).

Tilletei D. - Dsm. 226

umbellata. - [s. 490 .]

umbrina. - Bot. Zt. 1553. t. 7. f.9. Bonord

vastatrix Lib. - Wstd. 1193. ed. 15.57.

velutina. - Bon, t. 6. f. 113.

vera. - [S. 490.] - Letell. 709. 3. Spicu-

laria gemina $\mathrm{P}$.

- verticilloides. - Flora. 1s 17 . no. 1. f. 10. (Fres.).

. Viciae. - Journ, hortic. soc. I\$ $\$ 6$. I. t. 4.

f. 23. (Berk.) .

violacea. - Wstd. 1199

virella. - [S. 191 .

viticola. - C'arol. V. 90.

- vulgaris Fr. - S. 190.] - Wstd. 196. -

Bisch. f. $37+0$. - Willkomm, Wunder d. Mi-

krosk. 1556. p. 91. f. d. - Pringsh. Jahrb. II. t. 31). f. 26. Hoffm.)

Bovista. - [S. 142 .]

- ammophila. - Ann. sc. 11. 1S1s. IX. t. 9.

f. 5. 6. (Lér.).

_ caelata. (cf. Lycoperd.). - Berg, ('harakte-

rist. 1560 . t. 1. f. 3 .

craniifera. - [S. 442 : calv.]

favosa Rostk. - Sturm h. 1s. t. 3.

furfuracea P. - Wstd. 1261.

gigantea Grev. - Huss. I. 26. - Loud. f.

16512. - Sturm h. 1S. t. 10.

- miniata Dillen. - Wstd. 741.

- nigrescens P. - [S. 142.] - Loud. f. 16511. -

Kl. 1410. - Berk. Out. t. 20. f. 5. - Carol. V.

51. - Sturm h. 15. t. 15.

officinarum Dill. - Sturm h. 15. t. 1.

plumbea P. - [S. 442.] - Cd. Ic. V. t. 6. f. 47. - Ann. $\mathrm{Ig} . \mathrm{n} . \mathrm{h}, 1540$. IV. t. 5. f. $15-1 \mathrm{~s}$

Fructif. Jerk.) - C'd. Anl. t. C. f. 36:3-(; ; t. H. f. is: 11). 11. - Berk. Out. t. 20. f. 6. Ann. sc. n. 1539. XII. t. 2. Fruetif. Berk.). Pay. b. cr. p. 7. f. 20. Anat.) - Kil. 27. K1. II. 143. - Bot. Zt. I559. t.11. f. 20., (Keimg. Hoffm. . - Wstd. 1261. - Sturm h. 1S. t. 16.

Borista pusilla liostk. - Sturm h. 1^. t. 1.

- suberosa. - [S. $413^{\circ *}$.] - Sturm h. is. t. .2. tunicata. - S. 412.]

brachycarphium = Brachyclad. Thomasin. Berk.

Brach ycladium. - $[$ S. jot.

- penicillatum. - $[\$ .501]-$. D.m. III. 413 - Wstd. 591. zu Sphaeria Papaveris ex Tul Sel. 43. - R. f. $3>0$ Dendryph. Fr. - Pay b. er. p. it. f. 334 - Willkounm, Wunder il Mikrosk. 1456. p. 92 . f. f-h. - Kl. 136i. Dendryph. pen.)

- "Ihomasinum. - Ann. Mg. n. lı. 1s f. II t. 11. f, 2. Berk.

Bremia Lactucac. - ef. Peronospora ganglionit. Briarea. - [S. 459 t.

- aurea. - Fuck. rh. 141.

- elegans. - Cd. Anl. t. C. f. 21: 5. (i.

Pay. b. cr. p. 69. f, 295. - Sturm h. 11. t. 1.

Penicill. sparsum Lk.

- orbicula. - C'd. Ic. III. f. 34.

Hroomeia. - [S. H(t).]

- congregata, - Pay. b. cr. p. 112. f. 514. -

Hook. Lond. J. 1544. III. p. 193, t. VI. A.

Bryochista. - [S. 47.

Bry oc ladium, - [S. 406.503*.

Bryom yecs. - S. ju6.]

- elegans. - Nov. Act. 1s 12. XIX. II. t. ว5.

Miquel. - Kops Fl, bat. VIII. 619.

- Orthotrichi. - Cd. Anl. t. H. f, is: 1-2.

Bulgaria. - [S. $35 \%$.]

- A gaves Rbh. - Kl. 1223.

- Alobosa. - [S. 35\%.]

- inquinans. - [S. 354.] Wstd. 191. - Kops

F1. bat. XI. \$14. 4. - Berk. t. 22. f. T. - Cd.

Anl. t. G. f. 64: 20-23. - Bisch. f. 3359. -

Fuck s. iss. - Dsm. 569. - Ann. sc. 12. 15.3.

XX. t. 1j. f. 1-i. (Tulsn.). - Ioud. f. 16i2s11. - Bail S. t. 20. - Pringsh. Jahrb. II. t. 31. f. 31 .

Hoffm. - Kl. 115; Cent. VIII, suppl - Kl.

II. 599. - Voges. 197. (Pez. nigr.) - Carol. T.

43. - Huss. I. 32. - Ital. 235.

- nigrita. - \$. 3.54.]

pellucens Fr. - S. 35s.] - Fl. dan. XII.

21131. 2.

- pulla. - \$s. 35s.]

__ rufa. - Carol. IV. 23.

- sarcoides. - [S. 355.] - Brit. f. 273. - Cd.

Ic. V. t. 9. f. 6) Asci) - Berk, t. 14. f. 6. With.: Sturm h. 31 - t. 14 15. Szl !

- Loud. f. 16251. - Fuck. s. 757 .

- P.: K1. 723. - Kl. II. 115. - Sper-

matia: = Coryne sarcoid.

Bullaria Umbell. - [S. 505. 2.] - Wstd. 85i. Bulliardia. - [S. $43 \%^{\circ}$.]

- inquinans. - Linn. 1530. V. t. 6. f. 15 (Jungh.).

B yssocaulon. - [S. 465.]

$\mathrm{Byssocladium.} \mathrm{-} \mathrm{[S.} \mathrm{521.} \mathrm{524.]}$

- c cerevisiae. - Kl. II. 764 .

- dendriticum Ag. = Myxotrich, chart. FI. dan.

— fenestrale Lk. - Wstd. S66. - Kl. 454. -

Dsm. 102. - Voges. 1100.

- fenestrarum. - W'std, $\$ 66$. 
Byssocystis textilis. - zu Erysiphe lamproc.? Tul.). - Kl. 1726.

Byssothe eium circinans. - Bot. Zt. 1561 . t. 10. f. ร. p. 251. Fuck.) - zu Rhizoctonia Medicag. ex Tulsn. Sel.

Byssus. - [S. 521.]

- Aluta. - Rhacod. Al. 1'.

— antiquitatis. - [S. $5033^{\cdots}$.

-_ aurantiaca. - Wstd. 432.

- aurea J. - Wstd. 699.

— candida DC. - Ilimantia plumosa $\mathrm{F}$.

- cryptarum. - Wstd. 195.

— dolaris. — Wstel. 195.

- filamentosa. - Wistd. 5 Is

-... gigantea. - W'std. 100.

- mollissima. - Wstd. 145.

- petraea Dill. — W Wtd. 699.

- phosphorea L. - Wstd. 767.

— plumosa. - Cd. Anl. t. B. f. 1: 1 .

- purpurea Lam. - Wstd. 791.

- - septica I. - Wstd. 195. - Nov. Act. XI.

t. 59. Hedwig .

- velutina. - Wstd. 200.

Cacumisporium tenebrosum Preuss. - Sturm h. 35 . t. 11 .

Ca e om a. - cf. Aecidium, Uredo, Epitea, Ustilago.

- Absinthii. - Cd. Ic. VI. f. 2.

- aeriginum. - Wstd. 127.

- Alchemillae. - Cd. Ic. VI. f. 1.

-_ Alliorum I.k. - Wstd. 56 T.

allochroum Lk. - Wstd. 11 is

- antherarum Lk. = Ustilago violacea - Bon. Con. p. 192.

— apiculatun. - Wstd, $35 \%$

— apiculosum. - Wstd. $255 ; 1172$.

- - Bon.: R. f. 193. - cf. C. I'hyteuma-

tum Cd. : Bon. Con. p. 1 is.

Armeriae Lk. = Uredo'globoso-rufa Bon. Con.

— - S-l.: Wstd. 1166 .

aroideatum $L k$, = Aecid, aurant. Bon.

Artemisiae Lk = Credo globoso-fusea Bon.

Wstd. TtS.

- Asperifolii s-1. - Wstd. $\$ 34$.

- aurantiacum. - ef. C. lanceolatum.

- Betarum Lk. - Wstd. 2s6. - R. f. 192. =

Uredo globoso-fusca Bon.

__ berberidatum. - Wstd. 227 .

- betulinum. - ef. Dicaeoma bet B. - et Epitea bet. - Wstd. 129 .

Bistortarm Lk. = Ustilago destruens Bon Con, p. 192.

— borraginatum Lk. - Wstd. $\$ 34$.

- buniatum Lk. = Aecid. aurantiac. Bon.

- Caladii Lk. = UTedo globoso-fusca Bon

_- Calthae Lk. - Wstd. 1171.

- Campanulae. - Wstd. 35.5.

candidum. - Wstd. 125.329.330

- Capraearum S-1. - Wstd. 670.

- Carpini. - Schweiz. 5.

— Caryophyllacearum Lk. - Wstd. 674.

- Cerastii S-l: - Wstd. 67t.

- chenopodiatum Lk. = Aecid. aurant. Bon.

— Cichoracearum S-1. - Wstd. Si3.

- cinnamom. ef. Uredo cinn.

- Colchici. - [S. 516.]

- compositatum Lk. = Aecid. aurantiac. Bon. compransor. - R. f. is $=$. Cóleospor.

aureum Bon. Con. 157.-Wstd. 126.

- convallariat. Lk. - Wstd. 535 .

- cornutum S-1. - Wstd. T+2.

- crassatum - Aecid. crass, - Wstd. 55 \%
Ca e oma crassum S-l. - Wstd. 557.

- eressatum Lk. $=$ Aecid. aurant. Bon.

- cruciferatum Lk. = Accid. aurant. Bon.

cuminatum Lk. = Aecid. aurant. Bon.

cylintricum. - Wstd. 12!).

cylindrites $\mathrm{Lk} .=$ Aecid. lacerat. v. Crataegi

Ariae DC. - Wstd. 1156 .

- Dentariae Lk. = Uredo globoso-fusca Bon.

elegans $\mathrm{S}-1 .-\mathrm{Wstd}$. 535.

epilobiatum Lk. - Wstd. 559.

Epilobii Lk. - R. f. 192. = Uredo globoso.

fusca Bon.

- Epitea $\mathrm{S}-1$. - Wstd. 543.

- Epiteum Lk. = Physonema vulgare Bon.

- Euphorbiae S-1. - Wstd. 537.

- Euphorbiarum. - Wstd. 255.

- euphorbiat. Lk. = Aecid. aurant. Bon. -

Wstd. 837 .

— Falcariae S-1. - Wstd. 746. (Aecid.).

- falcariatum Lk. - Wstd. $7+6$.

— _ fallax. - Gd. Ic. V. t. 2. f. 7 .

- Ficariae. - S. 516.] - Ung. Ex. t. 6. f. 29.

Filicum Lk. - Wstd. 561 .

- flarum Bon. - Ital. 900. - R. f. 198. -

Bon. Con. t. 1. f. 4.

- Hosculosarum Lk. = Ustilago violacea Bon.

Con. 192.

__ formosum S-1. — Wstd. 515.

- fuscum Bon. - R. f. 196. - Bon. Con.

t. I. f. 3.

- Galii Lk. - Wstd. \$44.

- Geranii Lk. = Uredo globoso-fusca Bon. -

Wstd. 671

- Grossul. Lk. = Aecid. aurant. Bon. - Wstd.

745 .

— gyrosum. - Wstd. 346.

_- Helioscopiae. - Wstd. 255.

- Hellebori. - Lib. 291. Uredo Ranune. DC.).

- Hydrocotyles Lk.=Uredo globoso-fusca Bon. Hypericorum $\mathrm{S}-\mathrm{l}$. - Wstd. 565.

- - hypodytes S-1. : zu Ustilago segetum Bon.

Con. p. 192. - Wstd. 572 .

- Labiatarum Lk. = Lredo globoso-fusca Bon. lanceolatum Bon. cf. Fusisporium aurantiacum Ling.

-_ Leguminosarum. - Wstd. 254.

- leucospermum S-1. - W Wtd. 662.

_ leucoxum Bon. cf. C. Erythronii Cd.

- lineare Lk. - Wstd. 331. - Sturm h. 29-.

t. 25. Preuss.).-R. f. 200 - Bon. Con. t. 1. f. 9.

- Lini Lk. - Wstd, 1179.

- longissimum. - Wstd. 232. - (segetum)

S-1.: Sturm h:29-.t. 27. (Preuss.). - ef. Uredo

long. = Ustilago olivac. Bon. Con. p. 191.

Lonicerae S-l. - Wstd. 832.

- Luzulae. - Lib. 395. - Wstd. 566.

Lysimachiae S-1.: cf. Aecid. Iy.

- lysimachiat. Lk. = Aecid. aurant. Bon.

- inamillare Bon. Con. $176 .=$. C. Populi $S-l$.

- marginale Lk. = Ustilagö destruêns Bon.

Con. p. 192.

- Mercurialis Lk. - R. f. 192. - Fuck. s. i.

= Uredo globoso-fusca Bon. - Fuck, rh. 255.

miniatum. - Fuck. s. 5. - Fuck. rh. $25 \%$

minutum. - Lib. 292.

mixtum Lk. - Wstd: $\$ ₫ 2$.

olivaceum Lk. = Ustilago oliv.

Onagrarum. - Wstd. 570.674.

Ornithogali. - : R. f. 195.

Padi. - Lib. \$9.

pediculariatum Lk. = Aecid. aurantiac. Bon. penicillatum S-1. - Wstd. 556 . 


Caeoma periclymenatum Lk. - Wstd. $\ 32$. phaeum Bion. - Puccin. Tanaceti Fuck. -

Fuck. s. 53. - R.f. 199. - Bon. Con. t. 1. f. 1.

- Fuck. Enum. Nass. f. 4. p. 12.

- pineum Lk. - Wstd. 6(ji).

Polygonorum. - Wstd. 24:3.

Copuli. - Bon.t. 3.f.64. = mamillare Bon. Con.

- porphyrogeneta Lk. = Uredo globoso-rufa Bon. Con. S. 515. 14.

- Potentillae Lk. of. Alchemillae Cd. - Wstd. 672.

Poterii Lk. - Wstd. 56 .

Primulae int. Lk. = Uredo globoso-fusca Bon.

Pseudocyperi = Uredo globoso-fusca Bon. -

Wstd. 815 .

- pusillum. - Bon. t. 1. f. 19. = Ramularia p. Ranunculacearum. - Wstd. 5 .

receptaculorum Lk. = Ustilago purpurea $B$. rhamnatum Lk. - Wstd. j5s.

Rhinanthac. Lk. = Erannium aureum Bon.

- Wstd. 354.

— Ribis S-1. - Wstd. 745 .

- Rocstelites. - W'std. 123.

- Rosac. - Wstd. 229 .

Rosarum ef. Urerlo miniata.

rubellatum. - W'std. 32\.

rubiginosum Bon. - R. f. 197. - Bon. Con.

t. $1 . \mathrm{f} . \mathrm{s}$.

- Rubigo Lk. - Sturm h. 29-. t. 21;. l'reuss.).

- Bon. Con. t. 1. f. 2. a.

- Ruborum S-1. - Wstd. 5 ti3.

- rubrum Bon. - IR. f. 299.

- rufum 13on. - R. f. 191. - Bon. Con. t. 1.

f. 7. u. 10 .

Rumicis. - Wstd. 328.

Saliceti Lk. = Physonema aur. Bon. -

Wstd. S6.

Salicis. - Cd. Aul. t. B. f. 2: 3. 4

- Schlechtendalii. - Kl. $>$ 7. Cent. XVIII.,. -

ef. Ustilago violac.

Scorodizon S-1. - Wstd. 567 .

- scrophulariatum. - cf. Aecid. s. - Wistd.

1157.

scutellosum Lk. - cf. C. apiculos. Lk. (Bon.

Con. p. 179.

— segetumn. - Wstd. 333. 334 .

- Sempervivi Lk. - Wstd. 1173. -. R. f. 597.

- Senecionis. - Wstcl. 230.

sitophilum Lk. = Ustilago sit. - Wstd. 333. Smilacis Lk. = Uredo globoso-fusca Bon.

Spermacoces Bon. - cf. Puccinia Sp. Lk.

- suaveolens. - Wstd. Si. - R. f. 195. -

Bon. Con. p. 176 . t. 1. f. 6.

subapiculosum Bon. - R. f. 99.

- Symphyti Lk. - Wstd. 332. - R. f. 192.

= Uredo globoso-fusca Bon.

- Terebinthi cf. Pileolaria T.

tragopogonatum Lk. - Wstd. \$33.

Tussilaginis P. - R. f. $176 .-=$ Erann.

aurant. Bon. u. Aecid. 'Tuss. T.

Umbellatarum. - Wstd. 359.

urticatum Lk. = Aecid. aurantiac. Bon.

utriculos. Lk. - zu Ustilago segetum Bon.

Con. p. 192. - Wstd. 1163.

Violae. - Wstd. 228.

Violarum Lk, = Uredo globoso-fusca Bon.

- Wstd. $\$ 10$.

vitalbatum Lk. - Wstd. 664 .

Calathiscus. - [S. 435.]

- Sepia. - Ann. sc. n. 1841. XVI. t. 16.

(Mont.) - Cd. Anl. t. E. f. 49 : I. 2.

H of $\mathrm{m}$ an $n$, Index Fungorum.
Calloria cf. Peziza, Dacryomyces]. - [S. 359. $470 * * *$.

- atrovirens. - [S. 359.471:2.]

chrysocoma Fr. - [S. 359.] - Fuck. s. is2.

fusarioides. - Kl. II. 676 .

species. - [S. 359.

stillata. - [S. 359.]

C alocera. - [S. 339.]

- cornea Fr. - [S. 339.] - Voges. 6९2. -

Loud. f. 16153. - Fuck. s. \$99. - Kl. 639.

Clavar.). - Nov. Act. 1842. XIX. II. t. 57.

f. 135. Spor. Phöb.) - Carol. II. 45.

— divaricata. - Hook. Lond. J. I 42. I. t. 6. f. 2.

fasciculata. - Bon. t. 11. f. 235.

- flammea. - Bon. t. 11.f. 23\% cf. Cal. viscosa.

- furcata. - [S. 339.] - Bon. t. 11. f. 236.

- glossoides. - [S. 339.]

— palmata. - [S. 339.

— stricta P. - [S. 339.] - Kil. 1121.

— tuberosa Fr. - Loud. f. 16182. - cf. Cal, vise. viscosa. - [S. 339\%] - Voges, 196. (Clav.

cor.). - ('d. Ic. Y. t. 10. f. 7t. (Fructif.) -

Cd. Anl. t. G. f. $72: 12-1$ 1. - Fuck. s. 900 .

- Bail S. t. 27. - Amm. Mg. n. h. 1435. I. t. 5.

f. 17. Spor. Berk.) - Kl. 131. - Kil. II. 120.

- Krypt. Bad. 338. - Schacht, Phys. Bot. 1852.

t. 1. f. 7-9. Basid. - - ej. Lehrb. d. An. Phys.

1556. I. p. 161.f. 21 . Anat.) - ef. Cal. flammea.

Calocladia. - cf. Frysiphe, Microsphacra.

- Berberidis Lév. - Fuck. s. 141.

- Ehrenbergii Lév. - R. f. 55t. - Erys. penic. ('aprifol.

— Hedwigii Lév. - Fuck. s. 14:3. - Erys. penic. f. Viburn.)

- Ravenelii. - Carol. IV. 137

Calostoma. - [S. 4fil. 460 .]

— cinnabarinum. - Cd. Anl. t. C. f. 3\: 10 .

Calvatia. - S. 142.

Ca ly ci um. - ef. Sphinetrina,. - [S. 366.]

- microcephalum. - Voges. 1411.

- tympanellum. - Berk. In. p. 399. f. 81. b.

Calyssosporium. - [S. 15s.]

- bicolor. - Cd. Anl. t. C. f. 23: 1-3.-

Bisch. f. 3500 . - Sturm h. 12. t. 27.

Camillea. - [S. 3S2.]

- Bacillum. - Ann. sc. n. 14.5. III. t. 5. f. 3

(Iont.)

- Cyclops. - Ann. sc. 11. 1555. III. t. 5. f. 2. (Mont.)

- Labellum. - Ann. sc. n. I 555. III. t. 5. f. 5.

Mont.

Leprieurii. - Ann. sc. n. 1555. III. t. 5.

f. 1. (Mont.)

- mucronata. - Ann. sc. n. 1555. III. t. 5 .

f. 4. Mont.).

Campsotrichum album. - Bon. t. 4. f. 100.

- Bot. Zt. 155\%. t. 4. f. 14. Bon.).

— bicolor. - [S. $502 * *$. ]

species. - [S. $503 *$.]

Camptotrichum. - [S. $465^{* * *}$.]

Camptoum. - cf. Arthrinium). curvatum. - S. 502.] - Kl. 461. (Cent. 15.).

- Cd. Anl. t. B. f. 12: 6. - Kl. II. 179. -

Brit. f. 310 . - Lib. 79. - Wstd. 974.

- leucosporum. - Bon. t. 7. f. 150.

Cancellaria Pyri Hergt. - Wstd. 556. - Aecid. corn. P.

Cantharellus (ef. Merulius, Craterellus). Pay. b. cr. p. 117 , f. 545. albidus. - [S. 312.]

anthracophilus. - Ann. sc. n. 1841. XVI. t.

14. f. 2. (Lév.). 
Cantharellus aurantiacus Fr, - S. 311.] Ital. 331). - Kops Fl. bat. X. 719. - Kl. 23. Fuck. s, 1052. - 13ot. Zt. 1560). t. I3. f. 3. (Hoffm.). - Kl. II. 615. - Berk. Out. t. 11. f. 1.- Vent. mi. 79. f. 1. 2; 97. f. 11.

- - v. lactea. - Kil. 319.

— Jungh. : Guepinia [S. 331 ?]

- Brownii. - S. $312 *$.]

— bryophilus. - [\$. 312.] - Fuek. s. 1041. -

Dsm. 6.52 .

- cibarius. - S.311.] - Carol. II. T. - Kop.

Fl. bat. IX. t. fitil. - Ann. Mg. n. h. 153ง. I. t. 5. f. 36. Fruet. Berk.). - Hrz. t. 15. - Cd. Anl. t. H. f. $75: 5-11$ - Roq. t. 10. f. 1. 2. Vent. t. 7.f. 65-fit. - Bisch. f. 3414. - Fuck. s. 1053. - Ioud. f. 16053 . - Fries. Sv. t. T. - Bail S. t. 33. - Merg, Charakteristik. IS60. t. 2. f. 11. - Kl. 24. - Kl. II. I11. - Ann. sc. n. 1837 . VIII. t. 10. f. 21. Fruct. I.év.). Kützing, Phil. Bot. 1451. t. 4. f. 1. Fruct. .

Purton. II, t. 10. - Badham t. 9, 2. - Huss. 1. 4. - Vir 41. - Wstcl. 310. - Vent mi. 39.

f. 1. 2. Staude t. 2. f. 1. - Kirypt. Bad. 559.

- cinereus. - S. 31\%.] - Kl. 1 S6\%. - Fuck. s. 1047 .

- cinnabarinus. - Carol. II. \&.

- cochleatus. - Sturm h. 33-. t. 4. Strauss). - Coemansii R. - R. f. 209. - = Cyphella ampla Lév. (Tul. Sel. 135).

— confluens. - [S. 312 \%

- coriaceus. - [S. $315 *$.

Cornucopiac. - Wstd. 192.

- cornucopioides Fr. - Huss. II. 37. - Dsm. 161. - Loud. f. 16055. - Ann. Mg.n. h. 1535. I. t. 5. f. 37. 39. (Fruct. Berk.) - Sturm h. 31-. t. 5. (Schntzl.). - Wstd. 1y2.

- crispus. - [S. 3 I2.] - Carol. V. 13. - Fl. dan. X. 1739. 1. - Kl. 25. - Fuck. s. $1016 .-$

1I. 16.

- Crucibulum. - [S. 312.]

cucullatus Jungh.: Guepinia [S. $331^{*}$.]

- cupularis. - S. 312.] (Arrhenia).

- deliciosus. - Letell. 633.

- Dutrochetii Mont. = C. Crucibulum Fr. -

zu Ag. panuoides : (Berk.)

- fascicularis. - Sturm h. 33-. t. 3. (Strauss).

fasciculatus. - [S. $312 *$.]

— flabelliformis. - Carol. III. 5.

- floccosus. - [S. 312*.]

galeatus Fr. - Fl. dan. XII. 2027. 1.

- glaucus. - [S. 312.] - Kl. 1605. - Kl. II.

110 .

incarnatus. - [S. 312*]

- infundibuliformis. - [S. 312.] - Kl. 1211.

- Fuck. s. 1045. - R. f. 307. - Wstd. 1056.

- Kl. II. 121. - Vent. mi. 79. f. 3. 4.

— v. concolor. - Kil. 1912.

laeris Fr. - Fuck. s. 1043.

leucophaeus N. - Dsm. 163.

- lobatus. - [S. 312.]

- lutescens Fr. - Huss. II. 21. - Wstd. 1390.

- Loud. f. 1605 S. - Fuck. s.1049. - Kl. $\$ 12$.

- R. f. 114 . - Nov. Act. 1542 . XLX. II. t. 57 .

f. 93. (Spor. Phöb.).

- muscigenus Fr. - [S. 312.] - Dsm. 1050.

- Bisch. f. 324\%. - (cf. Kl. 1605, Nota).- -

Sturm h. 31- t. 3. (Szl.). - Kl. 242. - Fuck.

s. 1045 . - R. f. 115 . - Wstd. 764 .

nigripes Duby. - Dsm. III. 663. (aurantiac.

Fr. partim).

- olivaceus. [S, $312 *$.]
Cantharellus papyraceus. - Alger. t. $31,3$. partitus. - Ilook. Lond. J. 1842. I. t. XV.

(Berk.).

Petersii. - Carol. V. 12.

Ravenelii. - Carol. III. 4.

replexus. - [S. 312 .

retirugus. - [S. 312.] - Kl. 411. - Berk.

Out. t. 11. f. 2. - R. f. 113.

- sinuosus Fr. - (Craterellus) Dsm. 162. -

Brit. f. 2\$0. 294). - Letell. 6s4. spathulatus. - Tijdschr. v. natur. Geschied.

1510. VII. t. 4. f. 4. p. 259. (Jungh.).

- tubaeformis. - [S. 312.] - Brit. f. 140. -

Fl. dan. XII. 2050. 1. - Fuck. s. $1050 .-$

Bisch. f. 3412 - Kl. 109. - Krbh. t. 4.

f. $-10 .=$ infundiblf. Fr. - Bot. Zt. $1>60$.

t. 13. f. 2. (Hoffm.)

- umbonatus. - [ร. 312.] - Lib. 319. - Kll.

$191 \mathrm{i}$. (Merul.).

undulatus Fr. - Fl. dan. XIII. 2270. 3. -

Loud. f. 160.56.

- villosus P. - Sturm h. 2. t. 36 .

Capillaria. - [s. $\left.196^{* *} .521.\right]$

- grammica P. - Dsm. 1302.

Capitularia myelospora: - Kl. II. 28.3.

- pityrea. - Wstd. 65.

- Polygoni. - Kl. 1995. - R. f.1 \$5. - Fuck. s. 99.

Capnodium. - cf. Fumago.

_ australe. - Journ. hort. soc. 1549. IV. p. 259.

f. 9. (B. D.)

- caroliniense. - Journ, hort. soc. 1S49. IV.

p. 2.5. f. 7. B. D.!

- Citri. - Dsm.III. 99. - Ital. 345. - Berk.

In. p. 2ī. f. 63. b. elongatum. - C'arol. IV. 84. - Journ. hort.

soc. 1) 19. IV. p. 255. f. 5. (B. D.).

- expansum. - Journ. hort. soc. 1519. IV. p. 255. f. 4. (B. D.).

Footii. - Journ. hort. soc. 1519. IV. p. 259

u. 260. f. 10. (B. D.).

- Fuligo. - Journ. hort. soc. 1849. IV. p. 257.

f. 2 (B. D.)

- mucronatum M. - Ann. sc. n. 1560. XIV.

t. 10 f. A. p. 175 (Antennaria scoriadea Mont.). pelliculosum. - Carol. II. \$3́.

- Persoonii. - Journ. hort. soc. 1949. IV.

p. 25 ). f. 6. (B. D.).

- salicinum Mt. - Journ. hort. soc. 1949. IV.

p. 25 i. f. 4. (B. D.). - R. f. 6 S.

- Schweinitzii. - Journ. hort. soc. 1849. IV. p. 257. f. 3. (B. D.).

- sphaeroideum Lacr. - R. f. 352. - Dsm. III. 769 .

_- Thwaitesii. - Berk. In. p. 275. f. 63. a.

Carcerina. - 'S. 451.]

- spumarioides Fr. - [S. 451.] - R. f. 432.

- cf. Diderma).

- valvata. - [S. 451.]

Caries. - [S. 516.]

C arlia Oxalidis Rbh.-Kl. II. 567.-Fuck. s. 331.

Catach y um. - [S. 439.460.]

Catinula. - [S. 471.]

— turgida. - Dsm. 1818. - W'std. 1279.

Cauloga ster. - [S. 4S7*. 524.]

chordostyloides. - Cd. Anl. t. C. f. 25: 9-11.

Bon. t. 10. f. 204. - (ov. insector., Tul.). --

Sturm h. 12. t. 31 .

Cauloglossum. - [S. 440.] aegsptiacum. - Cd. Ic. VI. f. 44 transversarium. - Carol. V. 79. Sphaeria 


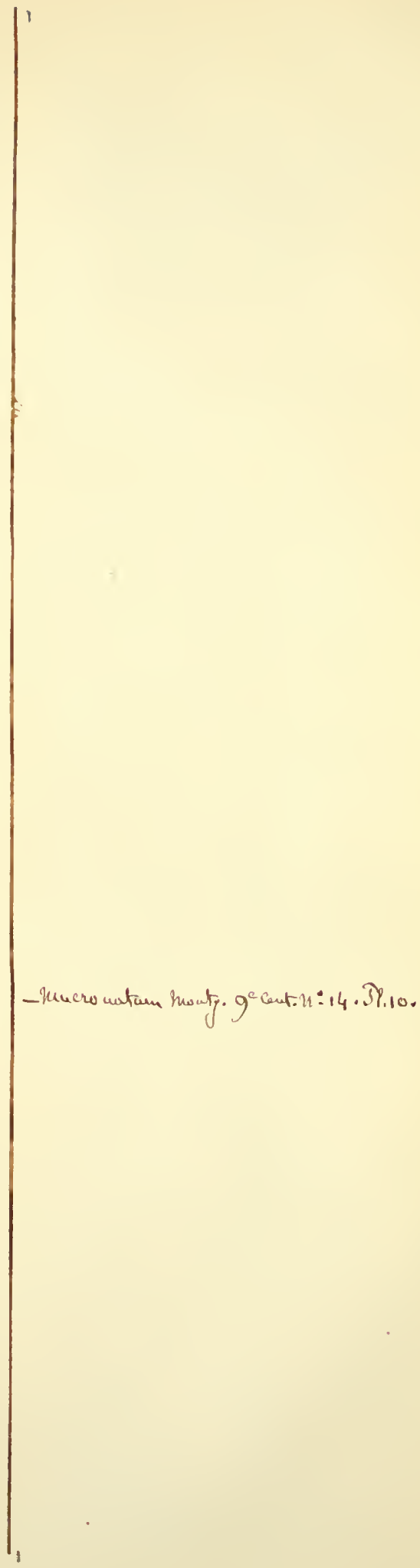




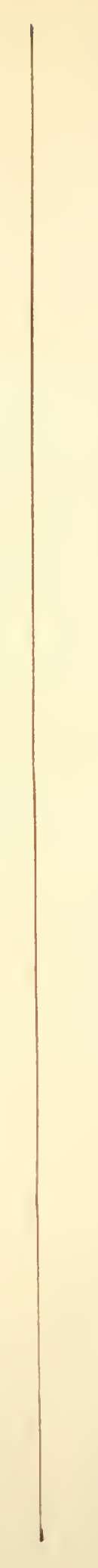


herculea Schw.). - Cd. Anl. t. C. f. 39: 5.-

Berk. In. p. 350 . f. 77 . a.

Cellularia. - is 3li.]

- cyathiformis. - C d. Anl. t. H. f. $75: 2$ l-26.

Cen angium. - ef. Peziza, Clithris pulv., Dermatea.

— Abietis Dub. - Wstd. 1275.

acerinum. - S. 361.]

acicolum - [S. 36i1.]

acutum. - [S. 36 t.] - Fl dan. XI. 1 sia!. 1.

asperum Dub. - Wstcl. 12:S.

- Aucupariae. - S. 36 1. - Bail S. t. 1!. -

Ki. 3.16 .

calyciforme. - cf. 'Triblidium eal.

Cerasi. - S. 36j.] - Brit. f. 11jl. - Iib.

130 - Wstd 491. 165. - Micr. J K5!\%. VII.

t. 11. f. 11. 12. Curr - D sin 5il- Fl.

dan. XIII. 2336. i. - Fuck s. 773. - Iun sc.

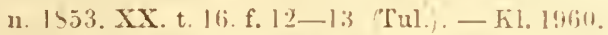

- huc Sphaer. dubia P. Tul.

—- v. Padi. - Voges. 6t;i splıaeria padina

- Coryli. -- Cd. Ic. V. t. 9. f. (i3.

Craterium: - Carol. I. 39 (ef. Peziza C"r.

difforme Fr. - Voges. b59.

Ericae. - [S. 361.] - Kil. 1357.

- ferrugineum. - Cd. Anl. t. G. f. $\{1\}: 3\{1-33$.

Pay. b. er. p. >6. f. 394 .

— ferruginosunı. - [S. 3151.] - Kil. 1615. -

Fuck. s. $766 .-$ lil. II. $51 \%$ - Dom. 1615. -

Letell. 613.3. - Wstd. 1275.

- Frangulae Tul. - Wstd. 127i. - Ann. sc.

n. 1 $53 . \mathrm{XX}$. t. 16. f. 1-4. "Tul.'

— Fraxini T'ul. - Ann. sc. 11."1433. XX. t. 16.

f. 14. Tul. - Berk. Out. t. 1. f. 13: a spor,

b. Spermat, ,

fuliginasum. - [S. 361. 391: 76., - Ii]. II.

710. - Norweg. 19().

- globulare. - S. 364.]

- ilicinum. - Alger. t. 29, 1.

- Labiatarum. - Kl. 1731.

- laminare Fr. — [S. 36il.] - Kl. 733.

Ledi. - S. 36it.

ligni. - [s. $\left.36 \mathrm{i}^{*}.\right]-\mathrm{D} \operatorname{sm} .1614$.

Padi. - S. 362.] - Lib. 131 .

paradoxum. - Jungh. pr. t. 4. f. 11.

P'inastri Fr. - Kli. 36.

- Populi Lasch. - Kil. 636.

- pruinosum. - Hedw. I. t. \. f. 1.

- Prunastri. - S. 362. $400,5]$.- K1. 1540.

(ef. Dermatea). - Brit. f. 162. 163.

pulveraceum: - [S. 364.].

- Pyri. - Palaeontol. VIII. t. 63. f. S. Ludw.

- quercinum. - [S. 361.] - Dsm. 571.- Fl.

dan. XIII. 2276, 1. - = Colpoma q. Wallr ex

Dub. . - Loud. f. 16253. - Kl. 116. - Fuck

s. $76 \%$ - Kl. II. 449 ! cf. Triblid. quere, Tul.

- Brit. f. $26 .-$ Wstd. 9 i.

Raineri, - Act. Turin. Is \$1. III. Dec. 1.

f. 1 (Notar.).

repandum. - [S. 364.] - Voges. $\$ 90$.

- Ribis. - [S. 364.] - Dsm. li13. - Ann. sc.

n. 1553. XX. t. 16. f. 9-1] (Tul.) - Il 345 .

- Norweg. 159. - Lib. 330. Pez. Ribesia P.).

seriatum. - Voges. 1479.

triangulare. - Carol. III. 12.

- turgidum. - Carol. IV. 21. - Wstd. 1279.

- Umbellatarum. - Kl. II. 312.

Urceolus. - [S. $362+364$.

—_ f. pezizaeformis. - Fuck. s: 769.

Cenocarpus. - [S. 342*.]

Cenococcum.- - [S. $\$ 15$.

geophilum. - [S. 445.] - Dsm. 1021.--
Act. Tur. 1 $\$ 43$. V. t. 3. f. 5. (Vitt.) - Linn.

1513. XVII. t. 1s. Schmitz. - Tul. F. hyp.

21. f. צ. - Lib. I 1. - Wstd. 99.

C'en ococeum geophilum v. byssisedum. - Bisch.

f. 3655 .

- heliophilum. - [S. $115^{* *}$.

Centridium. - [S. $510^{* *}$.] (cf. Aecidium).

- Ariac Dsm. cf. Aecid, lacerat.

- Crataegi Cst. ef. Aecid. cormut. 1 .

Mespili. - Wstd. 1156 .

Cephaleuros. - [S. 46 . $449 \%$.]

(ephalocladia. - [S 193.?

Cephalodochium album. - 130m. t 11. f. 22\%.

Cephalosporium. - S. 491 .

Acremonium. - Fresen. t. 11. f. 59-63.

p. 91.

- botryoides Bon. - Bot. Zt. Isfil. t. s. f. 1.

1. 202 an Stachylid pulchr. Iloffm. Bot. Zt.

1459. t. 11. f. . .

macrocarpum. - Cd. Anl. t. B. f. 19: 22.

Secptromyens. - Bon. t. 111. f. 2115/= Sceptrom. Opizii

Cephalothecium. -- [S 4!2. 3.

- candidum. - Fuck, rh II. 114. - Bon. t. 4

f. $\checkmark$. - cf. IR. f. $3 \checkmark$ 1. nota. - Fuck. rh. 152

- roseum. - Cd. Anl. t. B. f. 14: 1.2. - R.

f. 3\1. - Pay.b.cr. p. 104. f. 476. - Kl. 1275.

Cephalotrichum. - is. 16 .

curtum. - [S. 46 s: Ann. Ig. n. h. $1 \$ 11$.

V.I. t. 12. f. 13. Berk.

- Havo-virens. - [S. 167.] Bisch. f. 3761. -

Schntzl. t. 12. f. 46.47 .

- macrocephalum. - C'd. Anl. t. B. f. 19: $\$ .9$

monilioides. - S. 167 .

- rigescens Lk. - Bisch. f. 3763.

- Stemonitis Ns. - Bisch. f. 3765. [S. 167.

Pachin. sub.

Ceratitium ef. Aecidium.

('eratium. - [5. 165.5.$]$

- aureum. - [S. 465.

- hydnoides. -- [S. 465.] - Cd. Anl. t. G. f.

il: 1-1. Fuck. s. $25 \%$ - Loud. f. 16551 . -

Bisch. f. 3771 . - Kl. 572. - Fuck. th. 170.

- porioides A.S. - [S. 465.$]$ - Bisch. f. 3 i 55.

C'eratocladium. - [S. 454.$]$

- microspermum. - Cd. Ic. V. t. 2. f. 19. - Cd.

Anl.t. B.f. 15 : 1-3. - Pay. b. er.p. 74.f. 335

Ceratogaster. - [S. 445 .]

- maculatus. - Cd. Anl. t. C. f. $37: 7-10$.

- Sturm h. 19 -. t. 12.

Ceratonema. - S. 521 .

- capillare. - Letell. 650.1

- hippotrichodes. - Letell. 6£0. 2. (Rhizomor-

pha setif. DC.). - Wstd. 54 S.

Ceratophora. - [S. $\left.447^{*} .522\right]$

Ceratopodium. - [S. 467.2.$]$

- album. - Cd. Anl. t. B. f. 20: 10-12.

Ceratospora mammaeformis Rbh. - Krypt.

Bad. 54y.

Ceratosporium. - [S. 506.]

Ceratostoma Fr. - [S. 396.] ef. Sphaeria.

- brevirostris. - Bot. Zt. 1561 . t. X. f. 1 .

Fuck. p. 250.

- caprinum. - [S. 396.$]$

_ chioneum. - [S. 396.] cf. Melanospora ch.

Bail S. t. 17 .

- lagenarium. - [S. 396.]

__ leucophaeum. - S. 396.]

— lucifugum. - [S. 396.]

piliferum Rbh.

$-\beta$ Pinastri. - Kl. II. $5 \neq 9$

Stilbum. - [S. 396.$]$ 
Ceratostom a rernicosum. - [S. 396.]

vitreum. - S. 396.$]$

Zamiae. - S. $396.1 \%$

Ceraunium. - S. 445.] - cf. Elaphomyces. muricat. Wllr. = Elaph. mur. Cd.

- scabrum Trll. = El. muricat. Fr. Cd.

Cercospora Apii. - Fresen. t. 11. f. $16-54$. p. 91.

- ferruginea F. - Fuck. rh. II. 120. penicillata Fres. - Fuck, rh. II. $117-119$.

- lil. 155\%. = Passalora p. C'es.

Cerebell a Andropogonis. - Fil. II. 2) 1.

Cerebrina. - S. HU.

Ceriourtes, - [S. $315^{* * *}$.

Sturm. h. 14 f. 61 .

Ces a ti a Spartii. - Kl. 1113.

Ceuthospora. - S. 414 .

Asparagi Lasch. - Fil. 1063.

bifrons. - S. 414 .

- circumscripta. - S. $414 *$ ?

- concara Dsm. - Dsm. 1225.

- Lauri. - S. $414^{*}$.] - Brit. f. \$9.

phacidioides Grer. - Fres, t. ๖. f. 33-12.

- Bail S. t. 14. - R. f. 164. - D sm. 121. -

Dsm. III. $33-75 .-$ Wstd. 32. 21): immacul.

- Wstd. 527. - Ital. 5 t4.

—_

_ phaeocomes Rebt. - S.395. 3.' - Il. 995.

cf. Prrenoph. ph. Fr.

Cha eñocarpus setosus Reb. - Dsm. 1027.

- Simonini D. - Voges. 1241. - Dsm. 1025.

Chaeromyees. - cf. Choiromrees.

$\mathrm{Chaetocladium} \mathrm{Jonesii.} \mathrm{-} \mathrm{Fresen.} \mathrm{t.} \mathrm{12.} \mathrm{f.} 5-$

12. p. 97 .

Chaetomium. - S. 405. $495 \div$.

affine. - Cd. Anl. t. F. f. 55 : 21. 22.

atrum. - Dsm. III. -6. - Voges. 14>3.

Braunii. - Kl. 1554.

chartarum. - 'S. 405.

comatum, - S. 405.

elatum Kz. - S. 405. - Bisch. f. $367 \%$ -

Fuck. s. 490. - Dsm. 14ti. - Kil. 11132-Ki.

II. 5 - - Brit. f. 19. - Carol III. 66. W Wstd.

ธ3. - R. f. 529. - Ital. \i

epiphyllum. - Kl. 131i.

Fieberi. - Kl. II. 165.

rimeti. - Fuck. Nass. f. 21.

globosum. - S. $1115.7-\mathrm{K}$ - 959.

graminicolum Rbh. - Fuck. s. 492.

Graminis. - Kl. 15.55.

hispidum. - S. 405.7

- lageniforme Cd. - K1. II. 521

- murorum. - Cd. Anl. t. F. f 55: 1ヘ -20.

- R. f. 234.

— nivale. - Sturm h. 31-. t. 2. Strauss

- pannosum. - Kl. 1556. - Kl. Is56? - Kl.

II. ifs.

- pilosum. - S. 405.?

- pusillun Fr. - [S. 405.] - Sturm h. 31-.

t. 3 Straussi.

strigosum. - [S, 39S: phaeoc.]

- tomentosum. - Kil. 1556.

Chae topsis. - [S. 499.]

— fusca. - Cd. Anl. t. B. f. $12:$ s. 9. stachyobola. - Cd. Anl. t. B. f. 12: 10-12.

- Wauchii. - Pay. b. cr. p. 72. f. 315.

Chatoscypha. - S. $521^{\cdots}$.

- variabilis. - Sturm h.9. t. 63.

Chaetosporium. - [S. $51 !]^{*}$ -

Craminis. - Sturm h. 13 t. 33.

Chaetostroma. $-\$,+7$ ?
Cha etostrom a album. - Lotos. 1557. VII. t. 2. - Buxi. - Wstd. 49. - Flora 1547. n. 1.f. 14.

Fres. - Ann. sc. n. 1\55. IX. t. 5. f. $10-12$.

Debat. - Cd. Anl. t. G. f. 6 $\{: 25-31$. - Kl.

II. 2y0. - Fuck. s. 247 . - R. f. il. - Pay. b.

cr p. 75. f. 314. - Ital. 793. - Fuck. rh. 207.

IF std. 1296 .

- Carmichaelii. - Sturm h. 9. t. $5 \mathrm{~s}$.

Cyperacearum. - Ki] 1946.

isabellina. - [S. 472. 3.] - Sturm h. 9. t. 59.

- Rusci. - Wstd. 1296.

Solani. - Flora 1517. n. 1. f. 9. Fresen.'.

Cha etotrichum. - non est fungus.

- Graminis. - Kl. 1500.

Chalara. - S. $505^{* *}$.

- fusidioides. - Kl. 1761.

- Mycoderma. - Bon. t. 1. f. 27.

Cheilaria. - S. $4102 *$.

- - Aceris. - Lib. 255. - Dsm. 17\%. Didy-

mosp. Ac. M.). - Wstd. $\$ 23$. = Didymospor

Ac. Mtg. (Dsm.). - Fuck. rh. 474.

- Arbuti D. - Toges. 1267. - Dsm. 1025.-

Dothid. Arb. Spr.

Cor

- Cydoniae. - Dem. III. 79.

- Helicis Dsm. - Wstd. 526.

- Heraclei. - Lib. 25!.

- Mori. - Dsm. III. T. - Wstd. S2t.

- pulicaris. - Clile n. \&. f. 9.

Cheirom sces cf. Choirom.

Cheirospora Fr. - S. $\left.50 y_{.}\right]=$Myriocephalum botryospor. MItg.

Cli i s tosp or a parasitica. - Fres. t. 5. f. 1s-21. Chion rphe. - S. 4\$9.] - Nov. Act. XIX. I.

p. 19. Thienem.

Chloridium. - S. 199.] - cf. Haplaria.

atrum. - S. 499.

cylindricum Rbh. - Fl. 994.

Festucae. - Cd. Anl. t. B. f. 12:4.

viride. - rS. 199.1.]

Chlorosplenium. - S. 356 .

Choiromyces. - S. 437.] Chaerom. et Cheirom., Terfezia.

- Dormitzeri of Rhizopog. Dor. Cd.

- gangliformis. - Vitt. t. 2, 2.

- gangliodes Cd. Zbl. - Cd. Ic. VI. t. 14. f.

112 . sub. n. Rhizopog. gangliformis Cd.'.

- ganglioniformis Tul. = Khizopog. g. Cd.

Leonis. - Alger. t. 24. f. 22-30.

macroc. cf. Rhizopogon mac.

meandriformis ef. Rhizop. m.

- Vitt. : Cd. Ic. VI. t. 13. f. 110. - Bail

S. t. 13. - Micr. Journ. 16.57. V. t. 6. f. 45.

Curr.) - Tul. F. hyp. t. 19. f. T. - Vitt. t. 2,

1. u. t. A, 10 .

- melanoxanth. T. B. ef. Pachyphloeus m. stellatus. - Berk. In. p. 313 . f. 70 . c. d

Chondrom t ces crocatus. - Berk. In. p. 313.

f. 70 . a.

Chordostylum. - S. 469. 15:*.]

_ capillare. - [S. 469.]

- hispidulum. - [S. $469{ }^{*}$.]

- Todei. - Cd. Anl. t. C. f. 25:6-5.

Chromelosporium. - [S. $\left.496^{* *} .523.\right]$

- ochraceum. - Sturm h. 13. t. 41.

Chromosporium. - S. 520.7

- roseum. - Sturm h. 9. t. 56.

- viride. - Sturm h. 9. t. 57 .

Chroolepus. - S. 524 .?

- aureum. - Wstd. 699. 


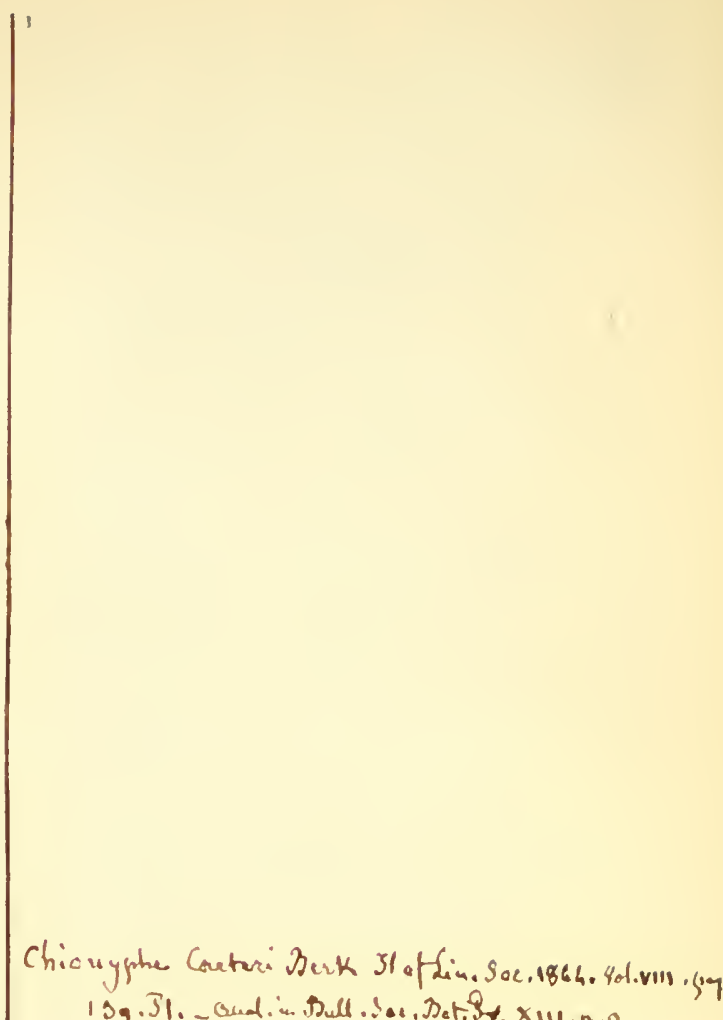

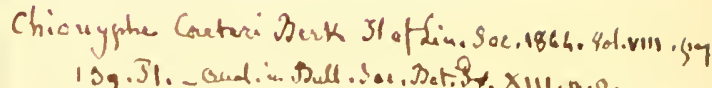

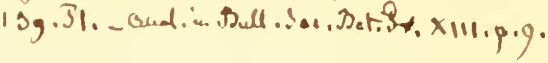




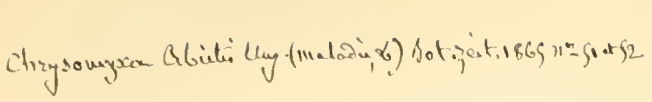

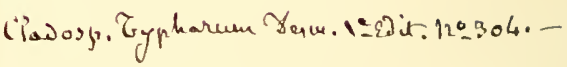

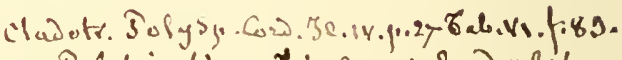

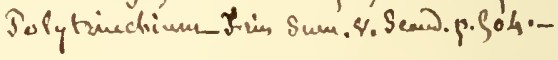


Chroostroma Pini. - [S. 141.]-Cd. Anl. t. G. f. $69: 16.17$.

Chrysom yxa. - [S. $519^{*}$.]

Chrysosporium. - [S. 19.5. 3.]

- Corii. - Cd. Anl. t. B. f. $7: 3$ - Sturm

h. 13 . t. 43 .

Cicinobolus Humuli. - Bot. Zt. 1554. t. 6. f. 12. 13. (Mohl.).

Oidii Tuckeri. - Bot. Zt. 1554. t. (i. f. 1-i. (Moll).

Ciglides. - [S. 5]10**.]

- laceratum Chev. ef. Aecid. 1. $\beta \quad$ C'rataegi Ariae DC.

Cilicia. - [S $5 \geq 1$.

Ciliciocarpus. - [S. fft*.]

- hypogaeus. - Cd. Anl. t. C. f. 40:6-11).-

Sturm h. 11. t. 3.

Cilic iopodiu in cf. Stilbum.

- sanguineum. $-\mathrm{Cd}$. Anl. t. B. f. 20: 13. I I

- violaccum. - - Sturm h. 12, t. 29.

Cionium. - [S. 451.] - cf. Didymium.

— farinaceum. - Kl. 1319.

— Iridis. Cd. Anl. t. C. f. 2s: 16. 1\% _ Sturm

h. 1.t. 7 .

- xanthopus. - [S. 152.] - Kil. 1350. - cf.

Physarum xanth. - Siurm l. 3. t. 13.

Circinotrich um maculiforme. - \$. (9)

- murinum. - Usm. III. 5.

- rufum. - Norweg. 179.

Cirrholus. - S. 45.

- flavus. - Cd. Anl. t. C. f. 29: 32. — Bisch.

f. $31 i-6$

Cladobotryum. - [S. 191*.]

- gelatinosum? - Fuck. rh. 2:7.

C'la doderris. - [S. $330^{* *} .33 \%$.]

Cladosporium. - [S. 4!y.]

- astroideum. - Kl. 174 i.

- atrum. - [S. 499.]

- bacilligerum - Ann. sc. 11. 1536. V. t. 12.

Kl. 16iti. - ef. Passalora

- Bellinckii W. - Bull. ac. belg. Sér. I. To.

21. 2. p. 246 . f. g Wstd. - Wstd. 10>9.

- caespititium. - R. f. 579.

caricicolum Cd. - [S. 500.] - Dsm. 1219.

- clavatum. - Schwahe, Fl anhalt. II. t. 6.

f. 13 .

- dendriticum. - Kll. I5>3. - Wstd. 1090. -

Kl. II. 766 . - Fuck. s. 165. - Dsm. 1543. -

Voges. 1152. - Wstd. 695. - Fuck. rh. II. 115.

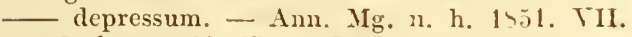

t. 5. f. \&. p. 99. (Spor., B. B.).

- desmitrichum. - Dsm. 1736-1735.

- epiphyllum Fr. - [S. 199.] - Kil. 157. -

Fuck. s. 164. - R. f. 7\%. - Wstd. 295. -

Fuck. rh. II. 113.

— fasciculare Fr. - [S. 199.] - Fuck. s. 160.

Cd. Ic. III. t. 1. f. 24. - Fuck, rh. II. 109.

fasciculatum Cd. - IIedw. 1562. II. t. 10.

f. II. 6. - R. f. $57 \%$.

Fumago. - [S. 500. 505, 9.] - Hedw. I.

t. 10. f. 13. 14. - Fuck. s. 163. - Kl. 65. et ex errore 1170. - Kl. II. 75. - Wstd. 149. Fuck, rh. 112.

- $\longrightarrow$ f. corticicola. - Kl. II. 330 .

- - f. Ulmi. - Kl. II. 329.

fuscum ef. Sporodum herb. - Wstd. S6.5.

- gracile. - R. f. 272 .

- graminum. - [S. 500.] - Kl. 136s. - Fuck.

s. 162 - Fuck, rh. 114.

helminthosporioides. - [S. 499.]

- herbarum Lk. - [S. 499.] - Fuck. s. 161.

Fl. dan. XIII. 2277,2 - Harrwitz, de Cl. h.
1515. - Krypt. Bad, 327. - Dsm. 231. - Cd. Anl. t. B. f. 10: 1. - Loud. f. 165.5. - Bisch. f. $373: \%$ - IIedw. I. t. 10. - Pringsh. Jalurb. II. t. 30. f. 27. (IIoffm.) - Kl. 67. - Kil. II. 333 . Letell. 637. 1. - Ökonom. Neuigk. v. Illubeck. IS.16. t. 3. f. S-15. - Fuck, rh. II. 111.110.

Cladosporium herbarum f. Mesembrianthemi. - Ki. II. 76 .

- - repens. - Fres. t. 3. f. 29.

- Lacroixii. - Dsm. III. 755.

- lanciforme. - Kl. ITs.

lignicolum Cd. - Kil. 1271.

Lythri. - Bull. ac. belg. Ser. I. 'To. 21.2.

p. 246 . f. 10. 7Wstd.). — Wstd. 1091.

- macrocarpum. - Sturm h. 25 - t. 14. (Preuss.).

- nigricans. - [S. 499.]

- nodulosum. - Kl. II. 76.5. - Euck. s. 157.

penicillioides. - Sturm h. $25-$ t. 16.

(Preuss.).

- personatum. - Carol. III. \5.

- polysporum. - ? Sturm h. 12, t. 211.

- profusum. - Dsm. III. 756. - R. f. $57 \mathrm{s.}$

- punctiforme F. - Fuck. s. II. 111 .

ramulosum. - Dsm. 1735.

- rectum. - Sturm h. 2.j - t. 15. (Preuss.).

- kil. Isiti.

— secedens. - [S. 499.

— - stromatum. - Sturm h. 25 - t. 1:3. (Preuss.).

- tortuosum. - $\$$. 499.]

- tuberculatum. - S. [19! ( $^{+4}$. 5(11).

- 'Yypharum Dsm. - Wstd. 1:391.

__ vagans. - Ism. III. 6.

- _ varium. - [S. 191. 1.]

- viticolum. - Ki. is $\%$.

Cladotrichum atrum. - [S. 504**]

- caesium. - K1. 1540.

— conjunctum. - Bon. t. 4. f. \$5. - Fuck. rh. 123 .

- polysporum. - [S. 504.] - Cd. Anl. t. B.

f. 11: 6. - Pay. b. er. p. 69. f. 395 . - Sturm l. 12. t. 20.

- ternatum. - Bon. t. 4. f. 8.. - zu IIypomyces t. Tul.

- triseptatum. - Ann. Mg. n. h. 1951. VII.

t. 5. f. $\div$. p. 99. B. B.)

Clasterosporium. - S. 506.

Clat lirospora Elynae. - IIedw. I. t. 15. f. 3. Clathrus. - [S. 135.]

- cancellatus. - Cd. Ic. V. t. 6. f. $49 .-\mathrm{Cd}$.

Anl. t. E. f. $45: 5-11$ - Bisch. f. 363s. -

Ilook. Lond. J. 14.15. IV. 4. 2. f. 7. (Berk.). -

Bail S. t. 23. - Ann. sc. n. 1545. IV. t. 1. f. 1-S. Anat. Lesplt. - Pay. b. cr. p. $11 \mathrm{~s}$. f. $556-559$. - R. f. 35 . - Jussieu Trait. él. Bot. 1542. p. 546 . fig. 509.511). (Spor. Lév.). - Alger. t. 23: 1- . (c. fruet.). - Huss. I. S6. - Vent. mi. 70. f. $2-5$. columnatus. - ct. Laternea. - Cd. Anl.

t. E. f. $15: T-s$.

- Bos. : Bisch. f. 3611. - Carol. II. 70. crispus. - [S. $435^{*}$.] Cd. Anl. t. E. f. 48 :

12. - Amn. Mg. n. h. IS 12. IX. t. 11. (Berk.). denudatus. - Wstd. 81 .

hirudinosus. - Pay. b. cr. p. 115. f. 554.

Alger. t. 23. f. 9-22. c. fruct.). - Colus h. pusillus Berk. - Cd. Ic. VI. t. 5. f. 56 . -

Hook. Lond. J. 1sł5. IV. t. 1. f. 6.

- recutitus L. = Arcyria cin. Fl. dan. ruber Mich. - ef. Ćl. cancell. 
Clathrus triscapus Fr. - Chile n. 10. f. 9. Bisch. f. 3640. - ef. Laternea.

- virescens IIuds. — Wstd. 5 Is.

Claustria. - [S. 151.$]$

Clavaria. - cf. Hericium.

- Mycelium. - Pay. b. cr. p. 55. f. 235. abietina P. - [S. 339.] - Dsm. 63i. - Fl.

dan XII. 2030. 2. - Fuck. s. 904. - Kl. 1:3.

- genuina et vireseens: R. f. 313. - Kl. II. 311.

— acuta Sow. - Loud. f. 16176.

afflata. - [S. 334. 14.]

albidula Lascl.. - Kil. 1219.

alutacea Lasch. - Krypt. Bad. 13i. - Kl.

1519. - Kl. II. I21

amethystea. - [S. 33ৎ.] - Roq. t. 1. f. 2

amethystina I3ull. - Tent. t. 12. f. 113. -

Berk. Out. t. 14. f. 2 - Badham t. 16. 1.-

Vent mi. 15. f. 3.

- angustata P. - Fl. dan. X. t. $17 \$ 3$.

- anomala. - [S. 334.]

apiculata. - S. 3.?.] - Kl. IS19.

- Ardenia Sow. - [S. 339.] - Loud. f. 16171.

- argillacea $P$. S. 335.$]$ - Fl. dan. XI.

15.)2. 2. - Kops Fl, bat. XI. S14. 3. - ef. Cl.

ericetor. - lil. 1215. - Kl. II. 315. - Krypt.

Bad. 155.

— aurantiaca P. - - Wstd. $3>5$.

aurea Schff. - [S. 3:\%.] - Vent. t 12.f. 112

- Kil. II. 123. - Wstd. 762 .

- Botrytis P. - [S. 33<.] - Vent. t. 12. f. 111.

- Fuck. s. 905. - Cd. Anl. t. G. f. 72: 15-

17. - Hrz t. 67. - Loud. f. 16159. — Biseh.

f. $3390 .-$ Cd. Ic. V. t. 10. f. 75. Fruct.) -

Kóutzing, Philos. Bot. 1452. t. 19. f. 14. (Fruet.)

- Carol. II. 41. - Viv. 54. - Staude t. 1.

f. 6. - Fries Sv. t. 35.

— bulbosa Schum, - Fl, dan, XIII. 2? 72 ?

__ brssiseda. - S. 335.] - FI. dan. XI. 1967.1.

_- canaliculata: - [S. 339.]

- ceranoides P. - - loud of 16173.

chondroides. - Hook. Lond. J. 1642. I.

t. 6. f. 3 .

einerea Bull. - [S. 335.] - Kíl. 639. - Le-

tell. 7us. 1. fuliginea $\mathrm{P}$.

- Badham t. 15.5 .

cirrhata. - Hook. J. VIII. t. 5. f. 5.

compressa. - Ann. Мg. n. h. 1\$42. X. t. 12.

f. 16. p. 351. Bcrk.). - ef. Acrospermum, Purton.

— condensata. - [S. 334.]

contorta Holm. - S, 334.] - Fl. dan. XI.

1 552.1 - I sm. 75. - Ki. 1125. - Kil II. 5115.

- coralloides. - [S. 33〉.] — Íl. II. 619. -

Ioq. t. 1. f. 1.

- Voges. 196. Calocera vis.) — : Badham

t. 16. 2. - Wstd. 692 . $7(i)$

— cornea 13. - Bisch. f. 3345. - Kil. 639. -

cf. Calocera: Voges. 642.

- corniculata Sch. - Bisch. f. 33५7. - Loud.

f. $16165 .-$ Sehnzl. t. 16. f. 3t).

cornuta S. - ef. Calocera Hammea

- crispula. - [\$. 33\$.] - FI. dan XIII $22 \%$.

1. - Bisch. f. 3472 .

- cristata. - S. 335.] - Sturm h. 31 -

t. 11. (Szl.) - Kl. 134 - Ann. se. 11. 193\%.

VIII. t. S. f. 15. (Fruct Lév.) - Anm. Mg. 11.

h. 1434 . I. t. 5. f. 46 . Fruct. Berk.). - Nor.

Act. I 42 . XIX. I1: t. 5\%, f. 123-12\%. (Fruct.

Phöb.) - Carol. II. 42 - Vent. mi. 73. f 3 ;

S2. f. 1.

— v curta.- Linn. 1530. V. t. 7. f, 2. b.

(Jungh.). = Theleph. crist.
Clava ria cristata v. flexuosa. - Linn. 1§30. V. t. i. f. 2. a. (Jungh.). = Theleph. crist.

- - r. ornithopodioides. - Letell. 70S. 2 dumaccornis Sclirk. - Wstd. 692.

delicata - [S, 334.]

- dendroides. - [\$.339*.] - Jungh. pr. t. 6 . f. 2 (9).

diaphana Sehum. = Pistillaria d. Fl. dan

eburnea $\mathrm{P}$. = fragilis Fl. dan. XI.

- a $\mathrm{P} .=\mathrm{Cl}$. fragilis a gracilis Fr. Ep.

clegans Bolt. - Wstd. 692 .

epichnoa. - [s. 3:35.]

- ericetorum P. - [S. 3335 35.] - Hrz. t. ia.

- argillacea Rbl. ; Fl dan. — Voges. 19:3.

- erythropus P. = 'Typhula er. F1. dan.

faleata. - [S. 339.]

- fascicul. P. - cf. Calocera f. Bon.

__ - ... v. aurantiaca Wilr. — Wstd. 955.

— fastigiatil. - [S. 335.] - Wstd 7 (i2. - Kops

FI. bat. XI. 41.1.- IIl. 516. - Kl. II. 315.

- pratensis B.

__ filiformis Schum - Fl dan. XIII. 2273. I.

- fistulosa. - [S. 339.] — cf. juncea.

- Haccida. - [S. 339.] - Kl 132.- Kil. II.

317. - R f. 314

flammea. - ef. Calocera $f$

- flava. - [S. 336, 17.337.] - Viv. 54. -

IIrz. t. †. b. - Loud. f. $1(6 / 69$. - Bail S. t. 27.

— Kl. 135. - Wstd. 762 - Vent mi. 70. f. - - flarescens Schff. - Wstd. 762 .

- Havipes P. - [S. 33\$. 35.] - Fl. dan. XI.

$196 i 6.1$.

flexuosa Lasch. - Kl. 1220.

— formosa. - [S. 334.$]$ - Til. II. 122

- fragilis. - $-[\mathrm{S} .334]-$. Fl. darr. X. $1793 .-$

= angustata P.: Fl. dan. XI. p. 11. sub fig.

1966. 2. - Loud. f. 16175. - Kl. 240. - Kl:

$1610 .-$ Vent. mi. 66 . f. $4-6$.

fuliginea. - Dsm. III. 660; grisea Krbh.

t. 53. f. 9. 10; rugosa Fr. partim (Dsm.). -

Letell. 70s. 1. cinerea Fr.).

_ fumosa. - [S. 335.]

_- fusiformis Sow. - [S. 336.] — Loud. f. 16172.

- Sow. 231. - Kl. II. 233.

__ gigantea. — $\$ \$ .33 \%>*]$

gracilis. - [S. 335, 37. 335.] - Carol.V. 34.

granulosa, - Act. Turin. Is03. XII. t. 11.

Balbis

grisea Krbh. - fuliginea $P$.

P. : Krypt. Bad. 56.

grrans Bolt. = 'Typhula g. Fl. dan.

helrola $\mathrm{P}$. - Wstd. 945.

herharum v. fungic. A. S. - [S. 401. 3.]

Voges, (i71. Sclerogl. lanc.).

IIypoxylon. - Wstd. 167.

inaequalis - [S. 334.] - Carol. IV. 17. -

Kops Fl. bat. XI. 11. 2. - R. f. 415 . - Huss:

I. Is.

— helvola Fr: - Wstd. 9ৎs.
v. aurantiaca. - Kl. 640. - Wstd.

$95 \times$.

incarnata. - [S: 339.]

juncea - [s. 33!).] - Voges. 118s. (fistu-

losa Bull. . - Dsin. T09. - ef Selerot. compl.

- - v. gracilis Dsm. - cf. Typhula pha-

corhiza. - R. f. 239.

Klotzschii. - Kl. 210, Cent XVII.).

Krombholzii. - [S. 3:35.]

Kunzei. - [S. 334.]

laciniata Bull. = Theleph. crist Fl dan. -

Wstd. 430 .

Wehff, : Wstd. 692 



$$
\mid
$$


Clavaria Ligula Schff. - Krypt. Bad. 156. Iil. 13:3. - R. f 125.

- luticola. - Kil. 1669.

- macropus. - [S. 335.]

- macrorhiza. - [S. 339.]

- merisnatoides. - [S. $339^{*}$.]

- miniata. - Purton. I. t. 15.

- minor. - cum Sclerotio: Ann. se. n. 1 \$ 13.

XX. t. 7. f. 2. (Lév.).

- Mucerdae Schum. = Stilbum M. Fl, dan.

- mucida P. - [S. 3:39.] - Carol. II. 43. -

ki. 1121 .

- muscigena Schum. = Cl crispula Fl. dan.

- muscoides L. - [S. 33..] - kil. 1123. -

K1. II. 125 .

—_ - Müll. cf. fastigiata Bull.

Mull. = C1. crispula Fl. dan.

nigrita. - [S. 334. 34.] - Wstd. 761.

obtusa. - Voges. 6.5.3.

ochraceo-virens. - Linn 1530. V. t. 7. f. 3.

(Jungh.).

- ophioglossoides I. - Wstd. F61.

— ornithopodioide:s. - [S. $339 \%$.]

- paludicola. - Lib. 322.

parasitica. - Wstd. 69.

penicillata Schum. - Fl. dan. XIII. 2273. 2.

Petersii. - Carol. V. 33.

phalloides. - W'std. 231.

- phosphorea Sow. - Wstd. 1251.

- pilipes. - [S. 339.]

pistillaris L. - [S. 334.] - Vent. t. 12.

f. 11.115. - Bisch. f. 3343. - Cd. Anl. t. G.

f. $72: 18-20$. - Schntzl. t. 16. f. 15. - Loud.

f. 16170 . - Dsm. 10-19. - Kil. $2+1$. - I. f.

127. - Cd. Ic. V. t. 10. f. 76 . (Fructif.) -

Nov. Act. ist2. XIX. II. t. 57. f. $125-134$.

(Fruct. Phöb.) - Huss. I. 62. - Vent. mi.

33. - Sturm h. 14. t. 55.

platyceras. - Viv. 54.

- pratensis P. - Lib. 23. - 1)sm. 351. -

K1. 516. — cf. fastigiata. - Wstd. $10 \$ 3$.

pruinella Ces. - R. f. $\$ 11$.

purpurea. - [S. 335.]

pyxidata P. - [S. 335.] - Kl. 100 \&.

radicosa. - Wstd. 69.

Resinosorum. - Wstd. 339.

rhodochroa. - Canar. t. 4. f. 3.

rosea. - [S. 334.]

rufa. - [S. 335.]

- rufo-violacea. - Nov. Act. 1560. XXVII.

t. 19. Barla).

rugosa Bull. - [\$. 33s.] - Badham. t. 15,

4. - Loud. f. 16169 - Berk. Out. t. 1S. f. 3.

- Dsm. it. - Kil. 1122. (Cent. XVII.).

Fuck. s. 905. - R. f. 129. - Brit. f. 149.

— v. grisea: Brit. f. 150. - Wstd. 692.

serpentina Müll. - Wstd. $\$ 63$.

setipes Grev. - (Typhula gyrans folior.)

Brit. f. 151. - Loud. f. 16151.

- spinulosa. - [S. $3399^{*}$. 33S.] - Kl.II. 124.

spiralis. - Jungh. pr. t. 5. f. 19.

- striata P. - Bisch. f. 3356.

- stricta. - [S.33S.] - Berk. Out. t. 16. f. 5.

- Kops Fl. bat. X. Ts5. - Kl.1121. (Caloc.). -

Kl. II. 316.

— subtilis. - [S. 335.] - Sichnzl. t. 16. f. 37. suecica. - [S. 33S.]

- Surculus, - Hook. Lond. J. 1842. I. t. 6.

f. 5 .

Swartzii. - [S. 335. 32.]

tenax. - [S. $339 *$.]
Clavaria tenuipes. - Anm. Mg. n. h. 1545. II. t. 9. f. 2. (Berk.).

- thermalis. - zu Agaric. tubaeform. (Tul.).

trichomorpha. - Kil. 1409.

- umbrina. - Berk. Out. t. 18. f. 4.

- uncialis Grev. - [S 339.] - Brit. f. 297.-

Loud. f. 16150 .

— vermicularis. - [S. 33ৎ.]
villosa Schum. = Typhul, v. Fl. dan.

- virgata. - [S. 334.]

— viridis Schrad. - Wstcl. G(i.3.

- viscosa. - ef. Calocera. - Kil. 131.

Claviceps. - of. Cordyceps et Cordyliceps, Sphaeria.

— in lepidopteris. Cramer, uitlandische Fiapellen. 1742. T. VI. p. 133. cr. t. 267. A. B.

- Euphorbiae - Fuck, s. S!ff. est Typhula. - microcephala. - Ann se. n. 1453. XX. t. 4.

f. 1-11. (Tul.). - Kühn, Krkh.t. 5. f. $21-30$.

- Kil. II. 430 .

- nigricans. - Ann. se. n. 1553. XX. t. 4.

f. $15-22$ ('lul.)

- purpurea. - Sclerotium et spermogonia:

Ann. sc. n. 1453. XX. t. 1 11. 2; t. 3. f. 1-20.

T'ul j. - Iühn, Krkh. t. 5. f. 1-22. - Berg,

('harakteristik. $1>60$. t. 1. f. - - II. II. 431 .

- Fl. dan. X. t. 1751 . f. I. (Sphaer.). - Wstd. 1201 .

- pusilla Ces. - Kil. II. 35. - Comm. soc.

er. it. 1561.2 . t. 4. f, 3. (Ces.).

Clavus secalis. - ef. Sclerotium Clavus.

Clethria. - cf. Clathrus. - [S. 435*.]

C'linterium. - S. 402. 415.

- Grepini ? - R. f. 265. - Wstd. 1235.

obturatum. - [S. 418.$]$

Clios tomum. - [S. 524.]

Clisosporium. - [S. $401^{*}$. 405.]

- herbarum. - S. 405.

- lignorum. - [S. 105. $522 *$.]

Clithris. - [S. 364 .

—_ degenerans. - [S. 36s.]

- pulveracea. - Bail S. t. 19.

C'lon ostachys. - [S. 490.6.]

- Araucaria. - Pay. b. cr. p. 104. f. 477. ef. Stachylid. Arauc.

Closteriosporium S. - ef. Sporidesmium atrum Cd.

Cnazonaria. - [S. $521^{* *}$.]

- setipes. - Sturm h. 7. t. 25.

Coceobolus. - [s. 407 i. $]$

Coccocystis. - [S. $\left.477^{*}.\right]-$ Cd. Ic. III. t. 3 . f. 49 .

C oc com y ces Dub. Hyst. 49. - cf. Hysterium.

- Phacid. coronat, trigon.

Coccopleum. - [S. $4 \%$ **.]

Coccospora. - S. 451.$]$

Coecosporium. - [S. 51)2.]

- maculiforme: - Cd. Anl. t. B. f. 10:5. -

Sturm h. 12. t. 25.

- Unedonis. - Comm. soc. crit. it. 1861. I.

t. 2. f. 5, p. 3\%. (Notar.).

Coccotrichum dichotomum. - Sturm h. 29-.

t. 35 (Preuss.).

Coccularia. - [S. 408, 1.522*.]

- rigida. - Sturm h. 9. t. 60 .

Coelosporium. - [S. 501.]

- ramosum. - [S. 501.]

- simplex. - [S. 501 .]

Coleosporium. - [S. 512.669.] - ef. Uredo.

- aureum Bon. - R. f. 157. - Caeoma Com-

pransor S-1. - Bon. Con. t. 1. f. 13.

Cacaliae DC. - R. f. $4 \$ 4$. 
Coleosporiu un Campanulae Lév. - Ital. (ifi2. - Fuck. rh. 303.

Compos. Lév. - ef. Uredo 'Tussilaginis. -

Fuck, rh. 3116. 307. Sonchor., 304, u 304. (Senec.).

_ flavum. - Bon. Con. t. 1.f. 1. - R. f. $2 \checkmark 6$. fulvum.

- f. Sonchorum. - Kil. II. 3is.

fuscum.

- f. I ussilaginum. - K1. II. 6995.

- Inulae. - Kl. I7t).

miniatum. - Bon. Con. t. 1. f. 16. ochraceum. - Bon. Con t 1. f. 15. -

Fuck. rh. 302.

- Petasit., - de Bary I3r. t. 2. f. 5. 9. pingue. - Berk. In. p. 10 f. 6. b. Pulsatillae. - \$. 512. - Kil. II. 193.-

Fuck. rh. 305 .

- Rhinanthacearum. - [S 512.$]$ - Ann. sc.

n. 155 i. II. t. 7. f. 11 . Tul. - Fuck. s. 32. -

Fuck. rh. 304.

- f. Euphrasiae. - Kl. II. 691. - f. Melampyri. - Kl. II. $37 \%$

- Senecionis. - [S. 512.] - Kil. II. 379. Wstd. 116.5 .

- Sonchi. - Ann. sc. n. I-51. II. t. S. f. 1-3.

(Tul. . - Lredo fulva.

- oleracei. - de Bary Br. t. 2. f. 10.

- Synantherearum. - [S. 5l2.] - Schweiz.

3. - Krypt. Bad. 512. 41. Prenanth.

- Tussilaginis Lér. - Amn. se. n. I \51. II.

t. 5. f. 1-9. Tul. - Kil. II. Tৎ9. - Ital. 799.

Coleroa. - [S. 42.2 .

Chaetomiun. - Kl. 1456.

Coleus. - S. $135^{\circ}$.

hirudinosus. - Anu. sc. 11. 1535. III. t. S. -

C'd. Anl. t. E. f. 15: 2-6. - Bail S. t. 23. -

Clathrus hir.

Collacystis. - S. $407^{*} *$.]

Collarium. - [S. 496.] - cf. Sporotrichum.

- Havum. - S. 496.7 - Kl. $157 \%$.

_- lyococcum. - S. 496.$]$

- melanospermum. - S. 496 .

— microspermum I.k. - Bisch. f. $3>05$

Colletosporium album. - Bon. t. 7. f. 151.

Colletotrichum. - S. 502*?.

- Lineola. - Sturm h. 12. t. 21.

Collyria. - [\$. 340.]

Colpoma. - cf. Sporomega, Coccomyces, Hysterium.

- quercinum. - Wstd. 96.

trigonum Wllr. = Coccomyces tumida $\mathrm{Nt}$.

Dub. Hyst. 49. - Wistd. 1121.

- verrucosum Wllr. - Kl. 107.

Comatricha alta. - Kl. 1759. a. - Sturm h. 36. t. 11 .

obtusata. - Kl. 1551. - Sturm h. 36. t. 10. Combodia. - S. 422 .]

Combosira reticulata Rbh. - R. f. 159. - R.

f. 549. Asteroma r. (hev.) v. Eryng - Dothidea Fr.

Cometella.- - [S. $\left.501^{*} .505.\right]$

- caricina. - [S. 506.]

Coniocephalum. - [S. 460 .]

Coniophora - [S. 335.]

$\longrightarrow$ arida. $-[$ S. 336.337, T.]

eradians. - [S. 336 .]

- laxa. - [S. 336.]

—_ inembran. - zu Merul, destr. (Tul.,.

_- puteana. - [S. 335.]

- stabularis. - [S. 335.]

Coniosporium, - [S. 522.]
Coniospori um Arnicae. - Lib. 352.

- circinans Fr. - [S. 126.] - Kil. II. 59. -

Fuck. s, 500. 'cf. Sphaeria!, - [S. 3!5.] -

Wistd. 35.

- fructigenum. - [\$. 522.]

Helmisporii. - [S. 523.]

olivaceum. - Schnzl. t. 14. f. 4.

quercicola Lasch. - Kl. 1055.

Verticillii. - [S. 523.]

Violae. - Lib. I 4. - Wstd. \$2S.

Coniothecium. - [S. 4s2*.523.]

- Amentaceorum Cd. - Dsm. 529. - Voges.

1259. - Melancon. conglomerat. Lk. - Wstd. 573.

— atrum. - Sturm h. 13. t. 36.

betulinum Cd. - S. 452.] - cf. Schizoderma b. - Jil. 1056. - Fuck. s. 115. - Dsm. 926. - Kil. II. is1. - Wstd. 5it. - Fuck. rh. 91. - Fresen. t. 13. f. 29-32. p. 103.

- chomatosporium Cd. - (variegatum) Sturm h. 29 - t. 30 . Preuss.. - Kl. 1379. - Fresen. t. 13. f. $8-1$ s. p. 102 .

- cffusum. - Bon. t. 2. f. 55.

- epidermidis C'd. - Kil. 1169. - Fresen.

t. 13. f. 1-i. p. 102 .

— phy̆llophilum. - Ǩl. 1795. - Dsm. 927.

_- Questierii. - Dsm. III. 40.J.

- Tiliae Lasch. - Kl. 1170. - Fresen. t. 13.

f $19-25$. p. 11):3.

— toruloides. - K1. $\$ 92$. (Cent. XVII.).

Conioth y rium cruciatum. - Fuck. rh. 92.

—_ glomeratum. - S. $522 *$.]

- olivaceun Bon. - Fuck. rh. 93.

— Pini. - S. 420.] - Cd. Anl. t. F. f. 54:

1-3. - Sacidium: Fr. Summ. - Bail S. t. 16.

Conoplea. - is. 475.]

- atra $\mathrm{P} .=$ Myxotrichum chartarum Fl. dan.

- Letell. $6 \% 2.1$.

- cinerea. - [S. 475.

— cylindrica. - Wstd. \$3. $\$ 61$.

- fusca. - S. 475.

- gilva. - [S. 495.] - Wstd 1196.

- grisea. - [S. 475.

- hispidula. - S. 499.] - Kil. 1161. - cf.

Polynema strig. St. - Sturm h. T. t. 31 .

- olivacea. - S. 475.7

- puccinioides. - cf. Goniospor. con.

Coprinarius. - cf. Agaricus.

Coprinus. - ef. Agaricus.

- Fructific. Ann. sc. n. 1537. VIII. t. 9.

f. 20 . Lév.).

- alternatus. - [S. 295.]

— aphthosus. - [S. 295.]

- astroideus Fr. - Bisch. f. 3249.

- atramentarius. - [S. 295.] - Berk. Out.

t. 12. f. 1 .

cinereus Fr. - [S. 295.] - Bisch. f. 3317.

- clavatus. - [S. 297 .

- comatus. - [S. 297.

_- congregatus sow. - Kl. U. 605.

coopertus. - [S. 29S.]

- cylindricus. - [S. 295.] deliquescens. - [S. 29S.] - Bail S. t. 35.

- digitalis. - [S. 29S.]

- dilectus. - [S. 295.]

- domesticus. - [S. 295.]

ephemeroides. - [S. 295.]

ephemerus. - [S. 295.]

extinctorius. - [S. 295.] - Bail S. t. 34.

filiformis. - Ann. Mg. n. h. 1S61. VII. t. 15.

f. S. p. 379 . (B. B.).

fimetarius. - [S. 298.] 


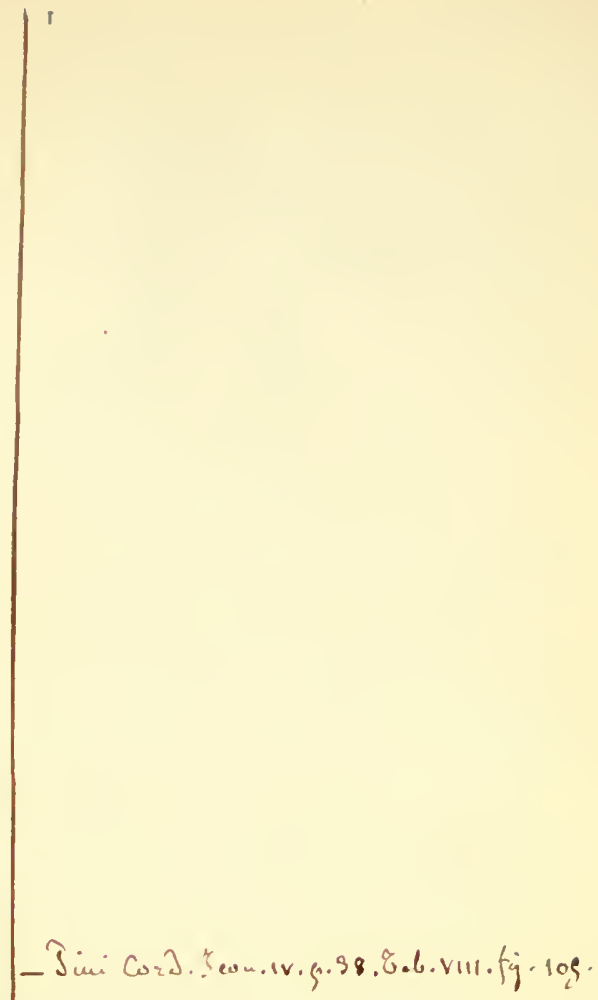



Coprinus fuscescens, - [S. 298.]

hemerobius. - [S. 299.]

- Hendersonii. - Berk. Out. t. 24. f. 8 .

lagopus. - [S. 29S.]

micaceus. - [S. 295.] - Bisch. f. 3306.

narcoticus. - [S. 295.]

niveus. - [S. 29S.?

- nycthemerus. - [S. 29S.]

ovatus. - [S. 297.]

papillatus. - [S. 298.]

- petasiformis IIumb. - Bisch. f. 3434.

- picaceus. - [S. 298.]

- plicatilis Fr. - [S. 295.] - Bisch. f. 3316.

- radiatus. - [S. 29S.j

rapidus. - [S. 299.]

- Sceptrum. - [S. 299.]

- soboliferus. - [S. 29S.]

- sociatus. - [S. 295.]

- stcrcoreus. - [S. 29S.]

- sterquilinus. - [S. $29 \%$.]

- tergiversans. - [S. 295.

- tomentosus. - [S. 294.]

_- truncorum. - [S. 295.]

varicus. - IS. 299.]

velaris. - [S. 299.]

(:oprotrich um cinereum. - Bon. t. 6. f. 133. purpurascens. - Bon. t. 6. f. 132 .

Cora. - S, 333,524.] - cf. Thelephora.

Corallodendron leucocephalum, - Jungh, pr. t. 1. f. 5 .

Corallomyes. - [S. 465 .

Cordana polyseptata. - Ki. 1579.

Cordicrites. - [S. 399.]

- guyanensis. - Ann. sc. n. 1540. XIV. t. 19.

f. 11. Mont.! - Ann. sc. n. 1S55. III. t. 6.

f. 6. Asci. Mont. - Cd. Anl. t. G. f. 64:

$10-42 .-$ Pay. b. cr. p. 62 . f. 270.

Sprucei. - Hook. J. 1556. VIII. t. 10. f. 5.

Cordyceps. - $\$$ s. 351.$]$ - ef. Clavaria granul.

Balb. - Claviceps, Cordyliceps, Hypocrea, Sphaeria.

- acicularis Berk. - Linn. Journ. 1556. I. t. 1. - alutacea 1'. - [S. 3S1.] - R. f. 132. - R.

f. 246. - Berk. Out. t. 23. f. 6. - Nov. Act. 1561. XXIX. t. 1. f. 6. 11.12. Bail.

- v. albicans $\mathrm{P} .=$ Sphacr, al. v. alb.

P. S., Sph. clavata Sow.

- armeniaca Berk. - Linn. Journ. 1556. I. t. 1.

- capitata. - [S. 381.] - Dsm. III. 379. -

('arol. V. 4s.

- carolinensis. - Carol. IV. 29.

- entomorhiza. - [S. 3S1.] - Robin v. par.

t. S. f. 6.5. t. 12 . f. 2. 3. t. 10. f. 3. 4. 6. -

Berk. Out. t. 23, f. 5 .

- falcata Berk. - Hook.J. 1554. VI. t. S. f. 2. ? fuliginosa. - Comm. soc. cr. it. 1\$61. 2.

p. 67. t. 6. f. 1. (Ces.).

gracilis. - Alger. t. 25, 2.

- Hügelii. - Cd. Anl. t. F. f. $56: 21-26$.

- microcephala. - Dsm. III. $3 \$ 1$.

- militaris. - [S. 381.] - Schnzl. t. 15. f. 35.

- K1. 47. - Berk. Out. t. 23. f. 4.

- myrmecophila Ces. - Kl. 1033. - Kl. II.

719. - Comm. soc. cr. it. 1861. 2. t. 4. f. 2. (Ces.).

- ophioglossoides Ehrh. - [S.381.] - Kl.655.

- Kl. II. 427 . - R. f. 442 . - Dsm. III. 378.

palustris Berk. - Linn. Journ. 1856. I. t. 1.

- pistillariaeformis. - Ann. Mg. n. h. 1561.

VII. t. 16. f. 22. p. 451 . (B. B.).

- purpurea. - cf. 'Claviceps. - [S. 381.] -

Hoff $\mathrm{man}$, Index Fungorum.
Berk. Out. t. 23. f. 7. - Dsm. III. 350. - Voges. $1450 .-$ Wstd. 1201.

Cordyceps purpurea v. Acus Dsm. - ef. Claviceps microc.

— racemosa Berk.-Hook. J. 1 554.VI. t. 5. f. 3.

Ravenelii Berk. - Linn. Journ. 1556. I.

t. 1. - Carol. IV. 28.

Robertsii. - Robin v. par.t. .. - ef. Splaer.

Rob. et Hügelii. - Bail S. t. 17.

- Sinclairi. - Berk. In. p. 73. f. 17. b.

- stylophora Berk. - Linn. Journ. 1 556 . I.

t. 1. - Carol. V. 49.

typhina. - [S. 3S1.]

Wallaysii. - Bull. ac belg. 1559. VII. p. 365.

Taf.). fig. 1. (W'std.).

Cordy li ceps. - cf. Cordyceps.

Coremi um. - [S. $459^{* * *}+465^{\cdots *}$.]

- bicolor. - S. 459.]

- coccineum. - [S. $165^{* \cdots}$. ]

glaucum. - André, Ökonom. Neuigk. 1S47.

t. 1. f. $14-16$. (Cd.)

- leucopus, - Bon. t. 11. f. 223.

- niveum. - Pay. b. cr. p. 10. f. 53.

- subiculatum. - [S. $465 \cdots$.]

vulgare $\mathrm{Cd}$. - cf. Coremium glaucum. -

Cd. Anl. t. C. f. $21: 13-16 .-$ Kl. $1377 .-$

Fuck. rh. 161. - Auersw., Bot. Unterh. 1563.

s. $3 \rightarrow 1$.

Corethropsis. - [S. $491 *$.]

- paradoxa. - ef. Stachylid. p.

Corticium. - cf. Thelephora, Stereum. [S. 333.]

- albido-carneum. - C'arol. IV. 14.

- amorphum. - [S. 333.] - Ital. 441. (Pe-

ziza P..

- arachnoideum B. - [S. $336 *$ *.] - Hy-

pochn. centrifug., Tul.

_- aridum. - [S. 336.]

— atrovirens. - [S. 335.]

Boltonii. - [S. 331 .

calceum. - [S. 335.] - Kl. 325. (Thel.).

— Pini. - Carol. IV. 15.

- sambucinum Fr. - Kl. II. 15.

ceraceum. - Carol. III. 29.

ciliatum. - [\$. 334.]

- cinereum P. Fr. - [S. 335.] - R. f. 20. -

Dsm. 823 .

—- cinnamomeum. - S. 335.$]$

- coeruleum. - [S. 335.] - Ital. 554.

- comedens Ns. (Leiostroma). - [S. 335.] -

R. f. 412.

- confluens. - [S. 335.]

- corticola. - Carol. III. 30.

- crocicreas. - Carol. V. 27.

- diminuens. - Carol. III. 31.

epichlorum. - Carol. V. 24.

- eradians. - S. 336.]

— evolvens Fr. - [S. 334.] - Sturm h. 31 -.

t. 7. (Szl.!.

_ flammans. - [S. 334.]

- flocculentum. - [S. 334.]

fumosum. - [S. 335.]

giganteum. - [S. 334.]

incarnatum P. Fr. - [S. 335.] - K1. II. 14.

laciniatum P. - Wstd. 597.

lactescens. - [S. 335.]

lacteum. - [S. 334.]

- laere P. - [S. 334.] - R. f. 120 .

laevigatum. - [S. 335.]

laxum. - [S. 336.]

Leveillianum. - ef. Stereum L. 
Cortic i u lividum. - [S. 335.]

- maculaeforme. - [S. 335.$]$

- Martianum. - Carol. V. 30.

- nigrescens (Schrd.) Fr. - R. f. 21.

- nudum. - [S. 335.]

__ Oakesii. - Carol. III. 32.

— ochraceum. - [S. 335.]

- ochroleucuri. - [S. 333.] - Carol. III. 33.

- Petersii. - Carol. V. 2\$.

— Platani. - K1. II. 215.

— polygonium. - [S. 335.]

- populinum. - [S. 334.]

- prasinum. - Carol. V. 29.

- puberum. - [S. 335.] - Kl. II. 216. a.

- - - effusum. - Kl. II. 216. b.

puteanum. - [S. 335.]

- quercinum. - [S. 334.] - Bisch. f. 3407.-

Fuck. s. 913: - K1. II. 214.

- radiosum. - [S. 334.] - K1. 1515.

- roseum. - [S. 334.] - Kl. 1516. - Ital.

488.

salicinum Fr. - [S. 334.] - Fuck. s. 914.

Sambuei P. - [S: 335.]- Dsm. \$24. - ef.

calceum. - Wstd. 589.

— sanguiueum. - [S. 334.]

- sarcoides. - [S. 334.]

- seriale. - [S. 335.$]$

- simulans. - Carol. V. 25.

- stabulare. - [S. 335.]

- sulfureum. - [S. $331^{* *}$. 335.$]$

- Typhae. - Ital. 442. (Athelia).

-_ uvidum. - [S. 335.]

- velutinum. - [S. 334:]

- versiforme. - [S. 334.$]$

— violaceo-lividum. - [S. 335.]

- viscosum. - [S. 335.]

— viticola. - Carol. III. 34.

Cortinarius. - ef. Agaricus.

Coryne. - [S. 341.]

— sarcoides. - Bon. t. 11. f. 233. - cf. Tremella sare. With.

- turbinata, - Cd. Anl. t. G. f. $70: 1=4$.

- unicolor. - Bail S. t. 22.

Corynelia. - [\$. 401.352**.]

Coryneum. - [S. 474.]

- brachyurum. - Kl. 1872.

_- caudatum Pr. - Kl. 1253.

depressum S. K. - [S. 474.] - Bisch. f.

3836. - Fuck. s. 232. - Bon. t. 12, f. 23 s. disciforme. - Ca. Anl. t. G. f. 69. 14-i6.

- R. f. 278. - Wstd. 1072. - Pay: b. cr.

p. 76. f. 356. - Bisch: f. 3593. - Bon. t. 12.

f. 239. - Voges, 573.

Kunzei. - Kl. 1360. - Fuck. s. 235. -

Kil. II. 779. - Brit. f. 312. - Fuck. rh. 228.

- macrosporium Berk. - [S. 474.] - R. f. 75.

- Micr. J. 1559. VII. t. 11. f. 19 b. (Fructif., Curr.).

- marginatum P. - [S. $402 * *$ 474.] - Bisch.

f. 3564

- oligosporum. - Cd.Ic. V. t. 9. f. 73.

- pulvinatum. - [S. 474.] - Bon. t. 12.

f. 240 . - Fuck. s. 231. - Kl. 1441. - Wstd.

183.

- umbonatum. - [S. 474.] - Dsm. 176. (Exosp. Til. ex Tul.). - Cd. Anl. t. G. -f. 69 : 11-13. - Bisch. f. 3S59. - Fuck. s. 233:

Corynites. - Linn. Trans. IV. 21. (Berk.).

- Ravenelii. - Linn. Trans. 1S53. XXI. t. 19. f. 4 .

Cosmarospora. - cf. Cosmospora.
Cos mospora coccinea R. - R. f. 459. - Hedw. II. 1562. t. 10. (soll heissen: f. $2: 1-5$ ). Sphaeria Granatum Wllr.

Couturea. - [S. 407.]

C Castänei. ODsh. 1020。- Iţal. 786.

Elaeanema. - Castagne, Marseille: t. 1.

Crat ere 11 us. - [S. 330.] - Bail S. t. 29.

ef. Cantharellus.

- clavatus. - [S. 330.] - Bisch. f. 3299. -

Staude t. 2. f. 2 .

- cochleatus. - [S. 330.$]$

— Comperi Lév. - Démid. Russ. t. 4. f. 2.

- Comucopiae. - Pay. b. cr. p. 107. f. 490.

cornucopioides. - [S. 330.] Cd. Ic. V.

t. 10. f. S0. (Fructif.) - Berk. Out. t. 19. f. 6.

- Cd. Anl. t. H. f. 78: 26-29. - Fuck.

s. 933: - R. f. 207 b. (Cent. IV. suppl.). -

Bisch. f. 3304 . - Kl. 626. - R.'f. 207. - Kl.

II. 11. - Voges. 393. - Carol. II. 27. - Ital.

232. - Krypt. Bad. 58.

- lutescens. - [S. 330.] - K1. II. 20S.-

Schweiz. 21.

- pistillaris. - [S. 330.]

$\therefore$ pusillus. - [S. 330.] - K1. 1S08. - Fuck.

s. 931 .

- sinuosus. - [S. 330.] - Kl. 625. - R. f.

205. - Fuck. s. 932. - Kl. II. 119.

$\because$ unicolor. - Carol. II. 26.

Craterium. - [S. 454.]

_- deoperculatum. - [S. 454.]

- Alavum. - [S. 454.]

_- leucocephalum. - [S. 454.] - Bisch. f. 3665.

- Loud. f. 16517. - Wstd.4S2. - Sturm h: 1.

t: 11 .

— minutum. - [S. 454.$]$

- mutabile Fr, - [S. 454.] - Fl. dan. XII.

2057. 2. - Alger. t. 22, bis. 3 .

nutans. - [S. 454.]

- pedunculatum. - [S. 454.] - Bisch. f. 3666.

- KI. II. 139. - Fuck.s. 253.

pendulum. - [S. 454.]

$\longrightarrow$ pruinosum Cd. - Cd. Ic. VI. f. $33^{\circ}$

- pyriforme. - [S. 454:] - Cd. Anl. t. C.

f. $29: 29,30$. - Sturm h. 1.t. 10.

- D Dtm.: Bisch. f. 3667. - Pay. b. cr.

p. 122. f. 578 .

- turbinatum. - [S. 454.] - FI. dan. XII.

2093. 3.

- vulgare. - Wstd. 4S2. - Cd. Anl. t.. C.

f. $29: 27.28$. - Sturm h. 1. t. 9.

Craterom yces. - [S. $45 S^{*}$. 524.]

— candidus. - Cd. Anl. t. 6. f. $23: 7-9 .-$

Bisch. f. 3795 . - Schntzl. t: 12, f. 42 . 43. -

Bon. t. 10. f. 207. - (ov. insector., Tul.). -

Sturm h. 12. t. 30 .

duplex. - Bon. t. 10. f. 210.

Cre opus. - Bail S. t. 17.

Cribraria. - [S. 456.]

argillacea. - [S. 456.]

aurantiaca Schrad. - [S. 456.] - Fl. dan.

XII. 2055. 1. - Cd. Ic. V. t. 3. f. 35: - Cd.

Anl. t. C. f. $31: 5-7$ - Pay. b. cr. p. 122.

f. 583 . - Bisch. f. 3678 :

didermoides Schum. = Dictydium d. Fl. dan. fulva. - [S. 456.]

intermedia. - [S. 456.]

intricata. - [S. 456.] - Berk. In. p. 335.

f. $75 . \mathrm{b},-$ Carol. II. 79.

macrocarpa. - [S. 456.]

micropus Schrad. - Loud. f. 16521.

Onygena ? - R. f. 26 S.

purpurea Schim. - [S. 456.] - KI. 930. 


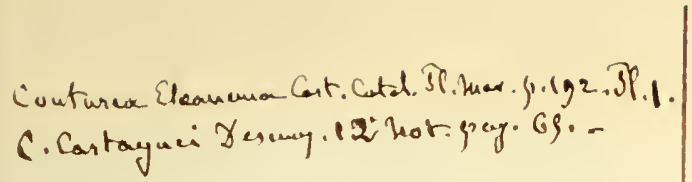

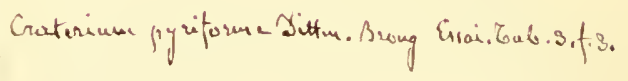


Gocierear Graminame Fries S.4.5e. p.h18.-

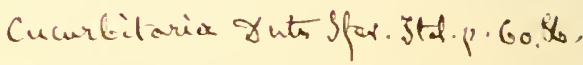


Cribraria pyriformis. - [S. 456.$]$

- 2 ubiginosa. - [S. 456.]

tenella. - [S. 456.$]$

- vulgaris. - [S. 456.] - K1. 1344.

Crinula. - [S. 339.]

- calyciiformis. - [S. 339.]

- Gayana. - Chile n. S. f. 1.

- nigra. - Bon. t. S. f. $17 \%$.

- paradoxa. - Carol. III. 35.

Crocicreas. - ef. Perisporium. - [S, 415.]

- giganteum. - Chile n. 9. f. $T$.

Crocysporium. - [S. $46 i^{* \cdots *}$.]

Aegerita. - Cd. Anl. t. G. f. $65: 15-20$.

album. - Kl. 1435.

fallax Bon. - R. f. 300. L= Oid. Tuckeri

Mohl u. Oid. leucocon. Sturm, - cf. Bot. Zt.

1\$61. p. 202: Bon.).

- torulosum. - Bon. t. 4. f. 90.

Cronartium. - [S. 510.]

- asclepiadeum. - [S. 510.] - Ung. Ex. t. 4.

f. 23 . - Bisch. f. 3513. 3544. - Fuck, s. 100.

- Dsm. 579. 1009: f. Paeoniae. - Voges.

1349. - Wstd. 454. - R. f. 559. - Ital. 547.

- Fuck. rh. 111 .

- Vincetoxici Fr. - Ann. sc. n. 1554.

II. t. 11. (Tul.). - K1. 95. - K1. II. 334. -

Bon. t. $3, f, 65$.

- Paeoniae. - Sphaeria flaccida A. S. -

Voges. 1050. - Wstd. 454. - Fuck. rh. 112.

__ populinum Fée. - Wstd. 1395.

Crucibul um. - cf. Cyathus. Nidularia.

vulgare. - Wstd. 451. - Ann. sc. n. 1541.

I. t. 6. f. $9-21$; t. 7 . f. 1 . $15-21$; t. $\$$, f. $13-$

17. (Tul.). - J3erk. Out. t. 2. f. 2. - Bot. Zt.

1555. t. 13. 14. Sachs'

Cryptococcus. - ef. Torula Cererisiae.

Absinthii. - Dsm. III. 515.

Cerasi. - Dsm. III. 516 .

Cerevisiae. - Rob. v. par. t. 6. f. 1. - cf.

C. Ferment. - Dsm. III. 514.515.

Clava. - Sitz. Ber. Wien. Ak. $1 \varsigma_{5} \varsigma$. XXIX.

7. p. 92. (Wedl.).

Fermentum. - Kützing, Philos. Bot. 1551.

t. 2. f. 4-6. - Willkomm, Wunder d. Mikr.

1856. p. S6. f. 39. - Schacht, Lehrb. d. An.

Phys. I. 1556. t. 1. f. 35. - Flora 1557. no. 27.

t. 1. f. $1-1 \bar{\gamma}$. Bail .

f. Urinae diabet. : Kl. II. 559.

glutinis. - Fres. t. S. f. $43-46$.

- v. Polyg. sar. - Dsm, III. 520.

- v.-Amyli. - Dsm. III. 521.

guttulatus. - Robin. t. 6. f. 2.

urophilus. - Dsm. III. 519.

Vini rubri. - Dsm. III. 517.

Cryptodiscus. - [S. 3i3. 42S.]

atrovirens. - Bail S. t. 19.

Cesatii Mont. - Kl. II. 522. - Hedw. I.

t. 14, f. F. p. 102.

lichenicola Ces. - Kl. II. 523. - Ital. 492.

pallidus. - Cd. Anl. t. G. f. $63: 1-4$.

phacidioides D. - Dsm. 925 .

Solidaginis. - Ital. 872.

Cryptomyces. - [S. 372.]

Betuli. - [S. 372 .]

disciformis. - - [S. 372.]

Wauchii. - [S.372.] - Cd. Anl. t. F. f. 61:

6-11. - Loud. f. 16259.

Cryptosphaeria. - cf. Sphaeria, Díplodia, Ceuthospora, Depazea.

_ acuminata Sow. - Loud. f. 16406.

acuta. - Wstd. 170 .

arbuticola Sow. - Loud. f. $16+1$ s.
Crsptosphaeria aurantia Grer. - Wstd. 113. Loud. f. 16403.

bifrons Fr. - Loud. f. 16391. (16402.).

capillata Grev. - [S. 39S: phaeoc.] — Loud.

f. 16112. - Pyrenophora phaeoc.

— confluens Sow. - Loud. f. 16397.

duplex Sow. - Loud. f. 16401.

glauco-punctata. - Wstd. 535.

Lauri Grev. - Loud. f. 16100.

- millepunctata Grev. - Wstd. 475. - I.oud.

f. 16396 .

nitida. - cf. Dothidea Robertiani Lib.

- Pteridis Sow. - Loud. f. 16404.

pulchella Grev. - Loud. f. 16390.

- Taxi Grev. - Loud. f. 16395. (cf. Diplodia

Taxi. - Wstd. 521.

Cryptosporium. - [S. 423.] cf. Fusidium.

acerinum. - [S. 423.424.]

Aesculi P. - [S.50S,] - Bisch. f. $3 \$ 34$.

atrum. - Sturm h. 9. t. 49.

- Caricis Cd. - Bisch. f. 3539. - Brit. f. 397.

- Sturm h. 9. t. 50.

- coronatum. - Fuck. rh. 102.

- deplanatum. - [S. $423^{* * *}$.]

- Equiseti, - cf. Hymenula.

graminis. - [S. 424.]

imberbe. - [S. 423.]

Neesii. - [S. 5uS. vulg.] - Cd. Anl. t. F.

f. 57: 1-1. - Bail S. t. 16. - Kl. 13s5. /cf.

Melancon. vulg. , - Kl. II. 577. - Fuck. rh. 101.

- Sturm h. 9. t. 51.

- Populi. - [S. $423^{* \cdots *}$.]

- Ribis. - [S. 424.]

- Sorbi Ces. - R. f. 160. 25i. (viride). -

Fuck. s. 361 .

- viride Bon. - R. f. $2 \varsigma \bar{i}$. cf. C. Sorbi).

Crypt th amniu m usneaeforme Wllr. cf. Thamnomyces Chamissonis.

Cryptovalsa. - cf. Sphaeria.

Cu cullaria helvelloides. - Cd. Anl. t. G. f. (j1): 11.12.

- infundibuliformis. - Cd. Anl. t. G. f. 66 :

13. 14 .

Cucurbitaria. - [S. 390.] cf. Sphaeria.

- Berberidis Grev. - Loud. f. 16384.

_- decolorans Grev. - Loud. f. 16357. (Nectria).

- elongata. - Cd. Anl. t. F. f. 55: 29-33. -

Loud. f. 1635s. - Pay. b. cr. p. 96. f. 439. -

lil. II. 727. - Ital. 646.

——_ simplex Fr. cf. Sphaeria.

Pteridis. - Hdw. I. t. 15. f. 6.

Rhamni R. = Sphaeria R., Ns.

Cudonia. - [S. 345.] cf. Leotia.

- circinans. - [S. 347.]

marcida. - [S. $345 *$.]

Cupularia leucocephala Lk. - Kl. \$21. -

Fuck. 5. 252.

C'y a th us. - [S. 435.] ef. Crucibul, u. Nidularia.

— agrestis. - [s. 43S.]

- byssisedus. - Ann. sc. n. 1844. I. t. 4. f. 8.

(Tul.).

- campanulatus Sibth. - [S. 435.] - Krypt.

Bad.332. - Cd. Anl. t. D. f. $42: 19-23$. -

Carol. III. 73. - Wstd. 252.

- cinereus. - Purton. I. t. 35. - Wstd. $4 \$ 2$. crucibuliformis: - Wstd. 451 .

- Crucibulum. - [S. 439.] cf. Crucibulum vulg.

- Dsm. 405. - Cd. Anl. t. D. f. 42: 10-1s.

Loud. f. 16314. - Fuck. s. 321. - Sturm h.

31 - t. 23. (Szl.) - Kl. II. 136. - Wstd. 4 \$1.

- dasypus. - Ann. sc. n. 184t. I. t. 5. f. 21 .

(Tul.). 
Cyathus farctus. - [S. 435. 2.]

Gayanus. - Ann. sc. n. 184. I. t. 1. f. $1 \mathrm{~s}$

-22 ; t. 5. f. 1. 2. (Tul.).

- intermedius. - Ann. sc. n. 1S44. I. t. 4.

f. $1-7$. (Tul.).

laevis $H_{.}=$C. Olla. - Wstd. $2 S 2$.

Lesieurii.

- major. - Ann. sc. n. 1S44. I. t. 5.

f. 5-10. (Tul.'.

- minor. - Ann. sic. n. 1544. I: t. 5.

f. 11-13. (Tul.).

- limbatus. - Ann. sc. n. 1511. I. t. 4. f. 12

- 17. Tul.).

- microsporus. - Ann. sc. n. 1S $\$$. I. t. 6 .

f. 6 -s. (Tul.'.

Montagnei. - Ann. sc. n. 1S44. I. t. 4. f. 9

-11. Tul.!.

__ nitidus. - Wstd. 252.

novae Zeelandiae. - Ann. sc. n. 1\$44. I.

t. b. f. $1-5$. Tul.).

Olla. - Voges. 152. - vernicosus Tul., -

laevis Hoffm. - Wstd. 2\$2. - Kops Fl. bat.

X. 760.2 . - Schnzl. t. 14. f. $36-11$. - Kl. II.

247. - Fuck. s. 319. - Ann. sc. n. 1s14. I.

t. 5. f. 14-23. (Tul.). - Dsm. 104. - Berk.

Out. t. 21. f. 1. - Nidularia vern. Bull., camp.

With.

- Pöppigii. - Ann. sc. n. 1S44. I. t. 4. f. 23 ;

t. 5. f. 3. 4. Tul. .

- scutellaris. - [s. 435.

striatus. - [S. $\left.43 \mathrm{~S}_{0}\right]$ - Dsm. 403. - Cd.

Anl. t. D. f. $42: 1-9$. - Linn. 1842. XVI. $t$.

6. 7. Schmitz. - Loud. f. 16342. - Berk.

Out. t. 2. f. 3. - Ann. sc. n. 184t. I. t. 3. t. 4 .

f. $1-3$; t. 8. f. 1-12. (Tul.) - Pay. b. cr.

p. S. f. 31 ; p. 109. f. $495-502$. (evolut.). -

Bot. Zt. 1559 . t. 11. f. 21. Keimg. Hoffm.). -

Kl. II. 135. - Fuck. s. 320. - Brit. f. 259. -

W'std. 121. - Ital. 23\%. - Krypt. Bad. 50.

— subiculosus. - Bull ac. belg. 1\$41. VIII.

2. t. 2. $\mathrm{Kx}$.).

- vernicosus. - ef. C. Olla P. - Wstd. $2 \$ 2$.

- Ital. $\$ \$ 6$.

C y clocon i m Oleaginum. - Castagne Marseill. t. 6.

Cycloderma. - [S. 410 .]

indicum Kl. - Linn. \$ $\$ 32$. VII, t, 9, B. -

(d. Anl. t. C. f. 39: 1-1. - Pay. b. cr. p. \%.

f. $43 ;$ p. 115 . f. 534 .

Cyclomyces. - [S. 324.]

- fuscus. - Linn. 1830. V. t. 11. f. 3. Fr.

- Cd. Anl. t. H. f. $76: 15-20$. Bisch. f.

3409. - Bail S. t. 319 .

- Greenei. - Hook. Lond. J. 1545. IV. t. 11.

- Pay. b. cr. p. 11 \%. f. 543.

Cylichnium. - [S. 459.$]$

Cylindri um candidum. - Bon. t. 1. f. 4.

- carneum. - Fuck. rh. 56 .

— elongatum. - Bot. Zt. 1553. t. 7.f. 1. Bon.'.

- Fuck. rh. 55.

- septatum. - Bou. t. 1. f. 16.

Cylindrocolla Urticae Bon. cf. Dacryomyc. Urt. Fr.

Cylindrodendrum album. - Bon. t. 5. f. 127.

Cylindrophora alba. - Bon. t. 6. f. 131 . tenera. - Bon. t. 5. f. 110.

Cy lindrospora concentrica. - Ung. Ex. t. 2. f. 9 .

- major, - Ung. Ex. t. 2. f. 11. - Kl. 1590. Phaseoli. - Kl. II, $32 \bar{\imath}$.

Cylindrosporium. - [S. $505^{* *}$.]

- concentricum Grer. - Loud. f. 16616.
Cylindrosporium Ficaria. - Brit. f. 212.

- longipes. - Sturm h. 29- t. 35. (Preuss.). majus. - Bon. t. 1. f. 22. - Fuck. rh. 75.

Cylindrotrichum album. - Bon. t. 4. f. 99. repens. - Bon. t. 4. f. 97 .

Cymatoderma. - [S. $332 *$.]

- elegans. - Tijdsk. v. n. Geschied. 1540.

VII. t. 8. f. 7. p. 290. (Jungh.). - ?'Thelepli. dendritica $\mathrm{P}$.

Cynophallus. - [S. 434.] (cf. Phallus'.

- canariensis. - Cd. Anl. t. E. f. $51: 8$. caninus. - Cd. Anl. t. E. f. 51: 1-7. -

Bail S. t. 26. - cf. Mutinus.

Cyphella. - [S. 336.]

- candida. - Jungh. pr. t. 5. f. 16.

- Capula. - [S. 336.]

- v. cernua. - Kl. 1612.

- Curreyi B. B. - R. f. 416.

- Digitalis. - Cd. Anl. t. G. f. 73: 23. -

Bisch. f. 3349. - Bail S. t. 2 S.

_ faginea. - Lib. 331.

- fissilis. - [S. 336.]

- fulva. Carol. IV. 16.

_ _ galeata. _ S. 336.$]$

- Gayana. - Chile n. 8. f. 3.

— Hoffmanni Tul. - Pez. anomala I.

— infundibuliformis. - [S. 336.]

— lacera Fr. - [S. 336. 4.] - Bisch. f. 3351.

laeta. - [S. 336.]

- Musarum. - Jungh. pr. t. 5. f. 15.

- muscicola Fr. - [S. 336.] - Kl. S24. - if.

Cy. Neckerae.

- - v. inaequilatera. - Fl. dan. XII. 2053.2. muscigena. - [S. 336.]

- Neckerae. - [S. 336.] - Bon. t. 10. f. 212. pendula Fr. - Polyporus cupulaef. B. C.

- Taxi. - Ann. sc, n. 1537. VIII. t. S. f. 11.

Fructif. Lév. , - Ann. se. 11. 1S41. XVI. t. 14.

f. f. Lév. . - Cd. Anl. t. G. f. 73: 24-26. -

Pay. b. cr. p. 10T. f. 455. - ? zu Pez. albo-riol. 'Tul. .

II. 10.

Cystophora. - [S. $501 * * *$.]

Cystopus. - [S. j̇12.] cf. Uredo.

Alismatis. - Bot. Zt. 1 \$61. t. S. f. 6. p. 194. Bon.

—_ Bliti Lév. - R. f. 595.

candidus. - [S. 512.] - Bon. t. 2. f. 41. -

R. f. 152. oospor., - de Bary Br. t. 2. f. 3- 7 .

Fuck. s. 4. - Ann. sc. n. 1560 . XIII. t. 13. f.

16-15. Zoospor. de Bar.) - Journ. hortic.

soc. 1 $\ 1$ ๖. III. p. 266. u. 270. (Berk.) - Fuck.

rh. 14. - Ital. 59S.

- f. C'ruciferarum: Sinapis. -Kl. II. $366^{4}$.

f. Lepidii. — Carol. IV. 93.

cubicus. - [S. 512.] - Kl. 1595. - An1.

sc. n. 1560 . XIII. t. 13. f. 10-15. (Bar.). - R. f. 450 . - Fuck. rh. 45.46 .

f. Cirsii oleracei. - Kl. II. 692.

Lepigoni de B. - R. f. 483. - Fuck. rh. 4 .'.

Portulacae, - Ann. sc. n. 1854. II. t. 7 . f.

1-5. Tul. - R. f. 151 - Kil. II. 799. -

Fuck. rh. 43 .

- quadratus. - [S. 512.]

- sphaericus Bon. - R. f. 156.

- spinulosus. - Fuck. rh. 47.

-1 de B. - R. f. 479.

Cystotricha Striola. - Ann. Mg. n. h. 1550. V. t. 12. f. 10 . p. 459. (B. B.).

Cytispora. - S. $344.410^{\cdots+} 413.7$ ef. Sphaeria.

- Abietis. - Schnzl. t. 15. f. 6-10. 


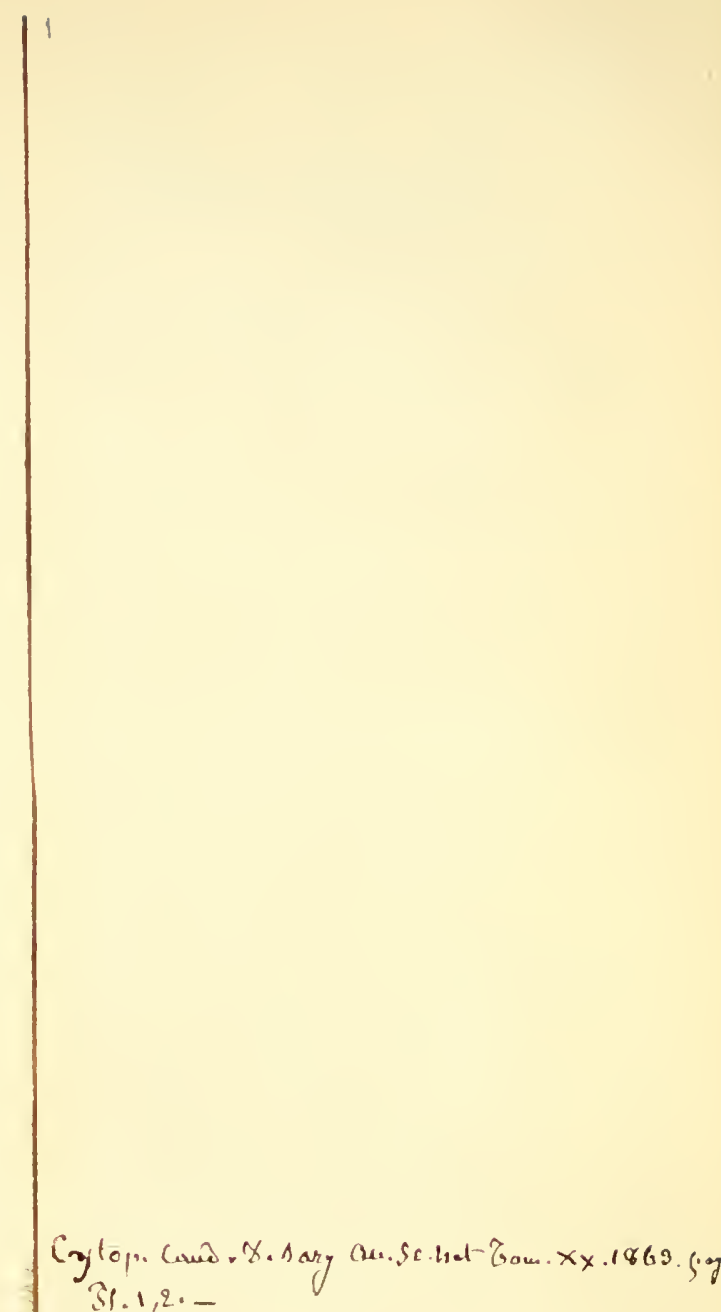

31.1,2. -

C. cubient te 1,at for.cit. It.2. Fy.14-21.

c. Sortulacor. Bar. Sol cit. S1. 3 . 

Cy tispor a Aesculi. Wstd. 721.

- atronitens. - Wstd. 362.

atrovirens. - [S. 413.]

- Aucupariae. - Lib. 16॰. (Sphaeria caespitosa Td.).

- Aurora. - Ital. 542.

- betulina Ehrb. - Kl. 764. - Fuck. s. 426.

brunneola. - [S. 413.$]$

Buxi D. - Dsm. 1499.

carbonacea Fr. (Synariza). - is. 413.] -

Kl. 371 . - R. f. 358 .

- carphosperma Fr. - [S. 413.] — Bisch. f. 3569. - K1.372. - Voges. 1340. - Wstd. 519. 1249.

- v. Tyri Mali Lasch. - Iil. 76.5.

chilensis. - Chile n. 9. f. 12.

chrysosperma. - [S. 413.] - Schnzl. t. 15.

f. 4. 5. - Fuck. s. 423. - Dsm. I-1. - Iil.

169 ; Cent. X. Suppl. - Voges. s51. - Brit.

f. 190. - Lib. 169 , W Wtel. 323 .

Corni. - Wstd. 1129.

Dahliae. - Lotos 1557 . VII. t. 2.

elegans. - [S. 40s.] - Kil. 1569.

endophylla. - [S. +13.]

enteroleuca. - ${ }^{-}$S. 113.

epinyces, - [S. 404.]

ferruginea I). - [S. 113.] - Dsin. 955. -

Lib. 265. - Wstd. $21 \varsigma$.

- foliicola. - Toges. $1176 .-$ Lib. 61. - Wstd.

21. (Ceuthosp. placid. v. immacul. Ism.

- fugax. - [S. 113.] - K1. 1351. - Fuck. s.

124. - R. f. 160 . - I) $s$. 142. - Wstd. 19.

globifera. - [S. 111.]

grisea. - Iil. 1112.

guttifera. - [S. 414 .

- incarnata Fr. - [S. 113.] - 1)sm. 190. -

Kil. 951. - Wstd. 520. v. Rosac. - Ki. 9S2.

Laurocerasi Fr. - Fuck. s. 437.

leiphaemia Awd. - Sphaeria Fr. - K1. 1326.

leucomyxa, - Kil. 1352. - Fuck. s. 425. -

R. f. $35 \%$.

leucosperma Fr. - [S. 113.] - Dsm. 159.

- II1. 169 ; Cent. X. Suppl. - Fuck. s. 422.

- brunneola. - Voges. J055.

macilenta Rob. - Dsm. 149s (ef. I)iscellis

Bk.). - W'std. 629.

melanospora Fr. - [S. 413.] - Fuck.s. 126.

microsperma. - Bon. t. 2. f. 61 .

microspora. - Kil. II. 166 .

Micula. - ef. Sphaeria.

Mougeótii. - Voges. 12:2.

nivea Fr. - Fuck. s. 431.

ocellata Fr. - Fuck. s. 432 .

- Oleae. - Act. Tur. 1519. X. Dec. ว. f. 8.

Notar.).

- orbicularis, - Ann. Mg. 11. 1. 1\$35. I. t. 7.

f. 6. (Berk.).

Oxyacanthae. - Kl. II. 75 1.

Persicae. - Carol. I. 1 l.

Peylii. - Lotos 1957. VII. t. 1. f. 6.

- Pinastri Fr. - [S. 413.] - Dsm. 1497. -

lil. 1S69. - Lib. 266. - Wstd. 521. (v. Taxi).

- Pini Fr. - Fuck. s. 433. - Dsm. 1500.

pinicola. - Wstd. 933.

pisiformis. - [S. 413. .]

- Platani Fr. - Fuck. s. 434.

- populina P. - Fuck. s. 129.

pulveracea. - [S. $114^{*}$.]

Pyri. - Fuck. Nass. f. 19. - Fuck. s. 435.

rhoina. - [S. 414.]

- Rosae Fr. - Kl. I1161. - Fuck. s. 421.
C y t is pora Rosarum Grev. - Loud. f. 16163.-I3ail S. t. 14.

- rubescens Fr. - [S. 413.] - Bisch. f. 3565 .

- Iil. 9S4. - Kl. II. 435. - Voges. 1084.

- Salicis Rbh. - K1. 763. - Fuck. s. 420. -

Kil. II. 439 .

- Scheidweileri, — Wstd. 1131.

_- sphaerosperma. - Wstd. 1250.! 1130 .

- Ulmi (Synariza) Awd. - Kl. 1061.

xanthosperma Fr. - Kl. 953.

( $)$ t t aria. - [S. 39) 5.1

Berteroi, - I,inn. Trans. XIX. t. 4. I3. 13erk.).

- Darwinii. - Linn. Trans. XIX. t. 4. p. 12.

Berk. !

- Gumnii. - 13erk. In. p. 259. f. 67. a.

- Ilookeri. - Crypt. Bot. antarct. voy. Ereb.

1551. t. 162.1 .

I) ac rin a. - [S. 466.$]$

- atra. - [S. 466.]

- fusco-virens. - S. 166 .

-_ lutescens. - Bon. t. 5. f. 122

- minuta. - S. J66.

I) acrobolus. - S. 404.]

Dacryomyees Dacrymyces.- [S. 342. 4711.]

cf. Tremella. - Jisch. f. :H 49. - Ann. se, n.

1 $3: 3$. VIII. t. \&. f. 16. Fructif. Lév.).

- acuum Iasch. - Kl. 571.

- - caesius S. - [S. $359,7.171$.$] - Act. 'l'ur.$

is 11. III. Dec. 2. f. 1. Notar.).

- contortus. - Iil. 1954.

_ - deliquescens Dub. - [S. 359*.] - Aun se.

11. 15.53. XIX. t. 12. 13. (T'ul.) - I Berk. In.

p. 350 f. 7 . c. (Keimg.) - Bot. Zt. IS59. t. 11 .

f. 6. (lieimg. Hoffm.). - Wstd. 139.

- Euphorbiae Iasch. - Kl. 1075.

- hyalinus. - Lib. 333.

- lacrymalis, - ('d. Anl. t. G. f. 65: 21-26.

- Vistd. $6<6$.

- Isythri D. - Dsin. 1015.

- moriformis Fr. - Loud. f. 16300. - Carol.

I. $>1$.

- Poae. - Lib. 135.

- roseus. - S. $359 *$.

- rubro-fuscus. - Ilook. Lond. J. 1S15. IV.

t. 1. f. 1 .

- stillatus Ns. - [S. 359. 171.] - F1. dan. XI.

1457.,2. - Berk. Out. t. 1 . f. 8. - Loud. f.

16301. - Sturm h. 31-. t. 10 (Szl.). - Bon.

t. 12. f. 242 . Bail S. t. 22. - Kl. 345. -

Kil. II. 276. - Verh. zool. bot. Ges. Wien. IS60.

X. t. p. S10. (Schulzer). - Dsm. 1545. -

Voges. 1191. - Brit. f. 161. - Carol. IV. 81. -

Schwabe, Fl. anhalt. II. t. 6. f. 3. - Wstd. 1 bै

- - v. lutescens: Wstd. 656 .

- Syringae. - [S. 171.] - Fl. dan. XI. I\$57.3.

- tortus. - [S. $359^{*}$, no. 6. 471.] - Ital. 598.

Urticae. - [S. 359, 9.4\% 4.] - Dsm. 402.

- K1. 148. - Fuck. s. 249.

- Fr. : Fusarium tremell. Gr. - Kl. II.

7:. - Ann. Mg. n. h. 1835. I. t. 7.f. 3. (Berk.).

- ef. Calloria fusarioid. - Lib. 31. - Wstd. 155.

- violaceus. - [S. 471.] - Ann. se. n. 185:3.

XIX. t. 12. f. 3. (Tul.).

- virescens Fr. - [S. 471.] - Fl. dan. XI. $155 \% 1$.

Dactylium. - [S. 491.504.]

- candidum Ns. - [S. 491.] - Bisch. f. 3517.

- Bon. t. 6. f. 139. - Letell. 655. 1. 
D a c ty 1 i u m dendroides. - [S. 191.] - Bisch. f. 3737 .

- fumosum. - [S. 504.] - Cd. Anl. t. C. f. 22: 3. 4. - Pay. b. cr. p. 69. f. $307 !$ - ef. Helminthospor. fum. Curr.

- macrosporum. - [S. 491.] - Letell. 655. 2. (varium).

- nigrum. - [S. 491.

- obovatum. - Ann. Mg. n. h. 1641. VI: t. 14.

f. 26. Berk. .

olivaceum. - Kl. $197 \varsigma$.

oogenum. - Robin v. par. t. 2. f. 6. - ? Mycelium in ovis: Hoffm. d. Pflzfamil. 1516. t. 1 . f. 2. p. $11^{*}$. - Verh. ph. med. Ges. Würzb. 1 5 0. t. 1. p. 73. S'Schenk).

penicillatum. - [S. 504. pyriferum. - S. 491 .

sphaerocephalum. - S. 491.] - Ann. Mg.

n. h. 1941. VI. t. 14: 27. Berk. .

tenellum. - [S. 491.]

tenuissimum. - Journ. hortic. soc. 1S46. I.

t. 4. f. 20. 21. p. 34. (Berk.).

- ternatum Cd. - Fuck. s. 156.

varium. - [S. 491.] - Letell, 655. 2. Cladobotr. var. Ns.).

I) a c dalea. - [S. 324.] cf. Polyporus, Trametes, Lenzites.

- - abietina Fr. - Kl. 114. - Ann. sc, n. $1 \$ 37$.

VIII. t. S. f. S. (Fruct. Lév. .

albida Schwz. = D. discolor Fr. - Purton.

I. t. $3 \mathrm{~S}$

asserculorum. - Voges. 191.

aurea. - Carol. III. 14.

betulina P. - Huss. I. 25. - Loud. f. 16063.

(Lenzit.) Kl. 27. - Wstd. 145.

biennis $\mathrm{Fr}$ - - Ioud. f. 16062. - Kl. 720 .

Nov: Act. 1S12. XIX. II. t. 57. f. Si-91. (Spor.

Phöb.).

- Bulliardi. - [S. 329. 19.$]$

cinerea Fr. - [S. 324.] - Kl. 113. - Kil.

II. 511 . Wstd. 1286 .

confragosa. - [S. 324.]

- erubescens. - Ann. Mg. n. h. 1840. IV. t. 9.

Berk.).

- ferruginea. - S. 324.] - Fl. dan. XII. 2029. gibbosa P. - Fl. dan. XI. 1964. - Loud.

f. 16066. - (Tram.) Kl. 29. - Purton. I. t. 14.

- Huss. II. 1.

- indica Jungh. = repanda $P$.

latissima. - [S. 324.]

mollis. - S. 324 .

- Pini Fr. Tramet. - Kl. 112.

- pruinosa Lév. - Bonite t. 136. f. 1. quercina P. - [S. 324.] - Kops Fl. bat. X.

†S9. - Berk. Out. t. 19. f. 5. - Loud. f. 16061 .

- Cd. Anl. t. H. f. $76:$ 1. 2. - Bisch. f. 3329.

Fuck. s. 962. - Bail S. t. 31. - Ki. 26. - ?cf.

Fibrillaria subterr, - Vent. mi, 19. f. 1.2. -

Ital. 142.

- repanda P. - Sagra, Cuba t. 14. f. 4.

sepiaria. - Cd. Ic. V. t. 10. f.90. (Fruct.).-

(d. Anl. t. H. f. $76: 3-6 .-$ (Lenzit.) Kl. 111.

- sepium. - Carol. I. 21.

- serpens. - [S. 324*.]

- suaveolens. ef. Polvpor., Huss.

- trabea. - Cd. Ic. V. t. 10. f. S9. Fruct.'

- unicolor. - [S. 324.] - Fl. dan. XIII. 2271.

1. - Fuck. s. 963. - Kl. 2S. - Kl. II. 116:

quercina. - Kl. II. 614. - Wstd. 12S5. -

Ital. 333, - Schweiz. 22.

- variegata. - Wstd. $9 \%$.

Dal din i a ef. Ifypoxylon.
Damnosporium. - [S. 474.]

Bulliardi. - Cd. Anl. t. G. f. $69: 7-10$.

I) apsilosporium. - [S. 521**.]

stromaticum. - Sturm h. 13. t. 38

$\mathrm{D}$ a rl u ca vagans. - Castagne Marseill. t. \&.

Delastreia. - [S. 43S.] (Delastria).

rosea Tul. - Cd. Ic. VI. t. 20. f. 145.-

'Tul. F. hyp. t. S. f. 1 ; t. 16. f. 1 .

Dem atium. - [S. 499.521.521.]

- album. - Letell. 637. 3 .

Aluta Lk. = Byssus Al. DC.

articulatum P. - Loud. f. 16557 .

atrum. - [S. 499.

aureum Reb. - Wstd. 699

cinnabarinum. - [S. 492 .

coelosporium. - [\$. 501.]

conicum Schum. = Cladospor. herb. Fl, dan.

decorticans. - [S, 499.]

echinobotryum Fr. - Bisch, f. 3154.

epiphyllum. - Letell. 637. 2. - Wstd. 295.

Erysiphe. - Wstd. 413.

fulvum. - [S. 492.]

_- fusco-virens. - [S. 466.]

_ fuscum Schum. = Myxotrich. fusc. Fl. dan. giganteum. - Wstd. 100.

graminum. - [S. 499.] - Lib. 284.

griseum P. - [S. 499.] - Bisch. f. 3720.

herbarum $\mathbf{P} .=$ Cladospor. herb. Fl. dan. -

Voges. 299

- hispidulum. - [S. 499.]

- lineatum. - [S. 499.]

muscorum S-1. - Kl. SS4.

olivaceum Schum. = Myxotrich. chart. Fl.

dan.

petraeum P. - Wstd. 699.

ramorum. - Carol. I. S7. (Glenospora ram.). sparsum. - [S. 499.]

- trichostylum. - [S. 499.]

virescens. - [S. 499.] - Letell. 637. 4.

vulgare P. cf. Cladospor. herbar. W' Wtd:

1394.

De ndrina. - [S, 49S.

— - Diospyri. - Carol. III. 84.

flava. - [S. 49S.]

pulla. - [S. $424^{*}$. 495.]

Dendrodochium aurantiacum. - Bon. t. 11.

f. 225 .

- Havum. - Bon. t. 11. f, 229.

Dendrosarcos Oleae Paul. = Agaric. olearius.

- phosphorus Paul. = Agar. olear.

Dendry i u m. - [S. 504.] ef. Brachycladium, Dactylium.

- atrum. - Cd. Anl. t. C. f. $22:$ 1. 2. - Pay.

b. cr. p. 69 . f. 306 ! coinosum W1lr. - [S. 501.] - R.f. 82. -

Fuck. rh. 57

- curtum. - Ann. Mg. n. h. $155 \mathrm{t}$. VII. t. 6.

f. 9. p. 176 . B. B.).

fasciculare. - Carol. III. $\$ 6$.

- fumosum. - [S. 504.]

griseum. - Ann. Mg. n. h. 1S51. VII. t. 6.

f. 11. p. 17\%. (B. B.) - R. f. 83 .

- laxum. - Ann. Mg. n. h. 1851. VII. t. 6.

f. 10 . p. 176. B. B.).

—- penicillatum Fr. - ef. Brachyclad. pen. Cd. - Resinae Cd. - Cd. Ic. VI. f. 29.

1) e paz e a.-cf. Phyllosticta, Ascochyta, Sphaeria. acericola Dub. - Dsm.17 (Didymospor. Ac.). Aceris D. - Dsm. $17 \%$.

aesculicola Fr. - [S. 423.] - Kl. 563. -

Fuck. s. 3\2. - cf. Ascochyta Aesculi. - Wstd.

1351 . 


Depa zea Agrimoniae. - Kl. 1356. alpina. - [S. 423.]

- Aquilegiae. - Kl. 1651.

areolata F. - Fuck, s. 393. - Fuck. rh. 436. Asperulae. - Kl. 1567.

- Atriplicis Lsch. - Fuck. s. 369.- - Fuck.

rh. 442.

- Bidentis Lsch. - Kl. $\$ 71$.

bifrons v. Aegopodii. - Wstd. 95.

— v. Cytisi. - Wstd. 124.

— v. chelidonicola. - Wstd. 425.

brassicaecola Fr. - Kl. 1142. - Wstd. 294.

Bupleuri. - Fuck. rh. 446.

- buxicola. - Voges, 974. - Kl. II, 265. -

Fuck. s.370. - Dothid. depazd. Dsm. - Fuck. rh. 429 .

Callae Lsch. - Kl. 365.- - Spilosphaer.

K1. II. 554 .

calthaecola. - Kl. 1650.

castaneaecola DC: - Fuck. s. 37 .

Clematidis. - Kl. 1647.

concava. - Ann. Mg. n. h. 1541. VI. t. 11.

f. 3. (Bk.).

convolvulicola DC. - Fuck. s. 36 s.

cornicola DC. - Wstd. 29. - Kl. 367.

cruenta. - Voges. 976. - Wstd. 649. -

cf. Phyllosticta cr.

- Dianthi. - Kl. 434. (cf. Sphaeria). - Fuck.

s. 365 . - R. f. 360. - Wstd. 293. 646. -

liuck. rh. 140.

- - . Saponariae. - Dsin. 290. (cf. Spi-

losphaeria Sap.).

fagicola Fr. - Fuck. s. 376.

ficariaecola Lsch. - Kl. 570. - Fuck. rh. 448.

- fragariaecola WIlr. - Kl. S69. - Kl. II. 152.

- Fuck. s. 364. - Krypt. Bad. 148.

- frondicola. - [S. 423.] - cf. Leptothyrium

Tremulae. - Wstd. 1354.

- geicola Fr. - Kl. 564 . - Fuck. rh. 449.

- gentianaecola v. Convolvuli, - Wstd. 295.

grossulariaecola Fr. - Kl. 1074.

hederaecola. - K1. II. 151. - Fuck. s. 350 .

- Septoria h. Dsm. - Wstd. 25. 1353. -

Schweiz. 11. - Krypt. Bad. 553. 554 (Ampelops.).

- hepaticaecola. - Kil. 165. - (Spilosph. 1i.)

KI. II. 555 .

Hieracii Lsch. - Kil. S72.

Hydrocotyles Rbh. - Kl. 1143.

ilicicola. - Voges. 975 .

Impatientis. - Fuck. rlı. 445.

- increscens. - Heer. Fl. tert. helv. t. 1. f. 7.

- juglandicola Fr: - Wsta. 37. - R.f. 153.-

cf. Leptothyrium Juglandis.

- juglandina. - Kl. 1649. - Fuck. s. 375. -

Fuck. rh. 432 .

- $f$, acerina Awd. - Kl. 9S7.

kalmicola. - Carol. III. 71 .

lichenoides v hederaecola. - Wstd. 25. 1353.

ligustrina F. - Fuck. s. 374.

Linnaeae Ehrb. - Kl. 363. - Kl. II. 149.

Lychnidis Fr.

- f. Saponariae. - Kl. 7555.

lyciícola Lsch. - Kl. 1252.

Lysimachiae Rbh. - Kl.9S9.

Majanthemi Rbh. - Kl. 752. - Kl. II. 51.

Mappa Berk. - Hook. J. 155̃1. III. t. 1. f. 3.

Meliloti Lasch. - Kl. 370.

myrticola Rbh. - Kl. 1146.

Nerii Rbh. - Kl. 1145, - Kl. II. 743.

nicotianaecola Rbh. - Kl. 1141.

Oenotherae Lsch. - Kl. 369. - Fuck. rh. 447.
Depazea Pallor. - Ann. Mg. n. h. 1541. VI.

t. 11. f. 2. (Berk.).

__ palustris. - Kl. II. 266.

_ paridicola Rbh. - Kl. $56 \mathrm{~s}$.

—- Petroselini. - Wstd. 135:

- phillyreaecola. - Kl. 1646.

- picta Ileer. - Palaeont. VIII. t. S. f. S. - (K.

Ludwig).

— polygonicola Lasch. - Kl. 566.

_- populicola. - [S. 423.]

- populina F. - Fuck. s. 372. - Fuck. rh. 130.

- punctiformis. - [S. 423.]

- purpurascens. - Kl. II. 557. - v. Fragariae:

Wsț. 175; Corni : Wstd..29.

- v. Scrophul. = Septoria Scroph. Wstd.

- Wstd. 13 s.

- Pyri. - Hdw. I. t. 3. f. 2. p. - Kil. 673; -

1755. - Kl. II. 451. - Fuck. s. 379.

— pyricola D. - Wstd. 130. - Dsm. 175.

—_ pyrina. - Fuck. rh. 431.

- Pyrolae Ehrb. - [S. 423.] - K1. 1140. -

Fuck. s. 371.

_- quercicola Wlir, - Fuck. s. 377: - Fuck.

rh. 433 .

— rhamnicola Lsch. - Kl.567. - Fuck. rh. 439).

- ribicola. - K1. 1652. - Ann. Mg. n. h.

1s.11. VI. t. 11. f. 1. (Bk.). - Fuck. s. 3\$1. -

Brit. f. 316. - Fuck. rh. 435

- Jobertiani Chev. - Wstd. 537.

— Rubi. - Wstd. 93s.

- Sagittariae Rbh. - Kl. 751.

- salicicola Fr. - S. 423.] - Kl. 366. - Kl. II.

566. - Septoria Capreae Wstd. - Fuck. rh.

435.

- Saponariae. - Kl.. 1653.

- Scabiosae. - ? Wstd. 30.

— scabiosaecola D. - Dsm. 179. - Wstd. 30.

—- Senecionis Fr. - Fuck. s. 367.

— smilacicola. - Kl. 1144. - Carol. V. iT.

- sorbicola. - R. f. 54 s.

— speirea Cd. - [S. 479.] - Kl. 1051. - Micro-

sticta vagans Dsm. - Perisporium sp.

- Spinaciae Fr. - Kl.754. - Fuck. s. 366. -

Fuck. rh. 441.

- stachydicola Lsch. $\rightarrow \mathrm{Kl}, 565$.

— stemmatea Fr. - Kl. 433. - Kl. II. 742.

— syringaecola Lsch. - Kl. 569. - Fuck. s.

355. - Kkl. II. 452. - Fuck. rh. 437.

- tremulaecola DC. - Kl.365. - Fuck. s. 373.

- K1. II. 52. - Fuck. rh. 431.

- Trientalis Lsch. - Kl. 364.

- Vaccinii. - [S. 422.] - Kl. II. 150.

— vagans Fr. - [S. 423.] - Kl. 754 u. 1566.

_- f. Armoraciae. - Kl. 567 . - Kl. II. 453.

- - f. Atriplicis. - Wstd. 960.

- f. Brassicae. - Wstd. 294.

- f..Dianthi. - ? Wstd. 293.

- f. glechomaticola. Kl. K70.

rh. 443 . f. Lamii, - Fuck. s. 354. a. - Fuck.

- f. scutellariaecola: - Kl. 1057.

rh. 444 . f. Urticae. - Fuck. s. 384 . b. - Fuck.

$-\frac{\text { f. Tineae }}{-}-$ Wstd. 31.

- Vincetoxici Schub. - Wstd. 1146.

Dermatea. - [S. 362.] cf. Peziza, Cenangium,

Tubercularia.

- carpinea. - [S. 362.] - Ann. sć.n. 1553. XX. t. 16. f. 17. 15. (Tul.) - - Tubercular. fascic. Td. - K1. II. 518. - Fuck. s. 775. - Wstd.

1274: 
233. - Krypt. Bad. 435. - (Cenang.) Ǐl. 1539. - Fuck. s. 773 .

Dermatea fascicularis. - [S. 362.] - Iil. II. 620. (cf. Peziza).

— fissa. - S. 362.]

_- furfuracea.- - [S. 362.] - Kl. II. 621. - Ital. 143.

- Padi. - S. 362.] - Kl. II. 711.

- Prunastri Fr. - S. 362.] - R. f. 125. - Cenang.) Fuck. s. $7 \div 2$.

- - - purpurea. - [S. 362.]

- rubiginosa. - [S. 362 .

- tegularis. - S. 362 †.

- tiliacea. - [S. 362 .

- tumida. - S. 362 .

-..- Urceolus. - [S. 362.]

Dermea. - Bail S. t. 19.

Dermosporium. - [S. 476.]

Desmazierella acicola. - Lib. 24. - Ann. sc. n. 1529. XVII. t. 6. B. (Libert).

- granulata. - Wstd. 50 .

Desmotrichum simplex. - Ann. sc. n. 1843. XIX. t. i. f. S. (Lév.).

Diachea. - S. 455.]

— elegans. - [S. 455.] - Cd.Ic. V.t. 3.f. 3S. R. f. 434 - Cd. Anl. t. C. f. $32: 7-11$. Pay. b. cr. p. 122, f. 5\$2. - Kl. 1229. - Fuck, s. 251. - Il. II. 36. - Dsm. III. 261. - Carul. I. 80 (leucostyla Schw.). - W'std. 1260.

_- fulgens. - [S. 455.]

- leucostrla, - cf. elegans.

Di a mphora. - [S. 4SS.

- bicolor. - Cd. Anl. t. C. f. 24: 17-19. Bon. t. 10. f, 211.

- Martii. - Bisch. f. 3739.

Di a ph anium. - [S. 450 .

Diatrye. - [S. 394.] cf. Sphaeria.

- aneirina. - [S. 3\$5.]

— aristata. - ${ }^{\text {S S. 3S5.] }}$

aspera. - [S. 355 .

bispora. - Carol. IV. 45.

- bullata E., Fr. - [S. 355.] - Kl. II. 4\%. -

Fuck. s. 654. - R. f. 536.

Callicarpae. - Carol. IV. 41.

capnostorna. - Carol. IV. 42.

ceratosperma. - [S. 3S5.

conspersa.

- - v. Juniperi. - [S. 355.] corniculata. - Carol. IV. 43.

decipiens. - [S. 3S5.

decorticata. - [S. 385.]

disciformis Fr. - S. $395,419^{* *}$.] - R. f. 137 .

- Fuck. s. 6\$1, a. - Krypt. Bad. 430. - Ital. 310.

euphorea. - [S. 355.

favacea. - [S. 3S5̃.]-Kîl. 1558. - Fuck. s.650. ferruginea Fr. - S. 3\$5.] - Fuck. s. 672. -

Wstd. 915.

flavovirens H. Fr. - [S. 355.] - Kl. II. 4S. -

Fuck. s. 679, a.

gastrina. - Carol. V. 59.

Grandinia. - Carol. IV. 40.

haustellata. - Carol. III. 53.

hypophloea. - Carol. IV. 3S.

Hystrix, - [S, 3S5.]

— insitiva. - [S. 355.] - Kl. II. 147.

- lanciformis Fr. - [S. 3S5.] - Kl. 1323. - R.

f. 248. - (Sphaer.) K1. 155s, a.

- lata. - [S.355.] - K1. 1565. - Fuck.s.675. leioplaca. - [S. 355.]

lenta. - [S. 354.]

ligniota. - [S. 3S5.]
Di a trype microplaca. - Carol. IV. 39.

- milliaria. - [S. 355.]

__multiceps. - [S. 355.]

obesa. - Carol. IV. 47.

occultata. - [S. 355.]

parallela. - [S. 355.]

podoides. - [S. 355.]

prorumpens. - [S. 355.$]$

- pyrrhocystis /versatiles). - Ann. Mg. n. h.

1 .59. III. t. 9. f, 10. (B. B.). - R. f. 136.

quadricocea, - [S. 355.$]$

quercina Fr. - [S. 355.] - Fuck. s. 67\%. -

R. f. 319 .

rhytistoma. - [S. 355.]

scabrosa. - [S, 355.

- Stigma Hffm, - [S. 3S5.] - Kl. 49. - Fuck, s.

liv2. - Kl. II. 529. (Stromatosph. S. Gr.) Ital. 591 .

- strumella Fr. - [S. 355.] - Kl. II. 49. -

Fuck. s. 676.

- subcutan. - [S. 355.$]$

- subferruginea. - Carol. IV. 44.

- undulata (lignosae). - [S. 395.] - Ann. Mg. n.

h. 1559. III. t. 9. f. 9. (B. B.) - Fuck. s. (is.3. velutina. - Fuck. s. 674.

verrucaeformis. - [S. 355.] - K1. II. 725. -

Fuck. s. 671. - R. f. 135. - Carol. IV. 46.

— verrucella. - [S. 355 .

_- versatilis. - [S. 3S5.]

— virescens. - Carol. IV. 45.

vitellina. - Chile n. 10. f. 4 .

Dia trypella. - cf. Sphaeria.

Dica e om a. - [S. 514.]

- Betonicae Ns. - Wstd. 690.

- betulinum. - Bon. t. 2. f. 54 .

Di chaena. - cf. Hysterium.

924

- fissa. - [S. 402.] - Kl. II. 724 .

- quercina. - [S. 102.] - Kil. II. 449.

rugosa. - [S. 402.] - Kil. 1472. - Kil. II.

4.) 1 - - cf. Psilospora faginea.

- Sambuci. - [S. 403.]

_- strobilina Fr. - [S. 403.] - Kl. 544 (Sphaeria).

- strumosa. - Carol. II. 67.

sulcata. - [S. 403.

Dichla en a Lentisci. - Alger. t. 22 bis, 2.

Dichonema aeruginosum. - Nov. Act. 1526.

XIII. I. t. 2: 3 (B1. Ns.).

erectum. - Hook. Lond. J. 1842. I. t. 7.

sericeum. - [S. $333 * *$.]

Dichospoxium. - [S. 452. 460 .

aggregatum Ns. - Loud. f. 16542.

album, - [S. 452.] - Cd. Ic. V. t. 3. f. 31. -

Cd. Anl. t. C. f. f. $27: 7-9$.

Dicladium graminicolum. - Kl. 1677.

Dicoceum. - [S. 4S4.]

dryophilum Cd. - Stigmella dryina Lév. -

R. f. 351 . - Ital. 298.

- minutissimum. - Cd. Anl. t. B. f. 4: 1-3.

- Sturm h. 9. t. 55 .

- Rosae. - Bot. Zt. 1S53. t. 7. f. 2 (Bon.).

Dic ty dium. - [S. 456.$]$

- ceruuum Ns. - Loud. f. 16522

—_ didermoides Fr. - Fl. dan. XII. 2085. 2.

microcarpum. - [S. 456.]

micropus. - [S. 456.]

splendens. - [S. 456.]

trichoides. - Kl. 1820 .

- umbilicatum. - [S. 456.] - Cd. Ic. V. t. 3.

f. 36. - Cd. Anl. t. C. f. 31: 5-11. - Bisch.

f. 36 \%2. - Krypt. Bad. 422. - Pay. b. cr. p. 


122. f. 573. - Willkomm, Wund. d. Mikrosk. IS56. \%. 92. f. 47. - Kl. \$20. - Carol. II. 7S. - Auersw. Bot. Unterhlt. 1563. S. 352.

D ict $\mathrm{y}^{-\mathrm{d}}$ i u menosum. - [S. 456.$]$

Dictyopeplos. - ef. Hymenophallus Cd.

Dictyophallus. -- [S. $434^{*}$.]

- aurantiacus Cd. - Cd. Ic. VI. f. 45. - Cd.

Anl. t. E. f. $51: 9-11$.

Dictyophora. - [S. 434*] cf. Hymenophallus $\mathrm{Cd}$.

- speciosa. - Nov. Act. XIX. Suppl. I. t. 6.

(lilotisch).

Dictyosporium. - [S. 506 *.523.]

- elegans. - Cd. Anl. t. B. f. 4: 7-9. - Pay.

b. cr. p. 61 . f. 255 .

Diderma. - [S. 450.]

__ acuminatum Schum. - Fl. dan. XII. 2091. 3.

atro-virens. - [S. 450 .

chalybeum. - [S. 450.]

citrinum. - [S. 450.]

conglomeratum. - [S. 451.]

contextum P. - [S. 450.] - Bisch. f. 3624.

- Sturm h. 3. t. 39 .

- crassipes Schum. = umbilicatum Fl. dan.

- eyanescens. - [S. 450.]

deplanatum. - [S. 450.]

depressum. - [S. 450.]

- difforme. - [S. 450.] ef. liceoides.

flavum. - [S. 450.]
globosum. - [S. 450.] -. Cd. Anl. t. C. f. $25:$

15-20. - Bisch. f. 3629 . - Loud. f. 16.530 . -

Sturm h. 1. t. 6 .

- granulatum Fr. - [S. 451.] - Fl. dan. XI.

1979. 2.

- hemisphaericum Fr. - Fl. dan. XI. 1972. 2.

- lepidotum. - [S. 150.] - Bisch. f. 3679. -

lay. b. cr. p. 122 . f. $57 \%$.

- Libertianum. - Fres. t. 4. f. 16-23.

- liceoides. - [S. 450.] - Lib. 276. (difforme).

- lucidum. - Ann. Mg. n. h. 1861. VII. t. 15.

f. 9. p. 350 . (B. B.).

- minutum. - [S. 451.] - Fl. dan. XI. 1979.1.

- Neesii. - [S. 454.]

nitens. - [S. 450 .

- oblongum Schum. - Fl. dan. XI. 1973. 1.

- ramosum. - [S. 450.] - F1. dan. XI. 1973.4.

- spumarioides Fr. - [S. 451.] - Fl. dan. XI.

1975.2. (ef. Carcerina).

_- : spurium Schum. (Didym \%). - Fl. dan. XII.

2015.2.

_ stellare P. - [S. 450.] - Bisch, f. 3615 .

- testaceum P. - [S. 450.] - Fuck. s. 296.

- Trevelyani. - [S. 450.]

_- umbilicatum P. - [S. 450.] - F1. dan. XI.

1972.1.

- vaccinum. - Alger. t. 22, bis. 1.

- valvatum. - [S. 451.] - Lib. 376.

vernicosum P. - [S. 450.] - Kil. 175. -

Wstd. 1063. (cf. Leocarp. v.). - Lib. 76.

Did y m ari a. - [S. 493. 3.]

— Helvellae Cd. - Cd. Ic. VI. f. 22.

- Ungeri. - Cd. Anl. t. B. f. 9: 1. - Pay. b.

cr. p. 73. f. 326 .

\begin{tabular}{l} 
Didyin i u m. - [S. 451.] ef. Physarum. \\
\hline albo-punctatum Fr. - Fl. dan. XII. 2092. 3.
\end{tabular}

- album. - Letell. 710.4.

- cinereum. - [S. 452.] - Dsm. 1120. - Carol.

I. 79 ; V. 83. - Letell. 710. 2. - Wstd. 382.

- complanatum. - [S. 452.]

- conglobatum. - Letell. 710. 3.

costatum. - [S. 452.] - Kl. 1547.

crustaceum. - [S. 452.$]$

$\mathrm{H}$ of $\mathrm{fm}$ an $\mathrm{n}$, Index Fungorum.
Did y m i u m effusum. - [S. 452.]

- farinaceum. - [S. 452.] - Fl. dan. XII. 2094.

1. - Fuck. s. 291. - K1. 423 (non 1349). -

R. f. 369 (Physar. P.) - K1. II. 135. - Krypt.

Bad. 424. - Ztschr. f. wiss. Zool. X. 1559. t. 6.

f. 11. (de B.). - Voges. 1457.

- fulvipes. - [S. 452.]

- furfuraceum Fr. - [S. 452.] - Fl. dan. XII.

$2092,2$.

- globosum. - Schnzl. t. 14. f. 23 ? $21-26$.

- hemisphaericum. - [S. 452.]

- herbarum Fr. - [S. 152.] - K1. S1s.

- leucopus Fr. - [S. 452 ; cf, ib. 22.] - Fuck.

s. 292. - Zeitschr. f. wiss. Kool. X. 1 559 . t. ti.

f. 10 . (de B.)

- liquidum. - Pay. b. cr. p. 122. f. 576.

- lobatum. - [S. 452.] - Bisch. f. 3654. -

Fl. dan. XII. 2094. 2.

- marginatum. - [S. 452.] - Fl. dan. XII.

2092. 1 .

—_ melanopus. - [S. 452.]

Michelii. - Jib. 1 so.

— muscicola. - Kil. 1545.

- nanum. - [S. 452.]

- nigripes Fr. - [S. 452.] - Bisch. f. 3612.

- Ztschr. f. wiss. Zool. X. 1559. t. 6. f. $4-9$.

(de B.)

- physaroides Fr. - [S. 452.] - Kl. 176. -

Letell. 711.1 .

- praecox de B. - R. f. $36 \overline{\text {. }}$.

_- reticulatum Fr. - Bisch. f. 3621.

- rufipes. - [S. 152.]

- Serpula Fr. - [S. 452.] - Ztschr. f. wiss.

Zool. X. 15.59. t. 6. f. 12-17. (de Bary).

- Sowerbyi. - [S. 452.]

- spumariodes. - [S. 452.]

— squamulosum. - [S. 452.] - Bisch. f. 3676.

- IIl. II. 455.

— terrestre. - [S. 452.]

- tigrinum. - [S. 452.]

_ versipelle. - [S. 452.

Weinmanni. - [S. 452.]

- xanthopus. - [S. 452.] - Bisch. f. 3692, -

Iil. 737 (non 1350). - cf. Physar. x.

Did y mocrater. - ef. Crateromyc. dupl.

- elegans. - Cd. Anl. t. C. f. 23: 10. 11. -

Bisch. f. 3796 .

Didy mosporium. - [S. 50S. 4S8.]

- Aceris Mt. - Dsm. 17\%. - Cheilaria Aceris

Wstd. - Depazea acericola Dsm. - Dep. Ace:

ris Dsm. - Sphaeria acericola Duby.

— betulinum. - Wstd. 132.

— bullatum Fr. - Wstd. 557 .

___ Carpini. - [S. 450.]

— complanatum Ns. - [S. 508.] - Bisch. f. 3839.

- Bon. t. 3. f. 66. - Kl. 5\$3. - Kl. II. 592.

(Stilbospora didyma Lk., fugax Ǩz. S.). - Letell.

704. 2. - Fresen. t. 13. f. $33-37$. p. 105.

- elevatum. - Letell. 704. 1. (Melancon. bet.

S.). - Lib. 391. - Wstd. 132.

exulatum. - Jungh. pr. t. 1. f. 1.

- macrospermum. - Cd. Ic. VI. f. 17. - Ann.

Mg. n. h. 1861 . VII. t. 15. f. 12. p. 381. -

Fuck. rh. S3. - Fresen. t. 13. f. 3S-12. p. 104. pezizoideum. - [S. 475.]

- profusum. - [S. 505.]

- pyriforme. - Kl. 1786. - Hedw. I. t. 3 .

f. 2 . (g. h.).

truncatulum. - Cd. Ic. VI. f. 16.

- truncatum Cd. - Bisch. f. 3540. - Sturm

h. 7. t. 23 . 
Dilophospora. - [S. 419.]

Alopecuri. - S. 419.

graminis. - Wstd. 719. - Dsm, 491. -

Cd. Anl. t. F. f. 5t: 5 9. - Ann. sc. n. 1410.

XIV. t. 1. - R. f. 311. - Pay. b. cr. p. 61. f. $256 .-$ Voges. 1157.

- Molci. - Bot. Zt. 1561 . t. 10. f. 5. p. 250. (Fuck.).

(a D. Alopecuri D. vix diversa).

Dinemasporium. - S. $367 \cdots \cdots$.]

- graminis. - Voges. 1260. - Pay. b. cr. p. Tร. f. 360 .

D iph therium flavo-fuscum.- Reticularia Fr. .

- Cd. Anl. t. C. f. 35: :3. A.

Diplocladium majus. - Bon. t. S. f. 16is.

- minus. - Bon. t. 5. f. 119.

Diploderma. - S. 441.460.]

Diplodia. - [S. 416.] - ef. Sphaeria. - Ann. sc. n. $1>34$. I. t. 13.

accrina. - [S. 416. 1.] - Wstd. 1127.

Aesculi. - [S. 417.]

aquilina. - [S. 41\%.]

- arachnoidea. - Hdw. I. t. 5. f. 1. - Kl.

1545 .

—_ arbuticola. - S. 417.]

- Buxi. - [S. 41\%.]

- chaetomioides. - Hedw. I. t. 5. f. 2. - Kl.

1547.

— elypeata. - [S. 417.] - Fuck. s. 410.

conigena Dsm. - S. H17.] - Macroplodia c.

Wstd. - Wstd. 1230.

- Corchori. - Wstd. 542.

- Crataegi. - Wstd. 1125.

— depazeoides Dur. - Dsm. 1455.

- Dianthi. - Kl. 1943.

- Empetri. - [S. 417.]

— Evonymi. - Wstd. 930.

— faginea. - [S. 417 .

— fibricola. - Hook. J. 1453 . V. t. 3. f. 12.

_- Fraxini. - [S. 417.] - Wstd. 932.

_- Georginae. - [S. 417 .]

- - Hederae. - [S. 417.] - Fuck. s. 406. - Wstd.

1126

- - herbarum. - [S. 417.]

- heteromorpha, - Bull. ac. belg. 2. Ser. II.

1557. p. 579. f. 5 (Wstd.).

Jasmini. - Wstd. 1226.

- ilicicola. - [S. 417.] - Ann. Mg. n. h. 1S41.

VI. t. 11.f. 7. Berk.j.- Dsm. 25\%. - Wstd. 931.

- Ilicis Fr. - [S. 41i.] - Fuck. s. 409.

- Juglandis. - [S. 417.]

- Kerriae. - [S. 417.]

- leguminis. - [S. 417.]

- Lilacis. - Wstd. 724.

- loculata. - Chile n. 9. f. 10.

- macrostoma Lér. - Dsm. $i \downarrow>1$.

- Magnoliae. - Wstd. 1225.

mamillana Fr. Su. 417. non $=$ Sphacria $\mathrm{m}$.

Fr. Sy. (Fuck. Nass. f. 16. p. 72). - Fuck. s. 411.

melaena Lév. - R. f. 34 S.

Mori. - R. f. 544.

mutila Fr. - [S. 416.] - Chromosp.) Dsm.

1450. - Act. Turin. 1845. VII. Dec. 4. f. $i$.

Notar.).

- Oleae. - Fuck. s. 413. - Act. Tnrin. 1545.

VII. Dec. 4. f. 10 (Notar.). $\rightarrow$ Kl. 1056. - Kl.

II. 543. - Ital. 148 (Sphaeria).

- oospora. - Hook. J. 1S53. V. t. 3. f. 11.

- perpusilla. - Dsm. 1494.

- polymorpha. - Act. Turin. 1545. VII. Dec.

4. f. 5 (Notar.).
Diplodia profusa. - Act. Turin. 1545. VII. I)ec. 4. f. s (Notar.).

— pustnlosa. - Voges. 1336. - Wstd. 541. ramulicola. - Dsm. 1479.

- Rosae. - Wstd. 1227.

Rosarum. - [S. 417.]

Rubi. - [s. 417.]

salicina Lév. - [\$. 417 \-Fuck. 8. 412. (cf.

Sphaeria signans Wllr.). - R. f. 450 .

- Salicis. - Bull. ac. belg. 2. Ser. II. 1557.

p. 579. f. 6. (Wstd.).

sarmentorum. - [S. 417.]

- Dsm. - R. f. 347. (Sphaeria). - Dsm

1143.

scabrosa. - Wstd. 1372.

- seriata. - Act. Turin. 1545. VII. Dec. 4. f.

i. (Notar.).

- subtecta. - [S. 41i.]

- 'Tamaricis. - [S. 41\%.]

Taxi. - [S. 41\%] - Act. Turin. 1945. VII.

Dec. 4. f. 9. Notar.). - Fuck. s. 4118.

[-[ tephrostoma. - S. $417 *$ *]

_- uredinaecola Dsm. - Dsm. 1456.

- Visci. - [S. 417.] - Fuck. 8. 40\%. - Wstd.

$1 \geq 31$

— viticola D. - [S. 417.] - Dsm. 259.

- vulgaris. - Voges. 1337.

Zeae. - Carol. 1. 74. (Sphaeria Maydis Bk.).

Diplodin a Salicis. - Wstd. 1229.

D) iplosporium. - [S. 492.]

album. - Bon. t. 5. f. 105.

Morsus Ranae. - [S. $493^{*}$.] - Cd. Ic. V. t. 2. f. 17.

D is ce ll a carbonacea. -- Ann. Mg. n. h. 1550. V.

t. 12.f. 1. d. (B. 13.) - cf. Phacid. u. Stilbospora.

- Desmazierii. - Ann. Mg. n. h. 1550. V.

t. 12. f. 1. a-c. p. 37i. B. B.).

_- microsperma. - Ann. $\mathrm{Mg} . \mathrm{n} . \mathrm{h} .1550 \mathrm{~V}$.

t. 12. f. 1. e. p. 37s. (B. B.).

Discina. - [S. 34 个.]

Disciseda. - [S. 442.]

Discosia. - [S. 123.] - cf. Sphaeria, Dothidea.

- alnea. - [S. 123.] - Kl. II. 154. - Fuck.

s. $362 .-$ R. f. 539 . - Fuck. rh. 452.

- Artocreas. - [S. 423.] - Fres. t. S. f. 1-3.

- Dsm. III. 61-64. (Sphaeria). - Carol.

V. $75 .-$ Fuck. rh. 45 \%

- clypeata. - [S. 423.] - Act. Turin. 1849.

X. p. 354. f. 4. (Notar.). = Sphaer. Artocreas

Tode. - Fuck. s. 363. - Fres, t. S. f. 4-6. -

Kl. 1735. - Fuck. rh. 453.

Cynosbati. - Fuck rh. 455.

elliptica. - Frés, t. 8. f. 9-11.

faginea. - Lib. 345. - Dsm. T47. - Wstd.

2i. - Ital. 592. (laurina).

f. 2. Notar.).

— impressa. - [S. 423.]

- minuta. - Kl. 1961.

_- pleurochaeta. - Alger. t. 27. 10.

- quercicola. - Act. Turin. 1849. X. p. 354.

f. 3. (Notar.).

- smilacina. - Act. Turin. 1549. X. p. 354.

f. 6. Notar.).

strobilina. - Lib. 346. - Fres. t. S. f. 7. S.

vagans. - Act. Turin. 1549. X. p. 354. f. 5.

(Notar.).

Discosphaera. - ef. Sphaeria.

- flarovirens. - Wstd. 113.

Discotrema. - [S. $397^{* * *}$.]

Ditiola. - [S. 363.] 


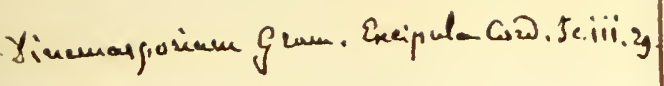

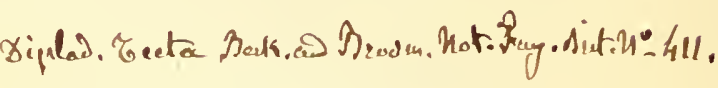

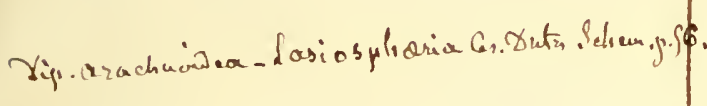

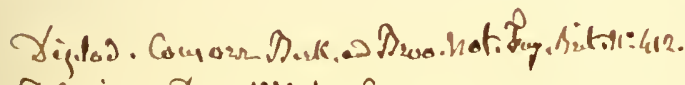

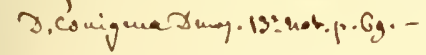

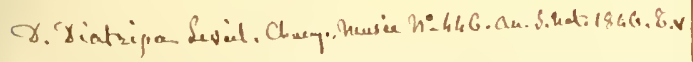

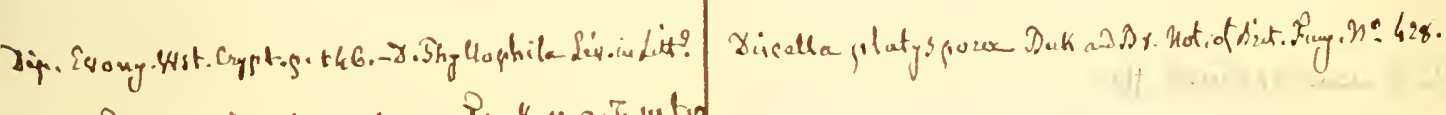

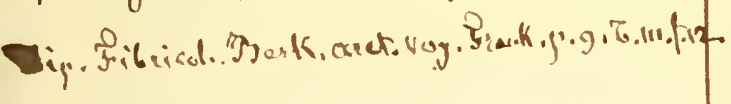

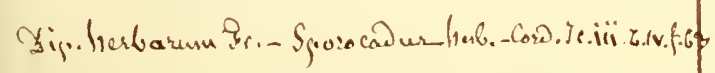

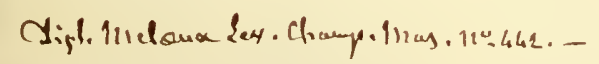

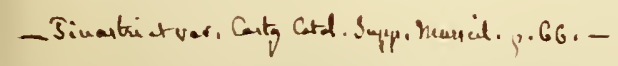



Ditiola fimetaria Awd. = Helotium fimetar. Fr. Su. Fuck. .

- inucida. - Verh. zool. bot. Ges. Wien.

1s60. X. p. S10. c. ic. Schulzer,.

- nuda B. B. ? = Dacryomyc. still. Tul. Bail s. p. 75.). - Ann. Mg. n. h. 18ts. II. t. 9. f.4.

13. 13.)

paradoxa Fr. - Kl. 956. - R. f. 4io.

radiata. - Kl. 1357.

- radicata Fr. - IS. 363.] - Fl. dan. 'XIII.

2:335. 1. - Col. Anl. t. G. f. 64: 41-11. -

Loukl. f. 16252. - Bisch. f. 3375. - Jail s. t. 22 .

- sulcata Fr. - [S. 363.] - Bisch. f. 33\%

volvata Fr. - Bisch. f. 3376 .

I) i topell a cf. Sphaeria.

1) oratom yces. - [s. 467 .

Desmazicri. - [S. 467 .

Neesii. - Sturm h. T. t. 31).

viridis. - ('d. Anl. t. 13. f. 19: 1\&. 19.

1) othidea. - [S. 356, 421. 122.] - ef. I'oly-

stigma, Sphacria, I'iggotia, Asteroma.

- abortiva. - Ism. III. 92. - Voges. 1172. advena Ces. - R. f. 516. - Ital. 195.

Alchemillae. - [S. 422.] - Kl. 191!. -

lib. 66 .

Alismatis Jasch. - Kil. 553. - Kil. II. lli2. alnea. - [S. 123.] - I.oud. f. 16169. -

cf. Ieptothyrium. - Wstd. 261 .

ambiens. - Lib. 366.

Anemones, - ef. sphaeronema.

Anethi. - [S. 357.] - Kil. II. 266.

Angelicae Fr. - [S. 425.] - Kl. 1iłl.

appendiculosa. - Hook. Lond. J. 1 -11. III.

t. 15 .

Arundinis Lév. - Démid. Russ. t. วั. f. 3.

Asteroma. - [S. 425, 6 $\vdots$ ]

Astragali Lasch. - Kl. 3 is.

lerberidis. - S. $3>6$.$] - Let. 'Turin. 1>11$.

III. f. 9. 1)ec. 1. Notar.

Betulae nanae. - $[\mathrm{S}, 3>i$.]

- betulina Fr. - [S. 35i.] - K1.376. 135...

Ii1. II. 656. - Krypt. Bad. 429. - Lib. 171.

(Xyloma DC.).

- Brassicae. - Dsm. HI. 95. - Kil. II. 751.

- Buxi. - Carol. I. 73.

Caricis. - S. 347.]

- Castaguei. - R. f. 545. - Ital. 343.

('haetomium Kz. - [S. 422.] - Stigmatea. -

Dsin. 935. - ef. Thacid. Rubi Wstd.

- circumvaga. - Dsm. III. 55.69.

- colliculosa. - Brit. f. 263. (Aecidii cancel-

lati status imperf.).

- - decolorans. - Dsm. III. 90.

- depazeoides. - Wstd. 916. - Dsm. 291. -

Depazca buxicola. Voges, 974.

deusta. - S. 35\%.]

- Drymidis. - Chile n. 9. f. 5.

- Epilobii Fr. - S. $425 *$.] -- Asteroma Ep.,

Fr. Su. - Toges. 10ss.

- etrusea. - Aet. Turin. 1557. XVI. Dec. 3.

f. 10. (Notar.).

- englypta Mont. - Ann. sc. n. 1540. XIV.

t. 19. f. 8. p. $32 \%$.

filicina. - S. 356.]

fimbriata. - Wstd. 467.

fulva Fr. - Kl. 259.- - Bail S. t. 16. - cf.

Polystigma.

fumago. - [S. 425.

- Galii Lasch. - Kl. 1153. - Mazzantia.

gangraena Fr. - [S. 357.] - R. f. 350.

genistalis Fr. - [S. 356.] - Fuck. s. 665.
Dothide a geographica. - Voges. 1179. (Asteroma). - Carol. I. 67. - Wistd. 265.

_- graminis. - [S. 35\%.] - Wstd. 523.

- Ilaydeni, - Carol. V. 59.

Hederae. - Voges. 561.

IIeraclei. - [S. $35 \%$.] Kil. II. 136. -

Dsm. \37. - Voges. 1057. - Wstd. 915.

- IIimantia. - S. 425.7 - Voges. 951.

- f. Solani. - Kl. 554 .

impatiens Matth. = Puccinia nolitang. Cd.

Impatientis. - Wstd. 1155.

insculpta. - Voges. 133s.

- Iridis, - Dsm. III. 94. - Wstd. 917.

- Juncagincarum Lasch, - Kil. 6i2.

- Junci. - S. 35\%. - Kil. II. 161. - Fuck.

s. lint. Sphaeria,

- Juniperi I). - Dsm. 491.

—— Lathyri Iév. - Démid. Russ. t. ว. f. 5 .

- latitans. - S. 34fi.] - Voges. $117 \mathrm{~s}$

- Lichenum. - S. 356.7

— Lycii I) ub. - iR.f. 55.56.-- Mém. soc.phys.

Genève. 1459. XV. I. p. 193 . (c. ic.).

__ maculacformis. - 1)sm. III. 93.

- melanoplaca. - Dsm. III. 96.

_- Mezerei. - [S. 3๑6.] - Kil. 1951.

millepunctata. - Dsm. 1II. 91. - Wstd. 16 is

— Napelli. - Kil. 1952 a.

- ocliracea. - S. 347.7 - Krypt. Bad. 53.

- Paliuri Lév. - Démid. Russ. t. (i. f. 6.

- pararloxa. - [S. 39צ.] - Mém. soc. phys.

fieneve. 1459 . XV. I. p. 193. (c. ic.'.

- Phlomidis Iév. - Démid. Russ. t. 5. f. 1.

- Pinastri. - S. 36ri.

- Pistaciae Lev. - Iémid, Russ. t. 5. f. 2.

- Podagrariae Fr. - S. 347.] - Fuck. s.670

- Wstd. 722.

- populina. - Wstd. $712 .=$ Splıaeria lamprotheca I)sm.

- Potentillae. - S. 122.] - Kl. S73. - Kl.

1I. 65. - ef. Stigmatea. - Voges. 1339. -

lib. 267.

- Prostii. - Ism. III. 57.

- 1'teridis. - [S. 357.] - Hdw. I. t. 11. f. A :

1-14. - Kl. 54. - Fuck. s. 665. (cf. Poly-

stigma . - Kl. II. 437. - Voges. 949).

— puccinioides. - [S. 356.] - K1. II. 67. -

1.ib. 36.). Sphaeria IC.). - Wstd. 165. -

Ital. (42.) 92 .

- pyrenopbora. - [S. 418.] - cf. Ascospora

pyr. - Brit. f. 252.

- Ranunculi Fr. - K̈l. 1538. - cf. Phacid.,

Lib. 69. - Wstd. 376.

— reticulata Fr. - Bisch. f. 3515. - Kl. 1950.

- Voges. 952. - Wstd. 636. - K. f. 549.

(Combosira, Asteroma). - Sturm h. 6. t. 13.

__ rhopalina. - ef. ropal.

- Ribesia. - [S. 356.] - Act. Turin. 1849.

X. Dec. 5. f. 7. Notar.). - Bisch. f. 3566 . -

Dsm. S35. - Kl. 436. - Kl. II. 655. - Fuck.

s. 664 . - Brit. f. 91 . - Letell. 656. 1. Wistd. 466 .

Ribis. - Schnzl. t. 15. f. 26-2S.

rimosa. - [S. 396.] - Kl. II. 267. - Fuck.

s. 661. - R. f. 349. (depaup.).

Robergei D. - Dsm. 493.

Robertiani Fr. - Dsm. 839. - Kl. 379.

ef. Stigmatea R. - Voges. S62. - Lib. 65.

('ryptosphaeria nitida Grev.). - Wstd. 537. ropalina Mt. - Ann.sc. n. 1540. XIV. t. 19.

f. 9. p. $32 \mathrm{~s}$. 
Doth idea Rosae. - [S. 3\$6. $402 * \cdots]$ - Carol. I. 65, ? 1 std. 116.

rubra. - [S. 3Si.] - Loud. f. 16170. - Polystig.) Kl. 377. - Fuck. s. 669. - Letell. 656. 4. - Wstd. 637.

— saligna. - cf. Sphaer. s. Ehrb.

Kx.: Wstd. 524 .

Sambuci. - [S. 356.] - Kl. 55.5. - Fuck.

s. 663. - Letell. 656. 2.

— - v. moricola. - Ital. $7 \varsigma 1$.

- - v. Hederae. - Act. 'Turin. 1511. III.

Dec. I. f. \&. Notar. - - Ital. T-1.

— Solidaginis v. Virg. — ef. Puecinia Virgaureae Lib. - Wstd. 651.

Stellariae. - Lib. 172.

- stellaris Fr. — Bisch. f. $3516 .-$ Kl. 260.

Voges. 563.

- striaeformis. - [S. 386.$]$

tetraspora. - Ann. Mg. n. h. IS59. III.

t. 11. f. 39. (B. B.).

_ - Trifolii. - [S.357.] - Kl. II. 657. - Fuck. s. 662. - Carol. IV. 50.

- typhina. - [S. 351.] - Bisch. f. 3567. Loud. f. 16466. - Kl. 55 ; Cent. XV., suppl. ef. Polystig. t. - Brit. f. 92. - Wstd. 522. kirypt. Bad. 4 .

- Ulmi. - [S. 357.] - Dsm. 836. - Loud. f. 16467. - Fuck. s. $66 \%$ - Kl. 3s0. Cent. XVI.! - Kl. II. 65 `. — Brit. f. 192. - Letell. 656. 3. - Wstd. 111. - Ital. 445 .

_- Vaccinii. - [S. 3\$6.]

_ valvata. - Bail S. t. 18.

- Veronicae. - Lib. 173., transit in Lasiobotrydem.

— Xylostei. - [S. 425.

— Zollingeri. - Ilook. Lond. J. 1544. III. t. 15.

Dothiora. - [S. 41s.]

Dryophilum. - [S. 484.515.]

D uben ia. - [S. 356.$]$

D u inortiera rostrata. - Bull. ac. belg. 1957. II. p. 579. f. 14. (Wstd.). - Wstd. 1251.

Fichyna. - [S. $146^{* * *}$.]

Echinobotrym. - [S. 523.]

- atrum. - Cd. Anl. t. B. f. 3: ว-7. - R. f. 353. - Bon. t. 10. f. 21 d d. - Kl. 1595. Sturm h. 12. t. 26.

Ectostroma. - [S. $371 * 519$.$] - non est fun-$ gus.

- Hederae. - Dsm. $145 \%$

- Iberidis. - Kl. S66.

- Iridis. - [\$. 520.]

- Liriodendri Fr. - Dsm. 1451. - Wstd. 4\$0.

- Oleae Cast. - Dsm. 1453.

_- Plantaginis Lasch. - Kl. 1393.

Quercus Rob. - Dsm. 1452.

- Sedi Fr. - Voges. 955. — Kl. 769. - Wstd. 1243.

- thoracella. - [S. 520.]

- Tiliae. - S. 520 .

Elaphom yces. - Ann. sc. n. 1811. XVI. t. 1.

f. 2. 3. (Anat. Tul.).

aculeatus. - Vitt. t. $3, \cdot 12$.

- anthracinus. - [S. 445.] - Tul, F. hyp.

t. 19. f. 5. - Vitt. t. 3. S.

- asperulus. - [S. 445.] - Tul. F. hyp. t. 3.

f. 9 . - Vitt. t. 4,6 .

atropurpureus. - Vitt. t. 4, 1.

citrinus. - Vitt. t. $1,16$. - Act. Turin.

1543. V.t. 3. f. 11. Vittad. .

— cyanosporus, - Tul. F. lı̣p. t. 3. f. 5.
Elaphomyces decipiens. - Act. Turin. I 413. V. t. 3. f. 4. (Vittad.). - Sturm 1.,19-. t. 11. (Cd.).

- echinatus. - Act. Turin. 1\$43. V. t. 3. f. 6. Vittad.). - Tul. F. hyp. t. 3. f. 6.

- - granulatus. - [S. 445.] - Dsm. 406. - Fl. dan. XI. 1969. 1. - gr. (et asperulus): Voges. 2\$2. - Brit. f. 279;306. - Ann. Mg. 11. h. 1S 11. VI. t. 11. f. 10. (Fruetif. Berk.). - Bisch. f. 3659. - Fuck. s. 302. - Act. Turin. 1543. V. t. 3. f. 7. (Vittad.). - Kirbh. t. 60. ex p. 1. cf. muricat. Fr. Cd. - Tul. F. hyp. t. 19. f. 4. Berg, ('harakterist. 1\$60. t. 1. f. 4. - Kl. 102. - K1. II. 114. - Kützing, Philos. Bot. 1452. t. 19. f. 13. (Anat. Fructif.). - de Bary, Ascomyc. 1563. t. 1. f. $24-33$. (Fructif.).

- hirtus. - Pay. b. cr. p. 100. f. 466.

- leucosporus. - Act. Turiı. IS43. V. t. 3.

f. 1. Vittad.

- Lereillei. - Tul. F. liyp. t. 3. f. 7 ; t. 19. f. 2. maculatus. - Vitt. t. 4, 5

Morettii. - Vitt. t. 4, 17.

- muricatus Fr. - [S. 445.] - Cd. Ic. VI. t. 10. f. 97 .

- mutabilis. - Vitt. t. 4, 14. - Act. Turin. 1-13. T. t. 3. f. 9. (Vittad.) - Tul. F. hyp. t. 19. f. 3 .

— v. flocciger. - Tul. F. hyp. t. 3. f. 1. papillatus. - Vitt. t. $4,3$.

Persoonii. - Vitt. t. 4, 1 .

pyriformis. - Act. Turin. 1543. V. t. :) f. 2.

Vittad. . - Tul. F. hyp. t. 3. f. 4.

- reticulatus. - Act. Turin. 1543. V. t. 3. f. 10. Tittad.).

- rugosus. - [S. 445.]

- rariegatus. - [S. 445.] - Tul. F. hyp. t. 3. f. S. - Berk. Out. t. 23. f. 3. - Act. 'Turin, 1४43. V. t. 3. f. S. (Vittad.).

- Vitt. $!=$ muricat. Fr. - Vitt. t. $4,4$. vulgaris.

- Cd. St. - ef. muricatus Fr.: Cd. Ic. - Schnzl. t. 14. f. 49. - Cd. Anl. t. C. f. $37: 11-13 ;-$ t. H. f. $78: 20-22$. (-25. ?. - vulgaris columellifer. - Sturm h. $19 \div$ t. 10. Cd.).

— - granulatus. - Sturm h. 19 -. t. \&.

(Cd.).

(C muricatus. - Sturm h.19-. t. 7. Cd.).

(d.).

Elvella cucullata Batsch. = Mitrula Heyderi Fl. dan.

— Brassicae. - Wstd. 182.

— ciliata. - Wstd. 141.

— lubrica Scop. - Wstd. Tti3.

-... mesenterica. - Wstd. 110.

- pulla. - Wstd. 191.

__ tubaeformis Schff. - Wstd. 1390.

Embolus. - S. 521.]

- crocatus. - Wstd. $\$ 1$.

—- sepulcralis. — Wstd. 18.

Fin ericella variecolor. - Berk. In. p. 311. f. iti. Empusa. - [S. 494.]

Culicis A. Br. - cf. Entomophth. C.

- Muscae. - S. 494. Spor.] - Nov. Act. XXV. I. t. 9-11. Cohn). - Kl. 1973. - Virchow's Arch. f. path. Anat. 1>57. XII. t. fi. f. 1--1\%. Leb.' : Myiophyton Cohnii. - cf. Entomophth. II. - Bail, Verh. d. 35. Naturf. Ters. Königsberg. 1960. Bot. t. 1. 2 : zu Achlya prolif. Ns. u. Muenr Mucedo.

Enchnoa. - S. 410.$]$ 


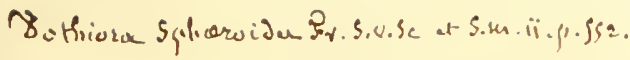




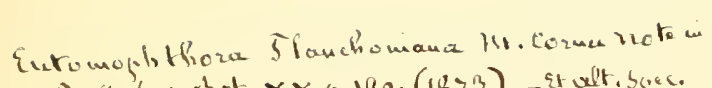

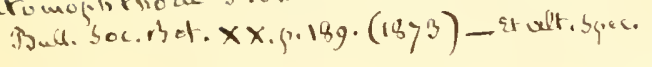


Encoelia. - [S. 362.]

Endogenium (rocq. = Erysiphe Tul.).

Findog one. - [S. 435.]

- macrocarpa. - ef. pisiformis Cd. - Tul. F. hyp. t. 20. f. 1. - Cd. Ic. VI. t. 9. f. $94:$ pisif. p. 49. - Hoffm, ic. t. 7. f. 1.

— microcarpa. - Tul. F. hyp. t. 20. f. 2.

- pisiformis. - [S. $\left.443^{* *}.\right]-$ Cd. Anl. t. C.

f. 40: 4. 5. - Berk. Out. t. 24. f. 7 .

- - B. 13. - ef, macroc.

Endophyllum. - cf. Uredo.

End op orus arenarius. - of. Lycoperdon arenar. Gaud.

End op tychum. - [S. 439.] - cf. Sccotium.

Fndothia. - [S. 355.410.] ef. Sphacria.

Endotrichu in xylogrammicum. - Cd. Anl, t. F. f. fio $): 4-6$.

Fnerthenema. - [S. 460.]

- elegans. - Linn. Trans. 1929. XVI. I. t. 16.

f. 152. Bowm.) - Ann. Mg. n. h. 1450. V.

t. 11. f. T. p. 367. Fructif. B. B.). - Berk. J.

p. 335. f. 75 c. - Bcrk, Out. t. 1. f. 6 c. - ef.

Stemonit. papill. de 13.

Finslinia. - [S. 399.]

En todes 11 i um rude. - Hedw. I. t. 4. f. I.

En tomophthora Aphidis II. H. - Fres. Ent. f. $59-67 .-$ Kl. II. 765 .

- Culicis. - Fres. Ent. f. 44-45.

Grylli, - Fres. Ent. f. 21-43.

Suscae. - Fres. Ent. f. 1-23. ef. Em-

pusa M.

- sphacrosperma. - Fres. Ent. f. li4-is.

Tenthredinis. - Fres. Ent. f. $51-56$.

Tipulae. - Fres. Ent. f. $1(i-50$.

Lill to mosporium brachiatum. - Voges. 1157.

- maculatum. - Voges. 1454.

Entomyelium. - S. 506.]

Ephebe. - S. 524.1

Ephedrosphaera coccinea. - Wstd. 366.

- decolorans. - Wstd. 361 .

Laburni. - Wstd. 363.

Ephelis. - [S. 370.]

Epichysium. - [S. $316^{*} .471^{* *} .521$.

argenteum. - Cd. Anl. t. H. f. $76: 11-17$.

leucoph. - [S. 564.]

Epiclinium. - S. 475.

Epicoccum. - [s. 176.]

- atro-sanguineum. - Fuck. rh. 231.

dispermuin c. M. - R. f. 472 .

Duriaeanum MIt. - R, f. 471 .

Equiseti. - Fuck, rh. 237.

- herbarum. - Cd. Anl. t. G. f. 64: 21-23.

- laeve Cd. - Fuck. s. 226. - Fuck. rh. $23 \%$.

lineolatum. - Dsm. 1044.

- Malinvernianum. - Ital. is9.

micropus. - Cd. Ic. III. t. 5. f. \$2.

neglectum.-Dsm. 127. (fase. XIX.; suppl.).

- Wstd. 492.

nigrum. - [S. 476.] - Wstd. 654.

pallens. - [S. 476.]

pallescens Rbh. - Fuck. rh. 235.

- Platani. - Fuck. Nass. f. 25. - Fuck. s.

227. - Fuck, rh. 233.

purpurascens. - [S. 476.] - Kl. II. 755. -

Dsm. III. 269. - Wstd. 65t.

- scabrum. - [S. 476.] - Fuck. rh. 236.

- sphaeroides Cd. - Fuck. s. 225. - Fuck.

rh. 231 .

— sphaerospermum. - Carol. V. ss.

vulgare Cd. - Wstd, 684.

Epidochium. - [S. 4ii.]

affine. - Dsm. III. 21.
Epidochium ambiens. - Dsm. III. 20.

- atrovirens Fr. - [S. 471.] - Dsm. 104i.

Maertensii. - Bull. ac. belg. Ser. 1. XXI.

p. 246. f. 6. (Wstd.). - Wstd. 107S. - R.

f. 433 .

melanochlorum. - Dsm. 1816.

nigricans. - [S. 471.]

- virens. - Bull. ac. belg. Ser. I. XXI. p. 246.

f. 7. Wstd.). - Wstd. 1079 .

Epitea. - [S. 511.] - cf. Uredo.

Alchemillae. - [S. 512.]

aurea. - Bon. Con. p. 201. t. 3. f. 33. -

R. f. 293.

— betulina. - Bon. t. 2. f. 45.

Carpini. - Kl. 1595.

- columellata. - [S. 512.]

- Euphorbiae. - [S. 512.] - Kil. II. 299. -

lirypt. Bad. 410.

- fencstrata Bon. - R. f. 149. - Bon. Con.

p. 203.t. 2. f. 31. = Ep. gyrosa p. pte.).

Fragariae Rbh. - Krypt. Bad. 411.

globosa. - J3on. Con. p. 204. t. 3. f. 32.

gyrosa. - Kl. II. 300. - Krypt. 13ad. 45.

- hamata. - Bon. Con. p. 203. t. 2. f. 30 . -

R. f. 497 .

- Lolii perennis - de Bary Br. t. 4. f. 1. -

? biventrale Bon. Con. 204.

- longicapsula. - Krypt. Bad. 405. -

Schwciz. J.

_- f. Betulac. - Kl. II. 3s4.

- f. Carpini DC. - R. f. 95.

- miniata. - [S. 512.$]$

oblonga Bon. - R. f. 190. - Bon. Con.

p. 201. t. 3. f. 31.

obovata. - R. f. 291.

pallida. - R. f. 292.

Potentillarum.

Bad. 412.

Rosarum. - [s. 512.]

liubi. - [S. 5|2. 1.]

Iuborum. - Kl. II. 697. - de Bary Br.

t. 4. f. 3 .

- Salicis aurit.). - de Bary Br. t. 1. f. 5. 6. (Salic. nigricantis). - de Bary Br. t. 4. f. 7. Vitellinae. - [S. 512.] - Krypt. Bad. 409. vulgaris Fr. - [S. 512.] - K1. 198. - K1.

II. 295 - Physonema v. Bon. - Krypt. Bad. 144.

— - v. carpophila. - Kl. 195. (Cent. XVI.).

Epochnium. - [S. 496.]

- fungorum. - [s. 496.] - Brit. f. 54.

- macrosporoideum. - Ann. Mg. n. h. 1535.

I. t. s. f. 14. (Berk.).

- monilioides Lk. - [S. 496.] - Bisch.f. 3809.

Erannium. - ef. Uredo, Caeoma.

- aurantiacum Bon. - cf. Caeoma 'Tussilag.

P. - Bon. Con. t. 2. f. 17. 15.

- aureum. - Bon. Con. t. 1. f. 12. p. 17. -

Uredo tremellosa Strss. u. Caeoma Rhinanth. Lk.

- segetum. - cf. Physoderma seget. Bon. Myk.

Ergota etia abortificans. - cf. Sclerotium Clavus Queckett.

Erinacella. - [S. 474.]

Erineum. - Pili degenerati; hic tanden omittendi.

Eriospora leucostoma. - Ann. Mg. n. h. 1850. V.t. 11. f. 1. (B. B.).

Erysibe.-cf. Erysiphe.

- Wllr. = Uredo et Ustilago.

Erysiphe. - [S. 405.] - cf. Alphitomorpha, Calocladia, Microsphaeria, Phyllactinia, Sphae- 
rotlieca, Uncinula. - Verh. naturf. Freunde. Berlin. 1 529. I. t. 1. f. 5. p. 46. (Sporang. S-1.). Erysiphe Aceris DC. - Tul. Sel. I. t. 2. f. 2. 3. Uncin. bicorn. Lér.). - Wstd. 551. = E. Mariss. Wstd. ; 550 .

— adunca Wllr. - [S. 406.] - Ki. 955. (ef.

Uncinula). - Schnzl. t. 14. f. 5-8. 10.

__ Grev. : E. Salicis Tul.

- Amentaceorum (Salic.). - Kl. II. I64.

- Dsm. 1445.

_- Mali. - Wstd. 971.

— Populorum. - Iil. II. 465.- R. f. 560.

- Prunastri Lk. - Dsm. 706. - Erys.

Prun. Tul. = Wstd. 969 .

- Rosacearum Prunastri). - Kil. Il. 755.

- R. f. 565 . b. v. Pruni).

- Ulmorum. - Kl. II. 466. (cf. Uncinula

Bivonae. - Dsm. 220.

Alchemillae Dub. - Sphaerotheca Cast.

Alni DC. - Tul. Sel. I. t. 2. f. 5-7. (peni-

cill. Alni Lk., Microsph. penicill. Lév.). IIstd. 531 .

- Aphanis Lk. - Dsm. 703.

- Apii Wllr. - Wstd. S41.

- Aquilegiae. - Wstd. 405.

_ _ areolata Wllr. - Wstd. 567.

— arillata v. Ranunc. Wlir. - Wstd.675. 1151:

v. Colchici.

- Armeriae Wilr. - Wstd. 1166.

Astragali. - Dsm. 221. - Kl. 411. - Tul.

Sel. I. t. 5. f. 2. - Wstd. 1059. (= holoseric.

L. . - Microsph. holos. Lév.

- baccata Wllr. = Ustilago purp. B. - Wstd. 750 .

_- Baryana Voigt. - cf. Podosphaera Kiunzei. Berberidis DC. - Wstd. 734. - Tul. Sel. I.

t. 5. f. 1. E. penic. Berb. Lk., Microsph. Berb. lév.)

- Betulae DC. - Kl. 144.

— betulina. - Wstd. 129 .

- bicornis. - [S. 406.] - Cd. Anl. t. F. f. 53:

14-18. - Kil. 179. - Uncinula Fuck. s. 46.

- Norweg. 1\$1. - Wstd. 550. - Krypt.

Bad. 51 .

- - f. Acerum. - Dsm. 512. - R. f. 559. biocellata Ehrb. - Wstd. 553 .

- Brayana Voit. - Kil. \$35. (Podosphaer.

Kunzei

- Capreae Dub. - Wstcl. $\varsigma_{0}$ _ — E. Salicis Tul.

- Caprearum WHr. - Wistd. 670.

- Cichoracearum. - Wstd. 226. - de I3ars,

Ascomyc. 1463. t. 1. f. 1-1 1. Fructif.)

__ cinnamonea W1lr. - Polygoni: Wstd. 2ऽ3;

Betarum 246; liumicum 2S5; Phaseolorum 357;

Fabarum 2s4; Valerianae 5i1; Viciar. S 46 ;

Violar. 40.

circumfusa S-1. - Dsm. 111.

- f. Bidentis. - Kl. 445. - Kl. II. 465. - f. Eupatorii. - Kl. II. 467.

clandestina, - [S. 406.] - Kl. 1637.

- Biv. = Erys. Bironae Tul. Sel. I. 200.

$-\quad$ Lk. =E. Oxyac. Tul. - Bivona t. 4. f. 4.

tata?. Oxyacanthae

_- comata Lk. - Fuck. s. 480. (Microsphaera com.) .

— communis. - [S. 406.] - Kil. 1313. - Ann. se. n. 15.51. XV. t. 11. f. 35. (Lév.) - Tul.

Sel. I, t. 5. f. 3. (E. comm. Hyper. Fr.). - Letell. 655. 1. - Journ. hort. soc. 1554 . IX. p. 65 . f. 1. 2. (cuin pyenidiis, Berk.).

- f. Aquilegiae. - Wstd. 40s:
Erysiphe communis f. Asperifol. - Dsm. 509. - Wstd. 553.

—— f. biocellaris Fr. - Bisch. f. 3695.

f. Calendulae. - Wstd. 972.

f. Calthae. - Fuck. s. 476 . f.

f. $2(12,269$.

f. Carduacearum. - Kl. 60. - Brit.

f. Caryophyllacearum: Lychniclis. Kl. 656 .

Wstd. 226 .

- - - f. Compositarum :

740.

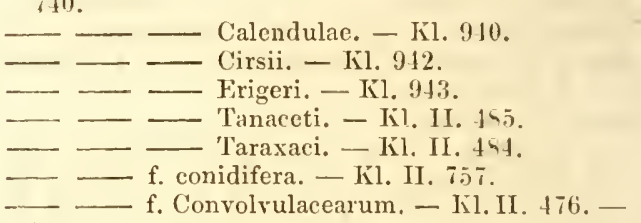

Fuck. s. 476. c.

- - f. Cruciferarum. - Kil. II. $15 \%$

- - - Hesperidis. - Kl. 935.

— - v. depressa. - ef. Er. dep.

- - f. Dipsacearuin. - Kl. 15.51. (cf. f. Knau-

tiae. - Dsm. 1515.

- — f. Geranii. - Kl. 64.

— f. Graminearum. - Kil. II. 759. - ef

Er. Graminis Lév.

— - f. Graminis. - Wstd. 5.5\%

— f. Heraclei. - Ki. 269.

— f. Humuli. - Iiolaczek, Lehrb. d. Bot.

15.56. p. 450. (ic.).

- - f. Hyperici. - Brit. f. 200. - Kl. 1134.

cf. E. Martii ; E. comm. Tul. Sel. I. t. 5. f. 3.)

[Dsm. III. 672.] - R. f. 563.

- f. Knautiae. - Fuck. s, 476. d.

— f. Labiatarum. - Wstd. 409. - Dsm.

1017

4:6. b. - R. f. 562 .

- f. Leguminosarum. - Kl.652. (cf. E.

Martii . Kl. II. 670. - Brit. f. 201. -

IV std. 120 .

- - Medicaginis. - Kl. 939.

479. - Fuck. s. 476. a.

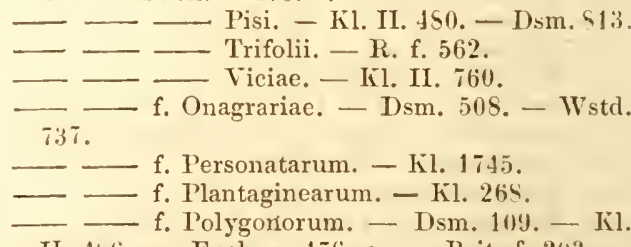

II. $4 \hookrightarrow 6$. - Fuck. s. 476 . e. - Brit. f. 203. Wstd. $\$ 30$.

- f. Potentillarum. - Brit. f. 313.

- f. Ranunculacearum. - Dsm. 1014. -

Fil. 61. - Dsm. III. 671. - Wstd. 404,553 , $13>9$.

- - Aquilegiae. - Iil. 947. - Kil.

II. $4 \nmid 3$.

- - Calthae. - Ki. II. 452.

- Ranunculi. - Kil. II. 4 \া.

Wstd. 1055. - cf. E. Martii.

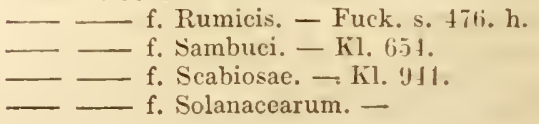




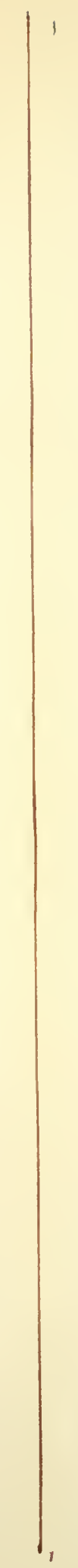




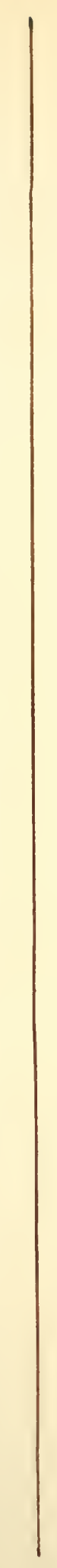


Erysiphe comm. f. Solan. Hyoscyami. - Kl. 944.

- - Verbasci. - Kl. 94j.

Wstd. 5.53 f. Thalictri. - Fuck. s. 476. i. f. Umbelliferarum. - Kl. 113\%. -

Ißbh. : ef. E. Martii. - Kl. II. 4i\%. Angelicae. - Kl. 946.

f. Urticacearum Rbh. - ef. Er. Martii.

Osm. 1547. - R. f. 564 .

Compositarum. -

- f. Carduorum. - Dsm. 1616.

f. Lappac: Wstd. 111 ; Artemis. : 111. confluens WIlr. - Wstd. litis.

Convolvuli DC. - Kl. 411 .

Corni Dub. - I. tortilis Tul.!. DC.: Wstrl.

Coryli DC. - Wstcl. 113. - W. guttata Tul.

- Voges. 53. Scl. Er.!.

densa B. C. - Berk. In. p. 27. f. 64. b. depressa. - Letell. (355. 2. - Kil. $1+11$. f. Artemisiae - hl. II. 471 . f. Bardanae. - 1) $\mathrm{sm}$. $14 .-\mathrm{Kl}$. II

170. - cf. E. Montagnei. - Wstd. 411.

detonsa Fr. - W'std. 555. - Bull. ac. belg.

Ser. I. XVIII. p. 417. f. 3. (Wstd.).

- Dipsacearum. - 'T'ul. Scl. I. t. 4. f. 4-5.

K. Knautiac Dub., Sphaeroth. Cast. I'., I ¿ér.;

- E. comm. Dips. Fr.; - E. IIumuli I) C. ?..

- divaricata I.k. - Kl. 618. - Fuck. s. 47):

Calocladia. - I.ctell. 655.3. penicill. - - Wstd. 135\%. Frangul.).

- v. Lonicerac Lk. - Dsm. 511. - E. Lo-

nic. Tul. - Wstd. 412 .

Duriaci. - Ann. Sc. 12, 1551. XV. t. 10.

f. 32 . (I.év.).

Epicaulon. - [S. 406.$]$

Epitea W7lr. - W'std. -43.

Erodii D. M. - ef. Sphaeroth. Cast.

- Evonymi DC. - Kl. 653. cf. Microsphaera comata.

- Fagi Dub. - Wstd. 739.

ferruginea $S-1 .-K 1.265$.

Filicum v. microspora W14. - Wstd. 561.

- Poterii Lk. - ef. Sphaeroth. Cast.

Flosculosorum Wlir. - Wstd. 2)i.

foetida W7lr. - ef. Ustilago sitophila Bon.

Con. p. 193; 'Tilletia Caries Tul. - Wstd. 333.

folliculata Wllr. — Wrstd. 333.

formosa Wllr. - Wstd. 545.

Fraxini DC. - E. guttata Tul. - Wstd. 655. fuliginea. - [S. 406.] Dsm. 702; - III. 673. - Wstd. 2S1. - Kl. 649. - cf. Sphaeroth. C'ast. Veronic. - Lib. 351. (Sanguisorbae, Ulmariae). - Wstd. 2S1.

v. Veronicarum Fr. - Wstd. 1056.

fulva v. Tussilag. W1lr. - Wstd. 126.

fumosa Rbh. Lk. cf. Sphaeroth. Cast.

Galeopsidis. - Lib. 153.

graminis. - Lib. 1\$2. - Ung. Ex. t. 3. f. 20.

- DC. : Tul. Sel. I. p. 212. - Ann. sc.

n. 1551. XV. t. 10. f. 33. (Lév.) - Fuck. s. 472. - Wstd. 554 .

guttata. - [S. 406.] - Bisch. f. 3693. 3694.

- Wstd. 413. - Schweiz. 14. - Krypt. Bad. 34\%.555. - de Bary, Ascomyc. 1563. t. 1. f. 15. (evol. fructif.).

Wlir.: Kl. 1 so.

Lk. : Tul. Sel. I. t. 1. - Brit. f. 205.

- Letell. 655. 4. - Norweg. 192. (Coryli et Fraxini).

f. Betulae. - Kl. II. 462 - Dsm. $70 \%$.
Erysiphe guttata f. Coryli. - Kl. II. 461. Dsm. 112. - Wstd. 413 .

f. Fraxini. - Wistd. 655.
f. Quercus. - Dsm. 1550.

gyrosa Wlir. - Wstd. $3 \triangleleft 6$.

Helioscopiae Wllr. - Wstd. $2 \backslash 5$.

holosericea Lk. - Kl.945; Cent. XV, Suppl.

- E. Astragali Tul. - Kl. II. 469.—Fuck. s. 442.

- Kbh.: Microsphaera hol.

- horridula. - Anı. se. n. 1551. XV. t. 11.

f. 37. Lév. .

Ki. II. 66,9.

- f. ('ichoracearum. - Kl. 1231.

— f. C'ynoglossi. - Ǩl. 953.

- f. Dryadearum. - R. f. 55 ᄂ.

- f. Spiracacearum. - Kl. 1135.

— f. Symphyti. - Kil. 6.5.

f. Ulmariae. - 1) 1516.

Humuli Lupuli. - Bot. Kt. Isa4. t. 6. f. ' 11. Mohl.

- DC. : 'Tul. Sel. I. 211. t. 4. f. 9. -

11 std. $40 \%$.

Hypericorum W71r. - Wstd, 565.

hypodytes W7hr. - Wistd. 572 .

insculpta Wllr. - v. Sempervivi: Wstd.

$1173 .-$ R. f. 597.

Iinautiae Jub. - E. Dipsac. Tul.

Labiataruu. - Wstd. 409.

- lamprocarpa. - Ann. sc. 11. $1651 . \mathrm{XV} . \mathrm{t} .10$.

f. 31. Lev.1. - Wstd. 109. - R. f. 561 .

f. Nolitg.

- - f. Cichorii. - Fuck. s. 471.c.

- f. Galcopsidis. - Fuck. s. 171. f.

I) $\mathrm{sm} .110$.

- - - Menthae. - Kl. 1133.

- f. Iycopi. - Fuck. s. 4ii. g.

Wistd. HII.

f. Plantaginis. - Fuck s. 4īl. d. -

- $\ldots$ f. Ranunculac. Thalictri. - Kl. 956.

- f. Scorzonerae Lév, - Fuck. s. iil. a.

- f. Stachydis. - Fuck. s. 4il.e.

f. Taraxaci. - Fuck. s. 471. b.

I.cguminosarum.

f. Pisi. - Kl. 1636.

Ienticularis $\mathbf{K x}$ : Wstd. 739 .

f. Carpini. - Dsm. I019. - Kl. 266.

f. Fagi. - kl. 449 - Kl. II. 463.

- f. Fraxini. - Dsm. 70s. - Kl. 647;

C'ent. X, Suppl. - cf. Phyllactin. gutt. Frax.

— f. Mespilorum. - Kl. $44 \%$

f. Ulmorum. - Dsm. 1519.

limbata v. Alliorum Wlir. - Wstd. 567.

- Linckii. - Ann. sc. n. 1\$51. XV. t. 10. f.

29. Lév.). - Fuck. s. 4io.

- linearis W1lr. — Wstd. 331.

Lini Wlir. - Wstd. 1179.

Lonicerae DC. - Kl.267. - Tul. Sel. I. t. 2.

f. 1. (divar. Lonic. Lk.). - Microsphaera Dubyi

Lêv. - E. ponic. Capr. : Kl. II. 473. - Wstd.

412.

Lyeii Lasch. - Kl. 950.

macularis Wlir. - [S. 406.] - Kl. 63.

f. Achilleae. - Kl. 739 .

f. Alchemillae. - Kl. 951 .

- Rob. : ef. Sphaeroth. Cast.

f. Humuli. - Dsm. \$12. - Kl. II. 460.

cf. Sphaeroth. Cast. Hum. - Wstd. 407.

R f. Poterii Rbh. ef. Sphaeroth. Cast. -

R. ค. รัวั. 
Erysiphe Mali Dub. - Wstd. 971.

Marissalii. - 13ull. ac. belg. Ser. 1. XVIII. p. 417.tf. f. 4. (Wstd.). - Wstd. 5i5l. (Ac.).

- Martii. - Ann. sc. n. 1451. XV. t. 10. f. 34. Lév. .

—— v. B. Lév, : E. communis Tul.

—_ f. Angelicae. - Fuck. s. 473. f.

- - t. Anthrisci. - Fuck. s. 473. c.

- f. (ralii. - Fuck. s. $473 . \mathrm{k}$.

- - f. Ieraclei. - Fuck. s. 473. d.

li. f. 56 i3.

f. Hypericorum. - Fuck. s. 473. m.-

-

- - f. Pisi. - Fuck. s. 473. i.

- f. 'Trifolii. - Fuck. s. 473. g.
f. Urticae - Fuck. s. 473 . a. - R. f.

564.

Mespili Dsm. - ef. Podosphaera cland.

__ miniata Wllr. - Wstd. 1167: v. Ulmariae.

- mixta Wllr. - Wstd. \$12.

Montagnei. - Ann. se. n. 1551. XV. t. 11.

f. 36. (Lév.). - Fuck. s. 475.

— muricella Wllr. - Wstd. 359. \$41, Apii.

_- myrtillina Podosph.). - [S. 406.] - Kl.

453. - Wstd. 1057. - Lib. 2 is. - R. f. 566. nervisequia. - Wstd. 970.

nitida. - Kl. 1743.

obtusata Lk. - Wstd. S0. - Kl. 1\%. (450).

- f. Salicis I.k. - Dsm. 113.

occulta f. Secalis Wllr. - Wstd. 325.

orbicularis Ehrb. - E. guttata Tul, - Lib. $\$ 2$.

- Wllr.: Wstd. 739.

Oxyacanthae DC. - cf. Podosph. clandest. ;

Erys. cland. Lk., Tul. - 'Tul. Sel. I. t. 4. f. 10.

— Wstd. 250.

- pachypus. - Wstd. 433.

_- Panicorum Wllr. ef. Uredo destruens; -

Ústilago olivac. Bon. Con. p. 191.

- pannosa. - [S. 406.] - Kl. 1415. - Kl. II. 4.59 .

— Lk. Fr.: Tul. Sel. I. t. 3. oben; t. 4.

f. $1-3$; t. 5. f. 9. 10. - Sphaeroth. pann. Lév.

- Oid. leucocon. Dsm. - Eurotium Rosar.

(irev. - Brit. f. 96. - Lib. S0.

- penicillata Wllr. - [S. 406.] - Kl. 653 ;

C'ent. IX, Suppl. - Carol. II. 65. (bid.) - ef.

divaric. - Lib. $\$ 1$.

f. Alni: E. Alni 'Iul. - Dsm. 221.-

(Caloclad.) Fuck. s. 479. a. - Wstd. \$31.

- f. Berberidis. - R. f. 296. - Kl. 954.

- Erys. Berb. Tul. - Fuck. s. 1S1. - Wstd.

73ง. - R. f. 555 .

— f. Betulae. - Dsm. 223.

— f. Caprifoliacearum, - K1. 1136. -

Kil. II. 473. - E. Lonicerae Tul., Microsphaera Iledwigii. - R. f. 556 .

- f. Grossulariae.
- f. Lk.: Dsm. 510. - Kl. 940.
- f. Lonicerae. - Wstd. 412.
f. Mespili. - Dsm. 705. - Wstd. 740.

1357.

f. Rhamni. - Kl. II. 474. - Wstd.

- f. Viburni (Caprifol.). - Fuck. s. 479.

b. 4\$3: Lantan. (Calocl. Hedw.) - Dsm. 222.

a. b. - Wstd. 412 .

Phlogis. - Carol. I. 69.

Pisi DC. - Loud. f. 16331. - Tul. Sel. I.

t. 5. f. 7. - Wstd. 120 .

Polygoni DC. - Kl. 445. - Wstd. $\ 30$.

_- polymorphav. Potentillae Wllr. - Wstd. 672.

- Populi DC. - Kl. 451.
Erysiphe Populi Wllr.: Wstd. 129.

- Potentillae. — lib. 279. - Dsm. 513. W'std. S2!.

- Poterii Dub. - Sphaeroth. Cast. Wllr.: Wstd. 564.

- Prunastri DC. - Wstd. 969. - Kil. 449. -

Uncinula Wallrothii Lév., 'Tul.

_- Pteridis Lasch. - Kl. 937.

__ pustulata v. Epilobiorum W1lr. - Wstr. 570;

- 67. : v. Caryophyll. et Epilob. ; - 1180: v. (ircacae.

_- Pyri Chaill. - Phyllactin. gutt. Lév.

_- quadrata Wllr. - Wstd. 430.

-_ quercina Schw. - penicillata, Carol.

- Quereus Mér. - cf. E. guttata Lév., Phyllact. gutt. Iév. - Fuck. s. 487. e.

— radiosa Fr. - W Wtd. 116.

- Ranunculacearum Cd. f. Clematidis. - K1. 452.

_ Rhinanthacearum Whr. - Wstd. 3\$4; 669:

v. Melampyri.

- Rosae W7lr. - Wstd. 229.

- rostellata f. Ficariae W7lr. - Wstd. \$S; 671: Geran.

- Ruborum Wilr. - Wstd. 563.

- Salicis DC. - Tul. Sel. I. t. 2. f. 1. -

Tistu. 40 .

- Sanguisorbae DC. - Sphaeroth. Cast. -

Wistd. 2\1.

- Senecionis Wllr. - Wstd. 230.

- _ sphaerica Whlr. - R. f. 1\$6. - Wstd. 125. f. Composit. - Wstd. 329.

- suaveolens Wllr. - Wstd. 87.

- suffulta Ns. - E. guttata Tul. - Wstd. 413. Symphyti Wllr. - Wstd. 332.

- Syringae Schw. - E. penicillata, Carol.

- taurica. - Ann. sc. 1. 1551. XV. t. 10. f. 30.

Lér. - Démid. Russ. t. 6. f. 5. - Fuck. s. 169.

- tortilis. - Ann. sc. n. 1\$51. XV. t. 11. f.

35. Lév.) - Kl. -36. - Fuck. s. 474. - Tul.

Sel. I. t. 5. f. S. (Spor.). - Kl. II. 472. - Brit.

f. 204 . - Wstd. 552 .

\section{f. Corni. - Dsm. $\$ 15$.}

tremellosa Wilr. - Wstd. 385.

tridactyla. - Dsm. 1014. 1S44. - Kl. $\$ 37$.

IIl. II. 175. - Podosphaera Kunzei.

- f. Pruni Dsm. = E. tridact. Tul. Sel.

I. t. 4. f. $11-13$. troglodytes Wllr. - Wstd. 335.

'Tuckeri Berk. cf. Oidium 'Tuck.

- typhoides Wilr. - Ustilago olivacea Bon.

Con. p. 191.

__ Ulm. Pers.? - ef. Sphaeroth. Cast.

- Ulmariae. - Dsm. 1015.

Urticae. - Kl. 65.

- utriculosa Whlr. = Ustilago violacea Bon.

Con. p. 191. - Wstd. 1163.

- vagans Biv. - E. guttata Tul. - Bivona

t. 4. f. 3. - Wstd. 413 .

— Valerianae offic. Whlr. - Wstd. 571.

vera Whlr. - Uredo segetum.

Hordei, Avenae: Wstd. 334.

Viburni Opuli. - Wstd. 412.

vitellina Wllr. - Wstd. 86 .

Xanthii. - Kl. 1113.

Eurotium. - [S. 405.] - Bot. Zt. 1553. t. 3.

f. 4-7. (Riess). - Bot. Zt. 1554. t. 11. (de B.).

— caulincola. - [S. 40S.]

- Cd. Anl. t. C. f. 27: 1-3. - Bisch. f. 3753.

- kl. 1270. - Kl. II. 45s. - Wstd. 400. nebulosum. - [S. 405.] 


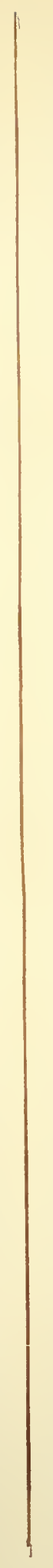



Eurntium Pyrobolus. - [\$. 10ৎ.]

Rosarum Grev. - Loud. f. 16ist9. - Ery-

siphe pannosa Tul., Spliaeroth. pann.

Fustegia. - ef. Stegia.

-_ arundinacea. - Voges. 993.

- Ilicis Fr. - K1. 131. - Lib. 15. - Wstd. 31\%. - Ital. 53s.

- v. Hederae. — Wstd. 45.

Fustilbum Rehmianum Rbh. - IIdw. II. 19 (i2.

t. li. f. 3: 2. rechts unten, bez.c. b.)

Exeipula. - [s. 1113.

L aspera. - [S. 103.] - Voges. S>6. - Wstd.

$12,1$.

Betulac. - Fuck. s. $49 t$.

chaetostroma. - Anu. Mg. n. h. 1 \50. V.

t. 11. f. 2. (B. B.).

- corvina. - [S. 403.]

- Einpetri. - [S. \{103.

- Eryngii. - Bail s. t. 15. - Fuck. s. 494.

fusispora. - Ann. Mg. n. h. 1559. III. t. 9.

f. 1. (B. 13.) - R. f. 155 .

- Ealii Lasch. - Kil. 6is.

Cientianae. cf. Peziza.

graminis. - Dinemasporiun.

graminum. - Brit. f. 326. - Wstd. Ist.

IIeraclei Fr. - Kl. \i. - Fuck. s. 197.

herbarum. - [S. 403.]

hirta. - S. 403.

_- immersa. - Dsm. III. 265.

melanopliaea. - [S. 403.]

- ornata. - [S. 367.] - Act. 'Turin. I 15. VII.

I)ec. 3. f. 2. Notar.).

— phaeotricha Rbh. - Kl. II. 515.

- punctiformis. - [S. 403.]

—- Ranmeuli? - Ǩl. 1534.

_ Rubi Fr. - [S. 103.] - Dsm. 1031. -

IVstd. 1277 .

- sphaerioides. - [S. 403.]

_- Stellariae Laseh. - Kl. 1235.

- strigosa Cd. - Kl. 1236. - Kil. II. 60. u. ili.

turgida. - [S. 103.] - Wstd. 1279.

'Typliae Laseh. - Kil. $67 \%$.

- Vermicularia. - [\$. 367. 1.] - Cd. Anl. t. F. f. 6i): 17-20. - Fuck, s. 495. - Bot. 1/t. 1559. t. 11. f. 9. Hoffin.

Exiclia. - [S. 340.] ef. Auricularia, Himeola.

- agaricina. - Chile n. 7 . f. 11 .

- ampla. - Nederl. kruidkdg. Arehief. 144\$. I.

p. 364 . c. ic. (Dozy.).

- auricula Judae. - [S. 340.] - Cd. Anl. t. G. f. $70: 12-1$ \% - Fuck. s. $\$ 90,-$ Dsm. 1279. - Loud. f, 16296. - Berk. Out. t. Is. f. 7. - Hrz. t. 45 . - Sturm h. $19-$ t. 6. (Cd.). - Schnzl. t. 16. f. 29-32. - Berg, Charakteristik. 1560. t. 1. f. 9. - Kl. 14\% - Kl. II. 301. - Voges. 993. - Carol. I. 31. - Huss. I.

53. - Wstd. 760. - Vent. mi. 27. f. 1-3. Ital. 337 .

- depressa. - Bon. t. 12, f. 24.

- Haccida E. B. - Loud. f. 16295.

- Glandulosa Fr. - [S. 340.] - Kl. 234. Dsin. 1278. - Kl. II. 241. - Pringsh. Jahrb. II. t. 31. f. 33. (Keimg, Hoffm.) - ef. Ex. spiculosa, Tremella. - Voges. 395. - Brit. f. 71 . - Carol. I. 35. - Huss. I. 42. - Lib. 332 Hoffm. Ic. t. 17. f. 5. (var.).

- impressa, - [S. 311.] - ef. Exid. depressa Bon.

- lobata. - Sommerf. in Tidsk. natur. Christian. 1527. c. ic. - Y. rurer. : 5.7.

- pithya. - 1 : : : : '

$\mathrm{H}$ off $\mathrm{m}$ an $n$, Index Fungorum.
Exidia plicata. - cf. Tremella plic.

- porphyra Lév, - Bonit. t. 139. f. 3.

-_ purpurascens. - Jungh. pr. t. 4. f. 13.

- recisa. - [S.340.] - Bisch. f. 3399. - Fuck.

s. \$ง9. - Loud. f. 16297. - Kl. 331! - Kl. Il.

416. - Dsm. III. 270. - Lib. 134. Tremella). - repanda. - [S. 341.] - Kl. 215. - Kl. II.

$24 !$.

- rosea. - Pay. b. cr. p. 75. f. 311.

- rufa. - Ann. Mg. n. h. 1542. X. t. 12. f. 17.

p. 34 4. Berk.

- sacharina. - [S. 340.] - Kl. 1611. - K].

1I. 212. - ef. 'Tremella sach.

spiculosa. (= glandulosi . - Ann. sc. n.

155\%. XIX. t. 11. (Iul.). - Norweg. 1st.

- straminea Berk. - Hook. J. 1551. III. t. 1.

f. 4

- truncata. - [S. 310.$]$

- vitellina. - Clile n. 7 . f. 12.

Ex o ascus I'runi. - Fuck. Nass. f. 26. p. 29. -

Fuck. s. 1s\%. - ?ef. Aseonye. bullat. Berk.

In. p. 2>1. f. 66 .

Exospori um. - [\$. $419 \cdots .175$.

- clavuligerum. - [S. 175.]

— Dematium Lk. — Wstd. 5ォ.

- depazeoides. - Dsm. 1549.

- Eryngii. - S. 175.] - Dsm. 115.

- Genistac. - [S. 475.

- hispidulum. - [S. 120.]

- hypodermium. - S. 120.

- Lilacis. - 1)sm. 1550. - Voges. 1311. -

Witd. $9>6$.

Lolii. - Wstd. 415.

- macrochactum. - Dsm. III. 23. - Wstd.

$1 \div 0$.

- maculans. - [S. 120.] - Wstd. 579.

- minutum Lk. - 1) sm. 14. u. 1515 . Lib.

3i11. Termicul.).

- Ononidis. - Fuck. rh. 230.

- pulvinatum. - Wstd. 153.

- Rubi Ns. - Lib. 95. - Kl. 576.

- Tiliae. - [S. 175.$]$ - Cd. Anl. t. G. f. 69 :

4-6. - Fuck. s. 22 . - Loud. f. 16604 . - Kl.

II. 782. - Krypt. Bad. 419. - Dsm. 176. (ex

Tul. - - Voges. 1099. (Helminthosp. Til. Lk.),

- 57! - Fuck. rh. 229.

__ trichellum. — Wstd, 133.

F a rinace a seminaria Sow. - Wstd. 435.

- sulphurea Sow. - Wstd. 400.

Favolus. - [S. 325.

— Boucheanus IKl. - Linn. 1\33. VIII. t. 5 ,

- Bail S. t. 31).

- brasiliensis. - Limn. 1530. V. t. XI. f. 1.

Fries! .

- ciliaris. - An1. se, n. 1543. XX. t. 15. f. 2.

(Mont.).

— cucullatus Mt. - Sagra Cuba t. 14. f. 2.

- fissus Lév. - Bonite t. 136. f. 3. - Pay. b.

cr. p. 10\%. f. 459 .

— glaber. - Cd. Anl. t. H. f. $75: 29.30$.

— nummularius. - Hook. Lond, J. IS42. I.

t. 15. Berk.).

— pusillus. - Limn. 1\$30. V. t. 11. f. 2. (Fries).

- Bisch. f. 3405.

- Sprucei. - Hook. J. VIII. t. 5. f. 8.

Femsjonia. - [S. 341.]

Fibrillaria. - [S. $\left.496^{* *} .521.\right]$

- subterranea. - ?Daedaleae quercinac status abnormis; Tul. F. hyp. t. 21. f. 12. - Wstd. 125.5.

— vinaria Sow. Wstel. 195. 
Fissidium arachnoidem Horn.-Fl. dan. XIII. 2329. 3. = Hysterium ar. Fl, dan.

Fistulina. - [S. 32.5.]

- lepatica Fr. - [S. 325.] -- Vent. t. 10. f. 97. 95. - Cd. Aul. t. II. f. 75: 1-4. - Bisch. f. 3124. - Loud. f. 16111 . - liog. t. 2. f. 4. - Bail s. t. 29. - Berk. Out. t. 17. f. 1. Ki. 1.10.1. - Fuck. s. 959. - Dsm. III. 272. Badham t. 12, 4. - Huss. 1. 6i5. - Viv. 53. Vent. mi. 16. f. 1. 2; 61. f. 1. 2. - Ital. 7 I1.

Foetidaria. - S. $43.1^{\star}$.]

Fracidia. - S. 352 .

Fuligo. - s. 419.$]$ - of. Reticularia.

- violacea. - Sturm h. 7. t. 26.

Fumago. - [S. 521.] - cf. Capnodium.

- foliorum. - Voges. 690. \&= vagans].

Mali 1. - Wste. 695.

- vagans 1 . - Dsm. 107. 157. 562. - Voges. (i90. - Torula Fumago Let. - Wstd. 114. 1195 (Alni, Populi. 1294 Armeniacae). - ’cf. Coniothec. Tiliae.

Fusarium. - [S. 472*] - Nov. Act. XIX. Suppl. I. t. 6. no. 5?

- amentorum. - Dsm. III. 657.

— aurantiacum. - [S. 473.] - Sturm h. 6.t. S. - Betae. - R. f. 69.

— Biasolettianum. - [S. 473.] - Kl. 1955.

- candidum. - [S. 473.$]$ - Wstd. 336.

capitatum. - [S. 4SI.]

Cesatii. - Kl. 1410.

ciliatum. - Kops Fl. bat. XI. \$55. 2.

- Lk. = Tubercularia cil. Kops.

- A. S. = Volutella eil. Fr. S.

- cinctum. - Cd. Ic. V. t. 9. f. 72.

- Equisetorum. - Dsm. $15+6$. - Wstd. 493. fissum. - Lotos Prag) 1559. t. 1. f. 17.

(Pejl.

- - flocciferum. - Sturm h. 6. t. 7.

fructigenum. - $[\mathrm{S} .472$.

- Georginae. - Cd. Anl. t. G. f. 65: 32-35.

-_ Graminearum. - S. 472.] Kl. 1656 ; -

11. 1791. b. - Ki. II. 155. - Fuck. s. 241. -

Schwabe, Fl. anhalt. II. t. 6. f. 7.

- Graminum Cd. - Dsm. III. 157. 155. -

IIstd. 415 .

- herbarum. - S. 472 .

- heterosporum. - [S. 473.] - Bisch. f. 3499. - Wstd. 415 ; - 416 : v. Enodii. - Pringsh. Jahrb. II. t. 29, f. 20. Hoffm. - - Kil. 100 ; Cent. XV, Suppl. - Dsm. 1124. - Kl. II. 157.

- F. Gramin. Cd.

- lateritium. - S. 172.] - Bisch. f. 3ऽ37. -

Fuck. s. 23s. - Kl. 1165; Cent. XV, suppl. -

Brit. f. 262. - Carol. III. 7S. - Letell. 691. 1.

- Schwabe, Fl. anhalt. II. t. 6. f. 6. - Wstd. 952. - Fuck. rh. 210.

t. 2. f. 7. - Dori. 53 .

- maculans. - Kl. 157 s. - Kl. II. $1>9$.

- microsporum S-1. - Wstd. 952.

- minimum. - Fuck. rh. 213.

- nivale. - Ital. 212.

- obtusatum. - Wstd. 336.

- oxysporum. - [S. 472.] - Kl. 1439. Fuck. s, 210.

— pallens. - Kil. 1559. — Wstc. 336. - Selenosporium pallens $\mathrm{Cd}$.

- Peltigerae. - Wstd. 414.

— pezizoides. - Dsm. 1>1\%. - Wstd. $9>3$.

- Platani Mont. - Dsm. 1349.

__ pulvinatum. - Wstd. 336.
Fusarium reticulatum, - Amn. sc. n. 1943. XX.

t. 16. f, 3. Mont.)

rhizophilum Cd. - Iil. II. $\ 2$.

roseum I.k. - [S. 172.] - Wistd. 95.1. -

Fuck. rh. 20 s.

- _ f. erumpens. - Kl. 258.

sambucinum. - Fuck. rh. 211.

septatum. - Wstd. 137.

Sphaeriae, - Fuck, rh. 212.

strobilinum. - Kil. $13 \$ 2$.

subtectum Rob. - Wstd. 117. - Dsm. 929.

tremelloides Grev. - Loud. f. 16603. - R.

f. $3 \varsigma 7$. cf. Dacrymye. Urtic. Fr.) - Letell.

(691. 2. - Wstd. $1>5$.

-_ violaceum. - Fuck. rh. 209.

Fusicladium fuscescens Rbh. - Kl. II. 558.

-. virescens. - Bon. t. 4. f. 94.

Fusicoceum. - [S. 5ts.]

- Aesculi. - Sturm h. 9. t. 52.

Fusicolla. - ef. Fusisporium.

- Betae. - Bon. t. 12. f. 245. - Fuck. rh. 214.

Fusidium. - [S. $\left.472^{* * *} .473 .523.\right]$ - cf. Fusarium.

- Adoxae. - Kl. II. 598.

- Ajugae N. - Fuck. s. 216.

- anceps. - Kl. 1557.

-.. Arundinis. - Sturm h. 25-. t. 2. (Preuss.)

$=$ Uredo alba Bon. Con. p. 196.

- aurantiacum. - [S. 473.] - Schnzl. t. 12.

f. 19-21. - Kl. 125\%. (Cryptosporium).

- Buxi Lk. - Wstd. 49. - Kl. 771; - u.

Cent. XV. - cf. Chaetostroma Buxi. - Fuck. rh. 217 .

candidum. - Kl. 15つ2. - R. f. 70 .

clandestinum. -- Pay, b. cr. p. 61. f. 257. -

Cd. Anl. t. B. f. $3: 12$. 13. - Kl. 1355.

coccineum. - Fuck. rh. 222 .

cylindricum. - Kl. 1556. - Kl. II. 328. -

Fuck. rh. 21s.

- Havo-rirens. - [S. 473.] - Cd. Ic. T. t. 2.

f. 10. - Kl. 1351. - Fuck. s. 244. a. - Fuck.

rh. 216. - Sturm h. 2. t. 15.

- foliorum. - Wstd. 70. (Geran. Ranunc. Cerast.).

_- Geranii. - Kl. 1443. - Selenospor. minu-

tiss. Dsm. (Wstd.). - Wstd. 770.

- griseum. - [S. 473.] - Loud. f. 16605.

- Bon, t. 1. f. 12. - Fuck. s. 245. - ef. Fu-

sisporium g. - Voges. \$94. - Sturm h. 2. t. 17. hysteriiforme. - Wstd. 1290.

inaequale. - K. 13s3.

- lycotropum. - Sturm h. 29 - t.29 (Preuss.). pallidum (Quercus). - Fuck, rh. 215.

parasiticum. - Wstd. 593.

patellatum. - Bot. Zt. 1561. t. S.f. 1.p. 194.

Bon.).

Pteridis K. - R. f. $3 \$ 9$.

punctiforme. - Kl. 1774.

pyrinum Cd. - Kl. 577 .

- Ranunculacearum Rbh. - Krypt. Bad. $14 \%$.

- Ranunculi. - Bon. t. 1. f. 7. - Fuck. rh.

$2 \cdot 23$.

roseum Lk. - Wstd. 954. - Fuck, rh. 219.

Septoria. - Wstd. 137 .

sericeum. - $[$ S. $473,11$.

Sphaceliae - Bull. ac. belg. Ser, 1. XIX. 3.

p. 132. f. 2. (Wstd.).

- stilbophilum. - Pay, b. cr. p. 76. f. $35 \%$.

- Vaccinii. - Fuck. rh. 220.

$2+3$. 


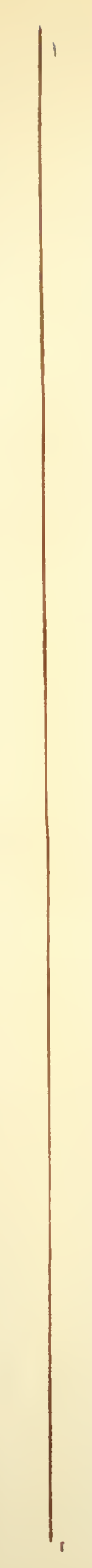



Fusisporium. - [S. $\left.172^{*+*}, 473.\right]$

aequirocum C'es. - Kl. II. 597.

- album D. - [S. 473.]-Dsm. 229. - Brit.

f. $321 .-$ Wstd. $\$ 67$.

- arachnoideum. - Cd. Anl. t. B. f. $7: 5$.

- argillaceum. - [S. 473.] - Fuck. rh. 226

- aurantiacum. - S. 473.] - Ung. Ex. t. 2. f.

13. - Bisch. f. 3504. - Carol. III. 79.

aureum. - [S. 473.]

bacilligerum Berk. - R. f. 17 .

Betae. - [S. 473.] - Ann. se.n. 1 \$3\%. XIX.

t. I†. f. 2. (1)sm.). - ef. Fusarium 13. Fusicolla

13. Bon. - Dsm. 104. - Voges. I095. - W'std. 496.

\section{J3iasolettianum. - S. 473.}

Buxi. - Flora 1nti. no. 1. f. 13. Fresen

cf. I'silonia. - Dsm. 224, - Toges. 11!11.

Brit. f. 5.5. - Wstd. 19. - Chactostr. B. Cil

II. f. $10 \pi$.

- calceun. - Dsm. (j:51. Oid. fusispord. Fr.

kil. II. 491.

- candidum. - Bon. t. 6. f. 110.

cinnabarinum. - Carol. III. 4).

concors. - Casp. Schimmel. f, 1 $4-21$

Iil. II. $27 \%$.

- didymum $\mathrm{Hg}$. - \%u F. Solani.

ebulliens. - - \$. 473.? K1. 1643.

Fquiseti. - Kl. II, 27つ.

equiv. cf. acq.

- Havidum. - Hot. Zt. 1561. t. ^. f. 3. 1) 191.

(Bon.).

- Havo-virens Fr. - S. 473. Bisch. f. 3777.

1) sm. 105. - Brit. f. 213.

- fungicolum Sporotrichum, - Cd. Ic. VI.

t. J. f. 4. d. u. $\bar{i}$.

Georginae. - Kl. 15 ti.

Graminum. - Fuck. rh, 225.

griseum Lk. Fusid. - S. 173. IIl. II. 596. - Dsm. 1730.1731. - ef. Polyscytalum fecundiss.

_- incarnatum Rob. - Dsm. 1303.

- inosculans = Keimung ron Tilletia Carics

(Berk. Intr. er. Bot, 321, u, Journ, liort, soc.

1) i7. II. p. 113. (Berk. .

- lacteum. - ef. Oidimu fusisporioides Vio-

lae). - R. f. $27 \%$. W Wstd. 1300.

- melamochlorum. - Casp. Schimmel. f. $1-17$.

- pallidum. - Verh. zool. bot. Ver. Wien.

1558. t. ๖. f. 2. (Niessl.).

Ns. ef. Fusidium cand.

Fr.

palustre Cher. - = I'silonia Arundinis Dsm.

pyrinum. - [S. 473.]

rhizophilum.

— v. Betae. - Wstd. 496.

— v. Solani tuberosi. - W'std. 496.

- sanguineun Fr. - [S. 473.] - Fuck. s. 230

(Fusicolla Bon.). - Fuck. rh. 224.

- Solani. - [S. 473.] - Indré ökonom. Neuigk.

14. t. t. f. 10. 11. - de Bary, Kartoffelkrkh.

1561. f. 12. - Flora. 1547. n. 1. f. 1-3-ff?

Fresen.\}. - Seubert, Lehrb. d. Pflanzenkunde.

I. p. S1. (ic.) - Kolaczek, Lehrb. d. Bot. 1656 .

p. 140. f. - - Schacht, Kart. t. 9. f. 20 ; t. 11 .

f. 1. - Kl. 995. - Müller, Pflzenstaat. IS61\%.

p. 265. 4. (Copie, - Wstcl. 196.

- udum. - Ann. Mg. n. h. 154l. VI. t. 11. f.

25. Berk.

Urticae Dsm. ef. Oid fusisporioid. r. - Wstd.

197. - Oid. concentr. J3. B.

- Zeae. - Wstcl. 5!)1.

Fusoma. - S. $423^{* *}$.
Fuson a glandarium. - Pay. b. cr. p. 61. f. 25.

___ Vaccinii. ef. Fusidium.

Gasteromyces farinosus. -.. Palaeont. X. t. 6. 1 661 . Ludivg.).

Gauticra. - S. 435.

graveolens Vitt. - Vitt. t. 1, 13. - Cd. Ic.

VI. t. T. f. 6.3.

— morchellaeformis Vitt. - Vitt. t. 3, 6. - C'd.

lc. VI. t. 7. f. 62. - C'd. Anl. t. D. f. 4 i: $1-3$

- Bail S. t. 27. - R. f. 2III.

Geaster. - S. 111 . ef. Irriostoma, Plecostoma. - Ann. sc. 11. 1 12 . XVIII. t. 5. 6. Fructif. 'I'ul. . - Flora 1\$13. L. t. 2. Tul. : Iüller I'tlzenstaat 1slifi. p. 26i: f. 5. 9.

- Bryantii. - Brit. f. 194.

- r'satii, - Kl. 1631. - Kl. II. 111. - Ital.

5.9i).

- colifurmis. - Loud. f. 16513.

- Irummondii, - Jlook. J ond. J. I415. IV

t. 1. f. 4 .

- fimbriatus Fr. - [S. 411.] Dsin. 256. - R.

f. 16i. u. 1:31. - Berk. Out. t. 21). f. 1. -

Fuck. s.315. hygr. - Ann. se. 11. 1 12. XVIII. t. 5. f. s-13: tunic. Fruet., 'Tul. - Voges. 1.21. - Vent. mi. 6.5. f. s.

— fornicatus Fr. - [S. 411 .] - Bisch. f. 3633. -

ki. 11. 111 . - R. f. 4311.

_ S Schff. : Voges. 1 14.5.

- hygrometricus. - [S. 141. - I)sm. 2.5.

Act. Turin, 1813. V. t. 3. f. 12. Vittal. Bisch. f. 3632 - Sehnzl. t. 11. f. 15. - K1. 311. - Fuck. s. 315: fimbriatus Fr. - Vuges. 1193. - Carol. I11. 75. - Auerswald, Bot. Unterlıaltung. 1 $>63$. S. 341

__ lageniformis. - Pay. b. cr. p. 113. f. 519.

- limbatus. - S.111.] - (d. Anl. tol). f. 4:3:

10. 11. - Bisch. f. 3636. 3637. — Purton. II.

t. 20 . - IIuss. 1. 2 .

X. 750.1

- minimus, - Carol. III. 74.

- mirabilis. - Ann. sc. n. 1\$55. III. t. 6. f. \&.

Mont.

multifidus. - Kindl. v. K. p. 29. f. 2.

quadrifidus cf. fornic.

rufescens. - [S. 141.] - Pay. b. cr. p. 101.

f. 469. - Brit. f. 275 . r. minor .

- saceatus. - Carol. I. 77.

- Schmidelii. - Pay. b. cr. p. 63. f. 251.

- Sowerbyi. - C'd. Anl. t. I). f. 43: 6-9.

stellatus. - Loud. f. 16516.

Wallr. : multifid.

316.

- triplex. - Tydsehr. v. nat. Geschied. 1510.

TII. t. s. f. 1-3. Jungh.'.

tunicatus. - Act. Turin. 1543. V. t. 3. f. 3.

Vittad.) - Tul.F. hyp. t. 21. f.9. ef. fimbr.).

umbilicatus. - [S.441.] - Chile n. 10.f. 16. vulgaris. - Cd. Ic. V. t. 4. f. 42. - Cd.

inl. t. D. f. $43: 1-5$.

Genabea. - S. 445.

- fragilis. - Tul. F. hyp. t. \$. f. 3 ; t. 16. f. 2.

Geuea. - S. 374.435 .]

bombycina. - Vitt. t. 3, 13. u. t. 4, s.

hispiclula B. - Cd. Ic. VI. t. 13. f. 119. pa-

pillosa ; p. 59.1.- Tul. F. hyp. t. 12. f. 2 ; t. 13.

f. 3. - cf. papillosa Cd.

Klotzschii Cd. - Cd. Ic. VI. t. 11. f. 101 .

- Tul. F. hyp. t. 13. f. 4, - Bail S. t. 13. 
G e ne a K̈unzeana Zob. - Cd. Ic. VI. t. 11. f. 102. (verruc. p. 56.).

_ Lespiaultii Cd. - Cd. Ic. VI. t. 12, f. I05. papillosa Berk. Vitt. = hispiclula. - Vitt. t. 3,15 .

- perlata Cd. - Cd. Ic. VI. t. 12. f. 104. - pulchra Cd. - Cd. Ic. VI. t. 11. f. 103. Tul. F. lyp. t. 13. f. 7.

- sphacrica. - Tul. F. lıyp. t. 4. f. 2 ; - t. 12. f. $1 ;$ t. 13. f. 6 .

- vermeosa. - Vitt. t. 2, 7. - C'd. Anl. t. 1$)$. f. $16: 5-7$ - Tul. F. hyp. t. 1. f. 1 ; t. 12. f. 3; t. 13. f, 5. - Pay, b. er. P. 100, f, 16.5.

- Kil. cf. G. Klotzschii Cd.

_ Kz. ef. G. Kunzeana Zob.

Geoglossum. - S. 347.] - Bail S. t. 21.

- aeruginosum S. K. - Wstd. 963.

- atropurpureum. - \$S. 317 .

_- difforme. - [S. $31 \%$.$] Kl. II. 124$ :glabr.

- Ann. Mg.n. h. 1539. I. t. 5. f. 50. Spor. Berk.)

- Brit. f. 256.

f. 1 .

- glabrum. - [S. 317.] - Kops Fl. bat. XI. \$2!.

3. - Kl. II. 121. diff.) - Cd. Anl. t. G. f. 6.) :

1-4. - Bisch. f. 3379. - Kl. 239. - Kl. II.

235. - Voges. 95. - Sturm h. 1f. t. 59.

- paludos. - Voges. 65-1 (Spliagnor.).

Wstd. 761. - Irypt. Bad. 55.

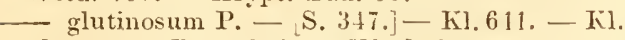

II. 319. - Dsm. 352. - Wstd. 863 .

L lubricum. - Voges. TSU.

hirsntum. - [S.317.] - Cd. Anl. t. G. f. 65:

5-s. - Berk. Out. t. 22. f. 2. - Bisch. f. 3350 .

3354. - Loud. f. 16154 . - Sturm h. 31 - t.

16 Szl. - Kil. 44. - Kil. II. 23\%. - Fuck. s.

\$70. - Wstd. 1084. - Voges. 91. - R. f. $52 \%$. a. b.

— v. capitatum P. - Kl, 612.

- mitratum viride Holmsks. - Wstd. 563.

- olivaceum, - Berk. Out, t. 22. f. 3.

ozonoides. - Letell. 672. huc Ozonium ful-

vum P. p. pte.).

__ rugosium Lasch. - Kil. \$16.

- Sphagnormm. - Voges. 651. G. glabr. paludos.).

- - viride. - [S. 317.] - Dsm. 353. - Bisch. f.

3359. - Fuck. s. 569. - Loud. f. 1615i.

Pay. b. er. p. 55 . f. 379 . - Schnzl. t. 16. f.

11.12. - Kl. 1613. - Kl. II. 239. - Nov. Act.

1542. XIX. II. t. 57.f. 146-115. (Fruct. Phöb.' .

- Müller, Pflzenstaat. 1 600. p. 271. - Voges.

994. - Lib. 123. - W'std. 563. - R. f. 524.

— Sturm h. 3. t. 45.

- viscosum P. - [S. 347.] - Loud. f. $161 \leqslant 6$.

Geotriehum. - [S. 521 .

Gibbera. - [S. 402.] cf. Spliaeria.

- glaucina. - [S. 402.]

- obturata. - S. 102.]

- obtusata. - [S. 391: 51 .]

- oppilata. - [S. 402 . plinthis. - S. 402 .

pulicaris Fr, - [S. 102.] - Fuck. s. 501. Vaccinii. - S. 402.] - Kl. II. 435.

Gibberidia. - S. 395.] cf. Sphaeria.

- Berberidis, - Iil. II. 653.

Spartii. - lil. I1. 726.

(i lacospor. cf. Gloeosp.

(ilenospora Curtisii. - ef. ramor. - Journ. hort. soc. I 19. IV. p. 260. f. 11. (13, D.

- ramorum. - Carol. I. $\checkmark$ i. (Demat. $r$. Schw.).

G i i ocladium. - [S. J!II.]
Gliostroma. - [S. 171.]

perizoides. - Cd. Anl. t. G. f. 6s: 13-15.

Gliotrich um. - [S. 466.$]$

- virescens Eschw. - Bisch. f, 3761 .

Gloe oporus. - [S. 325.]

— conchoides Mtg. - Sagra, Cuba t. 15. f.

1. - Carol. I. 22. (? Polypor. nigropurpurascens Schw.'.

Gloeosporium acerinum. - Wstd. 979.

- alnetun. - Wstrl. $17 \%$.

- andurnense Ces. - R. f. 95. - Ital. 317.

- Aurantiolum. - Wstd. 11 $\$$ S.

- Betuli. - W'std. 975.

- Carpini. - Ism. III. 1. - Wstd. 976. -

Fuck. rh. 2 ()2.

- Castagnei. - Toges. 1191. -- Castagne, Marseill. t. 9. - Fuck, rh. 200.

—_ concentricum. - Journ. hort. soc. \う1. VI.

p. 121 Berk.

- Delastri Lacr. - Dsm. III. 752. - R. f. 346.

-- Fuck. rh. 199.

- Fagi. - Wstd. 1157.

- Juglandis, - Fuck. rh. 201.

_- Mougeotii. - Dsm. 1725. - Voges. 1315.

— Populi. - Dsm. 1729; - III. 251.

— quercinum. - Wstd. IS1.

Hobergei. - 1)sm. III. 3. - Wstd. 975.

Rubi. - W'std. 950.

Salicis. - Wstd. 1269.

Veronicarum Ces, - R. f.97.-Fuck, rh.19

G $\mathrm{l}$ o m us. - [S. 43 4.

- macrocarpus T. ef. Endogone m.

Gloniops is decipiens Not. - Iil. II. 573. $\mathrm{Hy}$ sterium dec. Dnb.). - Atti VIII. riun, scienz. ital. cum icone Not. .

Gloni um. - S. 367 .

caenobituum. - Dub. Hyst. t. 2. f. 1 .

confluens. - Dub. Hyst. t. 2. f. 17. Hyster. aggregatum Wilr. ex Dub.).

- dives Not. = Hysterium div. Not. (Dub.).

graphicum Dub. = Hysterium g. Dub., con-

tortum Ditm. 'Sturm.

_- lepidum. - Dub. Hyst. t. 2. f. 19.

- lineare Not. - Fysterium angustatum Moug.

W11r. Dub. 35 .

- Mrúhlenbergii. - Cd. Anl. t. F. f. 59: 13 -17 .

stellare. - [S. 367.]

stellatun. - Carol.III. 43. - Bail S. t. 1s. Mühl. = Solenarium Mühl. Kz. (D)ub.

35$.$) .$

(i) utinium. - S. $400^{* *} .466$.

Gnomonia. - cf. Sphaeria.

fomphidius. cf. Agaricus.

- glutinosus. - [S. 306.] - Loud. f. 16019.

- Bail S. t. 34. - Schnzl. t. 16. f. 14-22.

Bot. Zt. 1556 . t. 5. f. 19. a. (Basid. IIoffm.) -

Letell. 617. - Sturm h. 14. t. 51.

-. rhodoxanthus. - [S. $306^{* *-4}$.]

rutilus Fr. - Loud. f. 16050 . Ann. Mg.

11. h. 153 \%. I. t. 5. f. $31-33$. Fructif. Berk.) -

ef. viscid.

stillatus. - Sturm h. $33-$ t. 2. Strauss'.

- viscidus. - S. 306.$]$ ef, rutil. - Letell. 6i:

Gom phinaria amoena Preuss. — Sturm. h. 35.

1. 12 .

Gumphus. ef. Gomphidius. [S. 330.: C'raterell.

Gonatobotrys. - S. $1 ! 12 . ?$

Hava. - Bon. t. 7 . f. 151 .

— ramosa. - Fres. t. 5. f. 22. 23.

- simplex. - Bon. t. 7. f. 153 - 1'ay. b. cr.

p. 104. f. 4i9. - Iil. $13 \% 3$. 


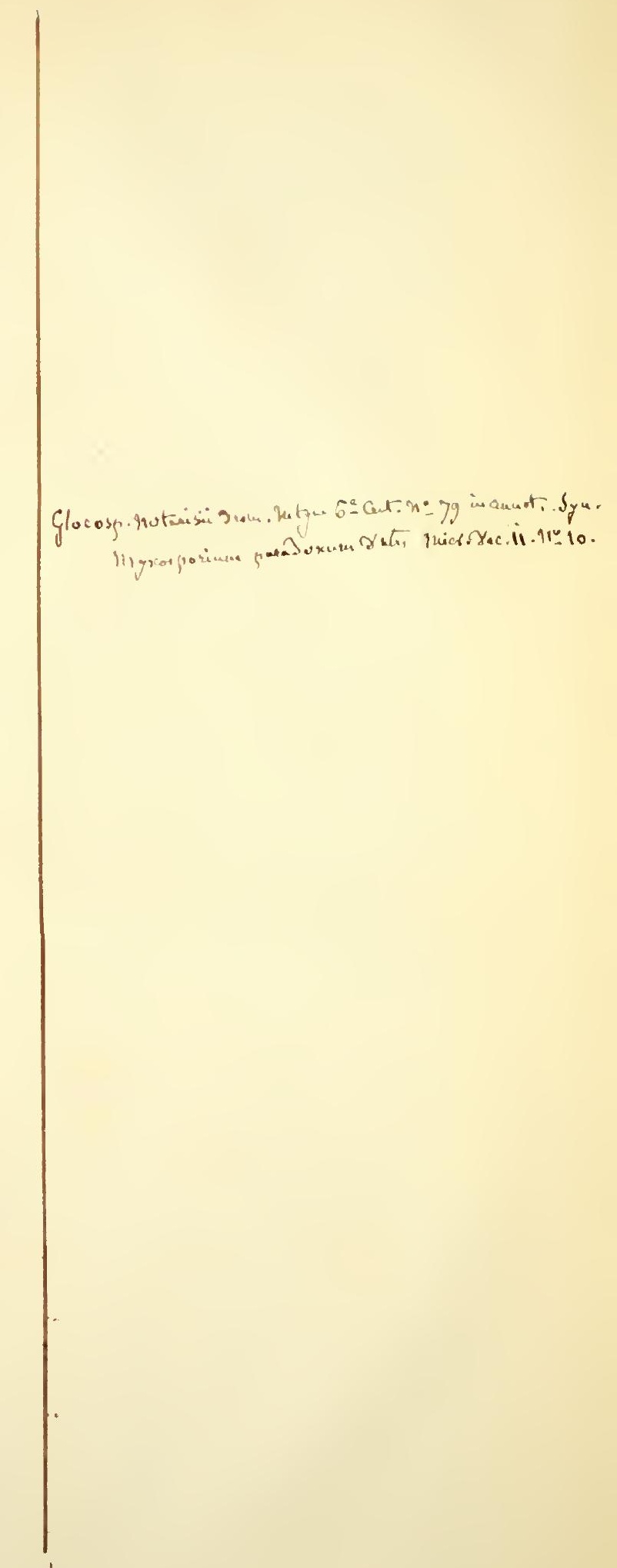




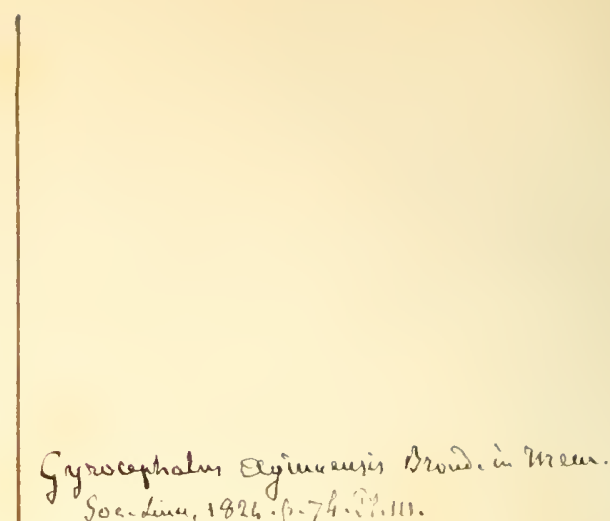

Syrocophatm aymurupis is 
Gonatorrhod um. - S. 4S9.] speciosum. - Cd. Anl. t. B. f. 14: 3. 4. -

l'ay. b. cr. p. 6. f. 15 ; p. 69. f. 3119. - Bon. เ. 9. f. 149

Gon a tosporium puccinioides. - C'd. Anl.t.B. f. $12: 7 .-$ Kl. 1575 .

(ronatotrichum caesium. - C'd. Anl. t. B. f. $8: \tau-9$.

- erectum. - Sturm h. 29 - t. t. 1. Preusis.

fiongylocladium atrum. - Iil. 13i:.

fioniosporium. - [S. 502.]

- conopleoides, - Lib. 243. C'onopl. puce.

- puccinioides. - _.51)2. - Kl. 1I.67\%. sul,

Arthrinio.-Fuck. s. 152.

(ionytrichum. - S. 191 .

— ceramicola f. simplex Hg. - Wstd. 92.

—_ ciliare. - Wstd, 4!2.

Grandinia. - [S. 32!)

- - Agardhii. - S. 3:!!.

___ alutacea. - Carol. 15. 11.

- crustosa. - S. 32!). - Kl.161)4. C'd. Inl.

t. G. f. $7 \pm: 1,2$.

f. $2(y !)$.

- mucida. - S. 32!.

—_ ocellata. — S. 32!!.

— papillosa. - \$.32!). - kil. (1)

- polycucca. - Chile n. 7. f. f11.

riranularia. - S. 521 .

liraphiola. - S. 122.]

Phönicis. - ('d. Anl. t. C. f. 2ti: 5...

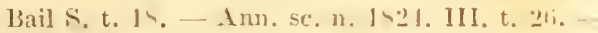

Kl. lorit. - Dsm. \i3. - Carol. IV. 湿. - Ital.

1511. - Trichodesm. Chev. - l'hacid: Fr.

Micr. J. 14.5!. VII. t. 11. f. 1-3. Curr. .

(iraphium. - S. 469 .

— atrum. - Dsm. 122.2.

- hyalosporum. - Carol. V. \$. .

- macrocarpum. - ('d. Inl. t. I3. f. 20: †-!).

Fuck. rh. 1i5.

- penicillatum. - Bot. /t. א17.t. †.

— penicilloides. - \$. $469{ }^{\top}$ - Kil. 16157.

- pliyllogenum. - Dsm. 1739.

ramosim Pr. - Iil. 1263.

- Rhizomorpharum Mont. - Mitth. nat. Ges.

Bern. J s.̆ti. Otth., sub. Rhiz, fragil. - cf.

Stilbum.

— stilboideum. - Kl. 1365. - : Kl. 17j1.

- Umbellatarum. - Iil. 1-5.j0.

rulgare. - S. $46,9.7$

Guepinia. - [S. 331.340 . Mail S. t. 27.

— aurantiaca. - [S. 331 *

- cucullata. - S. 331.

- dilatata. - Hook. J. K56. VIII. t. 10. f. 1.

- elegans. - Carol. V. 23.

- fissa. - Inn. Mg. n. h. 1542. X. t. 12. f. 15.

p. 3५3. Berk...

- helvelloides DC. - 5.331.\} - I. f. 131. -

Dsm. 11I. $66 \mathrm{I}$.

- palmiceps. - Ann. MIg. n. h. 1 \$2. X. t. 1:.

f. 14. p. 3\3. Berk.,. pezizaeformis. - S. 331. ?

spathularia. - Cd. Anl. t. G. f. $73: 16-1 \leq$.

- C'arol. III. 21.

(iymnoderma fayosum Hoffm. v. hereyn. subt.

t. 16. f. 1. = Merul. lacrymans Sehum

radiosum. - Hoffm, v. herevi. subt, t. i0.f. ?. rugosum. - Hoffm. v. hercyu. subt. t. I t. f. :-2.

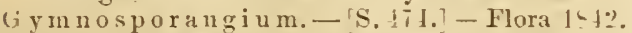

JI. t. 3. C. Miuller.

— fuscum. - Wstd. 339. I'odisoma .
Gymnosporangium juniperinum Ns. - Bisch.

f. 3551. -- Berk. Out. t. 2. f. 5. - Schwale,

Fl. anbalt. II. t. $6 . f, 1$.

- Juniperi. - S. 47 i.? - Carol. V. si. -

Fuck. rh. 413.

- f. Sabinae Fr. - Kl. J0st.

Gymnosporium. - S. 523.

Arundinis. - [S. 509.]-C'd. Anl. t. B. f. :3:

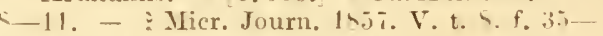

3.. Curr. .- ef. Papularia Ar. Fr. Su.

— aterrimum. - Iil. lis.1.

- entophytum. - Cd. Ic. V. t. 2. f. ?

- fulvum. - Berk. Out. t. I.f. \&. Frunt. .

- fuscum. - Berk. In. p. 73. f. 17. a.

- olivaceum. - D.m. III. 551. - Sturm h. 1:3.

t. 3.5 .

- Ihysciae II. - Fuck. rh. I(II).

- roseum. - Cd. Ie. Y. t. 2. f. 9)

Gyrocephalus. - s. $316 * *$ ?

Grocerus. - S. $17 \% \div .14 .7$

- Ammonis. - Cd. Inl. t. B. f. 5: 11 . -

Paỹ. b. cr. p. 64. f. 29t4.

- Celtis. - Iil. II. 27.

G y rographium Delilei. - Alger. t. 21, 2. -

l'ay. b. cr. p. !) f. $11-16 ;-$ p. 11 5. f. 531.

Ii romitra. - $\rightarrow$.3 16 - Helvella.

- suspecta. - S. 311 i.

(i yrophragmium. - - ‥ 13:.

(Gyropodium. - S. Aill.]

(ijrothrix. - S. K.

- podosperma. - (d. Anl. t. li. f. 1:: 1.

11 innia. - ‥ 3!7. ef. Sphaeria.

- salieclla $12 b h$. - Kl. 1115.

II alterophorit. - S. - tis!.

Il alysi um. - S. .ili.

- atrum. - Cd. Anl. t. Jj. f. 12: :3.

II an $\mathrm{zsch}$ ia l'hycomyees Awd. - R. f. 411 .

Iledw. I $>$;2. II. t. il. p. 6il. ? Phycomyces nit. K.

11 aplaria. - S. 1!) ef. Botrytis.

- brevis. - I3on. t. J. f. 96.

—_ Equiseti. - Bon. t. 4. f. 1112.

— grisea. - Letell. i(0!). 4. Botrytis .

— niven. - Ann. se, n. is 13 . Nix. t. 7 . f. 7 .

Lér.

— repens. - Bon. t. 1. f. 45.

- viridis. - Kl. II. 770 .

H a plocnemis ef. Battarea.

II a $\mathrm{l}$ ograph ium delicatum. - Ann. Mg. 11. h.

1 5.59. III. t. 9. f. 4. I3. B. .

Ha plospora. - 119 . $119 \cdots$

II a plosporium, - S. $11 \%$ ?

bulbosum. - Alger. t. $25,5$.

FI a lotrichum. - S. 190. 494 .

- capitatum. - S. 1911. - C'd. Anl. t. B. f. 19:3. 1.

- fimetarium. - Fresen. p. 105. t. 13. f. 5!

-1il.

glomerulosum. - S. 496 .

pullum. - Bon. t. 7.f. 1tid.- Fuck. rh. 145. roseum. - Pay. b. cr. p. 104. f. 174.

lielicobolus. - S. 426 .

Helicocoryne viridis Cd. - Cd. Ic. TI. f. $3^{\varsigma}$.

Helicoma Berkeleyi. - Carol. IV. 5.5.

- griseum. - Bon. t. 3. f. 7 .

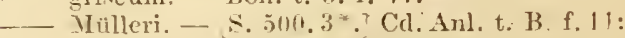

4. - Fuck, rh, II, 10. .

_- phaeosporum. - Fresen. p. 99. t. 12. f. 213 - i i).

Helicomyces. - 5.524 . 
II clicom ýces aureus. - C'd. Anl. t. B3. f. j: 11-13. - Fuck. rh. si).

—- tubulosus, - Bot. Zt. 15.33. t. 3. f. $11-13$. liess:

Hclicosporium. - S. 50u.

- Fuckelii. - Fresen. p. 101. t. 13. f. 55-.5

- Yülleri. . . S. 506 $\rightarrow$ ?

- obscurum Cd. - \$S. 5110. - Misch. f. 37! - Bon. t. 6. f. 136. - Cd. Anl. t. B. f. 11: i. - Sturm h. 11.t. 15.

pallidum ('es. - Kl. II. 62.

regetum Ns. - s. 5011.] - Loud. f. 16.56(1)

iil. 1133. - Sturm h. 11. t. 16.

II clicostylum. - is. 4!9?. $459=$.]

- elegains, - Cd. Ic. V. t. 2. f. 25. - C'd. Anl. t. ( . f. 2i: : - il.

If clicotrichum. - [S. 5(0) $\because]$

- candidum. - Kl. 1434. - Fresen. p. 101. t. 12. f. $31-33$.

pulvinatum. - Cd. Anl. t. B. f. 9: 1. -

I'ay. b. cr. p. 73. f. 331 .

II elion y ces decolorans. - Carol. V. i.

- elegans lív. - Bonite t. 13ti. f. 5. - Pay.

b. $\operatorname{rr.p.} 117$. f. 515 .

If loninthoph ora tenera. - Bon. t. 6. f. 13\%. Fuck. rli. 151 .

Hel minthosporium. - [S. 500. 504. 46ti: Iod. .

— altum. - Sturm h. 25 - t. 17. Preuss.). apicale. - Ann. Mg. n. 1. 1561. VII. t. 16. 15. p. 2-2. B. B.

appendiculatum. - Kl. 1369.

Artemisiae, - Kl. 1576.

_ a rundinaceum Cd. - S. 500.7 Dsm. 1220.

lil. 1171. - Kl. 11.' 7i. - Fuck. s. 151. -

Krypt. Had. 117. — Wistd. 661. - Fuck. rlı.

II. 107 .

- Arundinis. - Ann. sc. n. 1\$43. XIX. t. T.

f. 1j. Lév.

-_ atrichum. - S. 194.

- betulinum. - S. 5011 .

- bium (d. - Cd. Ic. VI. f. 26.

- brachyclaclum. - Fres. t. 6. f. 9-11.

bulbigerum. - Fres. t. 6. f. 22. 25.

camptotrichum. - Kil. 1134. - Kil. II. 749?.

carpophilum. - Ann. se. n. 1 43. XIX. t.

. Lév. .

('lavariarum. - Anm. se. 11. 1 431. II. t. 2.

(laruligerun. - Kl. 1370 .

- Fr. = Exosporium Rab.

concinnum. - S. 511!.

Crepini. - Bull. ac. belg. 2. Sex. XI. p. litio.

3. Wstd.

curvatum. - \$. 500 .

- cylindricum Cd. - Bisch. f. 3713. - Sturm

1. 1i. t. 11 .

delicatulum, - S. 50).] - Aun. MIg. n, h.

1)!1. VI. t. 13. f. 29. Berk. .

dendroideum. - Amm. Mg. n. h. I4ijl. III.

t. 16. f. 14. p. 351 . B. 13.

didymosporium kiz. - Kil. Ti!!

clorycarpum Mt. - Cd. Ic. VI. f. 27.

- Eryngii Fr. - S. 475. - Bisch. f. 3is 4.

fasciculare. - S. 5u1..

fructigenum R. - Ii, f. 46 .

fumosmm. - Micr. Journ, 15.5\%. V. t.

ii. Curr. .

fusisporium. - ร. 500 .

granincum, -... Kl. II. $3: 32$.

Iloffmanni. - Jerk. In. p. 24) f. lis. f.

intlatum. - Carol, III. 57 .

juncicola. - Kil. $167 \mathrm{t}$.
Hel m in thos por i um maeroearpum. - [S. 500.]

- Cd. Anl. t. 13. f. 11: \&. - Dsm. \$16.

Wistd. 17 .

- microtrichum C'd. - Biseh. f. 3716. - Sturm

h. 11. t. 12.

- nanum Ns. - S. 500.] - Bisch. f. 3717.

nodosum. - Berk. In. p. 294. f. 6ৎ. e.

obovatum. - S.5010.] - Ann. Mg.n.h. I\$11.

11. t. 13. f. 19. Berk.).

- obtusum. - Bon. t. \&. f. 169.

oligocarpum. - S. s!n; *.]

olivaceum. - C'arol. II. 56.

oosporum. - Kl. 17 9 .

pellucidum liz. - Fuck. rh. II. 108.

Pyrorum. - Lib. 155. - Wstd. 695.

Ravenelii. - Carol. I. S4.

rhizoctonum. - Kl. 1970. - ef. Rhizoctonia

viol. Medicg.

rhopalodes. - Fres. t. 6. f. 15-21.

- - septosporium. - Kl. 13i1. - hl. II. 7 .

- simplex Kz. - [S. 500.] - Bisch. f. 37 ij.

- Sturm h. 11. t. 13 .

Smithii. - Ann. Mg. n. h. 15.51. TII. p.95.

t. 5. f. ว. B. B.) - Ii. f. 271. - Nicr. Jounn.

V.t. \&. f. $1-5$. Curr.

sticticum. - Ann. Mg. n. h. 1554. XIII.

t. 15. f. 10 . p. 461 . B. B. .

subtile. - S. joli.

subulatum Ns. - S. 500.1- Bisch. f. 3712.

sturm h. 11. t. 14.

- 'Tela Cd. - Bisch. f. 3714. - Sturm h. 11.

t. (1).

- Tiliae Fr. - Bisch. f. 3757. - Ann. Mg. 12.

h. Is 11. TI. t. 13. f. 15. Berk.) - Exosporium $\mathrm{T}$.

truncatum C'd. - Cd. Ic. VI. f. 25.

- turbinatum. - Ann. Mg. n. h. 15j1. VII.

p. (1. t. 5. f. 6. Spor. B. B.

- Vaccinii Fr. - א. 466. - Bisch. f.37\$9.

velutinum Lk. - [S. 500.] - Bisch. f. 3719.

- Fuck. s. 155. - Schnzl. t. 12. f. 30-33. -

R. f. 7 . - Fuck. rh. II. 106.

II $\mathrm{clm}$ is porium $\mathrm{cf}$. Helminthosporium.

II lo lo tum. - S. 354.] of. Peziza.

_- aciculare. - S. 354.$]$ - Kl.1521. L Leotia

ac. P.

- acuum. - S. 35.5.

- aeruginosum. - S. 355.1 - Krypt. Bad. 136. agaric. - $[$ S. $355,7$.

album Schum. - S. 35.5.] - Peziza Fl. d.

Aspegreenii. - - [S. 355.]

aureum. - S. 35.] - Voges. is2.

Buccina. - S. 355 .

calopus. - S. 355.]

Calyculus, - [S. 355.]

carneum. - S. 356 .]

carpophilum. - S. 355.]

Cesatii. - Pez, ('es. Mont.

chionaeum. - S. 356.]

chlorellum. - [S. 35.5.]

chrysostigma. - is. 355.$]$

citrinum. - S. 35..]

clariculare - Kil. II. 703. - R. f. \#1!.

coccineum. - Wistd. 90.

conigenum. - S. 35.5.]

culmigenum. - S. 355 .

disciforme. - S. 356.]

elongatum. - [\$. 354.]

epiphyllum. - [S. 3.56.]

fagineum. - [S. 356 .

ferrugineum. - [S. 3.56.] 


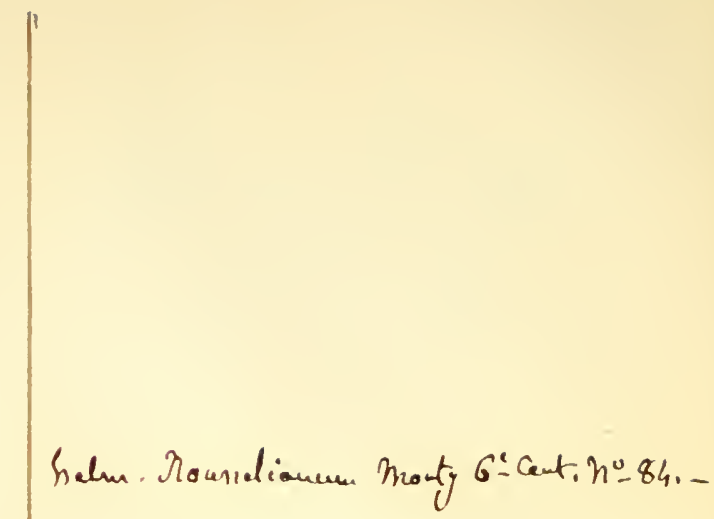

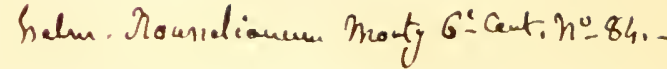




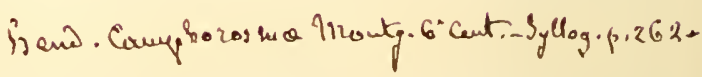

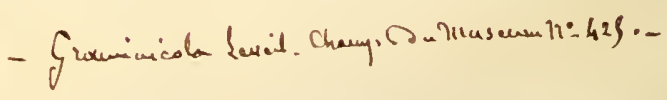

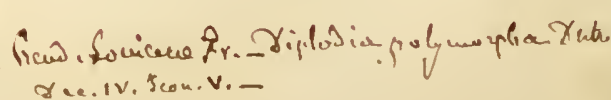


Helotium fimetarium Fr. Su. : Fuck. s. s0ว. = Pez. fimetar. Fr. S. m. - Leotia fim. P. - Stilbun erythrocephal. Rbh. lıb. myc., non Ditm, ap. Sturm. - Ditiola fimetaria Awd. - Helotium clavieulare W7lr. of. Kl. II. 703.-- [S. 355.]

_ flavovirens. - is. 35.5.

- glabrum. - [S. 351 * $]$ - C'd. Anl. t. G. f.

is: $19-22$. Kl. $122 \mathrm{~T}$. herbarum. - is. 354 .

- hirsutum. - [S.351\%]-An11. se, n. 1 52 ๖.

XIII. t. 11\%. f. 2. Tufour janthinum. - $[$ S. 355.] imberbe. - S. 356 . infundibul. - $\$$. $.35 \%$. kermesinum. - S. 356li. lenticulare. - s. 356 . luteo-virens. - S. $354 \%$ lutescens. - [S. 3355.$]$

Marchantiac. - S. 356.]

melanopus. - [5. 35t?] - Wstd. 54ti. nigripes Schum. = l'eziza lenticularis Fl.

dan. [S. 356. 33.]

- pallescens. - [S. 35.).

- perpusillum. - Kil. 1227. Ct. XVII.

- phascoides. - S. 355 .

- phyllophilum. - [S. 356.$]$

punctatum. - [s. 350 .

rhodoleucun Fr, - S. 355.] - K1. 1221.

roseum. - s. $35 \%$.]

- salicellum. - [S. 356.]

salicinum. - S. 355.$]$

serotinum. - [S. 355.

sublenticulare. - S. 355 .

subsessile Schum. = 1'cziza helotioides Fl.

dau. [S. 356.]

subtile. - [S. 354.

tenerrimum. - S. 355.$]$

titubans. - Chile n. ๖. f. 6 .

'luba. - [S. 355.]

uliginosum. - [\$. 35.

umbonatum. - \$. $354^{* *}$.

vaccinum. - [S. 355.$]$

f. 6 .

versiforme. - $[\mathrm{S} .356]-$. Berk. Out. t. 2.

virgultorum, - S. 355. - K1. 1416. Pez.

fructigena). - Fuck. s. 799 . a. b.

Helvella. - cf. Grromitra.

- acaulis Ifuds. - Wstd. Tfit.

_ agariciformis sow. = Leotia acic. 1. Tul. . albida $P$. = elastica Fl.-dan.

atra. - [S. 316.] - Cd. Anl. t. G. f. $l i \vec{i}$ :

12. - K1. 722. - Fuck, s. >it. - R. f. 236.

caryophyllea Bolt. - Wstd. 5 5.

cornucopioides Scop. - Wstd. 192.

costata. - [S. 34s**.]

crispa Fr. - [S.346.] - Vent. t. 11. f. 110.

- Berk. Out. t. 21. f. 1. - Cd. Anl. t. G.f. 67:

7, 4. - Lond. f. 16200. - Fuck. s. \is. a. b. -

I3isch. f. 327 . - R. f. 235 . - Brit. f. 264 .

Purton. II. t. 16.

clastica Bull. - [S. 346.] - Fl. dan. XI.

196 s. 2. - Cd. Ic. V. t. 9. f. 70. Asc.) - Cd.

Anl. t. G. f. $67: 4-6$. - Lindl. v. K. p. 33.

f. 3. 6. (Asc.). - Loud. f. 16204. - Berk. In. p. 36. f. 13. b. - Kl. 13\%. - R. f. 130. - Brit. f. 266 . - Ital. 233.

Ephippium Lév. - Dsm. 914. - Ann. sc.

11. 1541. XVI. t. 14. f. 7. (Lév.).

esculenta P. - Loud. f. 16202. - Hrz.t. 17.

- Berk. In. p. 36. f. 13. a. - Gyromitra) Kil.

135. - Schacht, Phys. Bot. 1552. t. 7. f. 10-13;

u. t, 2: 10. Fructif. - Schacht, Lehrb. d.
An. Phys. 1556. I. t. 1. f. 31, 32. - Berg, Charakteristik. I-60. t. I. f. T. - Badham t. 14,1 . - staude t. 1. f. 3.

II elvella esculenta v. suspecta. - Phöb. Gift. t. I. f. 1. 2 .

fistulosa A. S. - Fuck. s. $\$ 7$. gelatiuosa, - Bail s. t. 21. - Wstd. 7ß3.

grandis. - Act. Turin. I405. XIV. t. 2. ('umino'.

- inflata. - Act. Turin. 1 $>05$. XIV. t. 3. ('u-

mino . - Cd. Anl. t. G. f. 67: 9.

— Infula Sch. - \$. 3 !t.] - Bisch. f. 3313. -

foud. f. $162033 .-$ Ital. 334 .

Klotzschiana. - Cil. Anl. t. G. f. 6i : 10,

11. - Isisch. f, 3135 . - Schnzl. t. 16, f, 6-111. Sturm li. 11. t. 57.

lacunosa Afz. - [S, 346.$]-$ Fl. dan. XI.

1964. 1. - Fuck, s, 577. - Loud. f. 16201. -

3ails.t. 21. - Brit. f. 26.) - Badham. t. 14, 2. - - leucophaea. - of. II. Mitra? Rou.

- P. S. = II. crispa scop. Fr.

lutea Berg. - Wstd. 763.

mesenterica Dicks. - Wstd. 6993.

Mitra, - lioq. t. 1, f. 3.

_- Schff.: lacunosa F1. dan. - 'crispa?

Purton. II. t. 16. - Vent. mi. 61. f. 1. 2.

- mitraeformis. - I'ay. b. er. p. 45. f. 354.

__ monachella. - [5.346.] - Vix.52. - Wstd.

9!9ti. - Vent. mi. 52. f. 5. (5.

— pezizoides Afz. - [S.344.4.] - Kil. 413. -

li. 1. 237 .

Pineti. - Wstd. 557.

- pulla 1Iolmsk. - [S. 316.] - 11. dan. X11.

2011. 2 .

jumila, - Vent. mi. 62. f, 3. 4.

reflexa. - Act. Turin. 1505. XIV. t. 2. (Cu-

mino

- revoluta Girel. — Wstd. 763.

- sulcata. - - S. 316.

- suspecta. - S. 316.

- tabacina. - Chile n. 4. f. 2.

tremellosa. - S. $341 ; \cdots \cdots$.

tubaeformis Bull. = Cantharell. tub. Fl, d.ın.

velrosa. - S. 3-16.?

_- verpoides. - \$. 316 .

He m is cyphe bicolor. - Bon. t. 10. f. 205.

— stilboidea, - Cd. Anl. t. C. f. $23: 1-6$ - -

Bisch. f. $379 \%$. - Bon, t. 10. f. 206. - Sturun h. 12.t. 25 .

__ trigemina. - Bull. ac. belg. 1\52. XIX. 1.

p. 562. Spring. ?

Hendersonia. - [. 116 .] Ascospora, Sphaeria). acinosa. - - S. 116.]

- acuminata Lév. - Pestalozzia monoch.

_- conorum Lacr. -- R. f. 540.

- Corni. - Fuck. Nass. f. 14. - Fuck. s. 416.

Desmazieri Piestospora) Mt. - Dsm. 1191. elegans. - [S. 116.$]-$ Ann. Mg. n. h. $1>11$.

VI. t. 11. f. 9. Berk.

- eminens Piestospora - - Dsm. III. 65.

exilis. - S. 416.$]$

fibriseda. - Hook. J. 1553. V. t. 3. f. 10.

graminicola Lév. - Dsm. 1495.

- Ifumuli. - [S. 416 .]

- innumerosa (Piestosp.). - Dsm. III. 11.

- insidiosa (Sphaerospora) - Dsin. III. 66.

- Laburni. - Bull, ac. belg. 2. Ser, 155\%. II.

p. 379. f. 2. (Wstd.). - Wstd. 1370.

- lichenicola Somm. - [S. 116.] - Kil. 1166.

(Sporoc, ros.). Lonicerae. - [S. +16 . $]$ 
Hendersoni a Lupuli. - Toges. 1215.

- Luzulice. - Bull, ac. belg. 1\$5\%. II. p. 579.

f. 3. Wstd. .

macrospora Berk. - : Wstd. 1223.

- Mespili. - Bull. ac. belg. 145\%. II. p. 579.

f. !. Wstd. . - Wstd. 1225.

— untabilis. - IIook. J. 1551. III. t. 10. f. 6. neglecta. - Wstd. 1224.

:1odor. Laer. - cf. II. sarment.

Geae Sphaerospora D. - Dsm. 1tas.

(ari. 12ii!).

- v. Buplenri. - Dsm. 1494.

Philadelphi. - W'std. 1223.

- Phragmitidis Piestosp. - D - Dsm. III. 70.

polycystis. - R. f. 26.1.

Populi. - S. 416 .

- Pyri. - Fuck. Nass, f. 17. - Fuck. s. 415.

- Rosae Fr. - $[$ S. 416.$]=$ Sporocadus rosae-

cola Rib. - Fuck. s. 114 .

__ sarmentorum Wstd. - Bull. ac. belg. Ser. 1.

XFIII. 1. 17. t. 2. - R. f. 139 . H. nodorum

f.?r. - Dim. III. 763. - Wstd. 633.

_- suilacina Piestospora. - Dsm. III. 69.

_ subseriata Sphaerospora, - [S. H1li.] -

I) - in. 1193. - Wstd. 635 .

—_ Syringae. - S. 416.377. ?

Typhoidearum Sphaerospora - Dsm. 1191.

R. f. 45. - Dsm. III. 65, 762.

uredineacola (Sphaerospora . - Dsm. 1 14 7.

- ITstd. 634 .

- vulgaris Piestosporaj, - Dsm. III. 6i.

Hercospora. - cf. Sphaeria.

- Carpini. - S. $39 \%$.

__ circumscissa. - [\$. 397

erythropyrena. - [S. 39 .

-.. nigrescens. - Bon. t. 3. f. 72.

oculata. - s. 397.

Pupula. - [S. 39\% - R. f. 543. - Mas-

saria pyxidata Riss.

— rhodostoma. - [S. $39 \%$.

Tiliae Fr. - W'std. 533.

Hericium. - S. 325.] - ef. Hydnum.

alpestre 1 . - Ital. 146.

caput Medusae. - Roq. t. 2. f. 3.

- coralloides. - Cd. Anl. t. G. f. i2: 23.

- Erinaceus. - Cd. Anl. t. G. f. $72: 22$.

—- stalactitium Schrk. - Sturm h. 33 -. t. 9. Strauss.

It e rp otrichum. - [\$. 521.523.]

II terosphaeria. - S. 365.] - cf. Tympanis, Phacidium.

- laciniata. - S. 365.]

- laevigata. - S. 365.

- Patella. - [S. 36..] - Bail s. t. 19. - Kl.

II. 416. 417. - Fuck. s. 764. - Wstd. 321.

— phacidioides. - [S. 365.$]$

- pinicola. - [S. 365.$]$

- Plinthis. - S. 365. - Kl. II. 715.

Heterospori u circinale Kil. - Kl. 15\%.

- Oruithogali Kl. - Kl. 69.

Hex agona. - [S. 324.]

- apiaria. - Cd. Anl. t. H. $75: 3$ i. 32.

— Carmichaeliana. - Cd. Anl. t. H. f. 75 : $33-3.5$.

— ciliata. - Nov. Aet. XIX. Suppl. I. $1 \$ 43$.

t. 5. f. 1. Klotzsch.

- - erinigera. - Afzel. t. 4. f. 10.

- fasciata. - Amm. Mg. n. l. 1S42. IX. t. 9.

f. 2. Berk. .

- glabra. - Pay. b. cr. p. 10i. f. 191.
II exagoua gracilis (Pleuropus). - Hook. J. VIII. t. 5. f. 4 .

- nitida. - Alger. t. 33, 1.

-_orbiculata. - Afz. t. 1. f. 9.

- polygramma Fr. - Sagra, Cuba. t. 14. f. 3.

f. 1 .

- tricolor. - Afz, t. 10. f. S.

Wightii. - Bail S. t. 30 - Bisch. f. 3410.

'cf. Polyporus W.!.

He y den ia alpina. - Fres. t. 5. f. $37-45$.

He y deri a cucullata. - [S. 347.]

II im a ntia. - S. $496 *$. 521.] - Brit. f. 121 . 125 . Mycelium Agarici peronati et fusco-purpurei.

- clisticha. - Letell. 660. 4.

- _ fimbriata. - Letell. 661\%. 3.

__ Hammea. - Linn. 1530. V.t. 7.f.t. (Jungh.).

_- tulva. - Wstd. 432.

- globulifera Schum. - Fl, dan. XII. 2099. 1.

- lactea. - cf. Theleplora, Jungh.

___ laevis. - Letell. 630. Athelia).

__ nitens. - Letell. 660. 2.

-_ plumosa. - Letell. 660. 1. Byssus cand.!.

- pulchella. - Wstd. 116.

- ramosissima. - Fl. dan. XII. 2099. 2.

- sulfurea. - [S. $334^{* *}$.]

Hippoperdon. - [S. 442 .

II irne ola. - [S. 340.$]$ - cf. Exidia.

II irudimaria macrocarpa. - Hedw. I. t. 14. f. $(\div, 1.2$.

- Mespili. - Hedw. I. t. 1.1. f. G. 3. - Til. II. 269 .

H orm i a c tis alba Preuss. - Sturm h. 35. t. 9.

- fusea Preuss. - Sturm h. 35. t. 10.

II or mis e i u m. - cf. Torula.

- abbreviatum. - Bon. t. 1. f. 5 .

- album. - Bon. t. 1. f. 3 .

—— intennatum. - Bon. t. 3. f. $\checkmark 3$.

- caulicola f. Pelargonii. - Hedw. I. t. 7. f. 2 .

- conglutinatum. - Bon. t. 1. f. 10.

- disciforme. - Bon. t. 1. f. 11.

laxum. - Bon. t. 1. f. 17.

nlivaceum, - Bon, t. 1. f. 6 .

rhizophilum. - Bon. t. 1. f. \.

Sachari. - Bon. t. 1. f. 2.

'Iritici. - Bon. t. 1. f. 9 .

- Sini. - Bon. t. 1. f. 1. - Krypt. Bad. 141.

Hormodendron Bon, - ef. Penicill. Cd. atrum. - Bot. Zt. 1 533. t. 7. f. 7. Bon.,

- farinosum. - R. f. 173. - Bot. Zt. 1561.

เ. ?. f. 9. p. 196. Bon.). - Fuck, rh. 139.

Hornom yces aurantiacus. - Bon. t. 11. f. 234.

H ormospora. - S. 404.] - cf. Splaeria.

Husseia. - [s. 410.]

- insignis. - Hook. Lond. J. 1947. VI. t. 17.

15. f. 3. t. 19. f. 1. - Pay. b. cr. p. 112 . f. 517.

II y alopus. - [S. 491.$]$

- muscorum. - Cd. Anl. t. B. f. 19: 12.

Hydnangi um. - [S. 436.] - cf. Octaviania.

- candidum. - Ann. se. n. 1513. XIX. t. $1 \%$

f. 20. Fructif. Tul.) - Tul. F. hyp. t. 21.

f. 2. - Pay. b. cr. p. 114 . f. 527.

cameum. - Cd. Anl. t. D. f. $47: 11-13$.

Tul. F. hyp. t. 21. f. 3. - Bail S. t. 2i. - Kl.

131 .

carotaccolor. - Tul, F. hyp. t. 21. f. 4. -

Berk. Out. t. 20. f. 1. - Berk. - ef. Octav. car. lyysterangioides. - Tul. F. hyp t. 21. f. 5. liospermum. - Tul. F. hyp. t 21. f. 1. 


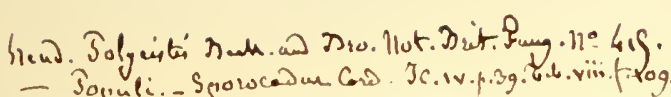

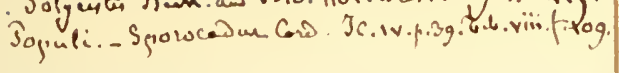

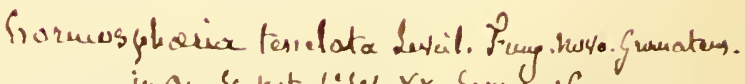
in ace. Sc. hat. L'S el. XX. Sear. Y, 16 . 

II y d n anginm Stephensii v. Ravenelii. - Carol. II. 7 I.

- virens Kil. - cf. Splanchnomyc. Kl.

Il y d n o bolites. - [S. 43९.] - cf. II ydnotria.

- cerebriformis 'Tul. - Cd. Ic. VI. t. 16 . f. $|2|$.

Oogast. e.) p. 60. - Tul. F. hyp. t. 4. t. 5 ;

t. 11. f. 2 .

Tul.

melaroxantlus C'd. - c. Pachyphloeus mel.

L'Tulasnei B. - ef. Irydnotr. 'T. - Brit. f. :3112.

Il y dnocaryon. - [S. 435.]

- fragrans Wlir. ? cf. Genea Klotzschii Zob.

II ydnoeystis. - [S. 434 . ]

- arenaria. - Tul. F. hyp. t. 1. f. 7 ; - t. 14. 1. 1.

- piligera, - 'Tul. F. hyp. t. 13. f. 2.

Il yduotria carnca C. - C'd. Ic. VI. t. 15. f. 11 J.

Rhizop. c. p. 61.)

'Tulasnei 13. - C'd. le. VI. t. 15. f. 166.

(Rhizop. 'Tul. p. 60. - - 'l'ul. F. hyp. t. S. f. 2;

一 t. 14. f. $3 ;-$ t. 21. f. 14. - Kl. II. 321. -

Brit. f. 302 .

H y d n u in. - $\$$. 326.] - ef. Odontia.

f. 3 .

alpestre R. - R. f. IS.

alutaceum. - [S. 327.] - Carol. III. 19.

argillae. - Palíontol. VIII. t. S. f. I. (R.

l.udwig.

-

- aurantiacum. - [S. 326.] - lil. II. 114. -

liuck. s. 951 .

- - aurcuin. - [s. 327 .

auriculatum. - [S. 32 \%。

- auriscalpiun1. - [S. 326.] - ('d. Anl. t. G.

f. 74: 12-15. - Risch. f. 32 \$1 - Fuck. s. 914.

- Ism. 254. - Loud. f. 16115. - Schnzl.

t. 16. f. 33-35. - Kl. 126. - li. f. 17. - Ann.

sc. 11. 1837 . Vili, t. 4. f. $\vec{i}$. Fruetif. Lév. -

Bot. Zt. 1559. t. 11. f. 29. lieimg. Hoftin..-

Voges. 777.

barba Jovis, - Loud. f, 16123?

- bicolor A. S. - S. 3227.] - K1. 1114.

Sturm h. 7. t. 29.

- byssinum schrad. - Kl. b/3.

castaneum. - [S. 32\%. 39 .

cervinum P. - Tent. t. 11. f. 101. 102. -

('d. Ic. V. t. 10), f. Sl. Fructif., - Cd. Anl.

t. G. f. $i \neq: 16-19$.

ciliolatum. - Carol. IV: I1).

cinnabarinum schw. - zu Polypor. sangui-

nolent. (Berk. Intr. 370.).

- cirrhatum $\mathrm{P}$. - [S. $32 \overline{\mathrm{l}}$ ] $-\mathrm{Fl}$. dan. $\mathrm{X}$.

17ง9. 2.

coeruleum. - S. 326.

- compactum. - [S. 326.] - Sturm h. 33-.

t. 7. (Strauss). - Ki. $12 \mathrm{~S}$.

concrescens. - Voges. 296. - Wrstd. 1392.

_ coralloides Scop. - [S. 326.] — Bisch.

f $3393 .-$ Loud. f. 16115 . - Kil. 125. - Kl.

II. 702. - Dsm. 1S10. - Carol. II. 24. -

Fries Sv. t. 34. - Auerswald, Bot. Unterhaltung. 1563 . S. 356 .

- corrugatum Fr. - [S. 326.] - Fries Sv. t. 16.

- crinale. - [S. 327.]

- crustosum Fr. - Fl. dan. XIII. 2271. 2.

- cyathiforme. - [S. 326. 18.] - Pay. b. cr.

p. $10 \%$. f. $45 \%$ - Kl. 124.

- Bull. : Fuck. s. 949.

Wistd. 1,392.

- Ulaphanum. - [S. 327.]
Iy du u discolor. - Ann. Mg, n. h. 1St2, X.

t. 10. f. 9. p. 3>1. Berk.).

- diversidens. - [S. 327.]

dryinum. -- Alger. t. 32. 2.

- Erinaceus Bull. - [S.326.] - Loud. f. 16117.

Fuck. s. 943.

- fallax. - S. 327.]

- farinaceum P. - [S. 32S*.] - K1. 623. -

Wstd. 1391.

- fasciculare A. S. - [S. 32S.] - Bisch.

f. 3295 .

- ferrugineum. - [S. 326.] - Kil. 1115. -

Fuck. s. 950. - Dsm. 1509. - Carol. III. 17.

- ferruginosum. - [S. 32\%.]

- fimbriatum. - Brit. f. 1.13 .

- flabelliforme. - ef. 1hhois

- Havum. - [S. 32১*.] - Ann. Mg. n. h.

[s 42. X. t. 111. f y. 1). 340. (Berk.).

— foctidum Secr. - Kil. 1116. - Fuck. s.956.

- fragillimum. - Carol. V. 21.

- fuligineo-album S. - [S. 326.] — Fuck.

s. 952 .

- fusco-atrum. - [S. 327.

_ gelatinosum. - S. $32 \%$ - - Journ. proc,

Lim. soc. V. 20. Mai 1561. p. 152. (Fructif.

('urrey.).

- gracile. - [\$. 326.

- graveolens. - [S. 326.] - Carol. IIr. 16. -

Ital. 145 .

- hortense. - [\$. $32 \overline{\mathrm{z}}$.

- imbricatum L. - [S.326.] - Loud.f.16112.

- Huck. s. 95\%. - Irz. t. 3. b. - Biscli.

f. 3214. - 13ail s. t. 29 - Kil. 127. - Kl. II.

112. - Fries Sv. t. 33 .

- Schum. = subsquamos. Fl. dan. -

Vent. mi. 32. f. 1. 2. - Staude t. 2. f. 4.

- Infundibulum Sw. - [S. 326.] - Fuck.

5. 954?

- lacticolor. — Carol. III. IS.

—_ laevigatum Sw. - [S. 326.] - Fuck. s. 955.

- leoninum. - [S: 327.]

- melaleucum - [S. 326.] - Kil. 122. - Kỉ.

II. 415. - Fuck. s. 916 .

- menibranaceum. - \$. 32.$]-$ Cd. Anl.

t. G. f. it: 10.11. - Brit. f. 112, - Carol. V.

20. - I'urton II. t. 15.

—. microdon. - Kil. I914.

- minimum Bolt. - Loud. f. 16121.

- minutum Schun. - [\$. 327.] — El, dan. X.

1759: 1.

- molle. $-[\mathrm{S}, 561$.

- molluscum. - [S. 327 .

- mucidum. - [S. 327.] - Kl. $191 \%$.

multiplex. - [S. j(61.]

- nigrum. - [S. 326.] - Fuck. s. $94 \%$.

- niveum. - [S. 32S.] - Bisch. f. 3321.

- obtusum Schrad - Kl. 515.

- ochraceum P. - [S. 327.] — Loud. f. 16120.

- olivaceum. - Carol. I. 27. (Irpex fusceses.).

- palmatum Vent. - Vent. mi. 24. f. 3.

Pinastri. - [s. 327.]

politum. - [S. 326.]

proliferum (Mesopus - Afz. t. 5. f. 13.

pudorinum. - [S. 32\%.]

Ramaria. - [S. 327.]

repandum L. - [S. 327.] - Vent. t. 11.

f. 103. 104. - Ann. Mg. n. h. 1838. I. t. 5 . 1. 42. 43. (Fructif. Berk.). - Loud. f. 16113. Berk. Out. t. 17. f. 2. - Roq. t. 2. f. 2. - Hrz. t. 23. - Fuck. s. 951. - Bisch. f. 3430. Fries Sv. t. 15. - Kl. 229. - Nov. Act. 1912. XIX. II. t. 57. f. 95-103. (Fructif. Phöb.) - 
Brit. f. 1.1. - Badham t. 12, 3. - JIuss. I. 16. - Viv, 39. c. v. rufesc. et ligul. .- Vent. mi. 24. f. 1. 2 ; \. . - Staude t. 2. f. 3. - Ital. 141. H y d n um repandun v. Havidum. - Ifr. t. fil.

- Rhois. - Carol. II. 25.

— rufescens. - S. 326.]

- scabrosum. - S. 326.

_ scrobiculatum. - \$. 326. - R. f. 211. -

:Fuck. s. 945.

septentrionale. - \$. 327.

—_ serpens Lasch. - Kil. 1311.

- setosum P. - I)sm. 6.53.

113. spadiceum I. - Bisch. f. 3131. - Kil. II.

- squalinum. - 327.

squamosum Bull. = subsq. Fl. dan.

— Schff. - S. 326.

- stipatum, - [s. 32\.] - Kl. $110 \%$.

striatum. - S. 326. 4.

strigosum. - S. $32 \pi$.

- suaveolens Scop. - \$.326. - Mrz. t.52. --

R. f. 104 - Sturm li. 33 -. t. S. Strauss.-

Kil. II. 11). - Dsm. 1〈1)

— subcarneum. - \$. 327.

- sublamellosum Bull. t. 453. f. 1. = Sistotrema confls. $\mathrm{P}$.

- subsquamosum 13. - [S. 326.] - Fl. dan.

XI. 196.5. - Ital. $7 \% 2$.

subtile. - S. 324.

sudans. - S. 401 .

- - tenuiculum. - Alger. t. 31, 3.

- thelephoroideum Dub. - Wstd. 1291.

- tomentosum I. - s.326.] - Hrz. t.3.a. -

Kl. 123,-- Kl. II. - - Fuck. s. 445.

- tormlosum. - \$. 561.?

- trichodontium. - ('arol. III. 20).

— udum. - S. 327. - Herk. Out. t. 17. f. 3.

variecolor. - S. 32 Ћ.]

- velutinum. - is. 326.3 - Ital. 334 .

- violascens. - \$. 326 .

- viride. - [5. 327.] - Bisch. f. 3293.

zonatum. - \$. 326.] - Kill. $1715 .=$ sero-

biculat. Fr. Ep. - K Kl. II. 9. - Fuck. s.94.

943. . - Dsm. III. 27!. - Carol. I. 25. -

Wstd. 1392. - Vent. mi. 1. f. 3. 4.

Hydrogera crystallina Wi. - ef. Pilobolus cr. Cd.

II ydrophora. - [S. A4i.j - ef. Mucor.

- fimetaria. - Jon. t. 9. f. 190.

- Mucerdae Fr. - S. 4\:.] - cf. Stilbum

leiopus Ehrb.

— murina. - S. 45i.] - Bon. t. 9. f. 191. -

Fuck, rh. 50 .

- stercor. T.: - cf. Ascophora sterc. C'd. -

[S. 4>7.] - Fuck. rh. 51.

tenella. - S. 4h $\mathrm{i}$.

II rgrocrocis. - S. J2 I.

Hygophorus. - cf. Agaricus.

II yme nang i u m. - \$. 136.] - cf. Splanchnom yoc

- album. - Cd. Anl. t. D. f. 17: $\bar{\imath}-11$. -

Kl. $736 .-\mathrm{cf}$. Ilymenogaster Klotzschii. griseum. - Par. b. cr. p. 114. f. j2s. virens. - Cd. Anl. t. D. f. 47: 4-li. Kl. : Sturm. h. 31 - t. 4. 9. Szl.).

11 ymenobolus. - is. 36ti.] Agaves. - Alger, t. 29, 2.

If y menoch a ete. - S. $330^{* * *}$.]

II y menogaster. - [S. 436.] — cf. Splanchnomyces.

- albus. - [S. 436.]

- arenarius. - Tul. F. hyp. t. 10. f. 2 .

- Bulliardi. - Vitt. t. 3, 5. - Cd. Anl. t. D. f. 15: $\bar{i}-9 .-$ Amn. sc. n. 1413. XIX. t. 17 .

f. 11-16. Fructif.'Tul. .-Tul, F. hyp. t. 10, f. 1;.

II ymenogaster calosporus. - linl. F. hyp. t. 10. f. 4 .

citrinus Vitt. - S. 136.] - Ann. sc. n. 1513. XIX. t. 17. f. 1. 10. Frnctif. Tul. - - 'Tul. I. lypp. t. 1. f. 1-; t. 10. f. 3. - I3erk. Out. t. 211. f. 2. - R. f. 34 .

- Tierk. - cf. Splanchnom. Citrus Cd.

- Vitt. - ef. Spl. citrinus Cd. - Irit. f. 2)1. - Titt. t. 3. f. 2. 3.

decorus. - Ann. se. n. 1943. XIX. t. 17

f. 1-ᄂ. Fructif. Tul. - 'Iul. F. hyp. t. I1). f. 9.

- griseus Vitt. - Vitt. t. 3, 15. - Anm. se. $n$.

14t3. XIX. t. 17. f. 1-3. Tructif. Tul.,

— Kilotzschii T. - S. 436 . alb. - Bail s.t. 27.

= Splanchnomyc. albus (d., Rhizopogon albus

Berk., IIymenangium album Kl. . - Tul. F. hyp.

t. 10. f. 12. - Kl. i967. - R. f. 212. - Hot.

Kt. 145 (5. t. 5. f. 30. evolut.), — Pringsh. Jahrl).

II. t. 31. f. 31. Hoffm. - IIoffm. Ic. t. \% f. 1 .

- lilacinus. - Brit. f. 36.). - Tul. F. hyp. t. 1. f. 2. - t. 11). f. ?.

— - Berk.? - ef. Splanchnom. Tul. Zol. - luteus Vitt. - Vitt. t. 3, !. - Ann. sc. n.

1 43 . XIX. t. 17. f, 11-13. Fructif. Tul. 'Tul. F. hyp. t. 1. f. '3.

- I3erk. et Vitt. - ef. Splanclinomye. Berk. C'd.

- Iycoperdineus. - Vitt. t. 2, 5. - Tul. F. hyp. t. 10. f. ร.

_- Vitt. - ef. Splanehnomyc. 1. Cd.

- muticus. - Tul. F. hyp. t. J0. f. i.

- niveus. - Vitt. t. 4, 9.

- oliv. Vitt. - cf. Splanchnom. ol. Cd.

_- populetorum. - Tul. F. hyp. t. 10. f. (1).

- - Berk. - ef. Splanchnomyc, olivac. Cid.

f. 301 .

- rufus. - Vitt. t. 3, 17 .

- tener. - Tul. F. hyp. t. 1. f. 1; t. 10. f. 1.

- Lerk. Out. t. 1. f. 3. Spor..

Berk. - ef. Splanchnom. t. Cd.

Thwaitesii. - 'Tul. F. hyp. t. 10. f. 11.

- vulgaris. - Tul. F. hyp. t. 10. f. 13. - ef.

Splanchnom. Tul. /ob.

II ymenogramme. - S. 315.]

- javensis. - Hook. Lond. J. 14 44. III. t. 11.

p. $331 \%$.

II ymenophallus. - S. $431 \% .7$ - (f. Thallus. - Bail S. t. 21.

- I)aemonum. - Cd. Anl. t. F. f. 52: 11. duplicatus. - Cd. Anl. t. F. f. 52: $\rceil$.

Hadriani. - Bail s. t. 26.

indusiatus Vent. - C'd. Ic. V. t. S. f. 51. -

(d. Anl. t. F. f. $52: 5-10$.

- suhiculatus IIont. — C.d. Ic. VI. f. 45.

II y m en op odium. - s. 506.]

- sareopodium. - C'd. Ic. I. 121.

HymenuIa. - S. 171. 342.]

alba. - [S. $4 \pi$ i $* \rightarrow$.]

Arundinis Fr. - S. 471.] - Kl. 347. (est

Sphaeria rimosa,

- ciliata. - S. 471.]

- Clarus. - cf. Sclerotium C.

- Desmazieri Cast. - Dsm. 13łs.

Ebuli. - [\$. 471.] - Fuck. rh. 1 \$.

- Equiseti. - Lib. 236. Cryptosporium Eq.

Fr.). - Wstd. 493.

Georginae Whlr. - S. 471.] - Kl. 580. -

Fuck, s. 252, - Fuck, rh: 153. 
$\mid$ 


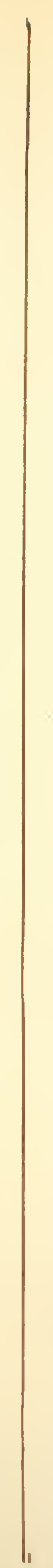


If y menula nigra. - [S. 171.]

1317. - Voges. 992.

- Solidaginis. - SS. 171.

- Spartii. - Kl. II. 75.5.

rubella. - lib. 137.

umbilicata. - Voges, $7 \varsigma$ S. Pez.).

Urticac. - [S. 471.]

vulgaris. - [S. 47l.] - Cd. Anl. t. G. f.lis:

1-4. - Kl 1390. - Fuck. s. 251. - Lib. 136.

(Tremella elliptica 1.. - Fuck. rh. 1 52.

- f. Pliytolaccae. - Kl. 1467.

II yperom yxa. - S. $409 *$ * 51)\%. stilbosporoides. - Cd. Anl. t. G. f. i0:

5-9. - Myriocephal. botryospor. Fres.

turbinata. - Bon. t. 12. f. 211 .

II yperrlıza. 一 [s. $43 \%$.

carolinensis Bose. - Bisch. f. 3(ilil).

liquaminosa. - \$s. $\left.4: 37^{*}.\right]$ - Bail s. t. 11.

— Kl. - ef. Mclanogast. İlotzschii Cd.

tuberosa. $-[$ S. 437 .

variegata. -- [S. $43 \%$.]

II y pli a arachnoidea. - Nov. Act. XI. t. 59. A. li.

IIelwig.

—_ argentea I'. - Letell. (i7 1. 2. - Kỉl. 4(i0.

bombycina. - Ietell. ti.1. 1.

- lanata. - Nov. 1et. XI. t. 59. C. Helwig.

_ papyracea P. - Kil. 773.

sulphurea, - Nov. Aet. XI t. 5!). D). (IIelwigr .

II y hasma asteriforme. (Iycelium). - I)sm. ji)0.

- Racodium - W Wtd. 155.5.

thelena. - Wstd. 115.

Il y pliclia. - S. 417.]

aurantiaca. - Lib. 379.

fusca. - [S. 447. ]

rosea. - Cd. Anl. t. C. f. 33:3. 1. - Kl.

679. - Iil. II. 773.

terrestris. - [S. 447.]

H yphoderina. - S. H7.

lintaceum Whr. - Wstd. 557.

roseum. - S. $41 \%$.

scirpinum DC. - Wstcl. 1221.

terrestre W'llr. - ef. 'Thelephora terr.

II y pochnus. - S. 336. $461^{*}$.]

anthochrous. - [S. 337.]

asterophorus. - Bon, t. 12. f. 252.

bombycinus. - [S. 337.] - Kil. 14115.

centrifugus Tul. = Rlizoctonia c. Lév.

cinereus. - Bon. t. 12. f. 2.19.

coeruleus Fr. - Wstd. 767.

confluens. - Bon. t. 12. f. 254 .

fuscus. - [s. 337.]

granulatus. - Bon, t. 12. f. 257 .

isabellinus. - S. 337.] - Kirypt. Bad. 157.

lacteus. - Bon. t. 12. f. 259.

laevis. - Bon. t. 12. f. 251 .

- Michelianus Cald. - R. f. 413. - Ital. 159.

Thelephora orbicularis.

mollis. - [S. 336.]

olivacens, - [S. 33\%.]

puberus. - Bon. t. 12. f. 256.

rubro-cinctus. - Cd. Anl. t. G. f. 73: 1. -

lisch. f. 3766. - Carol. I. 33.

- serus. - [S. 336.]

- strigosus. - Bon. t. 12. f. $25 \mathrm{~s}$.

tenuis. - Bon. t. 12. f. 250.

- umbrimus, - [S. 33\%.]

uvidus. - Bon. t. 12. f. 255.

violascens. - [S. 337 .]

Hypocopria. - ef. Sphaeria.

Hy pocrea. - [S. 3\$3.] - cf. Sphaeria.
H y pocrea armata. - [S. 3ৎ3.]

atra. - [\$. 564.]

atro-virens. - Chile 1r. 9. f. 1.

citrima Mont. - Sagra, Cuba t. 12. f. 1.

Ital. $-\leqslant 1$.

_- eupularis. - [S. 35.3.]

eupeliaca = Xylaria Guepini v. cupel. (Ces.).

floccosa. - [S. 561 .]

fuliginosa. - ef. Cordyceps.

gelatinosa. - [S. 3-3.]

hyalina. - 5.343 .

- inclusca. - Ann. Mg. n. h. |\fi|. VII. t. 17.

f. 23 . p. 452 . B. B. .

—- lactea Fr. - [S. 343.] - (Kl. II. 39: ovula in-

sectorum, t. Tul. Sel. I. 121.). - Fr. Obs. mye.

II. I>Is. $t$. f. 4. - Hoffm. Ic. ef. sub Sphaeria. lateritia Fr. - [S. 3\$3.] - R. f. 317.

luteo-virens. - [S. 3\3.]

plyylogena. - Ann. se. n. 1510 . XIII. t. 6.

Iont.).

— purpurea Cordyceps'. - Kil. 1534.

___ pusilla. - Kil. 1533.

—_ Ravenelii. - (arol. T. 51.

- riccioidea, - S. 3 33.$]$

- rigens. - S. 34.3*.]

- rufa. - \$. $3>3$.

__ rufo-viridis. - Carol. V. 53.

- scutellaeformis. - Carol. IV. 31.

—_ tremelloides. - [S. 3\3.39八 \%.]

—- tuberiformis. - Carol. V. 52.

- Vilalbae. - R. f. 43. - Ann. Mg. 1. h. 1与.5. III. t. 9. f. \$. B. I3.).

II yooderna. — cf. IIysterium.

- arundinaceum DC - Iil. 55\% = Lopho-

dermium ar. Chev. (Dub.)

- crispum 1$)($. = IIysterium elatinum $\mathbf{P}$. var.,

'Triblidium crisp. I'. (Uub.).

- Desmazierii. - I)ub. IIyst. t. 2. f. 22.

- Hederae Not. - Kil. 1954.

nitidum Not. = II. vulgare Fr. (Dub.).

- I'inastri. - W'std. 223.

- quercinum DC. = Colpoma Wllr. (Dub.).

- Iiubi Not. $=$ virgultorum DC: (Dub.). -

Wstd. 921 .

- rufilabrum. - Dub. Ilyst. t. 2. f. 21.

- virgultorum DC. $=$ II ksterium Rubi $\mathrm{P}$. (Dub.).

- Wstd. 921.

Fr. Dub

xylomoides DC: = Lophodermium $\mathrm{x}$. Chev.

(Dub.). - Wstd. 922.

II y podermium.- [S. 152.]

- pinastri. - [S. 452.]

sulcigenum Lk. - cf. Schizoderma Pinastri

Willk. - Fuck. rh. 235.

Hypodrys hepatica. - ef. Fistulina hep.

II y poglossus Mentagnei. - Hook. J. IS12. I. t. 6 .

Hypom yces. - [S. 3५3.] - cf. Sphaeria.

H y p op te is Luzulae libh. - Krypt. Bad. 33.5.

H y porh a mm a reticulatum $\mathrm{Cd}$. - ef. Trichia ret.

II ypospila. - [S. 421.]

- populina Fr. - [S. 121.] (Sphaeria p. Auctt.

- Sph. ceuthocarpa Fr. Rbh:). - Kl. II. 571. -

Fuck. s. 391. - Fuck. rh. 418.

- quercina Fr. - [S. 421.] - Fuck. s. 392.

(cf. Sphaeria bifrons Fr.). - Fuck, rh. 417.

Hypoxylon. - [S. 353.] - of. Sphaeria, Xylaria. [S. 3S1.]

- ambiens. - Wstd. $36 \%$. 
Hypoxylon annulatum. - Chile n. 10. f. 3. - argillaceum. - [S. 3\$.1.] (Sphacria. - Fuck.

s. 693. - Wstd. 530.905.

—_ atro-purpureum. - [S. 341.]

- Berberidis Kx. - Wstd. 1203.

- Berterii. - C'hile, u. 9. f. 3.

- Jomba Mont. - Sagra, Cuba, t. 12. f. 3.

bullatum. - W'std. 6 S.

Carpini. - Wrstd. 1206.

ceratospermum. - Wstd. 67.

chrysites. - Wstd. 365.

cinnabarinum. - Wstd. 361.

cirrhatum. - Wstd. 323.

coccineum. - [S. 354.] - Kíl. II. 116. 〈ef.

Sphaeria fragiform. I'. - Fuck. s. 695. a. b. --

IVstd. 366. 903.

- cohaerens. - [S. 354.$]$ - Carol. III. 15.

Fuck. s. 690: H. multiforme. - Wstd. 90ذ.

- collabens. - Inn. sc. n. 1510. XIII. t. 9.

(Mont.).

comosum. - Ann. sc. n. 1540. XIII. t.9.

Mont.

- concentricum. - [S.3S1.] - Kil. II. 600. -

Carol. III. 49. - Ital. 642.

- confluens. - Wstd, 166.

- coprophilum, - [S. 3 S.] - Kl. II. $25 \%$.

—_ corrugatum. - [S. 35 t. $]$

- crustaceum. - [S. 3\&4.]

__ cubense Mont. - Sagra, Cuba. t. 13. f. I.

cupreum. - [S. 354.]

- cupulare $\mathrm{Kx}$. - Wstcl. 525.

- Cyclops. - Ann. se. n. 1540. XIII. t. 10.

Mont.

- deustum. - Ital. 643.

-_ dichotomum Mont. - Sagra, Cuba, t. 13. f. 3.

- durissimum. - [S. 354.]

- effusum. - [S. 3S4.]

- enteroleucum. - [S. 354.]

__ ferrugineum. - Wstd. 915.

_ _ filiforme ef. Xylaria fil.

-_ flavo-virens. W Wstd. 113.

— fragiferum Kx. - Wstd. 903.

fragiforme. - Carol. III. 50. - ef. Institale acariforme.

—_ fuscum.- [S.3\$4.] - Berk. Out. t. 1. f. 12. a.

(Spor.). - Fuck. s. 691.

Galeotianum. - Bull. ac. belg. 1s11. VIII.

2. t. 2. (Kx.).

gastrinum. - [S. 353.] - Wstd. 266. -

Ital. 64t.

_ glomeratum Bull. = Sphaeria fusea, Fl. dan

— glomerulatum. - Tistd. 219.

- glomerulosum. - [S. 3 4.]

- gramineum. - Pay. b. cr. p. 62. f. 266 ;

p. 97. f. 452 .

1- grammicum. - Ann. sc. n. 1510. XIII. t. 9.

Mont.

granulatum Bull. - Wstd. 713.

granulosum B. - [S. 354.] - Fuck. s. 692. guyanense, - Ann. se. 11. 1S40. XIII, t. 9.

(Mont.).

- Heliscus, - Ann. sc. n. 1\$40. XIII. t. 10.

(Mont.).

jecorinum. - Carol. IV. 37.

investiens. - Carol. IV. 33.

irradians. - Ann. sc. n. 1810. XIII. t. 10.

(Mont.).

irregulare. - Wstd. 472.

—- Kickxii. - Bull. ac. belg. 1\$52. 1. Ser. XIX.

p. 132. f. 1. (Wstd.). - Wstd. 714 .

- Laburni. - Wstd. 363.
II ypoxylon I,eprieurio. - Ann. sc. n. Isto.

XIII. t. 10. Mont.) - Pay, b. cr. p. 97. f. 146. luteum. - [S. 353.$]$

macromplialum. - Amm. se.n. 1 \$5. III. t. 5.

f. S. Mont. .

- macrosporum. - Ann. sc. 11. 1810. XIII. t. 10.

Mont. .

Malleolus. - Carol. IV. 32.

Miclielianum. - Ital, 753.

microceras. - $\Lambda \mathrm{mn}$. se. n. 1510. XIII. t. 9.

Mont.

_- multiforme. - [S. 3\$4.] - Berk. Out. t. 21.

f. 1. - Fuck. s. 659 - Carol. V.56. - Wstd.

i13.

- notatum. - Carol. IV. 36.

_ nummularium. - S. 34.] - Act. 'lurin.

15.5. XVI. Dec. 9. f. 1. (Notar.). - Fuck.

s lisli. - Ital. 295.

oedipus Mont. - Sagra, Cuba. t. 13. f. 2

omphalostoma. - [S. 354.]

operculatum. - Wstd. 112. 529.

ostraceum. - [S. 524.]

_ perforatum. - Carol. V. 54.

_-_ platystoma. - Carol. V. 55.

-- polymorphum. - Kops Fl. bat. XI. S29. 2.

- c.f. Xylaria p. - Schnzl. t. 15. f. 36-3s. -

Jiail S. t. 17. - Nov, Act. 1961.25. t. 1 f. S.

spor. Bail .

__ punctatum. - Lindl. v. K. p. 29. f. $\vec{\imath}$.

— punctulatum. - Carol. III. 51.

repandum. - [S. 3\$3.]

- rhizicola. - Ann. sc. n. 1540. XIII. t. 9.

(Mont.)

Ribis. - Mstd, 474.

_- rubiginosum Fr. - [\$. 3\$1.] - Fuck. s. $6 \varsigma 5$. rubricosum. - [S. 351] - Chile n. 10. f. 2.

Sagraeanum Mont. - Sagra, Cuba. t. 12.f. 4.

salicinum. - Wstd. 473.

- scabrosum Bull. = Sphaeria sc. Fl. dan.

- Scleroderma. - Ann. sc.n. 1540. XIII. t. 10.

Mont.). - Pay. b. cr.p. 97. f. 151.

— serpens. - [S. 344.] - Fuck. s. 694. - Ca-

rol. IV. 34.

— Sphaerii. - Pay. b. cr. p. 62. f. 265.

sphondylinum. - [S. 353.$]$

spiculosum. - Wstd. 651.

steroides. - [S. 3S4.]

tabacinum. - Bull. ac. belg. 15ł1. VIII. 2.

t. 2. Kix.)

tubulinum. - [\$. 3\3.]

turgidum. - W'std. $90 \mathrm{~T}$.

udum. - S. 3S4.]

- ustulatum. - S. 353.] - Sphacria deusta

H. - Berk. Out. t. 21. f. 3. - Fuck. s. 65i.Krypt. Bad. 431.

__ velutinum. - Bull. ac. belg. 1 5.51 . Scr. 1.

XVIII. p. 11\%. f. 1. Wsta.). - Wstd. 17.

- virgultorum Fr. - [S. 3̈ 1.] - R. f. 31S. -

Ital. $\$ 73$.

- vulgare. - Cd. Anl. t. F. f. $56: 5-\varsigma .-$ c.

Rhizomorpha subcort. : Nor. Act. $1561.24, \mathrm{t} .1$.

f. $1-7$. Bail

Walterianum. - Carol. IV. 35.

xanthocreas. - Carol. V. 57.

II ysterangium. - [S. $43 \%$.] - Bails. t. 14. cf. Splanchomyces ('d.

clathroides. - Vitt. t. 4, 2. - Jail S. t. 14.

- Cd. Anl. t. D. f. 46: 1-4. - Tul. F. hyp.

t. 2. f. 2 ; t. 11. f. 6. - Pay. b. er. p. 114. f. $524-526$.

- Vitt. - ef. Splanchnomyc. el. - Alger, t. 24. f. $7-11$. 
| 

II ysterangium membranaccum Vitt. - ef. Splanchnomye. m. Cd. - Vitt. t. 4. 15.

- Pompholyx. - Ann. se. n. 151:3 XIX. t 17. f. 7-9. Fructif 'Tul.) - Tul. F. hyp. t. 2. f. $3 ; \mathrm{t} 11$. f. 6 .

- stoloniferum. - Tul. F. liyp. t. 11. f. 7.

II y s terites labyrinthiformis. - Cd. Anl. t. F. f. $59: 31) .31$.

II ysterium. - S. 365.372 , Tyl.; 102 . Dicl. ef. Colpoma, Aporia, Coccomyces, Angelina, IIypoderma, Lophoderma, Psilospora, Hysterographium, Gloniopsis, Aylograpluum.

- abietinum P. = Xylograplia parallela Fr. (I) ub. Hyst. 52.) - Voges. 656 .

- acerinum Wstcl. = pulicare $\mathrm{P}$. (Dub.). - acuninatum. - S. S365.

- acutum Sclı. = Cenangium ac. FI, dan.

- aggregatum. - Kl. II. 15S. = II. elatinum P. (Tub.).

- DC. (non Kl. II. 155.) = Mytilinid. aggr. Dul).

Wallr. = Glonium contluens $\mathrm{D}) \mathrm{b},-$

Norweg. 192.

Audromedae KJ. = Sporomega A. Dub

angustatum. - [S. 364.

- I', Kl. 1259. u. 1566 a. = II. pulicare var. Dub.

Moug., Wrllr., Ns. = Glonimm lineare Not. (Dub.). - Fuck. s. $745 .-$ Voges. 5ti3. -

Wstd. 926.

- apiculatum. - [\$. 3169.] - Kl. 1.165. - KI.

II. 144. - Fuck. s. $743 .-$ Cd. Ic V t. :1.

f. 5. = Lophodermium arund. v. (1)ub.). -

Lib. 73 .

—_ aquilimun Sclıum. - Fl. dan. XIII 2330. 2.

arachnoideum Schum. - Fl. dan. XIII. 2329.3

- Artemisiae Schum.

commune F'l. dan. - Wistl. 1115.

arundinaceum Schrad. - [S. 369.] - Dsm.

12S.1. - Kil. II. 575. - Fuck. s. 751. - I0-

phoderm. ar. Chev. Dub. . - Voges. 655. -

Brit. f. 91. - Wstd. 377.

- australe. - Dub. Hyst. t. 1. f. 13.

Berberidis. - Kl. II. 156. = Iophoderm.

xylom. Chev. (Dub.).

biforme. - [s. 365.]

breve.

- Berk. ; zu Lophium arund. Cher. (Dub.

- Berk. Fg. antarct. 3. t. 65. f. S.

- caricintum. - Kl. II. 723 . - I) $s m$. III. I6s.

- catemulatum. - Palaeont. VIII. t. ๖. f. 9

li. Ludwig'.

cinerascens. - Dub. Hyst. t. 1. f. 15.

cinereum P. - ef. Ostropa c. Not.

- cladophilum Lév. - R. f. 157. - Sporo-

mega el. Dub. - Dsm. HI. 561 . - Voges. 12.13.

- cocciferum Cast. = ('enangium I)ub.

- commune. - S. 365.297.$]$

- Fr. - Fuck. s. 710. - Fl. dan. XI.

1 $\rightarrow$ (j). 2. - Eeptostroma vulgare Fr.

- Dub. = Iypoderma c. Fr. (Iub.).

- Moug. : Hypoderma c. var. (Dub.). -

I.etell. 650.5.- - Vistd. 1115.

- - v. Humuli. - Kl. II. 570.

— v. nitidum. - Voges. 1071.

confluens $\mathrm{Kz} .=$ Triblidium rufulum Spr.

conigenum. - [S. 36s.] - Fr.: Fl. dan. XIII. 2330.3.

Voges. 75 .

Moug. : var. fgi. (Dub. Hyst. 52.). v. Fraxini: Wstd. 224. - Wstd. 92う.
Hysterium contortum, - [S. 365*.] Kl. II. 157.

Dub.

- Corni Kz. - [S. 36S.] - Kil. 562. = IIypoderma C. Not. (Dub.).

- corrugatum. - [S. 36s, 13.]

- corticolum. - [S. 36S.]

- crispum I'. - [S. 36 S.] - Var. v. elatin. P.

Dub.).

- culmigenun. - [\$. 369.] - K1. 3९」.

- Fr. : Fuck. s. 73\% - Dsm. 12s:3. -

Iil. II. 34. = Lophodernium ar. Chev. (l)ub).

- v. abbreviat. Ism. = I.ophodern.

arund. v. (Dub.). - I) sm. III. 171. - Wstd. 923.

_- f. Cerealium lasch, - KI. 1151.

Wstel. 1/:0).

- Cutisii. - Dub. Iyst. t. I. f. 10.

curvatum Dur. - $\mathrm{S}$. 36 b.] = pulicare $\mathrm{P}$. (I) nb.

- decipiens. - Iub. IIyst. t. 1. f. 16. (iloniopsis dec. Not. Rlbh.) . - Ioffm. Ic. t. I . f. I. _- degenerans. - [5. $3(j)^{*} .36$ i . $]$ - Kl. II.

260. = sporomega cladophila Dub.

- $\mathbf{F r}$. = Sporomega deg. (Oub.). - I) sm.

III. 1々2. - Voges. 762 . - lib. 270.

dives. - Act. Turin. 1s!5. VIH. Dec. 3. f. ..

Notar. . = Glonium (l. Not. Dub.)

- clatinum. - [S. 365.] - Voges. 47t.

— crispum Fr. - Bisch. f. 3546.

elevatum.

- - P.: Dsm. 296. - Triblidium hysterinum 1)uf. Dub.). - Voges. 1070.

_ ellipticum. - [S. 365.]

- elongatum. - [S. 365.] - Cd. Ie. V. t. ?.

f. $\{2$. - I) ub. Hyst. t. I. f. 9. - Fuck. s. $7\{!$.

- Moug.: II. Rousselii Not. (Nub.).

Voges. 1It.

Dub.

- v. Rousselii I $\mathrm{sm} .=$ H. Rousselii Nut.

Dub.).

- Epimedii. - Ǩl. 1547.

episphaericum. - [S. 368.]

flexuosum. - Carol. II. 45 .

foliicolum. - [S. 369.] - Wstd. 922. -

Voges. 7i1. - Brit. f. 196. - Lib. 72. - Dsm.

295. и. III. 179.562 .

- Fr.: Dub. Lib. = Lophoderm. $\times y l$.

C'lier. Dub.).

- v. maeulare.

- Will. = Aporia herbar. Fr. Dub.

- ISerk. = Hypoderma ilicinum Not.

Dub. . - Brit. f. 95 .

- v. Hederae Fr. = Hypoderma Hed.

(Dub.). - Voges. 1073.

- Fraxini. - [S.365.] - Schnzl. t. 15. f. 11-

15. - Fuck. s. 738. Hysterograph. Dsin.). -

Berk. In. p. 2\4. f. 66. a. - Dsm. 366. - Kil.

167. ef. Iysterograph. Fr. - Kl, II. 33. -

R. f. 55. - Voges. 267. - Letell. 650. 2. -

Purton. I. t. 32. - Wstd. 224.- Krypt. Bad. 153.

- gramineum. - P. : zu Lophium arund. Chev.

(Dub.).

Moug. Dsm.: zu Lophodermium ar.

Chev. (Dub.). - Dsm. III. 170. - Voges. 368 .

- Wstd. 1120.

grammodes Not. - Kl. II. 57 t. - Var, v.

II. Rousselii Not. Dub. - - Ital. $29 \%$. 
Hysterium graphicum. - S. 36 b.

-- I) ub. = (ilnnium gr. Dub).

- Fr.: Bisch. f. 3is.j.

Hederac. - ('d. lc. l. t. !l. f. ji.

- M.: Fuck. s, í. - 1'ay. b. cr. p. 9.1.

f. 121;. - Wistd. 1017. - Kl. 19.ji.

- Mart.: IIrpoderma IIed. Not. Dub...

- 1I. foliicol. v. II Ied. Fr.; Hypoderma sylo-

moicles $\checkmark$. Ifed. 1)(.; Hypod. IIederae Nut,

IV std. - 1)sm. III. 140.

— herbarum. — S. 365.?

- Juniperi Grev. - Loud. f. llilufi

juniperinum. - Kl. 1654. - W'std. 6.57.

— I.: Fuck. s. T47.

- Lauri. - Ital. $\$ 16$.

I.edi. - S. 365.7

- lenticulare - S. 365. 1.

_ lineare. - S. 3(is.] - Loud. f. 16;i6.

Hysterographium lin. - Wistd. 926.

- longum P. - S. 372 .

maculare. - S. 364. I.oud. f, $1617 \%$.

- Grev, = Hypoderma ilicin. Not. Dub.

- Fr. = Lophodermium mac. Not. Bul).

- J):1u. III. $5(11$. - Voges. 1072. - Jib. 370. Mali Schum. - Fl. dan. XIII. 23:9. 2.

melaleucum. - S. 365. - Loud. f. Itihil.

Fuck. s. 712. - Wstd. 104.).

- Fr. = Lophodermium mel. Not. Dub.

- Voges. 6.j4. - Lib. 17.

micrographum. - Act. Turin. 14t5. VII.

Dec. 1. f. 3. Notar. . = A ylograplium vag. Dsm.

Dub. - Kl. II. 52s.

- minutum DC. - ef. Dub. Hrst. 53.

- Mülleri. - Dub. Hyst. t. 1. f. 12.

naviculare. -

Wllr. = curvatum Fr. Dub.

- nervisequum. - Kl. 195.5; Kl. II. 722 : Hy-

poderma nerv. DC. Dub.

- nigrum Tode. $=$ ('olpoma quere. W'llr. Dub.)

- Wstd. 96.

- Oleae Cast. = Opegrapha 'Dub. .

__ opegraphoides, - Palaeont. VIII. t. 5. f. 7.

Ii. Ludwig.

- Osmundae. - cf. Leptostroma filicinum.

ostraceum $\mathrm{DC}$. = Lophium mytilinum Fr.

D) ub. ; Hypoxylon ostr. Hull.

- oxycoccos. - [S. 365.]

parallelum $\mathrm{Whl}=\mathrm{Xylographa}$ parallela $\mathrm{Fr}$.

(1)ub.).

pedicellatum schum. = 1I, pulicare $\mathrm{v}$. ped.

Fl. dan. - Ostreichnion europ. Dub.

_- petiolare. - [\$. 369.] - Kl. 1730 est Pe-

ziza, Dub. - Dsm. III. IS1, 563. - Lib. 74.

- Wstd. 35.

- Pinastio.

1 4.53. LX. Stein .

—- Schrad. : Fuck. s. 750. - Kil. 560. -

Dsm. = Iypoderma Desmazierii Dub. .

- Schrad. = Lophodermium I'. C'hes.

I) ub.,. - Voges. if. - Carol. I. 11. - Letell.

tisil. 1. - Wstel. 223. 1222. Inajor - Ital iั).

_ - v. juniperin. Fr. Dsm. = Lophoderm.

junip. Not. Jub., - Kl. 165. - Kil. II. 115.

- - Fr. Dsm. 367; III. 1\$3. — Wstd. (j5:.

1222

Pini Schum. - Fl. dan. XIII. 2331. 2.

- pithyum Kiz. - Kl. 561 . 'zu Phaeid. pith.,

Dub.

- Polygoni - [s. 3(is.]
IIysterium Populi Schum - Fl. dan. XIII. 2i331. 3.

- I'rostii lick $x=$ pulieare I. (1)ub.).; Opeeraplia l'rostii Nyl.

- 1)ub. - S. 3(is.] - Voges. 1145.

pulchellum Fr. ef. melaleucum Fr. Wstcl.). - pulicare. - $\$$. $31 ; 4$.$] - Fl. dan. XIII. 2:331.$ 1. - ('d. Anl. t. F. f. 59: 5-4. - Jisch. f. 3.561 - 1) sm. 365. — Iysterographium Iil. 1.169. u. 1.566. b.

—— 1.: Fuck. s. 752. - Voges. 266. -

Carol. IV. 25. - Letell. (i5). 1. - Norweg. 1.2.

- Lib. 1 1. W Wtd. 326. - Ital. テて. - Krypt.

l3arl. .joli.

- f. Hetulae. - Kl. II. 261.

2331.1

- punctiforme. - S. 369.$]=$ Jophodermium xylomoides C'lier. Dub., - Fuck. s. 756 . -

1i. f. 462 . I Iib. $2 i 1$. quercinum. - Cd. Ic. V. t. 9. f. 59.

_ L'. C'enang. q. Fl. dan, ; Colpoma $\mathrm{l}$.

Wllr. Dub., - Voges. 36i. - Wstd. 96.

repandum Bl. - Dub. IIyst. t. 1. f. 6.

- Robergei $\mathrm{Dsm}$. = Lophodermium arundinac.

v. 1)ub. - Dsm. III. 169.

Rochcanum. - Dub. IIyst. t. 1. f. 11.

rotundum Bernh. - Riöm. Arch. I. t. 1. f. 5 .

- ef. Ostropa cinerea Not.

Rousselii Not. - Dub. Hyst. t. 1. f. 7.

- - v. grammodes. - Dub. Hyst. t. I. f $\$. Rubi. - S. 36〉.] - Cd. Ic. I'. t. 9. f. sti.

- Cd. Anl. t. F. f. 59: 1-4. - Voges. 564. Letell. 6.50. 3. - Lib. 17 - Wstd. 921.

— Pers. : Fuck. s. 753. — Loud. p. 11333.

f. 16478. - Ann. se. n. 1 553. XX. t. 15. f. 10 - 14. Tul.) - K1. 5.59. - Iypoderma virgultorum DC. Dub. - Kil. II. j43.

P. = virgult. Dsm.

- rubrum Fr. = Hymenula Dub.

_- rufescens Fr. = Angelina ruf. Schw. (Dub.).

- rufulum. - Carol. II. 17.

- rugosum. - [\$. 402.] - Kl. II. 450. - Fuek.

s. $73 \%$ - Wstd. 925. 921. fagin. et querein.).

- $v$ fagineum Wllr. ef. Psilospora fag.

- salicinum. - [S. $36 \mathrm{~S}$.

- Salicis. - [S. 520.]

- samarae. - [S. 369.]

__ samararum Lasch. - Kl. 1259. (an H. samarae Fr. : = Phoma Dub.).

- Sambuci Fr. - [S. 103.] - Fl. dan. XI. 1460.3.

f. it.

- Fr. : = Hypoderma se. D('. Dub.)

I) $\mathrm{sm}$. 369. - Bisch. f. 35si. - Kil. 1152 ; Cent.

XV, Suppl. - Kl. II. 262. - Voges. 47j. Wstd. 1221.

- seriatum. - Iib. 371. - Wstd. 1016. -

Lophodermium ar. ('h. var. Dub.

- Smilacis. - Dub. IIyst. t. 1. f. I1. - Ca-

rol. II. 49.

Sorbi. - S. 415.]

- sphaeroides A. S. - S. 365.] - Bisch. f.

3.5র. - Lophoderm. sphaer. Dsm.

- f. Iihododendri. - R. f. 355.

striaeforme Irllr. = Phoma Dub.).

Striola. - S. $106 \%$ ?

Taxi P. - Kil. 291.

truncatum. - (5. 401.]

- tuberculosum Schuin. - [S. 520.] - ? Fl.

dan. II. 23:9. 1. 


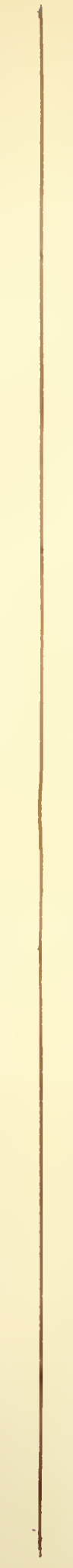



II ysterium tumidum. - [S. 364.] - (d. Ie. Y. t 9 . f. ว.ร.

1121. c. v. trigon.

- Typhae. - [S. 365.]

- Vaccinii Carm. = Sporomega clodophila Dub valvatum Ns. - Wstd. 1117.

- variegatum Berk. = Hypoderma var. Dub.

- varium. - S. 365] - Bail S. t. 15.

- versicolor. - [S. 36५.] - Whlbg. Fl. lappon. 1912 . t. 30 . f. 5 .

- virgultorum $\mathrm{Dsm}$. = IIypoderma virg. DC: Dub. - - I)sm. III. 172-175. - Wistd. 921. Rubi ; 1119 quercin.

— Wallrothii. - Dub. Iyst. t. 1. f. 5.

IIysterographium. - I. 36 cf. Ilysterium. - acerinum. - Bull. ac. belg. Ksis. VII. P.

366. f. 14. Wstd., - Wstd. 927.

- conigenum Fr. - Wstd. 925.

elongatum. - 1'ay. b. cr. p. 94. f. 42.5. -

Cd. Ic. I. t. 9. f. (f). = IIysterium el. Winl.

Dub. - Dsm. III. 154 .

- Eupatorii Lase?. - R. f. Jis!l.

73)

Fraxini Dsm. cf. Ilysterium $\mathrm{I}^{\mathrm{r}}$ - Fuck. s.

- Not. = IIysterium F. I'. Dub.. -

Dim. III. 155, 565. - Ital. 7\% - IIyst. Ole-

astri Cast.

- f. Oleae: li. f. 3isti.

- lineare Fr. Wstd. 926i.

I) ub.

Oleastri ('ast. Dsin. = Hysterium Fraxini

- Prostii $\mathrm{D}_{\mathrm{sm}}=$ Hysterium Pr. Dub. - D sm.

III. 156 .

- pulicare. - C'd. Ic. V. t. 9. f. (il ; Hyste-

rium pul. I. Dub. .

II ysteromyces. - S. 435.]

Hystricapsa trochiformis Preuss. - Sturm h.

36. t. 9.

Ileodictyon. - [S. 435.]

gracile. - Hook. Lond. J, 15-15. IV. t. 2.

f. ᄂ. - Cd. Ic. VI. t. 5. f. 57.

Illosporiums. - [s. 4-1.]

- aurantiacum Lasch. - R. f. 74. - Fuck. s.

206. - Fuck. rh. 239.

- carneum. - S. 4\$1.] - Kl. 414. - R.f.

73. b. Cent. IV, Suppl. - R. f. 73. - Brit.

f. 293. - Lib. 353. — Wstd. $\$ 9$.

—— coccineum. - [S. $151.452 \cdots$.$] - Fuck, s.$

204. - Toges. 996. - Lib. 241. - Fuck. rh.

240.

- corallinum Rob. - Dsm. 1151.

- fagineum. - [\$. 452*+.] Lib. 155.

- niveum. - Dsm. 1732.

- persicinum. - S. $4>1$.

- puniceum. - Lib. 252.

- roseum. - [S. 4\$1.] - Dsm. \$2. - Schnzl.

t. 14. f. I-3. - Willkomm, Wunder d. Mi-

krosk. 1556. p. 87. f. 40. c. - Kil. 957. - R.

f. 7 . - Schwabe, Fl. anhalt. II. t. 6. f. 5. -

Iib. 77. - Fuck. rh. 241.

Inoconia. - Michelii: Lib. 96.

Institale. - [S. 44\%.]

- acariforme. - Carol. V. 52. (status morbosus Hypoxyli fragiformis).

Irpex. - [S. 32S.]

- candidus Weinm. - Kl. 1214.

- canescens. - Cd. Anl. t. G. f. 94: 9. - Bail

S. t. 29.

carneo-albus. - [S. 32S.]

cinnamomeus. - cf. fuscescens.
Irpex coriaceus. - Carol. III, 21.

crispus. - [S. 324.]

deformis. - Hoffm. Ic. t. 17. f. 3 .

- fuscescens? - Carol. I. 27. cinnamom. Fr.

- fusco-violaceus. - [S. 324.] - Kl. 230. -

lil. II. 115. - R. f. 369. - Dsm. III. 794. -

Agar. decips. Willd., Sistotrema viol. b., I'ers.,

Hydnum Fr. S.

_ lacteus. - - S. 325.]

- maximus Mont. = Polypor. labyrinthif. Fr.

- obliquus. - S. 325 - Kl. 121. - R. f.

213.

- pallescens. - Carol. I. 26.

- paradoxus. - [S. 324, - Kl. 1405.

- pendulus. - C'd. Anl. t. G. f. 7t: 7. S. -

Bisch. f. 3411. - Kil. 621 - R. f. 19.

— sinuosus. - [S. 324.] - R. f. 116 .

— spathulatus. - - 32.32 - Bisch. f. 3322.

- tabacinus. - Carol. III. 22.

I - aria. - [\$. 464 .] - ef. Imphichorda [s. 164.]

¿auf Raupen. - Robin v. par. t. 9. f. 6.

— agaricina. - ef. Tul. sel. I. 113.

- arachnophila Dt. - S. 4ijt.] - Bisch. f.

3769. - Letell. fi!l 2. - sturm h. 4. t. 5.5.

- brachiata. - [S.461. - Fl dan. XIII. 2240.

3. - Kl. 1126. - Kil. II. 331. - Fuck. s. 25.

Fuck, rlı, $16 \%$.

- bulbosa Ns. - Bisch. f. 3753.

- calva Fr. - Bisch. f. 3775.

- candida. - S. $161^{*}$.

- carnea. - \$. 464.

- citrina, - [S. 161 l.] - Cd. Anl. t. G. f. il:

5-7. - Pay. b. cr. p. 76. f. 351. - Bisch. f.

3756. - Kl. II. 133. - zu I. agaric. T'ul. . -

sturm h. 3. t. 37 .

- clavata Ditm. - Bisch. f. 3̈tis. - S'chnzl.

t. 12. f. 4\$. 49. - sturm h. 1. t. 56.

- crassa. - l'ay. b. cr. p. 5s. f. 241.

- dendroidea, - Jungh. pr. t. 1. f. 6.

- eleutheratorum Ns. - s. 464.] - Bisch. f.

3i74. - Kl. 1543. - zu Claviceps entomorhiza

Dicks. Bail

- — r. racemosa Iwd. - Kl. S72.

epiphylla. - S. -164.] - zn I. agaric. T.

Tul.j. - Letell. 69y. 4.

— farinosa. - \$. 461.] - Ki. 1666i. u. 1749.

- zum Formenkreis der Empusa Muscae Bail.

- R. f. 575 .

- - velutipes Fr. - Bisch. f. 37 tell. 695.1.

- fasciculata. - [S. 464.]

- filiformis. - Kl. $166 ; 5.5$

- Friesii. - [S. $\left.461^{* \rightarrow}.\right]-$ Anm, sc. n. 1436.

V.t. 12 .

- glauca Ditm. - Bisch. f. 3767 . Kil. 1427.

- Sturm h. 2. t. 19.

- intricata. - [\$. 464.]

- leprosa. - [S. 464.]

- _ microscopica Grer. - Loud. f. 16.552.

- monilioides A. S. - [S. 467.] - Bisch. f.

3752. - Letell. 699. 3.

— puberula. - Ann. IIg. 1. h. 1S41. VI. t. 12.

f. 12. (Berk.).

_- ramealis. - [S. 464.]

- sphecophila Ditm. - [S. 464.] - Bisch.f.

3776. - Sturm h. 4. t. 57.

- Stemonitis P. ef. Stysanus St. [S. $467^{* *}$.]

- strigosa. - [S. 464.]

- sulphurea. - R. f. 60.

- terrestris. - [S. 464.]

- tomentella. - [S. 464.]

- umbrina P. - R. f. 172. - Fuck. s. 260. 
Is aria velutipes. - Sturm h. i. t. $\mathrm{j}$ i.

Isariopsis pusilla lires. -- Fuck. s. 2li2. Fuck, rh. 171. - l'hacellium lion. - Fresen. t. 11. f. 15-2s. 1). 4\%.

Is o thea. - $\$, 121$.

Junghulnia. - \$. 315. XXII

Kentosporium. - cf. Claviceps. mitratum Wllr. - ef. Clavic. purpurea. IV'std. 1201.

Kineiffia. - S. 536 .

— candidissima. - ('arol. T. 32.

— setigera. - Caro1. V. 31.

Kretschmaria. - S. Jiv9.

L aboulbenia Guerinii. - Robin r. p. t. !)

- Rougetii. - Robin v. p. t. \&. f. 1. 2; t. 10. f. 2. 9 .

Labrella. - S. 422 .

- cedrina. - Nlger. t. $2 \bar{i}, \overline{7}$

- Cruciferarum. - \$. 422.

- Fagi Rob. - Gloeospora F. Wstd. - Dsin.

III. $T \%$ - ITstd. 116i.

- inervisequa. - Fuck. rh. 427. 124

- Periclymeni, - Dsm, III, it.

Pomi. - is. 122.

- Mont. = Sphaeria hians Wllr. - Fuck. s. 3>6. - Dsin. 300. - Voges. $11>6$. Xyloma pyrig.'

- Ptarmicae. - Dsm. 117. - Kil. 1394. - Ann. Mg. n. h. 1৮3 . I. t. 7. f. 7. Berk. - Toges. 957. - Wstd. 375. - Schizothyrium Pt. Dsm.

- Punctum. - Cd. Anl. t. F. f. 601: 1-3.

Posacearum. - Bail S. t. 15.

Rosae. - S. $402 \% \rightarrow \rightarrow$.]

La chnella. - S. 365 . ef. Peziza.

Lachnobolus. - S. 457 .

Lactarius. - ef. Agaricus.

Lamyella. - S. 110 .

Langermannia. - S. 412. ef. Bovista; Sturm

h. 1s. t. $10-14$.

Lanopila. - [S. 411 .]

Lanosa. - s. 159 * ; 495 .

Laquearia. - S. 366.

Lasehia. - S. 315 . XXII; 325.

- infundibuliformis. - Ann. Mg. n. h. $15 i 2$

IX. t. 9, f, 3. 'Berk.

— papulata. - Chile n. 7. f. S.

Lasiobotrys. - S. 406.$]$ - cf. Dothidea Veronicae.

- Lonicerae. - $\$$. 406.$]$ - Anm. Mg. n. h. 1 52 . IX. p. 3>7. t. 12. f. 14. Ant. B. B. . Cd. Anl. t. F. f. $53: 1-\bar{i}-$ Bisch. f. 3701. Dsm. 257. - Loud. f. 1619s, - Schnzl. t. It. f. 9. - K1. II. 665. - Voges. $\$ 60$ - Brit. f. 45. - Ital. 19.

Lasioderma. - S. 446.

- flavovirens. - Alger. t. 20, 4.

Lasiosphaeria ef. Sphaeria.

L a ternea, - [S. $435^{*}$.] cf. Clathrus.

- columnata. - Bail S. t. 23.

- triscapa. - Cd. Anl. t. E. f. .1s: 1.

Leang i um. - S. 450.] cf. Polyschismium.

- floriforme Lk. - Loud. f. 16525.

lepidotum. - Cd. Anl. t. C. f. 29: 21. 22 .

- K1. 1514. - Sturm h. 2. t. 21.

— stipitatum. - [S. $45 \%, 2$.

- Trevelyani Grev. - [\$. 450.] - Bisch. f. 3650 .

I. ecanidion. - Bail S. t. 19.

- atrum Rbh. - R. f. 33. - Fuck. s. 765.

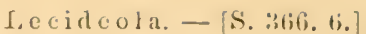

Leeythea, - [S. 511.] ef, Epitea, Uredo.

1, eeythes. - S. j]1.

1, eiophat 11 us. - S. $134^{*}$. . ] ef. II ymenoph.

I. em a 1 is. - S. Btit).

- Alismatis. - [S. 360.$]$

Le mbosia. $-\left[\right.$ s. $370^{-*}$.]

l, ent in us. - ef. Agaricus.

castoreus. - [s. 31\%.

- cirrhosus. - Afz. t. 10. f. 21.

- cochleatus. - [S. 314.

erinitus, - I.inn. Trans. XX. t. 9. f. 1.

Berk. . Ann. Mg. n. h. 1542. X. t. 9. f. 2.

Berk.

—- dactyliophorns Tév: - Bonite t. 136. f. 2. descendens Scleroma . - Afz, t. 10. f, 22.

— eugrammus Mont. - Sagra, Cuba t. 1\%.f. 2. flabelliformis. - \$. 314.]

friabilis. - S. 314 .

- fulvus. - Ann. IIg. n. h. 15-12. X. t.9. f. 1. Berk.

- giganteus. - Hook. Lond. J. 1547. VI. t.

17. 15. f. 2

- herbarum. - S. 314.

— inquinans. - Hook. J. VI. t. $i . f .1$.

—_ Lecomtei. - Carol. I. I.

_- lepideus. - [S. 314.]

—_ leucochlorus Lév. - Bonite t. 140. f. 1.

- Léveillei. - Linn. 'Traus. XX. t. 9, f. 5. Berk.

- maculatus. - Hook. Lond. J. 15 17 . YI. t.

19. f. 2 .

- Momnardianus. - Alger. t. 20, 2.

- Nicotiana - Hook. J. VIII t. 5 . f. $\bar{c}$

- nigripes Fr. - Linn. Trans XX. t. 9. f. 4.

Berk.

obnubilus. - Hook. Lond. J. 1S-17. VI. t.

19. f. 3.

_- praerigidus - Hook. J. 1 5 4. VI. t. \. f. 1. Ravenelii. -- Carol. II. 11

ringens. - S. 314.1

- Schomburgkii. - Limn. Trans. XX. t. 9. f. 3. Berk.

- setiger Lér. - Bonite t. 136. f. 4.

stenophyllus. - Ifook. Lond. J. 1547. VI.

t. $17.15 . \mathrm{f} .1$.

- suavissimus. - [S. 314 .

- tener K1. - Linn. Trans. XX. t. 9. f. 2.

Berk. .

tigrinus. - S. 314. - Bail S. t. 32.

- umbellatus. - S. 314.]

_. ursinus. - S. 314.]

- velutinus Fr. - Sagra, Cuba t. 17. f. 3.

vulpinus. - S. 314 .

s,c nzites. - S. 315.9 cf. Daedalea.

- abietina, - S. 315 - Disch. f. $343 \%$ -

Fuck. s. 102 ว. albida. - S. 315.

betulina. - is. 315.$]$ - Kops Fl. bat. X.

790. 1. - Berk. Out. t. 15. f. 3. - Bail S. t.

32. - R. f. 14. - Fuck. s. 1025. - Dsm. 1123.

- Carol. II. 14.

- bicolor. - Carol. II. 15.

— Haccida. - S. 315.$]$

- Guineensis. - Afz. t. 12. f. 26.

- heteromorpha. - S. 315 .

- Palisotii. - Afz. t. 11. f. 21.

- - sepiaria. - [S. 315.] - Kops Fl. bat. X.

790. 2. - Fuck. s. $11126 .-$ Usm, 1505. -

Daedalea asserculorum, Voges. 401.

- striata. - Carol. I. 7.

tricolor. - Ital. $1+1$. 


$$
1
$$




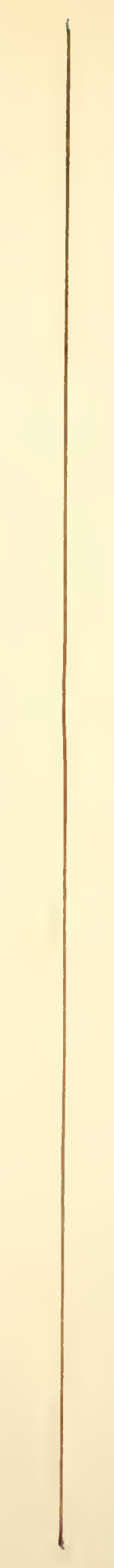


I. enzites variegata. - [S. 315.]

- verrucosa. - Bull. ac. belg. IS 11 . VIII. 2. t. 1. (Kx.).

L, e ocarpus. - [S. 450.]

- atrovirens. - [S. 450.]

— calcareus Lk. - Kl. $\$ 17$; u. dito Cent. XVII.

- Fuck. s. 293.

- chalybeus. - 'S. 450.]

- contextus. - is. 450.$]$

- cyanescens Fr. - [S. 450.] - Kl. 992. Diderma).

deplanatus. - [S. 450.]

- flavus. - [S. 150.$]$

- fragilis. - Purton. I. t. 24. f. 3. (vernic.)

granulatus. - [S. 451.]

- minutus. - [S. 451.]

_.. nitens. - [S. 450.$]$

ramosus. - [S. 450.]

- vernicosus. $-[\mathrm{S}, 450]-$. Cd. Ic. V. t. 3. f. 32. - Cd. Anl. t. C. f. $25: 14.15$. - Wstd. 1063. (Diderma). - Fuck. s. 294. - Loud. f. 16537. - Kl. II. 37. - Bot. Zt. 1559. t. 11. f. 15. (Keimg. Hoffm.) - Carol. I. 75. - Purton. I. t. 24. f. 3. - Krypt. Bad. 49.

Leotia. - [S. 357 . ]

- atropurpurea. - Cd. Ic. V. t. 9. f. 71.

- atrovirens. - R. f. 522.

- chlorocephala. - Carol. IV. 22.

- circinans P. - [S. 345.] - Kl. 139. - Fuck.

s. S65. (Cudonia). - R. f. 35. 312 .

- Clavus. - [S. 361.] - Voges. Tol.

elegans. - [S. 347**.]

- fimet. P. - cf. Helotium fim. Fr. Su.

gelatinosa. - Linu. 1542. XVI. t. 9. f. 32

-10. (Fructif. Näg.). - Wstd. 763. geoglossoides. - Pay. b. cr. p. 55 . f. $37 \%$.

-1 Cd.: = Geogloss. vir. Fr. Ep.

- infundibuliformis Fr. - Loud. f. 16206.

__ lubrica. - [S. 357.] - Nov. Act. 1542. XIX.

II. t. 57. f. $136-145$. (Fructif. Phöb.) - Cd. Anl. t. G. f. $66: 15-17$, - Ann. Mg. n. h. 1835. I. t. 5. f. 52. (Spor. Berk.). - Loud. f. 16205. - Dsm. 354. - Bail S. t. 21. - Berk. Out. t. 22. f. 1. - Kl. 911.- Kl. II. 29. a. b. Fuck. s. 759 . - Sturm h. 31 - t. 22. (flavovirens, Szl.). - Voges. 553. - Brit. f. 255. Wstd. 763. - Vent. mi. 53. f. 1. 2; :97. f. 4.

- v. lacunosa. - Kl. 1628.

Mitrula P. = Mitrula Heyderi Fl. dan. truncorum. - cf. Vibrissea tr. [S. 361*. uliginosa. - Voges. 655. - Cd. Anl. t. G.

f. $66: 15.19$. - Wstd. 234.

Leptiola. - [S. 496.]

J.eptomitus. - [S. 524.]

Leptosphaeria cf. Sphaeria.

Lep tospora Hübnerii Rbh. - Kl. M. 531. porphyrogona. - Hdw. I. t. 15. f. 1.

— rubella Rbh. (Sphaeria rub. P.), - Kl. II. 532.

Leptospori um tremellinum. - Bot. Zt. $155 \%$. t. 4. c. (Bon.).

Leptostroma. - [S. 371.521.] cf. Hysterium. acerinum. - Bail S. t. 15 .

_- Camelliae. - Flora 1\$34. I. t. 3. (Zenk.). caricinum. - [S. 371.] - Kl. 353. - Fuck.

s. 710 . - Wstd. 1247. - Fuck. rh. 156. v. Eriophori. - Kl. II. 665.

Cytisi. - Fuck. rh. 195.

filicinum. - [S. 371.] - Bisch. f. 3603. Fuck. s. 711. - Dsm. 299. - Kl.653. - Wstd. 1048. - Voges. 673. (Sclerotium Pteridis). Fuck. rh. 187.

- v. Osmuud. = Hysterium Osmundae

Hoffm a nn, Index Fungorum.
S.; Schizoderma filic. E.; Hypoderma striaeforme DC. (Wstd.).

L eptostrom a herbarum Lk. - Fuck. rh. 159.

- - v. Euphorbiae. - Kl. 765 .

hypophylla. - Carol. III. 45.

- hysterioides. - [S. 371.] - Kl. 1260. -

Lib. 374 .

- graminicolum. - Act. Turin. 1945

VII. Dec. 3. f. 6. Notar.).

- juncinum. - [S. 371.] - Kl. 43\% - R. f.

59. - Voges. 653. (Xyloma varium). - Brit. f.

197. - Lib. 260. - Wstd. 1247. - Fuck. rh. 193.

- laricinum. - Fuck. rh. 196.

- Liriodendri Lk. - Wstd. 450. - Kl. 1073.

_- litigiosum Dsm. - Dsm. 727. - ef, Sclerot.

Pteridis Moug. - Wstd. 76 .

— longissimum. - Lib. 259.

__ loniceraecola Rbh. - Kl. $\$ 65$.

- Luzulae Lib. - Lib. 75. u. 375. - Wstd.

1049 .

_ inaculans. - Fuck. rh. 194.

— maculare W. - cf. Melampsora Lini Dsm.

- Phragmitis Fr. - Kl. 766.

- Polygonati Fr. - Kl. 352. - Fuck. s. 717.

- Fuck. rh. 192.

- Pteridis Ehrb. - Dsm. 371. - Wstd. 150.

— punctiforme W1lr. - Fuck. s. 715. - Fuck.

rh. 190 .

- quercinum Lasch. - Kl. 1075.

- rugosum. - [S. 371.$]$

- salicinum I.k. - Fuck. s. 2s, d.

- Scirpi. - kl. II. 496. - Wstd, 1215.

- scirpinum. - [\$. 371.] - Bisch. f. 3559. -

Dsm. 3 70. - Kl. 351. - 1. xylomoides Fr. Obs.

- Schizoderma sc. Ehrb. - Stat. spermog. v.

Hypoderma scirpinum DC. : Dub.

scriptum. - [S. 373.]

— Sedi Lk. - Fuck. s. i13. - Fuck, rh. 191. sphaerioides. - [S. 371.]

Spiraeae. - [S. 371.] - Brit. f. 46.

stipatum. - [S. 371.]

_ vulgare. - [S. 371.] - Kl. 652. - Dsm.

295. - Spermogon. v. Hypoderma commune

Fr. "Tul. Dub. . - Voges. 674. Sclerot. nitid.

P.). - Carol. IV. 27. - Lib. 166. - IYstd.

1125 . - Fuck. rh. 197 .

—_- Verbenae. - Kl. II. 753. - Wstd. 1246.

Leptothyrium. - [S. 3:1*.423.424.] cf. Leptostroma.

- acerinum. - Kl. 1551.

- alneum. - Lib. 361. - Dsm. 1022. (Xyloma,

Dothid.).

—_ Betulae. - Lib. 163.

- Carpini. - Lib. 256. - Wstd. 976.

- decipiens. - Crypt. Bot. antarct. voy. Ere.

bus. 1554 . t. 163.3.

- Dryadearum. - Dsm. 1231.

Fragariae. - Lib. 162.

Helicis. - Dsm. 1333.

Juglandis. - Wstd. 37. - Dsm. \$40. -

Iib. 164. (Xyloma, Depazea) .

— Lunariae. - Kl. 767 .

- Mezerei. - Lib. 360.

Populi. - Lib. 25\%. - Wstd. 543.

Rhois. - Wstd. 544.

- Ribis Lib. - Lib. 25s. - Dsm. 1250. -

Wstd. 179.

Tremulae. - Lib. 161. (Xyloma concentricum P., Depazea frondicola Fr...

- Veronicae. - Lib. 362.

Leptotrichum. - [S. 495.] 
Leptotxichum glaucum. - Cd. Ic. V. t. 2. f. 16. - Cd. Anl. t. B. f. 10: 3. 4. - Pay. b. cr. p. 73. f. 325 .

Le ucina. - [S. $467 * *$.

Leucosporium. - [S. $472 * *$.]

- vesiculiferum. - Ns. j. Syst. t. 3. f. 1-3.

- Sturm h. 13. t. 34 .

Leucostroma Cast. = Erysiphe ('Tul.).

Leveillea. - [S. 409.]

Levicuxia. - S. 415 .

Libertella. - S. $\left.413^{* * *} .426.\right]$

acerina. - Wstd. 1190.

—_ alba, - Lib. 364. — Wstd. 955. 1252.

betulina. - [S. $426^{* * *}$.] - Ann. sc. n.

1530. XIX. t. 5.f. 4. (Dsm.). - Lib. 363. -

Ita1. 415. (Naemaspora aurea).

- crocea. - Bon. t. 3. f. 63. a. c.

Equiseti. - [S. 426.]

faginea. - [S. $426^{* * *}$.] - Dsm. 141. -

Ann. sc. n. 1530. XIX. t. 5. f. 5. (Dsm.).-

Wstd. 335 .

- fusca. - Bon. t. 3. f. 70 .

- macrospora. - Wstd. 1252.

nigrificans. - Bon. t. 3. f. 63. d.

- Rosae. - Ann. sc. n. 1530. XIX. t. 5. f. 6.

(Dsm.). - Bon. t. 3. f. 63. e.

Licea. - [S. 45S.] cf. Phelonitis, Physarum. alba. - cf. Didymium, Let.

Artocreas. - Carol. II. 82.

atra. - [S. 45 4. 30.]

badia. - [S. 459 .]

bicolor P. - Lycogala pariet.

caesia Scluum. = Physarum caes. Fl: dan.

contorta. - [S. $45 \mathrm{~S}$.]

cylindrica. - [S. 459.] - Kl. 349.

- fragiformis. - [S. 459.] - Loud. f. 16544.

- macrospora Schum. = Polyang. umbr. Fl.

dan. [S. $159^{* *}$.]

- nitens. - [S. 459.]

- pusilla. - [N. 453, 24.]

- serpula. - [S. 459.]

strobilina. - [S. 45S*] - Voges. 579. -

Norweg. 59. - cf. Perichaena. - Sturm h. 2. t. 20 .

- suberea. - [S. 459.] - Cd. Anl. t. C. f. 27 :

$14-16$.

- sulphurea. - Kl. 1545. - cf. Anixia trun-

cigena Hoffm. - Bot. Zt. 1559. t. 11. f. 12.

(Keimg. Hoffm.).

- tubulina. - [S. $449 * * *$.]

- umbrina. - [S. 459.]

- variabilis. - [S. $459^{*}$.]

Lichen aidaelus. - Wstd. 1254.

- hippotrich. - cf. Tul. Sel. I. 149.

- radiciformis. - Wstd. 1255.

Lichenopsis. - [S. 416 .]

- sphaeroboloidea. - Carol. I. 72.

Lignyota. - [S. 459.]

Lim bosa. - [S. 406 ; ast.]

Lind bladia. - [S. 449.]

Lituaria. - Bot. Zt. 1553.t. 3.f. S-10. (Riess.).

Lizonia. - cf. Sphaeria.

Lophiostoma. - cf. Spliaeria.

Lophium. - [S. 401.]

aggregatum. - [S. 401.] = Mytilinidion.

dolabriforme. - Dub. Hyst. t. 1. f. 4.

- elatum. - [S. 401.] — Ann. sc. n. 1842.

XVII. t. 5. (Dsm.).

- Grev.: Loud. f. 16455. - Dsin. 1030.

Lonicerae Fr. - ? Wstd. 909.

mytilinellum. - [S. $\$ 01$.

mytilinum. - [S. 401.] - Loud. f. 16459.
- Bisch. f. 3543. - Bail S. t. 18. - Schnzl. t. 15. f. 1 fi-20. - Pay. b. cr. p. 94. f. 427 . Kl. 1150. - Kl. II. T14. - Hysterium myt. P. Ns., ostraceum DC., Hypoxylon ostr. Bull. (Dub.).

L. o p hi um.unguiculatum Wrllr. - Ostreichnion europ. Dub.

Lophoderma. - S. 371.] ef. Hysterium Rubi ('lul.).

- Pinastri. - Wstd. 223.

- Rubi Chev. - Wstd. 921.

— xylomoides Chev. — Wstd. 922.

1. ophodermi um. - ef. Hysterinm, Phacidium.

- arundinaceum. - Wstd. 377.

culmigenum Not. = arundinac. Chev. (Dub.).

herbar. Chev. - Iypoderma commune Fr.

Dub.), Leptostroma vulg. Fr.

- Juniperi Not. = Hyster. Jun. (Tul.).

- laricinum Dub. - R. f. 158.

Neesii. - Dub. Hyst. t. 2. f. 23.

- Phacidium Not. - Placid. coronat. Fr.

—_ Pinastri. - Ital. 779; - Hysterium P.

— quadratum. - ef. Phacidium.

_- Rhododendri Ces. - R. f. 458. - Ital. 537.

- ? sphaeroides Dub. — cf. Hysterium sph. -

R. f. 355 .

I, undia. - [S. $409^{* *}$.

L ycogala. - [S. 448.$]$

- argenteum P. - Loud. f. 16539. - cf. Re-

ticularia ar.

- cinereum Schum. = plumbeum Fl, dan.

- _ conicum. - [S. 14\%.]

- contortum Dit. - cf. Trichia retic. - Sturm

h. 1. t. 5 .

— epidendrum. - S. 445.] - Fl. dan. XII. 20S6. 2. - Krypt. Bad. 330. - Kops Fl. bat. XI. Su5. 2. - Bisch. f. 3673. - Cd. Ic. V. t. 3. f. 40, - Cd. Anl. t. C. f. 36: 1.2. - Ztschr. f. wiss. Zool. 1\$59. X. t. 9. de Bary - Kil. 933. - Fuck. s. 300. - Iil. II. 243. - Carol. II. 74. - Huss. II. 3. - Wstd. 711. - Ital. SST.

- v. carnea Fr. - Fl. dan. XII. 2150. 2. - ferrugineum Sehum. = L. epidendrum Fl. dan. - Wstd. 741. globosum Mich. - Wstd. 741.

___ griseum majus Mich. ef. Reticularia arg. Cd. miniatum P. - Loud. f. 16535. - Verh. zool. bot. Ges. Wien. 1S59. Fbr. t. 1. f. 1.

Keimung. Bail). - Voges. 85. - Wstd. 741.

- minutum Grev. - Loud. f. 16540.

- parietinum Fr. - [S. 145.] - Dsm. 166. -

Voges. 1092.

- plumbeum. - [S. 449.] - ?Fl. dan. XII.

$20>6$. 1. u. 3 .

- punctatum Ns. - Wstd. 741.

— terrestre Fr. - [S. 445.] - Cd. Ic. VI. f. 37.

- cf. plumbeum Fl, dan. - Carol. IV. is.

_- verrucosum Batsch. - Wstd. 741.

Lycoperdon. - [S. 442.] - Ann. sc. n. 1942.

XVII. t. 1. f. 2. (Capillit. Tul.) u. 1\$37. VIII. - Flora 1543. t. 4. Fructif.). - cf. Langermaunia.

- Anemones Pultn. - Wstd. 662.:

- arenarium. - Gaudich. voy. 1526. t. 1: 1.

- areolatum. - Sturm h. 18. t. 5.

- Bovista. - [S. 442*. - Kops Fl, bat. IX.

t. 720. 2. - Vent. t. 12. f. 115. 119. - Bisch.

f. 3659 . - Loud. f. 16507 . - Schnzl. t. 14. f. 43.41 .

- caelatum Bull. - [S. 442.] - Kops Fl, bat.

IX. t. 715. 1. - Bisch. f. 3604. - Ann. IIg. 


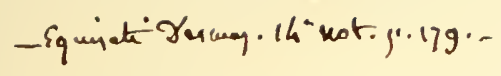



n. b. 1510. IV. t. 5. f. 1-6. Fructif. Berk., Hiz. t. 74. - Berk. Out. t. 20. f. T. - Kl. II. 512. - Fuck. s. 311. - Ann. sc. n. 1539. XII. t. 2. Fructif. Berk. - Berk. In. p. 335. f. 75. d. (Anat.) - Huss. II. 23.

L y coperd on cancellatum L. - Wstd. 123.

- cepaeforme. - Schnzl, t. 14. f. 4 S $\div$

Pay. b. cr. p. 111. f. 50 S.

- cinereum. - Wstd. 3\$2.

- constellatum. - [S. 412.$]$ - Kl. 1111. -

Fuck. s. 313. - Bot. Zt. 1559. t. 11. f, 15.

(Keimg. Hoffm.) - Sturm h. 15. t. 7.

- Corium DC. - cf. Mycenastr. Cor. Desv.

—_ corniferum Müll. — Wistd. 712.

- cruciatum. - Sturm h. Is. t. 8 .

cyathiforme. -- Carol. IV. 71 .

defossum. - Act. Turin. 1543. V. t. 3. f.

15. Vittad. - Purton. I. t. 19. f. 2.

epidendrum L. - Wstd. 741.

epiphyllum L. - Wstd. 663 .

Euphorbiae Schrank. - Wstd. $53 \%$.

_- excipuliforme. - Kops Fl. bat. IX. 715. -

Loud. f. 16509. - cf. L. gemmat. v. - Wstd.

1262. - Vent. mi. 65. f. $3 ; 74$. f. 3. 4.

- farogineum B. - Trichia chrysosp. Fl. dan.

—_ Fontanesii. - Alger. t. 22.

- fucatum Lés. - Bonite t. 140. f. 3.

- furfuraceum Batsch. - Wstd. 126il.

- gemmatuin B. - Kops Fl. bat. IX. t. 715. 2 .

- Fuck. s. 308. - Carol. IV. 73. - cf. L. plumbeum. - Huss. I. 54 .

- - v. echinatum. - Dsm. 1011.

Wstd. 1262 .

- v. perlatum. - Bisch. \&. 3611. - Kl.

5s. - Wstd. 1263.

giganteum. - [S. $\left.442^{\circ}\right]-$ Fl, dan. IX.

1920. - Ann. sc. n. 1539. XII. t. 2. Fructif.

Berk. . - Anm. Mg. n. h. 1s40. t. 5. f. T-14.

(it.) - Cd. Ic. V. t. 4. f. 40. - Cd. Anl. t. C.

f. 36 : $7-11$. - Berk. In. p. 335 . f. 75 e. Basid.

graniforme Sow. - cf. Cenococcum geoph.

- Wstd. 79 .

hirtum Bull. - Wstd. 1263. - Vent. mi.ti.

f. 4.5 .

-_ hypophyllum Ehrb. - Wstd. 663.

lineare Schrk. - Wstd. 331.

lumbricale Sch.: Trichia reticulata.

- Batsch: Trichia Serpula Fl. dan.

Mali. - [S. 520.]

molle. - Schnzl. t. 11. f. $45 *$ *

nigrescens $\mathrm{P}$. - Wstd. 1263.

oleraceum Poll. - Wstd. 152.

- pedunculatum L. = Tulostoma brumale Fl. dan.

penicillatum Müll. - Wstd. 556.

perlatum. - Schnzl. t. 14. f. 12. = L. gemm.

v. papill. Schff. t. 154. - Voges. 577. - Wstd.

1263. - Sturm h. 1s. t. 6.

pertusum Sow. - Wstd. 39.

Pini. - Wstd. 660.

— v. acicola. - Kops Fl. bat. XI. t. 555.1. pisiforme L. - Wstd. 741 .

plumbeum. - Badham t. 15, 6. 'gemmati

var. :ै.

poculiforme Jacq. - Wstd, 227.

polyrhizon. - cf. Sclerangium pol.

populinum Jacq. - Wstd. 129.

pratense P. - Loud. f. 1650 S.

- pusillum. - [S. 142.] - Kl. 1635. - Kl.

II. 513. - Fuck. s. 312, - Carol. II. 73.
Lycoperdon pyriforme. - [S. 112.] - Kl. 213. - Kl. II. 112. - Fuck. s. 30\%. - Fl. dan. X. 1650.1. - Loud. f. 16510 . - Carol. II. 72. Huss. I. 70. -- Schweiz. 10. - Krypt. Bad. 552. - Sturm h. 1S, t. 9.

— - v. excipuliforme. - Dsm. 1012.

- radiatum Sow. - cf. Agaricus radians Dsm.

- L.: [S. $363^{* * *}$.]

saccatum. - [S. 412.] - Bisch. f. 3605. -

Fuck. s. 309. - Huss. I, 14.

—_ sanguineum Rupp. - Wstd. 711.

- Semen Retz. - Wstd. is.

solidum. - [S. 44t†.]

- sphaericum Jich. - Wstd. 1261.

- tessellatum Schum. $=$ Scleroderma vulg. $v$. squamosa.

- truncatum I. - Wstd. 191.

- turbinatum P. - Wstd. 1264. livid.).

- umbrinum P. - Fl. dan. X. 1500.

- uteriforme. - Kl. 1412.

- variolosum L. - Wstd. 903.

- varium. - Trichia varia.

- velatum. - Pay. b. cr. p. 112. f. 515.

- verrucosum. - Bot. Zt. 1959. t. 11. f. 19.

Keimg. Hoffm.). - Wstd. 1262. (excipulif.).

I. ys urus. - [S. 434 .

- aseroëformis Cd. - Cd. Ic. VI. t. 4. f. 3-5.

- Gardneri. - Hook. Lond. J. 1\$ 16. V. t. 17. f. 2 .

Bail S. t. 24.

M acroon. - [S. 500.]

- obscurum. - Sturm h, 13. t. 47.

Nacroplodia. - ef. Diplodia.

- conigena Wstd. - R. f. 151. - Wstd. 1230.

- Visci. - Wstd. 1231.

II acrosporium. - [S. 456. 501.]

— atrum. - R. f. 273. (cf. Septospor.).

- bulbotrichum. - [S. 501 .

- caricinum. - [S.501 .506.] - Bon. t. 6.

f. 135 . a. b.

— Cheiranthi. - [S. 501.] - Carol. I. \$9.

- concinnum. - [S. 501.] - Ann. Mg. n. h.

14 11. VI. t. 12, f. 21. Berk.)

- Convallariae. - [S. 501.] - Fl. dan. XIII.

22;9. 3. - Bon. t. 6. f. 135. c.

- divisum. - Bon. t. 8. f. 175.

- echinellum. - Carol. III. $\delta \delta$.

- heteronemum. - Dsm. III. 7. - Wstd. 1194.

- heterosporum. - Dsm. III. S.

- pinguedinis. - Hook. Lond. J. 1815. IV.

t. 12. f. 2 .

- punctiforme. - Hook. Lond. J. 1545. IV.

t. 12. f. 1 .

—- Sarcinula. - Ann. Mg. n. h. 153૬. I. t. \&.

f. 10. (Berk.).

- tenuissimum. - [S. 501.]

Malinvernia. - cf. Sphaeria.

- anserina. - Hdw. I. t. 15. f. 4. - Sphaeria

Hypocrea ans. K'l. II. 526.

M am i ania. - cf. Sphaeria.

Mamm aria echinobotryoides. - Kl. 1895.

I an in a. - [S. 522.]

Marasmius. - [S. 313.] ef. Agaricus.

Martella. - [S. 313.]

M a s saria. - [S. 396.] cf. Sphaeria.

amblyospora. - Fres. t. 7. f. 24-33. -

R. f. $25 \%$ - f. M. foedans.

Argus. - Fres. t. 7. f. 7-17.

atro-inquinans. - Carol. IV. 65. 
II a s a ri a crustata. - [S. 396.$]$

- crypta. - [S. 396.]

epiphegea. - Fres. t. 7. f. 35-40.

- foedans. - [S. 396.] - Berk. Out. t. 1. f.

12. e. (Spor.) . = Sphaer. amblyospora B. B. inquinans 'Td. - [S. 396.] - Kl. 541. -

luck. s. 515.

mamillana. - Iil. 1644 .

Platani Ces. - R. f. 323.

pyxidata. - Fres. t. 7. f. 1-6. - R. f. 543.

Sphaeria Pupula).

- squalens. - [S. 396.$]$

II astigosporium album. - Kl. 1755.

$\longrightarrow$ Rss. = Bactrid. alb. Bon.

Mastomyces, - [S. 524.]

Fricsii. - Ann. sc. n. 1549. X. t. 6. f. 4.

Mont.)

Mazzantia Galii. - [S. 391.] - R. f. 537. 539.

II edusula. - [S. $493^{* * *} .521$.

_ ochracea. - Cd. Anl. t. 13. f. 13: 7.8.

II elampsora. - [S. 152.512.]

- areolata. - [S. 4\$2.] - Dsm. 1650.

- betulina. - Dsm. 1647. - Ann. sc. n. 1554.

II. t. 7. f. S. 9. u. t. S. f, $10-12$. (Tul.). -

Fuck. s. 29. - Voges. 1276. - Fuck. rh. 299.

- Carpini. - Fuck. s. 26. - Fuck. rh. 294.

Epilobii. - Fuck, rh. 300.

- Euphorbiae. - Fuck. s. 27. - Dsm. 1086. -

Castagne Marseille t.5. - Wstd. 973. - Fuck.

rh. 295. (cum Uredine Euph.).

- herbarum. - S. 452.

_ _ f. Epilobii. - Dsm. 1645. - Wstd.

1060. (Sclerot. h. Fr., Xyloma l. D.)

- Lini. - Dsm. 1649. - Fuck. s. 30. - Wstd.

1061. - Fuck. rh. 301.

- populina. - [S. 4S2.] - Ann. sc. n. 1554.

II. t. T. f. 10. Tul.). - Fuck. s. 25. a. u. c. -

Iil. II. 493. - cf. Sclerotium pop. u. Perispo-

rium p. - Fuck. rh. 291-293 cum Ured. longicapsulari).

— punctiformis. - Dsm. III. 405.

- salicina. - [S. 4S2.] - Ann. sc. n. 1551. II.

t. 7. f. 6-7. (Tul.)-Fuck. s. 2S. a-d. - cf.

Uredo Capraear. - Kl. II. 494. - Ital. 799. -

Fuck, rh. 296-295. (c. Uredine mixta).

— Vaccinii. - Wstd. 1259.

II elanconium. - [S. 5015 .]

- apiocarpum Lk. - Fuck. s. 126. - Fuck.

rh. 89 .

- v. Alni. - R. f. 469

atrum Lk. - Wstd. 573.

betulinum. - Wstd. 132. - K1. 655. -

Fuck. s. 124. - Voges. 670. - Letell. 704. 1

(Didymospor, elevat.). - Fuck. rh. 85.

- bicolor. - [S. 509.] - Bisch. f. 3835. -

Kl. 574. 1470. - Kl. II. (a.) 590. - Fuck. s.

123. a. - Letell. 704. 3. - Fuck. rh. 54.

(b.) 590 .

- conglomeratum. - [S. 509.] - Coniothec.

Amentac. Cd. - Wstd. 573. glomeratum Wlir. - Wstd. 573.

juglandinum. - Kl. 777. - Kl. II. 279. -

Fuck, s. 12S. a. b. - Pringsh. Jahrb. II. t. 25.

f. 19. (Hoffm.). - Wstd. 1189. - Fuck. rh.

87. 88.

- Juglandis. - [S. 509.]- Cd. Anl.t. F. f. 57:

10-14. - Krypt. Bad. 324. - Wstd. 1159.

— melaspermum. - [S. 413.] - Dsm. III. 753.

- microsporum. - Wstd. 140. - Ns. : Fuck.

s. $12 \bar{i}$.
Melanconium ovatum. - Lk.: Kl. 684. Dsm. 139. - Voges. 1257. - Wstd. 131. Ital. 655 .

- ovoideum Lk. - Kl. 776.

- Papularia. - [S. 509. P. F.]

-... pyriforme. - Wstd. 577.

- sphaeroideum Lk. - [S. 509.] - Dsm. 140.

- Letell. 704. 4.

sphaerospermum. - [S. 509.] - Kil. 573.-

R. f. 179 . - Fuck. s. 125. - Bot. Zt. 1859.

t. 11. f. 5. (Keimg. IIoffm.). - Voges. 1259. Wstd. 392.

-_ sphaerosporum Wllr. - Wstd. 392. - Fuck. rh. 86 .

—_ stromaticum. - Kl. 1359. - Fuck. rh. 90.

- vulgare. - [S. 50S.] - Kl. 1358. (Crypt.

Neesii p. ptc.).

Melanocybe. - [S. $400 *$.]

Melanogaster. - [S. 437.]

- ambiguus, - Ann. sc. n. 1843. XIX. t. 17.

f. 24. (Fructif. Tul.). - Tul. F. hyp. t. 12. f.5; t. 2. f. 5 .

Hyperrhiza liquaminosa.

- Broomeianus Berk. - Ann. sc.n.1843. XIX.

t. 17. f. 23. (Fructif. Tul.) - Cd. Ic. VI.

t. 9 . f. 90 .

Brit. f. 255 .

Cauvin Mont. - cf. Splanchnomyc. -

Klotzschii Cd. - Cd. Ic. VI. t. 9. f. S8. -

cf. Melan. ambiguus Cd.

- rubescens. - Tul. F. hyp. t. 2. f. 6; t. 12.

f. 7. - Cd. Ic. VI. t. 9. f. 92 .

tuberiformis. - Cd. Anl. t. C. f. 37: 1-3.

- Cd. Ic. VI. t. 9. f. 89. - Sturm h. 11. t. 1.

- variegatus. - Ann. sc. n. 1843. t. 17. f. 22.

(Fructif. Tul.) - Tul. F. hyp. t. 2. f. IV, 7 ;

t. 12. f. 6. - Pay. b. cr. p. 114. f. 530. - Cd.

Ic. VI. t. 9. f. 91 .

- v. Broomeianus. - Tul. F. hyp. t. 2.

f. IV. 1-6. - ef. Melan. Broom. B.

Melanosorus. - cf. Rhytisma.

Melanospora barbata. - [S. 396. Scop.] -

Alger. t. 25,3 .

- chionea, - [S. 396*.] - Cd. Anl. t. F. f. 55 :

S-10. - Bail S. t. 17.

— cirrhata. - Brit. f. 325.

- Coemansii. - Bull. ac. belg. 2. Sér. 1857. II.

p. 579. f. S (Wstd.).

— Zamiae. - Cd. Anl. t. F. f. 55: 11-12.

II ela nostroma. - [S. $363^{* * *}$.]

- fusarioides. - Sturm h. 9. t. 61. - minutum. - Cd. Anl. t. G. f. 6S: 11-12.

Helanotrichum. - [S. 495***. 503*.] microspermum. - Sturm h. 13. t. 45.

Melasmia. - Rhytisma.

- acerina. - [S. 423. 424.] - Voges. 1470. -

Wstd. 929.

Melidium. - [S. 4\$9.] subterraneum Eschw. - Bisch. f. 3735.

Meliola. - [S. 406. 503*.] - Froricp Tagesberichte. 1552. no. 592. 593. ic. (Bonnet).

- amphitricha Mont. - Sagra, Cuba. t. 12.

f. 2. - Berk. In. p. 273. f. 62. d. - Carol. I. 70. cymbisperma. - Berk. In. p. 273. f. 62. c.

I elit tosporium aeruginosum Cd. - Kl. 924. versicolor. - Cd. Anl. t. G. f. $63: 5-8$. -

Bail S. t. 19.

- $r$. lacteum. - Kl. 925.

Melogramma. - [S. 3S6.] — cf. Sphaeria. ambiguum. - Carol. IV. 49. 


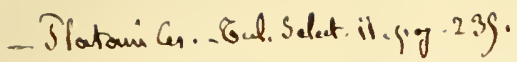


79

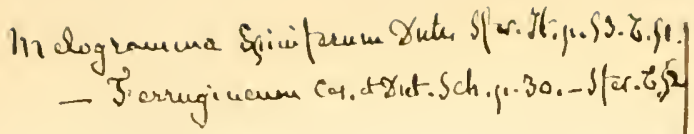


M elogramma oligospermum. - Ann. Mg. n. h. 1859. III. t. 11. f. 35 . (B. B.).

- vagans. - Act. Turin. 1557. XVI. Dec. 9. f. 2. Notar.).

M emnonium. - [S. 495***.]

- effusum. - Cd. Anl. t. B. f. 7: 4. - Fuck. rh. 140. - Sturm h. 13. t. 46.

Menispora alba. - Sturm h. 25-. t. 20. (Preuss.).

- ciliata. - Cd. Anl. t. B. f. 13: 2. - Kl. 1436.

(Preuss.).

- glauca. - [S. 495.]

- pyriformis. - Sturm h. 29-.t. 18. (Preuss.)

- Kl. 1760 .

- torulosa. - Fres. t. 3. f. 44-1s.

Merism a. - [S. $339 *$.] cf. Thelephora.

_ cristatum Schum. = Thelephora cr. Fl. dan.

- Wstd. 430.

- fastidiosum P. - Dsm. 76 .

- foetidum. - Cd. Ic. V. t. 10. f. 77. Fructif.). - Cd. Anl. t. G. f. 73: 11-13. - ? cf. Sparassis foetida.

- tremellosum. - Hoffm. hercyn. subt. t. 14. f. 1 .

truncatum. - Cd. Ic. V.t. 10.f. 78. (Fructif.)

- Cd. Anl. t. G. f. 73: 14. 15.

Merosporium minutum. - iS. 466.] - Cd.

Anl. t. B. f. 7: 6. 7. - Sturm h. 12. t. 23.

Merulius. - [S. 325.] — ef. Cantharellus, Craterellus.

- applicatus. - Ann. sc, n. 1543. XIX. t. 7 . f. 2. Lúv.

— aureus. - [S. 325.] - Wstd. 1390.

- brassicaeformis. - Carol. II. 23.

- bryophilus. - Cd. Anl. t. H. f. 76: 12. 13. cantharelloides Schum. = Cantharellus tu-

baef. Fl. dan. ; - ef. Cinth. cibar.

- Sow.: Wrstd. 1390.

Cantharellus. - Wstd. 310 . - cf. Canth. cib. cibarius. - Wstd. 340

collariatus With. - Wstd. 1252.

confluens. - Carol. I. 23.

Corium. - [S. 325.] - Kl. 1916. - Fuck.

s. 961 . - Brit. f. 19.

- Cornucopiae. - cf. Craterellus C.

cornucopioides. - Wstd. 192.

crispatus. - [S. 325.]

crispus $P .=$ Canthar. cr. Fl. dan.

cucullatus. - [S. $333^{*}$.]

cupularis. - Wahlenb. Fl. lappon. 1812.

t. 30. f. 6. - cf. Arrhenia c.

daedaleus. - [S. 312*.]

destruens. - Dsm. 125. - Cd. Anl. t. H.

f. $76: 7-10$.

- P. = lacrymans Fl. dan.

fugax. - [S. 325.] - Fl. dan. XII. 2027. 2.

(inf.). - Carol. I. 24.

galeatus Schum. = Cantharell. g. Fl. dan.

himantioides. - [S. 325.]

hoedinus. - Carol. IV. 8.

- incarnatus. - Carol. II. 22.

- lacrymans. - [S. 325.] - Fl. dan. XII.

2026. - Kops Fl. dan. X. 760. I. - Loud.

f. 16059. - Huss. I. 3. - Hrz. t. 77. - Berk.

Out. t. 2. f. 1. - Pay. b. cr. p. 107. f. 4 S4. -

Sturm h. 31 - t. 4. (Szl.) - Kl. 228. - ef.

M. destruens. - Lib. 320 . - R. f. 508 .

- lutescens P. - Wstd. 1390.

molluscus. - [S. 325.]

niveus. - [S. 325.]
M erulius pallens. - [S. 325. 10.]

I. petropolitanus. - [S. 325.]

porioides. - [S. 325.]

- pulverulentus Fr. - [S. 325. 13.] - zu 11.

destruens P. ('Tul.).

refluxus Gmel. - Wstd. 1390.

retirugus. - Fl. dan. XIII. 2340. 2. - Cd.

Anl. t. H. f. $76: 11$.

- serpens. - [S. 325;4.] - Kl. 1006. - Kl.

II. 6. - Carol. IV. 9.

squalidus. - [S. 325 .

__ Squamula With. - Wstd. $5 \$ 6$.

- terginus Fr. - R. f. 12.

- tortilis. - [S. $\left.329^{* *}.\right]$ - Fl. dan. XIII.

2270.2 .

tremelloides Schrad. - Kl. 110

tremellosus. - S. 325.] - Ann. sc. n. 1537.

VIII. t. 10. f. 22. (Fructif. Lév.) - Fuck.

s. 960. - Bail S. t. 30. - Kl. II. 7. - Carol.

III. 15. - Huss. I. 10. - Lib. 222. - Vent.

mi. 63. f. 4 .

—_ tremulosus Schrad. - Bisch. f. 3147.

- umbonatus. - cf. Cantharellus.

umbrinus, - [S. 325.]

Vastator. - [S. 325. 12.] - Fl. dan. XI.

2027. 2. (sup.).

villosus P. - Wstd. 1390.

- xanthopus P. - Wstd. 1390.

M esenterica. - [S. 521.]

II es ophellia arenaria. - Linn. 'Trans. 1859.

XXII. t. 25. (Berk.).

Metrem yces lucidus. - Hook. Lond. J. 1545.

IV. t. 1. f. 5. - cf. Mitremyces.

Mi a inom yces. - S. 495 .

- fungicolus. - Sturm h. 13. t. 42.

Microbotryon. - [S. 516. 7.]

- antherarum. - S. 516.$]$

Microcer a coccophila. - R. f. 269. (zu Stilbum od. Atractium Tul. Sel. I. 130.). - Dsm. 1350. - Sphaerostilbe cocc. Tul. in sched. Ital. 543.

Microcrater. - Bail S. t. 20.

Micropeltis, - [S. 395.]

- applanata Mont. - Sagra, Cuba, t. 12. f. 6.

Micropera. - [S. 413 **.]

- Drupacearum. - Kl. 1960. - Dsm. III. 697. - Carol. IV. i1. - Wstd. 465.

Microplo di a Aquifolii. - Bull. ac, belg. Ser. 2. 1557. II. p. 579. f. 7. (Wstd.).

Iicrosphaera. - cf. Erysiphe.

- Berberidis. - Ann. sc. n. 1551. XV. t. 10. f. 29. (Lév.) - Erys. Berb. Tul. - R. f. 555 . comata. - Ann. sc. n. 1851. XV. t.9. f. 23. (Lév.). - R. f. 43 S. - E. Evonymi DC., comata Rbh.

- divaricata. - Ann. sc. n. 1551. XV. t. 8. f. 18. (Lév.). - Wstd. 135i.

Dubyi. - Ann. sc. n. 1851. XV. t. 9. f. 26.

(Lév.). - Frys. Lonicerae Tul.

- Ehrenbergii. - Ann. sc. n. 1\$51. XV. t. 8 . f. 22. (Lév.). - Wstd. 1358.

Friesii. - Ann. sc. n. 1851. XV. t. S. f. 20.

(Lév.).

- Grossulariae. - Ann. sc. n. 1851. XV. t. 9. f. 25 . (Lév.).

- Hedwigii. - Ann. sc. n. 1851. XV. t. 8. f. 19. (Lév.) - R. f. 296. - Erys. penicill. Fr. - holosericea. - Ann. sc. n. 1851. XV. t. 9. f. 27. (Lév.) ; ic. mala ('Tul. Sel. I. 207.). - R. f. 439. - Erys. Astragali Tul.

- Mougeotii. - Ann. sc. n. 1851, XV. t. 9. f, 24. (Lév.). - Berk. In. p. 278. f. 64. e. 
Microspha era penicillata. - Inn. se. 11.1551. XV. t. S. f. 21. (Lév.). - Erys. Alni Tul. $\ell$ R. f. $43 \bar{i}$. . Alni.

- semitosa 13. C. - Berk. In. p. 2- s. f. 61. c.

II icrosticta l'omi. - Dsm. 300 .

- vagans D. - Wstd. 1062. - Perispor. speireum Fr., vagans Dsm. - Depazca speirea ('d. 1)sm. 254. $259 ;$ I1I. 674-67t.

Microstoma hyemale. - Nov. Act. XXIII. II t. I.XI. IIilde ; = Peziza protracta Fr. - Iil. 1719.

- vulgare Awd. - R. f. 253.

Iicrothecium. - S. 426.

- fallax. - Kil. 19 is.

— Zobelii. - cd. Ic. V. t. \. f. 53. - Pay. b. cr.p. is. f. 365 .

Microthyrium. - [S. 414.]

- microscopicum. - Dsm. 492; III. 696. Ann. sc. 11. 15H1. XV. t. 11. Dsm... - Cd. Anl. t. F. f. $54: 17-20$. - Voges. 1256.Vistd. 170.

- Smilacis. - Act. Turin. 1445. VII. Dec. 4.

f. 4. Notar. . - Kl, II. 654. - Dsin. III. 695. - Ital. 44. 94.

II c ula IIongeotii. - K1. II. 636. - Hedw. 1555.

II. t. 1. f. A. - Sphaeria Micula Fr. El.

- nivea. - Iil. I1. 635 .

IIidotis. - S. 362.]

Mitremyces. - S. 410.] - cf. Metremyces.

- coccineus. - Ann. nat. hist. III. t. $\bar{\imath}$. f. 1.

Berk.

- fuscis. - Ann. Ig. n. h. 1539. III. t. i.

f. 1. p. 326 . Berk.,

- Junghuhnii. - Bot. Zt. IS44. t. 3. B.

- lutescelıs. - Cd. Anl. t. D. f. $41: 15-19$.

Iisch. f. 3612. - Carol. I. 76.

- Ravenelii. - Linn. Trans. XXII. t. 25. (Berk.)

Mitrula. - SS. 34i.]

Abietis Fr. - Loud. f. 16191.

Perterii. - Chile no. S. f. 5.

eucullata B. - S. 347 .

Fuck. s. 573. - Brit. f. 254.

— - v. Abietis. - Dsm. 655. - v. Pini. - Biscli. f. 3375 .

globosi. - S. 34\%.

- Heyderi. - Fl. dan. X. 1670. 2. - Cd. Anl.

t. G. f. G6 : 5 .

— Minuta Fr. - Loud. f. 16190.

— paludosa. - -S. 317 . - M. phalloides Cher.

- Loud. f. 16159. - Dsm.952. - Bail S. t. 21.

- Sturm h. 31 - t. 13. Szl. - Kil. 23s. -

Fl. II. 132. - Brit. f. 275. - Carol. V. 36. -

Huss. I. 9. - Wstd. 234.

— phalloides, - Wstd. 234.

- spathulata Fr. - Bisch. f. 3266 . - Brit. f. $25 \%$.

Monas prodigiosa. - ? Fres. t. 0. f. 15. 19. -

Presburg. Verh. Verein. f Naturk. 1557. t. 1. Tiolaczek .

Ionilia. - [S. 459.]

_ auf Seidenraupen. Robin v, par. t. 7. f. $\subseteq$.

- antennata. - W'std. 433.

- aurea P. - Loud. f. 16.555.

- caespitosa. - Purtoll. I. t. 31 .

- - candida. - Bon. t. 4. f. $\$ 6$. - Letell.612. 2.

(Aspcrgill.). - : cf. Moniliger.

Celtis, - Birona t. 3. f. 6.

- cinerea. - Bon. t. 3. f. is. - R. f. 271.

digitata. - [S. 4 -9.]

fructigena. - Letell. 639. 2.
Monilia Graminum. - Letell. 638. 1. hyalina Fr. - zu Frysiplie Graminis (Tus.). -

Letell. 635. 3. - Wstd. 299.

- nidulans. - Wstd. 400 .

_ penicillata Fr. - Bisch. f. 3755 .

racemosa $P$. - Bisch. f. $3 \$ 05$.

virens. - ef. Aspergillus v.

vulgaris. - Wstd. 196.

II o n i lige r candidus, - Letell. 66`. 3. (Monilia !) . peniculatus. - Letell. 665. 4.

I on op lo dia Magnoliae. - Bull. ac. belg. 1559 VII. p. 366. f. 19. (Wstd.)

Ionosporium. - ef. Peronospora. Botrytis.

- acuminatum. - Bon. t. 7 . f. 165.

- agaricinum. - Bon. t. 5. f. 112. - Fuck.

rh. 154. cellarum. - Bot. Zt. 14.33. t. 7. f. 5. (Bon.). Chenopodii $\mathrm{S}-1 .=$ Peronosp. effusa $\mathrm{Rbl}$.

(asp.)

- corticola. - Bon. t. 5. f. 126.

- curvatum. - Bon. t. 5. f. 115.

- decumbens. - Bon. t. 5. f. 116.

- Havum. - Bon. t. 5. f. 107.

griseum. - Kl. $16 \varsigma_{5}$.

- macrocarpum. - Bon. t. 5. f. 125.

macrosporum - Bon.t. 5.f. 125 . (cf. p. 96.). membranaceum. - Bon. t. 5. f. 113.

niveum. - cf. Peronosp. paras.

- oxycladum. - Bot.Zt. 1853. t. 3. f. 15-20

Riess.

- reflexum. - Bon. t. 6. f. 134.

spinosum. - Bon.t. 7. f. $14 \mathrm{~s}$.

torulosum. - Bon. t. 5. f. 123.

viridescens. - Bon. t. 5. f. 111.

II onotos pora. - S. $497^{* * *}$.]

- megalospora. Ann. Mg. n. h. 1854. XIII.

t. 15. f. 11 . p. 462 . (B. B.)

- sphaerocephala. - Ann. Mg. n. h. 1559.

III. t. 9. f. 5. (B. B.).

Il ontaguea. - cf. Montagnites.

Montagnites. - S. 439 .]

- Candollei. - Cd. Ic. VI. t. 20. f. 116.

Cd. Anl. p. 196. ic. - Pay. b. cr. p. $11 \%$.

f. 550 - Alger. t. $21,1$.

- Dunalii. - [S. $439 *$ * ]

Morchella. - S. 346.$]$ - Müller, Ptianzenstaat. p. 2i1. Copiet.

bohemica. - Cd. Anl. t. G. f. $67: 14-16$.

- Schnzl. t. 16. f. 13.14. - Sturm h.14. t. 56.

- conica. - [S. 316.] - Cd. Anl. t. G. f. 67:

13. - Kl. 1009. a. ; cf. M. escul. v. - Dsm. III.

26.5 .

costata $\mathrm{P}$. - Vent. mi. 6.5. f. 4 .

deliciosa. - S. 346 - Iil. 136.

distans. - [S. 346.$]$

dubia. - Canar. t. 4. f. 4.

elata. - [S. 346.] - Ki. 1009. b.

esculenta. - [S. 346.$]$ - Kops Fl. bat. IX.

t. 670 . - Berk. Out. t. 21. f. 5. - Vent. t. 11.

f. $107-109$. - Roq. t. 1. f. 4. 5. - Hrz. t. 50 .

- Loud. f. 16197. - Bail S. t. 21. - Berg, Charakteristik. 1560 . t. 1. f. 6. - Dsm. III. 264. Carol. I. 36. - Badham t. 12, 2. - Huss. I. 13. - Tent. mi. 52. - Staude t. 1. f. 4. 5. Ital. S69. - de Bary, Ascomyc. I\$63. t. 2. f. 15-21. (Asci).

- v. conica Fr. - Bisch. f. 3302. - ef.

II. con.

hrbrida. - Dietr. Fl. boruss. t. 334.

patula P. - [S. 316.$]$ - Bisch. f. $3301 .-$

Loud. f. 16195 .

- praerosa. - Pay. b. cr. p. \$5. f. 352. 


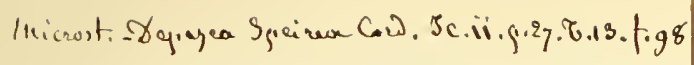




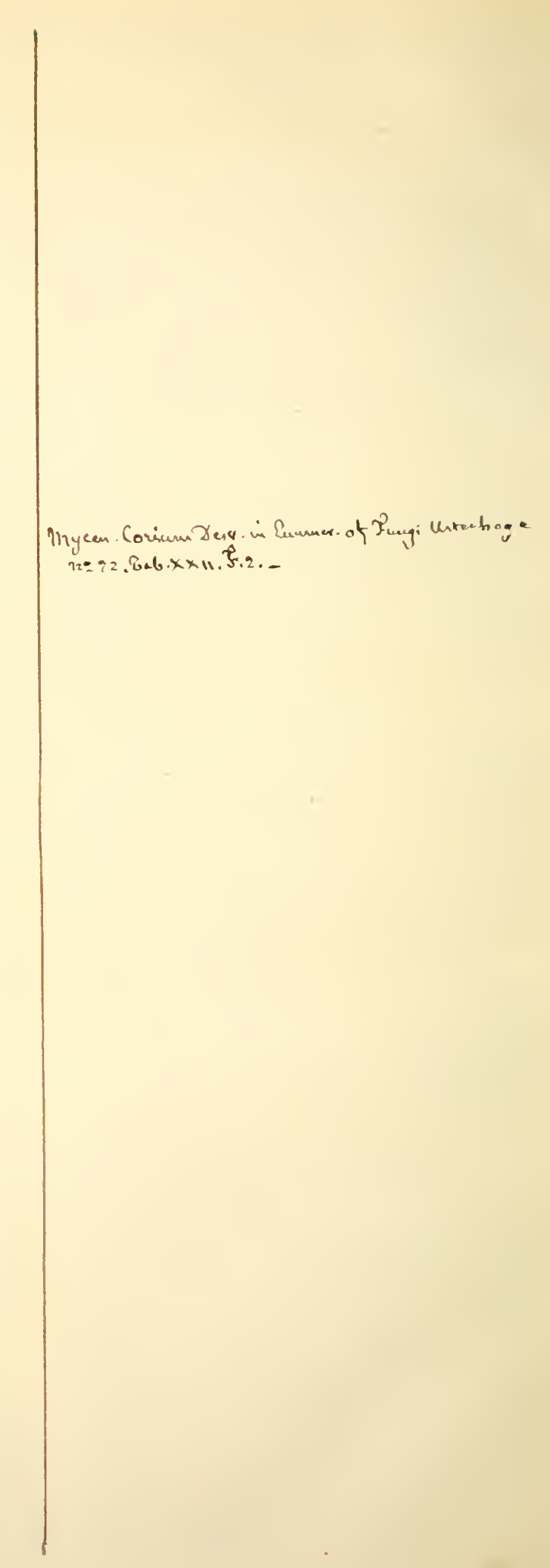


Morchella rimosipes DC. - Hrz. t. is.

- semilibera DC. - [S. 316.] - Dsm. \$25. -

Vent. t. 11. f. 105. 106. - Loud. f. 16199. -

Kl. 232. - Letell. 696. - Vent. mi. t. 96. f. 2-6.

Mucilago. - [S. 521.]

Mucor. - [S. 4ל7.] - cf. Ascophora, Hydrophora. - Flora. 1557. no. 27. t. 3. f. 50. (Bail). auf Pollen: C. Müller, Pflanzenwelt. 1557.p. 63. (Karsten).

- albo-virens. - [S. 457.]

- arcuatus Mart. - Bisch. f. $375 \%$.

- bifidus, - Fres. t. 1. f. 13-23.

- breviceps Riess. - Bot. Zt. 1553. t. 3. f. 1.2. - caninus. - [S. 45\% - Lindl. r. K. p. 29.

f. 3. - Bisch. f. 3732 . - Fuck. rh. 52.

- chrysospermus. - Wstel. $\$ 36$.

clathroides Scop. - Wstd. 51 .

clavatus. - [S. 457.] - Bon. t. 10. f. 202.

- Mag. Zool. Bot. 1§37. 2. t.11. p. 340, status

confervoideus Berk.

- coccineus Fr. - Bisch. f. 374 s.

- Leers. - Wistd. $\$ 1$.

crustaceus. - Wstd. 300 .

cyanocephalus Mart. - Risch. f. 3731.

dendroides. - [S. 491.]

elegans Fr. - Bisch. 3735 . - cf. Tham-

nid. el.

- Erysiphe. - Wistd. 407.550.

—_ ferrugineus Bull. - Wstd. 341 .

__ flavidus P. - S. 457.] - Bisch. f. 3742.

- flavus Fr. - Biscl. f. 3750 .

- flocculentus. - Act. Turin. 1S05. XIV. t.2.

(Cumino).

- fragiformis Schff. - Wstd. 711.

_ fruticulosus. - Act. 'lurin. 1605. XIV. t. 3.

(Cumino).

- furfuraceus L. - Wstd. 51 1 .

- fusiger. - [S. 4>i.] - Fuck. rh. 53. -

Mier. J. 1S59. VII. t. 11. f. 7. (Spor., Curr.,

- helminthophthorus (Hydroph.) de B. -

Ztschr. f. wiss. Zool. I>61. XI. 135. t. 15. A.

(Keferstein).

- herbariorum Web. - Wstd. $\$ 100$.

— lignifragus Bull. - Wstd. 353.

- lycogalus Bolt. - ef. Reticularia arg. Cd.

- macrocarpus. - Cd. Anl. t. C. f. $23: 12-15$.

melittophthorus. - Hedw. I. t. 16. (Hoffm.). nicrocephalus. - Bon. t. 9. f. 199.

- Mucedo. - [S. 457.] - Lindl. r. K. p. 30. f. 12. - Fres. t. 1. f. 1-12. (Ascoph. M. Cd. ? cf. p. 9.) - Dsm. III. 414. - ? Schnzl. t. 12. f. 26. 27. - Muller, Pflanzenstaat. 1\$60.265.6. (Copie). - Mycelium: Bisch. f. 3525.

Fres.: Terh. d. 35. Naturf. Vers. Königsberg. 1560 . t. 1. (Bail).

- murinus. - S. $45 \%$.

nigrellus. - [S. $45 \tau$. $]$

obliquus Sc. - ef. Pilobol. eryst. Cd. ; u.

oedipus R. f. 352 .

- oogenus. - Bull. ac, belg. 1§52. XIX. 1.

p. 565. (Spring).

- pygmaeus ? - cf. Muc. stolonifer. - [S. 457.] racemosus, - Fres. t. 1. f. $24-35$.

Bull. : Mstd. 435 .

ramosus. - [S. 457.]

roseus Bull. - Wstd. 437 .

rufus. - [S. 487.]

- sphaerocephalus? - Schnzl., Nachtr, t.2.f. 2.

spinaceus Sow. - ef. Botrytis effusa.

- stercoreus. - [S. 45\%.] — Loud. f. 16545. -

Bon. t. 9 . f. 195 .

- Ik. : Ascophora st. Cd.
Mucor stolonifer Ehrb. - [S. 457.] - Bisch. f.3S15. 3519. - Boll. t. 10. f. 201.

— subtilissimus cum Sclerotio Cepae'. - Journ. hortic. soc. I\$ 15. III. p. 9 i u. 99. (Berk.).

- succosus. - Ann. MIg. 11. h. 1S11. VI. t. 12.

f. 15 . (Berk. ?

- tenellus. - [S. 45\%.]

- tenuis. - Bon. t. 9. f. 195.

- urceolatus B. - ef. Philob. cryst. Cd.

— virens $\mathrm{Fr}$. - Bisch. f. 3747.

Mucronia. - S. 329. $464^{*}$.]

M utinus. - [S. 434 . - ef. Phallus.

Yy celia: in cellulis. - Linn. $1542 . \mathrm{XVI}$. t. 11.

f. 11-17. Nägeli - Ann. se. n. 1sł3. XIX.

t. 3. f. $11-1$ j. Näg. .

- in Hartgebilden ron Muscheln, Fischen etc.-

Ztschr. f. wiss. Zool. 1559. X. t. 15. 16. (Töl-

liker .

- in lacrymis Vitis viniferae. - Ann. sc. n.

1530. XXI. t. 1. p. 100. Amici).

—_ in ovis galiin. - ef. Dactyl. oogenum.

- Mycodermata. - Ann. sc. n. 1. Ser, 1527. I.

t. 3. - ef. Torula Cerevisiae Cd.

II ycenastrum. - [S, $443^{* *}$.]

- chilense Mont. - Cd. Ic. VI. f. 43. - Chile

11. 11). f. 8 .

- Corium Dsv. - Hook. Lond. J. 15.3. II.

t. 22. f. 2. Berk. - - ef. Lycoperd. Cor. Cd.

- leptoderinum. - Alger. t. 22, bis. 6 .

- radicatum. - Alger. t. 22, bis. 7 .

-. uteriforme Lév. - cf. Lycoperdon.

MI ycinema. - [S. 524.]

My cobanche cervina. - Schnzl. t. 12, f. 41.-

cf. Mycogone simplex et Sepedonium fuscum.

- chrysosperma. - Wstd. -136.

Mycoderma. - cf. Mycelia. - [S. 521.524.

- Inthemidis. - Dsm. III. 530.

- Favi Gruby. - Oidium Schönleinii Fr. -

Achorion Schönleinii Remak.

_- Grossulariae. - Dsm. III. 226 .

- lactis-butyri, - Dsm. III. 529.

- mesentericum. - Chalara Mycoderma.

- l'ersici. - Dsm. III. 524.

— Pyri. - Dsm. III. 525.

—- Ribis spinosi. - Dsm. III. 528.

- Rubi idaei. - Dsm. III. 527 .

— vini. - Dsm. III. 522. 523.

Ir yogone. - ef. Iycobanche.

alba. - Letell. 667. 2.

Cerasi Her. - Kl. 1592. - cf. Puccinia.

cervina. - 'S. 497.3.] - Kl. II. 672. - ef.

II. simplex. - Letell. 667. 3. - Sturm h. 4. t. 53.

- incarnata. - Letell. 66\%. 1.

- rosea Lk. - [S. 497.] - Kl. 686. - Bon.

t. s. f. $150 .-$ Fuck. rh. 142.

- simplex. - Cd. Ic. VI. f. 4.

Mrcomater. - S. $456.496 * .520$.]

II don osporium olivaceum. - Cd. Anl. t. B.

f. $11: 8$. - Sturm h. 13. t. 4 s.

II ydonotrichum. - [S. 503.]

- atrum. - Cd. Anl. t. B. f. $11:$ T. - Sturm

h. 12 , t. 19.

M y i ophyt on Cohnii Leb. - cf. Empusa et Fntomophthora Muscae.

II ylitta. - [S. 436. 44.]

- australis B. - [S. 444 †.] - Cd. Ic. VI. t. 9. f. 93. - Ann. Mg. n. h. 1\$39. III. t. i. f. 2. (Anat. Berk.).

- Pseudo-Acaciae. - [S. 436 *.] - Kl. 1549. - venosa. - [S. 436 .]

MIriocephalum. - S. 509.] 
Myriocephalum botryosporum. - Fres. t. 5. f. 1-9. - Fuck. s. 119. a., 120. - Microsc. Journ. IV. t. 11. f. 1-9. (Curr.). - Ital. 546.

- - v. Carpiui. - Kl. 1sS4.

densum. - Fuck. Nass. 23. = M. botr. Fres. t. 5. f. 4. 5. - Fuck. s. 119. a. b. - Fuck. rh. 96.97.

- hederaecolum. - Act. Turin. 1S45. VII.

Dec. 3.f. 10. (Notar.) =M. botryospor. (t. Mont.). - Kl. 1969.

—- Not. (Thyrsidii sp. Mont.) Kl. II. 593. - zu densum: Fuck. Nass, 23.

laxum. - Fuck. Nass, 23. = M. botr. Fres.

t. 5. f. 9. - Fuck. s. 120. - Fuck. rh. 98 .

Myriococcum. - [S. 438.]

- praecox. - [S. 439.] - Cd. Ie. V. t. 3.

f. 39. - Cd. Anl. t. C. f. 27: 4-6. - Bisch

f. 3697 . Kl. 929 .

My riophysa. - [S. 451.]

Myriophyton. - cf. Myioph.

Myriostoma. - [S. 441.] - cf. Geaster.

- coliforme. - Cd. Anl. t. D. f. 43:16.17. -

Pay. b. cr. p. 63, f. 279.

Myriothecium. - cf. Myrothec.

Myropyxis caricicola. - Kl. 1429 ; it. Ct. XVI.

- graminicola, - Kl. 1541.

II yrosporium. - [S. 45s*.

- coccineum. - Sturm h. 12. t. 32.

My rothecium. - [S. 44 s.

- fuscum. - Bon, t. 10. f. 216.

- gramineum. - Lib. 350.

— inundatum Td. - [S. 445.] - Bisch. f. 3625.

- Fuck. s. 301. - R. f. 571. - Fuck. rh. 165.

- Sturm h. 1.t. 3 .

- luteo-album. - Bon. t. 10. f. 214.

- _ roridum Td. - [S. 148.$]$ - Bisch. f. 3685. -

Fuck. rh. 166.

- scybalorum. - [S. 445.] - Fl. dan. XII.

2053. 1.

- Verrucaria. - [S. 445.] - Bisch. f. 3627. -

Kl. 1655 . - Cd. Anl. t. G. f. $65: 5-8$. -

Sturm h. 1. t. 4.

- Vitis. - Bon. t. 10. f. 215

Mystrosporium. - [S. 506.]

aterrimum. - Carol. IV. S6.

dubium. - [S. 506.]

pulchrum, - Hook. Lond. J. 1s45. IV.

t. 2 . f. 9 .

pyriforme. - Dsm. 1094.

Stemphylium. - Cd. Anl. t. B. f. 10:9.-

Pay. b. cr. p. 73. f. 324.

II tilinidion aggregatum. - Dub. Hyst. t. 1.

f. 3. (Hysterium a. DC. non Rbh. exsice.).

Myxarium. - [S. $477 *$.]

II y xocladium. - [S. $503 * * *$.]

Myxocyclus confluens. - Fres. t. 7. f. $41-45$.

- ? R. f. 264. - ef. Stegonospor. mur.

Myxomphalos. - [S. 359.]

Myxonema. - [S. 4s6. 496**.520.]

- assimile. - R. f. 280 .

My $\mathbf{x}$ ormia atroviridis B. B. - R. f. 63 . - Ann.

Mg. n. h. 1550 . V. t. 12 . f. 9. p. 457 . (B. B.).

Myxosporium. - [S. 413.] - ef. Nemaspora.

Cameliae. - Lotos 1S57. VII. t. 1. f. 3.

- corallinum. - Lotos. 1858. t. 1. f. 15.

(Peyl.).

- croceum. - Cd. Anl. t. B. f. $3: 14-16$.

nitidum. - Carol. TV. 70 .

- paradoxum. - Act. Turin. 1841. III. Dec. 2.

f. 10. (Notar.).

1. quercinum Lasch. - Kl. 1076.

If yothecium. - [S. 407.]
Myxotrichum.- [S. 502. 494.] — ef. Actinospira.

- aeruginosum Mg. - ? Kl. II. 179.

- chartarum Kz. - [s. 498 t. $502 * * .503$.$] -$

Dsm. 106. - Fl. dan. XIII. 2277. 1. - Actinospira ch. Cd. - Sturm h. 29 -. t. 40.(Preuss.). - Bisch. f. 3755. - Kl. 1675. - Kl. II. 179. -

Brit. f. 207. - Letell. 679. 4. (Sporotrich. ch.).

- Ann. Mg. n. h. 1562. IX. t. 6. p. 32. (Church).

- deflexum. - Ann. Mg. n. h. 1835. I. t. 8.

f. 9. (Berk.).

- fuscum. - [S. $502^{* *}$. 503.] - Fl. dan. XIII.

$227 \% .3$.

- molle. - [S. 503.]

- murinum. - [S. 502.

murorum. - [S. 495 t. $502 * *$.] - Cd. Anl.

t. B. f. $8: 4-6$.

- patulum. - [S. 502.$]$

- rarum. - [S. 503.]

- Resinae. - [S. 502.] - Kl. II. 780. - Fuck.

rh. 129 .

Na e mas pora. - [S. 413.] - Nemaspora.

— aurea. - [S. 414.] - Bisch. f. 35S4. - Kl. 171. - Kl. II. 594. - Ital. 449. (Libertella betul. Dsm., Naemasp. croc. Fr. Scler.).

- Carpini Sow. - Loud. f. 16613.

chrysosperma. - Fres. t. 4. f. $30-34$. -

Wistd. 323.

— coerulea. - Fres. t. 5. f. 24-30. - Kl. 1756.

- crocea. - [S. $413^{*}$. 414.] - Ann. sc. n.

1530. XIX. t. 5. f. 3. (Dsm.). - Cd. Ic. I.

t. 6. 7. - Fres. t. 4. f. $35-39$. - Bisch. f. $35 \$ 5$. - Fuck. s. 419. - Kl. 170.

- Fr. = Libertella cr. Bon. - Voges.

17\%. - Brit. f. 104. - Carol. V. 74. - Schwabe

Fl. anhalt. II. t. 6. f. 2. - Lib. 261. - Wstd.

335. - cf. N. aurea, - Schweiz. 12.

- encephaloides Spr. - Wstd. 138.

- epiphylla. - cf. Spilosphaeria Saponariae.

359 . grisea. - [S. 414.] - Bail S. t. 16. - Lib.

incarnata. - [S. 414.] - Ann. sc. n. 1930.

XIX. t. 5. f. 2. (Dsm.) - - Lib. 262. - Wstd. 438. inquinans Spr. - Wstd. 371.

leucomyxa Cd. - ef. Cytispora 1. - R. f. $35 \pi$.

- microspora. - Ann. sc. n. 1830. XIX. t. 5. f. 1. (Dsm.).

- Lib. : status spermogonifer Nectriae cinnabarinae. R. f. 324 . - Lib. 38S. - Wstd. 138

Mougeotii. - Voges. 1171.

orbicularis. - [S. 414.]

persicina. - Fres. t. 3. f. 53-55.

Plantaginis. - Kl. 1664.

Ribis. - [S. 414.]

Rosae. - Bisch. f. 3556 .

Rosarum Grev. - Loud. f. 16615.

Salicis. - Cd. Anl. t. F. f. 57: 5-9, -

Cd. Ic. III. f. 70 .

- Tiliae Reb. - Wstd. 533.

Naematelia. - S. $477^{*} .476$.

encephala. - Brit. f. 291.

frondosa. - Bon, t. 11. f. 232. - Fuck. s.

s57. (Tremella fr. P.).

- nucleata - [S. 477*.] - Carol. IV. 82.

- rubiformis Fr. - Bisch. f. 3449. - Bail S. t. 22 .

- virescens. Cd. Anl. t. G. f. 70:10. 11.

Naevia. - [S. 373.]

Lauri. - Ital. 491.

Nassula. - [S. 456.] 
viryxotrichum.

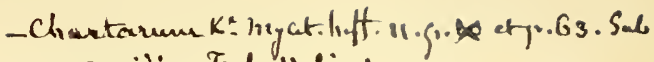
Oncidio. T.l. $11.5 \mathrm{y} .1$.

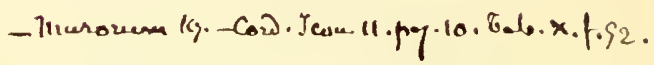

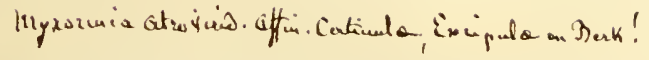




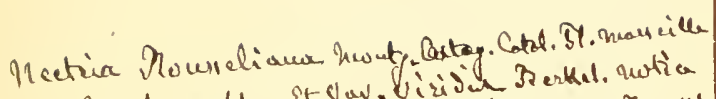

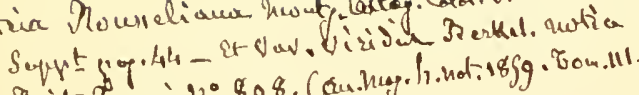

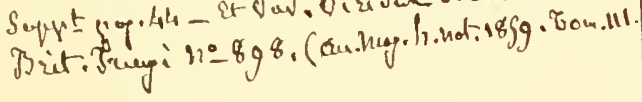


Natalia. - S. $415 * *$.]

Nectria. - [S. 357.] - Sphaeria.

- Albertinii. - Ann. Mg. 12. h. 1661. VII.

t. 17. f. 24. a., p. 152. (B. B.).

aurantia. - S. 355 .

aurigera, - Carol. V. 60.

- australis. - Chile n. 10. f. 1.

- chrysites, - Kil. 1444. - Kil. II. 632.

- cinnabarina. - [S. 3\$5.] - Kl. II. 633. -

Fuck. s. $660 .-12$. f. 324 . (cf. Naemaspora microsp. Lib. et 'Tubercularia vulgaris).

- citrina Fr. = SphaeriaCitrumWllr. - [S. 3 s ५.] citrino-aurantia Lacr. - R. f. 32.5.

- coccinea 1 . - [S. 355.] - Fuck. s. 659

- coruscans. - Afz, t. 12. f. 31 .

- cucurbitula. - [S. 355.] - Kil. II. 245. -

Carol. IV. 52.

Daldiniana. - Ital. $\ 2$.

-C diploa. - Carol. III. 55.

discophora. - Chile n. 9. f. 6.

episphaeria. - [S. 385.] - Fuck. s. 656. -

Ital. 539.

flávida. - [S. 35S.]

fulgens. - S. 355.$]$

graminicola. - Ann. Mg. 12. h. 1559. III.

t. 11. f. 40 . (3. B.)

Granatum. - Fuck. s. 655.

helıninthicola B. B. - R. f. 47.

- inaurata 13. I3. - R. f. 16. - Berk. Out.

t. 1. f. 11. unter S. - Fructif.

media. - S. 356.]

miltina. - hl. II. 631.

mobilis. - S. 35s.]

- ochracea. - $[$ S. 357 .

Oropensis. - Ital, 540.

parasitans. - \$. 38s.]

perpusilla. - Carol. IV. 51.

Peziza. - [S. 358.] - Berk. Out. t. 24.

f. 6. - Fuck. s. 657 .

polythalama. - Carol. III. う†.

- resinae Fr. - [S. 355.] — Fuck. s. 654.

rhodomela. - [S. 3SS.]

- rosella. - - S. 35S.] - Kil. 1557. - Ann.

Mg. n. h. $1 \$ 61$. VII. t. 17. f. 24, b. p. 452 .

(Spor.).

- sanguinea. - [S.3S . $]$ - Kl.1732. - Fuck. s. 653 .

— sinopica. - [S. 355.] - Ital. 493.

- violacea. - [S. 3SS.]

xantholeuca. - ef. Sphaerolina. - [S. 355.]

N ematogonium. - [S. $459^{* *} .496^{* *}$.]

aurantiacum. - Ann. sc. n. 1s34. II. t. 2.

f. 1. (Dsm.) - Bisch. f. 3729. - Pay. b. cr.

p. i2. f. 323 .

- byssinum. - Kl. 1752 .

- fumosum. - Bon. t. 9. f. 186.

149 simplex. - Bon. t. 9. f. 15\%. - Fuck. rh. 149.

Neottiospora. - [S. 426.]

f. 42 . - Voges. 1263.

Neuroecium. - [S. 370. 406*.]

Nidularia. - [S. 43S.] - cf. Cyathus u. Crucibulum.

(Tustralis. - Ann. sc.n. 1844. I. t. 7. f. 2-12.

(Tul.).

bissiseda. - Jungh. pr. t. 3. f. 12.

- campanulata Silth. - Bisch. f. 3652. -

Wstd. 2S2. - Loud. f. 16343.

- With. = Cyath. vernicos. Tul. - C.

Olla. - Brit. f. 255. - Purton. I. t. 17. f. 3.

confluens. - [S. 135 .]

II of $\mathrm{man} n$, Index Fungorum.
Nidu laria Crucibulum Fr. - Bisch. f. 3651. -

Foges. 776. - Brit. f. 167. - Wstd. 151.

- dentata. - [S, 435*.]

- denudata. - [S. 435.]

-- Duriacana. - Ann. sc. n. 1S41. I. t. 7.

f. 13-17. Tul.) - Alger. t. 24, 1.

farcta. - Kl. 1517. - Kl. II. 458.

— v. radicata Fr. - Bisch. f. 3651 .

- granulifera. - [S. 43S.] - Cd. Anl. t. D.

f. $42: 24-27$.

- laevis Bull. - Kl. 340. - Purton. I. t. 17.

f. 2. - Wstd. 451 .

Olla Ik. - Wstd. $2 \$ 2$.

- plumbea P. - Wistd. 252.

- radicata. - S. 435.]

- scutellaris Fr. - Bisch. f. 3653.

- striata Bull. - Bisch. f. 3650 . - Kl. 335. -

Voges. 2\3. - Brit. f. 259 . - Purton. I. t. 17.

f. 1. - Wstd. 121. - Vent. mi. 63. f. 1.

- vernicosa Bull. = Cyathus Olla P. - Voges. 152. - Wstd. 242.

Niptera. - [S. 359.] - cf. Peziza.

Nodulisporium album. - Sturm h. 35. t. 3.

- ochraceum Pr. - Kl. 1272. - Sturm h. 35. t. 2 .

- sphaerosporum. - Kl. 1975.

Nodulosphaeria dolioloides Awd. - R. f. 547.

- hirta. - Kil. II. T25.

Nosophloca. - [S. 520.

Nyctalis. - ef. Asterophora.

- asterophora. Bail S. t. 33. - Kl. 617. - Kl.

II. 235 . - Bot. Zt. 1559 . t. 13. f. 1-11. (Anat.

de 13.) - Müller, P'flanzenstaat. p. 274. (Spor.

Copic). - cf. Artotrogus ast. - [S.312.116**.]

— canaliculata Fr. - Bisch. f. 3415.

microphylla Cd - Fuck. s. 1042.

- parasitica. - [S. 312.] - Kl. II. 501. -

Berk. Out. t. 19. f. 2. - Bot. Zt. 1559. t. 13.

f. 12-19. Anat. de B.) - Nov. Act. 1861 .

XXTII. t. 1. f. 10. Anat. Bail:

- verpoides. - [S. 312 .

Octariania. - [S. 436.]

- ambigua Vitt. - Vitt. t. 4, 7. - ef. Melanogast. Kil. Cd.

- asterosperma. - Vitt. t. 3, 7. - Cd. Anl.

t. D. f. $45: 5.6$.

- Vitt. - Ann. sc. n. 1\$43. XIX. t. 17

f. 21. (Fructif. Tul.). - Tul. F. hyp. t. 11.f.1.

- Pay. b. cr. p. 114. f. 529. - Cd. Ic. VI.

t. 7. f. 61 .

- aurea. - Vitt. t. $3,14$.

- carnea. - Cd. Ic. VI. t. 7. f. 66.

- carotaccolor. - Cd. Ic. VI. t. 7. f. 65.

compacta. - Tul. F. hyp. t. 11. f. 3.

- depauperata. - Tul. F. hyp. t. 11. f. 2.

mollis. - Comment. soc. crit. it. 1561 . I.

t. 2. f. 4. p. 33. Notar.).

rubescens Vitt. - cf. Melanog. rub. Cd. -

Vitt. t. 4, 12.

Stephensii. - Tul. F. hyp. t. 21. f. 6. -

Cd. Ic. VI. t. 7. f. 67.

- variegata Vitt. - cf. Melanogast. v. -

[S. 437.] - Vitt. t. 3,4 .

- Webbiana. - cf. Splanchnomyc.

Octospora. - cf. Peziza.

citrina Fl. dan. = Peziza lenticularis Fl. dan. XI.

- hirta. - Wstd. 141 .

Odontia. - [S. 329.]

- albo-miniata B. C. - zu Polyporus sangui-

nol. (Berk. In. 370.). 
O dontia bugellensis. - K1.1915. - Kl. II. 210. - Ital. 536.

— barba Jovis. - [S. 329.] - Cd. Anl. t. G. f. $74: 3.4$.

- compacta $=$ Phlebia hydnoides. - [S. 329.] - crustulata. - [S. 324.]

- fimbriata $=$ Sistotrema f: P. $-[$ S. 329.]

- lateritia. - Carol. V. 22.

- Pruni. - Kl. 1514.

Odontostroma.-[S. 141.]

Oedemium. - is. 501 .

- atrum. - Cd. Anl. t. 13. f. 13: 3. - Bisch.

f. 3516 . - Sturm h. 6. t. 9 .

Oedocephalum album Prcuss. - Sturm.. h. 36. t. 2 .

- alienum Preuss. - Sturin h. 36. t. 3. 1974 .

_- elegans Preuss. - Sturm h. 36. t. 1 .

Oidium. - [S. 494.] $\div$ ef. Torula, Erysiphes conidia.

- in sero sainguinis: Ioetle, Chemie u. Mikrosk. 15 18. Anm. p. 29: ic.

in ovis gallin. - ef. Dactyliun oogein.

- in graminibus. - Limn. Trans. 1535. XVIII. t. 33. (F. Bauer).

—- Aceris. - Kl. 1892.

(Bon.).

acutatum. - Bot. Zt. 1561. t. S. f. 7. p. 196.

- aeruginosum, - $[\mathrm{S} .494$.

- anguinum. - Fres. t. 3. f. 40. - Bot. Zt.

1553..t. 7. f. 13. (Bon.).

— aurantiacum. - Dsm. III. 553.

‥- aureum. - [S. 449. 194.]

$\div$ Berberidis. - Fres. t. 9. f. 12.

- botryoides. - K1. 1671 .

— bulbigerum Fr. - ef. Torula.

$\div$ bullatum. - Joum. hortic. soc. 1S5̆. IX.

p. 51 . (Berk.).

- candidum. - Bon. t. 3. f. 82.

- chartarum Lk. - Dsm. 115. - Wstcl. 149. -

Torula ch. Cd.

- Chrysanthemi. - K1. 1763. - Dsm. III. 552.

- crystallinum. - Aun. sc, n. 1S43. XIX. t. 7.

f. 9. (Lév.)

- Epilobii: - Dsm. III. 256.

- erysiphoides Er. - [S. 194.] - Kil: 154: -

Dsm. 507. - ? zu Erysiphe. comm. (Tul.). -

Dsm. III. 15S. - Wstd. 435. - Krypt. Bad. 316.

- - f. Cucurbitarum. - R. f. 57.

f. Pisi sativi. - R. f. 276 .

Favorum, - Ann. Mg. n. h. is54. XIII.

t. 16. f. 14. p. 163. (B. B.).

fructigenum. - S. 494 - Bisch. f. 3522

3923. - Dsm. 155. - Kil. II. 50:- Carol. II. S4. - Lib. 83 .

— f. Prunorum. - Dsm. 111.

- fulrum. - [S. 494.]. - Kl. 147s. - Brit.

f. 295 .

- fusisporioides Fr. - - [S. 494.] - Kl. 1256. -

R. f. $27 \%$ - Dsm. 230. 631. - Dsm. 1542.

(Fusispor. lact.) u. 1734. - Wstd. 1300. Violae). - Krypt. Bad. 146. (Lamii). - Fuck. rh. II. $132-137$.

- f. Glechomatis. - Kl. II, 491.

- f. Lamii. - Kl. II. 192.

- f. Urticae. - Kl. II. 271.

- f. Urticarum. - R. f. 85. rium lacteum Dsm.).

lactis. - Fres. t. 3. f. $41-43$. - R. f: 5 s8.

- v. luxurians. - Kl. 1555.
Oidiun Lamii. - Kl. 1777.

Leuckartii. - ef, Mucor melitt.

leucoconium. - Ann, sc. n. 1829. XVII.

t. 6. A. Dsin.) - zu Tirysibe pannosa Tul. -

? hue Oid. Ros. Fres. - Sturm h. 29 - t. 34.

(Preuss. = Crocysporium fallax Bon.). $\rightarrow \mathrm{K} l$. 1778.

- D Dsm. : Sphaerotheca pannosa. - Brit.

f. 100. - Carol. IIY. 81. - Wistd. 434 - R.

f. 597 .

_- monilioides Ns. (Acrosporium). - [S. 494.] -

K1. 73. - R. f. 169: forma graminum. - Fuck.

s. 165. - Pringsh. Jahrb. II. t. 29. f. 22.

(Hoffm.) - zu Erysiphe graminis Tul. - Dsm.

III. 257. - Carol. II. S5. - Wstd. 299 ; v. Rosae: 431 .

- f. Leonuri. - K1. II. 272. (c. ic.) $=$

O. erysiphoid. (Tul. Sel. I. 215.) opuntiaeforme. $-\mathrm{Kl}$. 1672.

_- Polygoni avicularis. - Fres. t. 9. f. 17.

- Porriginis $\mathrm{Igh}$ : - ef. Achorion Schönleinii Rem.

— radiosum. - Lib. 295.

$\div$ Rosarum. - Fres. t. 9. f. 13. - ? leucocon. Dsm.

- rubens Lk. - Wstd. 309.
Schönleinii Fr. - ef. Mycoderma Favi Gruby.

simile. - Hook. Lond. J. 1S45. IV.t. 12. f. 4.

_- Tanaceti? - Fres. t. 9. f. 16.

- Tritici. - Lib. 355. - Kl. II. S1. - R. f. $47 \%$.

- Tuckeri. - Traubenpilze: J. Crocq, Rech. s. 1. malad. de la vigne, mit 3 Taf. MIém. d. sav. étr. de l'Acad.r. sc. Belgique. 1551-1953. Tom. XXV. Brüssel. 1S51. 1 ${ }^{\text {10 }}$.): - Journ: hort. soc. 15.53. VIII. p. 235. (cum Cicinobolo); u. 1554. IX. p..69: 6. 7 ; p. 70:5.'(Berk.). Fres. t. 9. f. 14. - Fuck. s."169. - Bot. Zt. 1853.t. 11. Mohl : = Crocysporium fallax Bon. - Willkomm, Wunder d. Mikr. 1\$56. p. 89. f. 42. - Regel, Gartenflora. 15ว2. t. 21. f.1-6. - K1. 1669. - Natur, v. Müll. u. Uhle. 1861. p. 29. (c. fruct., Bettziech). - Schacht, Baum. 1560. p. 314. - zu Erysiphe Tuckeri Berk. : Journ. hort. soc. London. $1 \$ 55$. IX. p. $6 \$-70$. ic. - Dsm. 1733. (ed. 1550.). - Wstd..771. Fuck. rh. 131 .

-Uredinis. - [S. 50j.]

- velutinum. - K1. 1670

- violaceum. - Ann. sc. n. 1846. VI. t. 6

f. 16.17 ; u. 184 S. IX. t. 9. f. 16. 17. (Harting)

- Schacht, Kart. t. IX. f. \&; t. ${ }^{\prime}$ X. f. 1.

- virescens, - [S. 494.$]$

Ombrophila. - [S. 357.$]$

Oncidiu m. - ef. Actinonema,

On comyces. - cf. Sphaeria. - Bail s. t. 22 .

- carneo-albus B. B.:- R. f. 134 .

Onygein a. - [S. 446:]

- agaricina S. - cf. Astezophora phys.

- corvina A. S. - [S. 446.] - Bisch. f. 3617.

- Pay. b. cr. p. 99. f. 457. - Berk: In. p. 273.

f. 62. b. - Ann, sc. 1. 1814. I. t. 17. f. $1-11$. (Tul.).

- equina, - - [S. 446.] - Ann. sc: in. 1544. I. t. $17:$ f. $12-17$. (Tul.) - Cd. Anl. t. C. f. $27:$ $20-22$. C C. Ic. VI. t. 10. f. 96. - Loud. f. 16501 - Bisch. f. 3611 - Bèrk. In. p. 273. f. 62. a. c. - K1. 645. - Voges. 775 .

- faginea. - [S. 446.] - Kl.1724. - ef. Pilacre et Botryochaete.

- hypsipus Ditm. - Sturm h. 1, t. 12. piligena. - [S. 416.$]$ 
Oid. Aurrantineum Leveit. Penicillium Sitoghilum quodt

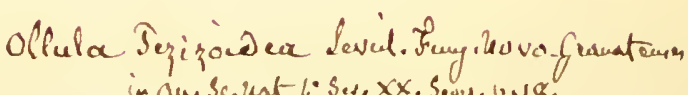

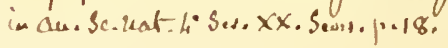




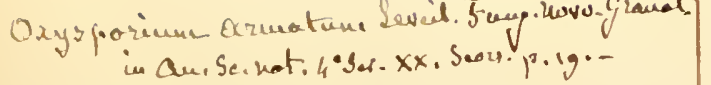


O og a ster algericus. - Cd.Ic. VI. t. XVI. f. 122.

$=$ Terfezia Leonis Tul. u. Tulasneinia Leonis. - Berkeleyanus. - Cd. Ic. VI. t. 16. f. 11 s. brumalis Cd. - Cd. Ic. TI. t. 17. f. 127. cerebrif. Cd. - cf. Hydnobolit. cereb. Cd. - -

Cd. Ic. VI. t. 16. f: 121 .

- cibarius Cd. - Cd. Ic. VI. t. 17. f. $126 . \doteq$ O. gulonum Cd. ef. p. T4. - Französ. Trüffel. $=$ O. melanospor. Cd. ?

- gulonum Cd. - Cd. Ic. VI..t. 17. f. 125. - Lespiaultii Cd. - Cd. Ic. VI. t. 16. f. 119. leucophloeus Cd. - ef. Oog. brumalis C'd. melanosporus Cd. - Cd. Ic. TI. t. 16. f. 124. - nitidus Cd. - Cd. Ic. VI. t. 15. f. 117 rufus Cid. - Cd. Ic. VI. t. 16. f. 123. Venturii. - Cd. Ic. VI. t. 16. f. 120.

Oom yces carneo-albus B. B. - Fuck. s. 501 . -

R. f. 131.

O ospora chartarum Wllr. - Wstd, 119. monilif. Wllr. - ef. Oidium monilioides. -

zu Erysiphe Gram. Tul - Wstd. 299.

U pegrapha. - ef. Lichenes.

- coccinea. - Wstd. 316. culmigena Lib. - Wstd. 66.

Epilobii I,ib. - Wstcl. (iti.

herbarum Mont. - Wstd. 66.

herpetica v. disparata. - Wstd. 315

- macularis Ach. - cf. P'silonia faginea. s. $102 * *$.

- Prostii Nyl. = Hysterium Pr. Dub.

rimalis Wahl. - Wstd. 315.

- rubella Duf. - Wstd. 315.

rufescens $\mathrm{I}^{\prime}$. - W Wtd. 315 .

rugosa Schaer, - ef. Psil. fag.

scripta Duf. - Wstd. 40.5.

verrucarioides: - Wstd. 315.

Op.hiobolus acuminatus Dub. - Kl. II. 5 i.

- disseminans: - Hedw. I. t. 4. f. \. - Kl.

1823 u. 1914. - Til. II. 5:30.

Ophiotheca. - (affinis Areyr. et Didermati). chrysosperma Curr. - Micr. Journ. II. p. 241.

t. 9. f. $1-5 .(155)$.

Ophiotrichum. $\ldots[$ S. 503.]

Orbilia. - [S. 35\%.] - ef. Peziza.

Ospriosporium. - [S. $\left.4>1^{* 2 *} .503.\right]$

- atrum. - [S.503.] - Cd. Anl. t. B.f: $13: 4$.

- Sturm h. 12, t. 24 .

Ostraoococeum. - [S. 46t).]

Ostracoderma. - [S. 147.44 .

-. pulvinatum. - Fuck. rb. 162.

Ostreichnion anericanum. - Duby Hyst. t. 1 . f. 1.

europaeum. - Duby IIyst. t. 1. f. 2. (Lophium unguiculat. Wllr.).

Ostropa. - [\$.401.] - cf. Sphaeria, Hysterium. cinerea. - [S. 401.] - Aet. Turin. 1 $\$ 49 . \mathrm{X}$.

Dec. 5. f. 5. (Notar.) = Sphaeria barbara Fr. s.

(Dub.). - Flor. alger. I. t. 26. f. 4. Dur.

Mont.). - Kl. 1525:- Kil. II. 527.

- truncata. - [S. 401.]

Ottaviania. - cf. Octaviania.

Ozon i um.- [S. 521.]

- - aurantiacum Lk. - Dsm. 15s. - Lond.

f. 16561. - Fuck. s. 204.

- aureum Dub. - Wstd. 699.

- auricomum Lk. - Wstd. 432 .

- candidum Mart. - Kl. 907. - Fuck. s. 203. fulvum. - ef. Agaric. ozonoides.

Pa chnocybe: - [S. 467.$]$ albida. - cf. Sporocybe alb.

- subulata. - cf. Periconia sub.
Pachyma. - [S. 44.]

Pachyphloeus. - [S. 437.]

citrinus B. B. - Cd. Ic. VI. t. 14. f. 11 1.

Rhizopog. pach.).

- ligericus. - Tul. F. hyp. t. 14. f. 5. t. 14. f. 4. - Cd: Ic. VI. t. 14. f. 113. (Rhizopog. mèl.).

Pactilia. - IS. 472.

Panhistophyton ovatum. - Virehow, Arch. f. path. Anat. 1557 . XII. t. 6. f. 16. 17; u. Berlin. entom. Zeitschr. 1s5s.t. 1 ; t. 2. f. $6.8-11$; t. 3. f. $13 ;$ t. 4 . 6 . (Lebert).

Pan us. - cf. Agaricus.

- areolatus. - Ann. Mg. 11. h. 1842. X. t. 9.

f. 3. Berk. .

- conchatus. - [S. 314.$]$

- cyathiformis. $\div$ Bail s, t. 32 .

- dealbatus. - Carol. V. 9.

- dorsalis. - Carol. II. 13.

- farinaceus. - [s. 315.].

- foetens. - Carol. I. 5 .

- griseo-tomentosus. - [S. 561 I.]

patellaris. - S. 315.

rudis Seer. - Ital. 140.

Sprucei. - Hook. Lond. J. VIII. t. 5. f. 6 . stypticus..-[S. 315.] - Kops FI, bat. XI.

415. 1. - Roq. t. 10. f. 5. - Kl. II. 207.

Fuck. s. 1030. - Bot. Kt. 1560. t. 13. f: 1. ILoffn. - Krypt. Bad. 339. - Carol. II. 12. Ital. 139.

torulosus. - S.314.]- Kil. 1910. - Agar.

carneo-tonentos. Bot.Kt.1856.t.5. f.31.(Hoffm.).

Pa jularia. - S. 509.

- Arundinis Cd. - [s. 509.] - Kl. 1456. -

Fuck. rh. 99.

- concentrica. - Dsm. $4 \$ 5$.

Fagi. - \$s. 509.]

Papulaspora sepedonioides, - Sturm h, 29-.

t. 15. P'reuss.) - Berk. In. p. 305. f. 69. b. I. f. $>0$.

Parmularia. - [S. 114.]

Passalora. - $[$ S. 500 .

- bacilligera. - Fresen. t. 11. f. $55-55$. p. 93.

Cladospor., Mont.).

- penicillata Ces: - Kl. II. 557... Cercospora,

Frese11. p..93.

- polythrincioides. - Fuck, th. 103, fse. 2.

Patellaria. - ef. Peziza.

arduennensis...-. [S. 365.]

atrata. - [S. 366.] - Kl. 521. - R. f. 33.-

Mag: Zool. Bot. 1\$37. II.' t. 7. f. h-k. p. 225.

(Eructif., Berk.). - Micr. J. 1559. VII. t. 11. f. 9. 10. (Curr.).

Carestiae. - Ital. 776.

- clavispora. - Nier. J. 15.59. VII. t. 11. f. \&.

(Curr.).

concolor. - [S. 365 .]

- connivens. - [S. 366.$]$

- discolor. - [S. 366. $]$ - R. f. 52S. (Pez. fallax Dsm.).

Lecideola Fr. Su. = Peziza Fr. - [S. 366.] lignyota. $=[\mathrm{S}, 366$.

melaleuca. - [S. 366 .

melaxantha. - [S. 366.] - Kl. II. 709. -

ef. Pez.: Ital. 7 74.

microsticta. - Alger. t. $2 \varsigma, 7$.

proxima, - Ann. Mg. n. h. 1861 . ViI. t. 16.

f. is. p. 451 : (B. B.).

rhabarbarina. $\rightarrow$ Carol. V. 46.

rhizogena. - Chile n. 8. f. 7 .

Rubi. - Lib. 231. 
Patellaria socialis. - Hoffm. ic. t. 17. f. 4 .

Paxillus. - cf. Agaricus, IRhymovis, Ruthea. S. 306.$]$

- atro-tomentosus. - [S. 306.$]$

extenuatus. - [S. 306.]

filamentosus. - S. 306.$]$

- involutus. - Bail S. t. 34. - Dsm. III.

45\%. - Ital. 635. - Hoffm. ic. t. 10. f. 2.

Lepista. - [S. 306.]

- panaeolus. - Hoffm. ic. t. 10. f. 1. (spilomelacus .

- panuoides Fr. - [S. 306.] - Kl. 1212.

sordarius. - [S. 306.

spilomelaeus. - cf. P. panaeolus.

Peltidi u m Oocardii. - Hedw. II. 1S62. t. 10.

f. $3: 1$. bez. a-e, links unten. Kalchbrenner). - R. f. 521.

I'em phidi um. - [S. 395.]

-. nitidum. - Cd. Anl. t. F. f. 55: 23-24. -

Ann. sc. n. 1540 . XIV. t. 19, f. 10. (Iont.).

I'enicillium. - [S. 459.]

—_ armeniacum. - Berk. In. p. 294. f. 65. c.

— bicolor. - S. 459.]

brevipes. - Cd. Anl. t. C. f. $21: 3.4$ -

Pay. b. cr. p. 69. f. 297.

- candidum. - S. $4>9.7$ - Pringsh. Jahrb. II.

t. 30. f. 25. Hoffm.). - Journ. hort. soc. 1 ts.

III. p. 9 \%. f. 6 . Berk.

chlorinum, - Fres. t. 3. f. 20, 21.

cladosporioides. - Fres, t. 3. f. $23-25$.

crustaceum Fr. - S. 4々9.] - cf. P.glaucum. - v. Coremium Fr. - Bisch. f. 3762.

- curtipes. - Ann. Mg. n. h. 1845. II. t. 11.

f. 1. "Thomas'.

- elegans. - Auerswald Bot. Unterhaltg. $1 \varsigma 63$. S. 355 .

- expansum Ns. - cf. crustac. Fr. - Letell.

665. 2. non glaucum P.

- fasciculatum. - [S. 459.] - Brit. f. 210.

- Fieberi. - Bon. t. 3. f. 79.

- finitimum Pr. - Kl. 1250.

- glaucum. - [S. 459. crust.] - Meyen, Syst.

Pflz. Phys. t. 10. f. 20. 21. - Loud. f. 1655 s.

- Bon. t. 3. f. So. - Willkomm, Wund. d. Mi-

krosk. Iรjf. p.91.f. b. - Kl. II. 675. - Pringsh.

Jahrb. II. t. 29. u. 30. f. 24. Hoffm.). - Berg,

Charakteristik. 1560 . t. 1.f. 1. - P. crustaceum

Fr.: Bisch. f. 3711 ; R. f. 66 . - in ovo Gallinae:

Bull, ac. belg. 15.2. XIX. 1. p. 569. Spring. .

- Fuck. rh. 143. - Fresen. t. 10. f. 17-29.

p. 54 .

- leucocephalum. - S. 4\$9.7

- morsus Ranae. - C'd. Ic. V. t. 2. f. 23.

- nigrescens. - Fres. t. 3. f. 22. - Jungh. pr.

t. 1. f. 3 .

olivaceum, - Fresen. t. 11. f. 1-10. p. \$5. plicatum. - Bon. t. 3. f. \$1.

racemosum P. - Wstd. 196.

roseum. - [S. 4S9.]

- sitophilum. - Ann, sc. n. 1543, XX. t. 16.

f. 4. (Iont.).

sparsum Lk. - Sturm h. 11.t. 6. Bri. el.).

- subtile. - Ann. Mg. n. h. is 11 . VI. t. 14.

f. 25. (Berk.).

- viride. - Fres, t. 3. f. 16-19.

Peribotryon. - [S. 465.$]$

P'erichaena. - [S. 45s.459.]

- abietina. - [S. 459.]

- angulata. - [S. $45 \mathrm{~S}^{* *}$.]

- congesta. - [S. 45S.]

- contorta. - [S. 455.]

- depressa. - [S. 455.] - Lib. 378.
Perichaena incarnata. - [\$. 459.]

lutcovalvis. - [S. $45 \mathrm{~S}^{* *}$.]

marginata. - [S. 455.]

- microcarpa. - [S. 455. 3.]

- populina. - [S. 45S.] - Dsm. 16\% - Brit.

f. 47 .

quercina Fr. - [S. 45S.] - Fl. dan. XII.

209. 3.

- strobilina. - [S. 459.] - Lib. 377. - cf.

Licea. - Cd. Ic. V. t. 3. f. 30. - Cd. AnI. t. C:

f. 2 T: 10-13. - Kl. 616. Phelonitis). - Dsm.

III. 259. - Brit. f. 292. - Schwabe, Fl. an-

halt. II. t. 6. f. 12 .

- vaporaria. - [S. 455.]

_- vermicularis. - S. $45 s * *$.]

Periconia. - $\left[\right.$ S. $\left.467^{* * *}, 465.\right]$

- atra Cd. - [S. 46i.] - Dsm. 1221. - Wstd. 45 .

botrytiformis. - Fres. t. S. f. $47-50$.

- - bulbipes. - Cd. Anl. t. B. f. 19: 10. 11.

— bulbosa. - Pay. b. cr. p. 74. f. 340.

— byssoides. - Cd. Anl. t. B. f. 19: 12. 13.-

cf. Sporocybe. - Wstd. 45. - Dsm. ed. 1. 1621. atra'.

__ capitulata. - Bot. Zt. 1553. t. 3. f. 32-35.

Riess.j.

chlorocephala. - Fres. t. 4. f. 10-15.

Desmazieri. - [S. 467.]

- - grisea. - [S. 467. Pachn.

-_ Helianthi. - Bon. t. 9. f. 191.

- hyalina. - Pringsh. Jahrb. II. t. 30. f. 2ㄱ.

(Hoffm.).

- lichenoides Td. - Bisch. f. 3793. - Wrstd. 45.

— minutissima. - Cd. Anl. t. B. f. 19: 14. 15.

- podospora. - Cd. Anl. t. B. f. 19: 16.17.

- pycnospora. - Fres. t. 1. f. 1-9.

- pyriformis. - Bon. t. 9. f. 196.

- ramosa. - Bull. ac. belg. 1\$52. XIX. 1.

p. 557. u. 55s. Spring..

- Stemonitis. cf. Stysanus St. - [S. 46i**.]

subulata. - Brit. f. 51. Pachnocybe sub.).

- thebaica. - Cd. Ic. V. t. 2. f. 22 .

- toruloides. - Fres. t. 6. f. $33-36$.

typhoides. cf. Sporocybe Desm.

Peridermi u m. - [S. $5 \mathrm{io.}]$

- Abietis. - S. 510.]

acicola. - Kl. $6 \$ 5$.

columnare A. S. - KI. II. 96.

corruscans. - [S. 510.]

- elatinum Lk. - Dsm. 139. - Kl. 3६9. -

Fuck, rh. 290.

oblongisporium. - Fuck. rh. 247.

Pini. - S. 510.] - Kl. 263. - Amn. se. n.

1554. II. t. I0. f. 13. (Tul.). - Bot. Zt. I 59.

t. 11. f. 4. (Keing. Hoffm.).- Lycoperdon Pini.

- Wstd. 660. - Fuck. rh. 2ऽऽ.

- — acicola. - Kl. II. 95. - Wstd. 660.

- corticola. - III. II. 94. - Fuck. s. 23. $\beta$.

Sempervivi. - Fuck, rh. 2-9.

Periola. - S. 472.]

candida, - S. 472.$]$

furfuracea Fr. - Kl. 657.

hirsuta. - [S. 472.] - Cd. Anl. t. G.f. 6๑: 27.

pubescens. - [S. 472.]

stipitata. - [S. 472.$]$

- tomentosa Fr. - [S. 472.] - Dsm. $119 .-$

Loud. f. 16320. - zu Fusispor. Solani (Berk.).

- Wstd. 43. - Fuck, rh. 203.

Perispori um. - [S. 404.] cf. Crocicreas.

- alienum. - Dsm. III. 765 .

alneum. - [S. 404.]

Arundinis R. - Fuck, s. 494. - Wstd. 350. 


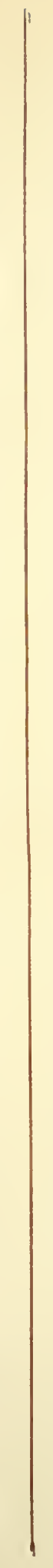




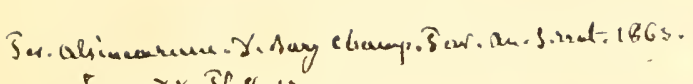
Evin. $x \times$. 3P $8-1 \% \cdot-$

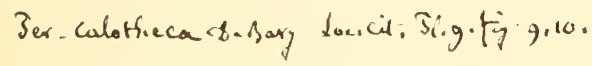

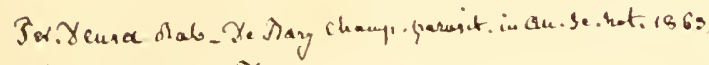
rom. $x \times 114.51 .7 \cdot-$

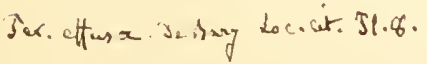

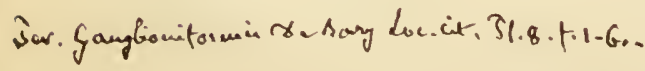

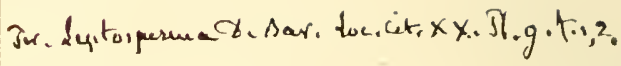

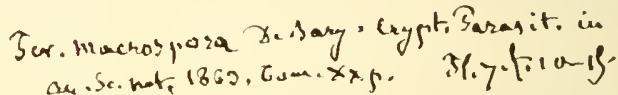

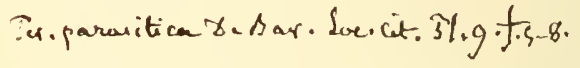

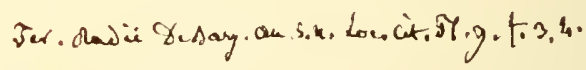

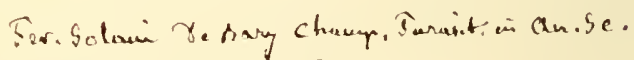
inat. 20u. $\times \times 1803 .-39.5,6 \ldots$

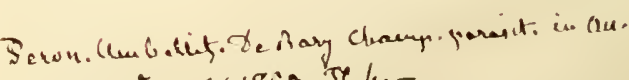
sc. luat. tom. $\times x \times 1863.57 .4 .-$

Ter. violacea Bark. in Mentand. Cryst. By.1. p. Bh3. 
Perisporium betulinum Fr. - [S. 104.] -

Bisch. f. 3691. - Wstd. 279.

—_ Brassicae. - Lib. 250.

- circinans. - Voges. 1097.

- disseminatum. - [S. 404.]

- elegans Fr. - ef. Sphaeronema el. Not.

exuberans Fr. - [S. 404.] Kl. 97.

fagineum. - [S. 404.]

?fibrillosum. - Dsm. III. 766. 767 .

Graminearum Fr. = Crocicreas Gr. Fr. Su.

- Fuck, s, 40.1.

gramineum. - [S. 415. 1.] - Kl. 1322.

Juglandis Lasch. - Ki. 1060.

?occultatum. - Kl. II. 721 .

poliotum Fr. - Dsm. 1021; III. i65.

- populinum Wllr. - Melampsora pop. -

Wstd. $T 7$.

princeps. - Brit. f. 257.

- speireum Fr. - Kl. II. 5io. Depazea sp. Cd.,

Microsticta vagans Dsm.

Tragopogi. - [S. 102 .

vagans Dsm. = Microsticta. - Dsm. 259.

vulgare. - [S. 40.1.6.] - Cd. Anl. t. F.

f. 55: 1-7. - Iay. b. cr. p. 61. f. 249 ; p. 96.

f. 142 .

$$
492 .
$$

Perona. - [s. 333.]

Teronospora. - [S. 493. 191*.| of. Botrytis,

Monosporium, Artotrogus.

- affinis Rossm. - Kl. 11. 149. - Fuck, rh. 22. Alliorum. - Fuck, rh. 11.

- Alsinearum Casp. - Kl. \Ћ゙! non Ung. .

- Fuck. s. 179. - R. f. 37\%. - Fuck. rh. 20.

- alta. - Fuck. rh. 39.

Arenariae. - Fuck, rh. 1s.

calotheca. - Kl. If. 673. - Fuck s. 17\%.

(Sporang.). - Fuck. rh. 2৬. 29.

candida. - Fuck. rh. $3 \mathrm{~S}$.

Chenopodii. - Kl. $1776 .=$ effusa Rbh.

(Casp.).

— conferta. - [S. 493.] - Bot. \%t. 1417. t. 4.

f. 7. - Fuck, rh. 16. 17, - Kl. 15is. P. Alsinear. Casp.

- conglomerata. - Fuck. rh. 25.

crispula. - Fuck, rh. 23.

- crustosa. - [S. 49:3.]

- curta. - Berk. Out. t. 1. f. 7. b.

- densa. - Casp. Schimmel. f. 27. - Fuck.

s. 171. - Kl. 1572. - Kl. II. 173̈. - Dsm. III.

112. - Fuck. rh. 34 .

- Dentariae. - R. i. \$6. - Fuck. rh. T.

destructor. - [S. 493.]

devastatrix. (cf. P. Solani), - Fuck. rh. 37.

Dipsaci. - Fuck. rh. 32.

effusa. - Kl. 1776, Iss0. - Fuck.s. 170.a.b.

Dsm. 1II. 254. - ef. Botrytis farin. Fr. u.

effusa Grev. - Fuck. rh. $11-11$.

- f. Fumariae off. - Dsm. III. 410.

f. Hyoscyami. -- R. f. 291 .

f. intermedia. - Kl. II. 490 .

f. major. - Kl. II 171 .

f. minor. - Iil. II. $172 ; 323$.

f. Papaveris. - Dsm. III. 411.

f. Ranunculac. - Dsm. III. 409 .

f. Ranunculi. - Krypt. Bad. 325.

Fuphorbiae. - Fuck. rh. 40.

farinosa. - [S. 493.]

Ficariae Tul. - R. f. 55. - Fuck. rh. 3.

Galii. - Fuck. rh. 30 .

ganglioniformis. - Bot. Zt. 1843. t. 3. f. B.

- Fuck s. 172. - Transact. hort. soc. 1946.
I. 31. (Berk.) - Kl. II. 169.326 ! - R. f. 290 ).

- ?cf. Botrytis geminata. - Fuck. rh. 33.

Peronospora grisea. - Kl. II. 322. (? var. n.

effusa. Kl. II. 323., Casp.). - Fuck, rlı. 9.

- - Medicaginis. - Ki. II. 775. minor. - K1. II. 323. ef. P. Papav.

veris 'Tul.). Trifolii. - R. f. 375 . (cf. et P. Papa-

- Ilepaticae. - Casp. Schimmel. f. 22-26. -

lil. 1972 .

Ilolostei. - Kl. II. Ti4.

- infestans. - ef. P. Solani.

- Lamii. - Kl. II. 325. - Fuck. s. 17ti. -

luck. rh. 36.

Lepigoni. - Fuck. rh. 21.

- leptosperma de B. - R. f. 571.

lilacina Fres. = effusa Kl. ISつ(1.

nacrocarpa. - Cil. Ic. V. t. 2. f. 21.

Ribl. non ('I.) zu P. Umbellif. Csp. -

lil. 1172 ; it. Cent. XV; it. Cent. XVII. I.

f. 373 . - Botrytis crustosa Fr. - Fuck. rh. 2 .

— f. elongata. - R. f. 37.

macrospora Casp. = Botrytis crustosa $\mathrm{Fr}$.

Iyosotidis de J. - R. f. 572 .

obovata B. - R. f. 24.9. - Fuck. rh. 19.

ochroleuca. - Ki. II. 175. -- Fuck. xh."

Papaveris '1'. - Fuck. s. 175. - Kl. II. 323.

l= grisen Ing.: Lasp. . - Fuck. rh. 4.

- parasitica. - [\$. 493.] - Bon. t. 5. f. 121.

- Fuck. s. 17\% - Kl. II. 324. - ef. Botrytis

par. - Fuck. xh, 5. 6. - Schweiz. 9. - kiryt.

liad. 516 .

pulveracea. - Fuck. rh. 1.

- pygmaea. - S. 193.] Bot. Zt. 1447. t. fi.

f. 4. - Fuck, s, Ist). - Fuck, rh. 26.

radii de $B .-$ R. f. 573 .

- Rumicis. - Cd. Aul. t. B. f. 17: 2*...-

Pay. b. cr. p. 72 . f. $317 .-$ Kl. 1455 .

— Schleideni. - [S. 193.5.]

- scleranthi R. -- R. f. 375. - Fuck. rh. 24. Sherardiae. - Fuck. rh. 31 .

Solani. - Flora 15.7. no. 1. f. 7. Fresen.)

- Ki. 149. - Fuck. s. 17 - Kl. II. I7. -

Pringsh. Jahrb. I1. t. 29. f. 23. (Hoffm.). - Bot.

Zt. 1s 17. t. 4. f. 1-6. - Regel, Gartenflora. 1554. t. 7 . f. $5-10 .-$ Willkomm, Wunder d. Mikrosk. 1556. p. 4 . f. 41. - Berk. Out. t. 1. f. 7. - Bull. Soc. Nat. Moscou. 1456. no. IIIV. S. 301-306. 1 Taf. so. (Mereklin). - de Bary, Kartoffelkrkht 1461. f. $1-11$; Ann. se. n. 1560. IIII. t. 13. f. 1-9. - Schacht, Kartoff. t. (i. f. 5-11. - Kühn, Krkh. t. 7. f. 1. 2. - Dsm. 1092. (ed. 1516j. - Kolaczek, Lehrb. d. Bot. 1 556 . p. 439. f. 7. P. trifurcata, devastatrix, infestans. - Carol. V. 92. - Journ. hortic. Soc. 1546. I. p. 9. t. 1-4. (Berk.). Fuck. rh. :37.

- sparsa Berk. - Gardener's Chronicle 1862.

p. 3!) . - Regel's Gartenflora 1463. p. 204.

stellata. - Dsm. III. 75 .

tomentosa. - Fuck. rh. 15.

torulosa. cf. Monospor.

trifurcata, cf. P. Solani. - [S. 493.]

Umbelliferarum. - Casp. Schimmel. f. 28

-32. - Fuck. s. 173. a. b. - Fuck. rh. 27.

- - f. Aegopodii. - Kil. II. 170.

- v. Berkelei Ces. - Kl. II. $5<5$.

f. Conii, - Kl. II. Ifig.

f. Mei. - R. f. 376 .

Valerianellae. - Fuck, rh. 35.

Pestalozzia. - [S. 415.]

Callunae Ces. - R. f. 161 . 
Pestalozzia Castagnei. - Dsm. 1090.

caulicola. - [S. 116.

conigena. - [S. 416.

funerea. Wstd. 491.

- v. heterospora. - Dsin. 726. - Kil. II. 63. - Voges. 1262.

- Guepini. - I)sm. 15.1. - Cd. Anl. t. F f: is: s-9. - Pay. b. cr. p. 7s. f: 361 . - Ann. sc. n. 1440. XIII. t. 4. - Voges. 1195. Prostenium Guep, M. . - Wstd. 337 .

- hypericina C'es. - Iti. II. 61.

- hysteriformis. - Carol. III. 70.

__ Ilicis. - 13ull. ac. belg. 1559. VII. p. 366 f. 21. Wstd.

- Laurocerasi. - Bull. ac. belg. 2. Sér. 1457.

II. p. 5i!) f. 1... Wstd.

- - macrospora. - Fres. t. 6. f. 41-15. - Kl.

$1663=$ Bactridium Bon. R. f: 173 ." Kl. II. 66.

- monochaeta. - Dsm. 1334. - Kil. 1452.-

Ital. 6.52. - Hendersonia acuminata Lév.

- pezizoides. - Act. Turin. 1S4l". III. Dec. 2.

f. 9. Notar.

phacidioides Ces. - Kl. II. 65.

Quepini. cf. Guepini.

ramealis. - [S. 416.

Peylia racemosa Opiz. - I Iotos. 1557. VII. t. 1 f. 1 .

Peziza. - [S. 315.] of. Helotium [S. 35j], Dernatea, Cenangium, Niptera, Lachnella, Patellaria. - Ann. se. n: 1537 . VIII. t. 11. f. 33. Fructif. Lév. - Linn. 1542, XVI. t. 9. f. 41 -45. Fructif. Nägeli. - Flora. 1547. n. 1.

f. 15. asci. Fresen.). - Müller, Pflanzenstaat. p. 2i1. Copie).

- abacina. - [S. 357:

- Abbotiana Sow. - Loud. f: 162;6.

- - abietina. — S 3.319 .7 - Fl. dan. XI. 1553.

1. - Cd. Ic. V. t. 9. f. 66. (Asci) - Pay: b. er. p. 7. f. 23.

[- Abietis. - S. 351.] - Voges. 399.-W Wtat. 1275.

Absynthii. - lil. 1317.

- Acetabulum Bull. - [S. 345;3.]. Loud. f. 16209. - Cd.. Anl, t. G. f. 64: 12-14.Cd. Ic. III. f. 6. f. 95. = bulbosà Schnzl. Sturm h. 31- t. 20. Szl. . - R. f. 316. I) $\sin$ III. 795. - Viv. 52. — Kl. II. 627. (sule.) - acicularis - Loud. f, 16275. - Helotium v. abietina: Fl. dan. XII. 2015. 1.

Aconiti. - S. 354.]

- acuum A. S. - Kl. 1022. - Dsm. 1036. Fuck, s. 797.

- aeruginosa. - [S. 520.7 - Loud. f. 16259. - Fuck. s. 501. - Krypt. Bad. 136. - Brit. f. 251. - Carol. V. 40. - Iib. 325 . aftinis. - Borez. fg. ingr. t. 3. f. 1 . Afzelii. - Afzel. t. 12. f..30.

agyrioides. - [S. 35.]. 360, 6.] - Ann. sc.

11. 1\$37. VIII. t. 2. f. 5.

alabastrina. - [S. 353.]

alba Fr. - Fl. dan. XI. 15ร5. 1.

albida. - Dsm. 1604

albo-badia - [S, 3.51.]

albo-marginata Schum. = canlicola, Fl, dan. albo-testacea. - Dsm. 915.

-- albo-violaseens. - [S. 365.] - Fl. dan. XI.

191\%.2. - Ki. 142. - [S. 365.] - Letell. 615.

1. fallax P.. - Lib. 126. - Wstd. 397. -

cf. P. relutina.

- - r. alba. $\leftarrow$ Dsm. 119.

- r. nigro-cacsia Fr. - Fl. dan. XII.

2052. 2. - lil. 918 .
Pe ziz a Nlismatis. - [S. 360.]

- alutacea. - Fl. dan. XIII. 2275. 1. - Fuck. s. $>62$.

- - amentacea. - Act. Turin. 1505. XIV. t. 2. (Cumino). - Kll. 1019.

- amentalis Schum. = P. Caucus Fl. dan.

amenti. - Kl. 1019, - Fuck. s. s02.

ammophila. - Alger. t. 2s, 2.

- amorpha. - Voges. 395:- - ef. Corticium,

amplissima. - [S. 319 .

ancilis. - [S. 3 i!.]

amularis. - Atractob. ubiq. 'Td.

- annuIata. - [S. 352.]

anomala. - [S. 352.] - Fl. dan. XII. 2052.

3. - Dsm. 459. - Fuck. s. \$23. - Kl. 524.

Kl. II. 307: - Brit. f. 260. - Carol. IV. 20.

- Lib. 227. - Wstd. 395.

Antzii. - Bull, ac. belg. 1\$6́l. p. 17t. ic.

(Münter).

apala B. B. - R. f. 25.

applanata. - [S. 3 19.] - Fl. dan. XII.

2051.3.

Aranea. - Act. Turin. 1S11. III. Dec. I.

f. 1. Notar. - Kl. II. I7.

- arảneosa Sow. - R. f. 30.

- ardennensis Mnt. - [S. 365.] - Dsm. $46 \%$.

- Anm. sc. n. 1s36. V.t. 13.' (Mont.), - R. f.

223. rhabarb), - Wstd. 992. 1251 (Rosaej.

- arenaria Osb. — S. 349.1 — Fl. dan. XI.

1 $551,2 .-\mathrm{kl} .732$.

areniraga (Phial.).- Dsm. III. 17.

- - arenosa Fuck. - Kl. II. 630. (t. Fuck.).

- arenula. - S. 354.]

- Ariae. - Voges. ss

armeniaca. - S. 352.]

Armeriae Laseb. - Kl. 1126.

Artemisiae Lasch. - Kl. 335 ; it. Cent. XVII.

- Kl. II. 623 .

- Artocreas. Reb. - Wstd. 919.

- Arundinariae. - Carol. III. 39.

Arundinis. - Kl. 1425. (cf. Cent. XVII.). ascoboloides. - S. 365 ***.]

Aspidii, - Lib. 226. - Ital. 5SS̀.

aterrima Lasch. - Kl. 336.

atrata. - [S. 354.] - Dsm. 123.713. - Kl

729. - Phial) Voges. 1190. - Brit. f. 6ৎ. .

Wstd. 99. 993: var. Leguminum.

—- v. Ebuli. Dsm. 1042. u. III. 451.

- Kil. 1923.

atrides. - [S. 354 .]

Atropae. - Voges. 656 .

atro-rufa, - S. 350.65 .

atro-virens. - [S. 359..]

Aucupariae, - Toges. 759.

aurantia. - [S. $349:$ — Loud. f. 16213. -

Bisch. f, 3365. - Hrz. t. 72, B. - Sturm h.

31 - t. 15. Szl.! - Iindl. v. K. p. 33. f. 5.

Spor.) - Bail S. t. 20. - Micr. Journ. 1557.

T.t. \&. f. 35. 39. (Currey). - Kl. 523. - Fuck:

s. $\$ 60$. - Pringsh. Jahrb. II. t. 31. f. 36. (IJoffin.)

- Huss. I, 37. - Wstd. 687. - Ital. 44 .

aurata. - Carol. III. 37.

- aurea $\mathrm{P}$. = chrysocoma Fl. dan.

aurelia. - [S. $351^{*}$.] - Voges. 753.

- aurella. - Carol. V: 11. - Lib. 127.

auricula Judae Hall. - Wstd. 760 .

arellana Lasch. - R. f. 25.

- azurea. - Ann. sc. n. 1S11. XVI. t. 15. f. 9.

Lév. .

- badia, - [S. $3 \$ 9.7-$ Cd. Ic. V. t. 9. f, 67.

(Asci - Kl. 337. - Berk. Out. t. 22. f. 4.-

Huss. 1t: i3. 


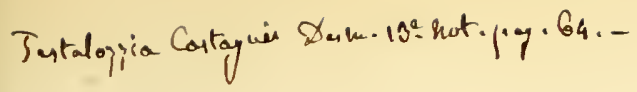



Yeziz a barbata Kz. - [S. 365.] - Fuck. s. 762. -. R. f. 429.

- Batschii. - Act. Turin. 1s(03. XII. t. 11. (Balbis).

- benesuada. - Anm. sc. n. 1553. XX. t. 15. f. 5. 9. (Tul.). - Schacht, Lehrb. An. Phys. II. 1559. t. VI. f. 4.

_- Betuli. - S. 372.]

— betulina A. S. - Wstd. 279 .

- bicolor. - [S. 351.] - Loud. f. 16242. -

Fuck. s. 836. - Dsm. 457. - Bot. Zt. 1562. t. 11. F. z. Solms - - Bisch. f. 3357. - Kil. 141. - Kl. II. 224 .

- Schum. = cerinea Fl. dan. - Brit. f.

15.5. - Norweg. 99." - Wstd. 159. v. caulicola: R. f. 227 .

blandula. - Berk. Out. t. 1. f. 13, c. Sppermat.).

- bolaris. - [S. 353.$]$

— brevipila Rob. - Dsm, 13.12.

- brunnea A. S. - [S. 350.] - Kl. 915. -

D́sm, 712. - ¿Schnzl. t. 16. f. 1-3. - Sturm h. T. t. $2 \varsigma$.

\section{- Batsch: Wstd, 191.}

- brunneo-atra. - [S. 33̀1.7 - D)sm. 26.

— brunneola Dsm. - Dsm. 656. - R. fo 126.

-_ bryophila. - [S, 353 .

- bulbósa. - S. 3 16.] - Bisch. f. 3341.

Sturm h. 31-. t. 21. (Szl.). - cf. 1'ez. Acetab.:

('d. Ic. III. t. 6. f. 95 . sec. Sclinzl.

— byssiseda. - [S. 352.]

- Cacaliae Phialea'. - Kl. 1525.

s. $\$ 22$.

_ calamaria. - Kl. 1926 .

calyciformis. - [\$. 345.]

- calycina. -- [S..35!.] - Fl. dan. XI. 1917.

1. - Fuck. s. 837 . - Cd. Ic. V. t. 9. f. 61 . -

Ki 1021, a. - Kl. II. 422 . - Bot. Zt. 1562.

t. 11. (F. z. Solms - - Brit. f. 261. - Lib.124.

- f. Abietis Fr. - KI. 237.

- v. flavo-coccinea A. S. - Kil. 1021. b.

Calycium. - [S. $356 * *$.]

calycula Schum. = P. Calyculus v. Infund.

Fl. dan.

calyculaéformis. - [S. 351.广 - Fl.dan. XII.

2032. 2.

Calyculus Sow. -

2275.3.

- Campanula. - S. $3.53 \%$ L Loud f : 16264 .

- Bisch. f. 3350. - Kll. II. 419 .

— Candolleana. - cf. Sclerotium Pustula.

cantharella. - [S. 349.] 지. 236.

- capillaris. - [S. 351.]

_ capula Holmsk. - [S. 353 *.] - Fl. dan. XI.

1970.3.

- carbonaria. - [S. 349.] - Kl. ПI. 622.

- Carestiae. - KKl. II. 704.

- caricicola. - Kl. 1531.

- caricina. - Lib. 230.

- Caricis. - Dsm. 455 .

- carnea Fr. ? = subcarnea Fl, dan.

- carneo-pallida. - Dsm. 1609.

- carpinea P. - Dsm. 462. - Voges, isi.

- fascic. = Dermatea carp. Fr. - Carol.

Y. 3s. - Wstd. 12it. (fascic.).

Catinus Holmsk. - [Ș. 349.] - Fl. dan.

XII. 2051.2. - Kil. 1720.

- Caucus. - [S. 353.] - Fl. dan. XII. 2054.

2. - Fuck. s. Sis, a. - Krbh. t. 5. f. 39.
Peziz a caulicola. - [S. 3ă1.] - Fl. dan. XI. 1915. 3. - Fuck, s. 532. - Kł. 416. - KJ. II. 220 .

- Cenangium. - Act, Turin. 1S45. VII. Dec.

1. f. 1. Notar - Ital. 637.

_ ceracella. - Is. 353.7 .

- Cerasi. - Voges. 494. - ef, Cenang. ('er. — Wstd. 494.

- Cerastiorum. - Kl. 1120. - R. f. 421 . -

Wstd. 10\1. - Dsin. 465, - Lib. 25.

- cerea Sow. - [S. 349.] - Loud. f. 16216.

- Vent. t. 12. f. 116.117.

- cerina P. - [S. 351.] - Kl. 525. - Fuck.

s. $535 \%$ - KI. II. 624 - - Bot. Zt. 1462. t. 11.

F. z. Solms. - Toges. lis?.

- cernua schúm. = capula Fl, dan.

- cervina P. - Wstd. $12 \%$.

C'esatii Ifont. - Hedw. I. t. 11. f. IV.

Kl. II. 125: Helotium.

— Chailletii. - Lib. 269, a.: Phacid. Patella camp.

Chauvini P. - Wstcl. 321.

- Charetiae. - Lib. 26.

— ehlora. - S. $356^{*}$ - - Carol. V. 39.

- chlorina $\mathrm{P}_{\mathrm{C}}=$ diversicolor $\mathrm{v}$, viridis $\mathrm{Fl}$, dan.

chlorinella. - Kil. 1 $\leq 12$.

chlorotica. - [S. 352.]

- chrysocoma,-_\$.359.] Calloria -Fl. dan.

XII. 2133. 2. - Loud. f. 162io.,- Dacrymy-

ces chr. 'Tul.

- chrysops. - [S. 351.$]$

chrysostigma. - K1: 1526.

ciboria Vahl. -

- _ - v. major. - Kl. 1011.

- ciborioides. - [S. 353.] — IIoffm. Ic. t. 16.

f. 1 .

—Ciborium. - [S. 344. 353.] - Ǩl. 913.

- cíliaris. - [S.351.] - Fl. dan. XIr. 2032.1.

Fuck, s. 540. - Kil. 1622. - Dsm. 456 .

- oiliata Hoffm. - Wstd. H1.

- cinerea Batsch. - [S. 353.] - Loud. f.

16271. - Kl, 52s; Cent. XV, Suppl.

_- cinnabarina schum. = subhirsuta v., Fl. dan.

[s. $365^{* * *}$. ]

- cinnamomea - - S. 362 .

- citrina Batsch. - Fl. dan. XI. 1971. 3. -

Toges. 754. - Kl: 333. - Fuck. s. 796. - R.

f. 29 ; 29. b. : Cent. III, Suppl. - Bot.Zt. 155 9.

t. 11. f. 2t. (Keimg. Hoffin.)

clandeştina: - [S. 351.] - Dsm. 1037. -

Kil. 1129. - Kl. I1. 21. - Voges. 1475.- Norweg. 100 .

- Y.patula A. S. - K1. i28.

- Clavariarum. - [S. $-352 \%]-$ Ann. sc. n.

1537. VIII. t. 2: f: 4.

clavata: - S. 353.] - Norweg. 1S؟.

clavellata. - Dsm. 122.

- Clavus A. S. - S. 35\% ombr.] - Bisch. f:

3367.

- clypeata. - [S. $345^{* *}$.]

coccinea. - $[\mathrm{S} \cdot 350]-$. Loud. f. 16225. $=$

Fuck. s. 545. - K1. 1020: - Huss. I. 44. -

Vistd. 657. - Vent. mi. S2. f. 3.

- coccinella Orbil. $:-[$ S. 357. 3.]

- cochleata. - [S. 349.] - Loud. f. 16215. -

Hrz. t. $72 . . A_{0}$ - Fuck. s. 563 . - Kl. 417. -

Ki. II. 25. - Wstd. 657. - Ital. 234.

- coelopus. - Chile n. S. f. 4.

- coerulescens Schum. $=$ P. Schumacheri. $\mathrm{v}$. coerulescens.

- comitialis Batscly. - Wstd..919.

compressa. - [S. 351,] - Kl. 530. (1417). 
Kl. II. 509. - Fuck. s. S06. - Dsm. 714. Voges. 556.

Pez iz a concinua. - Ictell. 609.

- contluens. - [S. 350.] - de Bary, Ascomye.

1563. t. 2. f. 1-14. evol.).

— conistea. - Alger. t. 24, 10.

- comivens. - [S. 366.$]$ - Wstd. 919.

- convexula. - [S.350.] - de Bary, Ascomyc.

1563. t. 2. f. $15-17$.

- convivalis Batscli. - Wstd. 152.

_- coracina. - Alger. t. 25, 4.

- coriacea. - zu Sphaeria Poronia (Lév., Ann.

sc. n. 1s+3. XIX. p. 220.) - [S. $366^{* *}$.]

- cornucopioides L. - Wstd. 192.

- coronata. - [S. $353.349,23$.$] - Bisch. f.$

3369. - Fuck. s. $515 .-$ Dsm. 72. - Kl. 1128.

- Kl. II. 219.

—- Jacq. : = repanda Fl. dan. corrugata. - [S. 349, 13.]

- corticalis I'. - [S. 365.] - Kl. 332. - Kl. II. 625. - Fuck. s. 763. (Lachnella) - Dsm. 120. 6.57 .

- P. = granulaeformis Fl. dan.

- corvina. - S. 403 ¿.]

_ Craterinm. - [S. 361. Urnul.] - Carol. I. 39. crenulata. - Bot. Zt. 1\$61. t. 10. f. 6. p. 250.

Fuck.).

crocina. - S. 359 *.]

dan.

_- Schaeff.: Wstd. $4 \$ 1$.

Crucibulum. - [S.353.] - Fl.dan.X.1754.1.

culmicola. - Dsm. 25.

cupressina. - [S. 351.] - R. f. 122. - Dsm.

III. 19. - Fuck. s. $\$ 35$. - Wstd. 689.

- cupularis L. - [S. 349.] — Loud. f. 16221.

- Fuck.s. 555. - Berk. Out. t. 1. f. 2. (Fruct.).

- Curreyana. - huc Sclcrotium roseum Mont. cyanoderma de B. - R. f. 516 .

eyathoidea. - [S. 353.] — Bisch. f. 3356.

- Kl. 140. - R. f. 224. - Dsm. 461. - Brit.

f. 155. - Wstd. 144. - Ital. 55\%.

- f. Lychnidis - Kl. 1616. (ef. 161\% b.).

_- ? v. platealis. - Kl. 1617. b.

Iil. 1I. 120 .

cyathula. - [S. 351.$]$

Cyathus. - S. 361.5 ?.

daedalea. - Carol. I. 3\%.

- Dehnii Rbh. - Kl. 334. - Kl. II. 23.

dentata P. - [S. 351.] - Bisch. f. 3365 .

Diadema Gmel. - Bisch. f. 3352.

Digitalis. - Voges. $5 \$ 5$.

diluta. - [S. 349.]

$72 \%$.

dilutella. - $[$ S. 354.] - Dsm. 1344. - Kl.

- diminuta Rob. - Dsm. $103 \mathrm{~S}$.

- disciformis. - [S. 356.] - Hoffm. Ic. t. 16. f. 2 .

diversicolor, - [\$. 351.] - Carol. V. 37.

- - v. luteo-subferruginea. - Fl. dan. XII. 20s2. 1. - Dsm. 1343.

- v. viridis Fr. - Fl. dan. XI. 191S. 1. Druminondii. - Hook. Lond. J. 1S45. IV.

t. 2. f. 10. (Ascus).

dumorum. - Dsm. 1602.

Ebuli. - Kl. 1424 .

eburnea. - Dsm. 1605; III. 656.

echinophila. - Kl. 1525.

effugiens. - Dsm. III. 16. (Phial.).

clatina A. S. - Bisch. f. 3355 .

elongata Schum. - FI, dan. XIII. 2275. 2.
Pe z i z a epiblastematica (Orbilia) Wllr. - Kl.1016. — [S. $35 \%$. 1.]

epiphylla I'. - FI. dan. XII. 2033. 1. -

Fuck. s. 792 . - Kl. 1920 - - R. f. 220.

epithelepliora. - Ital. 559.

equina Mill. - cf. stercorea $P$.

- Equiseti IIoffm. - R. f. 123. - cf. P. Persoonii.

— erecta Sow. - Loud. f. 16225.

- eriobasis. - [S. 352.]

crioloma. - [S. 353 .

erratica. - [S. 352.]

erumpens. - [S. 359.] - Dsm. 1345. -

Loud. f. 16273.

- erythrostigma. - [S. $359^{*}$.]

- eximia. - Alger, t. 28, 9.

_ expallens. - [S. 359.]

fallax. - Dsm. 920. - cf. albo-violascens u

velutina; Patellaria discolor R. f. 525.

- fascicularis. - S. 362.] - Bisch. f. 3363.

F'uck. s. 77\%. (cf. Dermatea). - Kl. 249. -

Dsm. 567. - Brit. f. 154. - Letell. 609. (populnea). - Norweg. 97 .

- fasciculata Fr. - cf. Dermatea carpin.

ferruginea Schum. - Fl. dan. XIl. 2033. 3. fibuliformis Fr. - Loud. f. 16277.

fimetaria Schum. - [S. 350.] - Fl. dan. X.

17ธ7. 1. - Fuck. s. 505 .

- Fr. $=$ Helot. fimet. Fr. Su. (Fuck.).

fimiputris. - [S. 350.$]$

firma. - [S. 353.] - Loud. f. 16251. -

Lib. 225.

fissa. - [S. 362.]

- flammea A. S. - [S. 365.] - Bisch. f. 334 \%.

- Kl. 1523. - Kl. II. 22.

- flavo-fuliginca. - [S. 351.]

flexella. - [S. 372.] — Voges. 1094.

floceosa Lasch. - Ḱ1. 1225.

Frangulae. - Voges. 557. - Wstd. 1276.

fructigena Bull. - Dsm. 460. - Loud. f.

16253. - Fuck. s. 799. a. - Bisch. 3335. -

Kl. \$25. - Kl. II. 510. — Wstd. 10S0. - cf.

Helotium virgult.

- f. Carpini. - R. f. 121.

fulgens. - [S. 349.]

fuliginea Schum. - [S. 349.] - Fl. dan. XI.

1854.1 .

— fumosa. - - S. 353 .

_- furfuracea. - [S. 362.] - Pay. b. cr. p. S.

f. 24-26. (Ascobolus). - Ital. 443. (Dermatea).

- fusarioides Berk. - Dsm. 463. - Kil. II. 2v.

- Voges. 1476. 396. (Trem. Urt.) - Brit. f.

67. - Mag. Zool. Bot. 1537. I. t. 2. f. 4. p. 46.

Berk.). - Wstd. 65s.

- fusca. - [S. 352.] - Loud. f. 16250.

Kl. II. 306. - Fuck. s. \$21.

- Schum. = P. Schumacheri Fl. dan. -

Brit. f. 2S6. (zu P. Rosae). - Letell. 615. 3.

fuscescens. - [S. 351.]

Galegae. - Kl. 1614.

gemmata Schum. - [S. 319.] - Fl. dan. XI.

1553. 2. - Pay. b. cr. p. 7. f. 22.

- Gentianae P. - R. f. 423. (cf. Excipula G.). globosa. - [S. 345.]

globularis. - [S. 364.]

glumarum, - [S. 352.] - Dsm. 454.

- Godroniana Mont. - Kl. II. 16. - (Lach.

nea) Voges. 1159.

- Graminis. - Dsm. 466. - Kl. II. 22S. -

Wstd. 95 .

granulaeformis Schum. - Fl. dan. XI. 1917. 3. granulata Bull. - [S. 350.] - Loud. f. 16223. 


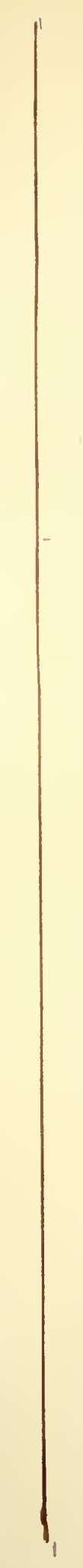



Peziza granuliformis P. - Wstd. 690. granulosa Schum, = repanda Fl, dan.

- haemastigma Fr. - Bisch. f. 3372.

- haematostigma Hedw. - Sturm l. 33-. t.

11. Strauss.

IIederae. - Lib. 229. Sphacria Crat. DC.).

- IVstcl. 45

- helodina. - Alger, t, 2<, 3.

helotioides Fr. - Fl, dan. XI. 1 N... 3

helvelloides. - S. 345.] - Kil. 11)!11.

- Fr.: Kl. II. 26.

IIelvellula. - Alger, t. 27, 11.

helvola. - Jungh. pr. t. 5. f. 17.

henisphaerica. - [S. 350.? - 1sim. 711. -

kil. 235. - Kl. II. (j30): 1'. arenosa Fuck., Bot.

Zt. 1\$61. 1. 250. - Fuck, s. S11. a. - liot. Zt.

1562. t. 11. F, z, Solms .

herbarum. - Bisch. f. 3313. - Kil. 633. -

Ki. 11. 227. - Fuck. s. 791. - 1)sm. 5iv. -

loges. $755 .-$ Lib. $2 i$.

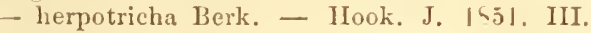

t. $1, \mathrm{f} .2$

heteromera. - Ann, sc, n. 1440. XIII, t. 6.

Iont.

IIindsii. - IIook. J. 1412. I. t. 15. Berk.

hirncolus. - $\$$. 349.$]$ - Borcz. fg. ingr.

t. 3. f. 2.

hirsuta Schff. - W-tcl. 121.

- hirta Schum. - -S. 351. - Loud, f. 11;232.

- Fl. da11. XI. 1970. 2. - 1i. f. 2i.

- hirtella I'. = subhirsuta Fl. dan.

liispicla Schff. - Wstd. (1)!!.

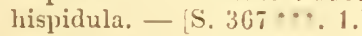

horridula. - Dsm. $1341 \%$ - Voges. 1471.

humilis, - Dsm. 13.16.

humosa. - S. 350.] - Loud. f. 16226. -

('d. Ic. Y. t. 9. f. 169, Asc..

Iumuli. - Kil.630.- Kil. II. 221. Tuck.

s. $>17 .-$ Ital. $>70$

- hyalina. - S. 352 .

- Hypnorum. - [S. 351.

- hysteriiformis. - [S. 36i.3.

janthina Fr. - Bisch. 1. 3333 .

ilicincola I3. B. Dasyscyph. - - Ann. Mg.

n. h. 1561. VII. t. 16. f. 17. p. 45u.

imberbis.

- v. cinerca. - Kl. 1524

inaequilatera Schum. = Cyphella muscicola

v., Fl, dan.

__ inclusa. - [S. 373.]

- infarciens Ces. - R. f.'515. - Ital. - 1

inflexa Bolt. - Loud. f. 16255. über 16259.$)$

Infula Rebt. - Wstd. 17\%.

infundibuliformis Hoffm. - Wistd. 191.

ingrica. - S. 350 .]

inquinans P. - Wstd. 191

insidiosa. - Dsm. 29. - Wstd, 45.

integra Schum. = abietina Fl. dan.

jucundissima. - Dsm. 1040.

juncicola. - [S. 351.]

juncina. - [S. 356: Dub.

Jungermanniae Ns. - [S. $353: 15 \% .359 \%$.

- Iil. 917.

Kerguelensis. - Crypt. Bot. antarct, voy.

Erebus, 15วั. t. 164.3.

Kneifi. - Kl. 1924.

Körberii, - Lotos Pragł 155̌s, t. 1. f, 20.

Pevl.)

labiata Rob. - Dsm. 1035.

lacera. - [S. $362 * * *$.]

lachnobrachya. - Dsm. II. 1603.

laciniata. - [S. 365.]

Hoffmann, Index Fungorum.
Peziz a lacustris. - [S. 359.] - Dsm. 464. Kl. 1623. - Kl. II. 231. - Toges, 11193. I'std. 14.

_ laetissima Ces. - Kl. 1024; 15:\%. - ef. P. Persoonii.

- laerigata. - S. 365.

- L laevis Huds. - Wstd. 451.

- lancicula Rebt. - Kl. 1229.

- laricina. - Act. Turin. 1503. XII. t. 11. Balbis.

- Lecideola Fr. = Patellaria Lec. Fr. Șu.

s. 36ii.

- Ledi. - [S. 361.

- lenticularis Bull. - Fl. dan. XI. 15.5. 2..

Fuck. s. ig..

- - lentifera I. - W'std. 121.

- Huds.: W'std. 252.

lepidota. - 'S. 319.

leporina Batsch. - S. 349.] - kl. 143.

lil. II. $24 .-$ Fuck. s. $865 .-$ R. f. 512.

- leucoloma. - S. 350.] - IBisch. f. 33.15. -

Fuck. s. 450 . C Cl. Anl. t. G. f. 6.1: 14-19.

- Sturm h. 31-, t. 17. Szl. - Kl. 1315. -

L. f. 513. - Krypt. Bad. 15.1.

- leuconcla P. - ? I'std, 1279.

- leucorrhodina Mont. - Sagra, C'uba t. 13. f. 1 .

- leucostigma. - \$. 35\%. 1.

leucotricha. - 5. 3.31.

- lignyota, - ? Kützing, Phil. Bot. 1652. t. 21.

t. 2. - [\$. 36fi.

- Ligustici. - Phacid. I'atella camp. - Wstd. 324.

- lilacina. - 4. 3\% *

- Linariae IRbl. - Kl. 22.1 a Pez. lerb, vix

diversa

litorea. - S. 353.] - Kil. 1125; 1(i19. -

Wistd. 112.

— livida. - \$. 351.] - Fl. clan. XI. 1915. 3

Fuck. s. 411

_- livido-fusca. -. S. 353.

- Lonicerac. - S. 35.3.

- lutea Schum. - Fl. dan. XIII. 2271. 2.

luteola. - S. 352 .

- luteo-virens. - Dsm. 1041. - Kl. 1s15.

- lutescens. - Kil. 1\$13.

- Ixchnidis. - Kl. 1616.

macrocalyx. - Fres. t. 9. f. $7-11$

macropus. - S. 345.? - Loud. f. 16219. -

Fuck. s. -66 . - Berk. In. I. p. 36. f. 13. c. -

Kl. 144. - Kl. II. 21i. - Wstd. 991.

- maculincola. - [S. $365^{* * *}$.]

- malatephra. - cf. melat.

- Marchantiae. - S. 354.]

marchica. - - $5.362 * *$.

- marginata Holmsk. = cerinea $\mathrm{Fl}$. dan.

- maritima. - S. 349.? - Dsm. 9/s.

- Marsupium P. - Kl. 1013.

- Martii. - Sturm h. 33-. t. 10. (Strauss).

melaena. - [S. 319.$]$

melaleuca. - [S. 366.]

- melaloma. - Kl. 1423; - it. Cent. XVII.

Fuck. s. 552 .

melanoloma. - [S. 350.$]$

melastoma. - [S. 350.]

— Sow. - Loud. f. 16229

—

- melatephra Lasch. - Kl. \$25. - Dsm. 1604.

- Wstd. 994.

melaxantha. - [S. 352. 366.] - Ital. Ti4.

- Patellaria mel. 
Peziza membranacea Schum. - [S. 350.] - Fl. dan. X. 17\4. 2.

- bovina $P^{\prime}=\mathrm{P}$. vaceina $\mathrm{H} l$. dan.

Messerschmidii. - [S. 351 \%]

micropus P. - [S. 3 i9.] - Bisclı. f. 3344.

- Berk. Out. t. 22. f. 5.

- miliaris. - K1. 1621.

- millepunctata. - Lib. 125.

- minima. - S. 354.]

mirabilis. - Borcz. fg. ingr. t. 1. 5.

misella Rob. - Dsm. 1039.

- mollissima. - Kl. II. Tos.

- mortuaria Ces. (Tapezia) - Kl. II. 15.

- Mougeotii P. - Biatora campestris Fr. (ex

Nyland.).

mundula: - Kil. 1530.

mussiva. - [S. 350.]

- mycopliila. - [S. 353.]

- Neesii. - Kl. 1.119.

— neglecta. - Lib. 29. — Wstd. 658.

- nervicola. - Dsm. 467.

- nervisequa P. - Dsm. 1612. - Toges. 756 .

- Nidulus S. K. - [S. 352.]-Kl. 112; it.

Dsm: 71. - Voges. 5Ss. - Lib. 25. - Wstd. 691.

- nigra Sow. - icf. Pez. Clavariarum.

- Schum. = nigrella Fl. dan. - Toges.

197. (Bulgar. inq.) - Wstd. 191.

- nigrella. - [S. 350.$]-$ Fl. dan. X. 175 . 2

- Krbh. t. 16. f. 17 . a. - Dsm. 70. - Bot.

Zt. 1562. t. 11. (F. z. Solms) - Kl. 1314; 1921 .

- Kl. II. 309. - R. f. 219 . - Schnzl. t. 16.

f. $1-3$.

- nigripes $\mathrm{P}$. - K1. 527 .

- nigro-caesia Schum. $=$ albo-violascens v.,

Fl. dan.

- nivea. - [S. 351.] - Kl. 1023. - Kl. II

705. - Bot. Zt. 1562 . t. 11. F. z. Solms).

Wistd. 397 .

obvelata Lacr. - R. f. 422 .

occulta. - Ki. 1922.

ochracea. - Act. Turin. 1505. XIV. t. 3.

(Cumino).

- Schum. = P. Tuba Fl. dan.

- Oedema (Phialea). - Dsm. 1607.

olivacea. - [S. 353 .]

Olla Batsch. - Wstd. 252.

- ollaris. - [S. 350.] - KI. 337. - Kl. II. 423.

omphalodes. - [S. 350.] - Kl. 1015.

Bull. : Fuck. s. $54 \%$.

- onotica P. - [S. 349.] - Fl. dan. XI. 1970.

1. - Fuck. s. 564. - Loud. f. 16212. - Bisch.

f. 3355 . - R. f. 215 .

Oxyacanthae P. - Wstd. $1 \$ 9$.

palearum. - Voges. 1473. - Wstd. 190. -

(Lachnea) R. f. 519. - Dsm. 917.

— pallescens P. - Kl. 526. - Kl. II. 226.

- Fr. = P. elongata Fl. dan.

- palustris Rob. - Dsm. 1043.

- papillaris. - [S. 352.$]$

papillata Schum. = diversicolor v., Fl. dan.

- P. [S. 351.]

- parvula. - Act. Turin. 1841. III. Dec. 1.

f. 2. Notar.).

- patula. - [S. 351.] - Dsm. 1034. - Fl.

dan. XI. 1454. 3. - R. f. 425 . - Lib. 225 . -

Wstd. $1 \uparrow 3$.

_- paulula. - Dsm. 1610.

- pedunculata Schum. = repanda Fl. dan. -

[S. 319 .
Peziza pellucens. - Kl. 1620 .

-- Schum. = Bulgaria pel., Fl. dan.

perlata. - [S. 34S.]

Persoonii Mlong. - [S. 353.] - Kl. 725. -

Kil. II. 229. - Dsm, 73, - Fuck. s. S16. - ef.

Pez. Equiseti et P. laetissima. - Lib. 329.

- petiolorum. - [S. 353 . - KI. II. 223. -

I) sm. 6.5.

— phacidioides Fr. - Kl. 1226. - Kil. II. 305.

_- phaeoloma Lév. - Démid. Russ. t. 4. f. 7.

- phaeotricha. - Alger. t. $24,5$.

_- phellophila. - Alger. t. $29,6$.

- Phragmiticlis. - [S. 355. I 5.$]$

phyllophila. - Dsm. 659.

- pilosa Schum.) Fr. - [S. 351.] - Fl. dan.

XII. 2032. 3.

— Pinastri. - Voges. 557.

— Pineti Batsch. - [S. 352.] - Kl. 631.

- v. Abictis. - R. f. 31 .

-- pinicola. - [S. 365.] - Kl. II. 50९. - R.

f. 124 .

- pithya P. - Bisch. f. 3340. - Bail S. t. 20.

- Voges. $29 \varsigma$.

placentaeformis. - Verh. zool. bot, Ver.

Wien, IS5S.t. S. f. 3. Niessl).

- Platani. - [? S. 367.5.$]$

_- platealis. - Kl. 161\%. b.

plumbea. - [S. 349.]

pocula. - [S. 319.]

266.

Polygoni Lasch. - KI. 1127. - Dsm. III.

- polymorpha Fl. dan. - Wstd. 191.

- Polytrichi Schum. - [S. 350.] - Fl. dan.

XI. 1916.1. Kl. 1316 - Kl. II. 310 - Anm.

Ig. n. h. 1554 . XIII. t. 16. f. 14. p. 464.

Fructif. B. B.).

- polytrichina $\mathrm{P}$. = rutilans $\mathrm{Fl}$. dan.

- populnea.- - ef. P. fascicularis.

— porioides A.S. - [S. 352.] - Bisch. f. 3479.

- protracta Fr. - Nor. Aet. 1552. XXIII. II.

t. 61. Microst. hy.).

- pruinata. - Carol. I. 35.

_- Prunastri. - Letell. 613. 1.

- $\beta$ A. S. - cf. Dermatea Prun. Fr. -

[S. 362.7.$]$

- Prnini Avium. - [S. $352 * *$. ]

- Prunorum. - [S. 352.]

— Pteridis - [S. 354.$]$ - Kl. 1615. - R. f.

424. - Dsin. 919.

— puberula. - K1. 1529.

- pulchella. - Lib. $32 \overline{7}$. - Cd. Anl. t G. f.

61: 15. - Wstd. 159.

- pulverulenta. - Lib. 125. - R. f. 514.

_- punctata Grer. - Loud. f. $1626 \%$.

- punctiformis. - [S. 352.] - Kl. 826. -

Fuck. s. 824.

- punicea. - Purton. I. t. 25.

— pura. - [S. 35\%.]

- purpurea. - [S.362.] - Fl. dan. XIII. 2274.

1. - Bisch. f. 3339 .

- pusilla. - [S. 353.]

— pustulata. - [S. 349.]

- pygmaea. - [S. 351.]

_ Pyri P. - cf. Tympanis conspersa.

_- pyriformis. - [S. 353.] - Aet. Turin. 1 S05.

XIV. t. 3. (Cumino). - Bisch. f. 3362.

- Pyxis Batsch. - Wstd. 4S1.

—_ quercina P. - Wstd. is9.

- Rabenhorstii Awd. - Kl.920. - Kl. II. 222.

radiata. - Cd. Anl. t. G. f. 64: 16.

radiculata Sow. - [S. 341 .] - Loud $f$.

162311. 


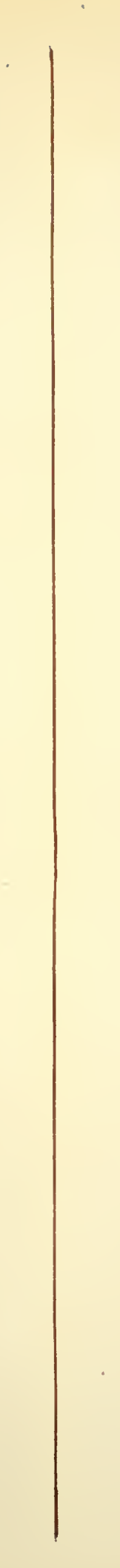




$$
\mid
$$


Peziza Radula. - [S. 319.]

-_ Rapulum Bull. - [S. 345.] - Bisch. f. 3312. Ravenelii, - Carol. II. 46.

- relicina. - [S. 352.] - R. f. 216. - Bot.

Zt. 1562. t. 11. F. z. Solms'.

- repanda. - [S. 349.] - Fl. dan. XII. 2031.

1. u. 20) 1. 1. - Fuck. s. 559 . - Grev. t. 59.

v. Fr. = P. alutacea, Fl, dan.

Resinae. - [S. 354.] - Ki. II 706. - Le-

ciclea) Biatorella M. Fr.

- reticulata. - [S. 349.$]$ - Berk. Out. t. 22.

f. 6 . - cf. venosa, p. 362. - Grev. t. $156:$ -

Huss. II. 7 .

retincola. - R. f. 225 .

_- rhabarbarina B. Lachnella . - [S. 365.] -

I. f. 223. - cf. ardemn. a Kosie Wstd - Pez. 1. Patell. Rubi Lib. ? - Brit. f. 271. - Wstd. 1251.

- rhizopus. - [S. 350.] - Fuck. s $\$ 46 .=$

Sphaeria monocarpa Fl. dan.

Khododendri, - Kl. 1 \11.

_- Ces.: R. f. 129. - Ital. $5 \$ 6$.

rhodoleuca Fr. - Kl. 12:1.

Ribesia P. - ef. Cenang. Ribis Fr.

Kobergei. - Dsm. 916.

rorida $W$. - Fuck. s. 431.

Rosae P. - [S. 352.] - K1. 527. - cf. Tympanis obtexta v. pezizaeform. IV. - Rrit. f. 157 ;

2`6. icf. fusca, - Letell. (3!5, 2.

- rosea Schum. = onotica Fl. das. - Niptera Chile n. 10. f. 7

- roseola. - [S. 352.]

- rubella. - [S. 353. - Wstd. $195=$ vinosa

Dsm.)

- Rubi Iasch. - K1. 635. - Kl. II. 191. -

R. f. 515 .

Rubi Lasch. v. herbicola. - R. f. 215.

- rubicunda Saut. - Kl. 16ils! P. Urticae

Awd! .

rubiginosa. - S. 362 .

rubricosa, - [S. 350.]

rudis. - Ann. Mg. 11, h. 15.51. VII. t. 6.

f. 13. p. 153 . B. B.

rufo-nigra Sant, - Kl. 914.

rufo-olivacea. - [S, 351. - Kl. II. 629.

rugosa Sow. = anomala Fl. dan.

- rutilans, - [S. 350.] - Fl. dan. Xl.1916.2.

- Fuck. s. 853. - Kl. $141 \mathrm{~s}$.

salicaria P. - Dsm. 921

salicella. - Act. Turin. 1 11 . III. Dec. 1.

f. 3. (Notar.) - R. f. 520.

salicina P. - K1. 919 .

- sanguinea. - [S. 352.] - Kl. II. 624. -

R. f. 226 .

_- sanguinolenta. - Bisch. f. 3315 .

- saniosa. - Ann. IIg. n. h. 1 83 S. I. t. i. f, 2.

(Berk.).

- sarcoides. - cf. Bulgaria sarc.

Sceptrum B. - Bisch. f. 3354.

- Schumacheri. - [S. 352.] - Fl. dan. X $1785,1$.

1756.1 .

Scirpi Rbh. 一 Kl. 739.

sclerotiacea. - Kl. 1532. - Ital. 339.

Sclerotii Fuck. : Bot. Zt, 1561, t. X. f. 1.

p. 249. (dazu Sclerot. compact. DC.).

Sclerotiorum. - Lib. 326. - ic. : Coemans,

in Bull, ac. belg. 1560 . IX. p. 79 ; dazu Sclerot.

varium (Münt. in lit.) ; it. aus Seler. compactum

DC., tectum Fr., bullat. DC., sphaeriaeforme

Lib., varium P. (Coems.).
Peziz a Sclerotium P. - Wstd. 690.

Serophulariae. - Kl. $1 \$ 16$.

scutellata. - [S. 351.] - Schnzl. t. 16. f.

4. 5. - K1. 1621? - Bot. Zt. 1562. t. 11. F.

z. Solms , - Wstd. 141. - Ital. 5ऽ5 macro-

chaeta), - Krypt. Bad. 54.

- scutula. - [S. 353.] - Letell. 615, 4.

scybalorum Schum. = Myrothec. sc., Fl. dan

- seinipurpurea Horn. = P. purpurea Fl. dan .

- sepium Phialea. - Dsm. 1606.

_ sepulta. - [S. 564 .]

- sericea. - [S. 35\%.] - Wstd. $2 \$ 2$.

- serotina. - Fuck. s. Su0.

- sessilis Sow. - ITstd. 690.

- setosa. - Cd. Anl. t. G. f. 64: 17. - Bisch.

f. $3373 .-\mathrm{K} 1.634$.

- - smaragdina. - Voges. 1345.

Solani P. - Fuck. s. S14.

- Solenia DC. - Voges. 96. Solenia cand.)

spadicea. - [S. 36.5.]

- sphacelata Schum. = P. Catinus Fl. dan.

sphaerioides P. - Dsm. 124.

sphaerocephala. - Kl. 1121. - KI. II. 230.

Spiraeac. - Dsm. III. 453.

spumosa. - [S. 351 .

- squamosa Schum. - [S. 350.] - Fl. dan.

Xi. 1915.1.

_ sterenraria Bull. = Ascobol. furf. Fl. dan.

- stercorea P. - [S. 351.] - Kl. 731. -

(rypt. Bot. antarct. voy. Erebus, 1554. t. 163,

1. - Norweg. ys.

stipata. - [S. 352.]

stipitata Huds. - Wstd. 991.

- Stitzenbergeri R. - Ri f. 427 . / cf. P. umbon.

v. epiph.) - Krypt. 13ad. 55 \%.

striata N. - R. f. 221 - Fuck. s. 413.

strigosa. - S. $367 \%$ - v. hysteriiformis.

iち. $36 \%$. 3.] - cf. Vermicularia str. u. Graminum Lib. - Wstd. $1>1$.

- strobilina. - [.. 353.] - Kl. 1925. - R.

f. 222. - Fuck. s. 519.

- subcarnea Schum. - Fl. dan XII. 2051. 1.

- subhirsuta. - [S. 350.] - Fl. dan. X. 17h5.

1. - Fuck, s. 551 .

X. 1757.2.

—_ sublicia Holmsk. - Wstd. 991.

— subplana Schum. = ehrysocoma Fl. dan.

subtilis Fr. - Fuck. s. So3. (Helotium).

- subtorta. - [S. $\left.356^{* *}.\right]$

- succosa. - Ann. Mg. n. h. 1941. VI. t. 11).

Berk.).

- sulcata P. - [S. 345.] - Kl. 1721. - Kl.

II. $62 \%$ = Acetabulum.

- sulphurata. - [S. 350.] - Fl. dan. XI.

\$915. 2 .

- sulphurea P. - $[\mathrm{S} .352]-$. Fl. dan. XI.

1915. 2. - Kl. 726; Cent. XV, Suppl. - Kl.

II. 707 . (cf. 705.$)$.

f. 156 .

- sulphureo-caesia Schum. = sulphurea $\mathrm{Fl}$. dan.

- sylvatica. - Palaeont. VIII. t. §. f. 12. ( $\mathrm{K}$. Ludwig).

- syringea. - Kl. 1122. - R. f. 32.

- Tami Ly. - Dsm. 27.

- te tegularis. - [S. $362+:$ ]

$\because$ testacea. - [ [S. $359.366^{*}$.]

- theleboloides, - [S. 351.] - Bisch. f. 336!.

-? Kl: II. 704 . 
Peziza tomentosa Schum. - [S. 350.] - Fl. dan. II. 1916.3. - trechispora I3. B. - R. f. 26.

- triblidioides Rbh. Iachnellal. - Kl. 1130.

- tricholoma. - Amm. sc. n. 1>31. II. t. 4.

_- Trifoliorum. - Lib. 321. Ascobol.) --

-.. Wstd. 399.

— triformis. - [S. 352.]

- Tuba Bolt. - Fl. dan. XI. 1971. 2. - Bisch.

f. $3360 .-$ cum Sclerotio: Ann. se, n. 1413.

XX. t. $i$ f. 6. Lèv ex Micheli.

tubaeformis. - [\$. 353.]

- tuberosa. - S. 34..] - Bisch. f. 3361. -

Fuck. s. S67. - Dsm. 1339. - Loud. f. 16220 ?

16225. - Kl. 1522. - Kil. II. 305. - Voges.

397. - Brit. f. 153. - IIuss. II. 10. - Wstd.

990.

- tumida. - [S. 362 †.

- tumidula. - Dsm. 1611.

- uda. - [S. 359.]

- Ulmariae. - Kl. 1723.

umbilicata. - Voges. 75 s. (Hymenula umb.) . umbonata. - [S. 354.]

— v. epiphylla A. S. - R. f. 127.

umbrata. - S. 351.

umbrinella. - Dsm. 922.

umbrorum Fr. S. Ind.]. - [S. 351. 71.

umbrosa Fr. - [S. 351. 71.] - Kil. 1011.

- Kl. II. 626. - R. f. 217.

- - Schrad. [S. 3\$9.39.

— v. pubescens. - Kl. 1012.

undella. - [5, 3\%̃.]

urceolus. - S. 362 .

urnula. - [S. 353.]

Urticae P. - R. f. 221. (non Kil. 161s. ef.

IIedw. 15.53. n. 4.

- ureata Schum. - [S. 353.] - Fl. dan. XII.

2034.1.

- vaccinea Schum. - Fi. dan. XI. 1971. 1.

varia. - S. 349 .

varians Saut. - Kl. 916.

variecolor. - SS. 352 .

velutina. - Wstd. 397 . - P. albo-violas-

cens $r$. alba Fr., fallax $P$.

venosa. - ef. reticulata Berk. Out. S. 349.

venustula. - Dsm. 45 与.

vernalis Schum. - S. 351.] - Fl. dan. XII.

2034. 2.

- Veronica Lasch. - Fil. 632.

versatilis. - S. 353 *.]

versicolor Phial. - Dsm. III. 15.

- versiformis. - [S. 359, 366*t.] - Brit. f.

274. - Wstd. 297.

- vesiculosa Bull. - S. 359.] - Loud. f.

1621\%. Cd. Ic. V. t. 9. f. 65. (Asc.) - Kl.

829. - Sturm h. 31-. t. 19. Szl. - - Bot. Zt.

1556. t. 5. f. 15. Isc. Hoffm.) - Bot. Zt.

1559. t. 11. f. 25. Keimg. Hoffm.). - Pringsh.

Jahrb. II. t. 31. f. 35 . Hoffm. - Vent. mi.

76. f. 7. 5; 97. f. 10. - Ital. 773.

— villosa P. - [S. 352.] - Kl. 331. - Kl. II. 225.

- Wstd. 690. - Fuck. s. \$26. - Dsm. 1311.

- Vincae. - Lib. 325.

rinosa. - [S. 359.] - Dsm. III. 1与. - Ca-

rol. IY. 19. - cf. rubella Wistd.

violacea. - S. 349.]

virescens Schum. - [S. 352.] - Fl. dan.

X. 1785.2

_ virginea Batsch. - S. 351.] - Fl, dan. XIII.

2274. 3. - Fuck. s. S39. - Kl. 1722. - R. f. 517 .

— viridi-atra. - [S. 353.$]$
Peziza viridi-brunnea Humaria). - Kl. 1919. viriclis Bolt. - Wstd. 919.

vogesiaca. - Voges, 5 s4.

Volutella Fr. - Bisch. f. 3366.

vulgaris Fr. - [S. 354.] - Dsm. 465.

Loud. f. 16272. - Kl. 1017. - Carol. IV. 18.

Weinmanni. -.. [S. 35!.]

xantholoma. - Kl. II. 21 ; cf. xanthomela. xanthomela P. - R. f. 315 . - Iil. II. 2 Is.

xantholoma Rbh. Handb.).

- xanthostigma. - [S. 35 ־. 2.]

P'l a cellium inhonestum 13on. - R. f. 24s.

$\mathrm{P}^{\prime} \mathrm{l}_{1}$ a c idiu m. - [S. 369.] — ef. Triblidium, 'Troclila.

-. abietinum. - S. 370.

-

alneum. - [S. 369.

- Andromedac. - [S. 369.]

- Aquifolii. - Voges. 562. - Wstd. 32.

- Aretii. - Lib. 369

_- Ari in Aro pertenue) : Palaeont. VIII. $t .63$.

f. ¡. (R. Ludwig

- arundinaccum Ces. - Hedw. I. t. 14. f. C

p. 102 .

Astrantiae. - KI. II. $25 \mathrm{~S}$.

- Buxi - ? Kl. 1154. - Kl. II. 717. -- Wstd.

119. - Septoria phacidioides Dsm.

calyciiforme Rebt. - Iil. 950. - Ii. f. 231. carbonaceum. - - S. 369.] - Kl. 13t6.

- Fr. = Discella carb. B. B. - Brit. $f$

44. - Lib. 160.

commoduin Rob. - Dsm. 1242.

compactum. - S. 369.

congener. - Kl. 172 \%. - Kl. II. 163

- coronatum. - [S.370.] - Dsm.416. - Mag.

Zool. Bot. 1ร37. 1. t. 15. f. 5. a. b. p. 513.

Fruct., Berk.). -- Fl. dan. XIII. 2340. 1. -

Fuck. s. 731. - Cd. Anl. t. F. f. 59: 25-29.

- Bisch. f. 3575. - Loud. f. 16474. - Fres.

t. S. f. 22. $23 .-$ Kl. II. 716. - Kl. 770. non

376. cf. Dothid. betul.) - Voges. 559. - Brit.

f. 195. - Lib. 175. Ayloma pezizoides P.).-

Wstd. 919. - Ital. 236. - Sturm h. 4. t. 63.

— craterium Mt. - cf. Sphaeria crat. - Vo-

ges. 9\$6.

Fr.: Wrstd. 15.

- Delta. - Berk. In. p. 2st. f. 66. b. dentatum. - [S. 370.$]$ - Fres. t. S. f. 12

16. - Fuck. s. 724. - Bail s. t. 1s. - Iil.

j5s; - it. Cent. XV. - Dsm. 117. - Voges.

561. - Brit. f. 93. - Carol. III. 41. - Lib.

365. - Wstd. 177. - Ital. 3.15.

divergens Rob. - Dsm. 1211.

elegans. - Carol. V. 17.

Friesii. - Kl. 1535. - Dsm. 1213.

geographicum. - Dsm. III. 699.

- Hicis. - Fres. t. 5. f. $17-21$ - Lib. 367.

- Wstd. 32.

Juglandis. - Palaeont. VIII. t. 63. f. 5. R.

Ludwig)

- lacerum. - [S. 370.] - R.f. 164. - Lib. 70.

- laciniatum Fr. - Bisch. f. $357 \%$.

- laevigatum. - S. 369.

- Laurocerasi. - Kl. 1465. - Fuck. s. 757.

"rochila. - R.f. 156. - Dsm. 292 Eustegia).

Voges. 955. - Brit. f. 45. - Wstd. 75. - Ital. 490.

_ - f. Hederae (Trochila). — Fuck. s. 757. b. ledi. - [S. 369.] - Bisch. f. 3579. - Kil.

II. 520 .

- leptideum. - [S. 370.] - Bisch. f. 3550. -

Tinges. 9\$4. - P. quadr. Schm.

__ litigiosum Rob. - Dsm. 1239. 

$\mid$ 
Phacidium Medicaginis, - Kl. 1729. - Kl. II. 311. - Fuck. s. 733. - Dsm. 719. - Alger.

t. 23, 1. - Lib. 176. - Wstd. 278.

- :microscopicum. - Dsm. III. 794.

- minutissimum Awd. - R. f. 225.

- multiforme. - Palaeont. VIII. t. 63. f. 1.

(R. Ludwig).

- multivalve. - Voges. 560. - ef. Ascochyta Aq. - Wstd. 32. 527 .

_- nervisequum Ces. - K1. 1051.

- Oxyeoceos. - [S. 370.]

- Patella Fr. - Bisch. f. 3512 . - K1. II. 116.

117. - cf, Tympanis Pat., Heterosphaeria Pat. - Brit. f. 259 .

- v. alpestre. - Lib, 269. a. (Pe\%. Chailletii $\mathrm{P}$.).

- - v. campestre Fr. - Dsm. 415. - Lib.

269. b. (Pez. Ligustici DC.) - Wstd. 321.

- 1'atellaria. - [\$. 369.]

- perexiguum. - Dsm. III. 793.

- Philadelphi. - Verh, zool. bot. Ges. Wien.

155s.t. S. f. 1. Niessl.

- Pini. - [S. 369.] = Melampsora punctiformis Mont. (Wstd.) - Kz.: Dsm. T.1s.

- - A. S.: Fuck. s. 730. - ? Fres. t. S.

f. $24-32$. - Wstd. 1117 .

- pithyum Fr. - ef. Triblidium.

Plinthis. - Kil. 1315.

- Populi Lasch. - Kl. II. 51!.

- Populorum Trochila). - Ism. III. 151.

_ pumilum. - Dsm. III. 792.

- punctiforme Wllr. -Kl. 9-9.

- pusillum. - Lib. 264 .

- quadratum S. - [S. 370.] - Fuck. s. 727.

- cf. Phac. leptid. (Voges. 951). - Ital. 555.

(Lophodermium).

- quercinum. - Dsm. 1214.

- radians. - [S. 370.] - Din. 750.

_- Raunneuli Lib. - Kl. 1534. - Vnges. 1142.

(Dothid.). - Brit. f. 347. - Lib. (i). J)othidea

Fr.). - Wstd. 376 .

— Ranunculorum, - D:in, 4 ऽ.

repandum. - [S, 37(t.] - Dsns, 1210.

v. Cerastii. - Fuek. s. 725 . a. r. Galii. - Kl. $375 .-$ Kil. 116 f6.

- rimosum. - Palaeont. VIII. t. §. f. $\therefore$. R.

Ludwig).

- Rubi. - [S. 370.] - Kl. 671. - Fuck.

s. 729 - Krypt. Bad. 431. - Wstd. 656 = Dothidea Chaetomium Kz.

- rugosum, - [S. 369.] - Fuck. s. 72S.

- salicinum. - [S. 370.]

- Saponariae. - Kl. 172\.

- schizoxylon. - [S. 369 .

- simulatum B. B. - Ain. Mg. n. h. 1561 .

VII. t. 16. f. 20 . p. 451 .

- sinuosum; auf Pyrus serrulata Göpp. - Pa-

laeont. VIII. t. 63. f. 6. (R. Ludwig).

- Taxi. - [S. 367.] — Fuck. s. 755.

- Tini. - [S. 367**.] D Dsm. 293. - Voges.

1151. - Wstd. 920 .

- trigonum Schm. = Coccomyces tumida Not.

(Dub.). - [S.370.] - Wstd. 1121. - Ital. 759.

- Vaccinii. - [S. 370.] - Voges. 1180.

— Wallrothii. - Kl. 1537.

Phacobolus. - [S. 116*.]

Phacorhiza erythropus Grev, = Typhula er.

Fl. dan.

-_ sclerotioides. - Kl. II. 231.

Voges. $\$ 85$.
Phall us, - [S. 431.] — ef. Cynophallus, Mutinus, Dictyoph.

- aurant. - [S. $434^{* *}$.] - Ann. sc. n. 1541, XVI. t. 16. (Mont.). - ef. Dictyophall.

campanulatus. - Bisch. f. 3337. - Ann.

Ig. n. h. 1812. IX. t. 10. f. 2. (Berk.).

canariensis Mont. - Webb et Berth. Ca-

nar. Bot. p. 4. t. 4. f. 2. (Cynophall. c. Cd.).

- caninus Huds. - S. 434.] - Loud. f. 16337.

- Bisch. f. 3335 . - Ann. se. n. 1539. XII.t. 2.

Tructif, Berk.). - Ann. Mg. n. h. 1\$40. IT.

t. 5. f. 19-26. (Fructif. Berk.). - ef. Mutin. et Cynophall.

- curtus Berk. - [S. $434^{* * * *}$.] C Cd. Ic. VI. f. 47 .

duplicatus. - IBisch. f. 3333.

IIadriani. - Cd. Anl. t. E. f. $51: 15-17$.

- hyemalis. - Act. Turin. 1503. XII. t. 11.

Balbis).

- impudicus I. - [S. 434.] - Bisch. f. 3330.--

Dsm. 1625. - Berk. Ont. t. 20. f. 3. - Mül-

ler, Pflanzenstaat, p. 269. f. 11. jung). - Abh.

naturf. Ges. zu Nürnberg. I. S. 95. t. 1. f. 1. 2.

(Szl.) - Loud. f. 16336. - Fuck. s. 321. -

Cd. Anl. t. F. f. 52: 1-6. - Pay, b. cr. p. I1s.

f. 551 u. 560. - IIrz. t. 65. 66. - Cd. Ic. V.

t. 7. f. 50. - Nov. Act. 1542. XIX. II. t. 7 .

Oschatz; erron.). - Kil. 21 1. - liail S. t. 25.

- Jiops F1. bat. X. 795. - Tul. F. lyp. t. 21.

f. 10. Fructif.) - Ann. se. и. 1545. IV. t. 1.

f. 9-13. (Anat. Lespiault). - Bot. Zt. 1553.

t. 4. Evolut. Rossm.). - Bot. Zt. 1551. t. 1.

f. 1-11. Anat. Bon.). - Verh, zool. bot. Ges.

Wien. 1559. t. 1. f. 4. Basid. Bail) - Tent. mi. 76. f. 1-4. - Ital. Ts

— indusiatus Vent. — Biscl. f. 3334.

- iosmus Berk. - Curt. Brit. Fntom. X. t. 169.

- marcidus. - [S. 3 H S.]

-_ novae Hollandiae Cd. - Cd. Ic. VI. ᄃ. 66.

- roseus. - Cd. Aul, t. E. f. 51: 15-21.

- rubicundus. - Cd. Anl. t. E. f. 5i: 11.

-_ senegalensis P. - cf. Staurophallus.

-_ xylogenus. - Ann. sc. n. 1455. III. t. 6.

f. 7 . Mont.)

Thellorinia. - [S. 114.]

- inquimans, - Hook. Lond. J. 1943. II. t. 22.

f. - . Berk...

Phelonitis, - [S. 459. - Palaeont. VIII. L. 6.

t. 62. f. 16-27. (Anat. Spor. Fres. .

- lignitum. - Palaeont. VIII, L. 6. t. 62.

f. 1-15. Fres.).

— minima. - [s. 159.$]$

- strobilina. - [S. 459.] - Kl. II. 215.

Phlebia. - [S. 329.]

- contorta, - S. 329.

hydnoidea. - [S. 329.]

- merismoides. - [S. 329.] - Cd. Anl. t. G.

f. 73: 2. - Bail S. t. 29. - Huss. II. 11. (v. aurant.). - R. f. 50 \%.

- miesenterica Fr. - Wstcl. 693.

_ radiata. - [S. 329.]

rubiginosa. - Carol. III. 23.

- tremelloides Fr. — Wstd. 693.

vaga. - [S. 329.] - Loud. f. 16126. - Ann.

Mg.n.h. ! $\$ 39.1$. t.5. f. 15.49. (Fructif. Berk.).

Pli lebomorpha P. - est Mucilago Myxomyce-

tum Mont.). - [S. 521.]

Phlebophora. - [S. 329 **.]

- eampanulata. - Ann. se. n. 1S41. XVI.t. 1 i. f. ว. (Lév.).

Thloeoconis. - [S. 520.

Phloeoscoria faginea Wlir. - Wstd. 924 . 
Phloeospora Aegopodii. - Wstd. 95.

- - Humuli. - Wstd. 1 \&\$.

_- Iyperici. - Wistd. 1153.

-_ Oxyacanthae. - Wstd. fo.

— Tetroselini. - Wstd. 135.

— Ribis. - Wstd. 92 .

Rosae. - W'std. 93.

Ulmi. - Wstd. 137.

Violac. - Wstcl. 94.

Phlyctaena. - [S. 426. Phlyctema.]

cheilarioides. - Dsm. III. 691.

- - vagabunda. - Dsm. 1224. - Wstd. 112\%.

Phly ctidi um. - [S. 123.] - Excipula; Sphaeria.

- catenulatum Wilr. - Wstd. 636.

clypeatum. - [S. 423.] - Act. Turin. 1545.

VII. Dec. 3. f. 7. (Notar.).

dendriticum. - - \$. $125,3$. ?]

Heraclei. - Wstd. 915 .

nitidum v. alneum. - Wstd. 264.

Ranunculi. - Kl. 1537.

Rubi Wllr. - Wstd. 1277.

Saniculae. - ? IIl. II. 259.

Phlyctospora. - [S. 426*.460.]

— fusca. - Cd. Anl. t. C. f. $37: 4-6 .-$

Sturin h. 19 -. t. 16. Cd.).

Pholiota. - ef. Agaricus. - Bail S. t. 36.

Phoma. - S. 421.] ef. Sphaeria. - ? Hook. J. 1551. III. t. 10. f. 6.

— Agaves. - Kl. 1662.

- albicans. - Dsm. 1465. - Voges. I341.-

Wstd. 631

—_ Andrachnes Lév. - Déınid. Russ. t. 6. f. 3. atriplicinum. - Wstd. 1233.

Aucubae. - Wstd. 1373.

Bellynckii. - Wstd. 609.

bicuspidata. - Hook. J. 1\$53. V. t. 3. f. 6.

cinereun. - Dsm. III. 356.

cirratulum. - 1 Usm. III. 53.

commune. - Dsm. III. 693.

complanatum. - R. f. 342. (Sphaeria).

— - v. acutata Awd. - R. f. 343. (Sphae-

ria acuta Pers. p. p.)

- concentricum. - Dsm. 4\$5. - Voges. 1177.

Convallariae. - Wstc. 1136.

Coronillae. - Wstd. 966.

decorticans. - Act. Turin. 1\$41. III. Dec. 2.

f. 7. Notar

denigratum. - Dsm. III. 3ã3.

Desmazieri. - Alger. t. 27, 6.

effusum. - Dsm. III. $35 \overline{7}$.

epileucum. - Hook. J. I 553. V. t. 3. f. 2.

Equiseti Dsm. - Wstd. 632.

errabundum Dsm. - Dsm. 1470. - R. f. 163.

- Depaz. vagans Fr. - Wstd. 113๔.

exiguum. - Dsm. Ił69. III. วั7.

1137

fibricolum. - Hook. J. 1553. V. t. 3. f: 1.

Ficus, - Krl. 1ST0.

Filum. - ef: Ascochyta Convolvuli Lib.

f. Chondrillae, - Kl. II. 44 s.

f. Iridearum. - Kl. 1959, a.

f. Loguminosarum. - Kl. 1959. b.

f. Umbellatarum. - Kl. $17+7$.

gramınicum. -- Voges. 1459.

Hederae. - [S. 421.] - Dsm. 149.- Voges. 979. - Brit. f. 90. - Wstd. 110. - Splacropsis Ilederae Iév. - Wstd. 1126.

-_- herbarum. - cf. Melamp. et Medioag. Wrst.

- R.f. 455. a. b. - W Wtd. 965. 1133 . Melainp. .

__ jasminicolum. - Dsm. III. 355 .
Phom a Ilicis. - Dsm. 790.

- lanceolatum, - Ism. III. $5 \S$.

—- Laricis I.év. - Démid. Russ. t. 4. f. 5.

Jeguminosarum IIstd. - R. f. 153.

- Leguminun. - Wstd. 1135.

_- lincolatum. - Wstd. 965.

-.. Lingam. - Dsm. 1177. - cf. Sphaeria.

-_ Lirella. - Dsm. 1471.

- Iiriodendri. - Wstal. 967.

- longissimum. - Dsm. III. 760. - Wstd. 1139.

maculare. - Dsm. III. 51 .

Malvacearum. - Wstd. 1232.

melaenum II. Dur. - Dsm. 1473. - cf.

Spluaeropsis. - Wstd. 961. - R. f. 34. (Sphae-

ria).

_- millepunctatum. - Dsm. III. 55.
_ muciferum. — I Iook. J. IS53. V. t. 3. f. 5.

- neglectum. - Dsm. III. 354.

— nitidum. - Dsm. III 355 .

- oblongum. - Dsin. III. 30.

— occultum. - Kl. $1664 . \mathrm{b}$.

- petioloruin. - Dsm: 1172. - Wstd. 471.

- Phascoli. - Dsm. 43. - Wstd. 470.

- phyllostictoides. - Dsm. III. 694.

- Pinastri Lév. - Wstd. 1230.

- Podagrariae. - Wstd. 722.

- Populi. - [S. 121.] - Loud. f. 16465.

- protuberans. - Toges. 1460.

- punctiforme. - Dsm. 1167.

- Pustula. - [S. 421.] - Bisch. f. 3565. -

Kl. 1746. - Brit. f. 40. - Wstd. 653.

rameale. - Dsm. $117 \mathrm{~S}$.

roseolum. - Dsm. III. 761 .

Rubi. - Wstd. 1234.

salignum. - [S. 421.] - Bisch. f. 3564. -

Fuck. s. 394. - Krypt. Bad. 333. - zu Sphae-

ria Capreae. (Bail). - Brit. f. 191. - Wstd. 524.

- samàrarum. - Dsm. 145;1475. - Wstd .16i9.

- Siliquastrum. - Dsm. 1174.

Spiraeae. - Dsm. 150.

striaeforme. - Dsm. III. 59. - Wstd. 1132.

Striola Mont. = Hysterii Striolae Fr. Sper-

inogon.

- strobiligenum. - Dsm. 1476.

- subnervisequum. - Dsm. III. 5 i.

- subordinarium. - Dsm. 1466. - Voges.

1:342. - Wstd. 723 .

- Tanni. - Voges. 10\$6. - Wstd. 1123.

_- tularostomum. - Bail S. t. 14.

—_ulmicolum. - Hook. J. 1553. V. t. 3. f. 3.

- vagum. - Dsm. III. 692 .

- vicinum. - Dsin. III. 352.

- Vincetoxici. - Wstd. 1134. (folliculor.).

- Violae. - Wstd. 525.

Westendorpii T. - R. f. 265. - Bull. ac.

belg. 2. Ser. 1557 . II. p. 579. f. 10. (Wstd.). Wistd. 1235.

Pliraguidi u m. - [S. 507. 512*.] cf. Aregma, Puccinia. - Linn. 1S2S. III. t. 1. f. A-F. Eysenliard).

- acuminatum. - [S. 507.]

- apiculatum T. - Fuck. s. 35. a.b. - Fuck.

Th. 311. 312. (c. Ured. Poterii).

- asperum Wilr. - Bisch. f. 3063 . - R. f.

176. - Bon. t. 2. f. 49. Wstd. 1071. Sturm h. 31-. t. 2 \%. (Szl.). - Kl. 1 I t. - Iil. II 83 - Fuck. s. 3T. - Pringsh. Jahrb. II. t. 25. f. 1\%. (IIoffin.). - Fuck, rh. 315. bulbosum. - [S. 507.] - Fl. dan. XIII. 2279. 1. - Cd. Anl. t. B. f. $4: 10$. Bisch. 


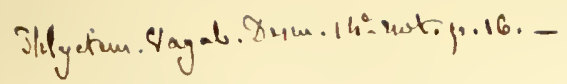

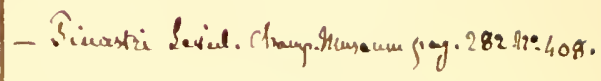

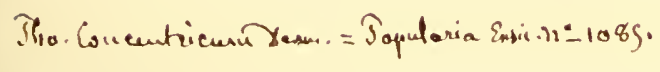

-Equireli Léviel. Chaup. hues. gog.282. -

$\hat{T} h_{1}$. Strobiligena Descu. $y^{2}=n_{0} 1:\{1 \cdot 8 \cdot 11=11$. 


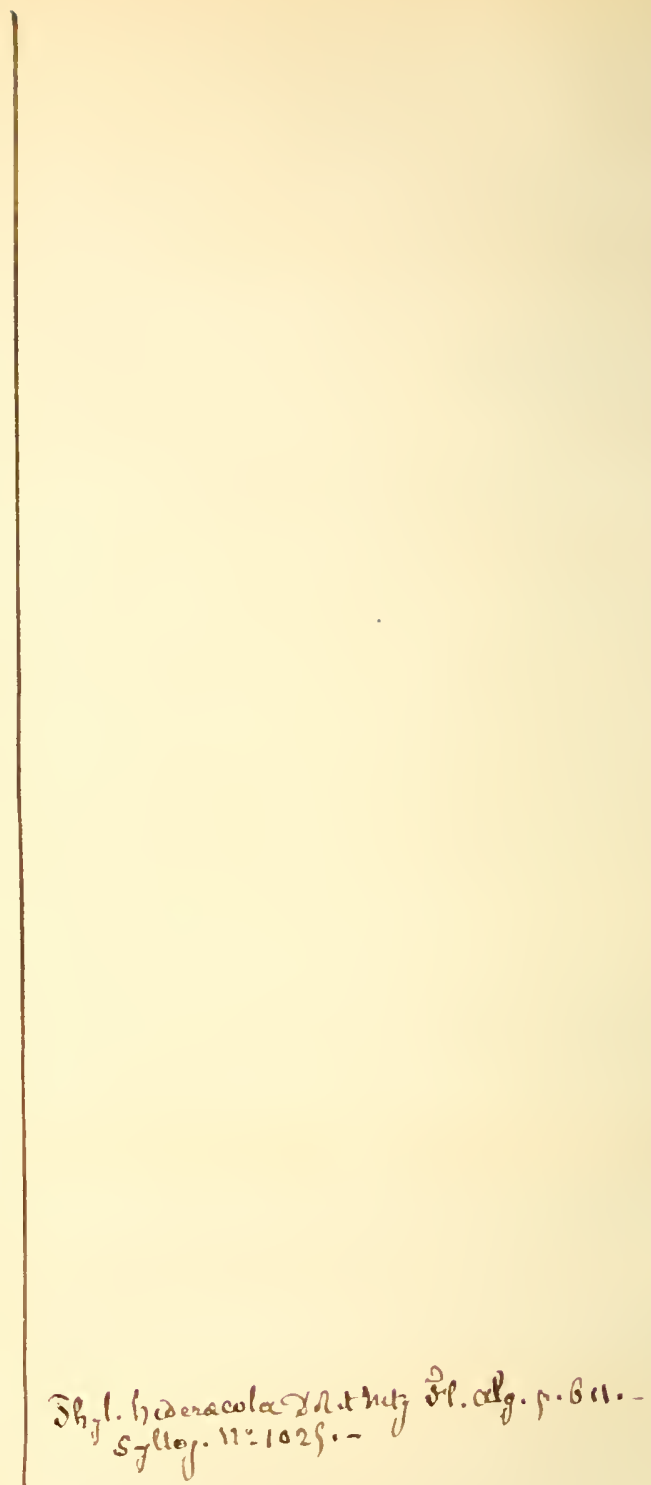


f. 3561.3900 - Fuck. s. 36. b. - Ann. sc. n. 1954 . II. t. 9. f. $15-17$. (Tul.) - Bon. t. 2 . f. 16 . - Wstd. 1070 - Microse. Journ. 1557. V.t. S. f. $7-16$. u. 1S-20. (Currey). it. VII. 18.99. t. 11. f. 4-6. (Curr.). - Kl. 76.

Phragmidium bulbosum Bon. Myk. = constrictum Bon. Con. - Letell. 661. 2. Puccin. Rubi\}. - Wstd. 157. - Schweiz. S. a-c. lirypt. Bad. 47 .

— bullatum. - Wstd. 1069.

- constrictum. - Bon. Con. p. 225. P. bulbos. Bon. Myk.).

— cylindricum. - Bon. Con, p. 226. t. 2. f. 25. R. f. $475=$ P. bulbosum Strauss.

effusum. - Kl. 1391. - Fuck. rh. 316.

Fragariae. - Kl. 1957.

- Rossm. = obtusum. - Kil. II. $2>1$.

Fragariastri Bellk. - Wstd. 85\%.

granulatum Rbh. - Kl. 1255.

- incrassatum Lk. - Müller, P'flanzeṇstaat. 1560. 264. (Copie). - Dsin. 413. - Ann. sc: n. 1547. VII. t. 7. f. $1-16$. (Tul.) - Schnzl. t. 12. f. $11-13$. - Fuck. s.36. a. b. - de Bary Br. t. 4. f. 9. 9. - Pringsh. Jahrb. II. t. 25. f. 16 . (Hoffm.). - Letell. 661. 1. Puccinia Rosae DC.). - Auersw. Bot. Unterhlt. 1563. p. 356 .

- v. Rosae. - Wstd. 131. - Fuck. rh. 313. (c. Ured. Rosae), 314. (c. Ur. Rubor.) bosum S. - Wstd. 15\%.

- intermedium. - Ung. Ex. t. 7. f. 36. f. -

kil. 77.

- mucronatum. - [S. 507.] - Fl. dan. XIII. 2279. 2. - Cd. Anl. t. B. f. 4: 11. - Bisch.

f. 3560. - Microsc. Journ. 1557. V. t. S. f. 17. (Currey). - cf. Puccinia m. - Voges. 293. (Pucc. Ros.). - Wstd. 131. -61). 'P'. obtus. Potent.) ; \$59. P. obtus. Fragar. ; \5̄'. (P. apiculat. Poter.) ; $\$ 60$. P. apic. Potent.). - Schweiz. 7 .

- obtusatum, - [S. 507.] — de Bary Br. t. 4.

f. 10. = obtusum. - K1. 6\$9. - Muller, I'flanzenstaat. 1560. 264. (Copie). - Brit. f. $105 .-$ Vstd. 660 .

- obtusum. - Ann. sc. n. IS54.II. t. 9. f. 1 \-

23. (Tul.). - Fuck. s. 31. - Dsm. 11159. cf. P. Fragariae et obtusuin. - Wstd. $\$ 60$. Fuck. rh. 310 .

f. Potentillae. - Kl. II. 679.

Potentillae. - Cd. Anl. t. B. f. $4: 12$. -

Microse. Journ. 1557. V. t. S. f. $25-33$. Wstd. S60. (Curr.).

_— Poterii Wllr. - Wstd. $\varsigma 55$.

- Rubi. - Voges. 193. Puccin. Rubi DC.!.

- Ulmariae. - Pay. b. cr. p. 61. f. 252.

Phragmotrichum. - [S. 474.] - affine Sirodesmio.

- acerinum. - [S. 474.]

- Bullaria. - [S. 505.] - Kl. 1173. (= Puccin. Umbellif.). - Brit. f. 57. (Pucc. Bullar.). W'std. $\$ 57$.

Chailletii. - [S. 474.] - Cd. Anl. t. F. f. $60: 21-23$. - Bisch. f. 356\%. - Nees j. Syst. d. Pilze. t. 3. - Pay. b. cr. p. 70. f. 310. u. 313. - Berk. In. p. 327. f. 74. c. - Kl. II. 756. - Dsm. III. 430. - Voges. 1261. - Norweg. 178. - Lib. 196. - Krypt. Bad. 54 s.

— lignicolum. - [S. 505.]

- populinum. - [S. 474.]

- quercinum. - Hoffm. Ic. t. 17. f, 1.

Phycomyces. - [S. 465. 456**. 524.]
Phycomyces nitens. - [S. 465. 458**.] Bisch. f. 3792. - cf. Hantzschia Ph. - Tóges. 1351.

— splendens. - [S. 469. 155**.]

Pli yllacea. - [S. 409.]

Phyllactinia. - cf. Erysiphe.

- Candollei. - Ann. sc. n. 1551. XV. t. f. 12 . Lév. .

- guttata. - Erys. gutt. Tul. - Ann. sc. n. 1 5. 1. XV. t. 7. f. 11. Lév.. - R. f. 166. Fuck. s. 1†7. a. - Erys. Pyri Chaill., Quercus Mére.

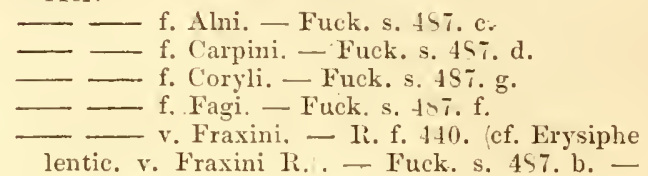
Ital. ว94.

- - f. Quercus. - Fuck. s. 197. e.

- Schweinitzii. - Ann. sc. n. 1551. XV. t. 7. f. 13. Lév.).

Phyllerinm. - [S. 519.] - Pili degenerati.

thy ylloedia. - [S. 452.]

Phyllopta. - [S. 342.]

I'b yllos phaera cornicola Dmtr. - Wstd. 29.

- hederaecola. - Wstd. 25.

scabiosaecola. - Wstd. 30 .

Phyllosticta. - [S. 425.] ef. Depazea.

Andromedae. - Wstd. 645.

Anemones, - Fuck. rh. 496 .

Argentinae. - Dsm. 1230.

Asclepiadearum. - Wstd. 137\%.

- Atriplicis. - Dsm. III. 653. 654. - Wstd. 960.

Berberidis. - Kl. 1S65. - Wstd. 1375.

Cheiranthorum. - Dsm. 1156.

Chenopodii. - Wstd. 959 .

Cirsii. - Dsm. 1232.

cornicola. - Kl. II. 451. - Fuck. s, 331. -

Fuck. rh. 495.

- Coryli. - Wstd. 731. = Cheilaria Coryli I) sm.

- cruenta. - [S. 426.] - W'std.649. - I'l. I. 263. - Fuck. s. 333. - Dsm. 1455. - R. f. 152. - Ital. 593. Depazea). - Fuck. rh. 194. - Cynarae. - Wstd. 1140.

Cytisi. - Wstd. 424. - Dsm. 1461.

- destructiva. - [S. 426.] - Dsm. 122i; III.

$6>0-652 .-$ Wstd. 962 . Lycii - R. f, 552 . destruens. - Ism. 1233.

Dianthi. - Wstd. 293.

- Digitalis B. - Wstd. 1053.

fragariaecola. -.Dsm. III. $6 \varsigma 6$.

— Laureolac. - Dsm. 1237. - Voges. 1266.

- Lauri. - Wstd. 650.

-_ Loniceràe. - Wstd. 961.

- melanoplaca. - Wstd. 1141.

- Mercurialis. - Usm. 1457. - Wstd. 458.

- Nerii. - Wstd. 963.

- Paviae. - Dsm. 1236.

- Potentillae. - Dsm. 1231.

- primulaecola. - Dsm. 1229. - Wstd. 457.

Rhamni. - Wstel. 95S.

rhamnicola. - Dsm. 1235.

ribicola. - [S. 425.]

Robergei. - Dsm. III. 655 .

Rosae. - Dsm. III. 65\%.

ruscicola. - R. f. $46 \%$. - Dsm. 1234. -

Wstd. 1374 .

Sagittariae. - R. f. 553. - Fuck. rh. 497.

Sambuci. - Dsm. 1235. - Wstd. $13 \% 6$. 
Pliyllosticta ranguinca. - Dsm. 1454.

- Syringae. - W'std. 617.

— Ulmi. - Wstd. 11.13.

- vagans. - [S. 426 .

— Violac. - Wistd. 456. - Dsm. 1228.

viteum. - I) sm. 561 .

Vitis. - Fuck. s. 19ti. - Krypt. Bad. 140.

$141 ? !$

- vulgaris. - Dsm. 1459. 1460. - Fuck, s. 332. - Wstd. 1112. (Philadelphi). - Fuck, rh. 493.

\section{- Cerasi. - Wstd. 730.}

- Lonicerae. - R. f. 1.j1.

Ph rmatostroma. - [S. $472^{*}$.

- fusarioides. - S. 472 .

- leucosperma. - [S. 472.] - Pact. lenc.

— stercorarinm. - [S. 172. fim.] - Cd. Anl.

t. G. f. $65: 9.10$.

Pl y mat otrich u memellum. - Kl. II. $1>3$.

Bon. t. 6. f. 134.

—- laneum. - Bon. t. 9. f. 197. - R. f. 394.

- pyramidale. - Bon. t. S. f. 181.

Physarum. - [S. 453.] - ef. Badhamia.

- albipes Lk. - [S. 453.] - Bisch. f. 3619. -

Zeitschr. f. wiss. Zool. 1 559 . X. t. 6. f. $1-3$.

de Bary). - R. f. 366.

- - v. sessile. - Krypt. Bad. 423.

- albo-punctatum Schum. = Didymium a. Fl. dan.

— album. - [S. 454.] - Kl. II. 456.

$\longrightarrow$ atrum, - [S. 151.$]$

aureum. - [S. 453.] - Cd. Anl. t. C. f. 25 :

¡. ร. - Loud. f. 16536 . Fuck. s. 255. sturm h. 2. t. 23.

- Schum. = Ph. nutans v. aur., Fl. dan. — bryophilum. - [S. 153.] - Fl. dan. XII.

2095. 2. - Cd. Anl. t. C. f. 25: 5. 6. - Pay.

b. cr. p. 122 . f. 571.575 .

__ bulbiforme Fr. - Fl, dan. XI. 1974. 3.

- bullatum Lk. - Bisch. f. 3618. - Sturm

h. 2. t. 22 .

- caesium Fr. - [S. 454.] - Fl. dan. XII. 2087.1 .

- cornuum. - [S. 454.] - Fl. dan. XI. 1974. 2.

- cinerascens Schum. = Didymium lobatum,

Fl. dan.

- cinereum. - cf. Didymium, Let.

coccineum. - [S. 453.]

- columbinum. - [S. 453.] - Kl. 1631. -

Terh. zool. bot. Ges. Wien. 1559. t. 1. f. 3. Keimg., Bail .

— confluens. - [S. 454.]

_ conglobatum Ditm. - [S. 453.] - Bisch.

f. 3620 . - Sturm h. 3. t. 40 .

- connatum. - [S. 455.] - Sturm h. 3. t. 41. cupripes. - Carol. III. 76.

- depressum Schum. = Diderma hemisphaer.

Fl. dan.

- farinaceum P. - cf. Didym. far. S. - R.

f. 369 .

- fasciculatum. - Jungh. pr. t. 2. f. S.

fimetarium Schum. - [S. 455.] - F1. dan.

XII. 2095. 3.

— Havum Fr. - [S. 454. 9.] - Fuck. s. $2 \searrow 6$.

fulvum. - [S. 453.]

furfuraceum Schum. - Didym. Fl. dan.

globosum Schum. = Didym. farin., Fl. dan. Somm. = Ph. subulatum Fl. dan. gracilentum. - [S. 453.]

- hyalinum. - [S. 453.]

Fl. dan. XII.

2093. 1 .

— hypnophilum. - S. 453.
Physar um leucophacum. - [S. 454. Til.]

- Licea. - [\$. 153.]

lilacinum. - [S: -153.]

- luteo-album Schum. - [S. 454. 5.] - Peri-

chaena quercina Fl. dan.

_- luteo-valve. - [S. $455^{* *}$.]

macrocarpon. - Kil. 1968. - Fuck. s. 259. -

Bot. 1/t. 1ব59. t. 11. f. 14. (Keing. Hoffm.).

- marginatum. - S. 45..]

- metallicum. - [S. 153.] - Mag. Kool. Bot.

I ; i. I. t. 3. f. s. p. 19. (Berk.).

— Nichelii. - Cd. Ic. V. t. 3. f. 33.

muscicola. - S. 453.]

nigripes. - Sturm h. 3, t. 42.

nigrum. - [S. 454.]

- nutans. - S. 153.] - Fl. dan. XII. 2096. 1.

- Bisch. f. 3616 . - Fuck. s. 259 . - R. f. 40. - v. aureum Fr. - Fl. dan. XII. 2096. 2. ovoideum Schum. = I'h. utriculare v. ses-

sile, Fl. dan. - [S. 453.]

paniceum. - [S. 153.]

pedunculatum Schum. - Wstd. $1 \times 2$.

piceum. - [S. 453.]

- Pini. - [S. 153.] - Fl. dan. XII. 2096. 3. -

Bisch. f. 3703 .

_- plumbeum Mich. - Zeitschr. wiss. Zool.

1459. X. t. 6. f. 4. 5. (de Bary).

- psittacinum. - [S. 453.] - Sturn h. 1. t. 62. pulcherrimum. - Carol. II. $7 \%$.

salicinum Schum. = P. bryophilum, Fl, dan. sinuosum Fr. - [S. 451.] - Kl.819. - cf.

Angioridium.

_- solutum Schum. - [S. 454.] - F1. dan. XI.

197. 1 .

[ striatum Fr. - S. 453.] ? = nutans Fl. dan.

- subulatum Schum. - [S. 453.] - Fl. dan.

XII. 2095. 1.

sulcatum Lk. = P. bulbiforme Fl. dan. -

[S. 453.]

- sulfureum A. S. - [S. 153.] - Bisch. f. 3613.

thejoteum. - [S. 453.]

turbinatum Schum. = Craterium t., Fl. dan. utriculare. - [S. 453.]

— v. sessile Fr. - Fl. dan. XII. 2093. 2. vermiculare. - [5. 155 **.]

vernum. - [S. 454 .

violaceum. - [S. 453.] - Fl. dan. XI.

1950.2.

- virescens. - Cd. Anl. t. C. f. 25: 9. 10.

Sturm h. 1. t. 61.

_._ viride. - S. 453.] - Sturm h. 2. t. 24.

xanthopus. - Schnzl. t. 14. f. $20-22$.

Physoderma. - [S. 515*.] - cf. Uredo.

- Eryngii. - Fuck. s. 117. - Dsm. 1075. Fuck. rh. 261.

- gibbosum. - Kles s5. - it. C'ent. XVIII. -

Fuck. s. 115. - cf. Protomyces macrosp. Fuck, rh. 259.

- Menyanthis. - Fuck. rh. 260.

- segetum. - cf. Uredo Rubigo $v$.

Physonema. - [S. 511.] — ef. Uredo.

aureum. - Bon. Con. t. 2. f. 25. p. 201

('aeoma Saliceti Lk.).

- intermedium. - Bon. Con. p. 201. t. 2. f. 27.

- minimum. - Bon. Con. t. 2. f. 20. p. 200.

pallidum. - Bon. Con. t. 2. f. 19. p. 200. -

R. f. 29 \%.

- vulgare. - Bon. Con. t. 1. f. 5. - R. f. 474.

cf. Epitea rulg. Fr. - Bon. Con. t. 2. f. 26. p. 201. (= Caeoma epiteum Lk.).

I'hysospora. - [S. 495.] 


$$
\mid
$$




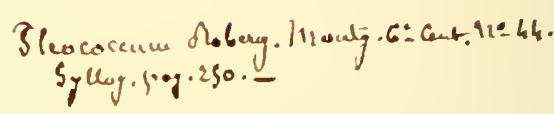

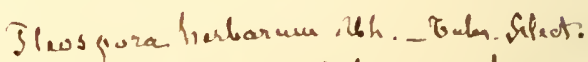

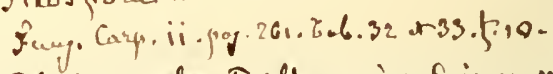
at IP. Sarcinulo, S. alterwario, Enicocenm

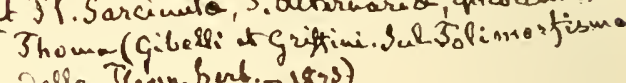
Jella Jranp. hert.-187s)

Siptostonncene Severt. Charey. Enot, p1.65.11:-316. 
Picoa. - [S. 435 .

— Juniperi Vitt. - Vitt. t. 2, S. - Cd. Ic. VI. t. 10. f.95. - Bail S. t. 13..f. 1. - Alger. t. 24. f. $12-21$.

Picrostoma. - ef. Sphaeria.

I'iggotia. - cf. Dothidea, Asteroma.

- astroidea. - Asteroma Ulmi Grev. - Ann.

Mg. n. h. 1\$51. VII. p. 95. t. 5. f. 1. a-c.

(I3. B.). - I sm. III. S4.

Pilaere. - [S. 361 .

- faginca. - Ann. $\operatorname{Mg} . \mathrm{n} . \mathrm{h}, 1550 . \mathrm{V}, \mathrm{t}, 11$

f. 5.1 . 365. B. B. :

- P'etersii B. C. - R. f. 269. - Carol.IIL. 39.

l’ileolaria. - [S. 5j!.]

- Incripes. - Carol. III. 91.

- l'erebinthi. - [S. 514.] - Dsm. I055. -

C'l. Ic. VI: f. 3. - Bon. t. 3. f. 76; - Kl. 1967.

I!!!i. - IKl. II. 54 ; 199. - of. Uredo Decaisneana. - Castagne, Marseille t. 3.

Pilidium. - S. 370. Phacid.

-. acerinum, - Dsm. III. sร̃.

- carbonaceum. - ef. Pliacidium c.

risseminatum, - Lib. 359.

aryophyllum. - Cd. Anl. t. F. f. 60: 11-13.

lemisphacriemm. - Cl. Anl. t. F. f. 60 :

$11-16$.

- inyrtinum. - Alger. t. 26. s.

Piligena. - ef. Onygena.

- lycoperdoides Schum. - Fl. dan. X. 17.10.2.

- Onygena piligena Fr.

Pilobolus. - S. 157.] - Proceed. Linn. Soc.

1457. I. 'c. ic. (C'urrey). - Bot. Zt. 1 $\backslash 42$, p. 17.

_- anomalus. - Kil. 1512. - Ascoploora C'esatii

Civems.

crystallinus. - [S. 15.] $]-$ Cd. Ic. VI. f. 32.

- Cd. Anl. t.C. f. 25: 1.2. - Bisch, f. $3721 .-$

R. f. $270 .-13 u l l$ ae. belg. 15.59 . VIII. p. i70.

216? f. 1-16. (coems. . - Loud. f. 163.4\%.-

Nor. Act. XXIII, I. t. 51.52 . - Cohn=l'. Oclipils R. f. 3\$2, Coems. - Iion. t. 10. f. 2033 : Ocdipus, Coems. - Kl, 1630. - Kl. II. is.

- Lév. (sub P'. rorid. : Mém. Soc. Linn.

Par. IY. t. 20. (ex Coems.)

- Coemans Monogr. Pilob. 1561. t. 2.

f. 1-20. (Ac. roy. Belg., mém. cour. XXX.) -

Fuck, ih. 49 .

lentigerus Cil. R. - ef. P. Ocdipus M.

- Oedipus Mont, - ? R: f. 270 . - R. f. 342.

AIucor obliquus Scop., Pyenopod. lentig. Cd., I'ilobol. lentig. R., Pil. crystallin. Cohn.) Mém. Soc: Linn. de Lyon. 152৬. 1) 1-7. c. ic. - Coemans Mon. Plitob. 1561. t. 1. f. $1-20$.

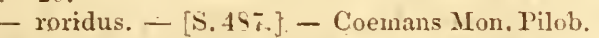

1st;1. t. 2, f. B. nach Bolton' - - Linn. Journ.

1 56. I. t. 2. (Currey) = crystallinus Coems...

- Lév. : crystall. (Coems.) - - Bisch.

f. 3725 : crystall. Coems.) - R. f. 270 .

ureeolatus Purt. = crystall. (fide Berk.). -

Purton. I. t. 31 .

Pilopeza. - [\$. 356.]

Pilophora. - [S. 4s5.]

- rorida Wllr. - ef. Ascophora Mucedo.

I'ionnotes. - [S. 4S0 **. 4S1.]

l'iptostroma. - [S. 109.]

l'irostoma: - [S. 395.]

Pis omyxa. - [S. $406:$ Bry: $]$

- racodioides. - Cd. Anl. t. F. f. $53: 11-13$.

— Lindl. v. K. p. 43: f. 27. - Pay. b. cr. p. ij.

f. 240 . p. 9 s. f. 456.

Pistillària. - $[$ S. 340.$]$ ef. Scleromitrà. Ann. se.n. is 37 . VIII. t. $5-11$, Fructif: Lév.).

Hof fmann, lndex Funvorum.
Pistillaria aciculata. - Alger. t. 32, 4.

Acrospermum. - B Bail S. t. 27.

coceinea. - Kll. 162. .

culmigena. - [S. 340.] - Dsm. 453. - Ann.

sc. n. 15:36. V: t. 12. - Kl. 1520. - Wstd. $10 \leq 2$.

f. 152 .

diaphana, - $[S, 340]-$. Fl, dan, X. 1783.1.

inacqualis. - Kil. 1930.

incarnata. - Dsm. 710 .

- micans. - [S. :3 11.] - Biseh. f. 3.135. -

Kl. $342,-\mathrm{kl}$ : II. 126. - Amn, se. n. $1+3 \%$

VIII. t. S. f. 14. Fructif. Lév.) - cum Sclc-

rotio: Ann. sc. n. 1513. XX: t. 7. f. 3. (Lér.).

- Isin. 1515.

muscicola. - Kil. 1626.

ovata P.- [S. 340.] - Kỉ.613.- Krl. II. 23 ï.

- Fr. $_{1}$ Typhula, Bail S. p. 19.

pubèrula. - [S. 340.]

pusilla. - Cd. Anl. t. G. f. $72: 1-3$. -

C'd. Ic. II. f. 123!

- quisquiliaris Fr. - [S. 3.10.] - Loud. f.

14196. - Kl. 1312. - Kîl. II. 12\%. - Typhuli

'Todei Fr. Tul. - Brit. f. 25.

- rosella. - [\$. 340.$]$ selerotioides. $\rightarrow$ Dsm. 1514.

- Syringae. - Fuck. Nass. f. 24. - Fuck. s. \$91.

Pit to a r p i um. - [S. 521.]

1'la c cint aria depressa. - Kl. 1446.

Plcoostom a. - [S. 111.] ef. Geaster.

— fornicatum. - C'd. Ic. V. t. 4: f. 43. - C'd.

Anl. t. I. f. $43: 12-15 .-1$-ay. b. cr. p. 63.

f. 25() .

Plecotrich um. - [S. $495^{* * *}$.]

- fuscum. - sturm h. 13. t. 44.

- Lauri. - Kil. $14 \$ 1$

Plegmatium. - S. 523.]

P'leu odom us Rabenhorstii Pr. - Kl: 1292.'-

stum h. 36. t. 12.

- sclerotioides Pr. - Kil. $12 \$ 1$.

Pleococeum Robergei. - Dsm: 133 S.

Pleasphaeria scimpicola. - K Kl. II. 256.

Pleospora. - ef. Sphacria.

- Asparagi. - Kl. II. 750 .

- Diantlit R. - R. f. 451 .

herbarum Rbh. - Ital. 496.

- v. Allii. Sphacria herbarum. Autt.) -

Iil. II. 517. a. - Fuck. s. 517.

- f. Salicomiae. - R. f. 145.

- Leguminum Rbh. - Iĩl. II. j19.

pellita. - Kl. II. it9.

Ple urage: - $\left[\right.$ S. $41 \mathrm{~S}^{+*}$.]

Pleurubotrya indica. - Berk. In. p. 314.

t. il. b.

Pleu rocera s ciliatum. - Hdw. I. t. 4. f. 5.-

Iil. 1s22. Kl. II, 7 i6.

Pleurocystis adscendens. - Bon. t. 9. f. 15:.

- - Fresenii Bon. - cf. MIucor racemos.

Ple urapyxis. - [S. - f 117.$]$

- microsperma. - Cd. Anl. t. F. f: $53: \varsigma \div 10$.

- Pay. b. cr. p. 9s. f. 455.

Podaxis. - [S. 440 .]

Podaxom.-[S. 410 .]

carcinomale - Berk. In. p. 8. f: 5. c. (fila). pistillare. - Hook. Lond. J. 1915. IV. t. 10.

Podisoma. - [s. 474.] - Flora, 1S12. II. t. 3.

(C. Müller).

-.- foliicolum. - Fuck, rh. 4.4.

-fuscum. - Cd. Anl. t. G. f. 69: 17-22. -

Berk. In. p. 10. f. 6. a. - Kl. 75. - Kl. II.

6\%. - Voges. 1347. - Wstd. 339. 
Podisom a Juniperi I.k. - Wstd. 339.

- - communis. - [S. 171.] - Dsm.

1016. - Bisch. f. 3s>n. - Pay. b. cr. p. it.

f. 351 - $-\Lambda m$ m. se. n. 14.)1. II. t. 10. f. $1-12$.

'Tul. - Brit. f. 106. - Ital. 65\% - Fuck.

rh. 415 .

— Sabinne I.k. - [S. 17.1.] - Bisch.

f. 3৬๖2. 3\$४3. - Jicrk. Out. t. 2. f. 4. - Brit.

f. $107,-$ Fuck, rh. 116 .

- macropus. - Hook. Lond. J. 1s.15. IT.

p. 315. t. 12. f. 6. - Carol. 1. -5.

Podocystis. - S. 512.7 ef. Uredo.

— aecidioides. - S. 512.] - Kl. II. 392.

- angulosa. - Bon. Con. t. 1. f. 11.

- Capraearum. - [S. 512.]

— Lini. - [S. 512.] - Iİ. II. 391.

pustulata. - Fuck. s $\$ 5$.

- v. Ptellariae. - Kl. II. 659.

Ranunculacearum: f. Anemones. - Kl. II. 690 .

Podosphaera. - ef. Erysiphe.

- clandestina. - Ann. sc. n. 1551. XV. t. 6.

f. 5. (Lér. ; Ery̧s. Oxyacanthae 'Tul.

- Kunzei. - Ann. sc. n. 1551. XV. t. 6. f. 6. Lév.

— - r. B. = Erys. tridactyla 'l'ul.

- v. myrtillina. - R. f. 566 .

f. joj. bis. f. Pruni. - Fuck. s. 1>9, a. b. - R.

1. myrtillina. - [S. 106.]

Schlechtendalii. - Ann. sc. n, 1551. Xr.

t. 6. f. 7. Lér. .

Podospora fimicola Ces. - Hedw. I. t. 14.

f. A. - K1. II. $25 \bar{i}_{\text {, }} 259$.

Podosporium. - [S. 166. 512.]

- Lini Lér. - Wstd. 1179.

rigidum. - Berk. In. p. 311. f. 71. a.

Vaccinii. - S. 466 .

I'udostrombium. - S. 4S1.515.]

Polis tophthora Antillarum. - Zeitschr. wiss.

Kool. 1 S5s. IX. t. 17. f. 1s. ff. Lebert)

Polyactis. - $\$$. 490.$]$ - ef. Botrytis.

- cana. - Bon. t. i. f. 156.

- coerulescens. - Fres. t. 5. f. 51-53.

divaricata. - Sturm h. 35. t. 6 .

epigaea. - Bon. t. 7. f. 161 .

- fulva. - Bon. t. 5. f. 125 ; t. i. f. 159.

geophila. - Bon. t. 7 . f. $16 i 3$.

IIucedo. - Cd. Anl. t. B. f. 16: 1. 2. -

1'ay. b. cr. p. 6. f. $17 ;-$ p. 104. f. 472 .

- nigra. - Bon. t. 7 . f. 162.

- repens. - Bon. t. S. f. 173.

sclerotiophila. - Til. 1668 . = Bot. (inerea

(Ces.; cf. Tul. Scl. I. 22S.). - Kl. II. $16 \%$.

- simplex. - Sturm h. 25-.t. 24. (Preuss.)

- vulgaris. - Letell. 665. 1. - Wstd. 196.

I'oly angi um. - [S. 139.]

umbrinum Fr, - Fl, dan. XI. 19人0. 1.

- ritellinum. - [S. 439.] - Cd. Anl. t. C.

f. 40: 1-3. - Loud. f. 1fi346. - Bisch. f. 362s.

Polycystis. - [S. 515.] - cf. Caeoma, Urocystis, Uredo, Ustilago, Sporisorium. P. est Algarum genus: Rbh.).

\footnotetext{
- Anemones. - [S. 516.]

Colchici S-1. - [S. 516.] - Sturm h. 34.

t. 11. Strauss', - Fuck. s. 113. - Wstd. 11 s1. Ficariae. - [S. j16.]

Holci. - Bull, ac. belg. 2. Sér. XI. p. 660.

f. 1. Wstd.).

Lolii. - Bull. ac. belg. 1. Sér. XXI. 2.

p. 246. f. 1.

- occulta. - Dsm. III. 65\%.
}

Polycyst is opaca. - Sturm h. 34.t. 12. (Strauss).

- parallela. - [S. 516.]

- pompholygodes Iév. - Y Wstd. 6i79.1191.

- Ranunetilacearum. - [S. 5]6.] - Wstd. ii:. $11>2$.

I'o 1 y desmus. - [S. 191. 505*] - cf. Sporidesmium.

- elcgans. - Alger. t. 20.5.

- exitiosus. - Kühn. Krkh. t. 6. - Fuck. rh. 79.

Polygaster. - [S. 413.]

Poly or phus fagin. querc. Cliev. - Wstd.921.

l'olymyces phosphorus Batt. - Agaric. olearius.

P'olynema. - [S. 36;.] - ef. Conoplea, Excipula.

- Betulae. - Fuck. rh. 215.5.

- hispidulum. - Fuck. rh. 2(16.

_- strigosum. - [S. $367.47 \%$. $]$ - Fuck. rh. 201.

I'olyplocium. - [S. 439.]

- inquinans. - Ilook. Lond. J. 1513. II. t. 6.

T. - Lindl. v. K. p. 42. f. 26 - C'd. Ic. VI.

t. 6. f. $1-9$.

Polsporus. - cf. Boletus, Trametes, Poria. Amn. sc. n. 1<37. VIII. t. \-11. (Fructif. Iév.). - ef. Icones Rostkovianas apud Sturm h.5. I1. 1i. 17. t. 1-6il; h. 27. 25. t. 1-21. et Fries summa p. $318-324$.

- - abietinus. - [S. 321.] - Fl. dan. XII. 2079. 2. - Fuck. s. 975. - Dsm. II. 662. Curol. I. 12. acanthoides, - Sturm h. 2i-. t. 19. liostk.'. — adustus. - [S. 320.] - D:m. 15\% - Fl. dan. XI. 1550. 1. - Fuck. s.996. - Kl. 6i20. - Kl. II. 412. - R. f. 212. - Sturm h. 11;. t. 3 ๖. et h. 17, t. 57 . murin. .

- aftinis. - Jor. Act. 1526. XIII. I. t. 4.

lilum, et Ns. .

Afzelii Suberosi - Afzel. t. 2. f. 5.

_- agilis. - Vir. 37. 35: v. rubesc.

_ albidus Trog. - Fuck. s. 957.

- alligatus. - [S. 319.

alutaceus. - [S. 320.] - Sturm h. 10. t. 27.

destructor). - Rostk. - 3.320 .42$.

- alveolaris Bose. - Sturm h. 27 - t. 1 i.

Rostk.

- amboinensis Fr. (Pleuropus). - Rumph. II]),

amboin. XI. t. 57. f. 1. - Bisch. f. 3312. -

Iil. Cent. IX. Append.

-_ amorphus. —[S. 320.] - Dsm. III. 159.

- amorphus. $-[$ S. 320.$]$
- ancirinus. $-[$ S. 322.$]$

- amnosus Fr. - R. f. 405.

— annulatus. - Jungh. pr. t. 12. f. 24.

__ apiarius Gaudich. Voy. 1526. t. 2.

- apophysatus. - Sturm h. 2 i-. t. 4. (Rostk.). applanatus. - [S. 321.]

arcularius Fr. - Bisch. f. 3320 .

- argenteus. - [S. 320.]

- armeniacus. - [S. $322 \%$.]

- asper. - Jungh. pr. t. 11. f. 31.

- Auberianus Mont. - Sagra, Cuba. t. 1 f. f. 1.

= Bolet. microporus Swartz.

- aurantiacus. - [S. 321\%. 45.] - K1. 1711. -

Sturm h. 1\%.t. 55. inidulans.

Auriscalpium. - Gaudich. Voy. 1<26. t. 1.

australis (Apus) Fr. - Ann. Mg. n. h. \& 434 .

III. t. S. Berk.! - Kl. Cent. XII. Append.

barbulatus. - Carol. II. 19.

bathyporus. - [\$. 321. \$2:]- Sturm h. 1\%.

t. 54. : subspadic.). 



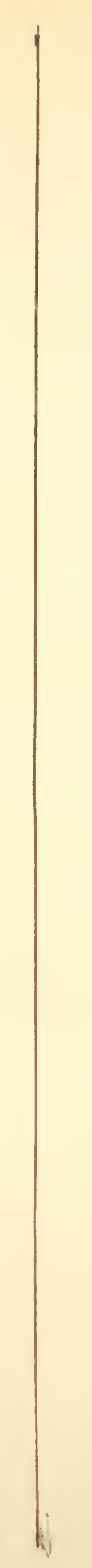


Polyporus betulinus. - [S. 321.] - Bisch. f. 3206. - KI. 32. - Fuck. s. 992. - Ital. 22!. Sturm h. 10. t. 22.

- bibulus. - Letell. 6!90. 1. - Wstd. 1257. - P. = medulla panis Fr.

- _ biformis. - Carol. II. 15. (carolinensis B. C.).

— Boltoni. - Sturm h. 27-. t. 21. (Rostk.). - bombycinus. - [S. 3:1.]

- borealis. - [S. 320.] - Kl. 716. - Fuck. s. 993: 'Theleph. mollissina. ? - Sturm li. 16. t. fi).

- Boucheanus. - [S. 319.]

brumalis. - [S. 319.] - Lotud. f. 1607]. -

K1. 204. - Sturm h. 5. t. 5. ciliat. - Rinstk. - [S. 319. \.] - Carol. I. 9. brumeus. - [S. 3:2.2. 4!.]

- byrsinus Mont. - Sagra, C'uba. t. 15. f. 3.

byssogenus. - Jungh. pr. t. 4. f. 23.

caesarius. - [S. 319.

- (aesius. - [S.320.] - Fl. dan. XI. 19(i3. 2. sup.).

callosus. - [\$. 322.]

carneus. - Carol. V. 11. - Nov. Act. 1526.

X1II. I. t. 3. Mlum. et Ns.?

- earolinensis 13. C. - ef. biformis Fr.

castaneus. - [S. 321.76.] - Sturn h. 16.

t. 47 . (zonat.)

- cellaris. - Lib. 223.

- Cerasi Fr. - Bisch. f. 3292. - Sturm h. 1 .

t. 61 .

- cervino-gilvus. - Jungh. pr. t. 9. f, 21.

-_ cervino-plumbeus. - Jungh. pr. t. 15 . f. 32. chioneus. - [S. 320.]

- ciliatus. - [S. 319.] - Bisch. f. 3314.

cinetus Berk. - [S. 322.] - Mag. Kool. Bot.

1 $\$ 37$. I. t. 2. f. 3. p. 44. 13erk.

- cinnabarinus Jeq. (Irametes). - K1. 22. -

Carol. II. 17. - Vent. mi. 61. f. 3-5. - Ital. $7 \pi 0$.

cinnamomeus. - [S. 321.]

cochlear. - Nov. Act. 1526. XIII. I. t. 6.

(13lum. et Ns.).

- conchifer. - Carol. V. 15.

- conthuens. - [S. 319.] - Sturm h. 16. t. 31 . (resinos.).

- Rost. - [S. 321. 59.] - Staude t. 3.

f. 1 .

conglobatus B. - ef. graveoleus.

contiguus. - [S. 321.] - sturm h. 27 -

t. S. (Rostk.). - Kl. 1007.-Carol. I. 16.

coronatus. - Sturm h. 27 - t. 17. (Rostk.).

corruscans. - [S. 320.]

- corylinus. - Viv. 1.

- crispus. - [S. 320.] - Sturm h. 16. t. 37. (adust.).

Rostk. - [S. 320.4S.]

- cristatus. - [S. 319.] - Bisch. f. 3297. -

Fuck. s. 1002 . - Vent. mi. 12. f. 1 ; 100. f. 1.2.

- Sturm h. 5. t. 16.

- croceus. - [S. 320.] - Sturm h. $27-.1$.

(Rostk.).

- cryptarum Fr. - Fl. dan. XI. 1963. 1. Bisch. f. 3259 .

-- cubensis Mont. - Sagra, Cuba t. 16. f. 3. cupulaeformis. B. C. - Carol. I. 10. $/ \mathrm{Cy}-$ phella pendula Fr., Sphaeria Pocula Sehw.).

- cuticularis. - [S. 320.] - K1.33. - Sturm

h. 10. t. 32. (rubiginos. Rbh., ? applan. Fr.).

__ Rost.: [? S. 321.66.] cyliscus. - Chile n. 7 . f. $T$.

cytisinus. - [S. 322*.]

dealbatus. - Carol. III. 10.
Poly por us Demidoffii Lév. - Démid. Russ. t. 3. dermatodes Iér. - bonite t. 135. f. 2.

dermoporus Gaud. $=$ Favolus brasiliensis Fr.

- destructor. - [S. 320.] - Bisch. f. 3422. -

lil. 22.5.

— dichrous. - [S. 321.] - Sturm h. 16. t. 39.

dryadeus. - S. $322^{*}$.] - Sturn h. $27-$

t. 9. (Rostk.). - Huss. I. 21.

— echinatus. - Hoffnı. v. hercyu. subt. t. s; t. 11 .

- elegans. - [S. 319.]

— epileucus. - [S. 320.]

- epixanthus. - [S.320. 33. alut. $]$ - Sturm

h. 10 t t. 30 .

- expansus (Stuposi). - Afzel. t. 5. f. 12.

- farinellus. - [S. 322.]

- fatiscens. - Carol. II. 21.

- ferruginosus. - [\$. 321.] - Fuck. s. 973.

sturm l1. 2\%- t. 6. liostk.).

- Flabellum Mont. - Sagra, Cuba t. 15. f. 2.

- flaveseens - Sturm h. 27- t. 23. (1Rostk.).

_- Havus Resupinatus Jungh. - Jungh. t. 10.

f. 25. 26. c. var. orbiculari).

— floccopus. - Sturm h. 27-, t. 13. (liostk.).

- Fuck. s. 1013.

- floccosus. - Jungh. pr. t. 11. f. 27.

- Horidanus. - Carol. I. 11.

- fomentarius. - [S. 321.] - Loud. f. $1609 \%$

- Schnzl. t. 16. f. 29. - Kl, 222. - Sturm h.

17. t. 52 .

- fragilis. - [S. 320.]

— fraxincus. - [S. $322 *$.]

- Tricsii KI. - Linn. 1\$33. VIII. t. 11. -

l3isch. f. $31 \geq \bar{i}$.

_- frondosus. - [S. 31\%.] - Kl.512. - Badham

t. 15, 3. - Viv. 36. - Vent. mi. 82. f. 4. -

Ital. 43. - Fries Sv. t. 41. - Sturm h. 10. t. 15. fuligineus. - [S. 319.]

_- fumosus, - [S. 320.] - Fl. dan. XI. 1963.

2. inf. - Bail S. t. 31. - Fuck. s. 997. -

ki. 320. 621. — Wstd. 997. - Sturm h. 16. t. 42 .

- funalis (Fumales). - Afzel. t. 1. f. 3.

- fuscidulus. - [S. 319.]

- fusco-carneus. - Carol. I. 1 S.

- Gaudichaudii Lév. (Apus). - Bonite t. 140. f. 2 .

- gibbosus. - Nov. Act. 15:6. XIII. I. t. 5 .

Blum. et Ns.). - Bisch. f. 3260.

-

giganteus. - [S. 319.] - IIuss. I, \$2.

- Fl. dan. $\mathbf{x} .1793=$ P. intybaceus Fr.

Ep. - Fuck. s. 1001. - Bisch. f. 332 1. - Hrz.

t. $32=$ P. squamosus. - Loud. f. 16079 . Sturm h. 10. t. 19.

- graveolens. - Carol. III. S. (conglob. B.).

- haematodus. - [S.322. S9.] - Sturm h. 17.

t. 62. (violaceus).

- helveolus, - Sturm h. 16. t. 36.

- helvolus (Stuposi). - [S. 320.] - Afzel. t. 6.

Herbergii. - Sturn l.. 27-. t. 1S. (Rostk.).

- heteroclitus. - [S. 322*.] - Loud. f. 16069 .

- hirsutus, - [S. 321.] - K1. S10. - Fuck.

s. 955. - Sturm h. 16. t. 44. (zonat.).

Rost. - [S. 321. 76.] - R. f. 510.-

Schweiz. 23. - Ital. 230.

- hispidus. - [S. 320.] - Kl. 1913. - Fuck.

s. $995 .-$ R. f. 406 . - Sturm h. 10. t. 31 .

- Rost. [S. $323^{* *}$.] - Huss. I. 29. 31.

- Ital. 12.

hydnoides Fr. - ef. Boletus hydnaticus.

igniarius, - [S. 321.] - Kops Fl. bat. X. 
744. - Fuck. s. 991. - Loud. f. 1609) 1. - Kl. 36. - I3erg, Charakteristik. 1560. t. 2. f. 13. - Dan. 1S06. 1S(17. - Sturm h. 17. t. 5t.

Polyporus igniarius y. Caryae. - Carol. IV. 5. ilicincola. - Carol. V. 17.

_ imbricatus. -.. \$.319.] - Sturm h. 10.t.21. impuber. - [S. $323^{\star *}$.

- incanus Lév. (l'rametes). - Bonite t 137.

f. 2 .

incarnatus. - - S. 322.]

—_ incomtus (Hormotini). - Afzel. t. 5. f. 11. infundibulif. - Sturm h. 10. t. 17.

intermedius. - [S. 319. 7.] - Sturm h. 16.

t. 33. (brumal.)

intybaceus. - S. 319.] - Huss. I. f.

- Inzengae. - Ital. 636.

- kymatodes. - [S.320.] - sturm h. 10.t.21. laceratus Berk. -- ef. pergamenus Fr.

- - lacteus. - [S. 324).] - Sturm h. 10. t 23.

- lanatus Coriacei. - Afzel. t. i: f. 15.

- lentus. - S. 32:.] - Sierk. $t .16$. f. 1

- lepideus. - [S. 31!.]

Leprieurii. - Anm, se. n. 1540 . XIII. t. 6.

(Mont.).

- leprodes. - [5. 561.] - Sturm h. 5. t. 1.5 leptocephalus. - [S. 319.

_- leptopus. - Gaudich. Foy. 1<26. t. 2.

- licnoides Mont. - Sagra, Cuba t. 16. f. 2.

- Lingua. - Nov. Act. 1526. XIII. I. t. 3.

(Blum. Ns.).

- lobatus. - [S. 320.]

- Lonicerae. - Anu. sc. ri. 1536 . V. t. 12. (Mont.).

- lucidus. - [S. 319.] - Roq. t. 2. f. 1. Berk. Out. t. 16. f. 2. - Loud. f. 16076. - cf. Bolet. dimidiat. "Thb. - Vent. mi. 17. f. 2. 3; t. 21. - Ital. 769. (estipitatus). - Sturm h. 5. t. 13 ,

t. 7.8 .

lutescens P. - Fuck. s. $9 \$ 6$.

luteus. - Nov. Act. 1526. XIII. I. t: 1.

(Blum. Ns.).

- macraulos, - Sturm h. 17. t. 55.

- marginatus. - $-[\mathrm{S} .321$.$] \div Sturm h. 16. t. 43$. mastoporus (Pleuropus) Lév. - Bonite t.

137. f. 1.

- medulla panis. - [S. 322.] - Kl. II. 5. Carol. III. 12. - Wstd. 125\%. - Fl, dan. XII. 2025. 1.- Kl. S14. - Fuck. s. 970. - Letell. 62S. - ef. bibulus.

- melaleucus. - [S. 319.

- melanopus. - [S. 319.]- Sturm h. 5. t. 1. membranaceus. - Ann. Mg. n. h. is $12 . \mathrm{X}$.

t. 10. f. 7. p. 37 s. (Berk.).

merismoides. - Sturm h: 11, t. 63 .

- micans. - [S. 322.] - Bisch. f. 325s.-

Fuck. s. 971. - K1.322. - Sturm h. 1j. t. 63. (nitid.).

$$
\text { Rost. - [S. 321. s6.] }
$$

Michelii. - Sturm h. 5.t. I.

micromegas Mont. - Sagra, Cuba t. 17. f. 5. - microporus. - A Aun. Ig. n. h. 1812. X. t.

I1). f. 6, p. 376 . (Berk.).

mollis.: - [S. 320 ; ef: $336:$ Hyp. m.] -

Sturm h: 10.t. 25

- molluscus. - [S. 322.] — Loud. f. 16099.

- Fuck. s. 967. - Sturm h. 17.t. 64 .

mons Veneris. - Jungh. pr. t. 15. f., 33 .

- Montagnei. - Alger. t. 33, 2.

Mori. - Viv. 58.

mucidus. - [S. 322.] - Carol. I. 17.
Polyporus murinus, - iS. 323 . 11. ? - 320 .

45. b.] - Sturm 1. 17. t. 57. (adust., argent.).

- nygrhimus lickx. - Bull. ac. belg. 15.35.

V. 1. 370, t. 15.

- nidulans. - [S. 320.] - Kl. S09; 1914.

niger. — Carol. I. 20.

- nigricans. - [S. 321.] - Bisch. f. 3426. -

k. f. 15. - Sturn 1. 17. t. 51.

nitidus. - [S. 321.] - Sturm h. 17. t. 63. mic.!

- Nordmanni Lév.:-Démid. Russ. t. 1. f. 1.

- nubilus (Laevigiti). - Afzel. t. 1. f. a.

- numuularius. - [S. 319.] - Sturm h. .5.

t. 12

obducens $\mathrm{T}$. - [S. 32.] - Bisch. f. 329) 1.

obliquus. - [S. 321.] - Sturm h. 27- t. 7.

(Rostk.)'.

- occidentalis. - Carol. V. IS.

, occultus Lasch. - Ki. II. GiL.

- ocellatus (Pleuropus): - Hook. J. 1556 .

FIII. t. 10 . f. 3.

$\therefore$ officinalis. - R. f. 211 . Ital. 291 .

- ovinus. $\longrightarrow$ [S.319.] $\rightarrow$ Cd. Anl. t. H. f. 75 :

$13-15 .-$ Cd. Ic. V. t. 10. f. S7. (Fructif.):-

Hrz. t. 57. - Fries Sv, t. S. - Kl. 116: -

Fuck. s. 1009. - Staude t. 1. f. 1.

— pachyus. - Slum h. 27- t. 5. (Rostk.). pallescens:- [S. 321.]

partitus Mesopus\}. - Hook. J. I556. VIII.

t. 10 . f. 1 .

- parvulus Kl. - Carol. I. $\rightarrow$.

- passerinus (Pleuropus). - Hook. J. 1556. VIII. t. 10. f. 2 .

- pédiformis (Fomentarii). - Afzel. t. 1. f. 2 . peremis Fr. - Loud. f. 16072? - Kl. II.

117 - Bisch. f. 3257. - Fuck. s. 1005 -

(Trametes) Kl. 31. - Dsm. 253. - Voges. 295.

IIuss. I. 51. - IVstd. 129. - Sturm h. 5. t. (6.

- - r. fimbriatus. - Dsm. 160.

- pergamenus. - Carol. I. 13. (lacerat. Bk.).

- pes Caprae P. - Vent. t. -10, f. 95. 96. -

Bisch. f. 3262. - Sturm h. 5: t. 11.

- Fr. Ep. - cf. scrobinaceus: - Vent.

mi. t. $23 ; 34$. f. 3 . 1 .

$\div$ petalodes: $-[\mathrm{S} .319$.

- picipes. - [S. 319.] - Sturm h. 27 - t. 20.

Rostk.).

_- Pini. - [S. 323. \$.] - Stum h. 17. t. 50.

pinicola. - [S. 321.] - Kil. 321.

Pisachapani. - Nor. Act. 1526. XIII. I.

t: 1. Ns.

politus: $-[\mathrm{S}: 319$.

polyc - [S. 319.19:]

- polygramus Mont. (Favolus). $=$ Hexago-

nia p. M.

- polymorphus Fr. - K1. 115. - Sturu h.

17. t. 56.

- populinus Fr. - F1. dan. X. 1791.

- poripes. - Carol. IV. 4.

- pterygodes (Discipedes). - Afzel. t. 3. f. 7

- pubescens. - [S. 321.] $\rightarrow$ Fl. dan. X. 1790.

1. - Sturm h. 2\%- t. 21. (Rostk.).

- pulcher Hornatini).-Afzel. t. S. f. 19.

- pủpureus. - - [S.322. $]^{-}$- Sturm h. 27-.

t. 3: Rostk.).

quercinus. - [S. 320.] - Huss. I. 52.

radians. - Dsni. III. 500 .

radiatus Scop. - [\$.321. 7\%.] - Kl. 622.

(an hirsutus?). - R. f. 509. - Sturm h. 16. t.

45. (rersicolor).

- Radula. - [S. 322.] - Kl. i1S. - Kl. II.

1]. - Dsm. III. 799 . 


Poly porus ramosus. - Letell. 626.

— -esinosus. - [\$. 321; if. n. 60.] - Sturm h. 10. t. 29. (rubiginos.); - sturm h. 16. t. 31 . (contl.).

[etículatus. - [S. 32?.] - Bisch. f. 3121.

- Loud. f. 16101.

$\longrightarrow$ Rhipidium. $\rightarrow$ Carol. III. 9.

rhodellus. - [S. 322.$]$

Ribis Fr. - Fl. diın. X. $1790: 2$ - Fuck. s. 949. - Dsm: 566. - Kopis Fl. bat. X. 745.

(Trametes' Kil. 11s - Sturm h. 1f. 't. 62. u. h. $17 . \mathrm{t}, 53$.

- rigidus. - Carol. I. 15.

roseo-poris. - Dturn h. 27-. t. 12. Rostk.).

- Fuck. s. 978.

- roseus. - [S. 321:] - Ki. 717.

- Rostkovii. - [\$. 31!.] - Biseh. f. 331).5. rubiginosus. $-5.321 .5(1)]$.- Stum h.

10. t. 32. (cut.); t. 20. res.

- rubripes. - Sturm h. 27- t. If. (lostk.) rufescens. - [S. 323: 1.] - Letell. ij]. si-

stotr. r. I*.). Stuin li, 5. t. 7.

- rufus sichrad. - Kil. 323. - lil. 11. 413.

- lir.: liuck. s. 972.

f. lilacina.- R. f. 30 s.

rugosis. - Nov, Act. 1>26. X111. I. t. 7 .

(Blum. Ns.).

- rugulosus lasch. - R. f. Ili.

rutilans. - [5.320.31. liostk.; - 320.] -

sturm b. 16. t. "3(i. testac.)

- - rutrosus. - Stum h: 2-i-. t. 2-2. (liostk.)

$\therefore$ saceatus. - Gaudich. Voy. 1 120, t. 1.

sacer (IIornotini). - Afzel. t. !.

Sagraeanus Mont. - Sagra, Cuiba t. 1ti. f. 1. salebrosus. - Ki. 1606.

salicinus. - [\$. 323 **:]- Fuck. 9. !)

- saliguus. - [S. 320.] - Sturu l. 27- t.2.

(liostk.). - Fuck. s. !)

sanguincus Fr. Pleuropuss. - Swartz, Obs.

bot. t. 11. f. 4. - Discipedes) : Afzel. t. 2. f. 1.

- Kixbh. t. 5. f. 6. 7. - Carol. II. 16.

- sanguinolentus. - [S. $322:$ - Kl. 1213.

- scaber. - Anu. Mg. n. h. Is 12. X. t.9. f. t.

(Berk.):

- Schweinitzii Fr. (Tranctes). - Kl. 513 ;

Cent. IX, Suppl. 1) pte. - Cd. Ic. V. t. 10.

f. so. (Fuctif.).

$\div$ scrobinaceus P. - R. f. 40\%, R. pes Caprac

Fr. Fp.).

- sector. - Ehrenb. hor. phys. berol. t. 1 s. f. 6 .

- serialis. - [S. 321. - 66.] - Sturu l. 17.t.

19. (zonat.):

serpens. - Gaudich. Voy. 1526. t. 2.

setiporus: $\div$ Hook. Lond. J. 15 17. VI. t. 20 :

f. 2. p. 505 .

sinuasus. - [S. 322.] - Kl. II. 303.

- spadiceus. - Juugh. pr. t. 13. f. 30 .

- spongiósus Fr. - Kil. S0s.

- spumeus. - [S. 320.] - Berk. Out. t. 16. f. I. - squamaeformis. - Ilook, J. VI. t. 7. f. 2.

__ squamosus, - $[\mathrm{S} .319]-$. Kops Fl. bat. X. 730. - Fuck. s. 1007. - Loud. f. 1606 : $^{-}$ Hiz, t. $32 ! \div$ Kl. $30:$ - Pringsh. Jalırb. II. t. 32. f. 40. (Hoffni.).- - Huss. I. 33. - Vent. mi. 27. f. 4-6;-t. 25. - Ital. (3S.) Ss. - Sturm h. 5 . t. 2.

- Stephensii (Resupinatus) B. 13. - R. f. 117.

-- Carol. IV. 7.

- stereoides. - [S. 321:] - Fuck. s. 976. -

Sturm h. 16. t. 46: (versicol.) Rostk. - [S. 321.77.]
Polyporus Steveni Lév. - Démid. Russ. t. 2. strobiliformis Dicks. - Loud. f. 16073.

- stypticus. - [S. 320.]

- suaveolèss Fr. - Fl. din. XI. 1449. - IIrz. t. 19. - Trametes Ki. 31. - Huss. I. 43. (Daedal. - Vent. mi. 5S. f. 5. 6. - Sturm 1. 16. t. 41 .

— subfuseus-flavidus. - Sturm h. 27- t. 11.

(Rostk.).

- subpileatus Weinm. - lil. 117.

-.. subspadiceus. - [S. 321.]

- subsquamosas. Fr. - [S.31S.] - Kl.119. -

Sturu l. 5. t. 3.

- substriatus. - Sturm h. 5. t. 9.

- sulphireus. - [S. 319.] - Bisch. f. 3257.

- Herk. Out. t. 16. f. 3.- Ki. 1005. - IIuss.

I. 16. Vent. mi. 11. f. 1. 2; 59. f. 4. 5. -

Ital. 143. - Sturm 1. 10. t. 20.

tabainus. - Chile is. 7. f. 6.

tenerrinuss. - Carol. 111. 13:

- tephroleucus - $[$ S. 320. $] \div$ Sturm h: 10.

t. 26.

- testaceus. - [5. 3201\%]

— tomentosus Fr. - Bisclr. f. 325\$. - Sturm

l. 5. t. S. (brunalis).

- tornatus Gaud. = iustralis Fr. (Gaud.)

- torridus ('oriacei), - Afzel. t. 7.f. 17.

- trabeus Iiost. - Fuck. s. 995. - Sturm h.

11. t. 25 .

- trachypus. - Sturm h. 27-. t. 14. (Rostk.!

- 'Trielioloma Mont. - Sagra, Cuba t. 17. f. 1.

tropicus Jungh. ? = lucidus Fr.

Tnheraster 12. - Biseh. f. 3174. - Badham

t. $11,3 .-$ Viv. 5) - Vent. mi. 36. f. 3. 1. udus, - llijdschr. v. nat. Geschied. 18.10,

VII. t. 5. f. 5. u. (i. p. 2\$!. Jungh.).

- ulmarius. - [\$..322*] - Kil. 11. 616.

Jierk. ()ut. t. 16. f. 5. - IIuss.I.64. - Ital. !1.

- umbellatus. - [S. 319.$]$ - Cd. Anl. t. If

f. $75: 16-14 .-$ Viv. $25 .-$ Bisch. f. 3273.

_- undulatus: - Brit. f. 294.

- - ungulatus Fr. - Vent.'mi. 19. f. 3. 4.

— unitus. - [N. 3?1.]

- Vaillantii. - [S. 322.] - Lib. 321.

- valenzuelianus Mont. - Sagra, Cubat. 15.

f. $\cdot$. = Bolet. resupinatus Swartz.

vaporarius. - [S. 322.] - Carol. I. 19.

- variabilis (Pleuropus]. - Hook. J. VIII.

t. $9 . f .1$.

- varius. - [S. 319.] - Kops Fl. bat. X. 755.

- II) 223. - Sturm h. 5. t. 11. (elegans).

$\because$ vegetus. - [\$. $321.322 *]$

- velutinus. - S. 321:] Kl. II. 701. -

Fück. 's. 954: - Carol. IV. 6.

$\longrightarrow$ r. Fr. - R. f. 15. u. 16.

f. 1 .

- versicolor, - - [S. 321.] - Ann. sc. n, 153\%.

VIII. t. s. f. 11 ; t. 11. f. 25. Fructif. Lév.). -

Cd. Anl. t. H. f. 75 : 19-22. - Loud. f. 16059.

$\longrightarrow$ Fuck. s. 9.52. - Kl.120; Cent. IV, Suppl.

- Kl. II. 209. - Pringsh: Jahrb. II. t: 32. f. 39. (Keimg. Inoffin:). - Carol. I. 11. - ILuss.

I. 24. - Wstit 156. - Krypt. Bad.59. - Stürm

h. 16. .t. 45 .

502 .

__ virgineus. - Carol. III. 11 .

vitreus. - [S. 322.]

vulgaris. - [S. 322.] - Kl. 619. - Berk.

Out. t. 16. f, 6. - Fuck. s. 968:- Carol. V.

16. - Letell. 690. 2. - Sturm h: 17. t. 60 . 
Polyporus Weinmami. - S. 320.]

Wightii Kl. - Linn. 1432. VII. t. 10. (Sce-

nidium $\mathrm{kl}$. ) = IIexagonia Wightii Fr.

- xanthopus (Ifornotinus , - Af\%cl. t. 3. f. 6. - xanthus. - S. 321.] - Carol. I1. 20.

- xoilopus Rist. - IBisch. f. 3269. - Sturm

h. 5. t. 10 .

— zonalis. - Ann. Mg. n. h. 1<12. X. t. 11 .

f. 5. 1. 375 . (Berk.).

zonarius. - Vent. mi. 90. f. 5.

zonatus. - S. 321.] — Fl. dan. XII. 2025.

2. - : Fr. : Sturm h. 27-. t. 11). (Rostk.).

- Ns... K Kl. 321?

- var. Fr. - Fl. dan. XII. 2179. 1.

P'ilysaccum. - [S. 411.] - Ann. sc. n. 1512.

XVIII. t. 5. 6. (Fructif. Tul.)

arenarium. - Dsin. 1223.

australe. - Ann. se. n. 15.15. IX. t. 9. f. 4.

lév. .

- crassipes. - Dsm. 1627. - Cd. Ic. V. t. 1.

f. 41. - Cd. Anl. t. D. f. 41: 7-14. - Bisch.

f. 360s. - Pay. b. cr. p. 4. f. 32. p. 9. f. 39.';

p. 110. f. 503. - Sturm h. 34. t. 5. 6. Strauss).

Tent. mi ↔. f. 1.

- - v. clavatum. - Kl. (it4.

degenerans. - S. $441^{* *}$.]

- pisocarpinm. - [S. H4.] - Act. Turin.

1543. V. t. 3. f. 13. Vittad.). - Bisch. f. 3606. $360 \%$.

- tinetorium. - Canar. t. 5. f. 1.

l'olyseh ismi un Trevelyani. - Cu. Anl. t. C. f. $29: 23-26$. Didlerma.

PolyseytaIum. - Bot. Zt. 1553. t. 3. f. 14.15. Riess.

- fecundissimum. - Fresen, t. 12. f, 1-4. p.

95. Fusispor, gris. Kll.

- - v. macrosporum. - Kl. 1573.

Polystigma. - S. 34\%.] v. Dothidea, Sphaeria.

- aurantiacum. - Bon. t. 3. f. 63 ; Aecicliol.

exanth. Ung. Tul. Sel. I. 221., - Wstd. 825.

- fulvum. - Cd. Anl. t. F. f. 51: 14-16. -

II. II. 69. - Voges. 271. - Norweg. 90. ef. Dothidea.

- f. ascigera. - Kl. II. 579.

1. f. 9. Bail.

v. maculare, - ef. Dothidea fulva.

pertusarioides. - Dsm. III. 72. - Wstd.

642. (Septoria Calystegiae!

- rubrum DC. - K1. II. 550. - Voges. 270.

- Letell. 6.56. 4. Dothiclea). - Purton. I. t. 33.

- cf. Ascochyta rubr., Lib. - Ital. 240.

- typhinum DC. - Dothidea typh. Fr. Kil.

II. 575. - Fuck. s. 703. Cordyceps!. - Wstd. 522 .

- Ulmi. - Fuck. s. 667.

I'oly thecium. - ef. Micropora.

I'olythrincium. - [S. 504.]

polysporum. - [S. 504.]

Trifolii. - [S. 504.] — Dsm. 560. - Bisch.

f. 3721. - Fuek. s. 151). - Loud. f. 16607. Kiryt. Bad. 326. - Cd. Anl. t. B. f. 10:6.-Iil. T0. - Cephalothecium Tr. Bon. - Voges. 65. - Brit. f. 97. - C'arol. V. 93. - Wstd. 1295. - Fuck. rh. 55. - Sturm h. 11. t. 9.

Pompholyx. - [S. 460.]

- sapidum. - Cd. Anl. t. C. f. $35: 1-5$. Sturm h. 19-. t. 15. Corda!.

Poria. - cf. Polyporus.

- cerea. - Hoffm, v. hereyn. subt. t. 11. f. 3 .

- Encephalum. - Ioffm. v. hercyn. subt. t.

12. f. 1.
Pori th favoginea, - Hoffm, v. hereyu. subt, t. 12.

f. 3.

scutata. - IIoffm. v. hereyn. subt. t. 9. 10. spuma. - IIoffm. v. hereyu. subt. t. 13. f. 2

['oronia. - [S. 342.] - Bail S. t. 1\%. (ef. Sphaeria Poronia).

-_ macropocla.

— - v. cladonioides. - Kil. 1\$16.

Oedipus.

- $r$. cladonioides. - Comm. soe, erit. it.

|४ij|. 2. t. 4. f. 4. Ces. .

— pezizoides. - ('d. Anl. t. F. f. $56: 1$ - -

- pilulifera. - [S. 3\2**.]

- punctata. - S. 3\$2.] - liops Fl. bat. XI.

45. 3. - Bail S. t. 1\%. - Kl. 255. - Kl. II.

15. - Nov. Aet. I (i) XXIX. t. 2. f. 11. 1\%.

(Frolut. Bail) . - Miiller, I'flanzenstaat 2\%0. (Co-

pie.- Tsm. III. 375.

- scutellata. - [S. 3,2.]

Porothelium. - [S. 325 .

- timbriatum. - [S. 325.]

— lacerum. - [S. 325.] - Carol. V. 19.

- rugosum Pleuropus,. - Hook. J. VIII. t. 9.

f. 2 .

- subtile. - [S. 325.] - Cd. Anl. t. II. f. 75:

23.

I'rismaria alba. — Sturm h. 3.). t. T. (Preuss.).

P'ropolis. - [S. 372.] - cf. Stictis.

_ alba. - [S. 372.]

- Berkeleyana. - Dsm. III. 14.

_. farinosa. - [S. 372.

- hrsterina. - [S. 372.$]$

_- nivea. - [S. 372.]

— phacidioides. - [S. 372.] - Cd. Anl. t. G.

f. $61: 1-4$. - Pay. b. cr. p. $\ 6$. f. $3>6$. - Bail

S. t. 19 .

- Pinastri Lacr. - R. f. 371. - Dsm. III. 791.

- quadrifida. - Chile n. §. f. $\varsigma$.

rhodoleuea. - S. 372.$]$

- versicolor, - [S. 372.]

- viridis. - [S. 372.$]$

Prosteni u m Guepinianum M. - ef. Pestalozzia Guep. - Wstd. 33\%.

Prosthecium ellipsosporum. - Fres. t. 7. f. $15-23$.

Prosthemium. - [S. 416.]

- betulinum. - [S. 416.] - Cd. Anl. t. F

f. 5s: 10-12. - Bisch. f. 3\$65. - Fuck. s.

417. - Pay. b. cr. p. TS. f. 36与. - Bail S. t. 15.

- Kl. 1356. - Voges. 1451.

- stellare. - Bot. Zt. 1553. t. 3. f. $25-31$.

Riess. - Ann. Mg. n. h. 1561 . VII. t. 15. f.

10. 1. 3\0.

Proteus. - cf. Amoeba.

Protomyces. - [S. 517.]

- endogenus. - [S. 51\%.] - Ung. Ex. t. 5.

f. 27 . - R. f. 400 . - Bisch. f. 3901. - Bon. t. 1. f. 21 .

Galii Rbh. - Fuck. s. 1. - P. endogenus

Ung. : R. f. 400. - Fuck, rh. 4 S.

- macrosporus, - Physoderma gibbosum Wilr.

- Ung. Ex. t. f. f. 25. - Cd. Anl. t. B. f. 2 :

1. 2. - de Bary, Br. t. 1. f. $9-12$; t. 2. f. 1. -

Bon. t. 1. f. 26. - Kl. 2000. - ? zu Peronosp.

Umbell. : Casp. Sehimmel f. $33-3$ i. - R. f. 600 . - Menyanthis. - de Bary, Br. t. 2. f. 2.

Psathyra. - ef. Agarieus.

Psecadia. - [S. H14.]

Pscudo-Erineum. - ef. Erincum.

Pseudographis elatina Nyl. - Hysterium el.

P. Dub. .

I'seudoralsa cf. Sphaeria. 


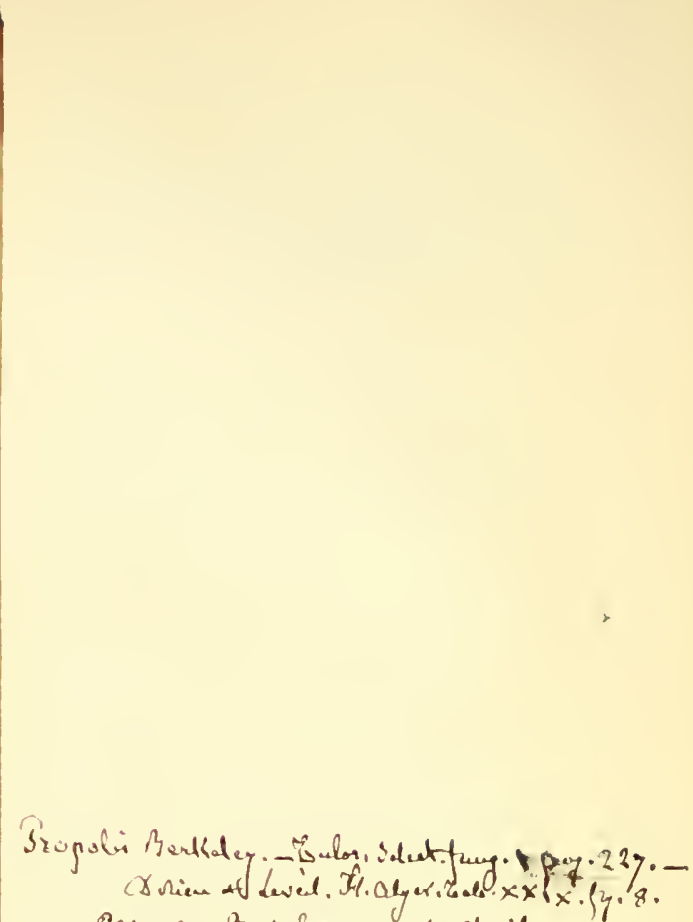

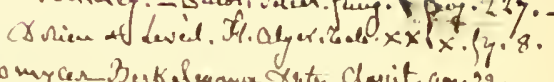

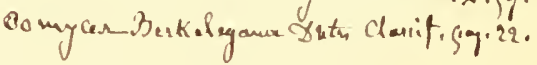




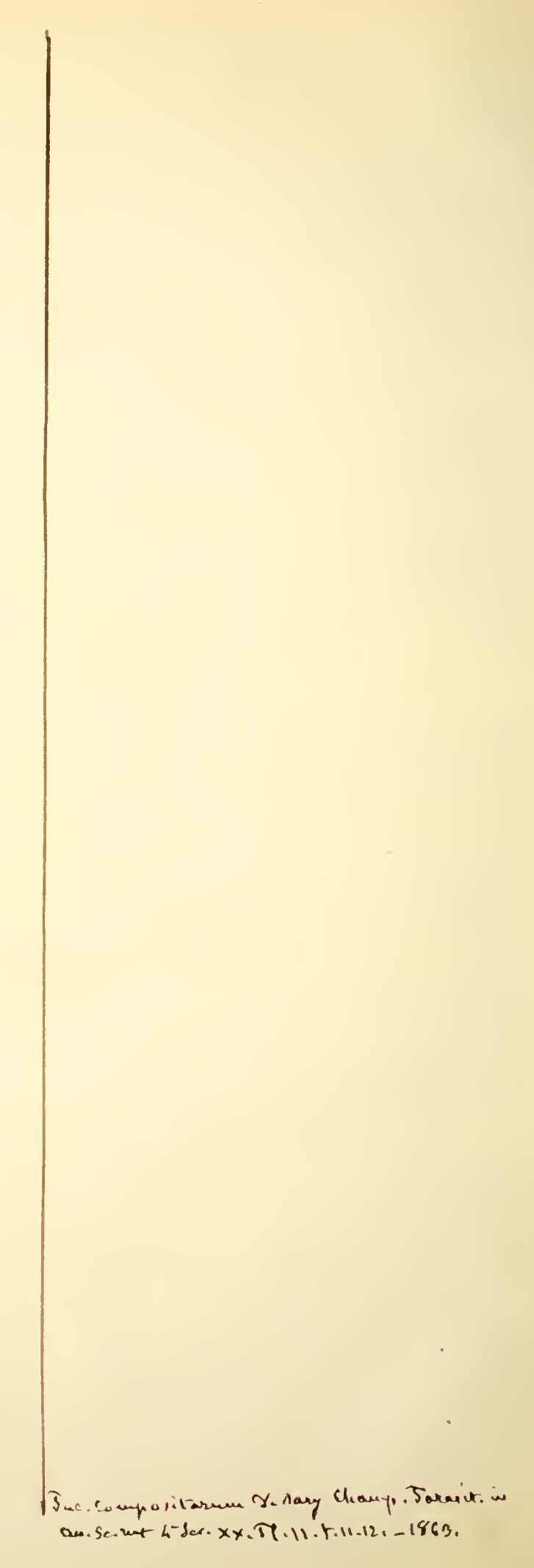


I'silonia. - [S. 495.]

- apalospora. - Carol. III. S2.

- Arundinis. - [S. 495.] - Dom. Jitil. -

lirit. f. 102.

— atra. - Kl. 1261.

- - aurea. - [S. 495.]

- Buxi. - Ann. se. n. 1555. IX. t. 5. f. 1-9.

1) cbat.). - ef. Fusisporium.

_- cinerascens. - Kl. 1781.

- Festucae. - Lib. 246.

- gilva. - [S. 49\%.] - 1'rit. f. 111

glauca. - [S. 495.

-_ Juzulae. L Lib. 3s(i.

_- maculiformis. - A. 19) - Bisch. f. 375li.

- nivea. - \$s. 49.3.] - Lib. 3 š $^{-}$

- pellieula. - Dsm. 911.

_- rosea. - Brit. f. 56.

- stipitatit. - Lib. $25 \overline{\text { I }}$

- tremelloides. - I3rit. f. 103.

Inilospora. - ef. IIysterium.

- faginea. - Kl. II. 150. - Rhb. Lichen cu-

rup. fsc. IV. n. 44. c. ic. . - ef. IIysterium rugustum.

Pterodinia. - ef. Isaria.

- nivea Cleve. = Isaria intricata v. suls.implex schum,

Pteropliyllus. - [S. 315.

l'terula. - [S. 339.]

- capillaris, - [s. 339.]

- flammea. - c'd. Anl. t. G. f. il: 11. 12.

- penicillata. - Cd. Anl. t. (i. f. il: lo.

- subulata. - Iimn. I 30 . V. t. 11. f. 4.

Fries). - Bisch. f. 3771. - Voges. 995. -

Carol. II. 4 .

J'tychogaster. - [s. 4.19.561.]

- albus. - Cd. Anl. t. C. f. 31: 7-10. -

Fuck. s. 993. zu Polypor. boreal. Fr. Su. 561.

P'll ceinella truncata, - Fuch. Nass. f. ১; ‘. b.

- Fuek. s. 57.

P'uccinia. - [s. 513.] - T3erk. In. 13. 325. f. i3. c.

- Absinthii DC. - ef. Cacoma A. Cd. - W'std. 4.1 .

- aculeata. - Cd. Ic. VI. f. 13. - Schw.

Carol. I. 96. Podophylli .

- Adoxac DC. - Fuek. s. 69. - Wstd. 5s1.

R. f. 590. - Fuck. rh. 371.

- A egopodii. - Dsm. 1533, - Kil. II. 65 - -

Kl. $273 .-$ Fuck. s. $60 .-$ Wstd. 679 .

- Actliusae Mart. - Kil. 275.

- I.k. : R. f. 491.

- Allii Rud. - Linn, 1s29. IV. p. 392. -

lil. 1093.

- Alliorum. - Dsm. 152؟ - Inl. II. 351. -

Robin r. p. t. 14. f. 13. - Wstd. 752.

- Alsineacearum. - [S. 513.]

- Amorphac. - Berk. In. p. 32j. f. 73. c. -

Carol. I. 97.

- Anemones Dicaeoma). - [S. 514.] - Dsm.

173. - Kl. 467; Cent. VII, Suppl. - Kil. II.

346. - Voges. 191. - Brit. f. 222 . - Wstd.

$550 ; 650$ : v. Betonicae. - Ital. 796. - Krypt.

Bad. $5+3$. - Fuck. rh. 372 .

- Apii. - Cd. Ic. VI. f. 11. - Wstcl. S56.

- Arenariae (serpyllifoliae). - Iñl. 1156.

- Aristolochiarum. - Ital. $\$ 93$.

Armeriarum. - [S. 513.]

Artemisiae Fuck. - Fuck. rh. 350.

- Artemisiarum S. K. - Wstd. S5l.

- arundinacea. - [\$. 514.]-KI. II. 24?. -

zu P. clavuligera Bon. Con. 50. - Fuck. s. 40.
- Carol. IV. 59. - Wstd. 186. 157: v. epicaula; 249: v. epiphylla. - Kirypt. Bad. 242. - Fuck. rh. 320.

Puceinia arundinacea $r$ epiphylla Wllr. - Bisch. f. $3 \varsigma ; 6$.

Asari Lk. - R. f. 195. - Kn̈ypt. Bad. 406. Fuck. rh. 3ifi.

- Asparagi DC. - [S. 513.] - Kil. 1181.

Ki. II. 690 - Fuck. s. (i-. - R. f. 394 . Vuges.392. - Lib. 291. - Wistl. 123. - Fuck. ith. 37 .

- aspera. - Ital. 49?.

- Asperulae. - Fuck, rh. 352.

- Asphodeli. - Ital. 79 \%.

- Asteris Dub. - Dsm. \il.

- Atragenes, - Ital. วう5).

-_- Ariculariae A. S. - Dsm. 473. - Wstıl. 120.

_- Balsamitac Str. - Kil. 163. 1290.

- Bardanac Willr. - Kíl. 997. - Kí. II. 343.

Ii. f. 492 . W Wttl. 1073.

— Betonicae. - Brit. f. 21 s. - Dsm. 115:3. -

Cd. Anl, t. I3. f. 4: 1. - Ki. 1590. - Kl. II. 355. - Wstd. 690.

— Bistortae. - Lib. 91. - Wstd. 755. - Fuck.

rh. 332 .

- Brachypodii. - Fuck. rh. 323.

- Brassicae. - Ann. sc. n. 1436. V. t. 12.

- Mullaria Lk. - Brit. f. 5\%. - Phragmotr.

B. Cd. - Wstd. $55 \bar{T}$.

- Buxi. - Brit. f. 109. - Dsm. 112. - Loud.

f. 16712. - Kil. 1992. - Kil. II. 6s1. - Wstd.

107t. - Voges. 676. - Ital, 214.

Calcitrapae DC. - Wstd. 757.

- Calthae Lk. - Kl. 465. - Kl. II. 341. -

Fuck. s. 67. - Wstd. 1156. - Fuck, rh. 370.

_- Campanulac. - [S. 513.] - Fuck. rh. 375.

- Caricis. - Kil. 169 ; Cent. XV, Suppl. -

Iil. 1591. - Kl. II. 339. - Fuck. s. 13. - zu

P. claruligera Bon. Con. p. 50. - Wstd. 290.

1272. - Fuck. rh. 321.

- Caryophyllacearum. - Dsm. III. 153.

- f. Arenariae. - Dsm. $17 \%$.

- f. Lychnidis, - Dsm. 17.

—- f. Spergulae. - Dsm. III. 2.51.

f. Stellariae Wlir. - Wstd. 1271.

- caulincola. - [S. 513.] - Kil. II. 495. -

Wstd. 420.

- Centaureae DC. - Wstd. 756. 757.

- - f. Cirsii. - Kl. 791.

- Cerasi. - Dsm. 1534. - Kl. 1592. - Ital.

245. - Mycogone.

- cerealis Mart. - Wstd. 91.

- Chamaedryos. - Kl. 1991.

Chondrillae Cd. - Fuck. s. 5\%. - Ital. 352.

luck. xh. $3 ₫ 6$.

Chrysophylli. - [S. วั13.]

- Cicutae Lasch. - Kl. is \%.

- Circaeae P. - [S.513.] - Dsm. 110. - Iil.

464. - Kl. II. $35 \%$. Fuck. s. 45. - Voges.

192. - Lib. 293. - Wstd. 11S4. - Ital. s94.

- Fuck. rh. 32S.

- Cirsii Lasch. - R. f. $\$ 9$ : P. Syngenesar. Cd.

Ic. - Fuck. s. 52. - Fuck. rh. 340.

- - oleracei Dsm. - Ann. sc. n. 1St7. VII.

t. T. f. 17-21. (Tul.).

- clavuligera Wilr. - R. f. 154. - Bon. Con.

p. 50. t. 3. f. 3ৎ. - cf. Lamyi, Stellatarum.

lior.); $5 \$ 3$ (v. Luzulac).

- Clinopodii. - Dsm. 235. - Wstd. ৎ.

— compacta. - Kil. II. 658. - Krypt. Bad. 544.

— Compositarum. - [S.513.] — Ung. Ex. t. :. 
f. 40, B. - Anm se, n. 1817. VII, t. T. f, $22-$ 2i. (Tul.) - Fuck. s. 56.-I) 5 m. 172. - Jil. 271. - Kl. II. 191. - Irit. f. 21!. - Wstd. 757. - Fuck. rh. 314. 34 .

Puceinia Compositarum f. Jellidis. - Prit. f. 225 .

f. Cardui. - Iil. igu.

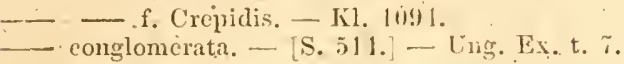
1. 35. - Schweiz. 2.

Convallaria Silnum. = Macrosporium Cons. Fl. dan.

- Controlruli. - Dsm. 1154. - Kl. 1692; 1955. coronata. - Amn, sc. n, 1st7. TII. t. 7 .

f. 24. Tul.) - Fuck. Nass. f. 3. - Fuck. s. 12. - de Bary, Br. t. 4. f. 2. - Dsm, 1152..Kühn, Krankh. t. 5. f. $43-15 .-$ Jand w. Annal. d. neeklenb. patriot. Ver, 1562, no. 2. kiklu - Dsm. III. 253. - Wstd. 759. - Fuck. rh. 32\%. v. Lolii. - Bull. ae. belg. 1. Ser. 1 -ăt.

XX1. 2.p. 216. f. 2. (Wstd:) - Wstd. 1076. - v. sertata. - Kl. II, is1. - cf. P. sert. Corrigiolae. - Dsin. 1155.

eristata Schmid. - Wstd. 339

Cirneianellae. - Dsm. 1155.

Cynodontis. - Dsm. III. 65.5.

Dianthi. - Iil. 191. - Kl: II. 342.

Wstd. 292 .

didyma.- Bon. t. 1. f. 23.

difformis. - Bon. Con. p. 216. t 3. f. 39.

Discoidearum Lk. - S.513. - Kil. 190. -

Til. II. 655. - Dsm. $1275 .-W$ std. 122 ; ?5. 1. Discoidearum Lk. f. Asparagi. - Wstd. 123.

— f. Millefolii. - Kl. 115s.

Dracunculi. - Iil. 145s.

Elymi. - Wstd. 291.

Epilobii. - S: 513.] - Dsm. 1159. - Kil.

115. - K1. II .337. - Fuck. s. 51 - R. f.

154. - Brit. f. 345 - Wstd. 642. - Ital. 596.

Fuck. rh. 339.

- Ervigii DC. - Dsm. 115\%. - Wstd. 1273.

- Fabae. - [S.514.] - Ung. Ex. t. T. f. 39.13.

- Wstd. 124.

fasciata. - Bon. Con. pi 21s. t. 3. f. 12.

$=$ P. Lychnidear. Cd. .

- Favi Ardst. - Robin. v. p. t. 11. f. 13.

ferruginea Lév. - Jonite t. 140. f. J.

Fieariae. - Voges. 294.- Wstd. SS.

Fragariastri I)C. - Wstd. S59.

Frankeniae. - Cd. Ic. VI. f. 9.

Galii Lk. - Kl. 470.

— Cruciatae. - Wstd. 544. verni Ces. - Kl. 11992. - Kl, II. 161.

- cf. Vaillantiac. - Ital. (19. 99.

Galiorum. - S. 513.] - Iil..II. 792. -

Fuck. s. 66. - Fuck. rh. 351.

- Gentianae Lk. - Kil. 166. - Kl. II. 334.

Geranii. - [S. 513.] - Fuck. s. 7.1: -

Fuck, rh. 3i3.

Gladioli: - Dsm. 1529.

Glechomatis DC. - Kl. 272. - Kl. II. 195.

- Fuck. s. 17. - Dsm. 236. - Wstd. 121. -

Finck. rh. 329.

globosa Gr, - Loud. f. 16711.

Globulariae DC. - Wstd. 1075.

glomerata. - Brit. f. 220.

Glycrrrhizac. - Kl. 1396.

Gonolobi. - Carol. IV. $! 1 !$.

Graninis. - [S. 51 $1.515,15$.$] - Bisch. f.$

in - - Lond. f. $16711 \%$. (p. 1017. u. 1015.

11119.). - Anu, sc, n. 1451. II. t. ?. f. $\longrightarrow$ -

('Tul.) - Bon. t. 1. f. 40. - Berk. Out. t. 1. f. 4. - Lindl. v. K. p. 35. f. 24. - de Bary, 1ir. t. 1. f. 1.-Kühn, Krankh. t. 5. f.37- Microse. Journ. 1\$.57. T. t. 8. f. 21. Cur1ey). - Ki. 79. - Kl. II. 3.17. - Fucks. s. 39. - I'ringsh. Jahrb. II. t. 2S. f. 15. (IIoffm.) Lamid. Anmal. d. mecklenl, patr. Ver. isfi2. no. 2. Kü̈hn. - Voges. 675. - Carol. I. 99. - Letell. (i61, 4. - Oekomom. Neüigk. v. I1lubeck, 14.16. t. 2. f. $9-11$ - W Wstd. 91. I il. 5. 2. 'v. epiphylla et spiculor.). - Krypt. I3ad.

113. - Fuck. rh. 319. (c. Ured. linear.).

Puccinia Graminis f. Avenae. - K. f. 3!! 2.

-.- _ - v. Arundinis. - Voges. 292.

- Graminum. - De Cand. Org. vég. 1527. II.

t. 60. f. 2. (IIedwig fil.).

granulata. - Kl. II. 499.

Helianthorum. - Carol. II. 90.

Heraclei Grev. - Loud: f. I 66939.

- Herniariane. - Ung. Einfluss d. Borlens: f.

1 $\overrightarrow{1} .-$ Kl. 139.

- heterochroa. - Dsm. 1532. - ef. Vaillantiae.

- Ilieracii T. - Fuck.s. 55. - Fuck. rh. 342.

hysterioides. - S. 513.$]$

- Jasmini. - Dsm. 1530.

- Impatientis:- [S. $51 \%$.

- incarcerata Lév. = Ustilago Cissi DE.

- inquinans Wlli.. - [S. 514.]. - Bon. Con. p. 21 . t. 3. f. 11: - R. f. 192 .

— - f. Aegopodii. - Wstd. 679; 6ऽ0: Betonicáe; 756 , u. \.5.5: Compos. ; 75. u. \$5 1 : Labiat.; 75j: Polygon.

- inquinans W7lr. f. Epil. - R. f. 493.

- - - .. Umbellifer. - Wstd. 11 . 553.

- Junci. - Dsm. 170. - Wstd. 55.5.

- Juniperi 1?. - Wstel, 339.

- Lamyi S. $=$ P. Valantiae P., punctata I.k., clavuligera v. Galiorum Willr.

— Lapsanae. - Fuck. Nass. f. 6. 7. - Fuck. $\therefore 59$. - Wstd, S5. - Fuck, rh. 317.

- lateripes. - Berk. In. 1). 325. f. 73. f. Carol. II. S9.

- lateritia. - Carol. III. 9;. Liliacearum. - [S. 513.

_.. f. Muscari comosi. - Iili II. 646. f. Ornitlogali unbellati. - KI. II. 791. Liliorum. - Cd. Ic. V. p. 511. t. 2. f. 12. Limonii DC. - R. f. 494.

linckii Kl. - Linn. 1-33. VIIT. t. 12. linearis. - Dsm. III. 152. - Wstl. 91. lineolatà.-Dsim: 152\%.

- Luzulac. - Lib. 91. - Kl. 1691. - Kl. II. 311 . = graeilis Bon. Con. 51 .

- Lib.: R. f. 393. - Dsin. III. 339. Hstd. 543. - Fuck, rh. 327.

- Lyehnidearum. - S. 513. - K1. 472. Iil. II. 653. - Ung. Fx, t. 6. f. 33: - Microse. Juurn. 15.7 . V. t. \&. f. 22. Currey!

- Cd. = P. fasciata Bon. - Brit. f. 221 .

Wstd. 292. - Ital. 6.56. - Fuck. rh. $366-36 !$.

- - Lk. - cf. Frankeniae Lk.

- f. Dianthi. - Kl. $114 \%$

f. Lychnidis. - Iil. $11+7$.

- f. Saginae. - K1. 593.

- macrospora Spr. - Wstd. 575.

- Malvacearum. - Cd. Ic. VI. f. 12.

Maydis I'oetsch. - R. f. 143.

Menthae. - S. 513.? - I)sm. 171. - Fuck. s. 51) - K1. II. 353. - Brit. f. 217: =- Ietell. (iti1, 3, - Wstd. 751. - Fuck. rh. $335-33 \%$ (Clinop., Calam., 'T'lỵm.)

Millefolii. - Fusk. rh. 3 I?.

mixta. - Fuck, rh. 377 . 


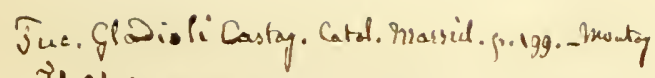
Ha. alyer sog.ing. 


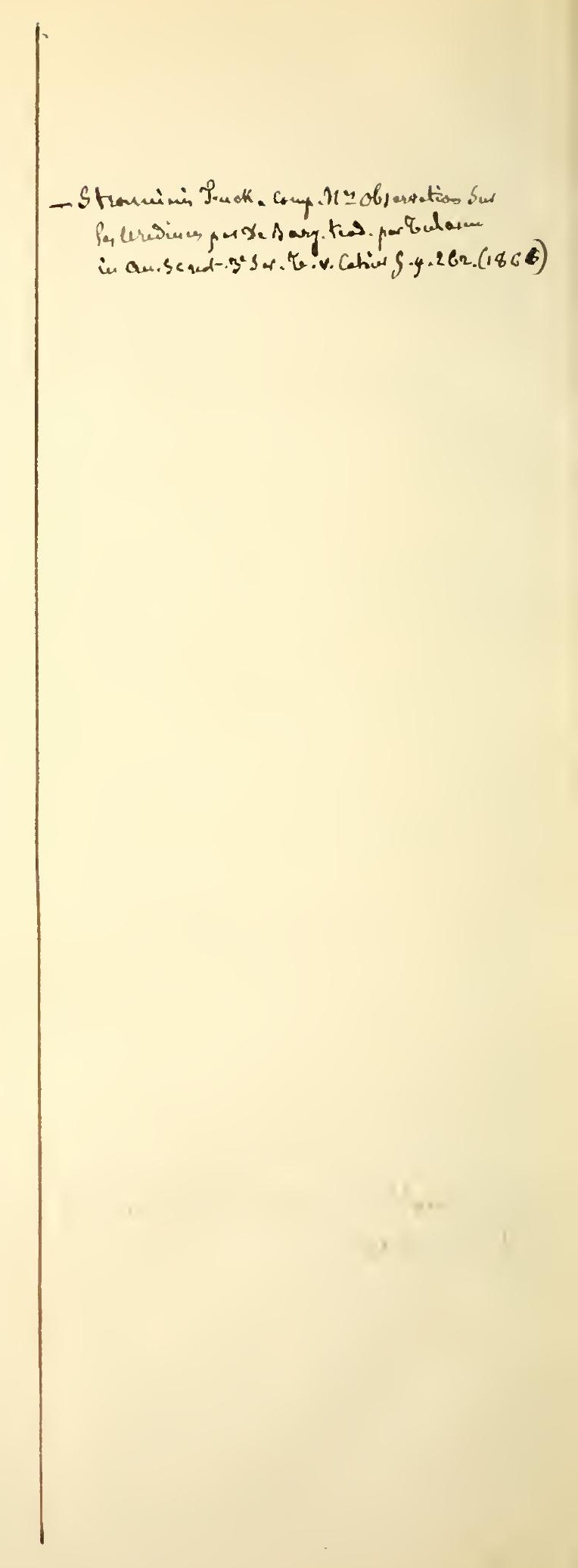


Puccin ia Möhringiae. - Fuck. rh. 364.

Moliniac. - Amn. sc. n. 165\%. II. t. 9. f. 9

-11. (Tul.). - Schacht, Lehrb. An. Phys. 1559.

II. t. 6. f. 6. - Ital. 495 .

- nucronata. — De Cand. Org. vég. 1927. II.

t. (ii). f. 4. (Iledwig fil.).

- 1 ' = Phragmid. bulbos. et mucron.

Fl. dan.

- L Rosae: Wrstd. 134; Rubi 157.

- Nolitangeris Cd. - Kl. 11S2. - KI. II. 340.

W.strl. 1155 .

obtegens. - Fuck. s. 59. - Fuck. rh. 319.

Urobi Kiz. -- Kil. 592 .

- Phaseolorum. - De Cand. Org. vég. 1 1327.

11. t. liv. f. 3. (Hedwig fil.). - Voges. 194.

[isedo append.).

— Pluagmitis Kz. - Wstd. 156.

- Phytcumatum. - Bisch. f. 39109. - Ung.

lis. t. 7. f. $35=$ Caeoma: Cd. Ic. $1 \mathrm{~V}$. p. $\mathrm{s}$.

I'impinellae Strss. - Wstd. 853.

J'isi DC: - W Wstd. $\$ .39$.

plagiopus Mont. - Sagra, Cuba t. 11. f. 1.

P'iatani. - Bivona t. 3. f. 5.

l'odophylli. - ef. aculeata Schw.

Polygonorum. - [s.513.] - Dsm. 105. -

Rubin v. p. t. 11 . f. $13 . \mathrm{m}$. n. - R. f. 4 s! -

kil. S1. - Fuck. s. 46. - Brit. f. 216. - Wstd.

419. - Ital. \$90. - Fuck. rh. 331.

- f. Bistortac. - Kl. 759. - Wstd. 755.

- f. Convolvuli. - Dsm. $970 .-\mathrm{K} 1.546$. Potentillae P. - Loud. f. 16651. - Wstd. S60. P'rimulae. - Brit. f. 350 . I $\mathrm{sm}$. 571 .

Prunorum. - S. 514.] - I)sm. 411. - Cd.

Ail. t. B. f. 4: 6. - Fuck. s.61.- (1)icacoma)

Kil. $590 .-$ Kil. II. 55. - Fuck. rh. 330 .

- pulverulenta. - Brit. f. 105. - ? Wstd.652. punctata Lk. - Wstd, 5\$4.

P'unctum. - [S. 514.] - Fuck. s. 11. -

Dsm. \$69. - Krypt. Bad. 407. - Wstd. 290. Fuck. rh. 325.

-_ Pyrethri. - Iil. $1569 ; 1990$.

recondita. - Usm. III. 252.

- Ribis. - [S. 513.] - Kl. 1545. - Norweg.

$17 \%$

- Kosac. - Voges. 293. - Iinn. 1\$2\$. III.

t. 2. f. 1-3. (Schwabe). - cf. I'luragmidium.

Loud. f. 16653.

- Schum. = Phragmid. mucronat. Fl.

dan. - Purton. I. t. 25. - Wstd. 134. 437.

rosea. - Cd. Ic. I. 9S. 7 Sporae Trichothecii rosei (Wstd.).

- Rubi Schum. - Wstd. 15i. = Phragnid.

bulbos. Fl. dan. - Voges. 193.

- Rumicis. - R. f. 496. - Wstd. 758.

- Salviae Ung. - R. f. 591.

- Saniculae. - Brit. f. 315. - Kl. II. 350. -

Carol. IV. 90.

- Saxifragarum S-1. - [S. 5 13.] - Dsm. 23 S.

- Scirpi. - [S. 514.] — Bisch. f. 3575. - Kl.

7S. - Kl. II. $345 .-$ Fuck. rh. 326.

- Scorodoniae - Lib. 93. - Kl. 1457. - Kl.

II. 356 . - Fuck. s. $49 .-$ Dsm. III. 154.654 .

— Wstd. 753 .

- Scrophulariae. - Lib. 193.

- Senecionis. - Lib. 92.

sertata. - Sturm h. 25-. t. 3. (Preuss.). -

Kl. 1395. - $\mathrm{cf}$. P. coronata var. Sisyrinchii. - Cd. Ic. VI. f. S.

- Soldanellae. - Ung. Ex. t. i. f. 3 \%, - Bisch.

f. 3910 .

Sonchi. - Ism. 1531.

Sorghi. - Carol. III. 92. - Ç. Ic. VI. f. 7.

H of $\mathrm{m}$ an $\mathrm{n}$, Index Fungorum.
Puccinia Spergulae. - Kl. 7S5. a. - Kl. II.

354. - Fuck. rh. 365.

- nodosae Iasch. - Kl. 759. b.

- Stellariae Duby (c. var.). - Kl. II. S6. -

Kl. S55. - Kl. Cent. X, Suppl. (v. gramineae).

— Fuck. s. 62. - W Wstd. 1271. - Ital. 499. -

Fuck, rh. 363 .

- - mediae. - Kl. 1186.

_- nemorum. - Kil. 1186.

- Stellatarum Dub. = clavuligera v. Galior.

W. ex pte. - ? Wstd. 5S4.

- Straminis. - Fuck. Nass, f, 2. - Fuck. s.

11. - Fuck. rh. 321 (c. Ured. Rubig. ver.).

striacformis. - Wstd. 1077.

- Striola. - [S.513.] - Dsm.407. - Kl. 165.

— Watd. 2s!. 1272.

- syngenescarum. - [S. 513.]

- 'Tanaceti. - Wstd. \$22. - Kühn, Kŕrankht.

t. 5. f. 31-36. - Fuck. Nass. f. 4. 5. - Fuck.

s. 53. - Ital. 795. - Fuck. rh. 341. (Caeoma placum Bon.).

- tenuis. - Bon. Con. t. 2. f. 40. p. 219.

- Teucrii. - Bivona t. 4. f. 2. - Fuck. rh. 333.331.

- Thalictri Cher. - Dsm. 409.

'Thesii Chaill. - Dsm. S72.

Thlaspeos. - Kl. II. 352.

Tragopogonis Cd. - Kl. 12S9. - Cd. Ic. V.

t. 2. f. 11 .

'Tripolii Wllr. - Iil. 591.

- 'Triptilii. - Cd. Ic. VI. f. 10.

- Ulmariae DC. - [S. 513.] - Bisch. f. 3599.

- 'Triphragm. Ulm. - Brit. f. 343. - Wstd.

1153.

Ulmi. - Iivona t. 3. f. 4.

Umbelliferarum. - [S. 513.] - Kl. 1185.

Fuck. s. 61: variae formae. - Ism. 1274. -

Kil. II. 315. 319 . - Kl. 1173. (Phragm. Bull.). - Brit. f. 221. - Wstd. 415. - Ital. 243. -

Fuck. rh. $353-362$.

—_ f. Conii. - Kḱl. II. 793.

Umbilici. - Brit. f. 329. - Wrstd. 1270.

Vaginalium. - [S. 513.] - Wstd. 420.

Vaillantiac r. pallida. - Wstd. 584. = P.

heterochroa Rob., Galii verni Rbh.; -v. Galii palustris: Wistd. 554.

- Valantiae. - Dsm. 1156. 876. - Kl. 697.

- Valerianac. - R. f. 490. - Ital. 500.

variabilis. - Cd. Anl. t. B. f. 4:5. - Ioud.

f. 1669s. - Wstd. \$52.

- Veratri Niessl. - R. f. 152.

- Verbesinae. - Caral. I. 99.

- Veronicarum. - [S. 513.] - Kl. II. 692. -

Kl. 1292 ; Cent. XIV, Suppl.

- verrucosa. - Wstd. 421. - Bisch. f. 3591.

- Ung. Ex. t. 6. f. 34. - Kl. 471.

- vesiculosa. - Wstd. 5 S0.

_ Vincae Cast. - Kl. 1091. - Kl. II. 197. -

Ital. 891.

Violae DC. - Wstd. 693.

- Violarum Lk. - [S. 513.] - Kl. 473. - Kl.

II. 196. - Fuck. s. 71. - Dsm. 1273. - Brit.

f. 223. - Fuck. rh. 374 .

- Virgaureae. - Wstd. 651. - Dsm. S75. -

Fuck. s. 54. - Iil. II. 19\$9. - Robin v. p. t.

14. f. 13. k. - Lib. 393. (Xyloma V., 1)othid.

Solidag. Virg.). - Fruck. rh. 343 . Xanthii. - Carol. I. 95.

- v. Ambrosiae. - Carol. III. 91.

Pudonia circinans. - ef. Leotia.

Pulveraria. - cf. Creopus.

Pycnopodium. - [S. 457.] 
Pyenopodium lentigerum. - Cd. Anl. t. C. f. 25:3-5. - cf. Pilobolus Oedipus R. f. 352 (zu Pil. Oed. ex Cocms.).

Pyrenium. - [S. 447.]

- lignatile. - Bail S. t. 19.

— lignorum v. vulgare Td. - Wstd. 353.

- terrestre. - [S. $170 \%$.]

Pyrenochaeta nobilis. - Act. 'Turin. 1819. X.

Iec. 5. f. 9. Notar.).

Pyrenochy um. - cf. Dothidea.

Pyrenodermium Bon. - cf. Sphaeria.

Pyrenophora. - [S. 397.]

- inclusa. - [S. 39S.]

- paradoxa. - [S. 39S.]

phaeocomes. - [S. 399. 414*.] - Kil. II.

717. (Sphacria). - (Ceuthospora ph. Rbh.,

Cryptosphaeria capillata Grev. t. 69, Sphaeria

ph. Berk.). - Fuck. s. 511.

Pyrenotrichum. - [s. 41s**. 377.]

- Splitgerberi. - Ann. sc. n. 1943. XX. t. 16.

f. 2. (Nont.).

ronema. - [S. 350.61.$]$

marianum. - [S. 350.] - Nov. Act. 1535.

XVII. I. t. 2\%. (Carus). - Fuck. s. S51. (? Pez. melalomae progeniens). - Cd. Anl. t. F. f. 61: 1-5. - Pay. b. cr. p. 61. f. 251. - R. f. 267.

Habenhorstia. - [S. 410.] - cf. Sphaeria.

- adpressa. - [S. 410.]

clandestina. - [S. 410.]

- personata. - [S. 410.]

- rudis. - [S. 410.]

- Tiliae. - [S. 410.]

Racemell a memorabilis. - Comm. soc. crit. ital. 1561.2 . t. 4. f. 1. (Cesat.).

Racodium. - [S. 521.] - cf. Hypha, Rhacod.

- Aluta P. - Byssus Al. DC.

aterrimum. - Schwabe, Fl. anhalt. II. t. 6 .

f. 15 .

- cellare - [S. 407.] - Kl. 29S. - Wstd. 195. - Fuck. s. 465. (Zasmidium). - Loud. f. 16556. - Bisch. f. 3700 . - Krypt. Bad. 426. Dsm. 1277. - Willkomm, Wunder d. Mikrosk. 15.56. p. S7. f. b. - Letell. 674. 3. - Schwabe,

Fl. anhalt. II. t. 6. f. 14 .

- fodinum. - Letell. 674. 4.

- Res. Fr. - ef. Dendryph. Res.

rubiginosum. - Dsm. 25.

rupestre. - Voges. 400.

Tela. - [S. 40\%.]

vulgare. - [S. 407.]

Xylostroma. - Wstd. 100.

Radulum. - [S. 325.]

_- aterrimum. [S. 329.]

botrytes. - [S. 329.]

fagineum. - [S. 329.]

- laetum. - [S. 329.] - Kl. 1607. - R.f.

21. - Wstd. 1291.

- orbiculare. - [S. 328.] - Cd. Anl. t. G.

f. $74: 5.6$. Bail S. t. 29 .

- palmatum. - Anm. Mg. n. h. 1S42. IX. t.

10. f. 1. (Berk.).

- pendulum. - [S. 325.]

- quercinum. - [S. 32S.]

- tomentosum. - [S. 329.] - Iil. 721. -

Fuck. s. 939.

R a ma ri a fimbriata Holmsk. = Clavaria byssiseda

F1. dan.

Ram ularia. - [s. 493.]

calcea. - Kil. 1691.

didyma. - [S. 19:3.] - Ung. Ex. t. 2. f. 10.
- Kl. 1679. - Fuck. rh. 153. - Fresen. t. 11.

f. $10-1 \%$ 1). $\{9$.

Ramularia dubia. - IIedw. I. t. 4. f. 9. kil. 1552.

- filaris. - Fresen. t. 13. f. 49-51. p. 90.

- macrospora. - Fresen. t. 11. f. $29-32$. p. S4.

— paludosa. - [S. $493^{*}$.]

-_ pulcliella. - Kil. 1751. - Fresen. t. 11. f.

11. 45. p. $91 \%$.

- pusilla. - [S. 493.] - Cd. Anl. t. B. f. 7 :

1. - Caeoma p., Bon. Con. - Ung. Ex. t. 2.

f. 12 .

tenuissima. - [S. 493.]

- Urticae. - Kl. 16\$0. - Fresen. p. \$. t. 11.

f. $33-34$. Oid. fusisp. p. pte.

Raphidospora. - [S. 401.]

- Lacroixii Mt. - Sphaeria herpotricha Fr.

- Ononidis Awd. - R. f. 359 . (Sphaer. fruticum Rob.'.

Ravenelia glanduliformis B. C. - Carol. III. 72.

Reticularia. - [S. 419.] - cf. Iycogala. Bisch. f. $36 \pi 1$.

- alba Sow. = Diderma spumarioides Fl. dan. - Bull. - S. 449.] - cf. Spumaria a.; Purton. - Wstd. $3>1$.

argentea. - Cd. Ic. VI. f. 36.

- atrd. - [S. 149.] - Kl. 174S.

- Carestiana. - R. f. 436.

- carnea. - [S. 449.] - Fl. dan. XI. 1977. 1.

— carnosa. - [S. $449 * *$.]

—- chrysosperma Bull. - Wstd. 436.

_ flavo-fusca. - [S. 449.] Diphtherium f.

- hemisphaerica. - Diderma hem.

Lycoperd. var. 1. Bull. cf. Ret. argentea.

- maxima Fr. - [S. 449.] - Cd. Ic. VI. f. 35.

- Fuck. s. 299

- muscorum. - [S. 449.] - Bisch. f. 3670.

3657. (= Lignidium griseo-flavum).

- olivacea. - [S. $449 *$.]

— plunbea. - [S. 149.] - Fl. dan. XI. 1976. 1.

- rosea. - Act. Turin. 1805. XIV. t. 3. (Cumino).

__ segetum Bull. - Wstd. 334 .

- sinuosa. - [S. 451.$]$

- umbrina Fr. - [S. 449.] - Fl. dan. XI.

1976. 2. - Cd: Anl. t. C. f. 35 : 1. 2. - Berk.

Out. t. 20. f. 8 .

- ungulina. - [S. $449 *$.$] - Fl. dan. XI.$

$197 \% .2$.

- Ustilago Gmel. - Wstd. 334.

___ versicolor. - [S. 449.]

R habdospora Alsines. - Kil. II. 744.

- Oenotherae. - Ital. 351.

- Senecionis. - Ital. 852.

Rhabdos porium diffusum. - Chev. Fl. envir.

Paris. I. t. 2. f. 13 ; t. 20. f. 3. $(=$ Myriocepl.

botryospor. Mont. Fres.).

- disseminans Rbh. - (Ophiobolus diss. Riess.).

K1. II. 530 .

- Urticae. - Kl. II. 745.

Rhap hidospora. - [S. 401.] ef. Sphaeria.

Rhinotrichum. - [S. 501.] - atrum. - Sturm h. 25 -. t. 21. (Preuss.).

- Bloxami. - Ann. Mg. n. h. 1\$51. VII. t. T.

f. 19. p. 177. (Berk. Br.). Opuntia. - Amm. Mg.n.h. 1534. XIII. t. 11 .

f. 13. p. 462 . (B. B.)

- repens. - Sturm h. 25 -. t. 22. (Preuss.). -

Bon. t. S. f. 171. - Kl. 1262. - Fuck. rh. 124. - simplex. - [S. 501.]-Cd. Anl. t. I3. f. 13: 5. 'Thwaitesii. - Ann. Mg. n. h 1551. VII.

t. 6. f. 12. p. 177. (B. B.). 

<smiles>C#CC#C</smiles> 
Rhizina. - [S. 347.] - Mail S. t. 20.

- lacvigata. - [S. 347.] - Bisch. f. 3253.

3115. - IKl. 233. - Kl. II. 27.

- undulata. - [S. 347.] - Tul. F. hyp. t. 21.

f. 16 . - Berk. In. p. 259 . f. 67 . c. - R. f: 39 .

— vaporaria. - [S. 3.7\%.]

- zonata. - Berk. In. p. 2\$9. f. 67. b.

Iihizoctonia. - [S. 479.]

- Brassicarum. - Lib, 210.

centrifuga. - Hypochnus c. Tul.

- Crocorum DC. - Ism. 1301. - Loud.

f. 16319 . - Bisch. f. 3400 .

- Muscorum Fr. - Bisch. f. 3101 - Schwabc

Fl, anhalt. II. t. 6. f. 9. - Lib. 141.

Napacae. - Wstd. 225.

Solani. - Kühn, Irrankh. t. 7. f. 17, ff. violacea.

t. 20. f. 3 .

. Crocorum. - Tul. F. hyp. t. S. f. 1 ; f. Medicaginis. - Tul. F. hyp. t. 20.

f. 4 ; t. 9. - ? Külin, Jírankh. t. 7. f. 3-16. huc: Byssothecium circin. I'uck.

Ill izomorpha. - [S. 3\$2. 520. 521.]; ef. Graplium Iilizom. Mont. (Fructif. ? G. Otth.).

aidaela. - Nov. Act. XI. t. 17.

angulosa. - [S. 3\2.]

capillaris Ach. - zu 'l'hcleph, sulphurea Fr.

corrugata. - [S. 352.] - Wstd. 125.

corticata. - [S. $464^{*,}$.] - Wstd. 1254.

dichotoma Sow. - Wstd. 1255.

divergens Grev. - Loud. f. 16563. - Schnzl.

t. 12. f. $36-35$.

fragilis. - [S. 352.] - Linn. 1843. XIII.

t. 16. 17. (Schmitz.). - Wstd. 1251.

- Bull. f. subcorticalis: Fuck. s, 697. -

cf. R. scandens.

Friesii. - Wstd. 1254 .

fusca. - Voges, $55 \mathrm{~S}$.

guyanensis. - $[\mathrm{S} .35 \%$ *.]

Harrimanni P. - Wstd. 1254.

hippotriclioides, - [S. 382.]

medullaris. - Linn. 'Trans. XII. t. 20.

(Smitl).

- muralis DC. - Wstd. 595.

— parallela (Iridis). - Dsm. III, 155.

_- putealis $\mathrm{P}$. - Wstd. 1255.

- Sambuci. - Wstd. 151. - Dsm. 701.

- seandens. - Letell. 650. 4. (fragilis $\varkappa_{\text {. }}$ ) -

Wstd. 1254.

- setiformis. - Letell. 650, 2. (Ceratonema

lippotr.). - Wstd. 545.

- simplex. - [S. 352. 7.]

- spinosa. - [S. 352.] - Wstd. 1255.

- subcorticalis P. - [S.352.] - Loud. f. 16562.

- Dsm. 951. - Kl. 229. 299. - Tul, Sel. I.

126-125. - Voges: 759: - Carol. III. 46. -

Letell. 6S0. 3. (fragilis $\beta$.). - Purton. I. t. 29. -

IVstd. 1254 .

-_ v. taen. c. Hypoxyl.: Nov. Act. 1561.

XXVIII. t. 1.f. 1. ff. (Bail).

subterranca $\mathrm{P}$. - [S. 3 2.] - Fl. dan. XI.

1919. 1. - Bisch. f. 3795. - Kl.300. - Xy-

lar. Iyypox. (Tul. Sel. I. 126.) - Wstd. 1255.

Tilletii. - Dsin. III. 156.

- trichophora. - Dsm. 1095. - Wstd. 549.

lihizopogon. - [S. 374. 435.437.]

albus. - [S. 436.]

- Cd. Ic. V. t. 5. f. 14. - Ns.j. t. 12, = Chae: romyecs meandriformis (ef. Bail S. p. 11:).

- Fr.: - (Tuber. Magn. Borchii) Sturm h. 19 - t. 14. (Cd.). - ef. Choiromyc. gangliod. Cd. - ef. rubescens.

Rhizopogon carneus Cd. - ef. Hydnotria carnea Cd.

- ganglioniformis. - Cd. Ic. VI. f. 112. (Choeromyc. g. Tul. F. hyp.).

Leonis. - Pay. b. er. p. 100. f. 462.

- luteolus. - [S. 435.] — cf. Splanchnomyces

Rbh. Cd. - Krrbh. t. 60. f. 13-15. - Tul. F.

hyp. t. 1. f. 5 ; t. 11. f. 5. - Kl. 420. - Fuck.

s. 323. - Dsm. 1013. - Voges. 1275. - Wstd.

39. - R. f. 570 .

— lutescens. - ef. R. rubescens.

Magnatum. - Cd. Ic. V. t. 5. f. 45. = Choe-

romyc, meandr, Tul. F. hyp. - Cd. Anl. t. D.

f. 46: 16-1s. - Pay. b. er. p. 61. f. 253 .

—_ Cd. (non Pico): Kíl. 735.

meandriformis $\mathrm{Cd}$. - cf. Chocromyc. $\mathrm{m}$.

- melanoxanth. Cd. - cf. Pachyphoeus niel.

'Tul.

— provincialis. - Ital. $350^{\circ}$

- rubescens. - Tul. F. hyp. t. 2. f. 1; t. 11 .

f. 4. - Dsm. III. 263. - Carol. I. 75. (lutescens Vitt., albus Schw.). - ? Sturm h. 11. t. 2.

_- Tulasnei Cd. - cf. IIydnotria 'Tul. Cd. Ic.

virens. - [S. 435.] - Bisch. f. 3657. -

lírbh. t. 60 . f. $16-20$. - ef. Splanchnom. vir. Cd.

Webbii Tul. — cf. Splanchnom. Webbii.

Rhizopus. - [S. 457.9.]

- nigricans, - Cd. Anl. t. C.. f. $23: 16-19 .-$

líl. 1351.

Rhizosporium. - [S. $497 * * *$.]

- Solani Rbh. - Kl. 900

Ithodocephalus. - [S. 489t.]

- candiclus. - Cd. Anl. t. C. f. $21: 1$. 2. -

Pay. b. cr. p. 10. f. 51 .

Iih op a li d i u m. - [S: 476.]

$\mathrm{Rh}$ h palom yces. $-[\mathrm{S} .490 *$. $]$

- candidus, - Ann. Mg. n. h. 1851. VII. t. 5.

f. 3. p. 9S. (Berk. Br.).

- elegans. - Cd. Anl. t. 13. f. 20: 1-3. -

Fres. t. 3.- f. $9-15$. - Bon. t. 9. f. 184. -

lay. b. cr. p. 7ł. f. 335 .

- pallidus, - Ann. Mg. n. h. 1S51. VII. p. 96.

t. 5. f. 2. (Berk. Br.).

Rh ymovis. - cf. Paxillus, Agaricus, Ruthea.

Rly tisma. - [S. 370.] - cf. Xyloma. Melanosorus.

- acerinum. - [S. 371.] - Cd. Anl. t. F. f. 59: 22-24. - ef. Xyloma. - Dsm. 420. Bisch. f. 3515.3519 . - Fuck: s. 719. - Bail S. t. 1S. - K1. 553. - Kl. II. 160. a.; 572. Dsm. III. 699. - Melasmia ac. - Brit. f. 194. - Carol. I. 41. (Aceris criocarpi). - Letell. 629. 2. - Lib: 67. - Wstd. 34. - Ital. 314. lirypt. Bad. 24 s.

Andromedae. - [S. 371.] - Kl. 261. - Kl.

II. 70. - Wstd. 1044. - D sm. 1335. - Sturm

h. 6. t. 16.

Bistortae. - Lib. 65. - Wstd. 1245.

- circumscissum. - [S. 371.]

confluens Fr. - Kil. 675.

corrugatum Fr. - Loud. f. 16473.

Cotini. - Kl. 1953.

Curtisii. - Carol. IV. 26.

decolorans. - Carol. II. 50.

Empetri. - [S. 371.] - Ital. 884.

gyrosum. - [S. 371.]

Heraclei. - Sturm h. 6. t. 15.

hysterioides. - [S. 371. Lophod.]

ilicincola. - Carol. II. 51. 
Rhytisma laciniatum. - [S. 371.]

Linnaeae. - Sturm h. 33 -. t. 12. (Strauss).

maximum. - [S. 371.]

Montiniae. - [S. 371.]

nervale. - [S. 3iI.]

- f. Bartsiac. - Kl. II. 666.

Onobrychis DC. - Fuck. s. 722.

Padi Rbh. - Kl. 761.

Pedicularis, - Kíl. II. 667.

Populi Heer, auf Popul. glandulifera. Mcy.

Palaeont. VIII, 3. t. 26. (1R. Ludwig).

Pseudoplatani. - [S. 37I.]

- punctatum. - [S. 371.] - Kil. 1072. - Kil.

II. 7 10. - Fuck. s. 718 . zu acerinum, status

junior, Fuck.). - Voges. 477. (Xyloma). -

Letell. 629. 1.

quercinum Rud. - Ism. 1337.

riccioides. - Letell. 629. 4 .

- salicinum. - [S. 371.] - Kl. 172; - it.

Cent. XV. - Kl. II. 159, 一 Fuck. s. 720.-

Dsm. ed. I. 1736. (umb.). - Ital. (13.) 93. -

Schweiz. 15. - Krypt. Bad. 433. - Ism. 419.

- Brit. f. 43. - Letell. 629. 3. (umbonat.). -

Norweg. 150. - Lib. 174. - Wstd. 33. 1241.

- Sedi. - Sturm h. 6.t. 14.

stellare. - [S. 371.$]$

- Ulmariae. - Voges. 865.

- Ulmi. - Palaeont. VIII. t. 8. f. 5. 6. (IR.

Ludwig).

- umbonatum R. - Dsm. 1336. - Fuck.

s. 721 - ef. R. salic. - Wstd. 33 .

— Urticae. - [S. 371.] - Lib. 62.

Vaccinii. - Carol. II. 52.

Riedera. - [S. 35s.]

Ri essia semiophora. - Fres. t. 9. f. 1-5. -

Kl. 1757 .

Robergea unica. - [S. 397.] - Dsm. 1026.

Robillarda. - [S. 415. Pest.

glandicola. - Castagne, Marseille. t. 4.

Roestelia. - [S.510.] - cf. Uredo, Aecidium.

- cancellata. - [S. 510.] - Dsm. S33. - Cd.

Ic. V. t. 3. f. 29. - de Bary Br. t. \&. f. 1. 2. -

Iil. 97. - Fuck. s. 21. - Cd. Anl. t. C. f. 26 :

3. 4. - Lib. 394. - Wstd. 123. - Schweiz. 6.

- Krypt. Bad. 545. - Fuck. rlı. 243.

f. spermatigera. - Kl. II. 285.

cornuta. - [S. 510.] - de Bary Br. t. \&.

f. $3-$ S. - Bon. t. 2. f. 45 . Kl, II. 97 .

Wstd. 742. — Ital. 50. - Fuck, rh. 254. 255.

(Ceratit. c.).

II. 497 .

exanthematica. - cf. Aecidium ex

lacerata. - S. 510.] — Wstd. 556. 1156.

(Mespili). - Fuck. rh. 256. (Ceratit. 1.).

penicillata. - [S. 510.] - Kl. II. iss. =

Aecid. cornut. f. Mali.

Phoenicis. - cf. Grapliola Ph.

Rosellinia Not. - [S. $395 *$.] Sphaeria.

Rota ea flava. - Kil. 1458.

$\mathrm{R} u$ bigo. - cf. Uredo, Erineum. acerina $\mathrm{Lk}$. - Wstd. 769.

- alnea. - Loud. f. 16590. - Wstd. 311.

- - faginea Ns. - Wstd. 1192.

- rosea Lk. - Wstd. 694.

IR ussula. - ef. Agaricus.

$R u t h$ ea. - cf. Paxillus.

Saccidium, - cf. Sacidium.

Saccothecium. - [S. 39S.] - Ann.sc.n. 1834.

I. t. 13 .

Corni Fr. - Wstd. 1108.
Sacharom y ces cerevisiae. - Meyen Syst. Pfl.

Phys. t. X. f. 22. - ef. Torula cer. Cd., Hefe (Oberhefe).

S a cidium. - [S. 420.]; cf. Coniothyrium. - alpestre. - Kl. 1952. b. - Pemphid. alp. Bail.

- Chenopodii. - Cd. Anl. t. F. f. 51: 1-5.

Desmazieri. - Dsm. III. 351.

foedans Ces, - Kl. II. 61.

microsporum. - [S. 420.]

- Pini Fr. - [S. 420.] - Fuck. s. 403.

umbilicatum. - [S. 420.]

versicolor. - Dsm. III. 52.

Sackea. - [S. 442.] of. Borista.

s. a ro leg $n$ i a capitulifera $\Lambda$. Br. ct ferax $=\Lambda$ clılya prolifera Ns. (f. Bail). - cf. Algae.

ferax u. capitulifera A. Br. = Achlya pro-

lif. Bail).

Sa rcopodium. - [S, 471.521**.] - cf. Tricholeconium roseum $\mathrm{K}]$.

- aterrimum. - Sturn h. 9. t. 61.

—- avenaceum. - [S. 472.]

- circinans. - [S. 472.]

- flavum. - [S. 4i2.]

- fuscum. - [S. 472.

— roseum. - [S. 472.]

- Vaccinii. - S. 466. Pod.

$\mathrm{S}$ a rea. - [S. 362.]

- difformis. - [S. 362.]

- filispora. - cf. Leptosporium tremellinum.

-- pithya. - ef. Peziza pithya.

Sartvelli a foveolata. - Berk. In. p.318. f. 72. a.

Sat yrinus. - [S. 434.]

Satyrus. - [S. $\left.431^{* *}.\right]$

Sceptromyces. - [S. $490^{* *}$.]

Opizii. - Cd. Anl. t. B. f. 16:10-13. -

ef. Cephalosporium Seeptr. - Sturm h. 11. t. 4. Schinzia. - [S. 497.]

- cellulicola. - Linn. 1842. XVI. t. 11. f. 2-10; u. Ann. se.n. 15.13. XIX. t. 3. f. 2-10. Nägeli)

- Iridis. - [S. 497. 3.]

—- penicillata. - Linn. 1S 42 . XVI. t. 11. f. 15

-21 ; u. Ann. sc. n. 1\$43. XIX. t. 3. f. $15-22$.

Nägeli).

Schismaturus. - cf. Lysurus.

$\mathrm{Schizocephalum}$ atrofuscum. - Kl. 1 iS5.

Schizoderma. - [S. 4S2.516**.]; cf. Coniothecium.

- betulinum Fr. - [S. 4\$2.] - R. f. 179.

- fagineum Chev. - cf. Psilospora fag. -

Wrstd. 921.

—_ filicin. - ef. Leptostroma.

- hypodermioides. - [S. 4S2.]

- Pinastri Fr. - [S. 4S2.] - Tharand. Jahrb.

1557. p. $15 \%$. u. 170 . c. ic. (Willkomm). - Kil.

II. 495 .

- - salicinum. - [S. 4S2.]

— scirpinum Ehrb. - Wstd. 1245.

sulcigenum Dub. - cf. Schizod. Pinastri Willk.

Schizoph yllum. - [S. 315.]

- conmune. - [S. 315.] - Ann. sc. n. 1537

VIII. t. \&. f. 13. (Fructif. Lév.) - Bot. Zt.

1860. t. 13. f. 1. (Anat. Hoffn.). - Cd. Anl.

t. If. f. $77: 1-4 .-$ Fl. dan. XIII. 2270. 1. -

Bisch. f. 3416.3440 . - Dsm. 252. - Loud.

f. 16060 . - Pay. b. cr. p. 117. f. 549. - Bail

S. t. 32. - Nov. Act. 1512. XIX. II. t. 57.

f. 5.5. S6. (Spor. Lamell. Phöb.). - Kl. 221. -

R. f. 206. - Voges. 195. - Carol. I. 6. - Ital.

331. - Krypt. Bad. 560. 


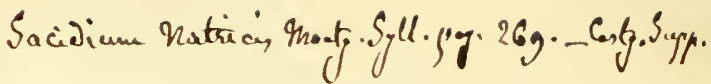

Bobugia albifrom 6al. Sal, fyy, ii, p. 258, $=59$. 


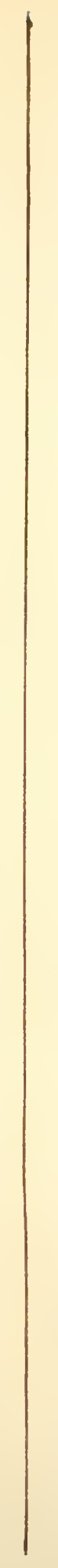


Schizophyllum flabellare. - Afzel. t. 11. f. 25. - umbrinum Berk. - Hook. J. 1S51. III. t. 1. f. 1 .

Schizopogon luteolus. - Kil. II. 320.

Schizostoma. - [S. 411.]

—- fimbr. Lév, - cf. Tulostoma fimbr.

Schizothecium. - [S. 41S.]

— fimicolum. - Cd. Anl. t.F. f. 60: 7-10. Bail S. t. 16. - Kl. II. 257. cf, Sordaria fimiseda.

- - Not. - Ǩl. II. 259.

Schizothyrium acerinum. - Dsm. III. 560.

- Ptarmicae. - Wstd. 375.

Schizoxylon sepincola. - Dénid. Russ. t. 1. f. 6. - Dsm. III. 129. - Voges. 174. - Alger. t. 27.4 .

Schmitzomia. - [S. 363.] - cf. Stictis.

Schweinizia. - [S. 361. 4 10.]

Sciniatosporium. - [S. $402^{* * *}$.

Sclerangium. - [S. 411, ] f. polyrhizon. - (Lév.). Ann. sc. n. 1S15. XI. t. 7.

Selerococeum. - [S.450.]

- sphacrale Fr. - [S. 450.] Kí. 95९.

Scleroderma. - [S. 44.] - Amn. se. n. 1412.

XVII. t. 1. 2. (Fructif, 'Tul.) - Flora. 1513.

I. t. 2. ('Tul.).

- album. - Carol. IV. 76.

— Bovista. - [S. 144.]

- Cepa Grev. - Loud. f. 16501.

- cervinum I'. - Elaphomye. granul, Fl. dan. citrinum. - Cd. Anl. t. C. f. 3५: ง. 9. -

Lond. f. 16505. - cf. S. vulgare var.

- Geaster. - Act. Turim. Is43. V. t. 3. f. 14.

(Vittad.). - Bisch. f. 3675 .

- Fr. - ef. Sclerang. polyrhiz I Lév.

- sinnamariense. - [S. 414*] - Ann, sc. n.

1S40. XIV.t. 19. f. 12. (Mont.).

— spadiceum Grev. - Loud. f. 16506.

- verrucosum. - [S. 44.] - Loud. f. 16503.

(cf. S. vulgare). - Fuck. s. 301 . (p. pte.). -

Cd. Anl. t. C. f. 35: 6. 7. - IIuss. I. 17.

- vulgare. - [S. 44.] - Kops Fl. bat. IX.

t. 720.1. - Fuck, s, 303. - Schnzl. t. 14 . f. $\$ 6$ -15. - Tul. F. hyp. t. 21.f. T. - Pay. b. cr. p.111. f. 506. - K1. 174. - R. f. 213. - Vent. mi. 10. f. 6 ; 61 . f. $6.7 ; 71$. f. $1.2 ; 76$. f. 5.6. — - v. squamosa Fr. - Fl. dan. XI. 1969. ‥ —— v. verrucosa P. - Kl. 419.

scleroglossuin conicun. - Voges. 672.

- lanceolatum. - Clavaria herbarum.

Selerographiu m aterrimum. - Hook. J. 1\$54.

VI. t. S. f. 4.

Scleromitra. - [S. $521^{* *}$.]

— cincreo-alba. - Bon. t. 10. f. 213.

- coccinea. - Sturm h. 7 . t. 27 .

- micans. - Cd. Anl. t. G. f. 72 : 4-\$. -

Typhula m. Fr. (Bail).

Sclerotium. - [S. 47..$]$; cf. Claviceps, Cordyceps, Sphaeria, Spermoedia, Mrelampsora. - Tul.

Sel. I. - Aegerita. [S. 47\%.]

- albidum. - Dsm. III. 567.

__ anthodiophilum. - Kl. 1550.

- areolatum. - [S. 452.] - Fuck. s. 213. -

Voges. S\$3. - Lib. 239.

- aurantiacum Lasch. - Kk1. 1234.

- aurantio-fuscum Rbh. - Kl. 936.

Betuli. - Lib. 336. - Wstd. 545 .

betulinum Fr. - cf. Melampsora bet. D. -

letell. 636. 2. - Wstd. 279.

- cum Botrytide erythropode. - Ann. sc. $n$.

1S43. XX. t. 7. f. 5. (Lév.).
Sclerotium Brassicae P. - [S. 479.]

- f. Georginae Lasch. - Kl. 1233.

— bullatum IC. - Kil. S32. - Wstd. 732. -

$\mathrm{zu}$ varium $\mathrm{P}$. (Coems.).

— carneolum. - Dsm. III. 162.

- carneum. - [S. 47S.]

- Castaneae. - Wstd. 739.

- Cepac. - Brit. f. 276. - cf. MIucor subtil.

- Lib. 235.

— cepivorum. - [S. 47९.] - Dsm. III. 569.

- Circacae. - [S. 479.]

- Clavus. - [S. $479^{* *}$. 519.] - Lib. 142. -

ef. Sphacclia segetum, Claviceps purpurea. -

Phöb. Gift. t. 9. f. 3-52. - Berk. Out. t. 23.

f. 7. - loud. f. 1 (i321. - Fuck. s. 209. a. c-1.

- Kolnczek, Lehrb. d. Bot. 1556. (5.) p. 430.

K1. 56. - Dsm. 5s1. - Voges. 1059. - Ökonom. Neuigk, v. Hlubeck. 1s 46 . t. 3. f. $21-30$.

— Wstd. 327. 379: v. Enodii. - Schweiz. 17.

- - conidia. - Linn. Trans. 1\$3\$. XVIII.

t. 32. 33. (F. Bauer). - Démid. Russ. t. 6. f. 7.

- Limn. Trans. XV1II. t. 33. B. p. 160.471 .

(Quckett). - Jot. Zt. 1558. t. 4. 13. (Bonord.),

- f. Clavicipitis purp. - Fl. dan. X.

t. 1791. f. 1. (Sphaer.). - Ann. sc. 1n. 1953.

XX. t. 4. f. $23-29$. (Tul.) - cf. Claviecps p.

__ - v. Agrostidis. - Kl. 1791. g.

_- v. Nlopecuri bulbosi.-Dsm. III. 167.C.

— v. Ammophilae. - Kl.1791.c. - Dsm.

III. 166. B.

- v. Arundinis. $-\mathrm{K} 1.1117$.
- v. Avenae. - Dsm. 1625.
- v. Baldingerac. $-\mathrm{K} 1.1791 . \mathrm{d}$.
167.13.

- v. Bromi gigantei. - Dsm. III, 166. C.

v. Calamagrostidis. - Dsm. III. 166. a.

167 .

III. 16.5 .

v. Glyceriae. - Kl. 1791. a. - Dsm.

f. Holei mollis. - Kl. 1791. e.

f. Lolii. - Kl. 1791. f. - Wstd. 1256.

f. Moniliae. - Kl. 1148.

- f. Phragmit. comm. - Ann. sc. 11.

19.53. XX. t. 4. f. $12-11$. ('Tul.).

— f. Poae. - Kil. $111 \mathrm{~s}$.

- f. Seirpi. - Kil. 935.

- f. (Secalis). - Pay. b. cr. p. 5९. f. 245. f. Tritici juncei. - Dsm. III. 161.

compactum. - [S. 479.] - Dsin. 1637-

1639. - Lib. 34. - Fuck. s. 216. - ef. Teziza

Sclerotii Fuck. - zu S, varium P. (Coems.).

—- Althaeae. - Wstd. 1257.

R. f. $5 \$ 6$.

Helianthi DC. - Bisch. f. 34 S1.34S2.-

- Zeac. - Wstd. 1257.

- complanatum Tode. - [S. 475.] - Bisch.

f. 3397 . - Dsm. 574. - Kl. 357. - Fuck. s. 220 . - vix Schnzl. t. 14. f. 12. - Brit. f. 72 . - Wstd. 37s.

- huc: Typh. phac.: R. f. 41S. (Wstd.) et ?'Typh. gyrans (Bail S. 76.); -- Clavaria juncea (Tul.).

- c. Clavaria juncea, - Ann. se.n. 1543.

xX. t. 7. f. 1. (Lév.).

f. Cucurbitae. - Kl. 428.

_. Helianthi. - K1. 1030.

concavum. - Ann. sc. n. 1837. VIII. t. 2.

f. 6. - Dsm. S1. 
Selerotium Convallariae. - Kil. 1552. cornutum. - Acrospermum corn.

- crustuliforme Rob. - Dsm. 1646. - zu Ty-

phula crytliropus P. (Bail S. p. 76 ; Tul.). - dorsale. - Dsm. 1611.

- durum P. - [S. 479.]-I.oud. f. 16315. -

Fuck. s. 210. - Bail S. t. 15. - Iil. 150. -

Dsm. 577. - Voges. 4\$9. - Brit. f. 74. - Lib.

35. - Wstd. 453. 739. Zeac, echinophila, Scolymi, Hippocast.'.

- - echinophilum. - Dsm. 1610.

echinatum. - Fuck. s. 215.

clongatum (liev. $-\mathrm{zu}$ varium $\mathbf{P}$. (Coems.).

emergens. - S. 47s.]

epixylon. - [S. 476.]

- Erysiphe. - ef. Erysiphe Coryli. - Wstd.

(1) $\%$. 405.113.

- Erythrinae. - Lotos 1557 . VII. t. 1.

Euphorbiac. - Wstd. 973.

eurotioides. - Lib. 13૬.

Evonymi. - Dsm、1613.

excentricum. - $\left[\mathrm{S} .47 \mathrm{G}_{.}\right]$

fibrillosum. - Lib. 139.

figuratum. - [S. 479.]

Hoccipendulum. - [.5. 175.]

- fructuum. - Wstd. 735 .

fulvum. - [S. 475.

- fungorum. - S.. $^{-}$i8.] - c. Clavaria mi-

nore. - Ann. sc. л. 1S13. XX. t. 7. f. 2. (Lév.). glauco-albuns. - Dsm. 1634 .

Graminum. - Dsm. 1615.

granulatum. - Lotos 1557.-VII, t. 2.

- herbarum Fr. - [S. 4\$2.] - Kl. 430. -

Voges. 990. - MeIarnpsora h. Dsm.

immersum. - is. 475.

inclusum S. K. - S. 395.] - Ki1. 102S. -

Trstd. 545 .

— f. pyricola Lasch. - Kl. 1029.

juucinum. - Dsm. 163‥ 1633.

- lacrymabundum Chev. - cf. varium.

lacunosum P. - $\mathrm{zu}$ Peziz: tuberosa Bull.

(Bail s. p. 76.).

— leiodermum. - Dsm. III. 56s. - Wstd. 1356.

- Liliacearum. - Wstd. 826. SScill. et Ama-

ryll.)

Linnaeae. - [S. 179.]

Lycopodii: - [S. 479.]

maculare. - [S. 4iৎ.] - Dsm. 1631.

545.

- medullare - [S. iis.] - Brit. f. 75. -

Mag. Zool. Bot. 183i. I. t. 3. f.5. p. 47. (IBerk.\}.

- medullosum Dsm. - zu durum ? (Tul.). -

1) $\mathrm{m}$. III. 160 .

- minutum. - Dsm. S0. 5is. (zu varium $\mathrm{P}$.

l.év. ['Tul.]). - Voges. 1192.

- imuscorum. - S. $47 \subseteq .479 \cdots \cdots$. Grev.

sc. t. 101. - Loud. f. 16309. - R. f. 370 -

Ann. sc. n. 15t3. XX. t. 6. f. 4. (Lév:) - Kil. b3:3.

- mycetosporum. - Iib. 334. - Kll. 425. -

Nov. Act. 1\$32. XTI..I. t. 5. (Ns.).

- neglectum. - [S. 175.] - Dsm: 1611. -

Brit, f. 165 .

nervale Tod. - Bisch. f. 339S. - Kl. 1551.

- A. S. - Iíl. $\leqq 31$.

nervorum. - Schnzl. t. 14. f. 13-16.

nitidum. - Voges. 674. (Leptostroma vul-

gare Fr. . - Wstd. 1125.

nudum. - [S.4is.]

occultum. - Hoffm. Ic. t. 16. f. 3.

olivaceum. - [S. 475.]
Sclerotium Orobanches. - [S. 179.]

142.

palliolatum. - [S. 47

Paspali. - Carol. II. 100.

perpusillum. - Iil. 1320.

- persicinum. - [S. 361.]

- pezizaeforme. - [\$. 47×.] - Lib. 140.

- populinum P. - [S. 152.] - Dsm. 550. -

Iil. 424. - Perisporium pop. Wllr.

- - Rbh. - cf. Melampsora pop. - Letell.

6317. 1. - Wstd. 7\%.

- populneum. - Voges. 355.

_- protuberans. - [\$. 4 79.]

- Iteridis Moug. = I.eptostroma litigosum

Dsm. - Voges. 672. (Icptost. filicinum Fr.). -

Wistd. 76.150.

- - pubescens. - [S. 475.] - Jil. 147...-Fuck.

s. 221 .

- Punctum. - Lib. 37. - W'std. 659.

— Pustula. - [S. 479.] - Dsm. 579. 1612. -

Wstd. 731. 1255. (petiolor.).

_- cum Peziza Candolleana. - Ann. sc.

n. 1s43. XX. t. 7. f. 4. (Lév.) - Fuck. s. 214.

- Letell. 636. 4 .

- DC. : zu Typhula sclerotioides (Bail S.

p. 76).

- pyrinum. - [S. 479.] - Brit. f. 166. -

Wstd. 735.

- quercinum P. - Voges. 575. - Loud. f.

16317. - Wstd. 734. 919.

_- radicatum 'Tod. - [S. 445. Anix.] - Bisch. f. 3395 .

- Renovii. - Dsm. III. 566.

- rhizoctonioides. - [S. 47. $]$

- rhizodes Awd. (Sarcidium). - Kl. 1232. -

I) sin. III. 5 ; 1.

- Rhizomorpha. - [S. $179 * *$. ]

__ rimosum Lasch.- Kil. 1149.

__ roseum. - [S. 47s.] — Kl. 356. - zu Pe-

ziza Curreyana (Journ. Proc. Linn. Soc. 1557.1.).

- Voges. Sst.

_.. Rubi. - [S. 479.]

- rugulosum. - [S. 4is.]

— salicinum. - S. 452.] - K1. 1176.

- - DC.- cf. Melampsora salicina_-Fuck.

s. 25. d. - Dsm. 953. - Voges. 356. - Brit.

f. 25 - Letell. 636. 3. - W'std. 736 .

sanguineum. - [S. 478.]

- scutellatum. - [S. 47..]. - Loud. f. 16313.

- Dsm. 1630. - Brit. f. 27.

- Selaginellae. - Lotos 155\%. VII. t. 2.

- Semen Tode. - [S. 475.$]-$ Dsm. 57. -

Bisch. f. 3394 . - Loud. f. 16307. $16307, \beta .-$

Iil. 426. - Kl. H. 507 - Fuck. s. 219. - c.

Tul. Sel. I. 11S. 2. - Wstd. TS.

- - v. Brassicae. - ? zu Typhula variabi-

lis Riess (Bail s. p. 75.).- Brit. f. 73.

- f. depressa. - K1. 1031.

Semini affine. - Ann. sc. n. 1S43. XX. t. (i.

f. 3. (Lév.).

- speireum. - [S.-479. 519.]

- spermoides. - [S. 47 . $]$

sphaeriaeforme Lib. - zu varium (Coems.).-

Lib. $23 \%$ - Wstd. $54 \%$.

_- sphaeroides. - [S. 419.]

- stercorarium. - [S. 475.] - Dsm. 576.-

lil. 1790. - Fuck. s. 215 .

DC. - zu Peziza tuberosa Bull. (Bail

s. p. i6.5.

- stipatum. - [S. 475.]

strobilinum: $-[$ S. $4 ; 8$. 


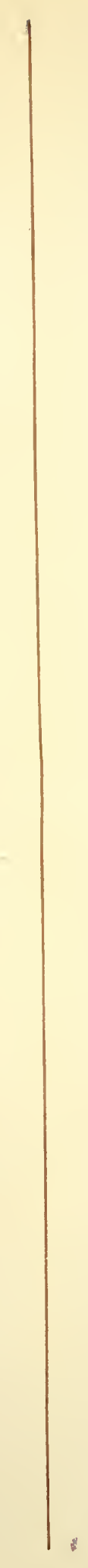



Sclerotium subterraneum a 'T. - cf. S. muscorum.

- trunc. - zu Agar. cirrat. P. Tul. . succineum. - Dsm. III. 163.

sulcatum Dsm. - zu Pez. Duriaeana (Tul.).

- Dsm. 1629.

- tectum Fr. - [S. 17s.] - Kl. 429, - Fuck. s. 212. - Lib. 335. - Wstd. 152.

— Tiliae Lk. - Kl. 575.

tomentosum Td. - Wstd. 43.

'Trientalis. - [S. 179.]

truncorum, - S. 47. $479 \cdots \cdots$

'T'ulipae. - Lib. 36. - Kl. 1321 .

umbilicatum Lasch. - Iil. 531.

- Rob. - Dsm. 1 (iig.5.

vaporarium. - [S. Ais.]

- varium. - [S. 179.] - Schnzl.t. 1.1. f. 11. -

Iil. 11\% - Fuck. s. 211. - Dsm. 7!) 16i36;

III. 570. - zu Pez. Sclęerotiorun Münter in

lit.t. - huc lacrymab. Chev, et Pcz. Sclelotior.

I.ib. Tul.). - Foges. 9\9. - Wstd. 1-2. 7i;1.

- velutinum. - Wstd. 516. - Bull. ac. belg.

1. Sér. XVIII. p. 117. f. 5. Wstd.)

vestitum. - Norweg. 163. (Acinula candicans Fr.).

- Vitis. - Lotos. 1557. VII. t. 2. -- Kil. 1319.

- vulgatum. - [S. 4\%.]

Scoleciocarpus. - [S. 113.]

Scolicotrichum. - S. 503.?

Graminis. - Fuck. rl. 130.

olivaceum. - Kil. 1675.

smaragdinum, - Bon. t. S. f. 176 .

tomentosum Bon. - Bot. '/t. 1433. t. T. f. 12.

venosum Bon. - R. f. 552 . (ć. ic.).

virescens. - Cd. Anl. t. B. f. 9: 3. - Bon.

t. 4. f. 93 .

Scopularia venusta Preuss. - Sturm h. 36. t. 4.

Scopulina. - [S. 396.]

Seorias. - [S. 466.]

spongiosa. - Carol. I. S1. (Batrytis? spongiosa). - Journ. hort. soc. 1549. IV. p. 25\%. 1. (B. D.).

Scorzonera pulverifera Mor. - Ustilago recept. Scorz.

Secale cornutum et luxurians. - cf. Sclerot. clav.

Secotium. - [S. 440.]

- acuminatum. - Alger. t. 22, bis. 1 .

- Basserrianum. - Alger. t. 22, bis. 5.

- coarctatum. - Hook. Lond. J. 1S45. IV.

t. 1. f. 3 .

- Berk. - Cd. Ic. VI. t. 6. f. $25-30$.

- erythrocephalum. - Ann. sc. n. 1545. IV.

t. 9. f. 5-17. Tul.) - Pay. b. cr. p. b. f. 33 ; p. 115 . f. 536 .

- Gueinzii. - Hook. Lond. J. 1543. II. t. 5.

- Cd. Ic. VI. t. 6. f. $10-1$ ๖.

- melanospermum. - Hook. Lond. J. $1 \$ 45$.

IV. t. 1. f. 2.

- melanosporum Berk. - Cd. Ic. VI. t. 6 .

f. $19-24$.

(Tul.).

- Queintzii. - cf. Gueinzii.

Seimatósporium. - [S. $\left.402^{* *+} .171^{* *}.\right]$

- Rosae. - Sturm h. 13. t. 40.

Seiridium. - [S. $401^{* *} .50 \%$. ; cf, Siridium, Coryneum.

- marginatum. - [S. $102 *+4.4 \% *$.

Selenosporium. - [S. 173*.] - cf. Fusisporium.
Selenosporium Asperifoliarum. - Bull. ac. belg. 2. Sér. XI. p. 6ibo. f. 2. (Wstd.).

- fuscum. - Bon. t. 10. f. 220. herbarum. - Cd. Anl. t. G. f $69: 1-3$.

Pay. b. cr. p. 76. f. 359 .

- lateritium. - Dsm. III. 796.

- minutissimum. - Dsm. III. 456 .

pallens. - Wstd. 336.

- pyrochroum. - Dsm. 1547; III. 22.

- sarcochroum Dsm. - Wistd. 655.

- tubereularioides. - Bon. t. 11. f. 230.

—- Urticarum Cd. - Ii.f. 3५4. - Dsm. III. 659.

Sepedonium. - [S. 497.] ; cf. Mycobanche.

- agglomeriferum. - Letell. 667.4.

- angulatum $\mathrm{Kl}$. = Nyctalis asterophora Fr.

caseorum. - Wstel. 300.

- cervinum. - [S. 4yT:] - Bisch. f. 3507.

- chrysospermum. - S. 297.$]$ - Bisch. f. 3506 .

- Bon.t. 4. f. 103 . - Ioffin. Ic. t. S. f. 1.

fuscum. - Bon. t. 4. f. 106.

- mycophilum. - [S. 497 . myc.] - Berk. In.

p. 295. f. 6s. d. - Fuck. s. 1-3. - Loud.

f. 16567 . Dsm. 103. - Schnzl. t. 12. f. 39 .

40. - Kl. 66 ; Cent. X., Suppl. - Kl. II. 1 s.

- Wstd. 436. - Fuck, rh. 111.

- osteophilum. - Bon. t. 5. f. 109.

roseum. - [S. $49 \%$.]

Septaria. - cf. Septoria.

Septocolla adpressa. - Jon. t. 12. f. 247.

Septonema. - [S. 504.]

— alba. - Sturm. h. 29 -. t. 37. (Preuss.).

elongatispora. - Sturm h. 29 -. t. 36.

Preuss.)

- irregulare B. B. - Anm. Mgg. n. h. 1561.

VII. t. 15. f. 13 . p. 351 .

radicans. - Carol. II. 5 i.

- secedens. - [S. 504.] - Kl. $19 \$ 1$.

- spilomeuin. - Hook. Lond. J. 1515. IV.

t. 12. f. 5. - Carol. IV. Sт.

- strictum. - Cd. Ic. VI. f. 22

-.. - virescens. - [S. 5t4.]

- - viride. - Cd. Anl. t. B. f. 6: 3. - Pay. b. cr. p. 69 . f. 302 .

Septoria. - [S. 426.] ; ef. Ascochyta, Spilosphaeria, Sphaeria, Depazea. Aceris. - Kl. 1560. - Fuck rl. 499.

- Berk. Br.: (S. incondita v. acericol.

Ds., Ascochyta Ac. Lib.) : Iil. II. 565. - Fuck. s. 329 .

- Aegopodii. - Dsm. 143. - Wstd. 95.

- Aesculi. - R. f. 550 .

aesculicola. - Wstd. 1394.

- Anemones. - [S. 126.] - Fuck. s. 326. -

Dsm. 240. - Wstd. 615.

Antirrhini. - Dsm. 1525.

Ari. - Dsm. 130 T.

Astragali Rob. - Dsm. 737 .

- Badhami. - Ann. Mg. n. h. 1854. XIII.

p. 460. t. 15. f. 9. (B. B.).

- bellidicola. - Dsm. 1S33.

- Bellidis. - Dsm. 1532.

Betae. - Wstd. 296.

- Brassicae. - Wstd. 294.

- Bupleuri. - Dsm. 131\%. - Wstd. 1144.

- Calamagrostidis. - Wstd. 1055.

Calystegia. - Wstd. 612. = Polystigma pertusarioides Dsm.

- Capreae IVstd. - Depazea salicicola Fr. Rb.

- Carthusianorum. - WVstd. 939.

Castaneae Lér. - Wstd. 725.

castaneaecola. - Dsm. 1332. - K1.1957. -

Wstd. $i 25$. 
Septoria caulinm Matth. - Wstd. 1123.

Cerastii Rob. - Dsm. 1321. - Wstcl. 916. Cheiranthi Rob. - Ism. 1321. - Wstd. $1: 379$.

Chelidonii. - Wstd. 424. - Kil. II. 552. -

Dism. 6i6.

- Chenopodii. - W'std. 6.13.

- Clematidis. - Jsm. 1536.

- Convolvuli. - Dsm. 6i2. 1527. - Kl. Isfi2.

— Wstel. 295.

— cruciata Rob. - Dsm. 1316. - Wstd. 1:341. Cytisi. - Dsm. 1810 .

Daplunes Riob. - Dsm. 729.

- Dianthi. - R.f. 36\%. (Sphaeria 1)., A.S.).Dsm. 1323. - Wstd. 293. 6łli.

951

- Dictamni. - Fuck. s. 330.

_- Dulcamarae. - I)sm. 1s.

- Ebuli Rob. - Dsm. I3is.

effusa. - Dsm. 132 $\%$.

Elacagni. - Dsm. 6\%0.

- Epilobii. - Dsm. 1534. - Wstd. 727.

- Equiseti. - Voges. 1261. - Dsm. 1306. -

Iil. II. 73 ร.

- Eryngii. - Wstd. 13>2.

- Eupatorii. - Dsin. 1531.

- Euphorbiae. - Dsm. 15il. - Voges. 1465.

- Evonymi Rbl. - Kil. 1071.

- Ficariac. - Dsm. 157. - Voges. 1265. -

Wrstd. 233.

- Fragariae. - Dsm. 6s0.

_- Frangulae. - Voges. 1467.

- Fraxini Fr. - Wstd. 952. - Dsm. 156. -

Bisch. f. 3517. Dothidea Fr. S., Sphaeria Echiuns Biv.).

- Galeopsidis. - Wstd. 1151.

- Gei Rob. - Dsm. 733 .

—- Geranii. - Dsm. 15:3\%.

- Grossulariac. - Wstd. 911.

— Hederac. - Wstd. 24. 1343.

II. 555 .

- herbarum. - Wstd. 11.15 (Iolii).

heterochroa. - [S. 426.] - Dsm. 1522. -

Wist. 949. (Lamii). 1351I (Antirrh., J'lantag.).

- lieterotricha Rob. - Dsm. 1320.

- Hippophaes. - Dsm. 1421.

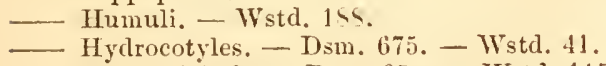

- Hyperici Rob. - Dsm. 67- - Wstd. 1153.

- incondita, - ef. S. Aceris. - Dsm. 1542.

1513. - Wstd. 953 : quercina.

- insularis. - Ann. Mg. n. h. 1554. XIII.

p. 460 . t. 15 . f. S. (Spor. B. B.).

- Junci. - Dsm. 1520. - Wstd. 936.

— Lavandulae. - Dsm.1523. - Wstd. 1149.

- Legunninum Rob. - Dsm. 736. - Wstd.

941. (Scopar.). - R. f. 551.

- Pisorum. - Brit. f. 323.

— Lepidii. - Dsm. 67\%. - Wstd. 726.

- Levistici. - Wstd. 935.

- Lituus. - Ann. Mg. 11. h. 1554. XIII. t. 15.

f. 5. p. 459. (B. B.).

— Lolii. - Wstd. 1149.

- Lychnidis. - K1. 1563.

- Lysimachiae. - Ascochyta L., Lasch. - Kl.

II. 561 . - Wstd. 950 .

— macrospora. - Alger. t. 27 9.

— maculosa. - Toges. I lii!.

- Majanthemi. - Wstd, Yili.
Septoria Medicaginis Rob. - Dsm. 1325.

Melissae. - D:m. 1524.

Menyanthis. - Dsin. 1529 .

Mori. - Hdw. I. t. 5. f. \&.

Lév. : Fuck. s. $32 \%$ - Kühn, Krankh.

t. 5. f. 53. (Keimg.). - Kl. 1861. - ? Berlin. entomol. Zcitschr. 15.35. t. 3. f. 1.1; t. 6. f. 27. 25. (lebert). - Voges. 1466. - Wistd. S24. nebulosa. - Dsm. 731. 1830. - Wstd. 127.

Oenotherae. - Wstd. 1152.

- Orchidearuin. - Dsm. III. 65£. - Wstd. 639.

- Oxyacanthae. - [S. 426.] - K1. 456. - Kl.

11. 563. - Fuck. s. 325. - cf. Ascochyt. Ox.

u. Cratacgi. - Wstd. 40. - Fuck. rlı. 5011.

- Padi Lasch. - Kil. 457. - R. f. 466.

- Paconiae. - Wstd. 95i.

_- I'astinacac Wstd. - R.f. 165. — Wstd. 639.

- P'etroselini. - Wsta. 135. - Dsm. 67t.

- phacidioides. - Wstd. 119. - Dsm. 1319.

jphaerops. cand. B. B.).

- Pisi. - Wstel. 1378.

_. Pistaciae. - Dsm. 6s1.

- Podagrariae Lasch. - Kil. 45ৎ.

Polygonorum Dsm. - Ki. II. 412. - Dsm. fiis. - Wstd. 12.

- Populi. - [S. 426.] - Dsm. 1331. - Kl.

$195 \mathrm{~s} .-\mathrm{Wstd} .500$.

-_- princeps. - Ann. Mg. n. h. 1s61. VII. t. 15.

f. 11. p. 350 . (B. B.).

- Prismatocarpi. - Jsm. 1529.

- Pseudoplatani Rob. - Dsm. 1322. - Wstd. $95 \overline{7}$.

Pyri. - Wstd. 136.

pyricola. - Ism. 17s. quereina. - Dsm. 1330. - Wstd. 644.

Ralfsii. - Ann. Mg. n. h. 1554. XIII. t. 15.

f. 6. p. 459. (Spor. B. 13.).

__ ramcalis. - Dsm. 1539. - Wstd. 934.

- Ranunculacearum Lév. - Démid. Russ. t. 6. f. 4 .

Ranunculi. - Wstd. 937.

- Rhamni cathartici. - Kl. 1956.

- - Rhois Lév. - Démid. Russ, t. 5. f. 4.

-- Ribis. - Voges. 11S3. (Ascochyta). - Dsm. 6i9. - Kl. 991. - Ascochyta R. Lib. - Kl. II. 5!i1. - Fuck. s. 325. - Wstd. 92. - Fuck. rh. 495 .

- Robiniac. - Dsm. 1329. - Wstd. 917.

—_ Rosae. - Wstd. 93; 426. - Dsm. 112.

_. Rubi. - Wstd. 935.

- Ruborum. - W'sta. 934.

rubra. - Wstd. 637 . f. Amygdali. - Dsm. 731 .

salicella. - Ann. Mg n.h. 1851 . XIII. t. 15

f. 7. p. 460 . (Spor. B. B.). Salicis. - Wstd. 611 .

- Saponariae. - Ital. S\$1. (Depazea, Sphaeria, scabiosaecola. - Dsm. 179.

Scandicis. - Dsm. $1 \backslash 34$.

Scillae. - Wstd. 1054.

Scleranthi. - Dsm. III. 65?.

Scrophulariae. - Wstd. 13ৎ5.

Sedi. - Wstcl. 943.

Senecionis. - Wstd. 729.

sepium - Dsm. 1426.

Sii. - Dsm. 1535. - Wstd. 9.12.

Silenes. - Wstd. 955.

Sorbi Lasch. - Kl. 459. - cf. Cryptospirium.

Spartii Rob. - Dsm. 735.

Spergulae. - Wistd. 1155.

Stachydis Rob. - Dsm. 1312. 


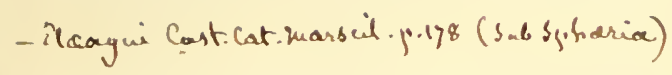

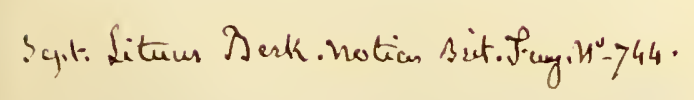

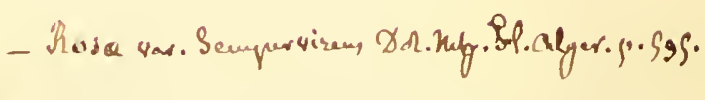

- Scabiosoeslorrym.21 not.g. 12.

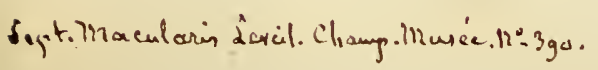




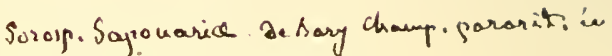

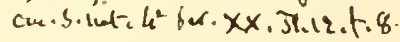


Siep t oria Stellariae Rob. - Dsm. 1325. - Wrstd. 915.

- srlvicola. - Dsm. III. 1990.

Tiliae. - Wistcl. 9.56.

'Tormentillae Rob. - D)sm. 1326. - Wstd.

11.1\%. Potent.1.

- Tritici Rob. - Dsm. 669.

- Tussilaginis. - Wstd. Glo.

- Ulmi. - S. 42(i.] - Cd. Anl. t F. f. 51:

111. 11. - Lond. f. 16161 - K 1.45. - K1. I1.

¿(i2. - c. Pliloeospora W. - Voges. 49:- -

Brit. f. 214. - ef Ascochyta, Lib. - W'ste 1:3i.

- Umbellatarum. - Kil. I'fil.

- Uredinis Rob. - Dsm. 1313.

- Urticac Rob. - Dsm. 1309. - Wstd. f49.

- Verbenae Rob. - Dsm. 1311. - W:td. 1145.

- Veronicae Rob. - Dsm. 1310. - R. f. 361.

-.. Tiburni. - Wstcl. I150.

— Viciae. - Wstd. 1151.

- Tillarsiae. - Dsm. 473. - Wistd. 9.5.

- Vincae, - Dsm. 730.

-_ Vincetoxici. - Wstd. 1110.

- Violac. - Kl. 145\%. - Wstd. 41. Cheila-

ria $V$, ex $D \mathrm{sm}$.

- Virgaureat. - [S. 426.] - J)sm. 1314.

- Vitis Lér. - W'st. 724

Xinthii. - D sin. 1315.

septosporium. - S. 501).]

— atrum. - R. f. 273 ; Sturm I. F1. IIl. 12.

p. 33. t. 17. 'ef. Macrosporium!

- bifurcum. - Fres. t. 6. f. I-ৎ.

- bulbotrichum. - [S. 501.] - C'd. Anl. t. R,

f. 11: Ћ. - Pay. b. cr. p. 73. f. 332

- caricinum, - ef. Macrospor. c.

- curvatum. - Verh. Yer. z. Beförd. des Gartenbaues in 1'reussen. 1 55 1. I. t. 1. A. (Bramm, C'usp., de Bary'. - kil. 1;79.

- Graninis. - Ökonom. Teuigk. v. Illubeck.

1) 16 . t. 3. f. $16-20$.

- instipitatum.- Sturm h. 25- t.12. Preuss.

myrmecophilum. - Fres. t. (6. f. 29-32.

- nitens. - Fres. t. 3. f. 30-30. Form von

C'ladospor.: = Sporidesm. ; Bail .

Septotrichum. - [S. 519.] - pili degenerati. - cf. Erineum, Phyllerium. - Cd. Ic. IV.

Sim b 1 u m. - s.. 131 .

- gracile. - Hook. Lond. J. 15.16. F. t. 17.

f. 1. Berk.'.

- periphragmaticum. - Cd. Anl. t. E. f. 51:

12. 13. - Bail S. t. 23.

- sphaerocephalum S-1. - Limn. 1s61. XXXI. t. 1.p. 154 .

Siridium marginatum. - Bon. t. 4. f. sऽ. -

Cd. Anl. t. 13. f. 5: 1-3. cf. Seiridium.

Sirodesmi um. - affine Phragmotricho.

- granulosum. - Kil. 1962. - Act. Tur. 1519.

X. Dec. 5. f. 10. Notar., - Ital. 45.

Sistotrema. - [S. 32\}.

carneum Bon, - Bot. Zt. 1557. t. 5. B. cinereum Schum: = Daedalea unieol. F1. dan. — P.: Wistd. 12S5.

confluens P. - [S. 325.] -. Hydnum sub-

lamellosum Bull. - Cd. Anl. t. G. f. it: 2(i-2?.

- Fuck. s. 941. - Loud. f. 16125. - Bail S.

t. 29 . - K1. $111 \%$ - R. f. 310 , a. b.

fimbriatum P. - Odontia f. Fr.

occarium. - Cd. Anl. t. G. f. 7. : 23.

pes Caprae. - Pay. b. cr. p. 107. f. 4hs.

rufescens. - Letell. 645.

spongiosum Schwtz.-Polypor. labyrinth. Fr.

violaceum P. - Voges. 6:5.

Hof $\mathrm{fm}$ ann, Index Fungorum.
Skepperia convoluta. - Limn. 'Irans. 1>59.

XXII. t. 25. lierk.

Soleciocarpus tener. -- Hook. Iond. J. 1543.

1I. t. 2.2. f. 3. Berk. .

Solenarium Mühlenbergii. - Bail s. t. J〕. cf. Glonimm stell. Dub.

Solenia. - [s. 35\%.]

— candida P. - [\$. 35\%.]-Kil.922. - Voges.

96. - Bisch. f. 3391.

-_ M. N. : Pez. Solenia I)C., Solenia fasc.

- Tul. Sel. I. 136 .

- epiphylla. - Alger. t. 29, 1.

- fasciculata. - s. 3.5.] - Bail S. t. 211. -

Pez. Solenia I) ( - ('arol. IT. 21.

- ochracea IIoffu. - \$\$.357.] - Bisch. f, 3392.

Fuck. s. 7!!t. - Kl. II. 232 - - cd. Ic, III. t. di.

f. 96. P. K. - Pez. anomala P'., P'. Iloftimanni Spr., Cyphella IIothmanni 'Tul.

_ villosil. - S. 357.$]$ - Camol. V. 42.

Solenod ont a Cast. - $\$$ \$. 514.] - I'uccinia sertata I'r.

— Flotowii. - K1. 1345. Cent. XVII1.j.

- Graminis. - Castagne, Marseille. t. 2.

Histd. $75 \%$.

Sophronia. -- [s. 431 . ] ; ef. Hymenoplallus. - brasiliensis. - Gaudich. Voy. 1426. t. 1:2.

Sordaria. - ef. Sphaeria.

- fimiseda. Not. Sfer, 19. p. 22. Schizothec.

fimicol. Cd, et I'odospora fimicola ('es.\}.

Soredospora. $\left.-[S .50]^{*} .505.\right]$

- Graminis. - Cd. Anl, t. 13, f, 9: 6 .

sorocybe. - [s. 46 .

sorosporium. - \$. $516^{*}$.

- Saponariac. - Limn. 1529. IV. t. 2. f. 1.

Rudolphi . - Fuck. s. 114. - Bisch. f. 34\%. -

Kil. 179; - 145U v. Gypsophilae. - Kl. II.

42. - Fuck, rh. 253.

- schizocaulon C'es. - Kil. 1043. - Kil. II. 93.

- v. Violae. - Iil. II. 190.

- Seleranthi. - Kl. I $\mid>1$.

Spadonia. - S. 36il.?

Sparassis - S. 33\%

- brevipes. - Col. Anl. t. G. f. i2: 21.

- crispa. - \$. 33\%.] - Bail S. t. 27. - Kl

517 . - Fries Sv. t. 1\% - Nov. Aet. 1512. XIX.

II. t. 57. f. 112-122. Fruetif, Phöb.

— foetida. - Pay. b. cr. p. 111. f. $1>3$.

- laminosa. - [\$. 337.]

spathulata. - [S. $33 \pi^{*}$.

spathularia. - cf. Spathulea.

$S$ pathulea. - [S. 347 .

- crispita. - S. 347.$]$

- flarida. - S. 317.$]$ - Cd. Anl. t. G. f. 66:

6-10. - Loud. f. 1615s. - Ann. Mg. 11. h.

1535. I. t. 5. f. 51. Spor. Berk. - Pay. b. er.

p. 7. f. 21 ; p. 55. f. $375 .-W^{\top}$ std. 11155. --

Bail s. t. 21. - Berk. t. 21. f. 7. - kil. 315. -

Kl. II. 2๖. - Krypt. Bad. 337. - R. f. 126.

- Fuck. s. $5: 1$. - Bot. Zt. 145\%. t. 11. f. 23.

Keimg. Hoffm.) - Dsm. IH. 455. - Voges.

$14 i \%$.

- rufa Ns. - R. f. 235.

Speira. - [S. 523.]

- toruloides. - Cd. An1. t. B. f. 5: †. - Pay.

b. cr. p. 6s. f. 295.

Spela eomyces Heydeni. - Fresen. t. 13. f. 43 -45. p. 106 .

Spermodermia. - [S. 450.519.]

Spermoedia. - $\left[\mathrm{S}, 49^{* *}\right.$.] cf. Selerotium.

- Clarus. - Kops Fl, bat. VIII. 626. - Wstd. 327.

- r. Scirpi. - K1. 935. ('ent, XVIII.). 
Sphacelia seget. - [S. 479**.] - Wstd. 327; ef. Sclerot. Clav,

Spliaerella. - [S. 395.]; ef. Sphaeria.

- conglomerata TRbl. - - alnicola IR. f. 1.,0.

- maculaefornis f. Carpini. - R. f. 512.

- myriadea Rbh. - R. f. 149.

- punctiformis. - Kl. II. $2(i)$.

- recutita. - Kil. II. 659.

sphaeria. - \$S. 3\$4.] - Pay. b. cr.p. 97. f. 419. - Ann. sc. 11. 1534. I. t. 13. Fruetif.). - ef. Bertia [S. 39.5.], Bombardia, Camillea, Cerato stoma [S. 396.], Cryptosphaeria, Cucurbitaria, Depazea, Diatrye [S. 344.], Diplodia [S. 416. , Discosia, Dothidea [S. 346.$]$, Endothia S.355.], Gibbera [S. 402.], Halonia [S. 397.], Hercospora [S. 397.], Hypocopria [S. 397.], Hypocrea [S. 3५. .], Iypospila, Hypoxylon [S. 3৬3.], 1.cptospora, Malinvernia, Massaria [\$. 396.], Melogramma [S.366.], Microstoma, Nectria [S.357.], Nodulosphaeria, Oncomyces, Oomyces, Ophiobolus, Picrostoma, Plcospora, Poronia, Pyrenophora, Rabenhorstia, Rhaphidospora $/=$ Ophiobolus), Sphaerella, Sphaerolina, Sphaeropsis, Spilosphaeria, Sporormia, Staurosphaeria, Stromatosphaeria, Valsa [S. 410.], Termicularia, Xylaria.

- Abietis (Valsa). - Kl. 1331. - Linn. 'Tr.

XXII. t. 4ด. f. 147. 146.? p. 279. (Curr.). acclinis Valsa). - Not. Sfer. 39.

— accumulata. - Voges. 665. (Vacenii Sow.) - acericola (Diatrype). - Not. Sfer. 27. p. 24. - acervalis M. Nectr.) - Linn. Tr. XXII.

t. 49. f. 152. (Curr.) - Dsm. 760. 1405. 1409. - acervata Fr. - [S. 39l.] - Fuck. s. 634. (caespit.) Fr.: Linn. Tr. XXII. t. 19. f. 144. 155. Curr. - Brit. f. 174. Achilleate - Kil. 144. - R. f. 531. achroa DC. - Kl. 1960). - S. dubia $\mathrm{P}^{\prime}$. acinosa. - [S. 416.1.] - Voges. 769. aculeans. - Carol. I. 55.

acuminata Sow. - [S. 393.] - Ann. Mg.

n. h. 1552. IX. t.11.f. 26. p.324. (Spor., B. B.).

- Ophiobolus Riess. - Linn. Tr. 1559. XXII.

t. 59. f. 133. Curr.).

Acus Trog. - cf. Claviceps microcephala.

- Blox. : Iinn. Tr. XXII. t. 5s. f. 93.

p. 325. (Curr.

- acuta Hoffm. - [S. 394. 376.] - Fl. dan.

XII. 2040. 3. - Cd. Aul. t. F. f. 55 : 12-11. -

Kl. II. 14. - Kl. 153. - Fuck. s. 550.

- P.: ef. Phoma complan. - Voges.

151. - Letell. 6\$6. 2. - Wstd. 170.

adpressa. - [S. 410.$]$

adunca. - D sim. $17 \%$.

Aegopodii P. - [S.425.] - Kl. 25\%. - Fuck.

s. 599. - Voges. 251. - Wstd. 95.

Aesculi Schum. - maculaeformis v., Fl. dan.

aesculicola. - [S. 423.]

affinis Grev. - Loud. f. 16423.

Agaves. - [S. 37\%.] - Voges, $117 \%$.

(Depazea). - (Pleospora) Not. Sfer. 75. aggregata Iasch, - Kl. II. 5.11.

agnina Rob. - Dsin. 1415 .

agnita. - Kl. 1935. - Wstd. 1111.

alba Schum. - S, ovina v. glabrata, Fl. dan. albicans P. - Fuck. s. 564 .

v. confluens. - Wstd. 166.

albo-punctata. - Bull, ac. belg. 1559. VII.

p. 366 . f. 16. (Wstd.). - Wstd. 1216.

Alismatis (Depazea) . - Iinn. Tr. 1 59. XXII.

t. 59. f. 15\%, (164), (Curr.).

Alliariae. - R. f. 261. -- cf. Bilimbiospora. siph a eria allicina. - [S. 394.] alligata Fr. - [S. 3\5.] - Linn. Tr. 15.59.

XXII. t. 5\%. f. 10. (Curr.).

Allii Rob. - Kl. 839. - Wvstd. 719. alnea. - [S. 391. 423.] - Lk. : cf. I)iscosia alnea Not. - Wstd. $4 \%$ - - I., f. 539.

- Alopecuri Fr. - [S. 419.] - R. f. 311. (D)i-

lophospora Graminis Dsm.). - Wstd. 717 .

alutacea. - cf. Cordyceps al.

- ambiens 1'. - Dsm. 967. - Fl. dan. XII.

2039. 1.-Kl. 1325. - (Valsa) : Linn. 'T'r. XXII.

t. 4. f. 135. (Curr.) - Voges. 572. - Brit.

f. So. - Wstd. $36 \%$.

- amblyuspora. - Berk. In. p. 2S1. f. 65. b.

= Massaria s. Sph. foedans Fr. (ex Berk.).

— - B.: R. f. 11. - ef. Splanchnonema

pustulatum Cd. bei Sturm h. 9. t. 51. - Kl.

1\$30. - (Subtectae) : Ann. Mg. n. h. 1552. IX.

t. 10. f. 11. p. 32\% (B. B.). - Micr. Journ.

1557. V. t. \&. f. 46. - u. IV. t. 11. f. $11-25$.

(Curr.). - Linn. Tr. 1559. XXII, t. 59. f. 99.

(Curr.). - Fresen. t. 7. f. $21-33$. - Fl, dan.

2160.2.

- Ammophilae. - Kl. 1340.

amoena Ns. - Dsm. 759. - Voges. 1251.

Amorpliae. - Kl. 1544. - Kil. II. 732. -

Fuck. s. 576 . - Hedw. I. t. 5. f. 7.

- anarithma (Foliicolae). - Ann. Mg. 1. h.

1559. III. t. 11 f. 37 . (B. B.).

Andromedarum. - Carol. I. 5 S.

- andurnensis (Rosellinia). - Not. Sfer. 17.

p. 19.

aneirina Diatrype. - Not. Sfer. 29. p. 29. Anemones DC. - Kil. 517. - Toges. $447 .-$

Lib. 167. Sphaeronema .

Anethi Dothidea P. - Dsm. 76.1. - Kl. 262.

- v. Petroselini. - Dsm. 1411.

Angelicae. - [S. 393.] - Brit. f. SS. -

Mag. Zool. Bot. 1与37. I. t. 3. f. 7. p. 1s. (Berk.).

— angulata Fr. - Bisch. f. 3503. - (Valsa)

Linn. Tr. XXII. t. 47. f. 122.

- Schum. : Fl. dan. XII. 2160. 1.

angustata. - [S. 391.] - Kl. 1241. - Fl.

dan. XII. 2154.1.

- angustilabra (Platystom.). - Ann. Mg. n. h

1559. III. t. 11. f. 27 . (B. B.)

- annulata M. - Linn. Tr. XXII. t. 45. f. 31.

Fruct., Curr.) - Carol. I. 47.

- anomia Fr. - Fuck. s. 643. - (Valsa) : Linn.

Tr. XXII. t. 47. f. 115 . (Fruct., Curr.).- Dsm.

III. 754. - Wstd. 472 .

- anserina P. - Bisch. f. 3595. - Malinver-

nia ans.

Not.

- Aparines Cast. - R. f. 535.

apiculata Curr. - Linn. Tr. 1559. XXII.

t. 55. f. 90. p. 326 . (Fruct.). - R. f. 250 . (cf.

S. Curreyi Rbh.).

apiospora. - Alger. t. 25, 1.

appendiculosa B. B. - R. f. 52. - Ann.

Mg. n. h. 1651. VII. t. T. f. 20. p. 159 . (Fruct.,

B. B.) - Iinn. Tr. 1559. XXII. t. 59. f. 97.

Fruct., Curr.) - Wstd. 474. - R. f. 331 .

(clyp.).

- applanata. - [S. 390.]

aquifolia. - cf. Nectria inaurata.

- Aquifolii Fr. - Linu. Tr. 1S59. XXII. t. 49.

f. 153 . (Fruct., Curr.) - Voges. 579. - Lib. 146. Aquila Fr, - S. 355.] - Bisch. f. 3599. -

Fuck. s. 618. - Kl. 15\% - Limn. Tr. 15.9.

XXII. t. 5i. f. 4. Fruct., Curr.) - Voges. $9(i 5$. 
<smiles>CCC(C)C</smiles> 


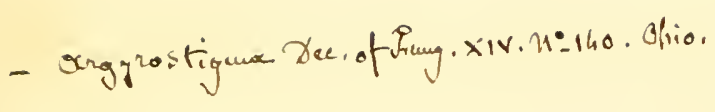


- Carol. V. 65. - (Rosellin. Not. Sfer. 15. p. 21.

Sphaeria aquilina Fr. - [S. 117.] - R. f.249.-

Hdw. I. t. 11. f. A. a--k.

araneosa Schum. $=\mathrm{S}$. atrostoma $\mathrm{Fl}$. clan.

- P.: [S. 390.]

Arbuti. - [S. 117. 423, 9.]

arbuticola (Diplodia Fr. - Kl. 113.

Dsm. 276 .

Archangelicae Lasch. - K1. 552.

Arctii Lasch. - Kl. 10-16.

- arcuata. - Linn. Tr. 15.59. XXII. t. 14. f. 167.

p. 251. (Fruct., Curr.)

Arecae. - [S. 422**]

arenula Denudat. - Inn. Mg. n. h. 15.52.

IX. t. 9. f. 5. p. 320. I'ruct., B. 13. .

areolata I'res. Mey. - Palaeont. VIII. t. s.

f. 11. R. Ludwig.

argillacea. - I. f 247. Hypoxylon. -

Linn. Tr. 1859. XXII. t. 15. f. 35. (Fruct., Curr.).

- P. = multiformis, Fl, dan. - W'std.

530. 713.905.

- Argus (Hereospora) - Kl. 1531. - Subtectae) Ann. Mg. n. h. 1552. IX. t. 10. f. 9 . p. 322. (13. B. - - R. f. 259 - Linn. Tr. 1559.

XXII. t. 5\&. f, 95. Fruct., Curr.

— Artocreas 'Tode. - [S. 423.] - Fl. dan, XII. 2100. 1. - Bisch. f. 3570. - cf. Discosia faginea Lib., u. clypeata Not. - Kl. 360. - Fuck. s. 363. - Discosia Linn. Tr. 1459). XXII. t. 59. f. 143. Fruct, (urr.) - - I)sm. III. (i1-(j)

(Discos. Art. liob.). - Wstd. 2i.

— arundinacea Sow. - S. 391. 119.] - Fuck. s. 605. - Dsm. 762. 973. - (Seriat.) I inn. 'Tr.

1559. XXII. t. 49. f. 200. Fruct., Curr. Voges. 1165. - Brit. f. 42. - Carol. III. 57

- Arundinis Fr. - [S. 391.] - Kl. 1037. -

Kil. II. 6+1. - Fuck. s. 590. - Linu. Tr. 15.59 .

XXII. t. 59. f. 121. Curr.). - Voges. 1172.

lirit. f. $\checkmark$.

- Asari Kl. - Kil. 250.

Ashwelliana (Obtect. . - Linn. Tr. 1559.

XXII. t. 59. f. 107. p. 32\%. Fruct., Curr.

- Aspegreenii Fr. - [S. 390.349**] - Fl.

dan. XIII. 2334. 3. - (Pertusae) Ann. Mg. n. h.

1559. III. t. 11. f. 26 . (13. B.).

— aspera. - ? Linn. Tr. 1559. XXII. t. 17 . f. 111.

p. 275: 117. (Diatryp.). Fruct. Curr. - Wstd.

167. 365. - Ital. j91. (Ielogr.). - Hoffm. Ic.

t. 15. f. 3. - Not. Sfer. 51.

- Aspidiorum. - Lib. 342.

- Asteroma. - [S. 125.] - Ascospora Fr. Su.

— f. Polygonati. - Kil. 1153.

f. Saponariae. - Kl. 1454 .

- f. Violae. - Kl. $\$ 16$.

asteromorpha. - Lib. 13.

Astragali. - Kl. II. 639. - Linn. Tr. 1559.

XXII. t. 59. f. 132. p. 331. (Fruct., Curr.).

- astroidea. - S. 392.]

- Atomus. - Wstd. 277. - Dsm. 793. - R. f. 532 .

\section{atramentosa - [S. 359.]}

- atrata Dsm. = Diplodia melaena Lév. - $\mathbf{R}$.

f. 345 . - Dsm. 775 . - Voges. 1270.

atriseda. - [S. 390 .

- atrofusca. - [S. 35S.]

- atropunctata (Diatry.) S. - Linn. Tr. 1559.

XXII.t. 16. f. 70. (Fruct., Curr.).-- Carol. I. 62.

- atropurpurea '1. (Hypoxy.).- Limn. Tr. 1559.

XXII. t. 45. f. 44. (Fruct., Curr.). - Voges.

765. (vogesiaca).

atrorubens. - [S. 405. 2.]
Spla eria atrostoma Schum. - F1. dan. XIII. 2333. 2 .

- atrovirens A. S. - Bisch. f. 3596.

__ - v. Rusci. - Brit. f. S6. — Wstd. 535.

- f. Visci. - Dsm. 42. - Kl. 535. -

Voges. 456. - Wstd. 1231.

- var. b. - K1. II. 510.

- v. Taxi. - Brit. f. 150.

- v. Mirebili Fr. - W'std. 26.

Aubertii, - Bull, ac. belg. 1459 . VII, p. 365.

f. 10. Wstch.

- aucta B. B. - R. f. 143. - (Obtect.) Ann.

Ig. n. h. 15ว2. IX. t. 10. f. 11. p. 323. Fruct.,

B. I3.

Aucubae. - Bull. ae. belg. 1559. VII. p. 360.

f. 14. (Wstd.) - Wstd. 1217 .

- aurantia P. - Bisch. f. 3601. - Fuck. s. 621. - Carol. V. 64.

- v. fulgens. - R. f. 134. - Nectria

Jinn. Tr. 1 559 .XXI t. 57 . f. 6. 7.? Fruct., Curr, .

- Aurantii. - Act. 'Turin. 1553. XUI. Dec..

f. 3. Notar.

- Avellanae. - [S. 394.] - Brit. f. 182.

- baccata. - S. 390.]

Badhami. - Diatry.) Linn. 'Tr. 1859. XXII.

t. Hi. f. S0. p. 270. (Fruct., Curr.).

- Balsamiana. - Act. 'lurin. 1553. XIII. Dec.

7. f. 7. (Notar.). - Ital. 649.

- barbara Fr. S. - S. 101. cin.? - cf. Ostropa cinerea Not. - (P'latysto.) Linn. 'I'r. 1 559 . XXII.

t. 54. f. 63. Fruct., Curr. - - Voges. 966.

barbata P. - 13isch. f. 3561 .

Barbierii. - Bull. ac. belg. 155\%. VII. p.

365. f. 11. Wstcl

- barbula Caespitos. - - Ann. Mg. n. h. 1459.

III. t. 10, f. 20. (B. B.).

- bellula. - Dsm. 774. - Ann. sc. 11. 1540.

XIII. t. 4.

- Bellynckii. - Bull. ac. belg. 1S59. VII. p. 366. f. 13. (W'std.).

— Berberidis P. - [S. 395.] - Bisch. f. 3571.

(Gibberidia) I.: Kl. 155: - it. Cent. XIX. -

Fuck, s. 632. - Caespitos.) Linn. Tr. I 559.

XXII. t. 49. f. 17\%. (Fruct,, Curr.). - Voges. 6.1. - Wstd. 1203.

- Berengeriana (Botryosph.). - Ital. no? -

Diatry.) Not. Sfer. 26. p. 27. - (Botryosph.) Not. Sfer. 90.

- Berkeleyi. - Dsm. 1419. - Wstd. 912.

- Betulae Schum. = S. lanciformis Fl. dan.

- nanae. - Wahlenb. Fl. lappon. IS12.

t. 30. f. 3 .

- Betuli. = Valsa Carpini Fr. - R. f. 322. -

Dsm. 966. - Wstd. 1206.

- betuligna Chev. - cf. S. favacea.

- bicolor DC. - Wstd. 903.

- biconica (Valsa). - Linn. Tr. 1859. XXII.

t. 4ร. f. 142. p. 279. (Fruct., Curr.).

- biformis P. - [S. 359.] - Ann. Mg. n. h.

15.11. VI. t. 11. f. S. b. (Berk.). - (Denudat.)

S. non Fr, : Linn. 'Tr. 1559. XXII. t. 57 . f. 41.

(Fruet., Curr.).

-_- v. terrestris. - Kl. $66 \mathrm{~S}$.

bifrons. - [S. 421. 1.] = Hypospila popu-

lina Fr. Su. - (Confert.) S. K. : Linn. Tr. 1859.

XXII. t. 49. f. 204. (Curr.). - Bivona t. 3.

f. 3. - Wstd. 52i. - (Hypoxyl.) Not. Sfer. 15. biturbinata. - Alger. t. 26, 7 .

blepharodes B. B. (Subtect.). - Ann. Mg.

n. h. 1561. VII. t. 17. f. 31. p. 451

bolbitia. - [S. 390.] 
Sphaeria Bombarda Batsch. - Bisch, f. 3527. Fuck. s. 5.54. - Berk. Out. t. 24. f. 5. - Ann. sc. n. IS 11. XIV. t. 19. f. 万. Mont.) - Denudat.) Linn. Tr. 19,59. XXII. t. 57. f. 29. Fruct., Curr.). - Brit. f. 20, - Ital. 576. Bertia.

- botryosa Fr. - [\$. 349.] - Limn. 'Tr. 15.59. XXII. t. 46. f. 46. Fruet, Curr.

- Brachreladii Lacr. $=\$$. Papaveris Tul. Sel. I. 43. u. 22.3; pellita Kil., Wstd., Walls. - I $\mathrm{sm}$. III. Thti. - Jil. 1246; II. 719. - Voges. 1456 . _ brachytele Pertusae , - Am. Mg. n. h. 14.59.

III. t. $10 . \mathrm{f}, 2$, (I3. I3.

- Brassicae. - [\$. 391. \$7.] - K1. 550. -

Linn. Tr. 15,59. XXII. t. 5\%. f. 23. Fruct.,

Curr. - - Foliic. Ann. Mg. n. h. 1452. IX. p. 344. t. 12. f. 42. Fruct., B. I3. .

- Braunii. - Heer, Fl. tert. IIelv. t. 1. f. 2

- Palaeont. VIII. t. \&. f, 2. R. Ludwig

- brevirostris Fr. - S. 392.7 - Fl. dan. XII.

2010. 1. - Ceratost. Iimn. Ir. 1 599. XXII.

t. 59. f. 65. (Fruct., Curr.).

- brunneola Fr. - 5.425.$]$ - Dsm. 266.

Kil. 662. - Voges. 1253. 970. - Brit, f. 34.

- - bryophila. - Dsm. 1755.

- bufonia Obtectac). - Ann. Mg. n. h. I 52.

IX. t. 16. f. 13. p. 323. Fruct., 13. B.). - Linn.

Tr. 1559. XXII. t. 59. f. 102. Fruct., Curr.

bulbosa 1 ?. Bisch. f. 3154 .

- bullata. - Yoges. 466. - Act. Turin. 15.53.

XIII. Dec. 4. f. 2. Notar. . - cf. Diatrype. -

Dsm. 961. - Bisch. f. 3501. - Kl. 719. -

Trstd. 65.

- Buxi Diplodia' DC. - [S. 417. \$19, >.1 -

Dsm. 740. - Kl. 549. - Caesp. B.: Linn.

'Tr. 1559. XXII. t. 49. f. 156. Fruct., Curr. -

Dothid.) Carol. I. 73. - Wstd. 26. - R. f. 531.

— byssiseda Tode. - [S. 355.] - Bisch. f. 3597.

- Kil. 1013. 123 \%.

_- caelata. - [S. $409:$ Lév.]

- caesia. - I.inn. Tr. 1559. XXII. t. 57. f. 17.

Fruct., Curr.

- caespitosa T. - S. $\left.399^{+4 .}.\right]$ - cf. C'enang.

Aucup. u. Cytispora Aucup.

- callicarpa Pertus. . - Lim. Tr. 1559. XXII.

t. 59. f. 62. p. 321. Fruct., Curr. callimorpha Mont. - Wstd. 1110.

callista. - Carol. V. 67.

- Callumae Sphaerella;. - Not. Sfer. 100.

calostroma. - Dsm. III. 365.

calva Tode. - [S. 349.] - Bisch. f. 3571

calvescens Diplodia Fr. - \$.394.] - Iinn.

Tr. 1559. XXII. t. 59. f. 134. Fruct., Curr.

- calvula. - Wstd. 367. - Wahlenb. Fl. lap-

pon. 1512. t. 30 . f. 4 .

Calycanthi Contlu.' Fr. - Linn. Tr. 1559.

XXII. t. 49. f. 195. Curr.

- Calyculus. - Ann. sc. n. 1911\%. XIV. t. 19.

f. 2. Mont.).

Campanulae DC. - Kl. 670.

campylospora. - [S. 356.] Melogr.]

cancellata $\mathrm{Td}$. - Wstd. 473.

candida. - [S. $399 \div$.

canescens P. - [S. 359.] - Bisch. f. 3593.

- Villos. Linn. Tr. 1559. XXII. t. 5\%. f. 14.

(Curr. - Brit. f. 31)1.

- capillata Ns. - Bisch. f. 3575. - Schnzl.

t. 15. f. 34 .

- capillifera (Villos.). — Linn. Tr. 1-59. XXII

t. 5 i. f. 26. p. 317 . Fruct., Curr.).

- capistraria Talsa - Not. Sfer. 40. p. 36.

— capitata. - [S. 39\%.] - Linn. Tr. 1\$5\%. XXII t. 45. f. 10. Fruct, , Curr. ef. Cordreeps, Voges. 763. - W'std. 21.

Sphaeria capitellata Ki. - Kl. 161. - Fuck.

s. 576. - 13ot. Zt. 1\$62, t. 12. f. 1-22. p. 37 .

Erolut., Sollmann.

[ capnodes B. Diatry.). - [S. 390.] - Limn.

'Tr. 1459. XXII. t. 46. f. 73. Fruct., Curr.)

- ('apreae. - Iib. 215. - Kil. $1741 \%$.

('aprifoliorum. - Dsm. 794.

(aprina Fr. - Fl. dan. XI. 1559. 2.

- Carduontum. - S. $393 \%$ - Dsm. 751.-

lil. 1 blil).

Wstd. 325

Caricae (Iatry.. - Not. Sfer. 26. p. 24.

Cariceti Foliicol. ]3. B. - Ann. Mg. 12. h.

[\&61. VIL.t. 17. f. 35. p. 15.5

caricina. - cf. Neottiosp. car.

Caricis. - Dsm. 175\%. - R. f. 535.

_- carnea Nectr.). - Dsm. III. 373.

carneo-alba - Lib. 241. - cf. Oomyces c. carpinea Foliicol. Fr. - [S. 425.] - Dsm.

2\1. - Anm. Mg. n. h. 1 552. IX. p. 3>1. t. 12.

f. 11. Fruct., B. B. . - Wstd. 275. - cf. $\Lambda$ scospora

— Carpini. - [S.397.] - K1.15\%. - ef. Valsa :

Iimn. 'Tr. 145\%. XXII. t. 47 . f. 11'. Fruct.,

('urr):

Hoffm. - cf. S. fimbriata Not.

Fr.: R. f. 322. - Wstd. 467.1206.

carpophila P. - Hrit. f. 76. - Fl. dan. XI.

15.5\%. 1. - ef. Xylaria, Hypoxylon. - I imn.

1643. XVII. t. 15. Schmitz, - Dsin. 260. -

I.inn. Tr. 1 599. XXII. t. 45. f. 23. Fruct.,

Curr. - - Voges. 1245. - Wstd. 111.

C'astagnei. - Dsm. 1764.

rastaneae Schum. - F1. dan. XIII. 2333. i.

castorea Td. - Wstd. 219.

- caudata Schum. - Fl. dan. XII. 2155. 2.

- Denudat. Curr.: Linn. Tr. 15.59.

XXII. t. 55. f. 50.

caulifraga. - [S. 391.

caulincola. - Voges. 1452. - Wstd. 221.

_- Wllr. = modesta Dsm. ex Wistd. .

- caulium Fr. - S. 391.] - Fuck. s. 5!1.

Ann. sc. n. 1s41. XT. t. 14. f. 2. a. p. 116.

'Lophiostoma' - Not. Sfer. 70 .

— cellulata Fr. Valsa, - - Iinn. Tr. 1559. XXII.

t. 17. f. 120. Curr. .

- Celtidis. - Carol. IV. 59.

- cenisia Valsa. - Not. Sfer. 11. p. 34.

- cenobitica Valsa, - Not. Sfér. 11. p. 37.

(erasi Schum. - F1. dan. XII. 2153.1.

('erasorum 'Obtect. Curr. - Iinn. Tr', 1459.

XXII. t. 55. f. 50. p. 323 . Fructif. .

- Fr. - [S. 393.]

cerastis. - Hdw. I. t. 3. f. 2. m--o. - hl

1711. - R. f. 337 . (petiolor. .

- ceratosperma 'Tode. - Bisch. f. 3500. - Y Via-

try. Lim. Tr. 1559. XXII. t. 47. f. 93. Fruct.,

Curr. - Voges. 567. - Lib. 35. - Wstd. 67. ceriospora Subtectae - Kil. 1937.

- cerviculata Valsa,. - Not. Sfer. 33.

- Cesatii Mont. - Not. Sfer. 5. Nectria.

- ceuthocarpa Confert. Fr. - [S. 121.] -

Linn. Tr. 1559. XXII. t. 49, f. 213. Fruct, Curr. - Dsm. 270. - Wstd. 531. - ef. Hypospila populina.

- ceuthocarpoides. - Heer, Fl. tert. IIelv. t. 1. f. 1 .

- ceutho-poroides Berk. - $13 \mathrm{sm} \mathrm{if13.} \mathrm{-}$

Wstd. 1116.1211 . Berber., Ilicis/. 



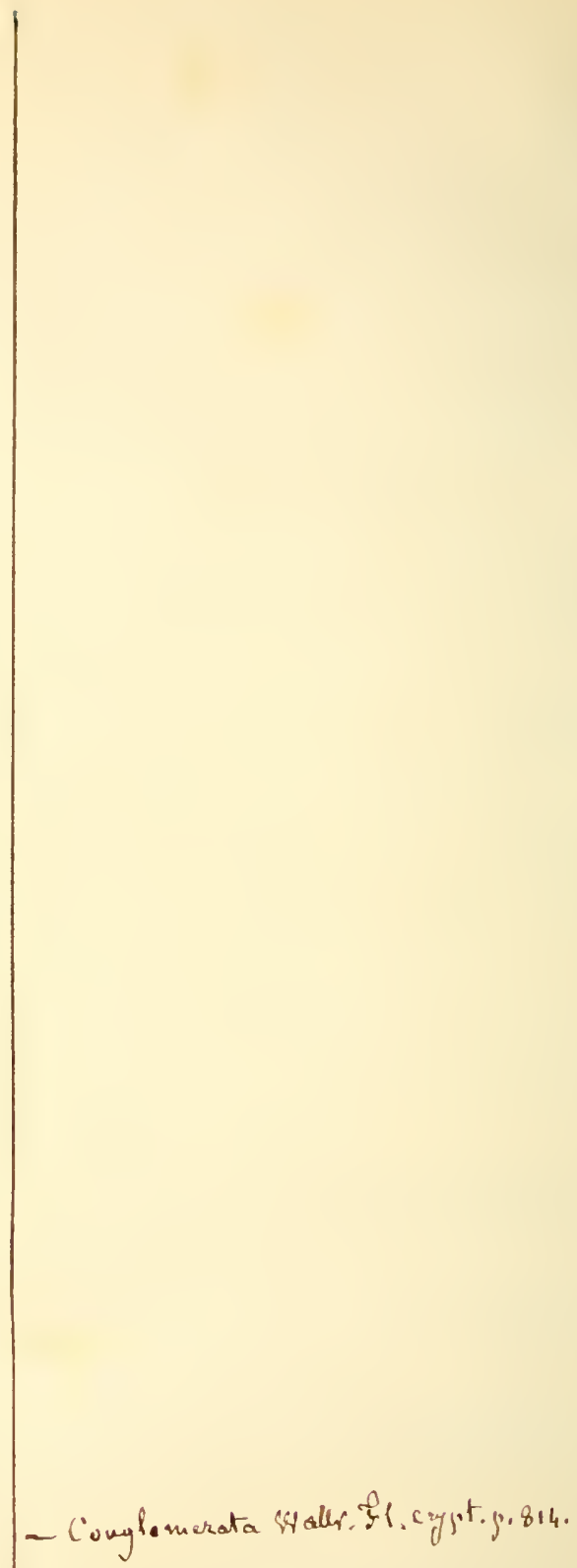


Sphaeria Chaetomium (villosa) Cd. - [S. 105. 7. pu.3.] - Dsm. 1759. - Lib. 311. Sphaeria comata Tode:, Chaetomium clat. anct : - Ann. Mg n.h. 15.52. IX. t. 9. f. 3. p. 319. B. B. Chamaemori. - S. 39)4.

chionea. - S. 37\%.] - Kops, F1. bat. XI. 5.5.5. 3. - K1. 361. - Ceratost. Fr. : Linn. Tr. 14.59. XXII. t. 57 . f. 22 . Fruct., Curr.

- ehlorospora c'es - R. f. 4h. - Itul. 296. Sphacrella) Not, Sfer. 9\%.

1932. b.

- chrysentera ('urr. Conflu.). - Limn. Tr. 15.\%. XXII. t. 4\%. f. 1\%, a. p. 2\$1. Fruet. - ehrysites. - S. 357. 1. - Wstd. 36i.

- chrysostroma Valsa M. - Iinn. Tr 1 $15 ., \%$ XXIJ. t. 48. f. 161\%. Fruct., Curr.,

Cibostii. - Act. 'Turín. 1653. XIII. I)ec. (i. f. 5. Notar.,

- Cieutae. - Kil. 1615.

- ciliaris Curr. - Journ. microse. Soc. 1<,j9.

VII. 1). 231. t. 11. f. 15. ciliata I'. - Bisch. f. 3534. cf. Tal:al eilicifera. - [s. 391.]

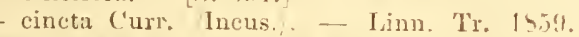

XIII. t. 4. f. 135. p. 27\%. Fuct.

713 .

- einerco-nebulosa. - Dsm. III. 370.

- eingulata.- Ann. se. n. \$ \$ 1. I. t. 12. Mont.

- cinnabarina 'Td. - Isum. !) il. - Bisch. f. 35111. - Schnzl. t. 15. f. 29.30. - Nectria Iinn. Tr. 14.39. XXII. t.19. f. 175 . Fruet, ('u1r. . - Kl. I.j(j; Cont. IT, Suppl. - Voges. 5i0 decolorans, - Wstd. 364.

- eireinans. - [\$. 3!15. 421, 3.] - cf. Coniosporium c. Fr.

- circinata. - [S. [10.5.]

- eircumelusa. - Not. Sfer. 63.

- circumseissa P. - S. 3!7.? - Fl. dan. XII. 2110.2 .

- circumscripta. - [S. H11**- Kl. 411.-

Valsa Fr.: Limn. Ir. 1 959 . XXII. t. 14. f. 132.

Fruct., Curr.

- cirrhata Sow. - Wstd. 323.

- cirrhosa P. - S. 392.] - Fuck. s. 19211.

Ceratost. Curr. L Limn. Tr. 15.59 . XXII. t. 54. f. 71 . 4. 72.2 p. 32 . Fuct. F

- citrina. - Lib. 311. - K1. 1 \41. - Hypocrea) $P_{0}:$ Iinn. Tr. 14.59. XXII t. 16. f. Jl.

Fruet., Curr. - Hypocr. Not. Sfer. f. 2.

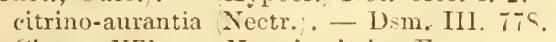

Citrum WI1r. = Nectria citrina Fr.

clandestina. - [今. $411 \%$.]

clara Seriat. Curr. - Limn. 'I'r. 15.59. XXII. t. 49 . f. $20 \% 3$. p. $2 \geq 5$.

- elausa Schum. - Fl. dan. X111. 2335. 2. = circumseissa Fl, dan.

clavacformis. - [S. 395.]

Clavariae Dsm. - R. f. 252.

clavata Sow. - ef. Cordyceps alutacea.

Clavus. - [S. 409.]

clivensis Caulicolae - Ann. Mg. n. h. 1552. IX. p. 379. t. 11. f. 29. Fruet., B. B. Limn. 'Tr. 1\$.59. XXII. t. 59. f. 135. SFruct., Curr.).

clivulosa. - Ann. sc. n. 1455 . III t. 6. f. 4. Mont.).

- elypeata Ns. - S. 417.423 .3]$.- ef. Diplodia. - (Sordaria) Not. non alior. : Not. Sfer. 2.2. p. 24 .
Sphaeria clypeata Moug. : R. f. 331. (clypeiformis Laer.

- Ns. : Limn. Tr. 1 9.59. XXII. t. 55 . f. $\$ 3$

Fruct., Curr. - Voges. 114s.

- elypeiformis. - Act. Turin. 1553. XIII.

Dee. 7. f. ?. Notar. - K Kl. 11. 645. - R. f.

33 i Sph. appendiculosa B. R.

- coarctata Schum. - F1. dan. XII. 2153. 2.

coecinea. - Lib. 212. - Fl, dan. Xl1. $210 \%$.

2. - Nectria : Linn. Tr. 14.59. XXII. t. -19.

f. 173. 174. Fruet., (mrr.) - K1. 535.

Dsm. 14117; III, 777. - Voges. 15(). - Letell.

(ii) 1. - Wstd.3166.- Hypoxyl. Not. Sfer. 10. - eonopus Hурохуl. М. - I,imn. 'Tr. 1459.

XXII. t. -15 . f. 11 . Fruct., Curr. .

cohaerens P. - Iypoxyl Ki. 253. - livt.

\%t. 14.9. t. 11. f. 111. Keimg. Hoffm.j. -- Jimm.

'1r. \&.3\% XXII. t. 15. f. 11 . Fruct. ('urr)

- Schun . = fusca F1. dan. - Voges.

761. - Wstel.901. - IIpoxyl. Not. Sfer. 1:.

-_ - var. : F'l dan. XII. 2155.1.

- eollabens Curr. Denudat.'. - Limn. Tr.

15.59. XXII. t. 54. f. 51. Fruet. .

- collapsa Sow. - Wstd. 96.

Coluteac Iibh. - Kil. 12391.

IX comata. - S. I1.5. - Anu. Mg.n. l. 1 jo.

IX. p. 32. t. 11 . f. 25 . a. Fruct., B. 13. .-

Kil. $53 \%$ - Bisch. f. $35 \%$ i.

$\longrightarrow$ v. capillatia Fr. - Kl. 410.

- commanipula (aulicol. - Anm. Ng. n. h.

14.52. IX. t. 11. f. 31. p. 3413. Fruet., 13. I:

- complanata Td. _ _. 3!)!.] - Bisch. f. 3.jt.)

- Ii. f. 342. P'loma - ('aulicol. Iinn. Tr.

14.59. XXII. t. .9. f. 125. Fruct., Curr. . - Voges. 2. v. minor: Brit. f. 2৬. - Letell. 1in $(i$. 3. - Norweg. 19!). - Iih. 211. herbarum «. P.j. - Wstd. 17:; var. 31. 1111. - Micr. J 1455. 111. t. 12. f. 11. 11. Curr.

- compressa P. - S. 391.] - Fuck. s. 555.

Wistd. 1205.

— compuncta. - Jungh. pr. t. 3. f. 11.

- eoncamerata Talsa ('urr. L Limu. Tr. 1 5.5\%.

XXII. t. 1\&.f. 13i. Eruct., ('urr.)

- concentrica. - S. 354.9. - Fl. dan. III. 203!j. 2. - Bisch. f. $3193-3196$. 11 ypoxyl. Linn. Tr. 1459 XXII. t. 15. f. 32. Fruct, Curr. . - Kl. 162. - Loud. f. 16360.

- eonfertil - - ‥ 21 .

- contluens Td. - \$\$. 309.7 - Bisch. f. $35: 6$.

- Fuck. s. 1jt7. - Dsm. 959. - Kl. II. 132 .

- Wstd. 219.166.

conformis Obtect. . - Am. Mg. n. in. $14, j 2$.

IX. 1. 325. t. 11 f. 19 . Fruct., B. B.). - Jinn.

Tr. I-59. XXII. t. 54. f. S5. Fruct., curr. - eonglobata Fr. Denudat., - [S. 391.$]$

Linn. Tr. 1459. XXII. t.57. f. 33. Fruct, ('urr). - eonglomerata. - S. 391..] — Dsm. 1419. -

Ki. \$1 - Fuck. s. 595 . - T. f. 150. - Wstd. 539.

- conica. - $[$ S. $400.6 \%$.

- coniformis. - S. 394. - Dsm. 953. - Kl.

1526. - Kl. II. 729. Caulieol. Fr.: Linn.

Tr. 1559. XXII. t. 59. f. 126. Fruet., (urr.). Wistd. 369 .

- conjugata. - Not. Sfer. 61.

- conjuneta. Ns. - Dsm. T5s.

- conorum. - Dsin. 1423. - Wstd. 913.

- contecta (Depaz.). - Dsm. III. $3(11$.

- controrersa. - \$. 111.$]-$ Dsm. 75. -

Wt $=$ td. 910 . convergens $\mathrm{Td}$. - Bisch. f. 3557 . - Pay. b. 
cr. p. $\varsigma .2 \varsigma, 29 .-$ Valsa' Linn. 'Tr. 14.99. XXII. t. 14. f 15j. Fruct., Curr.).

$\$ p$ ha eria cooperta. - Dsm. 1 $\$ 53$.

-- coprophila Hypoxyl. - Ildw. I. t. 11.

f. 13. 2. - Sordar. Not. Sfer. 2(1). p. 22.

Corchori. - Dsm. 951\%. - Wstd. 512.

Corni. - [S. 395*.] - Fuck. Nass. f. 15.

- Fuck. s. 591. - W'std. 1104.

- Corni suecicae. - S. 391.

cornicola LC. Depazea, Hendersonia. -

Linn. 'Tr. / 59. XXII. t. 59. f. 116 . Fruet.,

('urr.,. - lirit. f. 155 .

- comiculata Ehrb. - ef. Talsa corniformis. - Act. Turin. 1453. XIII. Dec.

(i. f. 1. Notar.). - Xylaria M. : Linn. 'Tr.

1-it. XVII.t.45. f. 20. 21 ! Fruct., Curr., -

lirit. f. $27 \%$.

_-_ cornu Damae. - Carol. 1. 45. cornuta Hoffm. - Wstcl. 16i.

coronata Hoffm. - Kl. 543. - Valsa Linn.

'lr. 1459. XXII. t. 4. f. 136. Fruct., Curr.

- Coronillae. - S. 391.] - Dsm. 779. -

Wstd. 966.

Corrigiolae. - Iloffm. Ic. t. ๖. f. 1.

- corticis. - [S. 393.] - K1. 1336. - Wstd.

47. - Fuck. s. 57s. - Obturat, Linn. Tr.

15.5\%. XXII, t. 59. f. 105. Fruct., Curr. .

— - Sow - S. 393: 113. - Fraxiui Brit.

f. 54 .

Coryli. - [S. 394. - F1. dan. XIII. 2332.

1. - K1. S15. - I. f. 333. - Dsm. 1412. -

Joges. 47. - Lib. 141. - Wstd. 219. - Ital.

ij).

- crassicollis. - Act. Turin. 1553. XIII. Dec.

. f. 2. Notar.

C'raterium. - S.367.] - Act. Turin. $1>41$.

III. Dec. 2. f. 6. Notar.). - Lib. 229. Peziza

Hederae Lib.. - Wstd. 15. 173. - R. f. 527.

Trochila

- crenata Fr. - [S. 991.] - Bisch. f. 35.53. -

Fuck. s. วัว.

- crenulata I3. IIypoxyl. . - Linn. Tr. 14.59.

XXII. t. 15. f. 15. Fruct., Curr. .

- C'repini. - W'std.911. - Bull. ae. belg. 1459.

VII. p. 3166. f. 17. Wstd., - R. f. 111. 1ycopodina IIont. : .

- crinita. - S. 349. - Fl. dan. NIII. 2331.

2. - Iil. 1242. - Wstd. 70. hirsuta P., ex Dsm.

- cristata Platyst. P. crenata Fr. - - Linn.

Tr. 1559. XYII. t. 5`. f. 67. Fruct., Curr.'

- - v. fallax Fr. - R. f. 252.

Cruciferarum Fr. - S. 422.7 - Ism. 245.

- Wstd. 1115.

cruenta Kz. - Wstd. 649. Depaz.'

crustacea Sow. = serpens Fl. dan.

crustata. - [S.396.] - 3 Hdw. I. t. 11. f. E. crypta. - [S, 396 .

cryptoderis. - Dsm. UI 366 .

- Cryptosporii. - Microsc. Journ. IV. t. 12.

f. 12-14. Curr. .

- eryptostoma. - Ann. sc. n. 1543. XIX. t. 7. f. 10. Lév.

cubicularis Fr. Halonia' - S. 397.] -

Linn. Tr. 14.59. XXII. t. is. f. 76. Fruct., C'urr. - Lib. 338.

— cucurbitula 'Td. - Bisch. f. 35uT. - Nec-

tria, Linn. 'Tr. 1>59. XXII. t. 19. f. 17s. p. 242.

Fruct., ('urr. - - Kl. \$6- - : Voges. 1455. -

Wstcl. 32-. v. nigrescens,.

- culcitella. - Carol. IV. 53.

- culmicola. - [S. 393 .
Sphaeria culmifraga Fr. - [S. 391. 120.] -

Dsm. 7Ьf;. 142 i. - K1. 964; 1339. - Fuck. s. 567 .

—_ culnorum Will. — Wstd. 373.

cupressiformis Wood. - Wstd. $36^{\circ}$.

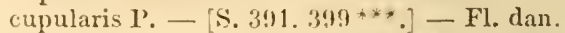

X11. $2159.2 .-$ Ann. sc. n. 1916. XIV. t. 19.

f. 1. Mont.). - Caespit.) Linn. Tr. 155!?.

XXII. t. \$६. f. 170. Fruct., Curr.) - Dsm. III.

741). — Toges. 115. T11. - Wstd. 525.

- Curreyi. - R. f. 250. (apiculata Curr.). eurta. - S. 391 .

currirostra. - S. 393.] - Letell. 656, 1.

cuspidata. - [S. 392.$]$

eyanogena. - Dsm. 1760. - Kil. II. (j+1.

- Vistd. f116.

- cyathoidea P. - Wstd. 75.

cytisporea. - S. 393.]

_- Jaldiniana Nectria - Not. Sfer. 7.

__ decedens Fr. - S. 392.] - Fuck. s. 540. decidua Td. - Wstd. 366.

decipiens Fr. - R. f. 14. - Bull. ac. belg.

15.5. VII. p. 365. f. 5. (Wstd.). - (Diatry.)

I)C. : Lim. Tr. 1459. XXII. t. 49. f. 196. (Fruct., Curr.

decolorans Nectria' P. - Wstd.364.366. -

Linn. 'Tr. 1559. XXII. t. 49. f. 176. Fruct., Curr. .

$\ldots$ Schum. = coccinea Fl. dan. - Voges. 57\%. cinnab. Td. .

decorticans. - Wstd. 529. - ef. Valsa.

- Sow. = S. Stigma Fl. dan. - Voges.

765. Penic., ; $: 373$ v. zu Stigma. - Wstd. 112. - decorticata. - Loud. f. 1636j5. - Wstd. 529. defossa. - Alger. t. 26, 5.

dehiscens 1 . - Wstd. 'Yu9.

delitescens. - [S. 419. §.] - Act. Turin.

1553. XIII. Dec. \&. f. 9. Notar. - - Kl. 1039.

Delphinii libh. - Kl. 747 .

- Dematium P. - [S. \$20.] - Kl. 154. - ef.

Vermicularia. - Caulicol. Linn. Tr. Is5?.

XXII. t. 59. f. 123. Fruct., Curr.). - Brit. f.

151. - Wstd. 575.

dendroides. - Carol. II. 61.

denigrans Curr. - Diatry. s. Valsa, Linn.

Tr. 1559. XXII. t. 46. f. is. p. 270. Fruct.). depazeaeformis. - Kl. 1611.

depazeoides. - \$. 395.]

depilata. - S5. 359.]

deplanata. - [S.394.] - Kl. 1737. (cf.Valsa depressa. - Wstd. 6- - Diatrype bullata,

R. f. 536 .

- Desmazieri. - Dsin. III. 369. - Nectr.) = coccinea $r$. cicatric. Dsm., Not. Sfer. 4.

deusta Whlb. - Wstd. 713. - Carol. V. TII. devexa. - Dsm. III. 367.

Dianthi. - S. f26.? - Ital. 617. - Depaz

Brit. f. 157. - Wstd. 293. 646.954. - Cucur-

bit.) Not. Sfer. 59. - Plcospora, Not. Sfer. 60.

- Venturia Not. Sfer. 42.

dichroa. - Alger. t. 26, 6.

digitata Bolt. - Wstd. 167.

diminuens. - [S. 391.] - Wstd. 1205.

dioica. - [S. 391.]

diplasia. - Alger. t. 27, 5 .

disciformis. - S. $35 t^{*} .419^{* *}$.] - Brit. f.

172. - Voges. \&1). - Carol. 1. 45. - Norweg.

195. - Diatry. Wstd. 1106. 'grisea.

— discreta. - Carol. IL. 57. Diatry. .

_- dispersa Botryosph.) Not. Sfer. 92. dissepta Fr. - Wstd. 910. 
118.

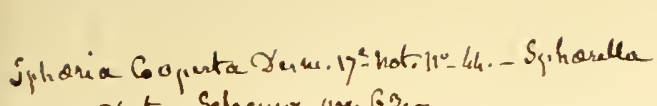

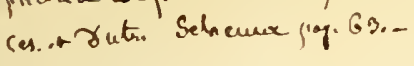


119

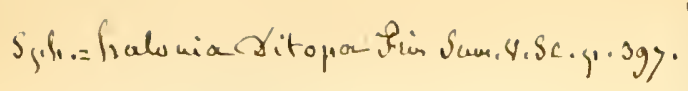


Sphaeria ditopa. - [S.397.] - Voges. 1245. Wstd. $47 \overline{7}$.

- ditricha. - [S. 420.]

dolabriformis Lophiostoma, - Not. Sfer. 69. Doliolum. - [\$. 391 **. 391.] - Voges, 571. Letell. (j\$6, 4.

- conoidea. - Ital. Sig.

- dolosa. - [S. 392.] - et $\beta$. Massarae (Valsaria Not. Sfer. 54.

f. Iothidea b. Rosac. - Voges. 971. - Brit.

f. 32 .

dryina. - [S. 392.]

dubia P. - Cenang. Ceras. Fr.

Dulcamarae. - S. 391.]

duplex. - [S. 394.] - Yoges, I1151. Nardi.

IVstd. 1214.

- Duriaei. - Alger. t. 25, 7.

Echinus. - IBivona t. 4. f. 1.

ectypa. - [S. 392.]

elliptica. - Loud. f. 16373. -- Stroma-

tosph.) Grev.: Wstd. i13.

- elongata. - [S. 391.] - Voges. 57... - Ca-

rol. III. 56. - Letell. 692, 3.' 'scabra S.) -

Wstd. 715. - Cucurbit. Not. Sfer, 5li.

- emperigonica. - Auerswald, Bot. Unter-

haltg. 1S63. S. 379. - (Lizonia Not. Sfer. 77.

- Empetri. - [S. 417.]

endochlora. - [S. 359.

entomorhiza. - [\$. 351. 5.] - Wstd. 1201.

epidermidis. - [S. 390.] - Wstd. 3it.

epimyces. - [S. 405.]

episphacria. - ef, sanguinea.

equina. - [S. 359.

Equiseti. - [S. 397.] Malon. - Wstd. 632.

Eres (Villosae). - Ann. Mg. n. h. 1552. IX.

t. 9. f. 4. p. 320. 13. B.

Ericae. - [S. 391.

errabunda Rob. - Dsm. 1411.

erubescens Rob. - Dsm. 1116.

eructans W. - Fuck. s. 5it.

- Eryngii. - Voges. 1052. - Dsm. So0. -

Kl. 1639. - Fuck. s. 525. - Wstd. 11 S.

- erysiphina. - Journ. hort. Soc. 1 154 IX.

p. 65. f. S. (Berk.).

erythropyrenia. - S. 397.]

erythrospora. - Kil. 1527.

erythrostoma P. - Fuck. s. 523.

escharoidea. - Ann. Mg. n. h. 14t2. X. t.

12. f. 19. p. 345. Merk. - [S. $3 \triangleleft 6^{*}$.] Melogr. eucrypta Foliicol... - Ann. Mg. 11. h. 1552.

IX.p.353.t. 12, f. 40. Fruet., B. 13.). - R. f. 49. eumorpha. - [S. 35\%.]

eunomia Fr. - S. 392.] - Fuck. s. 579.

euomphala. - Carol. IV. 54.

Euphorbiae. - Fuck. Nass. f. 2\%. - Fuck.

s. $5-19$.

$$
\text { eustoma. - [S. 395\%.] }
$$

eutypa Obtect. Fr. - Voges. 96 $\overline{\text {. }}$ - L Linn.

Tr. 185ั9. XXII. t. 5ร. f. 73 . Curr. - Brit.

f. 178 .

Eronymi Kz. - Voges. 1450. - Kil. 756. -

Fuck, s. 602. - Dsm. 797. - Wstd. 930.

- examinans. - Hook. Lond. J. 1S+2. I. t. 7.

- Nectria) Linn. Tr. 1559. XXIr. t. 49. f. 172.

(Fruct., Curr.).

- excipuliformis Fr. S. (Platystom.) - - [S. 391.] - Dsm. 1763. - Aun. Mg. n. h. 1559. III. t. 11 . f. $26^{*}$. B. B.) - Kl. 962 . - Voges. 1164.

exosporioides Dsm. - R. f. 1 10. - Dsm. 769. extensa (Valsa) Fr. - Limn. Tr. 1539. XXII.

t. 4i. f. 116. (Fruct., Curr.).
Sphacria fagicola. - [S. 395.]

- faginea Valsa) Fr. - [S. 11 \%.] - Linn. Tr. 15.59. XXII. t. 45. f. 16s. Fruct., Curr.). Wstd. $90 \%$.

- fallax Whlb. - [S. 362. 6.] - Kil. 1960. -

(Amphisphaeria Not. Sfer, 72 .

- farcta (Obtect.). - Ann. Mg. n. h. 1s,2.

IX. t. 10. f. 15. p. 324. (Fruct., B. B.).

- fasciculata (liombardia). - [S. 354.] - Ki]. 1939.

- favacea. - : Kl. 1323. - ef. Diatrype. -

Dsm. 1751. - 13rit. f. 77. - Norweg. 92. -

Diatrypella Not. Sfer. 30.

_- fenestrans Caulicol., - K Kl. 1933. - Wstd. 12114 .

- ferruginea P. - Dsm. 1753. - Kl. jł2.

Diatry. Linn. Tr. 155!. XXII. t. 47. f. 91. Fruct., Curr.). - Voges. 377.17 . (ef. quaternata.- Letell. $703,5 .-$ Wstd. 215.915. -

Melogr. Not. Sfer. 52.

- Festucae. - Lib. 246. - Wstd. 1220.

- fibritecta. - Ilook. J. 1453. V. t. 3. f. 11.

fibrosa Concresc. . P. - Brit. f. 30. - Limn.

Tr. 1 -5!1. XXII. t. 17. f. 94. Fruct., (urr.). filicina Fr. - S. Pteridis Kz., rimosa b. A.

S. - Dsm. 266. - Brit. f. 33. - Lib: 243.

I'teridis Sow.

- Filicum Dsm. - Kil. 10\%1. - Kil. II. 5i31.

- Fuck. s. 531. - Dsm. 243.

- filiformis A. \$. - Biscl. f. 3491. - Carol.

II. 5.. Xylar. .

- Filum. - Bivona t. 3. f. I. C. D. E. - ef.

Ascochyta Convolvuli.

fimbriata 12. - [S. 391.] - Bisch. f. 3.562.

- Krypt. Bad. 427. - Lib. 40. - Act. 'Turiu.

1519. X. Dec. 5. f. 1. (Notar.). - Kl. 53 . -

Iil. 11. 512. - Fuck. s. 601. - Dsm. 269. -

Confert.' Linn. 'Tr. 1559. XXII. t. 49. f. 212.

Fruct, Curr. - Brit. f. 36. - Wstd. 167. -

Schweiz. 15.

- - Carpini. - Voges. 2i 7 .

fimetaria. - Kl. 1733. - ef. Sporormia.

fimeti. - S. 397. $41 \mathrm{~s}^{* *}$.] - Hdw. I. t. 14.

f. B. 1. - Hypocopria; Massaria Berk - -

Fuck. s. 514. = S. stercoris DC. - [C. 397 .

Hypocopr.]

_ v. equina Concresc. P.: Linn. 'T'r.

XXII. t. 47. f. 102. Fruct., Curr.)

fimicola. - Dsm. 1761.

fissa. - [S. 102.$]$

flabelliformis. - Carol. II. 51). Xylar.

- flacca W. - Fuck. s. 6:33.

- flaccida A. S. - Kl. 551. - Voges. 1150.

Wstd. 451.

- flavescens. - is. 35\%.]

flavovirens P. - Kl. 1243; 1643. - Dia-

try. Linn. Tr. 15 59 . XXII. t. 46. f. 65. Fruet.,

Curr. . - Voges. 375. - Wstd. 112.

- Hoccosa. - [S. 393.]

- floriformis. - [S. 35.5. leiopl.]

foeda Amphisph. - Not. Sfer. 75.

- foedans Fr. - [S. 396.] ef. S. amblyospora.

- foraminosa. - Voges. 7 it (lanciformis Fr.).

- foraminula P. Valsa. - Linn. Tr. 15 \%.

XXII. t. 4i.f. 127. Fruct., Curr.).

- foveolaris Fr. S. 419.] ef. Sphaeropsis.

- Fragariae Lasch. - Kl. 1250.

- fragariaecola Wilr. - Wstd. 175.

- fragiformis P. - Wstd. 364. - Dsm. 95\%.

- Voges. 273. - Wstd. 903. - Kl. 165. - Fl.

dan. XII. 2149, 2. - Hypoxylon coccineum 
Bull. - Iypoxyl., Limu. Ty. 1 55!) XXII. t. 15.

f. 3i. Fruct., Curr.) - Loud. f. $1633(6 i$.

splia c ria fragiformis v. radians Fr. - Fl. diu.

XII. 215\%. 2 .

fraxinca Sow. - S. concentrica Fl, clan.

Fraxini. - s. 417.] — Wstd. 9332.

- fraxinicola M. S. Depazea). - Linn. Tr.

小.5!. XXII. t. 59. f. 1 in liruct, (mix.

- finticun Riob. - I)sm. 1771). - of. Raphli-

dospora Ononidis Awd. - Wstcl. 1207.

- fuliginosa P. - [S. 391.] - Kl. 1210. -

Toges. Till.

— fulva Fr. (Byssis.). - Linn. Tr. 1 559 . XXII.

१. : i. f. 5. Fruct., ('urr.)

- fulvo-pruinata B. Valsa) - Iimn. Tr. Is,is.

XXII. t. 17. f. 125. Fruct., Curr.

- furfuracea Fr. Valsa). - Linn. 'Tr. 14.ัง.

XXII. t. 15. f. 151. Fruct., Curr.)

— fusariispora. - Ann. sc. n. 1455. III. t. 6.

f. 5. Mont.)

_- fusca Pers. - Toges. 1is. - Fl, dan. XII.

2151.1; - XII. 2036. 3. ef. IIypoxyl.). -

Schnzl. t. 15. f. $21-25$. - Bot. Zt. 1556. t. 5.

f. 15. b. Fruet., Hoffm.) - Bisch. f. 3495. -

Kl. 247. - Pringsh. Jahrb. II. t. 3! f. 30 ,

(Hoffin.). - Hypoxyl., Linn. 'Tr. 1559. XXII.

t. 45. f. 3५. 39. (Fruct., Curr.). - W'std. 219. Krypt. Had. 151.

- - f. byssiseda. - Kil. 96i1. - Dsm, III

79. - Carol. I. 46. - Norweg. 194.

- fuscella Obtect.). - Amn. Mg. n. h. 1552.

IX. t. 11. f. 20. p. 325. Fruct., 13. B.) - Linn.

Tr. 15.9. XXII. t. 59. f. 103. Fruet., Curr.

fuscospora Hypox. ?) S. - Linn. Ir. 1659.

XXII. t. 46. f. 55. Curr.

— fusispora. - Ki. 1532. - [S. 356, ] Melogr. -

Ditopella) Not. Sfer. 15. p. 42.

- futilis Obtect. - - Ann. Mg. n. h. $1<\xi 2$.

IX. t. 11. f. 23. p. 326. Fruct., B. B.)

- Gagliardi Sordaria). - Not. sfer. 21. p. 23. galbula. - $[$ S. 404.]

Galii. - S.391.] - K1. 362. - Dsm. 1425.

- Toges. 1171. - R. f. 535 .

Galiorum Rob. - Dsm. 1435.

- Gangraena Fr. - Dsm. 767. - cf. Dothidea

R. f. 350.) - Voges. 1166. - Carol. II. 59.

— gastrina Fr. S. - Wstd. 266. = irregularis

Sow. (Curr.). - Dsm. 754 .

_- gelatinosa Td. - Fl. dan. X. 1752. - Fuck. s. 696. - Bisch. f. 3499. - Hypocr.) Not.

Ster. 1.

_- genistalis. - Voges. $4 \$ \$$.

- geographica. - [S. 425.] - Kil. 1311. -

Wistd. 265. - Fuck. s. 351. - ef. Asteroma!. Geranii. - [S. 121.] - Wstd. 537.

- gigantea, - Alger. t. 26. 3. - Ann. se. n.

1 3 3!. I. t. 11. Mont.) - Confert, Lim. Tr.

1559. IXII. t. 19. f. 206. Fruet., Curr. .

- gigaspora Obtect.) Dsm. - Wstd. 1104. I) $\mathrm{sm}$. 1765. - Limn. Tr. 1559. XXII. t. 59. f. 100. Fruct., Curr.]. - [S. 39s. Saccothec. Corn.

- glaucina. - [S. 402 .

- glanco-punctata Grev. - Linn. Tr. 1559.

XXII. t. 59. f. 114. Fruct., Curr.). (ileditschiae Schw. Confluent.).- Kil.II. $\$ 1$. Glis (Byssised.) Curr. - Lim. Tr. 18.59.

XXIl. t. $5 \%$. f. 1. p. 314. Fruct.)

_._- gloeospora I3. C. (Caulicolae). - Ann. Mg.

n. h. 1sii. TII. t. 1\%. f. 33. p. 454. glomerata DC. - Wstd. 219.
Spluaeria Ginomon. - [S. 391.] - Act. Turin. 1 tisi. XIII. Ilec, (i) f. 10. (Notar.). - Fuck. s. $531 .-1) \mathrm{sm} .279$.

Schum. = S. Coryli l'l. dan. - Voges. 37!. - Brit. f. 34.

- Godini Dsns, - Voges. $121 \%$. - Sow. t. 336. ex Berk. Ont. p. 397.). - (Seriat.) Iinn. 'l'r. 145\%. XXII. t. 19. f. 202. (Fruct., Curr.). I) $\mathrm{sm} .974$ - Wistr. 270.

- graminicola B. (1)epazea). - Lim. Tr. \\59. XXII. t. 59. f. 151. (Fruct, Curr.). - Brit. f. $1 \&$ i.

- Graminis 1. - Lib. 143. -.. Bisch. f. 3591. - Dsm. 26-; III. -\1. - Kl. II.617. - Fuck. s. tivi3. - (Confert.) Linn. Tr. 1559. XXII. t. 49. f. 211. (Fruct., Curr.) - Voges. 876 . - Wstd. 523.

- grammodes. - [S. 391. \$6.] - Act. 'Turin. 1541. III. I)ec. 2. f. 4. (Notar.). - K'1. 19111.

- Granatum. - cf. Nectria.

gramulosa. - Wstd. T13. - ef. IIypoxylon. gregaria. - Lib. 145 . - Wstd. 316.

- grisea DC. - Wstd. 1106. - Kl. 539. Sphaeria disciformis var. (Wstcl.).

- Grossulariae. - [\$. 394.] - Kil. 1563.

Gunnii (Cordyc.) B. - Linn. 'Tr. 1559. XXII.

t. 15. f. 1. 2. (Fruet., Curr.).

- guyanensis (Xylar.) M. - Lim. Tr. 1454.

XXiI. t. 45. f. 9. (Fruct., Curr.).

- Gypsophilae Lasch. - K1. 1050. - Fuck. s. 547 .

gyrosa. - [S. 35.5*] - Carol. I. 29.

- haematites, - Dsm. 177.1. - Wstd. 911.

- haematorhyncha. - [S. 392.]

- hapalocystis (Circinatae). - Ann. Mg. n. h.

1 52 . IX. t. 10. f. 12 . p. 31 \% (B. B.). - IR. f. 251 .

- Hedcrae Sow. - [S. 417.] - Act. Turin.

1५41. III. Dec. 2. f. 5. (Notar.) - Ism. 790. cf. Diplodia H. - Wstd. 173.

- hederaecola Fr. - Bisch. f. 3602. - (Depazea) Dsm. III. 362. - Wstd. 29.

- Helenae (Caulicol.j. - Linn. Tr. 1559, XXII.

t. 59. f. 137, p. 331. (Fruct., Curr.).

- helicicola. - Dsm. 1755. - Wstd. 1113.

helicospora B. B. - R. f. 141 .

- Heliscris (Hypoxyl. s. Poronia) M. - Lim.

Tr. 1559. XXI1. t. 15. f. 13. Fruct., Curr.).

- Irellebori Chaill. - ? Dothidea Prostii Dsm.

(IVstd.).

- helminthospora. - K1.1735. - Ital. 651. Leptosph. Not. Sfer. Ss.

- hemitapha (Obtect.). - Ann. MIg. n. h. I 559.

III. t. 11, f. 36 , (B. B.).

- herbarum I'. - [S. 394.] - Dsm. III. 363. -

Voges. 51. - Letell. 619.4. - Wstd. 271. 172;

717 (Allii, 1112 (Aspar. Eryng. tecta), 1213

(I)ianthi), - Micr. J. 155.5. III. t. 12. f. 1-?). (Currey). - Fl. dan. XII. 215s. 2.

- Fr.: Ism. 279. 1429-1433. 1772. -

Act. Turin. 1515. ViI. Dec. 3. f. 1. (Notar.). -

Schnzl. t. 15. f. 43. - (Diplodia) P.: Kil. 52.

(cf. Pleospora). - Kl. 1564. - Microse. Journ.

IV. t. 11. f. 32. (Curr.). - Kil. II. $545-54$.

Fruct., Curr.).

- Fr. : Mesascium Stockii Berk. (Wstd.).

v. Anethi. - Kl. II. 547. d.

- v. Brassicae. - Iil. II. 547. b.

- - v. Euphrasiae - R. f. 254 .

- v. Tabae. - Kil. II. 517. c.

v. IJyoscyami. - Kil. II. 514. 



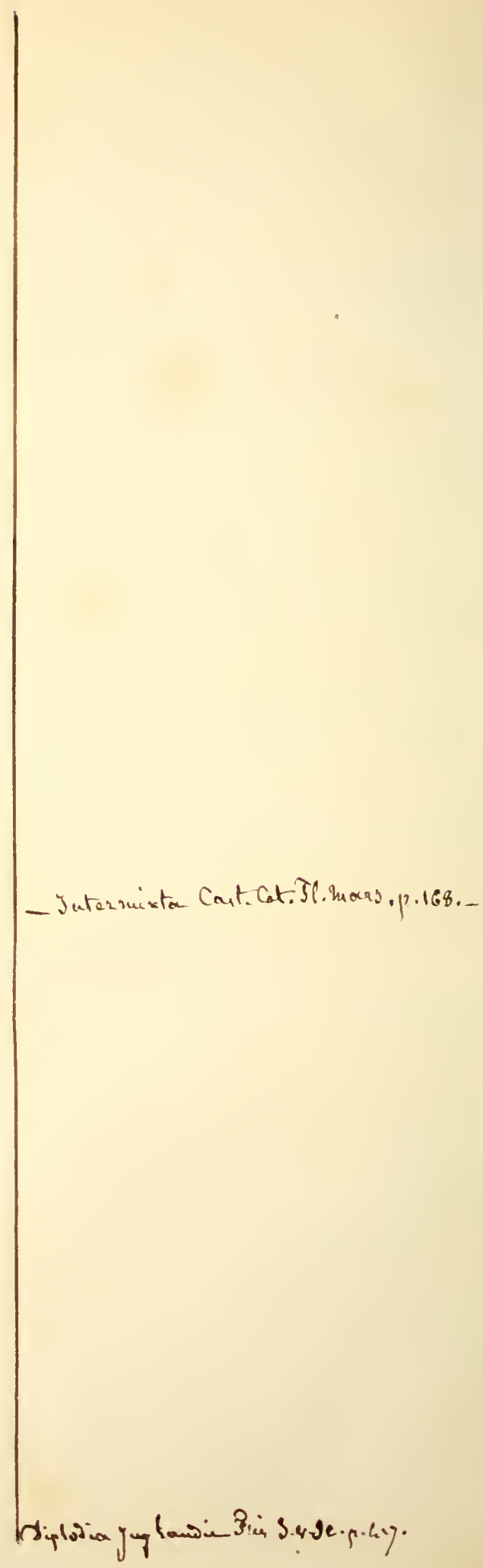


Sphaeria herbarum v. minor. -. Brit. f, $26 \pi$. f. spermogonifera! - lil. II. $5 ! 6$.

IIerculea Schw. - Cauloglossum transversarium, Carol:

- herpotricha. - S. 393. 3\%氵.] -- Act. Turin.

1557. XVI. Dec. 9. f. S. (Notar.). Fuck. s. fil7. - Usm. 7\3; III. 790. - Kl. II. (i52. Voges. 1147.

- herpotrichoides (Leptosphaeria). - No.t. Sfer. $4 \vec{\imath}$.

- heterospora Pleospora . - Not. Sfer. 1 ; 19.5.

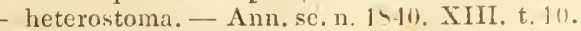

(Mont.).

_ hians Wlir. - [S. 122. 3.] - Labrella Poni

Mont.

-_ hiascens. - [S. 392.

- Fibisci. - Carol. I. 60).

- Ilimantiae P. - K1. 7.16. - Fuck. s. 354. (Asteroma).

- Hippophaës. - Bot. '/t. 1462. t. 12. f. 23. p. 37 . (Sollmann).

- hirsuta. - [S. 349.] - Loud. f. 16157. Fuck, s. 1;13. - Lsm. 1114. - (Villos.) Iinn. Tr. 1459. XXII. t. 57.f. 14. (Fruct., (Curr.)

Alger. t. 27, 1. (Spor.). - Wstcl. 70. - Lasioph. Not. Sfer. 1.

- hirta Fr. - S. 393.] - Fuck. s. 609. -

Wstd. 16\%.

- hirtella. - [S. 395.

- hispida Td. - [S. 359.] - Misch. f. 353.3. -

[ruck. s. (i) 1].

—_ hispidula. - Kl. 535.

- IIoffuanni Fr. Massar.). - IIdw. I461. Il.

no. 9. t. 9. B.

- holoschista P. B. (Subtect.). - R. f. 116.--

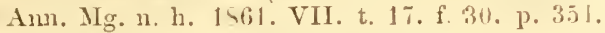

- homalea, - [S. 3<6.] - Melogr.

- II ügelii. - Cd. Ic. IV. t. 9. f. 12!) = Sphaeria

Robertsii. - Cd. Anl. t. F. f. 56: 21. (Cordyceps,

- hyalina S. IIypocr.).- I Iinn. Tr. 1 5.59. XXIT.

t. 46. f. 49. Fruct, Curr.).

- ivalospora. - Hdw. 1559. II. t. 2. f. 11.

(Ces.).

- hydrophora Sow. - Loud, f. 16.136.

hyetospilus Mart. - W'std. 637.

II yperici. - Carol. I. 59.

- hypodermia Fr. (Talsa). - 13rit. f. \&l. -

R. f. 3211 - Linn. Tr. 1559. XXII. t. 15. f. 156.

Fruct., Curr.

- hypotephra (Obtect.). - Ann. MIg. n. h.

is 52 . 1X. t. 9. f. 7. p. 321. Fruet., B. B.).

- hypoxyloides (Diatry.) - Not. Sfer. 21.

p. 26.

- Hypoxylon. - Foges. 272. - Kops Fl. bat.

VII. 510. - Bisch. f. 3149. - Xylaria) Kl. 251.

- Miuller, Pflanzenstaat. 270. - Linn. Tr. 1559.

XXII. t. 45. f. 17. Fruct., Curr.j. - ef. Rhizomorpha sube., Hypox. vulg. - Carol. I. 4\%.

- Wstd. 107.365: v. cupressiformis.

- Ivsteriformis Sehum. - Fl. dan. XII. 2 156. 2. hysterina. - [S. 402. Di. fiss.]

- Hystrix Td. - Voges. 959. - Bisch, f. 3502.

- (Diatry.) Limn. Tr. 15.99. XXII. t. 47. f. 92. Fruct., Curr. - Mamiania) Not. Sfer. 50. p. 13.

- janthino-velutina (Xylar.) M. - Linn. Tr.

1559. XXII. t. 45. f. 22. Fruct., Curr.).

- icterodes. - Hdw. I. t. 3. f. 2. (i-1). -

Kl. 1739.

L ? idaea Rob. - Dsm. 1446.

$\mathrm{H}$ off $\mathrm{m}$ ann, Index Fungorum.
Sphaeria Jenynsii (Pertus.). - Ann. Mg. n. h. 1559. III, t. I1I. f. 2.2. I3. B.).

- Jerdoni B. B. (Platystomae). - Am. Mg.

n. h. 1461 . TII. t. 17 . f. 2- p. 453.

ignavis Cueurbit. - Not. Sfer. is.

- Ilicis $\mathrm{F}_{1}$. - [S. 417. 367,3.] - (Diplod.)

Linn. Tr. 1559. XXII. t. 59. f. 117 . Fruct,

Curr. - Carol. IT. 63. - Wstd. 273.

- immersa. - Loud. f. $1637 \%$.

- impressa. - S. 423.

- inaequalis (1)iatry.) Curr. - Linn. Tr. 1559.

XXII. t. 16. f. 79. p. 270.

— imearcerata. - Dsm. 1121.

—_ inclinata. - I)sm. 17 1 .

- inclusa Lasch. - Kil. (i6).

- inconspicua. - Dsm. 7i6.

- incrustins. - \$. 3. 3,9.]

- incurva Śchum. - Fl. dan. XIII. 2332. 3.

—- Inne ij Valsa Curr, - Linn. 'Tr. 15.59 . XXII.

t. I4. f. 16iti. p. 2\1.

- inquilina. — \$. 393.? -- Act. Turin. 14 11.

III. I)ec. 2. f. 3. Notar.). - Obtect.) Linm.

Tr. 1954. XXII. t. 54. f. 92. Fruct., (urr.). ... Dsm. 176ti; III. Tร..

_ inquinans. - S. :996;.] - lierk. In. p. 251

f. 6.5.c. - Fres. t. 7. f. 3 1. Massaria - Ilook.

J. is.i1. III, t. 9. f. It, u. t. 10. f. 2. - III.

511. - (Obtectae) Limn. 'Tr. 1 59. XXII. t. 5\%.

f. 101. Fruct., Curr. - Norweg. 200.IIstd. 371.

— insiden; $\mathrm{S}$. ? Conflu. . - Linn. Tr. 1599 XXII, t. 4\%, f, 193. Fruct, Curr.

- insidiosa. - Dsm. is.. - Ann. se. n. IS11. XV. t. 14. $\mathrm{D}=\mathrm{m}$. ?

- incitiva. - IIdw. I. t. 15. f. 与. - Lisch.

f. 3523. - Aet. Turin. I - 33. XIII. Vec. 7. f. 1 .

Notar. - Auerswald, Bot. Unterhaltg. 1563.

p. :91. f, 132. Fruet.

- inspersa Obturat. Sz. - Limn. 'Ir. 1559. XX1I. t. 59. f. 112. (Curr.). - (Denudat.) B. : Lim. 'l'r. 1 54. XXII. t. 57. f. 27 . (Fruet., Curr. - insularis $\mathbb{W}$. - Fuck. s. 597. - Diatry. 3. : Linn. Tr. 1559. XXII. t. 49. f. 210 . Fruct. Curr.

- intermedia. - [S. $399^{*}$.]

- intermixta isubtect.) - Ann. Mg. n. l.

1552. IX. p. 32\%. t. 11. f. 24. Fruet., B. B.).

- interpungens, - IIeer, Fl. tert. Helv. t. 1. f. 3.

- interrupta. - Ann. se. n. 1s34. I. t. 11.

Nont.)

- intexta Valsa?, Curr. - Lim. Tr. $155 \%$.

XXIl. t. 45. f. 169. p. 279. (Fruet.).

- inverecunda. - Act. Turin. 1553. XIII.

Dee. 6. f. 7. Notar.'.

- inversa. - S. 391.]

- involuta Xylar. Kl. - Iinn. 'Tr. 15.59. XXII.

t. 45. f. S. Fruet., Curr.).

- irregularis Diatry.) Sow - gastrina Fr, S. -

Linn. 'Tr. 1559. XXII. t. 47. f. 91\%. Fruct, Curr.). - Wstd. 266. 4i2.

- isariphora. - Dsm. 791. - Toges. 1453. -

Wstd. 1T.

- ischnostyla. - Dsm. 17S . - Kíl. 1834. -

Fuck. s. $5.3 \%$. (petioli).

- ischnotheca. - Dsm. 1795.

_ _ isiaca C'es. - Kl. II. 40.

__ juglandicola S. Diplodia ?). - Linn. Tr. 1559.

XXII. t. 47. f. 121. (Fruet., Curr.). juglandina Botryosph.). - Not. Sfer. $\$ 9$. Juglandis (Obturat.) Fr. - [S. 117.] - Linn. 
Tr. 1559. XXII. t. 59\%, f. 111). H1. Fruet, Curr. - Kl. 1 $2-1$.

sphaeria Juglandis I)C. non Fr.) - KI. II. (ii; 9 . - Valsa) S.: limn. 'lr. 1 5!!. XXII. t. At.

f. 145 . Fruct., Curr.

- Jinci seriat. Fr. - Voges, Y(i.1. - Iinn.

Tr. 1 $54 \%$ XXII. t. 49. f. 199. Frnct., Curr.

- Iil. 6ifi. - Fuck. s.fil) (Dothid.) - I) $\mathrm{sm}$.

972. - Brit. f. $35 .-$ Wstd. $221 \%$.

— juncina. - Carol. T. 71.

— Juniperi. - S. 345. 22.] - Kil. 1533.

— Kierriac. - Wstd. 542.

— lietmiae Concresc. - Kl. 1934.

— Fretsclmari Rbh. - Kl. 574.

- Kunkleri. - Heer, Fl. tert. IIelv. t. 1. f. 6. - Tínzei Valsa Fr. - Limn. Tr. 1559. XXII.

t. 1.. f. 13\%. Curr. .

- lahiata. - Wstd. 713.

- Laburni P. - [S. 390.] - Kl. 960. - Fuck. s. 139\% - Dsm. f11. - Cucurbitar.; Kl. II. 12. - Caespit. Lim. Tr. 1-59. XXII. t. 19. f. 179. Fruct., Curr.. - Voges. \$73. - Letell. 619. 2. - Wstd. 363. - Ital. S75.

- lactea Iypocrea Fr. - Kl. 351. - IIoffm.

Ic. t. S. f. 3.

— Lactifluorum. - Carol. I. 5 1.

— lievata. - S. 390.]

— laevis Sow. - Loud. f. 16429 - lagenaria. - Hdw. I. t. 15. f. 2. - Ceratostoma P. : Kil. 967.

— lageniformis. - Bot. Zt. 1562. t. 12. f. 21.

p. 34(). Sollmann.

- lampadophora. - Ann. Mg. n. h. 1559. III.

t. 11. f. 29. B. B., - R. f. 139.

- lamprotheca. - Dsm. 17:9. - cf. Dothidea populina Wstd.

Lamyi. - Dsm. 39. Nectria .- Not. Sfer. 9.

- lanata. - 5. 393. - Act. Turin. 15.53. XIII.

1)e. $\div$ f. 9. Notar.;

- lanciformis Fr. - Dsm. 1401. - Fl. dan.

X11. 2156. 1. - Bisch. f. 3513. - Kil. 1323. -

Diatry. Fr. mon Kl. 1323. an Sphaeria faracea

Fruct., Curr. . - Voges. 77. foraminosa, -

I.etcll. 703. 2. Betulae . Pseudovalsa Not.

Sier. 53.

- Landeghemiae. - Bull. ac. belg. 15.59. VII.

p. 365. f. j. Wstd.

- laivata. - S. 359.

lasioderma. - Alger. t. 2i. 5.

lata. - Yoges. 565. - Kil. 1560 - Diatry.

Linn. 'Tr. 1559. XVII. t. 47. f. 110. Fruct., Curr.

— polycoeca Fr. — Bisch. f. 3505.

lateritia Hрpocrea Fr. - Kl. 254. - R.

f. $31 \%$ - Linn. Tr. 1459 . XXII. t. 46 . f. 47.

(Fruct., Curr.). - Dsm. III. 776. - Toges. 1334.

- Laurocerasi. - Dsm. i\$2. - Wstd. T4. -

Sphaeropsis epiphylla Lér.

—- Lebiseyi. - Voges. 1254. - Dsm. 73.

- lecanodes Nectria Ces. - Kl. II. 525.

- Lecythea Schw. Diplod.) - - Linn. Tr. $1>59$.

XXII. t. 55. f. 55. (Fruct., Curr.).

Legurn. - cf. Pleospora. - Kl. II. 518. W Wlir.: Wstd. 72.

Leguminis.

plodia Lér.

Leightoni (Foliicol.). - Ann. Mg. n. 1. 1552.

IX. t. 12. f. 43. p. 35.5. 'Fruct., B. 13.?

- leioplaca Fr. Diatry. - - Iinn. Tr. 1559.

IXII. t. 47. f. 101. Fruet., Curr.,
Sphaeria leiphaemia Fr. - Voges. $961 ;$ u. S69. = leiphaem. Ir., exTul., - Kl. 1326. (= Cytispora 1.) - 1).m. i5li. - (Valsa Fr. (=taleola ?) Limn. 'Ir. 15.5!. XXII. t. 48, f. 137. Fruct., Curr. . - Ital. ST.

lenta Td. - Bisch. f. 3j11. - (Iypocrea)

Linn. Tr. 1 引9. IXII. t. 46. f. \$3. Fruet., Curr.). - leprosi Fr. Diatry.) - Limm. Tr. I 559. XXII.

t. 46.f. 57. Fruct, ('urr.). - Dsm. 753.

- leptidea Fr. - S. 395.] - Kl. 1018. Fuck. s. 522.

- leptospora ('ucurbit.). - Not. Sfer. 61.

- leptostyla Fr. - Kl. 1521. - Kl. II. 63פ. Fuck. 8. 534.

- leucocephala P. - Wstd. 15.

- leucoplaca. - Carol. IV. 61.

- Icucosticta DC. - Wstd. 173.

- leucostoma. - Brit. f. 31. - Voges. 659. pustul.). - Letell. 692. 2. - Talsa Not. sfer. is.

Wstd. 909 .

lichenoides. - ef. Spilosphaeria Chelidonii). - Kl. II. 5.52.

_- v. hederaecola. - Voges. 663. - Wstd. 124 Chelidon. ; 295 Convolv. ; 20 u. $13 \triangleleft 3$ hederaecola ; 649 (Convallar.)

— lichenopsis. - Flora. 155. t. 3. f. 6. 7. Massal.

- ligneola C'eratostom., - Ann. Mg. n. h. is 39 . III. t. 11. f. 29. B. B.).

- ligniaria Platystom., Gr. - Linn, Tr. 1559. XXII. t. 5y f. 6ij, Fruct., Curr.

— Ligustri Rob. - Dsm. 796. - Wstd. 6.) f.

- limaeformis S. 'Sphaeria, - I Imn. 'Tr. IS59. XXII. t. 47. f. 107. Fruct., Curr.) - Carol. III. 55 .

- Liminghii. - Wstd, 1202. - Bull. ac. belg. i 5.5 . VII. p. 365. f. 3. Wstd. - - R. f. 263 .

- limitata P. - Fuck. s. 5 il.

- linearis. - S. 391.] - Kl. II. 2.51.

- lineata A. S. - [S. 392.] - Bisch. f. 3591.

- lineolata Seriatae. - Ann. Mg. n. h. 1852.

IX. t. 10. f. $*$ * p. 3is. B. B., - Dsm. 763.

_ Lingam Td. - [S. 394.] - Bisch. f. 3563. Voges. 1271.

- lintearia Aposphaeria, - Chile n. 10. f. 6.

- Lirella. - [S. 393.] - Kl. 1332. - Brit.

f. $37 .-$ Kl. II. 539. - Fuck. s. 60\%. - Dsm. 9:2. - Foges. 66s. - Lib. 42.

- Liriodendri S. Diatry.! - Linu. Tr. 1859.

XXII. t. 46. f. 72. Fruct., Curr.).

Lisae. - Act. Turin. 1545 . VII. Dec. 3. f. 3.

Notar.. - Kl. $1 \leqslant 46$.

- livida Fr. Obtect. - - [S. 392.] - Linn. Tr.

15.99. XXII. t. 55. f. 77. Fruct., Curr. - - Alger. t. 26,5 .

- lobata S. Hypocrea,. - Linn. Tr. 1559.

XXII. t. 16. f. 52. (Fruct., Curr.).

- longissima P. - [S. 119.] - Kl. 966. a. b. -

ef. Sphaeropsis.

- Lonicerae Fr. Obtur.). - [S. 39].] - Linn.

Tr. 1 59. XXII. t. 59. f. 115. Fruct., Curr.,

- Lonicerarum Depaz. - D Ds. III. 360. longispora Curr. Ceratost. - Linn. Tr.

15.59. XXII. t. 55. f. 69. p. 322. (Fruct., Curr.). longissima P. - Wstd. 1139.

- lugubris Rob. - [S. 394: 1S0! cf. 197.] — Dsm. 1 442 . W Wstd. 1219.

- Lunariae Caulicol. . - Amn. Mg. n. h. I 4.59

III. t. 11 . f. :3i. B. 13., : 
- Isewo valia Lancitormin Cos, Atsut. Schavera

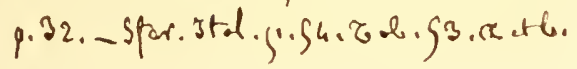

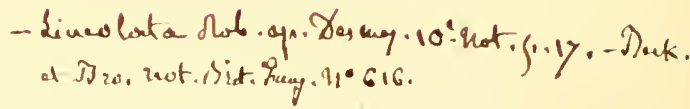



Sphaeria lutea A. S. ! - Bisch. f. $3511 .-$ (Hypox.) Linn. Tr. 15.9. XXI1. t. 46. f. 56. (Eruct., Curr.).

- luteola. - Dsm. 17is.

- luteo-virens Fr. IIypocrea' - Limn. Tr.

ム.59. XXII. t. 46. f. 53. (Fruct., Curr.!.

- Luzulae Rbh. - R, f. 533.

- lycoperdoides Weig. - Wstd. 219.

- lycopodina Mont. - ? = Crepini Wstd

macrospora. - Dsm. 1756. - Micr. J. 15.5?.

VII. t. 11. f. 19. Fruct., Curr.).

- macrostoma. - [S. 391.7 - Bisch. f. 3552. -

Dsm. 772. - (Platystom. Td.: Linn. Tr. 14.5!)

XXII. t.54. f.64. 65? Fruct., Curr. . - Wstcl. 909. 1205 (Aceris).

- macrostomoides. - Act. Turin. 1553. XIII.

Dec. T. f. li. Notar.

- macrotricha Villos . - Ann. Mg. n. h

1552. IX. t. 9. f. 2. p. 319. 13. B.). - Berk

Out.t. J.f. 12. 'Spol.

- maculaeformis $\mathrm{Fr}-\mathrm{D} \mathrm{\textrm {n }}, 1757-1792$.

Fl, dan. Xll1. 2335. 1. - Voges. 661. — v. Aesculi Fr. - Fl. dan. XII. 2100. 3. maculans Dsin. - Wstd. 531. - R. f. 330. macularis. - Toges. 969.

- - Fr. - [S. 345.] - Dsin. 1131. - (Pi-

crostoma Fuck. s. 5iff.

- maculiformis ispluaerella P. - S. 395.] -

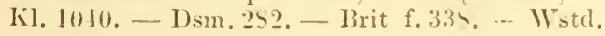

535. 1212. Castan.

- Malorum 13. Diplod. - Linn. Tr. 15.59.

XXII. t. 49. f. 205. Fruct., Curr.

Malvac Alecae Opitz. - W Wtd. 1232.

mamillana Fr. - S. $117 . \mathrm{T}-\mathrm{I}$ sm. 775.

Ki. 1331.

Fr. S. = S. Comi Fuck. Nass. p. 72; non Diplod. mam. Fr.

Tr. : Isull. ac. belg. 1409. VII. p. 365

f. 6. (Wstd. - - Wenders. Fr. : Linn. 'Tr. 145:).

XXII. t. 54. f. -5. Fruct., Curr.). - Voges. 1250.

- mammaeformis P. - SS. 3\$9.] - Fuck.

s. 557. - Denud.) Limn. Tr. 1559. XXII, t.5\%.

f. 37. Fruct., Curr.) - Voges. 3॰0.

mammillaris Schum. = S. umbrina Fl, dan. mammosa. -- Purton. 1I. t. 22.

- marginata Fr. Hypoxy1.! - Linn. Tr. 155?.

XXII. t. 16. f. 60. Fruct., Curr.). Schw.: S. annulata Fr. - Wste. 90ৎ.

Massalongii Mont. - Flora. 1.555. t. 3. f. 9. Massal. .

Massarue (Iypox.). - Not. Sfer. 14.

Massariana Valsal. - Not. Sfer. 3T.

mastoidea Fr. - S. 390.] - Dsm. 771.

Mathieui. - Bull, ac. belg. 1539. VII. p. 3136.

f. 12. (Wstd. . - Wstd. 1209.

Maydis Bk. - Diplodia Zeae Lér.

mediterranea. - Act. Turin. 1553. XIII.

Dec. 6. f. 2. (Notar.). - (Valsa Not Sfer. 43. p. 3 s.

- megalospora. - Ann.sc. n. 1540. XIV. t. 19

f. 4. Mont. . - Conflu.) Linn. Tr. 1659. XXII.

t. 49. f. 192. Fruct., Curr.).

- melaena Fr. - [S. 419.] - Kl. 533. - R.

f. 344. Phoma . - Voges. 1 15\%.- Dsm. 766 . cf. Sphaeropsis.

- nelanaspis. - Ann. sc. n. 1410. XIII. t. 10.

(Mont.) - Hypoxyl.) Linn. Tr. 1559. XXII.

t. 46 . f. 7. Fruct., Curr.

- melanoplaca. - Dsm. 1797.

- melanostigma Lév. - Démid. Russ. t. 5. f. 6. melanostoma Bernh. - Wstd. 1331.
Sphaeria melanostyla DC. - Fuck. s. 53.. Dsm. 143s.

- melanotes (Obtectae). - Ann. Mg. n. h.

1552. IX. t. 9. f. 6. p. 321. (Fruct., B. 13.).

— melasperma Fr. - Bull. ac. belg. 1559 . VII.

p. 36.5. f. \%. Wstd.). - Valsa) Limn. Tr. I s.5!.

XXII. t. 17. f. 123. Fruct, Curr.) - : Letell.

703. 4.

_ melastoma Fr. - cf. Valsa.

- melastroma Fr. - R. f. 254.

- Meliae Fr. Diplod.).- Carol. IV. 56.- I.inn.

Tr. 145. XXII. t. 4h. f. 11i2. (Fruct., Curr.). melina Obtectac). - Ann. Mg. n. h. 1 5!

III. t. 11. f. 33. B. I3. .

- melogramma P. - Jil. \$12. - Fuck. s. 625.

- Dsm. vagans Not.

- Schum. = S. rlodomela, Fl. dan. -

cont. Linn. Tr. 185 !. XXII. t. 49. f. is!.

Fruct., Curx.

Mereurialis Lasch. - Kl. 1251. - Fuck.

s. $5: 32$.

— merdaria I'ertus.) Fr. - [S. 397. Hypocopr.]

- Iinn. Tr. 1559. XXII. t. 5้. f. 56. Fruct., Curr.

- merlarum Fr. - Dsm. 176\%.

Mesascium. -- Not. Sfer. fi2.

- Michcliana. - [S. $\left.390^{*}.\right]-$ (Iypox. Not.

sfer. 13.

- Michotii. - Bull. ac. belg. 1559. VII. p. 366.

f. 15. Wstd. . - Wstd. 121\%.

- micraspis. - Hook. Lond. J. 15 42 . I. t. 7. -

I'ertus., Iinn. 'Tr, 1 .)!. XXII. t. 54. f. 59.

liruct., Curr.

- microceph. WVllr. - ef. Claviceps micr.

- microceras M. Xylar.). - Linn. Tr. 145?.

XXII. t. t5. f. 15. Fruct., Curr.).

microscopica. - S. 392.

microspila B. B. (Foliicol.) - Ann. Mg.n.h.

1 4 ; 1. VII. t. 17. f. 36. p. 155.

- microstoma P. - Kl. 1041. - Valsa) Linn.

Tr. 1459. XxII. t. 17. f. 130. (Fruct., Curr.). -

R. $f$ 253. (Microstoma rulg. Awd.).

- Micula Fr. - S. 391.62.] - Kl. II. 636. -

Dsm. iti. - (ytispora Linn. Tr. 1459. XXII.

t. jॅ. f. 70. Fruct., Curr. . - Voges. 1075.

militaris Elurh. - Bisch. f. 3453 . - (Cordy-

ceps L. : Kil. 47. - Míller, Pfanzenstaat. $2 i 0$.

- Limn. Tr. 1499. XXII. t. 45. f. 3. (Fruct.,

Curr. - Purton. I. t. 23.

millegrana. - Carol. IV. 55.

inillepunctata. - [S. 393.] - Lib. 41. -

Wstr. 475. - ('ryptosph.) Not. Sfer. 47.p. 40. milliaria Kz. - Kl. 1565. - (Diatry.) Fr. :

Linn. Tr. 1559. XXII. t. 47. f. 106. (Fruct,

Curr.!.

miniata Bolt. - Wstd. 90.

Iirbelii. - S. 419.] — Voges. 972.

mixta Schum. = ambiens, Fl. dan. - Wstd.

367 .

- mobilis Td. - Bisch. f. 3544 . - Tode II.

t. 9. f. 71 .

- modesta (Caulicol.) Dsm. - cf. caulincola. -

Dsm. 1436. 1773. - Ann. Mg. n. h. 1552. IX.

t. 11. f. 30. p. 379. (Spor. B. B.). - Fuck.

s. 515. - Wstd. 221 .

molybdina. - Voges. 1451.

monadelpha. - Kíl. 1245. - Dsm. 261.

cf. Talsa.

monocarpa Schum. - Fl. dan. XII. 2159.1.

Montagnei. - Ann. sc. n. 1534. I. t. 11.

(Mont.). - Alger. t. 2i. 2. (Spor.). 
Splua ria Mori Sow. = coccinea, Fl. dan. With: Wistd. 3iti.

-.. albae ('haill. = I)iplodia Mori Awd.

- moricola. - lil. 1561. - 1tal. 311. - 130tryosph.) Nat Sfer.

—_ moriformis. - [S. 395. 377. - Loud. p.1030. f. 16its. - Bisch. f. 3537. - Fuck. s. 5.5. Kil. II. 637. Bertia). - Bot, Zt. 165\%, t. 11. f. i). (Kicimg. Iloffin.) - Denud. 'T. : Linn. Tr. A 5!) XXII t. 5\%. f. 31\%. (Fruet., Curr. Voges, 3\2. - Wist. 25.

- moroides Denudat, ('nr'x. - Linn. 'Ir. 14.59.

XXII. t. $5 \%$, f, 34, p. 31t.

- Mougeotii Diatry.). - Iinn. Tr. $1559 . \mathrm{XXII.}$

t. 47. f. 97. Fruct., Curr)

mucida Fr. - Bisch. f. 3551.

Mï̈lleri. - Iil. II. $6+2$.

multiformis Fr. - Brit. f. 17\%, - Fl. dan. XII. 2149.1 . - Dsin. 75 1. - Kil, 163. - II $\mathrm{Y}^{\circ}$ poxyl.) Linn. Tr. 15.59 . XXII. t. 45. f. 25. (Fruct., Curr.). - Norweg. 193. - Wstd. i13. - - r. eoaretata. - Kil. 745.

_- v. rubiformis Fr. - Kl. 216.

- multiplex Kz. (Xylaria). - Limn. 'Tr. 1559

XXil. t. 45. f. 19. Fruct., Curr.

- muscivora. - Carol. I. 5\%.

- mutabilis P. - [S.349.] - Bisch. f. $35 \pm 1 .-$

Villos.) S. : Linn. Tr. 1459. XXII. t. 57. f. 211.

(Fruct., Curr.) - Sturm h. 4. t. 6-1.

_ mutila Fr. - [S.417.] ef. Diplodia m. Fr. S.

- Conflu.) Linn. Tr. 1559. XXII. t.49. f. 191.

(Frntet., Curr.) - Carol. IIT. 62.

__ mycophila. - S. 390.]

- myriadea (Sphaerella) DC. - [S. 396.] -

Dsm. $94.1410 .17 \checkmark 6$. - K1. 1042. - Fuck.

s. 1000. - Toges. $1175 .-$ Wstd. 73

_- myriocarpa Fr. - [S. 390.] — Fuck. s. 543.

- myrtillina Schub. - cf. Podosphacria Kun-

zei. - [S. 406.] - R. f 566.

- Nardi. - [S. 394.] - IIdW. I. t. 15. f. 7. -

W1. 1337. - Kl. II. 610. - Wstd. 1214.

— naucosa. - Kl. 1452.

- - Fr. : Amn. Mg. n. h. 1561. VII. t. 17.

f. 2\%. p. 453. Caespitos.'.

_- navicularis W7lr. - Tharand. Jahrb. 1853.

p. 111 . c. ic. Stein).

- nebulosa (sphaeropsis) I'. - [S. 393. 119.]

- Dsm. 267. 765. - Kl.540. - (Seriat.) Linn.

Tr. 1559. XXII. t. 19. f. 201. Fruct., Curr.). -

Voges. $107 \%$.

- nervisequa DC. - [S. 425.] KI. S49.

niclulans. - Carol. I. 50. - ? Wstd. 1110. Nidulus. - [S. 391.]

nigerrima (Diatry.) Blox. - Lim. Tr. 1559.

XXII. t. 47. f. 95. p. 272. Fruct., Curr.).

— nigrans (Caulicol.).- [S. 393.] - D sm. 1424.

- Ann. Mg. n. h. 1452. IX. p. 37\&. t. 11 , f. 27.

(Fruct., 13. 3.).

- nigrella. - S. 394.] - Kil. 1936. - Fuck.

s. 552. - Dsm. 956. - Caulicol.) Fr.: Linn.

Tr. 1459. XXII. t. 59. f. 130. (Fruct., Curr.).

nigro-annulata. - Carol. V. 73.

- nitens Holl. - Iil. 166.

- nitida. - [S. 391.] - Schum. = foedans,

Fl. dan. - Wstd. 901.

—- nivea Hoffm. - Voges, 27. - Fl, dan. XIII.

2155. 1. - (Valsa) Linn. Tr. 1559. XXII. t. 47.

f. 12 (\%. (Fruct., Curr). - Bisch. f. 3520. - Kl.

161. - K1. II. 635. - Krypt. Bad. 149. -

Talas Not. Sfer. 36.

- Notarisii. - Alger. t. 25, 6.

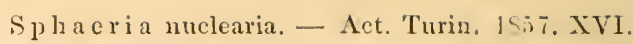
1)ec. 9. f. 1. (Notar.).

- nucleata (Diatry.) Curr. - Linn. 'lr. IS.j!.

XXIT. t. 46. f. T6. p. 2รT. (Fruct.).

- moula Fr. - S. 390.$]$ - Fuck. s. 563. Schweiz. 10.

- nummularia. - Voges, 371. - (IIpoxyl.)

Linn.'Tr. 1 59. XXII t. 16. f. 59. (Fuct., (ur.).

- obducens Schum. - [S. 34.4.] - Kil. 357. -

Fuck. s. 5.33.

— - Fr. : [S. 359.] - Brit. f. 1 $\%$. IIoffm.

Ic. t. 1ऽ. f. : - Not. Sfer. $66 ;$. obesa. - Alger. t. 27. 3. (Fruct.).

obliqua. - [S. 392 .

obliquata. - S. 392 .

_- obliterans (Obturat.). - Amn. Mg. n. l. 155!).

III. t. 11 . f. 31 . (B. B.).

- oblitescens (Obtect.) - Ann. Mg. n. h. 1459.

III. t. 11. f. 32. (13. B.). obstrusa Rbh. - Kl. 973.

obtecta (Obtect.) Curr. - Ann. Mg. n. h.

1)(il. VII. t. 17. f. 32. p. 45.1. -- XIicr. J. 15.99.

TII. t. 11. f. 16. (Curr.).

obturata. - [S. 4(12., 11 S.]

_- ocellata (Halonia) Fr. - [S. 397.] ef. Aglaispora. - Kil. 1327.

- P. Disp. - ef. Melogramma vagans Not.

-Obtect.) Fr.: Linn. Tr. 1559. XXII. t. 5-

f. 4 . (Fruet, Curr.).

ochralca. - Dsm. 1799. 1s!10

oculata. - S. 39 i.]

Dedema. - Voges. $\$ \$ 0$.

Ogilviensis (Canlicol.). - Ann. Mg. n. h

15.5. IX. t. 11. f. 24. p. 379. (Fruct., J3. B.).

Oleae DC. - ef. Diplodia Ol. - ISphae-

ropsis ? DC. : Linn. Tr. 1559. XXII. t. 59. f. 141 .

u. t. 55. f. 7,. (Frnet., Curr.). - (Diplod.). -

I1. II. 543. - Letell. 619. 3. - Ital. 14 '.

Olearum. - Act. Turin. 1553. XIII. Dec. 6.

f. 6. Notar.). - Dsm. 1117. - Kl. II. (j16. -

(I'ertus.) Cast. : Linn. Tr. 1459. XXII. t. 54.

f. 57. Fruet., Curr.). - S. umbrina Mont.

Olerum. - S. 591.] - Voges. 1076.

oligosperma. - ef. Melogramma.

olivacea Willd. - W'std. $3<3$.

oncostoma Obrallat.). - Ki. II. 253

opereulata. - [S. 392.] - (Obtect.) A. S. :

Lim. Tr. 1859. XXII. t. 55. f. 75. Fruct., Curr.

__ ophioglossoides Eberh. - Toges. 565. -

Bisch. f. 3491. - Ann. Mg. n. h. 35.35. I. t. 7 .

f. 4. (Fruct., Berk.) - (Cordyc.) Linn. Tr. I६

XXII. t. 45, f. 7. (Fruct., Curr.. - $\mathbb{N}$ std. (i!).

- Sturm J. 14. t. 61 .

oppansa. - [S. 393.]

oppilata (Hendersonia ?) Fr. - [S. \{112.] -

Linn. Tr. 1559 . XXII. t. 59. f. $11 \%$. Fruet., Curr. .

- opulenta. - Act. Turin. 1511. III. Dec, I.

f. i. (Notar.).

- orbicula. - Carol. IV. 64.

Not.

orbicularis P. - ef. Hyposylon nummular.

Not. ordinata Fr. - R. f. 329 . - Ann. Mg. n. h.

1561. VII. t. 17. f. 26. p. 453. (Denudat.). orcades Fr. - Voges. 1165. - Dsm. 169.

- Orni, - Act. Turin. 1553. XIII. Dec. s. f. 5.

Notar.).

- oropensis Nectria) Ces. - Kl. II. 521.

orthoceras (Ceratostoma). - Kil. 1455. -

Fuck. s. 56 s. 



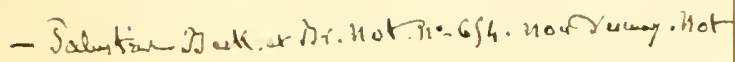

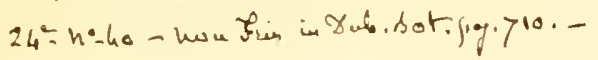

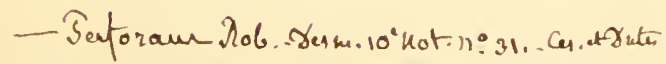
Schana p.6h. Sul Sphordlox.

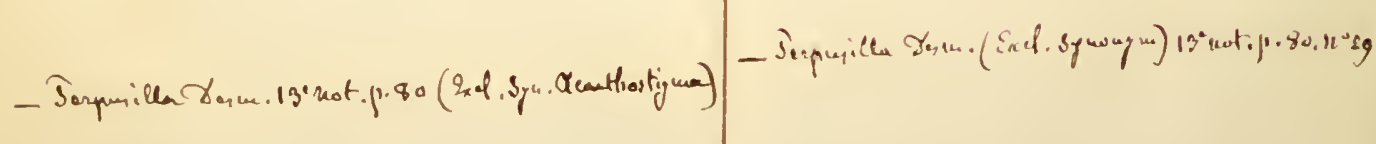


Sphaeria Ostruthii Fr. - [S. 125.] - Dsm.2Si. 1453. - Voges. 1053. - Brit. f. 330. - Wstcl, 372.

Ostryae Gnomonia.- - Not. Sfer. 49. p. 12. ovina P. - S. 34y.] - Biseh. f. 3573. Ki. 532. - Kl. II. 730. - Fuck. s.615. - Villos.) Limn. Tr. 155?. IXII, t. 57. f. 16. (Fruet., ('urr.). - Dsm. 1II. 95.

— v. glabrata Fr. - Fl, dan. XIII. 2333. 1. - ovoidea. - [S. 3६9.

- oxystoma - Voges. 767.

- padicola. - Lib. 119 .

_ padina. - Voges. 667. (C'enang. Cer. v. Pad).

- paccilostoma (Pertusae). - Amn. Mg. 11. h.

1459. III. t. 111. f. 23. (B. I3.).

- paetula. - [S. 393.]

palina. - S. 395.] - Voges. Sis

- Pallor (Depazea) Berk. - Linn. Tr. 145 9.

XXII. t. 5\%, f. 150. (Curr.

- palustris (Foliicol.). - Dsm. III. 36.5. Ann. Mg. n. h. 1552 . IX. p. 34t. t. 12. f. 3!. (Fruet., B. B.). - Berk. Out. t. 1. f. 12. c. (Spor.).

_- pantherina. - Brit. f. 3 !.

— papaveracea Cucurb. . - Not. Sfer. 60.

- papaverea. - Ann. Mg. n. h. 1551. VII.

t. T. f. 11. p. 15!. (B. 13.)

- Papareris Tul. - S. Brachycladii Lacr. huc: Brachrelad. penicill. Cd. /'I'ul. Sel. I. 43. .

— papillata. - S. 3901 - Fuck. s. 559.

Amplisph.). - Not. Sfer. il.

- parabolica Fr. - Bisch. f. 35.31.

- parallela Fr. - [S. 392.7 - F]. dan. XII

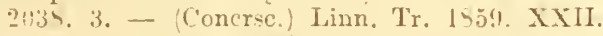

t. 17. f. 1111. Fruct., Curr.). pardalota. - Ann. sc. n. 153!. I. t. 12.

(Mont., - Brit. f. 175

- parmelioides. - Anm. sc. n. 1436. VI. t. 1S. (Iont.).

-_ parmularia B. Hypoxyl. ?) - - I.inn. Tr.

14.9. XXIl. t. 15. f. 41). Fruct., Curr.\}.

Patella. - [S. 365.] - Voges. 145. - Wstd.

32. 1211). (Limar.

- peduneulata Dieks. Aylar. - Linn. Tr.

1 99. XXII. t. 45. f. 5. Fruet., (urr.) - Brit.

f. 16 is. - IIg. Zool. Bot. 1 33 \%. Il. t. T. f. d--g. p. 225. Frurt., Berk.).

- pellita Fr. - S.359.] - Til. TI2. (var: : -

Fuck, s. 6!9) - Kl. 12!6; Kl. II. $i 19$. Pleo-

spora : Sphaeria Brachycladii Lacrx. - (Cau-

licol. Linn. Tr. 1\59. XXII. t. 59. f. 129.

Fruet., Curr.). - Wstd. 652.

- peltata. - Hook. Lond. J. 15.2. I. t. 7. -

Vistcl. il:?.

penetrans v. Patella. - Wstd. 32 $\frac{1}{4}$

- Penicillus. - KI. 1942. - Toges. $765 .(=\mathrm{S}$. decorticans Fr.'

- peregrina. - Ann. sc. n. 1\$34. I. t. 11.

ilont.

- perforans Rob. - Wstd. 27. - Dsm. iss.

- Ann. Mg. n. h. 15.52. IX. t. 12. f. 36 . e.

p. 3५3. ispor., 13. B.).

- perforata S. (Hypoxyl.) - Linn. Tr. 15.59.

XXII. t. 15. f. 45. Fruet., Curr.,. perisporioides. - Carol. III. (6).

perpusilla. - Dsm. 9,7. - Wstd. 1111. -

A anthostigma, Not. Sfer. 91.

- persistens, Obtect. - - Ann. MIg. n. h. 15.52 .

1I. p. 326. t. 11. f. 22. (Fruct., B. B.).

- Persolina Rhaphidoph.) Not. Sfer. 55. personata. -- S. 410.$]$
S phaeria pertusa P. Pertus.) - [S. 39n.]

Linn. Tr. 1559. XXII. t. 5५. f. 53. (Fruct., C'urr. - Ann. Mg. n. h. 1ร59. III. t. 10. f. 2.. B. B.)

Weigg.: Wstd. 1331.

perversa. - Act. Turin. 1553. XIII. Dee \&.

f. S. Notar.

Petasitidis Rbh. - Kl.97\%. - Kl. II. 733.-

Tistd. 1215.

- _ f. Digitalis. - Fuck. s. 530. b.

- petiginosa Fr. Diatry. ?'. - Limn. 'Tr. 1559.

IXII. t. 16. f. \6. Fruct., Curr.)

- petioli. - Fuck. Nass. f. 22 ; Fuck. s. 537;

cf. ischnostyla. - IR. f. 337 . eerastis liiess

— petiolicola. - Dsm. 1777. - Kl. 1 135.

- petiolormm. - Carol. I. 61. - Lib. $14 \mathrm{~s}$

- Petrucciana Cald. - R. f. 335. - Comment.

soc. critt. ital. $1>61$. no. 1. t. 2. f. 6. p. 111. (aldes. . - Ital. 342 .

_- Peziza Td. - Dsin. III. 371. — Wisch.

f. 3510 . - Berk. Out. t. 24. f. 6. (cf. Nectria).

- Toges. 4>3. - Brit. f. 176. - Sturm h. li. t. 12 .

- Pezizae Td. Neetria. - Kl. 53\% - Linn.

Tr. 1 54. XXII. t. 57.f. 4. Fruet., Curr.

- pezizoidea DC. - W'std. 364 .

- Pezizula. - Cárol. V. bl.

- phaeocomes. - Lib. 44. - Ann. Mg. n. h.

1411. VI. t. 11. f. S. Berk.) - Fuck. s. jll.

- ef. Pyrenophora ph.

- phacospora Villosac). - Kl. 1934.

- phaeosticta Berk. - Ann. Mg. n. b. I j2.

1X. p. 343. t. 12. f. 34. Fruct., B. I3.). - IR.

f. 112 . Linn. Tr. 1559. XXII. t. 59 . f. 127.

Fruct., Curr.

- phaeostroma Mont. - K. f. 51. - Byssis.!,

S. tristis $\beta$. engl. Fl.: Linn. Tr. 15.59. XXII.

t. 57.f. 12. Fruet., Curr. - Carol. V. 63. -

Alger. t. $26,2$.

- phaselina - Ann. sc. n. 1555 . III. t. 6. f. 2. Mont.

- phaseolicola Depaz.). - Dsm. III. 359.

- phomatospora C'aulicolae, - Ann. Mg. n. h.

15.52. 1X. t. 11. f. 33. p. 341. Fruct., B. 13. -

Obtect. B. 13. : Limn. Tr. 1 59 . XXIl. t. js.

ก. 94. Fruct., Curr.

- Plraguitis. - Palacont. VIII. L. 6. $t$ 6.3.

f. $2 ;$ t. 6 i. f. 1.5. 'R. Ludwig).

phyllogeneta. - [S. 422.$]$

- picastra Fr. (Pertus.) - S. 390.] - Limn.

Tr. 1559. XIII, t. 5\%. f. 51. Fruct., Curr.). picea. - $\left.{ }^{\mathrm{S}},+19^{*}.\right]-\mathrm{K}$. 351 . (cf. Sphaeropsis).

- pileiformis. - Hook. Lond. J. 1S 12 . I. t. 7.

- Xylaria Linn. Tr. 1559. XXII. t. 45. f. 4.

Fruet., Curr.)

- pilifera. - [S.392.] - Fl. dan. XII. 2039.3.

- Bisch. f. $3560 .-$ Kl. 547.

- v. dryina. - Kl. it3.

pilosa P. Till. - [S. 405.] - Linn. Tr.

1559. XXII. t. 57. f. 21. (Fruct., Curr.).

pilulifera. - S. $352 \rightarrow * *$.]

- Pinastri. - [S. 413. 392. 393.] - Toges. $7 \pi 2$.

DC. (Obtect.). - Linn. 'Tr. 1559.

XXII. t. 5S. f. \$2. (Fruct., Curt.).

pinea. - Dsm. 77\%.

Pini A. S. - Bisch. f. 3522 - Kl. 655.

f. 3 .

pinodes B. Bl. Caulicolae). - Ann. Mg. n.

h. 1561. VII. t. 17.f. 34. p. 45 . 
Splu a eria Pisi Sow. - [S. 394.] - Dsm.94. Loud. f 16151. - Kl. 1339. - Fuck. s. $540 .-$ cf. Sphaeria herbarum Not. - ('aulicol.) Iinn. Tr. 15.5!. XXII. t. 59. f. 131. Fruct., (urr.,

- Brit. f. 143. - Wstd. 272.

- pisicola Depazea B. - Linn. Tr. 1559. XXil. t. 5\%. f. 152. Fruct., ('urr.).

_ pithyophila. - [S. 391.] - 'C'ucurbit.) Not. sifer. $5 i$.

- Placentr Td. - Wstd.69. - Diatrype bullata R. f. 536 .

- planiuscula (Caulicol.). - Ann. Mg. n. h.

1 55 !). III. t. 11. f. 35. B. B3.) - Hdw. I. t 4.

f. $7,-$ Iil. $1 \leq 29$.

- Plantaginis. - Kl. 17\$2. a.

- Platani Massaria - Carol. T. 62. - Kl.

15.12. - R. f. 323 .

- plateata Denud. P. - Limn. Tr. 1559. XXII.

t. 5.. f. 35. Eruct., Curr.).

- platystoma Diatry. - S. 390.45.] -

Jinn. Tr. 1=59. XXII. t. 46. f. 66. Fruct., Curr.).

- pleuronervia. - Act. Turin. 1 $55 \%$. XVI.

1)ec. 9. f. 9. Notar.

- pleurostoma. - [S. 392 .

- plicata Td. - Bisch. f. 3549

- Plinthis. - S. $365 ; 102,6.3$

- Pocula - [S. $399^{*}$.] - ef. Polypor. cupulaeformis 13. C.

- Podagrariac R. - cf. Dothidea. - Wstd. 722 .

- podoides P. Diatry. - Linn. Tr. 1559.

XXII. t. 46.f. \$5. Fruct., Curr. - Toges. 1074.

— polita. - [S. 356.] Melogr.). - [S. 395.]

Picrost.

polycocca. - Carol. IV. 62

Polygoni. - [S. 394.]

Polygonorum. - [S. 421 .

- polygramma Fr. - S. 419.] - Kl. 1247. -

Dsm. 975. - ef. Sphaeropsis p.

polymorpha. - (Xylaria Linn. Tr. $1 \$ 59$.

XXII. t. 45. f. 12. Fruct., Curr.). - Sturm

h. 6. t. 11 .

- Polypodii Rbh. - Kl. II. 533.

- polytrichia Wllr. - R. f. 325. - I)sm. III.

7๑7. - Voges. 1449.

— pomiformis P. - [S. 359.] - Fuck. s. 556.

- Voges. 4\2.

- Populi Schum. - Fl. dan. XII. 2151. 2.

- populina. - [S. 390.421.] - Kl. 1045. -

Mstd. H - - ef. Iypospila p. - Mag. Zool.

Bot. 1ऽ37. I1. t. 7 . f. a-c. p. 22\%. Fruct.,

Berk.)

porphyrog. Td. - ef. Leptospora p -

Wstd. 171 .

- Posidoniae Mont. - Dsm. 1762. - R. f. 447.

- Alger. t. 25, S. - Ital. 650

- Potentillae. - [S. 39 1: 171.] - Carol.III. 61.

- praemorsa Lasch. - Kl. 1219.

proboscidea. - S. 115.$]$

- profusa Fr. - Voges. Si1. - Dsm. 262. -

cf. Aglanspora pr. Not. - (Valsa) Linu. Tr.

1559. XXII. t. 1\%. f. 12๙. (Fruct., Curr.). -

Ital. 235 .

- prominula. - [S. 391.]

- prosiliens. - [S. 391.$]$

- protracta Vermicularia) P. - [S. 120 ] -

Ki. 160. - Fuck. s. 619. - Cryptoralsal Not.

sfer. 46. p. 10.

— protusa. - [S. 391.]

- pruinata K. - Linn. Tr. 1559. XXII. t. 45.

f. 37. (Fruct., Curr.).
Sph a eria pruinosa. - [s. 393.] - Voges. 965.

- Brit. f. S5. - Wstc. 26\$.

- Prumastri l'. - Kl. 35'. 'cf. Valsa). - Voges. 3\%. - 13rit. f. 29. - Valsa Not. Sfer. 3i. - Pruni Schum. = eupularis Fl. dan. - Wstd ., 24 .

Pseudo-Bombarda Mont. - Ann. sc. n. 1911.

XIV. t. 19. f. 6. p. 321. - Caespit., Jinn. Tr.

15.5. XXII. t. 45. f. 171. (Fruct., Curr.).

— pseudo-maculiformis. - Ism. 14!s.

Psoromatis. - Flora 15.55. t. 3. f. 13. Massal.).

Pteleac Rbh. - Kil. 1236,

Pterirlis. - Dsm. 79\%. - Kil. 971.

K\%. ; cf. Sphacria filicina Fr.

J)sm.: S. punctiformis r. Pter, Fr.

Scler. - Sphacrella) Not. Sfer. 99.

- puccinioides. - Voges. 5ffi. - cf. Dothid. Wstd. 16.5.

pulchella P. - Voges.27. - Bisch. f. 3517.

- Valsa Linn.Tr.1559. XXII. t. 45. f.150-153.

Fruct., Curr.). - Schnzl. t. 15. f. 39-12. -

1i1. 152. - Dsm. 263. - Carol. I. 56. - Letell.

692. 1. - Wstd. 21. V. minor. - Schweiz. 13.

- pulchra Valsa Curr. - Linn. Tr. 1559. XXII.

t. 4٪. f. 133. p. 279.

- pulicaris Fr. - S. 402. - Kl. 74. - K1.

II. 735. - Gibbera p. - Caespit.) Linn. Tr.

14.59. XXII. t. 19. f. I>0. Fruct., Curr.). -

13rit. f. 253. - Carol. V. 65

- Pulsatillae Isasch. - Kl. 759.

pulveracea Ehrl. - [S. 359.] - Dsm. 976.

- R. f. 335. - Tenud. Linn. Tr. 1559. XXII.

t. 57. f. 42. Fruct., Curr., - Voges. 773.

pulvinata IIdw. - Wstd. 713.

Pulvinulus 13. (Villos.). - Linn. Tr. 1559.

XXII. t. 57. f. 24. Fruct., Curr.\}.

- pulviscula Denudat.) Curr. - Linn. Tr.

1559. XXII. t. 55. f. 52. (Fruct.).

- Pulvis pyrius. - [S. 399.] - Schnzl. t. 15.

f. $31-33$. - Berk. In. p. 2\1. f. 65. a. - Kil.

515. - Fuck. s. 552. - K1. II. 650. - (Denu:

dat. Iinn. Tr. 1559. XXII. t.57. f. 32 . Fruct.,

Curr., - Voges. 3\$1. - Wstd. 71.

punctata. - Brit. f. 109. - Bisch.f:3147.-

Poron.) Linn. Tr. 1559. XXII. t. 45. f. 27.

Fruct., Curr. . - Kil. 255. - Voges. 955. punctiformis Sphaerella, - [S. 396.423.]

- Dsm. 244. 1443. 1794. 1795. - Kl. 972. -

R. f. 22 s. - Fuck. s. 515. - Voges. 662. Carol. III. 59. - Wstd. 276. 373. 177. 173. 1111. - Hoffm. Ic. t. S. f. 5 .

Fr.: Fl. dan. XII. 2036. 1. _ P.:

Linn. Tr. 1459. XYII. t. 59. f. 142. p. 333.

Fruct., Curr.).

I) $\mathrm{sm}$. Wstc.

punctulata. - Dsm. 17Sf).

punicea. - cf. Nectria. - Wstd. 1109.

Pupula. - [S. 39\%] - Dsm. 1764. - R.

f. 543. (Hercospora).

- purpurea - Fl. dan. X. 1751. 1. - Claricepsp. - Cordyceps Fr. : Linn. Tr. 1559. XXII.

t. 45. f. 25. Fruct., Curr.). - Wstd. 1209.

- Purtoni Grev. Nectria!. - Linn. Tr. 1559.

XXII. t. 49. f. 14]. (Fruct., Curr.!. pusilla Wahl. - Wstd. 21.

Pustula Curr. Denud.). - Linn. Tr. 15.59.

XXII. t. 5\%, f. 31. p. 31\%. Fruet.). P. - [S. 121.$]$ - Wstd. 653.

— pustulata. - Wstd. 19. - Dsm. 1405. -

Voges. 659. (leucostoma.P.). - Wstd. 1107. 
126.

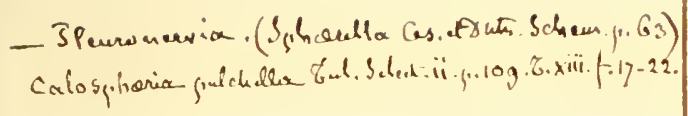

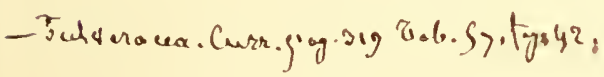

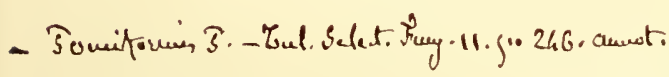




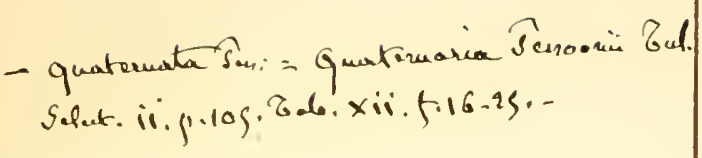

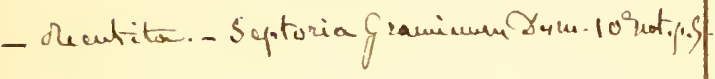


Sphaeria putaminum (Pertus.) Schw. - Linn.

Tr. 1559. XXII. t. 55. f. 69. 61. (Fruct., Curr.).

- Carol. I. 61.

- pyrenula. - [S. 390.]

- pyrochroa Nectr.). - Dsm. III. 372.

- Pyrolae. - [S. 423.]

quadrinucleata Curr. (Obtect.) - Linn. Tr.

1554. XXII. t. 55. f. 90. p. 325. (Fruct.)

quaternata I'. - Brit. f. 300. - Dsm. 969.

- Wstd. 269. - Fl. dan. XII. 2039. 2. - Bisch.

f. 3546 - Act. Turin. 1553. XIII. Dec. S. f. 7 .

Notar. - Pay. b. er. p. S. f. 30. - Talsa

Linn. 'Tr. 1559. XXII. t. 45. f. 161. 165. (Fruct.,

('urr.). - Voges. 179. (S. ferrug.).

- quercina (Diatry.). - [S. 121.] - Dsm.

1752. - Letell. 703. 1. - Kl. 249. - R. f. 319.

- Linn. Tr. 1459. XXII. t. 47. f. 90. (Fruct,

Curr.). - Voges. S65. (leiphaemia, Tul.)

- Quercuum. - [S. 356.] (Melogr.) - Carol.

II. 55 .

querna Curr.(Vals.). - Linn. Tr. 15.59. XXII.

t. 15. f. 141. p. 279. Fruet.

Racodium P. - 5.3 \9.] - Kil. H1. 649. -

Fuck. s. 616. - (Villos.) Linn. 'Tr. 1559. XXII.

t. 57. f. 15. (Fruct., Curr.). - Brit. f. 253. -

Carol. V. 66. - Wstd. 655.

— radians Td. - Bisch. f. 3512 . - Wstd. 903.

-.. radiata $S c h u m .=$ fragiformis $v$ rad., $\mathrm{Fl}$. dan.

- radicalis. - Kl. 11. 254 . - Fuck. s. 640. -

(1)iatry.) Lim. 'Tr. 1459. XX11. t. 17. f. 59.

Fruct, Curr.). - (Endothia) Not. Sfer. 3.

- radicosa DC. - Wstd. 1,9.

_- Ramalinae. - Dsm. 1769.

Ranunculi. - S. 121.

- Rebissia Pleospora - Not. Sfer. 79.

- recutita. - [\$.394: lugub., no. 197. - 396.]

- Fuck. s. 519. - Wstd. 373.

- redimita Wllr. - Kil. 1211.

— regia I ypoxyl. - - Not. Sfer. 12.

relicina. - [S. 420 .

repanda IIypoxyl. Fr. - Lim. 'Tr. 1>59.

XXII. t. 46. f, 54 . (Fruct., Curr.

- repens. - Schnzl. t. 15. f. 45.

- reptans Sow. - Loud. f. 1642s.

- Resinae Fr. - ef. Nectria.

- reticulata DC. - S. 12.5.] cf. Combosira,

Asteroma. - Wstd. 636.

- revelata (Obtect.). - Ann. Mg. n. h. I 452

IX. p. 325 . t. 11. f. Is. (Fruct., 13. B.). rhabdospora Valsa). - Not. Sfer. 15. p. 39. Rhamni Ns. - S. 391.] - Bisch. f. 3572 .

Fuck. s. 631 . - R. f. 339 .

- rhaphidosperma. - Ann. sc, n. 1510. XIV.

t. 19. f. 3. p. 325 . (Mont.).

Thinanthi. - [S. tos.] ef. Sphaeronaema. rhizogenea B. Confl.). - Lim. Tr. 1559.

Xxil. t. 49. f. 15s. p. 2\3. (Fruct., Curr.).

Rhizomatum. - Kil. 1539 .

Rhizomorphae (Foliicol.) Kz. - Linn. Tr.

1\59. XXII. t. 59. f. 139. (Fruct., Curr.).

- Mont.: [S. $352^{*}$.]

rhodochlora. - Ann. se. n. 1\$31. I. t. 12.

(Mont.). - Lim. Tr. 1559. XXII. t. 57 . f. 19.

Fruct, , Curr.).

Rhododendri. - Kl. 1536. - (Sphaerella)

Not. Sfer. 96.

rhodomela Fr. - Fl. dan. XII. 2154. 2.

- rhodomphalos B. (Denud.). - Linn. Tr. is.59.

XXII. t. 5\%. f. 25. (Fruct., Curr.).

rhodopis. - [S. 359 .]

rhodostoma A. S. - [S. 397.] - Bisch. f. $3509 .-$ (Iercosp.) Linn. Tr. 1559. XXII. t. 59. f. 79. (Fruet., Curr.).

Sphaeria Rhois (Diatry.) S. - Linn. Tr. 1859.

XXII. t. 46. f. 71. (Fruct., Curr.).

- rhopaloides II. - Linn. Tr. 1559. XXII.

t. 45. f. 1.1. (Fruet, Curr.).

rhytidotes. - (Denud.) Ann. Mg. n. h. 1-59.

III. t. 10, f. 21, 13. B.,

rhytismoides Confert.) 13. - Brit. f. 321. -

Limn. Tr. 1459. XXII. t. 49. f. 209. Fruct.,

(urr.). - Ann. Mg. n. h. 1s11. VI. t. 10. Berk.).

- Ribesia P. - ef. Dothidea R. Not. - Voges. $275 .-$ Wstd. 466.

- Ribesii Schum. = cohaerens var.? Fl. dan. ribicola (Depazea) Fr. - Lim. 'l'r. I 5 !).

XXIl. t. 59. f. 153. (Fruet., Curr.).

- Ribis 'Td. - [s. 390.] — Bisch. f. 3532. -

Wstd. 47. - K1. II. 35\%.

_-_ - Wllr. - ef. Iothidea Ribesia Not. riccioidea. - S. 343.

rigida. - S. $\left.100^{*}.\right]$ - Bisch. f. 37s4. Stil-

bum r.).

— rimarum Lasch. - Kl. 969 .

- rimosa A.S. - Voges, 963. - Bisch. f. 3590. Uim. 265. - ef. Dothidea r. Fr.

- Fr.: Ki. 347

v. b., A. S. = filicina Fr. s.

Roberoana. - Dsm. 1755.

Robergei Nectr. . - Dsm. III. 37.

Robertiani. - - 421 .] cf. Stigmatea R.

- liobertsii. - Lindl. v. K. p. 40. f. 25. -

Pay. b. cr. p. 5s. f. 242. - of. S. II ügelii, Cor-

dyceps Rob. - Limn. I'. 1 59. XXII. t. 45.

f. 2ii. Fruct., Curr.

_ ? Robiniae S. - Kil. 15.13.

Liosac schum. - Fl. dan. XIII. 2334. 1.

- Schl.: Wstd. \$76.

- Rosarum. - [S. 417.] - Valsa Not. Sfer.

12. p. 37 .

- rosea Pers. - Kil. 101 t.

- rosella A. S. - Loud. f. 16146 . - Bisch.

f. $3536 .-$ Nectria Linn. 'lr. 1559. XXII. t. 57.

f. 3. Fruct., Curr.

- rostellata Fr. - S. 393.] - Kl. 749. -

Dsm. III. - $\$ 3$. Lib. 147.

— v. minor Dsm. - cf. S. Vepris.

— rostrata. - [S. 392., ib. 103 ?] — R. f. 256.

- Dsm. 273. - Bisch. f. 3555. - Carol. IV.

$55 .-$ Wstd. 532 .

- v. tenuior Fr. - Bisch. f. 3559.

- Rousseliana. - Dsm. 17\2. - Voges. 1462, 1255.

Rousselii Diatrypella). - Not. Sfer. 32.

rubella P. - S. 394.7 - Bisch. f. 3554 .

Wistd. 171. - Fuck. s. 593. - Kl. 1640.-

Berk. Out. t. 1.f. 12. b. Spor. - Caulicol.)

Linn. 'Tr. 1 59. XXII. t. 59. f. 136. Fruct.,

(uur.) - Brit. f. 252. - (Rhaphidophora) Not. sfer. 56 .

- v. porphyrogona P. - Dsm. 27\%. Rubi Curr. - cf. S. Vepris. - (Obtect.)

Linn. 'Tr. 1559. XXII. t. 58. f. 91. p. 325.

- Fr.: [S. +17.$]$

- Dub.: Wstd. 939.

rubicola Curr. (Denudat.). - Linn. Tr. 1559.

XXII. t. 57. f. 15. p. 319. (Fruct., Curr.).

rubiformis P. - Wstd. 73.904 : cohaerens. rubiginosa $\mathrm{P}$. - (Iypoxyl.) Linn. Tr. 1559.

XXII. t. 45. f. 43. Curr.). - Loud. f. 16378.

Ruborum. - Lib. $340 .=$ callimorpha M.?

(Wstd.). 
sipharia rubra Fr. - Wste. 637. 903.

- rubricosa F. IIypoxyl.). - Limn. Tr. 1459. EXII. t. 45. f. 42. (Fruct., Curr.).

-

Fuck. s. 167. - (Diplod. ?) Limu. 'Tr. 1559. XXII. t. 47. f. 129. Fruct., Curr.).

— rufa P. - 13rit. f. 171. - Fl. dau. X. 1781. 2.

- (Iypocrea) I.inn. Tr. 1559. XXII. t. 45. f. 36.

Fruct., Curr.

_-_ rufescens Schw. - S. aculeans, Carol. I. 55.

- rufo-fusea Fr. - Wstd. 1203.

- rugosa Grev. - Loud. f. 16150.

- Rumicis. - Dsm. 795.

- Rusci Wlir. - Dsm. isl. - S. atrovirens

b. Fr. - Kl. II. 540 . - (Subtect.) Linn. Tr.

15.59. XXII, t. 59. f. 120. (Fruct, , Curr.). Voges. 1170. - Wstd. 535. - Ital. 5\$0. (Cryptosph.). - (Sphaerella) Not. Sfer. 95.

_- Ryckholtii. - Bull. ac. belg. 1559. VII.

p. 365. f. 4. Wstd.) - Wstd. 1201.

- sabuletorum (Foliicol.). - Ann. Mg. n. h.

is 52. IX. p. 353. t. 12. f. 36. a-d. (Fruct..

13. B.)

Sacculus. - [s. 112. Tors.]

Sagraeana M. Hypoxyl.) - Linn. Tr. 1559.

XXII. t. 45. f. 21 . (Fruct, Curr.).

_ - salicella Fr. - S. 393.] -- Dsm. 39. - Kl.

1445. (Halon.) - Voges. 1163. - Wstd. 370.

- salicina P. - Bisch. f. 3525 . - Act. Turin.

1553. XIII. Dec. 6. f. 4. (Notar.). - (Valsa)

Linn. Tr. 1ऽ59. XXII. t. 45. f. 149. Fruct.,

Curr.). - Carol. IV. (it). - Norweg. 196. (vibralis Somm.). - Wstd. 473.

- Sow, : cf. S. saligna.

- saligna Ehrb. - Wstd. 524. Dothid.). - S. Capreae DC., salicina Sow., Variolaria Salicis Bouch.

_- Sambuci. - Ital. 754. (Dothid.).

sanguinea S. - Nectria) Limn. Tr. 15.59.

IXII. t. 57 . f. 45. 46. (Fruet., Curr.). - Nect.)

Not. Sfer. 5 .

dia, Fr.)

_- - v. cicatricum. - Brit. f. \$3. - Mag.

Zool. Bot. 1ऽ37. I. t. 3. f. 6. p. 45. Berk.).

- sapinea. - [S. 391.]

- Saponariae. - ef. Spilosphaeria, Septoria, 1)epaz. — Wstd. 954.

- sardoa. - Act. Turin. 1 553 . XIII. Dec. 7. f. 5. Notar.).

- sarmentorum Fr. - [S. 117.] - (Diplod.)

Linn. Tr. 1 559. XXII. t. 59. f. 11s. Fruct.,

Curr.). - Voges. 1463.

- Sarraceniae. - Carol. I. 65.

- Sassafras (IIypoxyl.) S. - Carol. I. 53. -

Linn. Tr. 1559. XXII. t. 46. f. 50. (Fruct., Curr.).

- Saturnus. - [S. 411. 23.]

Saubinetii Caespitos.) Mont. - Ann. Mg.

n. h. 1>59. III. t. I0. f. 19. (B. B.).

scabiosaecola (Depaz.). - Wstd. 30.

scabra. - Kl. 147 .

Schmidt: Krypt. Bad, 42 ?.

(Villos.) Curr.: Linn. Tr. 1859. XXII.

t. 57. f. 13. p. 315. (Fruct.).

- Schum. - ef. elongata, Let.

- scabrosa (Diatry.) DC. - Limn. Tr. 1559.

XXII. t. 40. f. S4. (Fruct., Curr.). - Fl. dan.

III. 2035. 1.

_- scabrosae affinis Not. (Versatiles). - Kl. II.

$53 \%$ - Wstd. 1372 .

- scarlatina Schum. = rufa Fl. dan.
Splua eria seatigena (Villosae) 13. 13. - Aun. Mg.

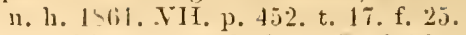

schistostroma 1)ub. - R. f. 53.

Scirpi. - [S. 393: 131.] - Kil. 1837. -

l'ringsh. Jahrb. I. 1559. t. XXIV. A. (Pringsh.).

- kl. II. 256. - scirpicola DC., Fr. - DC:

1125.

— scirpicola. - [S. 393.] - Wstd. 373.

- Sclerotium. - [S. 415. Clint.]

_ scoriadea Fr. (Confluent.). - [S. 391.] -

Jinn. 'Tr. 1559. XXII. t. 49. f. 157. (Fruet., ('urr.).

_- Scrophulariae. - [\$. 394: 155.]

- scutellata P. - Bisch. f. 3 J 3. - Kl.963. -

ef. Valsa.

- Secretani Heer. - Fl. tert. Helv. t. 1. f. 1.

- semilibera Rob. - Dsin. 1437.

- seminuda. - [S. 390.] - Act. Turin. 1553.

XIII. Dec. 7. f. 4. (Notar.)

- sentina. - [S. 394 .] - Kl. 1562. - Fuck.

s. 524. - Dsm. III. 364; $7 \$ 9$.

- sepincola. - [S. 395. Sace.] - Ann. Mg.

n. h. 1552. IX. p. 326. t. 11. f. 21. (Fruct.,

B. B.). - Dsm. 27t. - Toges. 107s.

- septorioides. - Dsm. 144\%. - Wstd. 150.

septospora. - [S. 356.] (Melogr.).

- seriata (Subtect.). - [S. 3\$9.] - Linn. Tr.

1459. XXII. t. 59. f. 121 . (Fruct., Curr.)

serograpta. - Dsm. 1793.

- serpens P. - Fl. dan. XII. 203\%. 1. - Hy-

poxyl. Lim. Tr. 1559. XXII. t. 46. f. 45.

Eruct., Curr. . - Bisch. f. 3505.

— - v. lumbricoides. - Kl. $163 \mathrm{~s}$.

- setacea. - [S. 394.] - Act. Turin. 1553.

XIII. Dec. 6. f. 9. Notar.) - Fuck. s. 536. -

Til. 545. - Dsm. 757. - Foliicol.) P. : Liun.

Tr. 1559. XXII. t. 59. f. 145. (Fruct., Curr.). -

Voges. 1173. - ef. Thamnomyces Cllamissonis

E. - Brit. f. 1 s.

— Alni : Norweg. 197. - Wstd. 536.

- signans W7lr. - [S. 41\%, 3 ?] cf. Diplodia

salicináa Iév.

- - simplex. - [S. 391.]

- sinensis. - Lindl. v. K. p. 39. f. 34 . -

Ilook. J. 1s 13. II. t. S. f. 1.

- sinopica Fr. - Dsm. 759. - Toges. 1335. -

(Nectria) Not. Sfer. 6.

-_- siparia (Obtect.). - Ann. Mg. n. h. 1652.

IX. t. ?. f. ᄂ. p. 321 . Fruet., B. B.) - Lim.

'Tr. 1\$59. XxiI. t. 55. f. 95. (Fruct., Curr.).

— Smilacis Cast. - Dsm. 1420.

- socialis. - S. 412.49 .]

Solani. - S. 394.]

solida Sow. - Wstd. $4 \backslash 3$.

Solidaginis Fr. - S. 425.] - R. f. 332. -

Carol. II. 6\%. (Dothid.).

- Sorbi. - Voges. S69. - Bisch. f. 3556.

Norweg. 93. - Lib. 339. - (Valsa) Not. Sfer. 34. - sordaria Fr. - [s 3\$9.] - Act. Turin. 1 557.

XVI. Dec. 9. f. 3. (Notar.). - (Denudat.) Linn.

'Tr. 1s 59. XXII. t. 5\%. f. 43. Fruet., Curr.).

- sordida. - [S. 356.] (Mclogr.). - var. b.:

Wistd. 520 .

- Spartii Ns. Gibberidia). - [S. 395.] - Kll.

355. - Fuck. s. 624. - (Conflu.) Linn. Tr.

1 559. XXII. t. 49. f. 159 . (Fruct., Curr.). -

Toges. 66.1. - Tetell. 692. 4. - Wstd. 716.

Micr. J. I 559 . VII. t. 11.f. 19. Fruct., Curr.!

_- sperinoides. - S. 359.] - Loud. f. 16420.

- Bail S. t. 17. - Kil. 531. - Fuck. s. 551 -

Iil. II. 651 - Dsm. $975 .-$ Denudat. Hffm. : 



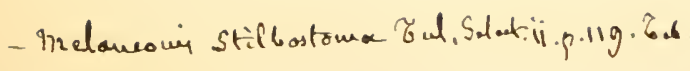
xiy. fig.1-12..

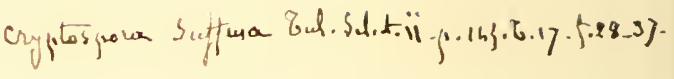

$-$ 
Linn. Tr. 1559. XXII, t. 5\%. f. 36. (Fruct.,

Curr.). - Voges. 45 1. - Krypt. Bad. 150. -

Lasiosplaeria Not. Sfer. 53 .

Spluacria sphaerocephala. - [S. 410*.]

- sphacrococea. - [S. 355. 23.]

— spiculifera Sow. - Wstd. 522.

_ spiculosa. - S. 393.] - Dsm. 1 f113.

- - 13t.; ef. Sphaeria fimbriata Not. -

Fuck. s. 614. - Linn. Tr. 1559. XXII. t. 47.

f. 111. Fruct., Curr.). - Wstcl. 467. (j,j1.

- v. Robiniae. - IIdw. I. t. 5. f. 6. -

li. f. $336 .-\mathrm{Kl}, 1736.1543$.

Spinaciac. - S. 125.$]$

spinifera W. - Fuck. s. 646 .

- spinosa P. - [S. 392 ; ib. 117.] - F]. dan.

XII. 203৬. 2. - Fuck. s. 645. - Act. Turin.

1\53. XIII, Dec. 7. f. 3. Notar.) - Bisch.

f. $3555 .-$ Kil. 356 . - Lim. Tr. 1859. XXII.

t. 47. f. 10s. Fruct., Curr.). - Voges. 376.

_ spurca (Diplodia Willr. - [S. $117: 11]$.

Kl. 915.5. - Fuck. s. 5s 1. - ? Diplod. Rosar.

Fr. Wstd. .

- squalens. - [S. 396.

— stamea. - [S. :359.]

- Steinheilii. - Ann. sc. 11. 1931. 1. t. 13.

— Stellariarum Rbl. - Kl. 975. - Fuck.

s. 5 lli. a.

_- f. IIolostcac Awd. - Kil. 97f;

- f. Spergulac. - Fuck. s. 51 li. b.

- Stcllarinearum Rbh. - R. f. 145. (r. Ceristii

stellaris. - [S. 37 .

- stellata Td. - Bisch. f. 3613. Splacrobo-

lus s.

stellulata. - 13rit. f. 79. - Act. Turin.

1५53. XIII. Dec. S. f. 6. (Notar.). - Kl. 65).

- c.f. Talsa. - Carol. 1. 51.

stercoraria. - S. 359 .

- - Fr, : non Fuck. s. 54. (t. Fr.! - De-

nud.) Fr.: Linn. Tr. 1559. XXII. t. 57. f. 40.

Fruct., Cur'r.

- Sow. : Micr. J. 1559. VII. t. 11. f. 17.

(Spor., Curr.)

- Sow.: Xylariae pedunculatae Sow.

t. 437: forma reducta cx Berk. In. p. 279, -

(Denud.) Sow. : Limn. T2. 1559. XXII. t. 57.

f. 35. 39. (Fruct., Curr.'.

_- stercorea Sow. - Kl. 432.

- stercoris. - [S. 390* . 405.] - Kil. II. 433.

— stercoraria Sow. var., Fr. Sy.

- Fr. El. = S. (Denud.)' stercoraria Fr.

(Curr.). - Dsm. III. 97. - Voges. 1169.

Stigma. - Diatry.) Norweg. 91. - Wstd.

112.529 . (c. v. decorticata). - Not. Sfer. 25. p. 26. - - Hoffin. - Voges. 372. - Fl. dan. XII.

2037. 2. - D $\sin$. 963. - Diatry.) II. : Kil. 49.

- Linn. Tr. 1859. XXiI. t. 16. f. 22. Fruct.,

Curr.) - cf. decorticans. - Carol. I. 63.

— stigmatella Wllr. - Wstd. 35 .

- stilbostoma. - Dsm. $75 \%$. - Valsa) Fr, :

Linn. Tr. 1559. XXII. t. 45. f. 110. u. 140. a. Fruct., Curr.).

906. v. Platani. - Voges, 569. - Wstd.

- r. papula. - Kl. 159. - Voges, 962.

- stipata Curr. = Valsa hypodermia Fr. -

Lib. 313. - R. f. 320 - Coneresc.) C. : Linn.

Tr. 1559. XXII. t. 49. f. 197, p. 27.1. Fruct.,

Curr.).

striaeformis Fr. - Wstd. 1132. - Dsm. 1410.

stricta. - [S. 342.]

strigosa. - [S. 359.]

Hof $\mathrm{mann}$, Index Fungorum.
S'phaeria strobiligena. - Dsm. 142.2.

- strobilina H. S. Dichaena . - Kl.514. lil. 1I. ว39.

— - Fr. [S. 403.] - Voges. 572.

- strumella Fr. - Voges. 960. - I sin. 1102.

- Kl. 757. - Kil. II. 255. - (Diatry.) Linn.

Tr. 1 59. XXII. t. 47 . f. \$s. (Fruct., Curr.) -

Brit. f. 173 .

- strumellacformis. - Act. Turin. 1 153. XIII.

Dec. S. f. 4. Notar.).

- stylophora B. B. Ceratostom., - Ann. MIg.

n. h. 1 61 . VII. t. 17. f. 29. p. 453.

subcongregata. - Carol. IV. 57.

- subiculata S. Byssised.). - Iinn. Tr. 14j!).

XXII. t. 57. f. 9. (Fruct., Curr.) - Carol. T. 72

subradians. - Dsm. 1796. - Voges. 12.52.

subscripta. - [S. 397.] - (IIalon.).

subsolitaria S. (Diplod.). - Linn. Tr. IS.59.

XXII. t. 49. f. 194. (Fruct., Curr.).

- subtecta. - S. 117 .

subterranea. - [S. 3>2 $\%]$

- subulata. - [S. 392.]

- succenturiata ' $\mathrm{T} d$. - Bisch. f. 3497. - Kl.

1535.

succincta W. - Fuck. s.572. - ¿Stauro-

sphaeria Rhamni Wstd.

- succinca liob. - R. f. 326. - Dsm. 1441.

- suffulta. - Kil. 1329. - Fuck s. 512.

suffusa Valsa F'. - Voges. 1216. - Linu.

Tr. 15.59. XXII. t. 15. f. 114. Fruct, C'urr.)

sulcata. - S. 103.] - Wstd. 224.

superficialis Villos. Curr. - Linn.'Tr. 15.59.

XIII. t. 57. f. 25. p. 317. Fruct., Curr. .

- superflua $\Lambda$ wd. $=$ Splh. licrbar. v. minor.

Iibl. - Fuck. s. 515.

- surculi. - S. 390.]

- sycomophila. - Act. Turin. 1453. XIII.

Dec. 6. f. 5. Notar.).

symeephala. - [S. 112.45.$]$

- syngenesia. - Voges. 40. - Iil. II. 613. -

Talsa Linn. 'Tr. 1599. XXII, t. 17. f. 119.

Fruet., Curr.

- Syringae Fr. Hendersonia, - Linn. Tr.

1459. XXII t. 59. f. 116. Fruct., Curr.).

- Talcola Fr. Valsa - Dsm. 1754. - Lim.

Tr. 14.59. XXi1. t. 47. f. 124. (Fruet., Curr.)

Talus $v$. licheniformis. - Wstd. 367.905:

v. plicata.

- Tamariscinis Grev. (Obtect.). - Limn. 'Tr.

1 559. XXII. t. 5`. f. 81. Fruct., Curr.

taphrina Fr.? cf. Diplodia polymorpha. -

[S. 392.]

-Tassiana (Sphaerella). — Not. Sfer. 95.

— Rosellinia. - Tassi, Flora di Siena.

III. 1462. c. ic. - Not. Sfer. 16. p. 19.

- Taxi Diplodia Sow. - [S. 417.] - Dsm.

9\1. - Kl.1036. - Voges. 1079. - Brit.f.179.

- Wstd. 718 .

Taylori. - Hook. Lond. J. 1s43. II. t. ५. f. 2.

tecta. - Kil. 353.543.

tectae proxima. - Kl. 745 .

- tenacella. - [S. 391.]

tenebrosa (Caulicol.).-Am. Mg. n. h. 1552.

IX. p. 352. t. 12, f. 35. Fruct., B. B.).

tephrotricha. - [S. 3S9.]

tessellata P. - Kil. 1035.

- tessera Fr. - [S. 392.] - Fuck. s. 642.

tetraspora (Valsa) Curr. - Linn. Tr. 1859.

XXII. t. 45. f. 148. (Fruet., Curr.).

thelebola (Valsa) Fr. - Linn. Tr. 1559.

XXII. t. f5. f. 157-159. (Fruct., Curr.) 
splaceria thelena Fr. - $\$$ \$. 34..] - Muller, I'flanzenstaat 270. - Brssis. Iinn. 'Mr. L hi!!'. XXII. t. 5i. f. Truct, Curr., - ]3isch. f. $36011 .-$ D sm. $271 .-$ Wstd. 115. therophila. - Msm. 17\%ti.

thoracella Rustr. - Wstel. 1213. 'Thwaitesii ('aulicol.) - Ann. Mg. n. h.

15.22. IX. p. 340 . t. 11, f. 32. Iruct., 1: 1:.).

- 'Thvrsis. - Ann. Mg. n. h. 1412. X. t. 1:. f. 14. p. 345. (13ark.

- Tiline P. - S. 416.7 - Dsm. \$7!. - Spi-

lobolus 'T., Rabenhorstia 'T'. - Kil. 25f. -

Obtect. Linn. 'Tr. 1-5!) XXII, t, 5!) f. 111. Fruct., Curr. - - (Valsaria) Not. Sfer 5.5

II. et Yist.: I iun. 'Tr. 1459. XXII.

t. 59. f. 1115. 106. Fruct., Curr. . - Voges, (5610.

- Wistd. 533.

tiliaginea, - Mier. J. 14.59. VII. t.11. f. 11.

Curr.).

__ Tocciaeana Diatrypella. - Not. Sfer. 31

- Tomicum Iér. - Ann. Mg. n. h. 15.52. IX.

t. 11. f. 1i. 1, 321. (Fruct, B. 33.. - I 1 .m. 1426. - Obtect. Fr.: Linn. 'Tr. is59. XXII.

t. 5S. f. ST. Fruct., C'urr.!

— torquata T'ers. - Wstd. 472.

- tortuosa. - S. 392.

- Tosquinetii. - Bull. ac. belg. 1-59. VII.

p. 3is. f. 2. W'stel.)

- tosta Caulicolae. - Ann. Mg. n. 1. 19.52.

IX. 1. 3>1.t. 11. f.31. Fruct., 13. B.. - Kl. II. 252.

- translucens, -- Act. Turin. is i!. X. Dec. 5.

f. 2. Notar.. - Ital. i 5.

- transversalis Sclww. Obturat.; -- Linn. 'Tr.

1-59. XXII. t. 59. f. J119. Fruet, curr. .

- tremclloides Schum. — S. 343.] - Fl. dan.

I1. 1455.2.

- Weiss.: Wstd. 91. - IIoffm. 1c. t. 15.

f. 2. Hypocrea

trichella Fr. - [s.420.] - cf. Termicula-

ria. - Wstd. 133.

trichina. - Toges. ribli.

trichostoma Fr. - [S. 391.$]$ - Kl. 1049. a.

- Kl. I1. 535. - Usm. III. ボー.

trichostroma. - [S. $40 \mathrm{~T} *$ ?]

- v. globosa. - Kl. (1) 19. b.

Tridactylitis. - Kl. $1 \$ 51$.

Trifolii I'. - Voges. 1167 . Dsm. 976.

- tristis Ta. - S. 354. - Biseh. f. 3n!19. -

(Byssis.) Linn. Tr. I 59. XXII. t.57.f. 11 Fruct.,

Curr. - Alger. t. 26. f. 1.

- tritorulosa Caulicol. - - Ann. Mg. n. h.

15.54. XIII. t. 16. f. 15. p. 466. Fruct., B. B... - trivialis Obtectae, - Ann. Mg. 11.h. 19.5.

IX. t. 10. f. 16. p. 311. Fruct., B. 13.

- Trogii. - Ileer, Fl. tert. Helv. t. 1. f. 5.

- tubaeformis Td. - [\$. 39 i.]. - R. f. 54. -

Kl. 359. - Fuck, s. 533. - Foliicol. Linn. Tr.

1559. XXII. t. 59. f. 141). Fruct., Curr. -

Voges. 240. - (Ceratost.) Not. Sfer. 23. 1. 24.

- Tubercularia, - [S. $35.5 \rightarrow$. $]$

- tuberculata Schum. = scabrosa Fl. dan.

- tuberculosa. - [S. 119 :.].- Loud. f. 16126.

- fusca, Fl. dan. - Wstd. 219.

_ tubulina A. S. IIrpoxy.) - [ef. S. 325.

11.]. - Linn. Tr. 1559. XXII. t. 46. f. 55.

(Fruct., ('urr.

- tumida P. - Cytispora t. Lib. - Wstd. 11311. tumulosa. - 1 0 sin. 1775.

- turbinea.- Palaeont. Vill. t. \. f. 10. Iradwig'.

— turgida I'. - \$. 112. 3\%.] - k1. 516.
Ialsa, Jinn. Tr. 1559. XIII. t. 15. f. 139

Fruct., Curr.). - Wstel, !y)T.

Sphaeria turpis Not. - Iil. II. 536.

—_ Typhac I.asch. - Kil. 660).

— Jypharmin. - lil. II. 731.

typhina. - of. Jothiclea t. - Nov. Act. 1561.

XXIX. t. 1. f. $1-5.7-10$. t. 2. f. $1-11$.

Bail. - Dsm. 95̃. - Toges. 79. - Wstd.

522 . - Ii. f. ill .

uberiformis Fr. 'Topospora'. - S. 11\%.]

- Ki. 112. Splateropsis?, Linn. '1r. 14.5!!.

XXXI. t. 5!!. f. 113. Lruet, Curr. - Mastomyeres lriesii.

- ulurina Mont. - Ann. se. n. J S5.5. III. t. 6.

f. 1. Fruct.

- uda liypoxy. I'. - Linn. 'Tr. 1559. XXII.

t. 4ti. f. 1 il - (i3.) Fruct., Curr.). - v. salica-

ria l'. : Wstd. 16 (i).

- Ulicis Fr. - Bull. ac. belg. 1४59. TII. p. 365.

f. 7 . T'std.

uliginosa. - [S. 359.] - Dsm. III. T>2.

ulmaria Sow. - Wstd. 111.

- vlmea. - Carol. II. 63. Xyloma).

- Umi Fr. - Kl. f69; C'ent. IX. Suppl. -

Voges. F66i. - Wrstd. 111.

- uhnicola (urr. Pertus.). - Linu. 'Ir. Thit.

XXII. t. 5\%. f. 55. p. 321. (Truct., (urr.).-

livona t. 3. f. 2.

- Bern: Wistd. 13i.

__ umbonata. - [S. 390 .?

- umbrina Fr. - S. 3911.] - Fl. dan. XIII.

2332. 2. - R. f. $32 \%$ - Act. Turin. 1s 11 . JII.

Dec. 2. f. 2. Notar.!

- Mont. $=\mathrm{S}$. Olearum Cast. cf. R.

f. 32:

- 13.: [S. 348. $17:$ ] Ital. 239.

- umbrinclla. - Aet. Turin. 1457. XVI. Uee.

9. f. 5. Notar.

undulata Schum. = serpens Fl, dan.

- P.: ef. Diatrype. - Loud. f. 16:36is.

- Tinges. 371 .

- Unedonis. - Act. Turin. 1457. XVI. Dec.9.

f. 6. Nistar. .

unguiculata. -- Chile n. 9. f. א.

- unicaudata Obtect.). - Amn. Mg. n. h. I 5.59.

III, t. 11. f. : 1. B. B.). - I I. f, 5i).

_ urceolata. - [5. 397. 2 :]

- Irticae libh. S. herbarum Pers. s.j. - Kl.

11. 5.15.

- Uvariac (Depazea, Berk. -- Linn. Tr. I \$і9.

XXII t. 5!) f. 1.1\%. Fruct., Curr.)

- Taceinii Gibbera Sow. - S. 102.] - Linn.

Tr. 145!) XXII. t. 54. f. 19. Fruet., Curr. . -

Wstcl. 11143. - Toges. (66.5. (accum.). - Lilb. 34.'

- Gibbera Not. Sfer. 93.

- vacillans. - Not. Sfer. 65.

- vagabunda, - Ism. 1415.

vagans. - cf. Melogramma v. - (Depazea)

Iirit. f. Is4. - W'std. (t5!).

Taginae Lasch. - III. 663.

varia. - S. 395.?

Variolariae. - Flora 1555. t. 3. f. 10. 11.

Míassal.

vasculosa Fr. - ef. Valsa.

Vectis Caulicol. - Ann. Mg. n. h. 1551.

XIII. t. Ili. f. 16. p. 467. Fruct., B. B.).

- velata. - [S. 392.] - K1. 132! - Corn-

cresc. T': Linn. Tr. 1-5!). XXil. t. 17. f. 111.

Fruct., Curr. - Brit. f. is.

- relutina W. - Diatrype Fnek, s. fiT.1.-

Wistd. 475 .

[rentricosa. - [S. 115.. Top.] 


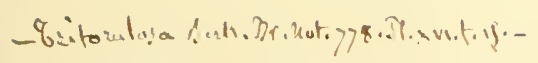


- Verveciur Serm. Ceratostoma Ver. Ca. Dut Schema

i. 55 . 
Sphaeria venulosa. - Kl, 132,. - Kil. II. 7:3. - Fuck s. 35. 'Asteroma.

Vepris Lacr. - R. f. 143 (S. Rubi curr, rostell. min. Dsm.).

_- verbascicola, - Carol. II. 62.

vermicularia. - [S. 421).] - (arol. V:6!).

verminosa. - Ann. se. n. 1455 . III. t. 6 .

f. 3. (Mont.).

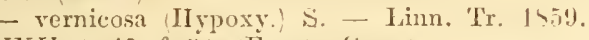

XXI1. t. 15. f. 3!. Fruct, C'urr.

- veronensis. - Flora 155., t 3. f. 12. Massal.).

verucaeformis Diatrype Ehrh. - Dsm. 752.

- Fl. dan. XIl. 2037. 3. - א̄l. 14. - Linu. 'T'r.

1) 59. XXII. t. i6. f. \1. Fruct, Curx., - Toges. 5157.

[-verrucaria. - [\$\$. 390.] - Not. Sfer. fit.

- verrucosa. - Carul. I. 52.

- versiformis. - [\$. $100 *$.$] - Wstd. 127f.$

lihamnil.

- vervecina. - Ann. sc. 11. 1612. XVII. t. 5.

I) $\sin$. .

— restita Falsa) Fr. - I.im. 'Tr. 1559. XXII.

t. 14. f. 16il. Fruct., curr.

vibratilis. - [S. 3933.] - Act. T'urin. 1453.

XIII. Dec. \. f. 10. Notar. - Obtect. Berk. :

Lim. Tr. 15.9. XXII. t, 54. f. T1. Fruct,

C'urr.l.

- Somm. : salicina I'. Mont.).

Tiburni Seliw. - Kl. lici.

Ticiae Lasch. - IIl. 133\%. = S. herbar. . -

Fuck. s. 551 .

vilis. - Kil. II. 431. - \$. 39!.

Vincac Fr. - Toges. 117.1. - Usm. 2\0.-

Wstcl. 31.

- Vincetoxici Inphisphacria - Not. Nfer. 76. - virescens Schw. - S. disciformis II. Fr. virgultorum $\mathrm{Fr}$. - Kil. bitit. - R. f.33 s.

- Hypoxy, Lim. Tr. 1559. XXII. t. 46. f. 69.

Fruct., Cirrr. .

- Visci DC. - [S. $41 \%$.] - ef. Diplodia. -

Wstcl. 1231 .

Vitis Lasch. - I1. 1017.

libh.: Fuck. s. 606 .

vogesiaca IIypoxy.) $\mathrm{P}^{\prime}$ - - Yoges, 765 . atro-

purpurea Tode, - Lins. Tr. 1459.7 III. t. 46 .

f. 6. 67. Fruet, Curr. .

Wahlenbergii. - Dsm. 964. - Wstd. 21.

- Widdringtonia. - Palacont. TIII. L. 6.t.63.

f. 1 ; t. 66 . f. $1-6,22$. R. I.udwig:

- Xanthii Lasch. - K1. 760.

- xantholeuca Fr. - Fuck. s. 645. - Norweg.

$19)$.

xanthostroma. - [S. 112 ; ib. 32.] - Ann. sc.

1. 1534. I. t. 12. (Mont.). - Valsa Linn. 'Tr.

1 59 . XXII. t. 45 . f. 160 . (Fruct, , Curr.). -

1) $\mathrm{sm}$, 1106. - Brit. f, 296.

- xylomoides. - Letell. 656.3. (Dothid. Ulmi).

- Wistd. 111.

-— Xylostei P. - [S.-392.] - Fuck. s. 573. -

1) sm. 1415.- Obtect. Iinn. 'Tr. 1559. XXI1. t.

5s. f. \4. Fruct., Curr.). - Voges. 1219.

(Amphisph.) Not. Sfer. Tt.

- Inccae gloriosae S. (Ienders?). - Lim.

'Tr. 1 559. XXII. t. 19. f. 205. (Fruct., Curr.). -

Carol. I. 6if.

Zeae Schw. (Hendersonia' - - Linn. Tr. 1559.

XXI1. t.5!. f.125. Fruct, Curr..- (Diplod.) Carol. I. 71 .

Zerbina (Amplisplateria). - Not. Sfer. 73.

Zobelii. - 'Iul. F. hyp. t. 13. f. 1. - Mier.

J. I ๖.9. VII. t. 11. f. 13. (Curr.).
Sphacriacei. - Fructif.: Ann. sc. 11. IStu. IIV.t. 19. Nont.).

Splı a erid i um flavovirens. - Fuck. rh. I 60.

- vitellinum. - Fres. t. 5. f. $31-36$. - Fuck. s. 253. - Fuck. rh. :79.

Sphaerila erythrospora. - IIdw. I. t. 5. f. 5.

Sphaerobolus. - [S. 439.] - Pay.b.cr.p. 10 s. f. 493 .

- crustaceus. - [S. 416 . ] - Carol. I. 72.

(Licheropsis spliaerobol.).

- eyclophorus Fr. B Bisch. f. 36-15.

impaticns lioudier. - Bisch. f. 361 t.

- stellatus T'rl. - [S. 434.] - Cd. Ic. V. t. 3.

f. 45. - lierk. Out. t. 21. f. 2. - Cd. Anl. t.

J). f, 11: 1:-20.- Loud. f. 16350. - Bisch.

f. $3643 .-$ Tul. F. hyp. t. 21. f. 11.- R. f. 36 .

- ef. Sphaeria. - Bot. Zt. 1451. t. I. f. 12.

liasid, Bonord. - Dsm. 1626. - Purton. I.

t. 31). - lib. 337 .

— tubulosus. - S. 439 .

spluacrocarpus fragilis. - ef. Leocarpus.

Sphaerolina. - cf. Sphacria, Nectria.

L Georginae. - Fuck. s. 650.

_- pellita. - Fuck. s. 619.

- Tanaceti. - Fuck. s. 6.51.

- xantholeuca. - Fuck. s. 6is.

sphaeromyecs. - [\$. 491.]

- algeriensis. - Alger. t. 21), 3.

spliacerona ema. - [S. 100.$]$

- aciculare Fr. - [S. 400.] - Bisch. f. 3529.

__ acrospermum Fr. - Bisch. f. 3525.

___ anenticolum. - Kl. II. 410.

- Anchusac. - Ki. 1162.

- Anemones. - Lib. 167. Sphaeria, Dothidea .

- blepharistoma. - Brit. f. Is 4 . - Mag. Zool.

Bot. 1 337 . I. t. 15. f. 4. p. 513 . Berk.).

Cerasi. - Kl. 1464 .

- circinans. - Lotos Prag, 15.55. t. 1. f. 1 .

Peyl

— Cirsii Lasch. - Kl. 1069.

_ cladoniseum. - [S. 400.] - Voges. 977.

- clavatum. - C'lille n. 9. f. 9.

- colliculosum. - [S. 100.] - Voges. 975.

_ columnare. - [S.100.4.]

- comatum. - Ki. 1535.

- conicum. - [S. 100.] - Bisch. f. 3530.

- consors. - Carol. II. 61.

cylindricun. - [S. 400.] - Jisch. f. 3526. - v. affine Fr. - Kl. lo6s. - Kl. II. 53. - elegans. - Act. Turin. IS15. VII. Dec. ¿.

f. S. (Notar.).

epicecidium Berk. - Hook. J. 1519. I. t. 9. I3.

feros. - S. 10 S.] - Act. Turin. is 15 . VII.

Dec. 3. f. 9. (Notar.).

Georginae. - [S. Hok.]

- Geranii Ces. - Kl. 1161.

lielicomyxa, - Fres. t. 1. f. 21-29.

- hemisphaericum. - [\$. 400.] - Bisch. f.

3539.

— macrosporum, - Carol. III. 61.

Mercurialis. - Lib. 264.

parabolicum. - [S. 400.

- Piceae. - Kl. II. 661 .

- Pini. - Ism. HI. 761.

- pistillare. - Kil. 1549.

- pithyophilum. - Kl. II. 1159.

- polymorphum. - Kl. 1160.

- pyriforme. - [S. 4(1)().]

- Rhinanthi. - Lib. 263.) - Wstd. 1052.

(Sphacria F. Somm.).

rufum. - [S. 400.] 
Spha erona em a Serratulac. - Kil. 1161. - Kil.

II. 663. - Ital. (6.5).

Surbi Lasch? - Kil. 1162.

Spinella k. - K. f. 15 i.

squarrosum Ricss. - Bot. K/t. 1453. t. 3.

f. $36-39$.

sticticum, - Crypt. 13ot. intaret. roy. Erc-

bus. 1551. t. 163. 1.

subtile Fr. - S. 100.$]$ - R. f. 162.

subulatum. - S. 101). - Bisch. f. 35.32. -

Fuck. s. 507. - Bail S. t. 1\% - Kil. 1163.-

Ki. II. (i62. - Loud. f. 16160. - Mag. Kool.

Bot. \५37. I. t. 15. f. I. e. p. \$13. Berk.

Ital. 653 .

- truncatum Fr. - [S. 106.] - Bisch. f. 35 15.

- Kil. 1536.

- Uredineorum. - Kl. 16.59. (cf. 1747.).

- verrucosum. - Cd. Anl. t. F. f. 5t: 12. 13.

- Pay. b. cr. p. \&. f. $2 \bar{\gamma}$.

Sphaeropsis. - [S. 419.] - cf. Sphaeria.

abscondita. - Chile n. 10. f. 5.

- Aceris. - Dsm. III. 6is.

aequiroca. - Dsm. III. 679.

arundinacea. - [S. 419.

Atomus. - Voges. 1465

Buxi. - [ะ. 4. 419. ๙.]

cid.).

Candollei B. B. - Dsm. 1319. Septoria pha-

- Castagnei Mont. - Dsm. $1+62$.

conica Lév. - Démid. Russ. t. 5. f. 9.

conigena M. - Wstd. 1230.

- cylindrospora Dsm. - Wstd. 540.

clepressa. - Wstd. 1124.

elevans. - Carol. V. 75.

Evorymi. - Dsm. 1II. 67i.

fimicola. - Bull. ac. belg. 2. Sér. 155\%. II

p. 579. f. 9. Wstd. .

- foreolaris Fr. - [S. 419.] - Kl. 755. (Sphae-

riał. - Fuck. s. 39s.

Hederae. - Wstd. 1126.

Juniperi. - Dsm. 1.16il.

longissima P. - S. 419.] - Kl. II. 50. -

Fuck. s. 399. (ef. Sphaeria) - Wstd. 1139.

- mclaena Fr. - [S. 419.] - Kl. 533. Sphae-

ria). - R. f. 154. - Fuck. s. 100 .

- Mirbelii Lév. - [S. 119.] - Dsm. 1163. nebulosa P. - [S. 119.] - Kl. 5 10. (Sphaeria.

picea. - S. \$19.] - Fuck. S. 101. Icf.

Spliaeria .

— polygramma Fr. - [S. 119.] - Kl. $124 \%$.

sphaeria).

—— Lév. : K1. II. $5 \$ 3$.

Vaginae. - Kl. II. 737.

Sphaeropus fungorum Guib. - ef. ('lariceps purpurea.

sphaerosoma. - [S. 374. 134.]

- fuscescens. - Bail S. t. 19. - Cd. Ic. VI

t. 11. f. $100 .-\mathrm{Cd}$. Anl. t. D. f. $16: 7-11 .-$

Anixia (cf. Hoffm. Ic. p. 70.).

- ostiolatum. — Tul. F. hyp. t. 19. f. 1.

Sphaerosporium. - S. Isl.]

Sphaerostilbe coccophila Tul. in sched. cf.

Iicrocera coec.

Sphaerotheca. - cf. Erysiphe.

- Castagnei. - Ann. sc. n. 1551. XV. t. 6.

f. 9. 10. 10! Lév. ef. Erys. fumosa, macularis etc. Rbh. - Fuck. s. 445. a. - Berk. In. p. is. f. 20. - Bot. Zt. 1851. t. 6. f. 14. JIoh] .

—— F. Lév. : Erys. Dipsacear. Tul. - Ital. Sis.

f. Agrimoniae. - R. f. 5.5\%.

f. Mlehemillae. - Fuck. s. 45s. a.
Sphaerothec a Castagnei f. Cucurbitac. - Fuck. s. 1)?. h.

— - f. Fpilobii, - Fuck. s. J4s. c.

_ f. Euphrasiae. Fuck. s. lis. d.

f. Humuli, - Fuck. s. Isi. k.

f. Nolitangeris. - Fuck. s. 154. e.

f. Potentillae. - Fuck. s. 154, b.

li. f. 557 .

f. Sanguisorbae. - Fuck. s, 144.

f. Senecionis. - Fuck. s. 154. f.

f. Veronicac, - Fuck. s. 144. i.

pamnosa. - Ann. se. n. $14.51 . \mathrm{XT}^{\circ}$ t. $6 . \mathrm{f} . \varsigma$.

(Lev.). - Erys. pann. Tul. - R. f. 5511.

Sphacrozosma. - ef. Sphaerosoma.

Splinctrina. - [S. 366;.] - ef. Calycium.

ciliata. - [S. 36ti.

microcephala. - Dsm. MI. 267. Calycium).

Wstd. 132\%.

turbinata. - [S. $366^{*+*}$.] - Bail S. t. 15.

Lichen'. - Fuck. s. 759.

Sphinctrosporium. - [S. 501.]

Spicaria Solani. - de Bary, Kartoffelkrankh.

$1 \leqq 61, f .13$.

Spicularia. - [S. 490.] Botrytis.

- gemina. - Botrytis vera, Let.

Solani Harting. - Schacht, Kartoff. t. X. f. 3 .

Spilobolus. - [S. $396^{* *}$.]

- Oleae. - Act. Turin. 1553. XIII. Dec. ?.

f. 11. Notar.

Tiliae. - Hedw. I. t. 11. f. B. 1. - Kl.

1932. a.

Spiloca a. - [S. 452.]

- alba. - Bon. t. 2. f. 57.

- epiphylla. - S. 452 †.]

— fructigena. - Carol. 1. 56.

- Pomi. - S. 452.]

- Scirpi. - [S. 4<2.] - Sturm h. 7. t. 32.

spiloma, - ef. Lichen. spbaerale. - [S. 450 .]

— tumidulum Ach. - Dsm. 541. - Wstd. 316. viridans Schaer. - Wstd. $\$ 22$.

Spilosphaeria. - cf. Sphaeria, Depaz., Ascoehyta, Septoria.

- Legopodii. - K1. II. 741. - Fuck. rh. 451.

- Callae Rbh. - (Depazea C. Lasch.) Kl. II.

551.

Cannabis R. - Ascoch.) Ki. II. 559.

C'lelidonii Rbh. - Sphaeria lichenoid. $v$.

DC., Ascochyta (hel. Lib., Septor. Chel. Dsin.)

II. II. 552.

- Comari. - R. f. 554.

- Hepaticae Rbh. - Depaz. hepaticaecola

Dub., Septoria Hep. Dsm.) - K1. II. 55.5.

- Petroselini R. - Kl. II. 560. - Fuck. rh. 150.

- Polygonorum. - Kl. II. $4+2$.

- Ruborum Rbh. - Kl. II. 533.

-.- Saponariae (DC.) R. - (Depaz. Dianthi A.

S., Nemaspora epiphyll. DC., Sphaeria Sap.

UC. - Kl. II. 555 .

- Scabiosae Rbh. - Ascoclyta S. Rbh., Depaz. purpurasc. r. Ií., Septoria Scab. Dsm.). -

Kil. II. 557.

Tiliae Rbh. - Kil. II. 556.

Vincetoxici Awd. - R. f. 362.

SpIanchnomyces. - [S. 436. g. 13. u. *.]

albus Cd. - Cd. Ic. VI. t. S. f. $82=\mathrm{Hy}^{-}$ menogaster Klotzschii 'Tul.

- Berkeleyanus Cd. - Cd. Ic. VI. t. ऽ. f. ५.). - Broomeanus Cd. Mpt. ef. Splan. olivac. r'd. - Cauvinianus Cd. - Cd. Ic. VI. t. i. f. 72. citrinus Cd. - Cd. Ic. VI. t. 9. f. 4-. - c.

IIymenogast. cit. Vitt. 


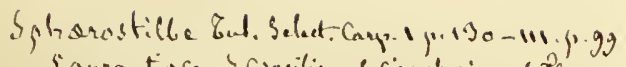

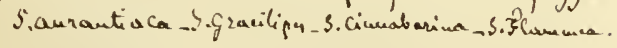



Splanehnomyees Citrus C'd. - Col. Ie. VI. t. \%. f. $>6$.

clathroides Cd. - Cd. Ic. VI. t. S. f. 77.

- Cordaeanus Zob. - C'd. Ic. VI. t. צ. f. st).

- cf. Splan. olivaceus Cd.

dubius Cd. - Cd. Ic. VI. t. 7. f. 70.

Klotzschii Cd. - Cd. Ic. VI. t. S. f. 75.

luteolus C'd. - C'd. Ic. VI. t. 7. f. 69.

luteus Cd. - Cd. Ic. VI. t. 8. f. 76.

lycoperdineus C'd. — Cd. Ic. VI. t. S. f. SI.

nembranaceus Cd. - Cd. Ic. VI. t. S. f. Th. nephriticus Cd. - Cd. Ic. VI. t. ๖. f. 79.

olivaceus Cd. - Cd. Ic. VI. t. J3. f. 107, -

uf. Spl. Broomeanus Cd., Cordacanus \%ob. populetorum C'd. - C'd. Ic. VI. t. s. f. \$.3.

Rabenhorstii C'd. - Cd. Ic. VI. t. ๖. f. 73. R. f. 571). (Rhizopogon luteolus Fr.).

roseolus Cd. - Cd. Ic. VI. t. 7. f. 6). -

Cd. Anl. t. D. f. $45: 1-1$ - Ns.j.t. $10=$

Rhizopog. rubescens Bail S. p. 11. - Sturm

h. 11. t. 2. lihizop. rub. Tul.?.

- tener Cd. - C'd. Ic. VI. t. 13. f. lus; t. \&.

f. G1. - ef. S. Tulasneanus Zob.

Tulasneanus Kob. - Cd. Ic. VI. t. S. f. \1.

- ef. s. tener C'd.

_- virens C'd. - Cd. Ic. VI. t. 7. f. 71.

Webbianus Cd. - Cd. Ie. VI. t. - f, 7 I.

splanehnonema. - S. $396 \ldots$.]

- pustulatum (d. - R. f. 41. - Sturm h.9.

t. $54=$ Sphaeria amblyospora B. B. Currey,

Massar. ambl. Fres.

Spondylocladi un fumosum Mart. - Kl. 1279.

- Sturm h. 35. t. 5 .

Sporendonema. - [S. 49!.

- Achlya prolif. - [S. 191.]

(asei 1)sm. - Dsm. 116. - Bisch. f. $37 \%$

Bon. t. 2. f. 51 - Ann. se. n. 1 127 . XI. t. 21.

— Voges. 995. - Wstd. 300. - Torula C'asei C'd. Nuscae. - S. 491 .]

Sporidesmium. - 5.506.$]$ Tolydesmus.

adscendens. - Ann. Mg. n. h. Isfo. IV. t.

ऽ. f. 1. Berk.

angustatum. ..- Sturm h. 7. t. 22.

antiquum. - [S. 5116**

asperum. - [S. $5116^{*}$.]

atrum. - [S. 506.] - (d. Ic. VI. f. 11. -

l.oul. f. 16612 - Sturm h. 7. t. 17.

bryounces. - [\$.506.]

- caulincola. - Bisch. f. 3592. - Sturm h. 7 .

t. 19 .

cellulosum Ǩl. - Kl. 159.

ciliatum. - Cd. Anl. t. B. f. 1: 14. - Bisch.

f. 3595 . - Sturm h. T. t. 20 .

Cladosporii C'd. - Fuck. rh. 77.

clavaeforme. - Sturm h. 25-. t. 4. (Treuss.)

clavatum. - Ann. sc. n. 1513 . XIX. t. 7.

f. 4. Lév. .

compositum. - Carol. T. 94.

- concinnum. - Cd. Ic. YI. f. 15. - Hook.

Lond. J. 1s45. IT. t. 12. f. 3. - Carol. I. 90.

(Termic. clav. Schw.).

congestum. - Sturm h. 29-.t. 31 . (Preuss. .

copulatum. - hil. $15 \mathrm{St}$.

curvatum. - Carol. III. \$9.

- epiphyllum. - Ann. sc. n. 1513. XIX. t. 7.

f. 3. (Lér.).

Eremita. - [S. 506.] - Kl. J วৎ?.

exitiosum. - Hdw. I. t. 12. f. B. C. (Küuhn).

- Fuck. s. 133. - Bot. Zt. 1556. t. 2. 3.'-

Kl. II. 151 .

- v. Dauci. - Kl. II. 152.

Havum Bon. - Bot. Zt. IS53. t. 7. f. 3. sporidesmium folicolum. - Dsm. $1 \geq 3$. Stigmella dryina Lév.

- fuscum. - Bon, t. 2. f. 43. - Fuck. s. 135.

- fusiforme Ns. - Sturin h. T. t. Is.

-_. Lepraria. - Berk. In. p. 75. f. A ‘. a.

— - nigerrima. - Hook. J. 1553. V. t. 3. 1.9.

macrospermum C'd. - Sturm h. i. t. 21.

marianum Wstd. - ef. Sp. nyriaumm.

- microscopicum Bon. - Bot. Zt. 18.33. t. †. f. 1 .

myrianum. - Dsm. III. 2. - Wstd. -61.

nodlosum. - Kil. 1175.

- pararloxum. - [S.506.] - Pay. b. cr. p. 76.

f. $35 .-$ Kl. 1171.

polym. - cf. Stemphylium polym.

- pyriforme, - ef. Stemphylium pyr.

scirpicola. - Fuck. rh. Th.

- septorioirles Wstd. - Bull. ac. belg. I. Śér.

XXI. 2. p. 216, f. 1. - Wstd. - 62.

simillinum. - S. 50 .

sparsum.-Fres. t. ti. f. 12-11.

stygium. - Carol. IV. Sᄂ.

uniseptatum I3. I, - Ann. Mg. n. h. I-5:!.

III. t. 9. f. 2.

vagum. - [S. 506.] - Kil. 1362.

- vermiforme. - Fres. t. 6. fo 56-(i)

Fuck. s. 131.

- Inck. rl. i6.

- vulgare. - Kl. 1361 .

siporisorium. - S. 516.

Colchici I.ib. - ef. Uroeystis, Uredo. -

W'stel. 1141.

_ Maydis C'es. - Iil. 1070.

muricatum. - Kl. 1693.

Sporocid us. - is. $\$ 1$ i. $]$ Hendersonia.

- eytisporoides. - Lotos (l'rag) 1455 . t. 1. f.

16. Peyl

diversisporus. - Kl. 1 557.

- Fiedleri Rab. - Kl. \$2. - Hend. Kil.

II. iI

- Georginae. - [S. 117.]

- herbarmu. - $\$ \$ .417$.$] - Bail S. t. 16$.

lil. IS5).

- maculans. - ('l. Aıl. t. F. f. 5s: 1-1. -

Pay. b. cr. p. is. f. 36 i. - Kl. 1167.

- Opizii. - Lotos. 1557. VII. t. 1. f. 1.

- rosaecola Rab. - lil. 1166. - Ienders. Ro.

sae $\mathrm{Fr}$. Su.

— ruscicola. - Kl. $165 \%$

- Sophorae. - Lotos. 15.7. VII. t. 1. f. 5.

subglobatus, - Kil. 1947.

Sporocybe. - [S. 167. 164. 191, 21.] Periconia Bon.

_ - albicla. - [S. 167. T'achnoc.] - Brit. f. 5\%. atra. - [S. 167.$]$

byssoides. - [S. 467.] - Bon. t. 10. f. 217. - Fr.: R. f. 64.

calycioides. - [S. 467.$]$ - Carol. I. $\$ 3$.

- Desmazieri. - [S. 467.491, 2 ].] - Ann. se.

n. 1537 . VIII. t. 1. f. 3. - Cd. Anl, t. B. f. 19:

5-7. - Kl. II. 156. - cf. Stysanus Stem.

- C'ol. (non Desm.) Cd. Ic. IV. t. 7. f. 90.

(ex Berk. ?).

- Iobulata Berk. - R. f. 171. - Ann. Mg. n.

h. 1ธ 11 . VI. t. 13, f, 17. Berk. .

nigrella. - [S. 467.] - Ann. Mg. n. h. $19 ! 1$.

TI. t. 13. f. 16. (Berk.).

- Persicae. - Carol. IT. 79.

- Resinae Fr. - [S. 46॰.] - KI. 151. - Kl.

II. 155 .

sporodinia. - [S. 158.] zu Syzygites meg. 
Sporodinia grandis. - [S. 14-.]- Bon. t. T. f. 160 .

h. 36. t. 4 .

Sporot um. - [S. ]9!.] asperum. - Kl. I491.

- conopleoides. - [S. J9!). gram.] - ('d. Anl.

t. Ii. f. 14: 1. 2. - Fuck. s. 1liti. - lil. 771.

- Fuck. rh. II. 101 .

- gramincum. - [\$. 19!).

- Cherbarum. - Bon. t. 3. f. (i?).

_- lineatum. - S. 499.

- sparsum. - [^. 1919.$]$

Sporomega Audromedae. - lub. Hyst. t. 2. f. 2 !.

degenerans. - [S. 364, 15. - ('d. Ic. V.

t. !. f. lill. - (d. Anl, t. F. 1. 5!: :-12. -

l'ay. b. cr. p. 91. f. 123.

śpuionema. - S. 123.

- glandicola. - Dsm. III. 3. - Voges. 1.j61.

- hycmalis. - Insm. III. 4I.

- plracidioides. - D sn . 12 !

— ramealis. - Dsm. III. 52.

strobilina. - Fil. II. 756 - D D.m. III. Tot?.

- Voges. 1343.

sporophle um. - [S. $502,1$.

- Graminum. - ef. Arthris. spor.

śporormia fumetaria. - Act. Turin. Int?. X.

Dec. 5. f. 6. Notar.' - Kil. II. 257. - ef.

Splizeria.

Sporoselis ma mirabile. - Fres.t. (i. f. 26-

24. - Berk. In. p. 327 . f. 7.1. a. - Kil. 1554 .

- Kl. II. $7(i 2$.

Sporotheea. - [S. 50 T.]

- Carpini. - [S. 507.] - Sturn 1. 9. t. 5.

Sporotrichum. - [S. 492.495.] 'Trichosporium. 'luburcinia.

- ? in unguibus hominis. - Archiy f. pluys.

Ileilk. XII. p. 193. ie. G. Meissner. - in ab-

domine piseis: Bull, ac. belg. 1555. V. 2. p. 29.

P'oelman.

- aeruginosum. - cf. Sp. viride.

agaricinum. - cf. Monospor. ag. Bon.

aurantiacum. - [S. 496.$]$

- caesiellum. - \$S. 496.$]$

- candidum I.k. - W'std. 592.

chartaceum. - Letell. 679, 4. Mysotrich. .

Wstcl. 119 .

chlorinum. - [\$. 195.]

(ollac. - Kil. 16-2.

-_ conspersum Kz. - Bisch. f. 375\%. (ef. Acladimm).

- densum Fr. - Jisch. f. 3710.

— fallax. - Iib. I> 7 .

- - fencstrale Ditun. - Wstd. S66. - Sturm

h. 1.t. 1 .

ferrugineum, - [s. 495.]

- Fiedleri. - Kil. 1573.

Havissinum. - Letell. 635, 3 .

Havo-rirens. - Kil. 17>1.

foliorum.

- v. Brassicae. - Dsm. 227

fungorum. - Letell. 639, 1.

- fusco-album. - [S. 4!6.] - Kl. is].

- fuscum. - Bon. t. 6. f. 134. - Letell. (7)!),

$3 .-$ Fuck. rl. 139 . griseum. - S. 44t;.]

- Hippocastani. - C'd. Anl. t. B. f. 7 : 2.

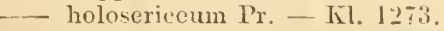

__- Iolithus Ficin. - Wistd. 6999.

- lanatum W'llr. - Kl. 127.

laxum. - [S. 496.] - Ki. 1.13\%. sporotriclium lyococcon. - [s. 196.] - Kl. 157.1.

- maculare 1.k. - Wstrd. 116.

- merdarium. - [S. J96.]

- minutum Grev. - Inoud. f. 165ill.

- muxinum. - Bon. t. fi. f. 1.15.

- nigrum lir. - Bisch. f. 3715 .

- mitens. - Ism. 1511.

- olivaccum. - lil). JSti.

- pannosmm. - IIedw. I. t. 4. f. t. - Kl. I!) î.

- poly:sorum. - [S. 4!li.?

- pulchellum Dub. - Witd. 110.

roseum, - [s. 4!9i.]

- rubcrimun. - S. 196 i.] - R. f. 174. - cf.

Terticill. ruberr. - Letell. (i35, 5.

_- rubiginosum. - [S. 195.]

salicinum, - [S. 4!)

— sporulosum. - I.etell. (63.5, 1-3.

_- sulfureum Grev. - [s. 196.] - Loud. i.

i6571. -- Brit. f. $211 .-$ Iiull. ac. belg. 1552.

XIX. 1. 1. 万(i). Spring.

torulosum. - Bon. t. 4. 178.

- virescens. - [S. 495.]

— viride. - Ietell. (i79, 2. (aeruginos. P. ?).

— vitellimm. - [5. 196.$]$ - I.etell. 635, 2.

Spumaria. - S. 449.]

- alba. - 5.149 .7 - Fl. dan. XI. 197. 1.-

Fuck s. 297. - Bisch. f. 3630. - Dsm. 119. -

Loud. f. 16511. - Bot. \%t. 1515. t. 5. f. 1-1;.

_- Schum. = Diderma spumarioides, 1 ll.

dau. -- Purton. II. t. 21.- Lib.179. - Wiste.

351.

-_ - v. laminosa Kil. - Kl. 59.

cornuta Schum. = alba, Fl. dan.

- didemoides 1 . = Diderma oblong. Fl. dan.

[s. 451 .

- granulata Schum. = Diderma gr. Fl. dan.

- minuta Schum. = Diderma min. Fl. dan.

Nueilago. - W'std. 341. - Cd. Anl. t. C'.

f. $34:$ J-3. - Schnzl. t. 15. f. 52-56.

- physaroides. - [S. 452. 2.] ef. Didymium, Let.

— ramosa Schum. = Diderma ram. Fl. dan.

s'tacluylidium. - [S. 490.] - Botrytis.

- agaricinum. - S. 490 .

- araucarium. - Bon. t. 7. f. 155.

_- Bassianum. - [S. 490 .

_- bicolor. - [S. 190.]

- candidum Grev. - Loud. f. 16546.

- characeun. - Cd. Anl. t. B. f. 16:3-5.

- diffusum. - [S. 490.] - Bisch. f. 374\%. -

Ki. 1349. - Berk. In. p. 31 1. f. 71. c. - cl.

Botryospor. diff.

- paradoxum. - Bon. t. 7. f. 152.

pulchrum. - Bot. Zt. I\$59. t. 11. f. $\uparrow$.

Iloffm.). - cf. Botryospor. p., u. C'eplialospor.

botryoid.

— Sceptrum Fr. - Biseh. f. 3746. - ef. Secptromyces.

- terrestre. - [S. 496 .

stachyobotrys. - [S. 491.]

alternans, - Bon. t. 9. f. $1 \$ 5$.

- atra. - Cd. Anl. t. B. f. 15: 5-5. - Pay.

b. er. p. 7t. f. 333 .

st a urophallus. - [S. 43.1*.

-.. senegalensis Mont. - Cd. Ie. VI. t. 4. f. I.

St a urosp ha eria Lycii. - 1bull, ac. belg. 1\$54. VII. p. 366i. f. 211. (Wstd.).

- Rosarum. - Wstd. 137l.

steganos porium. - S. 504.] ef. Stegonosp)rium.

Stegasma. - [S. $455^{\circ}$ ] 


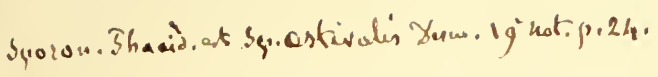

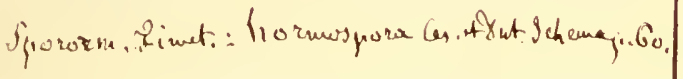




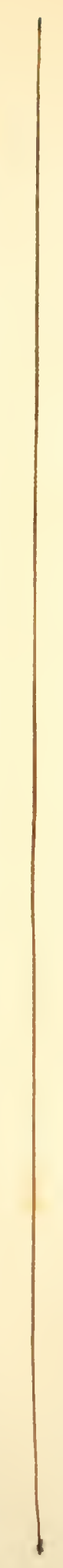


St c g a sm a depressum. - C. Ic. V. t. 3. f. 3.1. - Cd.Anl. t. C. f. $29: 31$.

Stegia. - [S. 370.]

II. 32 .

- Ilicis. - [S. 3711.] - Kil. II. 141. - D:m.

411. - Fuck. s. 723. - R. f. t13:3. Ital. 534.

- Eustegia. - Schweiz. 1!. - Tiypt. Bad. 5.5.

Stegilla arundinacea Fr. - Kl, 552.

- discolor. - Bail s. t. 14.

stegobolus. - [S. 52].]

stegonosporium. - [个. 50s.] - Fres. t. 7 . f. $5: i-5 \%$.

- cellulosum. - Micr. Journ. IV. t. 11. f. 10

-25. Curr. - Kl. II. 14. - Ital. 117

- clavatum Riess. - Bot. Zt. 1553. t. 3. f. 21 $2 i$.

elevatum. - K1. 1453. - Dsm. III. 255. -

Fuck. rh. 95. - 1i. f. $5>3$.

muricatum, - Bon.t. 2. f. 52, - ? R. f. 264. - pyriforme. - Cd. Anl. t. F. f. ji: li. -

Fuck. s. 121. - I3ail s. t. 11;. - Wstd. 5i . -

lick. rh. 91.

Steiroch a te Malvarum. - Verh. Ver. z. Jic-

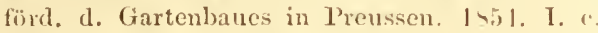

l'raun, Casp., de B.!. - Kl. 1!7!).

—- Solini. - Kil. 1950

Stem maria C'arestiac. - Ital, 4৬ל.

- eyathiformis. - Wistel. 142.

— globosa Preuss. - Sturm li. ifi. t. 7.

Stemonitis. - S. 155.]

- arcyrioides. - [s. 155.]

- enceinea Rioth. - Wstd. \$1.

- decipiens. - Nov. Aet. 14:32. XII. J. t. 7. l.

livolut., Ns.).

- elegans Roth. - Wstd. 12(i).

- fasciculata P- W'std. 42. - 1.0u(l, f. 1(i.) 19.

Voges. 490 .

- ferruginea. - S. 15.5.] - FI. dan. XII. 21116.

1. - Fuck. s. 27 - — Jisch. f. 3701 - 1'ily.

b. cr. 1) 122, f. 5\(1. - Keitschr, f. wiss. Zool.

1५i.. X. t. X. f. 1-13, de Bary, - ('arol. II.

i5. — Wstd. $\$ 2$.

Havescens Sehrk. = Arejria umbrina, F' dan.

Friesiana de $\mathbf{B}$. (oblonga et obtusata. - I:

f. 5lis. - lirypt. Barl. 55i.

furfuracea Gmel. - Wstd. i) IS.

- fusca Roth. - [S. 4.j..] - Fl. clan. XII.

2iil6. 2. - Kl. 374. - Fuck. s. 279. - Bisch. f.

3litil. - Jil. II. 157 . Zeitschr. f. wiss. Kool.

I5.59. X. t. 6. f. 20,25 ; t. 10. f. 1 1. de Bary.

- Bot. Zt. 15.9. t. 11. f. 13. Teing., Itofln./. globosa Schum. = obtusata v. glob., Fl. dan. - lencocephala P. - Wstd. 1>2.

- Iencopodia DC. - Wstd. 1260.

- leucostyla P. - Wstd. 126ii.

- lutea Trent. = Arcyr. umbr, Fl. dan.

- mammosa. - [S. $15 \overline{5}$.

nigra Schum. = St. ovata v., Fl. dan.

- oblonga. - [S. 155.] - R. f. 56s. Friesiana $\mathrm{r}$. ).

- obtusata Fr. - [S. 155.] - Zeitschr. f. wiss.

Zool. 1 559. X. t. 6. f. 21-21. de Bary). Carol. V. $81 .-$ R. f. sfis. Friesiana).

- v. globosa Fr. - Fl. dan. XII. 2091. 2. - ovata. - [S. 455.] - Cd. Anl. t. C. f. 32: 5. 6. - Fuck. s. $27 \%$ - Kl. 1132. - Zeitschr. f. wiss. Zool. 1 $\$ 59$. X. t. 6. f. 15. 19. de B.). - ef. Enerthenema eleg. - papillata P. - [S. 455.] - Loud. f. 16.j20.
- N. f. 114. - Zeitschr. f. wiss. Zool. 15.59. X. t. 10. f. 17-19. (de Bary). - cf. Enerthenema el.

Stemonitis physaroides Fr. ? = Trichia phys., F1. dan. Ss. t5.5.

- protracta, - S. 155.

- pulchella. - Ann. Mg. 1. h. 1s 11. VI. t. 12.

f. 11. Berk.'.

- pumila. - S. 455.] - Cd. Ic. V. t. 3. f. 37. tubulina. - S. 45.5 .]

— typhina Willd. - Wstd. >2.

- typloides. - S. 45..] - Kil. 423. - Fuck.

s. 250 . C C. Anl. t. C. f. 32: 1-1. - t/eitsehr.

f. wiss, Zool. 1 4. Y. X. t. 10. f. 15. 16. de Bary! - Carol. II. 76.

- violacea Schum, = fermuginea, Fl. dan. IVstd. \$2.

Stemphylium. - S. 506.] - e Cladosporio:

IIedw. I. t. 10. f. 7 .

- botryosium. - [ค. 50 (i).

— cordae. - \$. 506.]

_- dubiun, - S. Stui.]

- ericoctomm. - Verh. d. Ver. z. Beförul. „1.

(iartenbaues in 1'renssen. 1 5 1. I. t. 2. Liram,

('asp., de I3.). - Iil. 1444.

- polymorphum. - Jion, t. 3. f. 7i. - Fuck.

rh. $1 \geq 1$.

— pyriforme. -.. IBon. t. :3. f. 71.

- subtile. - S. $50(0.7$

Steplıanoma strigosum Wrlu. - of. Asteroph. lezizae ('d.

Stephensia bombyeina. - 'Tul, F. hyp. t. 12. f. 1 .

Sterbeeckia. - [S. 143.]

- cornucopioides. - Nov. Act. 15.32 . XVI. I.

t. 11. f. 33. I) Inortier, - Wstd. 1!).

Stereun. - S. 332. $330^{\cdots+4}$. ] cf. Thelephora.

- abietimum. - [S. 3332.$]$

— acerinum. - [\$. 333.] - carol. II. $3 \%$.

- albo-badium. - ('arol. I. 29. 'Theleph. Schw.). alneum. - [S. 333.]

atratum. - Amm. Mg. 11. 1. 1S12. X. t. 11.

f. 12. p. 3\2. 13erk. .

_ aurantiacum. - [\$. 333.$]$

avellanum. - [S. 332.$]$

- bicolor. - s. 332. - Carol. II. 33.

- candidum. - Carol. I. 32.

- cinereo-badium. - Nov. Act. XIX. Suppl. I.

t. 5. Klotzsch.

- coeruleum. - Carol. III. 27.

- complicatum. - Carol. II. 30.

conchatum. - [S. 332.]

- Curtisii. - Carol. III. 26 .

- disciforme. - [5. 332.$]-\mathrm{J}) \mathrm{sm} .22$.

dryinum. - [S. 33:2.

- ferrugineum. - [S. 332.]

- fistulosum. - Carol. II. 31.

- frustulosum. - [S. 33:3.]

giganteum. - Carol. II. 35.

- lirsutum. - [S. 332.] - Kl. 42. - Kl. II.

211. - Ital. 292.

hydrophorum. - Hook. J. 15.36. VIII. t. 6.

- Ann. Mg. n. h. 1544. XIV. t. 9. (Berk.).

- laeve. - Carol. II. 39.

-_ Leveillianum. - Carol. II. 35. (Corticium). lilacimum. - Ital. 336. (Thel. purp. v. pinea).

lobatum. - Carol. I. 24.

- ochraceo-flarum. - Carol. II. 31.

- papyrinum. - Carol. II. 36.

- pergamenum. - Carol. III. 25.

Pini. - [S. 332.] - K1. II. 213. 
Stereum purpurcum Schmm. Fr. $-{ }_{\mathrm{L}} \mathrm{S} .330^{\cdots}$. 3:32.] - Ǩl. II. 504. - Ital. 335.

- Ravenclii. - ('arol. IV. 13.

- reniforme. - Ann. Mg. 11. 1., 14t2. X.t. J1.

f. 11. 13. 342. licrk.).

repandum. - \$. 333.

- rubiginosum. - S. 332. - 1) sm. S21. -

kil. II. 212. - Wstd. 1249. - Ital. 293. - rugosum. - s. 332.] - Kil. 11. 503.

— sanguinolentum. - S. 332.] - R. f. 210.

— ¿Bail s. t. 24.

spadiccum. - [S. 332.] - Carol. II. 32. -

- Wstcl. $12 \% 0$.

- spectabile. - Nor. Act. 15.13. X1X. Suppl. I.

t. 5. Klotzsch .

- striatum. - Carol. I. 31. Theleph. sericea Schw.'

- subpileatum. - Carol. I. 30.

— tabacinum. - [S. 332.] - W'std. 1255

- vorticosum. - S. 332 .

Stcrigm a toe s s is antacustica ('ramer. - Vicrteljalnrschr. nat. Gies. Zürich. 1 599. IV. t. 2. p. 337. - ef. Aspergillus dub. Berk.

- sulphurea. - Fresen. t. 111. f. 311-33.) p. 43. Stictis. - S. 372: Xyl.373.] - ef. Propolis,

Schmitzomia.

alba Fr. - Fuck. s. 707.

- arundinacea. - S. 373.]

atrata. - Dsm. 92\%.

atrocranea. - s. 373.

Berberidis. - Aet. Turin. 1>\$1. III. Dec. 1.

f. 6. Notar.

Berkeleyana D. I. = Propolis Berk. Dsm.

'Tul.). - Voges. $1346 .--$ Alger. t. 2!!, S.

— chrysophaea. - [S. 363.] - Ann. Mg. 11. M.

1561. VII. t. 16 . f. 19. p. 451.

circinata. - Lib. 232.

- coccinea. - is. 356.

cxigua. - Voges. 1241. - Dsm. 1033. -

IIstd. $9>9$.

- fenestrata. - Dsm. 16011.

furfurella. - Kl. 1929.

- graminicola. - Kl. II. 713.

- Graminum. - Dsm. 471.

hemispliaerica. - S. 373.

hysterioides. - Voges. 1242. - Dsm. 717.

- Brit. f. 305 .

- inclusa, - S. 373.

Lecanora. - S. 373.] - Kil. 923. - R.f.

15\%. - Dsm. 69. - Lib. 30 .

- licheniccla. - [S. 373.] - Ann. sc. n. 1536.

V.t. 13. Mont.!

Luzulac. - Lib. 133.

macroloma, - Alger, t. 29, 5.

nigrella. - S. 373.$]$

- nivea I'. - Voges. 1095. - Act. 'Turin. $1>11$.

III. Dec. 1. f. ว. Notar. - F Fuck. s. Til. -

Kil. 1550. - Kil. II. 712 . - Wstd. 1240.

osellata. - S. 373.$]$

Oleac. - [S. 367*. $]$ - Act. Turin, 14. 5.

VII. Dec. 3. f. 1. Notar.

pallida. - [S. 373.] - Norweg. 157.

Panizzei, - Act. Turin. 1S45. VII. Dec. 1.

f. 2. Notar. - Kl. I1. 313. - Ital. 44.

pelvicula. - [S. 373.]

Pupula. - S. $364 ; 363^{* *}$.] - Carol. II. 54. radiata. - [S. $361 ; 363^{\cdots+*}$.] - Loud. f.

$11 ; 25$. - Dsm. 471). 11132. - Bisch. f. $3404 .-$

Kl. 637 ; 192s. - Brit. f. 70. - ('arol. I. 43.

rosacea. - S. $3(1) 1,3$.

rubicunda. - Alger. t. 29,-6.

seriata. - Lib. 233.
St ict is Seslcriae. - Carol. I. 12. - Lib. 132.

- sphacralis, - 's. 366.$]$

stellata W. - Fuck. s. ills.

Thelotrema. - Alger. t. 2!), 3.

Tiliae Lasch. - Kil. 63ৎ.

valvata. - Kl. 1927. - Dsm. 921. - Wstd.

235.

versicolor. - Cd. Anl. t. G. f. 6l: 5-ৎ. -

Bail S. t. 19. - J'ay. b. cr. p. 60. f. 3!n,. -

Dsm. 7І6. - Bisch. f. 3ł05. — Fuck. s. 7॥k.

Propolis - Kl. 1633. - Carol. II. 53.

Stigmatea. - is. 121.1 Dothidea.

- Alchemillae. - [S. 422.] - Fuck. rh. 125.

- Chaetomium. - iS. 422.] - Fuck. s. 3yu.

- Fuck. rh. 12 t.

- circinans, - Fuck, rh. 121.

conferta. - S. 421 .

Geranii Fr. - [S. 421.] - Fuck. s. 355. -

Fuck. rh. 120.

_ maculacformis. - [S. 421.] - K1. II. 72. -

Fuck. rh. 12:.

- Petasitidis. - Fuck. rh. 426.

- Polygonorum. - S. 121.]

- I'otentillae ('oleroa). - [S. 422.] - Kl. II.

155. - Dothidea P. - Fuck. s. 459. p. pte.).

- Dsm. III. 55\%. - Fuck. rh. 423.

- Ranunculi. - S. 421 .]

Robertiani Fr. - [S. 421.] - Sphaeria I.

Rbh., Dothid. R. Fr. - Kl. II. 569. - Fuck.

s. 34\%. - Krrpt. Bad. 425. - Fuck. rh. 419.

Stigmatella aurantiaca. - Berk. In. p. :313.

f. 71$)$ b.

- dryina. - ef. Stigmella dr.

Stigmatosphaera Artocreas Dmtr. - Wstd. 27.

- Racodium. - Wstd. 655.

_ rubclla Dmtr. - Wstd. 171.

Stignella. - S. 426.]

-__ dryina Lév. - R. f. 351\% 'Stigmatella, Spo-

ridesnium foliicolüm Dsm., Stilbospora dryo-

phila (d., Dicoccum dryophilum Cd. Ic.). -

1)émid. Russ, t. 5. f. 5. - Voges, 1461.

Stilbin um. - cf. Stilbum.

Stilbospora. - S. 50s.]

- affinis. - Not. Sfer. 53.

angustata $\mathrm{T}$. - [S. 50S.] - Kl. II. 591. -

Fuck. s. 130.

- asterospcrma. - [S. 505.] - cf. Asterospor.

Ioffmamni Kz. - Wstd. 5if. - R. f. 5S4.

- atherosperma Hoffm. - Bisch. f. $3 \varsigma ! 16$.

botryospora. - Ann, sc. n. 1536. VI. t. 15.

Mont, $=$ Myrocephal. botryospor. Fres.

- bullata Lk. - Wstd. $\$ 5 \overline{7}$.

cellulosa. - [S. 50 - . ]

ceratospora. - Act. Turin. 1S 11. III. Dec.

1. f. I11. Totar. chartarum. - Wstel. 149.

conglomerata Lk, - Wstd, 573.

didyon. - cf. Didymosporium complanatum. dryophila Cd. - ef. Stigmella dryina lér.

fugax. - cf. I)idymosp. compl.

Juglandis. - Wstd. I 159.

Tickxii. - Bull. ac. belg. 1. Sér. XVIII.

p. 417. f. 6. (Wstd.). — Wstd. \$50.

- macrosperma P. - [S. 50 S.] - Bisch. f. $35 i 9$.

- Fuck. s. 131. - Schnzl. t. 12. f. 16-1s. -

Fres. t. 7. f. 46-52. - Hook. J. I-51. III.

t. 9. f. 9. u. t. 10 . f. 2. - Pay. b. cr. p. 61.

f. $251 .-$ Kl. $993 ; 1571$ - R. f. 150 - Dsm.

III. 75. - Voges. 353 . - Schwabe ?. an-

halt. II. t. 6. f. 16. - Lib. 390. Wstd. $575 .-$

Fuck. rh. $>2$.

- macrospora Lk. - W'std. 575. 



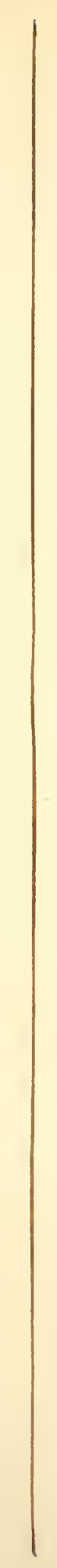


Stilbospora microsperma Johnst. = Discella carbonacea.

3. B. - Voges. 354. - Wstd. 132.

- militaris. - Mier. Journ. IV. t. 1\}. f. 1-9. (Curr., .

- ovata P. - [S. 504.] - Bisch. f. 3490. -

Wstd. 1:31.

- profusa Grev. - Loud. f. 1660 .

- pyriformis. - [.5. 505.] - Carol. V. 9.5. -

Lih. 295. - Wstd. 131.57\%.

- rhabdospori Fr. - Bisch. f. 3459.

__ spermatodes L.k. — Wstd. 132.

- sphacrosperina. - Melanconium, Wstd. 392.

Credo DC. - Wstd. 137.

- utriculosa. - Schwabe Fl. anlalt. II. t. C. f. 17 .

Stilbum. - [S. 165. 169.] ef. Griphium.

- in larvis: Cramer, uitlandsche Kapellen.

T. VI. p. 133 . cr. t. 267 . A. B.

- aeruginosun. - Ann. sc. n. 1530. IIX. t. 1 s.

f. 1. Dins.).

- anomalum. - Mag. Zool. Bot. 1437. 1. t. 3.

f. 9. p. 49. Berk.).

- aurantiacum. - Ann. MIg. n. 11. 1411. VI.

t. 12, f. 14. Berk. .

- bicolor P. . [S. 469.] - Fl. dan. XIII.

$22>0.1$.

bulbosum Td. - Bisch. f. 3\$13.

Buquetii M. R. - Robin v. p. t. S. f. 3. 1. ,

t. 9. f. 4. 5., t. 11. f. 1. 3 .

- byssinum. - [S. 469.]

- byssisedum. - [S. 469.

- byssoides sprg. - Wstd. 45.

- cateliatum. - Kl. 1753.

- cinnabarinum Mont. - Sagra, Cuba. t. 11.

f. 3. - Carol. I. \2.

- erystallinum $=$ Pay. b. cr. p. 71. f. 336.

- erythrocephalum Ditm. - [S. 469.] - Biscl.

f. 37-0. - Dsm. 912. - Wistel. 59. - Kil.

1425: Helotium fimetar. Fr. Su. t. Fuck.). -

sturm h. 3. t. 4j.

gelatinosum I'. - Bisch. f. '3515.

- herbarum. - Kl. II. 763 .

- hirsutum. - [S. 469.] - K1. 17\3.

hyalinum. - is. 469.]

- incarnatum. - Jungh. pr. t. 1. f. 4.

- lateritium. - Anm. Mg. n. h. IS fl). IV. t. S.

f. 2. (Berk.).

- leiopus. - [S. 45\%. MIucerd.] - Fuck. rh.

178.

leucocephalum. - Carol. IV. so.

micropus. - [S. 469.]

- Incerdac Fr. - Fl, dan. XI. 1552. 3. - S.

leiopus Ehrb. ; ef. Hydrophora Muc.

—- parasiticum, - Sturm h. 3. t. 46.

- pellucidum S. - [S. 469.] - Fuck. s. $251 .-$

Fuck. rh. 176.

- piliferum. - [S. 469.]

piliforme. - Cd. Ani. t. B. f. $20: 4-6 .-$

Brit. f. 50.

- pubidum P. - Bisch. f. 3511.

- Rhizomorpharum. - Hdw. I. t. 10. f. 15. -

(Atractium) Kil. 1931.

Ces. ; cf. Graphium Rhiz. Mont.

- rigidum P. - [S. 469.] - Fl. dan. XIII.

2250. 2. - Bisch. f. 3754. - Fuck. s. 255. -

Fuck. rh. 177. - Sturm h. 4. t. 59.

- sanguineum. - [S. 469.]

- smaragdinum A. S. - [S. 469.] - Bisch.

f. $3 \$ 12$.

- tomentosum Schrad. - [S. 469.] - Bisch.

f. 3751 .

Hoff $m$ a $n$, Index Fungorum.
Stilbu m turbinatum Td. - $S$ S. 469.$]-$ Bisch.

f. 3511 . - R. f. 61 .

- ventricosum Schum. = bicolor, Fl. dan. vulgare Td. - S. 469.] - Loud. f. 16553. -

Schnzl. t. 12. f. 41. 45. - K1. 1752. - Sturm h. 4. t. 59 .

_- xanthocephalum. - [S. 469.] - Sturm h.4. t. 60 .

- xanthopus Rbh. - R. f. 170.

Streptoth rix atra. - Carol. III. $\$ 3$.

- fusca. - ef. Monosporium curvat.

- spiralis. - Ann. Mg. 11. h. 1545. II. t. 12. Berk.

Strigula. - S. 1(1) *. 24 4.]

strobilomyces nigricans. - Hook. J. 1552.

IV. t. 6 .

_- strobilaceus Berk. = Boletus str. Fr.

Stromateria. - S. $470^{* *} .521 \cdots$.

Stromatosphaeria. - cf. Sphaeria, Gibberidia.

Strongylium fuligin. Sturm l. 3. t. 3S. = Re-

ticular, umbr., Fl, dan.

Strumella. - S. 442 .

Stylobates. - S. 30\%.]

morchellocephalus. - Chili. n. T. f. 1.

stylonites. - [s. 456.]

Stysanus albo-rosellus. - Usm. III. 10, -

îstd. 1200 .

— caput Medusae. - Pay. b. cr. p. 10. f. 52.

- Mandii. - Ann. sc. n. 15.5. IV. t. 14. f. 2.

Iont.

- parasiticus. - Dsm. 1304.

- pusillus. - Fuck, rh. 17.

- Reichenbachianus Preuss. - Sturm h. 36 . t. 6 .

- sphaeriaeformis. - Fuck. rh. 173.

- Stemonitis. - [S. $46 \bar{i}]$.- Cd. Anl. t. C.

f. $21: 9-12$ - R. f. 35.3. (Isaria S., P.). -

Bon. t. 11. f. 21<. - Fuck. rh. 172.

sy ncla di um Nietneri R. - IIdw. II. t. 1. f. D.

1459.

Sy sporium biguttatum Pr. - Sturm h. 35.

t. 4. - K. f. 275 .

s'yz ygites, - [S. 4bs.]

- megalocarpus. - SS. 4>t.] - Bon. t. 10.

f. 200. - huc: Sporodinia (de B.).

T ap e in ospor i um viride Bon. - Lot. Zt. 1553. t. 7. f. 6 .

Taphrina. - [S. 515.] Pili degenerati.

Terfezia. - \$. 137.] - - cf. Tulasneinia.

- Africanorum, Lco afr. - cf. Tulasneinia Leonis Zob., Terf. Leonis Tul.

— berberiodora. - Tul. F. hyp. t. 6. f. 4.

- Leonis. - Tul. F. hyp. t. 7. f. 5. - R.

f. 241 '= Oogaster alger. Cd.) - Tul. F. hyp.

t. 15. f. 3. - Y Rhizopog. Leonis. - Dsm. III. 670. - Ital. (1). 91. - Tuber arenar. Moris.

- leptoderma. - Tul. F. hyp. t. 15. f. 4. -

Willkomm, Wunder d. Mikr. 1556. p. 95. d.

(Spor.).

_- olbiensis. - Tul. F. hyp. t. 15. f. 5.

-_- oligosperma. - Tul. F. hyp. t. 21. f. 15.

Testicularia. - [S. 460.$]$

Cyperi Kl. - Linn. 1\$32. VII. t. 9. A. -

Cd. Anl. t. C. f. 40:11-14.

Tetradium. - ef. Botrytis.

Tetraploa aristata. - Ann. Mg. n. h. 1550.

V.t. 11. f. 6. p. 460. (B. B.).

'Ih am nidium. - [S. 4, 7.$]$

- elegans Ns. - [S. 457.] - Loud. f. 16546.

$-\mathrm{Lk}_{\mathrm{C}}=$ Ascophora el. Cd.

Thamnomyces. - [S. 352.] 
'I li a m n in yces amnulipes. - Amn. se, n. I 31 .

II. t. 1 .

- Chamissonis. - Mail S. t. 1\%.

- chordalis. - cf. Chamissonis.

—_ fucifornis Berk. - Ilook. J. VIII. t. ?. f. 3. hippotrichodes. - S. 342, 2.]

- rostratus. - Ann. sc. n. 1540. XIII. t. 6 .

(Mont.). - Cd. Anl. t. F. f. 56: У.-12.

'Th ecaphora. -- \$. 516.] - Uredo, Ustilago.

- aterrima. - Ann. sc. n. 15ti. VII. t. 1.

f. $20-22$. Tul. .

- deformans. - Ann. sc. n. 1447. VII. t. 4.

f. 23. Thul. - Pay. b. cr. p. 120 . f. $566 .-$

Alger. t. 25.4 .

- Delastrina. - Ann. se. n, 1547. VII. t. 1.

f. 24. Tul.) - Dsm. 1367. hyalina. - [S. 516.]

Thelactis. - [S. 1 15 . .

coccinea. - Bisch. f. 3715.

flara. - Cd. Anl. t. C. f. 24: 5. 6. - Bisch.

f. 3750 .

violacea. - Cd. Anl. t. C. f. 24: †. ᄂ. -

Bisch. f. 37.19. - Bon. t. 10. f. 209. - Mucor. - virens. - Bisch. f. 3747 .

'Thelebolus. - [s. 403.]

- stercoreus Td. - [S. 401.] - Loud. f. 16345.

- Cd. Anl. t. D. f. 14: 1-与. - Bisch. f. 3615.

— sudans. - [S. 104.]

f. $44: 9-11$ - K1. I1. 71 S. - Bisch. f. 3646 .

Thelephora. - [S. 330.] - cf. Auricularia,

Corticium, Merismium, Stereum, Hypochnus. -

Fructif.: Ann. sc.n. 1537. VIII. t. S-11. (Lév.).

- acerina. - Dsm. 1512. - Voges, 991. -

Brit. f. 65.

- adglutinata P. - Wstd. 1393.

- adusta Lér. Stereum). - Bonite t. 139. f. 2.

alnea Fr. - : Kl. 125 hirsuta ?

- anthocephala. - [S. 331.] - Berk. Out.

t. 17. f. 4. - Brit. f. 244.

arida. - Brit. f. 14 S.

aurantiaca P. - Sagra, Cuba t. 14. f. 1.

- biennis. -- [S. $330 * * 331,11$.$] - Loud. f.$

16151.

_ byssoides. - S. 331.] - F1. dan. XIII:

2276.2 . Brit. f. 20 .

caesia. - [S. 331.330*.]

- calcea P. - Kl. 151\%. (Cortic.). - Wstd. 55s.

- - acerina. - Kl. 325

- v. buxigena. - Kl. 1119

— v. illinita. - Kl. 909.

v. Sambuci. - Ki. 231 .

carbonaria. - [S. $\left.350^{* * *}.\right]$

carnea Humb. - Wstd. 1393.

- caryophyllea. - [S. 331.] - Cd. Ic. V.

t. 10. f. 79. (Fruct.) - Cd. Anl. t. G. f. 73:

3-5. - Sturm h. 31 - t. (6. (Szl.) - Kl.

1115. - R. f. 22. - Fuck. s. 930. - Brit.

f. 241. - Carol. II. 25. - Wstd. 557: v. laciniata.

— ciliata Fr. - Wstd. 1393.

- cinerea. - Wstd.1055. - Ioud.f. 16159. -

(Corticium) Kl. 62\%. - (Fraxini) Brit. f. 63. -

(Tiliae) Brit. f. 64 .

- cinnamomea. - Lib. 122.

- clavularis. - [S. 331.]

- coccinea Schw. - Hypochnus rubro-cinc-

tus E.

- coerulea. - Letell. 630.2 - Huss. I. 20, B.

- Lib. 22. - Wstd. 76,

- comedens Ns. - Kl. 329. - R. f. 412 . -

Brit. f. 2.2. - Norweg. 16.
'Thelephora contiuens. - cf. IIypochums.

— Corium I'. - Ioud. f. 16143.

corrugata Fr. - I3rit. f. 249. 295.

- crassa Ićv. - Bonite. t. 139. f. 1.

- cretacea b. Fr. - Wstd. 5s

cristata Fr. - [S. 331.] - Fl. dan. XIII.

2.272. 3. - 1) $\mathrm{sm}$. >19. - 13isch. f. 3290.-

3rit. f. $213 .-$ Wstd. 130.

- cruenta 1'. - Fl, dan. X. 173S. 2. - Voges. $5 \triangleleft 1$.

- crustacea Schum. - [S. 331.] - F]. dan.

XI. 1551. 2. - Wstd. 735.

- crustosa. - Alger. t. 33, 5 .

-_ dendritica. - Gaudich. voy, 1526. t. 1.

- diffusa. - [S. 331)**.331.]

- disciformis. - Voges. 5๖?.

—_ dubia P. - ? Wstd. 765.

elegans. - [S. $\left.330^{* * *}.\right]$

fastidiosa. - [S. 331.]

- ferruginea. - Voges. 394. - Wstd. 125s. u. 1249 .

— filiformis l'r. = Clavaria fil., Fl. dan.

fimbriata lioth. - Wstd. 767 .

frondescens. - [S. 331.]

- frustulata. - Ism. 1813. - K1. 1510. -

Fuck. s.915. - Voges. 650. (huc T. sinuans P.).

—_ gigantea Cortic.). - K1. 326.

- gyrolophia (Cora). - Cd. Anl. t. G. f. $73: 10$.

hepatica Fr. - Loud. f. 16136.

- hirsuta W. - Fl. dan. X. 1739. 1. - Fuck.

s. 924. - Cd. Anl. t. G. f. 73: 6-ヶ. - Loud.

f. 16137 . Linn. 1543. XVII. t. 14. (Schmitz).

- Stercum Kl.42. - Pringsh. Jahrb. II. t. 31.

f. 37. Keimg., Hoffm., - I3erk. Out. t. 17.

f. 7. - 3rit. f. 116. - Huss. I. 55. - Wstd.

766.1290. dryina.

- lydnoidea P. - Wstd. 1291.

- _ janthina. - Alger. t. 33,3 .

- incarnata (Corticium) P. - Letell. 607. -

Iil. 324. - K1. II. 14. - Dsm. 7T.

— incrustans. - [S. 33I.] cf. T. sebacea.

— intybacea P. - [S. 331.] - K1. 1215.

— isabellina Fr. - R. f. 23.

__ laciniata. - [S. 331.330**.] - Dsm. S20. -

Wstd. 5\$7. - Loud. f. 16130. - Fuck.s.927.-

Kil 41. - Kl. II. 129. - R. f. 311. - Ann.

Mg. n. L. 1535. I. t. 5. f. 41. (Fruct., Berk.).-

Brit. f. 212.

—— v. Vaccinii. - R. f. 410.

- lactea Fr. - Loud. f. 16157. - cf. Hypochnus. - Brit. f. 251). - forma pr. : Jungh. pr.t. 6. f. 21. (Himantia).

- lactescens. - Brit. f. 21.

laevis. - ef. Iypochnus $1 .=$ P.? = Th.

Pruni, Fl. dan. - Brit. f. 246. - Lib. 20.

- Laurocerasi. - Brit. f. 145.

- lilacina. - Nov. Act. 1S42. XIX. II. t. 57.

f. 111. (Phöb.) - - ef. T. purpurea v. lilacina,

Fuck. s. 925. b.

- R. : Kl. $171 \mathrm{~S}$.

- mesenterica. - Voges. 492. - Cd. Anl. t. G.

f. $73: 9$ - Auricularia, Merulius. - Wstd. 5ร 7.693 .

Micheneri. - Carol. IV. 12.

miniata. - Brit. f. 251.

mollissima. - [S. 331.] - Berk. Out. t. 17.

f. 5. - Brit. f. 245 .

multifida. - Kl. 171 . (cf. T. palmata).

nigricans Lév. (Stereum) - - Bon, t. 139. f. 5. ochracea Corticium) Fr. - Kl. 520.

ochroleuca Fr. - Fl. dan. XIII. 22 1 I. 3. - 



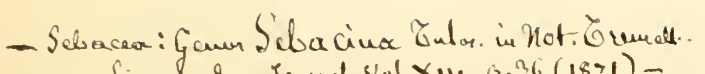
Limuean Soe. Journd yol X111. j. $36\left(187^{1}\right)$ - 
Loud. f. 1613\$. - Bail s'. t. 2`. - cf. 'T. serieea Sehmitz.

'Thelephora orbicularis. - Alger. t. 33, 7 . ef. IIypochnus Miehel.

- Ostrea. - Nov. Act. 1526. XIII. I. t. 2. (Blum et Ns.).

— pallida. - [S. 330****.] - Carol. II. 29.

- palmata Fr. - [S. 331. 330**.] - Loud.

f. 16131 - Bisch. f. 3311. - Fuck, s. 929. a. -

Jil. II. 13; 131. - ef. 'I', anthoeephala var., -

R. f. 119. - Sehwciz. 20. - Krypt. Bad. ј̇.

- $-\mathrm{v}$. angusto-incisa. - R. f. 411.

- pannosa. - [S. 330.] - Loud. f. 11;127. -

- Dsm. III. 79i.

- papillosa. - Lib. 21.

papyracea Fl. dan. - Wstd. Titi.

- paradoxa Lév. - Bon. t. 139. f. 1.

- pergamena. - Voges. 75.

- l'iceae. - Voges. fisl.

Pini Fr. (Stereum). - Kil. 906. - Fuck. s. 9211 .

_- polygonia P. - Dsm. is.

- princeps. - Jungh. pr. t. 7 .

Pruni Schum. - Fl. dan. XII. 2035. 2.

pubera. - cf. Hypochnus puber.

purpurea (Stereum) P. - Kil. 50. - Fuck.

s. 925. a. b. - Ann. Mg. 11. 1. 1>35. I. t. 5.

f. 15. (Fruet., Berk.) - Brit. f. 14i. - IIuss,

I. 20. A. - Wstd. 431. - v. pinea: Ital. 336 (Stereum lilaeinum).

- puteana Fr. - Fl. dan. XI1. 2035. 1. -

(Corticiun) Schum.: Kl. 624.

- quereina I'. - Lib. 2201. - Loud. f. 16147.

(Corticium) Kil. 129. (162!) - - Bot. Z/4. 1559.

t. II. f. 26. (Keimg., Hoffm ). - Voges, (75). -

Wstd. 1393.

radiata Fr. - [S. 331.] - Bisch. f. 3310.

(cf. Th. earyophyllea .

- Fl dan. R. f. 109

radiosa. - Pringsh. Jahrb. II. t. 32. f. 39.

(Fruct., Hoffm.)

- retieulata Fr. - Kil. 1216.

- rosea. - Letell. 630.3.

roseo-cincta (Resupin.). - Linn. 1-30. V.

t. [1. f. 5. Fries). - ef. IIypochnus.

Rubi. - Lib. 323.

rubiginosa (Stereum) Sehrad. - Kl. \$11. -

Fuck. s. 922 .

Wstd. 1249.1290.

— — v. resupinata. - K1. 905.

- rugosa Stereum) P. - Kl. 32\%. - Fuck.

s. 919. - Laurocerasi Brit. f. 145.

- Sambuci, - Voges. 779. - Wstd. 555.

sambucina. - Letell. 607.

sanguinaria $\mathrm{P}$. - Wstd. 791

- sanguinolenta Fr. - Loud. f. 16146. -

Fuck. s. 923. - (Stereum' A. S.: K1. 51.

-..- sebacea. - Letell. 605 (inerustans). - Bon.

t. 12. f. 253. - Berk. Out. t. 17. f. 6. - Iil.

I) 11 .

- serieca. - [S. $\left.3333^{* * *}.\right]$ - Linn. 1S13. XVII.

t. 12. 13. (Schmitz); ochroleuea Fr.

- Schw. - ef. Stereum striatum Fr.

- setosa. - Ann. Mg. n. h. 1542. X. t. 11.

f. 10. p. 3 \&1. (Berk.).

- sinuans. - [S. 333. 17.] - ef. T. frustulata:

Voges. 6 $>0 .$, var. crassior.

¿ Kl. 910.

- Sehw.: Wstd. 1290.

spiculosa Fr. - [S. 331.] - : Fuck. s. 926.
Tlle le phora suberosa. - Alger. t. 33. 6. sulphurea (Corticium) Fr. - Kl. 519.

tabaeina Fr. - Lib. 121. - Loud. f. 16135. -

Stereum Kl. 1120. - Brit. f. 245. - Norweg. i>6. - Wstd. 1290. 1258.

- terrestris. - [S. 331.] — Kops Fl. bat. X.

7ò). 3. - Bail S. t. 2S.

- Ehrh.: Fuck. s. 925, - Ann. sc. 11.

1537. YIII. t. 11. f. 29 ; t. S. f. 12 . Fruet.,

Lév. - Nov. Act. 1512. XIX. II. t. 5\%.

f. 11) $1-110$. Fruct., Phöb.). - Kl. 40. - Kíl.

II. 130. - Voges. 297. - Wstd. 765.

_ tremelloides DC. - Wstd. 693.

- tuberosa. - Lond. f. 16133.

- Typhae. - Kl. 15ls. - Corticium Kil. II.

411. - Dsm. 1411.

— Ulmi. - Kl. 1 ill.

- umbrina $\mathrm{P}$. - Wstd. 765.

- undulata. - [S. 331 .

- uvida. - Bot. Zt. I559. t. I1. f. 27. (Anat,

Iloffm.). - if. Hypoehnus uv.

_ variegata Schrad. - Wstd, 125s.

— viscosa P. - Fl. rlan. XI. 14.51. 1.

— vitellina. - Alger. t. $33,4$.

— rorticosa Fr. - Wstd. 131.

vulgaris. - [S. 536.]

Theleporus. - [s. 325.]

'Thermutis. - [S. 524 .]

Thyrsidium. - ef. Myriocephalum hederaecolum.

'Thysanopyxis pulchella. - Kl. 1132.

'l'illetia. - [S.5]6.] ef. Uredo.

- Caries. - [S. 516.] - Kl. 1999. - lierk. Out. t. 1. f. 5. - Fuck. s. 112. - Krypt. l3ad. 401. - Ann. sc, n. 1517. VII. t. 5. f. 1-16; 1553. XX. t. 3. f. $21-26 ;$ t. 4. f. $23-29 ;-$ 1451. II. t. 12. f. 1-26. (')ul.). - Schnzl. t. 12. f. 1-15. Ust. sit.) - Kolaezek, Lehrb. d. Bot. 1556. 6: c-g. - Kühn, Krankh. t. 4. f. 1-6; t. 1. - Berk. In. p. 319. f. 72. b-f. - cf. Uredo sitophila. - Fuck, rh. 252. - Journ. hort. soc. II. Is IT. Fusispor. inoscul. Keing. Berk.)

cndophylla. - Kil. II. 500.

Lolii. - Kühn, Krankh. t. 2. f. 1-12. -

K1. 1999.

- Sorghi vulgaris. - Ann. se. n. 1917. VII.

t. 5. f. 17-22. (Tul.).

Tilmadoehe. - [S. 45.1.]

'T'ip u laria. - [S. 459.]

Tophora - [S. 521.523.]

Topospora. - [S. 415.524.]

Torsellia. - [S. 412 .

Torula. - [S. 505.] ef. Oidium. - Bonord. in : Bot. Zt. $155 \%$. t. 4. f. 13.

abbreviata Cd. - ef. Xenodochus brevis. -

Ann. Mg.n. h. 1950. V. t. 11. f. 4. p. 161. (B. B.). Aerosporium Cd. - Sturm h. s. t. 34. -

cf. Oidium monil.

aequiroca. - ef. Alysidium aeq. alta. - [S. 505.] - Cd. Anl. t. B. f. 5 : 5. 6.

- Kl. 1376 .

alternata Fr. - Biseh. f. $3 \$ 69$.

antennata. - [S. 505.] ef Hormiscium a. -

: Bisch.f. 3570. - Brit. f. 215. - Letell. 673, 4.

- Wstd. 433. - Sturm h. 8. t. 39.

antiqua. - Fuck. rh. 60.

arbuscula. - [S. 505**.]

aurea. - Kl. 1374. - Sturm h. 8. t. 36.

botryoides. - Sturm h. 8. t. 35.

bulbigera Bon. - R. f. 175. - Bot. Zt 
1561.t. S. f. \&. p. 195. Bon.). - zu Erysiphe Graminis, Tul.

Torula carbonaria Cil. - Fuck. s. 101.

- casei. - Ki. 1565. - Kil. II. 671. - Wstd. 300.

\section{_ caulicola (Hormiscium).}

- f. Pelargonii. - Kl. 1942.

- Centaurii. - Fuck, rh. 66.

- cerevisiae. - Cd. Ic. VI. f. 21.

chintarum Cd. - Wstd. 119.

- cinerea Bon. - IBot. Zt. 1 5.33. t. 7. f. 5.

- coccinea. - Sturm h. S. t. 10.

- composita. - Sturm h. 29 - t. 33. Preuss. .

- conglutinata Cd. - Fuck. s. 139. — Fuck.

rh. 6 i.

Correac. - Kl. 1771.

- eulmicola. - Stum h. S. t. 13.

- curta. - (d. Ic. VI. f. 21).
disciformis. - cf. IIormiscium d.

- ellipsospora. - [S. 505.] - Kl. 17\%0.

—_ Epilobii. - Kl. 1769.

- epimyees. — ef. Alysidium ep.

epizoa. - Sturm h. S. t. 44.

expansa. - KI. 1569 . - Fuck. s. $116 .-$

Fuck. rh. 63.

— farinacea. - Sturm h. 25-. t. 6. (Preuss.).

- ferruginosa. - Sturm h. `. t. 11.

Flagellum. - Bot. Kt. 1553. t. 3. f. 16. 17.

liiess.).

—_ fructigena P. - Kl. 1266. - Fuck. s. $1+2 .-$

I'ringsh. Jahrb. II, t. 24. f. 1s. Keimg., IIoffm.).

- ef. Monil. u. Oid. - Fuck. rh. (i).

- fuliginosa. - Voges, 5\9. - Letell. 673, 2.

Antemn. pin. Ns.).

— fulra. - Sturm h. 5. t. 37.

- Fumago. - [S. 505.] - I Letell. 673, 1. Fu-

mago ragans). - Wstd. 1.14.

__ fumerea Ces. - Kl. II, 79.

- geotricha. - Sturm h. s. t. 33.

- glauca. - Sturm h. 29 - t. 32 . (Preuss.).

- graminicola. - Sturm h. \. t. 12.

-- Graninis. - [S. 505.] - Dsm.117. - Ann.

sc. 11. I 34 . II. t. 2. - Lib. 392. - Fuck. rh. 69.

— guttulata. - Robin. t. 3. f. 5. Loud. f. 1655 t. - R. f. 47. - Dsm, 67.-

bisch. f. 3s7. - Cd. inl. t. B. f. 5: 1. -

Fres. t. 6. f. 55. - Kl. 1769. - Wstd. 312. -

Siturm h. . t. 1s.

- heterospora. - Kzl. 126ৎ. - Wstd. 1692.

Bull. ac. belg. 1. Sér. XXI. 2. p. 2\{6. f. 7. Wstd.\}.

-_ hysterioides. - R. f. 242.

- longispora. - Sturm h. 25 -. t. 5. Preuss.)

- Kil. 1767.

- maculans. - bon. t. S. f. 170.

- Menzelii. - [S. 505.]

- monilioides. $-[$ S. 505.] - Bon. t. 2. f. 5 s.

- Sturm h. S. t. $3 \varsigma$.

- ochracea. - Kil. II. 150.

- olivacea. - Kl. 1375 ; it. Cent. XVII.

- papillata Bon. - Bot. Zt. 1561. t. \&. f. 10.

p. 195. - ? zu Erysiphe Graminis, Tul.

pedicellata. - Sturm h. 25 -. t. \&. (Preuss.).

- Kl. 17 iis.

pinophila. - S. $407^{*}$ ] - Kl. 1050. -

Fuck. s. $145 .-$ Cd. Ic. V. t. 2. f. 11. - Kl.

II. 331. a. - Fuck. rh. 71 . f. ericaccola. - Kl. II. 331 . b. placentaeformis. - Cd. Ic. VI. f. is. Plantaginis. - S. 505.] - Kil. 1761. -

Fuck. s. $1+9-$ Iil. II. 270.- Dsm. III. $15 \%$.

- Fuck. rh. 6.5.
Torula pulveracea. - Fuck, rh. 6ৎ.

- rhizophila. - Kl. II. is2. - Fuck. s. 13\%.-

Wstd. 999. 1197 (arundin.). - Fnck. rh. 59.

Thododeńdri. - Sturn h. \. t. 11.

rosea. - Sturm h. 25-. t. T. (1'reuss.). -

lil. 1267 .

rubella B. - R. f. $2 \$ 1$. - ? zu Erysiphe

Graminis, 'Tul.

— rufescens. - Fresen. t. 11. f. 11-17. p. Si.

Sachari. - I'ay. b. cr. p. 69. f. 29:3.

septonema. - Kl. 1४९l.

stilbospora. - Cd. Ic. V. t. 2. f. $1:$. -

Fuck. s. 143. - R. f. 79. - Fuck, rh. 62. -

Sturm h. 8. t. 16 .

— tenera J.k. - Kl. 1269. - Letell. (i73. 3.

- tenerrima. - Kl. 176 ili.

- Tritici. - Cd. Ic. V. t. 2. f. 15. - Fuck.

s. 145. - Fuck. rh. 70 .

tubercularia Ns. - Bisch. f. 3903. - Stum

h. S. t. 17 .

- tuberum. - Cd. Ic. VI. f. 19.

- ulmicola 1ibh. - Fuck. s. 14\%. - Fuck.

rh. 64 .

- Uredinis. - [S. 505.]

- vermienlaris. - Kil. 1593. - Kil. II, 753.

Trabecularia villosa. - Bot. Zt. 1\$57. t. 1. f. B. (Bonord.).

'Tra chys pora Alchemillac. - Fuck. (zu Ured"

Alch.) Bot. Zt. 14(j). t. 10. f. 3. p. 219. — ? ef.

Thecaspora deform. Pay. b. cr. p.126. f. 566 . -

Fuck. rh. 318 .

'Trametes. - [S. 322.] of. Polyporus.

— albida. - [S. 324.]

- annosa. - S. 323.

- benzoina. - [5. 323.]

- Bulliardi. - [S. 323 .

- cinnabarina. - [S. 323.$]$

- circinata. - [S. $3 \cdot 3 \cdot 3]$

- conchata. - [S. 323.]

_- connata. - [S. $323: 21 \div$.]

_- corticola. - [S. 324.]

[ fulva. - S. 323.]

- gallica. - Cd. Anl. t. II. f. $75: 35$ - Bisch.

f. 3324 .

- gibbosa Fr. - [S. 323.] - Bisch. f. 3125. -

I. : Sturm h. 33--. t. 6. (Strauss).

- hydnoides Fr. = Polypor. li. Fr.

- incondita (Sessiles). - Afzel. t. i. f. 16 .

—_ inodora - [S. $\because 23$.

- laevis. - S. $3233^{*}$.

—. lignicola. (popul.) Kíl. 14(1).

- 1.onicerae. - S. 323.

-_ Montagnei. - $[$ S. 323\%.]

_- Neesii. - [S. 324.]

[ nodulosa, - [S. 323.]

- occidentalis Fr. - Polypor. occ. Krl.

- odora. - [S. 323.]

- odorata. - [S. 323.]

- paleacea Sessiles'. Afzel. t. S. f. 1\$.

- perennis. - Kl. 11. 117.

- Pinj, - [S. 32\%.32. .] Cd. Anl. t. II.

f. $75:: 6.3 \%$ - Bail S. t. $31 .-$ Kl. II. 115.

- populina. - [S. 323.]

- radiata. - [\$. 323.]

- radicata. - [S. $323^{4}$.

- Ribis. - [S. 323.]

- rubescens. - $[$ S. 323.$]-$ R. f. 118.

- rubiginosa. - $S .564$.

- rufescens. - [\$. 322.]

- Schweinitzii. - [S. 322 .

- serialis. - [S. 321.]

serpen. - $[$ s. 324. 


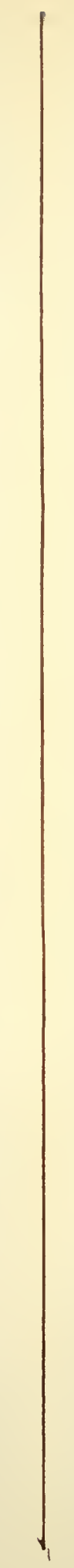



Tram t es suaveolens. - [S. 323.] - Bot. Zt. 14.3!. t. 11. f. 29. Keing., Hoffur.) - Kops Fl. bat. X. 600. - Fuck. s. 965 . - Ital, 231. - subpileata. - [\$. 323.] tomentosa. - [S. 323.] triquetra. - S. 323.

'I'remella. - [S. 3+1.] cf. Exidia. abictina 1'. = Dacryomyces stillatus, Fl. dan. agaricoides Rtz. - Wstd. 191. albida IIuds. - [S. 311.] - Loud. f. 16291.

- Boll. t. 12. f. $216 .-$ Kl. 731. arctica. - S. 311.]

atro-virens Bull. - Wist. T00.

auricula Judac. - (f. Exidia. - Wstd. 760.

XVI. t. 15. f. 10. Lév. auriformis Hoffin. - W std. Ifl. cerebrina. - Inn. sc. 11. 1537. VIII. t. 11.

f. 31). Fruct., Lév.). - Bisch. f. :342. clavata. - [S. 341. cruenta Fingl. bot. - Wstd. 791. doliquescens Bull. - [\$. 359.] = Dacryom. stillatus, Fl. dan. disciformis. - S. 372 .

elliptica 1'. - IIymenula vulgaris. epigaca. - Inn. Mg. 11. h. I 4 ᄂ. II. t. 9.

f. 3. Jork.). exigua. - Voges. 1273. - I) sm. 1017.

- ferruginea schum. = ('lavaria contorta, F1. dan.

fimbriata. - 5. 311 .

foliacca P. - S. 3 H I.] - Fuck. s. 4.3.

- frondosa P. - [S. 3.11.] cf. Nacmatelia fr

Bon. fusceseens. - S. $341 . \bar{T}$.

gemmata Lér: - Démid. liuss. t. 4. f. 1. geophila. - Afzel. t. 12, f. 24.

glandulosa. - cef. Exidia gl.

gramulata Huds. - Wstd. 50.

guttata. - Bon. t. 12. f. $24: 3$.

indecorata Somm. - Kil. \$23. - Lib. 234.

intumescens Engl. bot. - Loud. f. 16292.

juniperina IIucls. - W Wtd. 140.

lacrymalis P. - Wstd. $6>6$.

lutescens. - [S. 341.] - Bisch. f. 3103.

liuek. s. s\$2. - Bail S. t. 22.

- mesenterica Rtz. - [S. 341.] - Loud. f.

I (i-990. - Ann. sc. n. 1553. XIX. t. 1l. Tul. . Berk. In. p. 350 . f. 77 . d. Basid.)

Schacht, Lehrb. An. Phys. 1559. II. t. 6. f. 5. -

But. Zt. 1 599 . t. 11. f. 22. (Keimg., Iloffin. . -

? 'f. Naematelia frondosa Bon. - Huss. I. 27.

- Wstcl. 140.

- miniata Rbt. - cf. Fusarium tremelloides

Grev. - Wstd. 155.

inueoroides. - S. 47.*]

nigrescens. - [S. 341 .]

nivea IId $\pi$. W Wstd. 242.

Nostoc L. - Wstd. 700 .

palustris Dill. - Wstd. 50.

plicata. - Bail s.t. 22.

purpurea L. - W'std. 90.

recisa. - cf. Exidia. - Sturm h. I. t. 13.

Sabinae Dicks, - Wstd. 339.

sacharina. - Bon. t. 12.f. 215 . - Exidia Fr. sarcoides. - [S. $341.357^{* *}$. ] Loud. f.

16294. - Berk. Out. t. 2. f. 7. - Bisch. f. औ102. (cf. Bulgaria). - Fuck. s. s\$6, a. b -

Iil. 515. - Kil. II. 41 \% - ef. Coryne s.

—_ sepincola Willd. - cf. Fusarium tremelloi-

des Grev. - Wstd. 139. 155.

__ spicul. - cf. Exidia gland. - Voges. 395.
Tremell a Syringae Schum. = Dacryomyc. Syr., Fl. dan.

— terrestris Dillw. - Wstd. T00.

- torta Kx. - W'std. 139.

- turbinata IIuds. - Wstd. 191.

-.. unicolor. - [S. 341.]

- Urticac 1. - of. Fusarium tremelloides Grev.

— Voges.396. I'ez. fusarioides). - Wstd. 145.

- ustulata Lull. - W'std. 735.

— verrucosa L. - Wstd. 75\%.

- violacca. - Ann. sc. n. 1553. XIX. t. 12.

u. 13. Tul.

- virescens Schum. = Dacryom. v., Fl. dan.

- riscosa. - Ann. Mg. n. h. 1 551 . XIII. t. 15.

f. 1. p. 106;. Basid., 13. B.). - Berk. In. p. 3.50.

f. 77. b. Masid.!

'I'rentepohlia. - [S. 524.] - aurea Mart.: Wistd. 6i99.

Triblidium. - [S. 369.] - of. I'lacidium, Ilysterium.

—_ arcticum. - [S. 369.]

— bullatum. - 5. 369.

- calyciiforme. - \$. 369.] - Letell. (31:3. 2.

- confluens Not. = 'Trib. rufulum Spr. I) ub. .

- crispum I' - Var. von Iysterium clatin.

P. Dub.

- liysterinum, - Anm. sc.n. 1629. XIII. t. J11.

f. 3. 'I. Dufour . - Iysterium el. Moug., l'ers.

— P'illastri. - [S. 36!!.]

- pineum P. - Wstd. 1275 .

- pithyum. - [S. 369.]

- quercinum 1'. - Aun. sc. n. 1\$53. XX. t. 15.

f. $15-19$. ('lul.). - Wstd. 96 .

- rufulum spr. $=\mathrm{T}$. conflucus Not., IIy $\mathrm{s}$ terium confl. Kiz. Dub.

— seriatum. - \$s. $36 \%$.

Triehaegum. - S. 503.

- atrum. -- Sturm h. 25-. t. 15. Preuss. .

— cladosporioides. - S. 503.]

Tricliam phora. - S. 454.

- pezizoides. - 1ierk. In. 1. 335. f. 7., a.

Spor., - Jungh. pr. t. 2. f. 9.

Trichaster. - [S. 411 .

Triehia. - [S. 45\%.] - Mier. Journ. 14.5\%. V.

t. S. f. 43. - Linn. 'Tr. IV. 21. p. 221; ib. 1 51.

IXI. Elateres, Henfrey. - C. Müller, Pllinzenstaat. 269. f. 10.

- abietina Wigd. - Pringsh. Jahrb. 1 661 . III.

t. 2. f. 11. Fruct.) - Anm. sc. 12. 1562. XYI.

t. 15. f. 11. Nigand

- affinis de B. - Fuck. s. 265.

angulata. - S. $455 *$.

- applanata. - De Cand. Org. vég. 1527. II.

t. 60. f. I. Hedwig.

_ aurea Schum. = Craterium mutabile, Fl. dan.

- axifera Bull. = Stemonitis ferrug., Fl. dan.

botrytis. - [S. $45 \mathrm{~S}^{*}$.]

- cerina Ditm. - Sturm h. 2. t. 25. (clavata I'.).

- cernua Schum, = Physarum c., Fl. dan.

chrysosperma. - [S. 457.] - Fl. dan. XII.

2059. 2. - Cd. Anl. t. C. f. 30:5. - Micr.

Journ. 1 54. III. t. 2. f. 1-3. (Anat., Curr.). K1. 373. - Schacht, Phys. Bot. 1552. t. XVI. f. 14. (Capillit.). - Pringsh. Jahrb. 1561. HI. t. 2. f. $13-17$; t. 3. f. 1-). Anat., Wigand, cf. T. nitens. - R. f. 567 . - Ann. sc. n. 1\$62. XVI. t. 15. f. $13-17$. u. t. 16. f. $1-5$. Migd. .

cinerca Bull. = Aresria c., Fl. dan.

Trent. : Wstcl, t5\%.

circumscissa. - [S. 457. S.7 - Kl. 1314.

citrina Schum. = clavata, Fl. dan. 
Trichia clavata P. - S. iji. - Fl. dan. XII. 2(19. 1. - Bisch. f. 366 (is. - Voges. 251. -

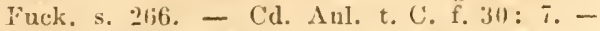
I'ringslı. Jahrb. 1561. III. t. 2. f. J-3. (Anat., Wigd. - Ann. sc. n. 14li2. XVI. t. 15. f. 1-3. Wigd. .

- I3eck. = 'T. obtusa Wigd.

coccincal Bull. - Wstd. $\$ 1$.

coerulea Trent. - Wstd. 34".

- crassa Schum. - Fl. dan. XII. 20s5. 2.

- craterinides. - Auerswald, Bot. Enterhal-

tungen. Isti:3. S. 35:

- denudati.-Purton. I. t. 21. f. 2. - Wstd. bl.

difformis. - \$\$. $157 * *$.

- fallax P. - S. 457.] - Fl. dan. XII. 2055.3.

- Krypt. Bad. 420. - Bisch. f. 36i 4. - Fuck.

s. $26 \%$ - I.oud. f. 16529 - Kl.931. - Kl. II.

137. - Voges. 575. - Ann. se. n. 1562. XVI.

t. 11. f. 11. 15. Wigd, aus Pringsh. Jahrb.

1'-iil. III.'

- faroginea Schum. = chrysosperma, Fl. dan. - Hava IIall. - Wstd. j̇

- flexuosa Schum. - Fl. dan. XII. 205S. 1.

furcata Wigd. - Pringsh. Jahrb. 1561. III.

t. 1. f. 1-11. (Structur). - Ann. se. 11. IS62.

XVI, t. 11, f. $1-11$. Wigd. ?

- furfuracea With. -- Wistd. 51 s.

- leucopodia Bull. - Wstd. 1260.

Neesiana. - Cd. Anl. t. C. f. $30:$ 1-6. -

IIier. Journ. 1554. III. t. 2. f. 5. 6. Capill., Curr. .

_ nigripes. - [S. $456 * 457$.] - Micr. Journ.

i5.54. III. t. 2. f. 4. Capill., Curr.) - Kil.

1025. - Pringsh. Jahrb. 1s61. III. t. 2. f. 5.

Anat., Wigd.). - Ann. sc. n. 1562. XVI. t. 15.

f. 5. Wigd.

nitens Lib. - Fuck. s. 264. - Lib. 277

(chrysosperma a Fr.).

- notata. - F1. dan. X. 1650.2.

— nutans Grev. - Loud. f. 16524.

- Bull. = Areyria n., Fl. dan. -. Pur-

ton. I. t. "1. f. 1.

obtusa Wigd. - Pringsh. Jahrb. 1561. III.

t. 2. f. 1. Spor.). - clavata de B. - Ann.

sc. n. 1sti2. XTI. t. 15. f. 4. (Wigd.).

ovata Y. - Loud. f. 16525 . - Dsm. III. 260.

- physaroides Schum. - Fl. dan. XII. 2057.3.

- pyriformis. - [S. 457.] - Cd. Anl. t. C.

f. 31): 1-3. - Micr. Journ, 1454. III. t. 2.

f. 9. 10. Capill., Curr.) - Kl. 1026; - 1632.

- Krypt. Bad. 329. - R.f. 214 bis . - Pringsh.

Jahrb. 15til. III. t. 1. f. 12. 13. (Anat., Wigd.).

- Ann. se.11. 1562. XVI.t. 11.f.12.13. Nigd..

- Schum. = clavata, Fl. dan. - Carol.

II. 51 . - Schwabe, Fl. anhalt. II t. 6. f. I0. -

Ital. $6 ! 0$.

- ramulosa Rud. = Arcyria r. Wigd.

- reticulata P. - [S. 457.] - Loud.f. 16527. --

Cd. Ic. VI. f. 34 .

— retiformis. - Pay. b. er. p. 122. f. 574.

rubiformis P. - \$. $457.455 \%$. $]$ Bisch.

f. 3664 . - Fuck. s. 264 - Iirypt. Bad. 421 . -

Pringsh. Jahrb. 1\$6l. III. t. 2. f. 12. Fruct.,

Wigd.) - Purton. I. t. 19. f. 1. - Purton. I. t. 3\%. - Anu. se. n. 1562. XVI. t. 15. f. 12.

Wigd.).

- serotina. - [S. $45 \overline{\mathrm{T}}$.]

__ serpula - [S. 45\%.] - Fl. dan. XII. 2059. 3.

- Schacht, Phys. Bot. 1552. t. XVI. f. 13.

Elater.) - Micr. Journ. 1551. III. t. 2. f. 7. S.

Capillit., Curr.). - Bisch, f, 362.2. - Pringsh.

Jahrb. 1561. III. t. 3. f. 5*. (Fruet., Wigd.). -
Schwabe Fl. anhalt. II. t. 6. f. 11. - Aun. sc. 11. /4i2. XYI. t. 11;. f. 5* (Wigd.).

'Trich i a serpula v. reticulata Fr. - ef. 'Tr. reticul. _ turbinata With. - S. 15\%.] - Kops Fl. bat.

XI. \09. 2. - Kl. 762. - Micr. Journ. 1557.

V. t. \&. f. 12. Curr.). - Pringsh. Jahrb. $15(j 1$.

III. t. 2. f. (j. (Capill., Wigd.) - Ann. sc. n.

1562. XVI. t. 15. f. 6. (Wigd.).

- varia. - S. $45 \%$ - De Cand. Org. vég. 1527.

II. t. 60. f. 6. IIedwig f.). - Sclinzl. t. II.

f. 27-33. - Zeitschr. f. wiss. Zool. 1559. X

t. \$. f. $10-20$ de Jary - Krypt. Had. 419.

Kil. 422. - K. f. 245. - Fuck. s. 2(i.3. - I'ringsh.

Jahrb. 1 61 . III. t. 2. f. T-10. (Fruct., Wigd,

- Ann. se, n. 1562. XVI. t. 15. f. $7-11$.

IVigd.

- venosa Schum. = T. serpula, Fl. dan,

Trichiamphora. - ef. Trichamphora.

Trichobasis. - ef. Uredo, Caeoma.

- linearis. - Kolaczek, Lehrb. d. Bot. 1556.

9: 1. u. 3. - de Bary, lir. t. 3. f. 5.

Trichocoma. - [S. 446.] - paradoxa: Carol.

IV. T. - Jungh, pr. t. 2. f. 7 .

Trichoconium glutinatum. - Sturm h. 13 . t. 37 .

Trichoderma. - S. 447.

— carneum Schum. - Wstd. 137.

- dubium. - [S. 14〉.]

flavum. - S. 445.]

laeve. - [S. 417.]

- roseum. - [S. 492.] - cf. Trichothec. r.

- viride. - [S. 145.] - Cd. Anl. t. C. f. 33 :

5-4. - Nees j. Syst. t. 10. - Schnzl. t. 1.1.

f. $17-19 .-$ Kil. 772 . Loud. f. 16559. $-\mathrm{H}$

f. 67. - Wstd. 343. - Fuek. rh. 161.

Trichodesmium. - [S. 122.] — cf. Graphiola.

Tricholeconium. - [S. 472.]

- fuscum. - Cd Ic. V. t.2. f. 20. - Cd. Anl.

t. B. f. 15:5-7.-(Sarcopodium) Kl. 13is.

— roseum. - [S. 472.] - Fuck. rh. IS5.

Trichospori um. - [S. 492.]

Trichostroma. - [S. 466.]

- decipiens. - Sturm h. 34. t. 7. (Strauss).

- olivacea. - Sturm h. 25- t. 23. (Preuss.).

- purpurascens. - Cd. Anl. t. B. f. 13: 6. -

Sturm h. 9. t. 62.

'Trichoth c ci um. - [S. 492.] cf. Verticillium. agaricinum. - Bon. t. 5. f. 114.

- anodotrichum. - [S. 492.]

- candidum. - Cd. Anl, t. B. f. 9: 2. - Bon.

t. 5. f. 167 - Yay. b. er. p. 73. f. 331 . - Re-

gel, Gartentlora. 1 552 . t. 21. f. 7-10.

capitatum. - [S. 492.]

clavatum. - Letell. 65.5. 4.

diffusum. - ef. Fusisporium Solani tub. Dsm. domesticum. - [S. 492.]

mutatum. - Jungh. pr. t. 1. f. 2.

nigrescens. - [S. 192.]

- roseum Lk. - [S. 492.] - Dsm. 156, Loud. f. 165\%3. - Fuck. s. 151. - Bot. Zt. 1554. t. S. A. (Hoffm.). - Kl. 152. 439. - Kl. II. 176. - Disch. f. 3503 . Pringsh. Jahrb. II. t. 29. f. 21. Keimg., Hoffin.) - Voges. 997. - Fuck. rh. 150. - Brit. f, 99. - Letell. 655. 3. - Lib. is (Trichoderma r. P.). - Wstd. 43\%. - Krypt. Bad. 547.

Trichulius. - [S. 455*]

- Schmidelii. - Cd. Anl. t. C. f. 25: 11-1s.

Triglyphium album. - Fres. t. 9. f 6.

Trin matosporium Salicis. - Cd. $\Lambda \mathrm{nl}$. t. $\mathbf{1}$. f. $(j: 4.5$.

Trimmatostroma. - [S. $\left.47_{1}^{* * * *}\right]$ 


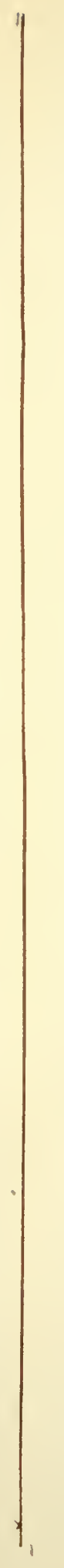


1 
Trim matostrom a Salicis. - Pay. b. er. p. 69. f. 3113 .

'Trin acrium subtile. - Fres. t. 5. f. $11-17$.

Triphragmium. - \{S. 51:3.]

deglubens, - Ijerk. In. p. 325. f. 73. d.

- echinatum. - Kl. II. isi. - Krypt. Barl. 313.

—- Isopyri Moug. - Dsm. J057. - Voges. \$9?2. Ulmariae. - [S. j]3.] - Cd. Anl. t. ]3. f. 4: 13. - Anu. sc. n. 165\%. II. t. J11. f. 14-1<. Tul.). - Mier. Journ, 1557. V. t. 8. f. 23. 21. Curr. - Pay. b. cr. p. 61. f. 250 - Fuck. s. $34 .-\mathrm{Kl} .271 .-\mathrm{Kl}$.II. 336. - R. f. Is1.Dsm. 1054. - Voges. S91. - Wstcl. 1163. Ital. S59. - Fuck, rh. 317.

'Iriposporium. - [S. 491 .

elegans. - Cd. Anl. t. B. f. 11: 2. 3. -

Pay. b. er. p. 73. f. 329. - Bon. t. 3. f. 75. -

Wstd. $100(10$.

- Ficinusium. - Sturm h. 29-.t.11. Preuss.)

- Mier. Journ. 1557. V. t. \&. f. 40. Curr.

- Gardneri. - Journ. hortic. soc. 1 S 19. IT.

p. S. (Berk.).

- strepsiceras. - Hdw. I. t. 4. f. 2. - Iil. 1874.

'I'ripotrichia. - [S. 451**. 153.]

'I'rochila. - ef. Phacidium, Sphacria.

'rogia. - [S. 315.]

Trullula Leguminum. - Kíl. 1661.

- Oreoselini. - Kl. 1660.

Spartii. - K1. II. 752.

Tuber. - [S. 437.] ef. Melanogaster, Oogaster.

(Anat.) Willkomm, Wunder d. Mikrosk. I s56. p. 91 .

- aestivum. - Vitt. t. 2, 1. - Tul. F. hyp. t. 7. f. 3 .

- Vitt. : Kl. 1222. - Berk.Out, t. 느. f. 2. Tul.: Cd. Ic. VI. f. I29) ( nare Zob., p. S2 . - Pringsh. Jahrb. 1460. II. t. 33 -35. (Copulat., IIofmst.) - cf. 'T. bohem.

- Vent. mi. 35. f. 4.5. - Ital. 63s.

— affine Cd. - Cd. Ic. VI. t. 19. f. 140. albidum Cd. - Loud. f. 16340.

- Pien: ef. Tuber Borchii Vitt.

album. - Ann. se. n. IS 14. II. t. 6. (Lespiault).

$-\frac{1}{\text { Vitt. }}$ Bull. (t. 404. f. A. B.) : cf. T. Borcliii Vitt.

V. (ex Cd. Ic. VI.). - Staude t. 1. f. 1. 2 . algericum Mont. - ef. Tulasneinia Leon, Z. arenarium Moris. - ef. Terfezia I.eonis T.

Asa. - Tul. F. hyp t. 5. 2.

bohemicum Cd. - C'd. Ic. VI. t. 1s. f. $12 \mathrm{~S}$

(zu culinare p. S2). = T. aestivum Tul. F. hyp.

Borchianum Zob. - Cd. Ic. VI. t. 19. f. 137.

(T. Borehii).

- Borchii. - Vitt. t. 1, 3. - Tul. F. hyp. t. 5. f. $1 ;$ t. 21 . f. 13 . Vitt.: Cd. Ic. VI. t. 21, f. 139 (T. elegans). - Ital. (40.) 90.

- brumale. - Vitt. t. 1, 6. - Tul. F. hyp. t. 7. f. 2 ; - t. 17. f. 3. - Kl. 1966. - de Bary, Ascomycet. 1563. t. 1.f. 16-23. (evolut. fruct.). - Vitt. : ef. Oogaster brum. Cd.

castaneum Cd. - Cd. Ic. VI. t. 1S. f. 132 (?). cibarium. - Cd. Anl. t. D. f. $46: 19-22$.

— Sibth. : Loud. f. 16339. - Cd. Ic. V.

t. 5. f. 46 (mesenteric. Tul. F. hyp.).

- - Sibth. : Sturm h. 19-. t. 13. (Cd.). Roq. t. 24.

- $\longrightarrow$ Sibth.: Bisch. f. 3655. - 'Truffe du
Perigord: Oogaster melanosporus, C'd. Ic. V1. p. 74 .

'Tuber cibariun St. Iŕrbl. : cf. Oogaster cibarium, Cd. Ic. VI. t. 17. f. 126. p. 71. - Schacht, Phỵs. Bot. 1552. t. 2. f. 1-11. (Fruct.). - id., Lehrb. d. An. Phys. I. 1 Siti. t. 1. f. 23-30. (Fruct.).Schwabe Fl. anhalt. II. t. 6. f. S. - Huss. I. 11.

- cinereum 'Tul. - cf. Oogaster rufus Cd.

- culinare Zob. - Cd, Ic. VI. t. Is. f. 12s

131. 134. t. 19$) \cdot$ p. S1.

-

- dryophilum. - Tul. F. hyp. t. 5. f. 3;

t. 19 . f. 5 .

- elegans Cd. - cf. 'luber Borchii Vitt.

- excavatum. - Vitt. t. 1, 7. - Tul. F. hyp.

t. 6. f. 1. cf. 'T. fuscum (d.), - t. 1\%. f. 5. Vitt. : ef. Vittadinion Montagmei $Z$.

ferrugineum. - Vitt. t. 3, 10.

foctidum Vitt. - Vitt. t. 1. \$ ; u. t. 3,

11. - Cd. Ic. VI. t. 19. f. 135. - 'Tul. F. hyp.

t. 17. f. 7 .

fuscum Cd. Mpt. ef. Vittadinion Montagnei

\%. - Cd. Ic. VI. f. 112. = 'I'. excavatum 'T'ul.

F. hyp.

- gallicum Cd. - Cd.'Ic. VI. t. 19. f. 135.

zu culinare $Z$ ob, p. $>2$

- griseum P. - ef. 'I. Magnatum Pico.

- macrosporum. - Vitt. t. 1.5.- Tul. F.

hyp. t. 17. f. $\checkmark$.

—- Vitt. : C'd. Ic. VI. t. 15. f. 131. - T. macrospermum.

- maculatum. - Vitt. t. 3. 16. - u. t. 4, 11.Tul. F. hyp. t. 19. f. 9.

— Vitt.: Cd. Ic. VI. t. 15. f. 133.

Magnatum. - Vitt. t. 1.4.- u. t. 2. 9. -

'lul. F. hyp. t. 6. f. 3., t. 17. f. 6. - Lindl. v. K. p. 33. f. 4. Spor.).

- I'ico: Cd. Ic. VI. t. 19. f. 136. - Vent.

mi. 67. f. 7. S. - Ital. 545 .

melanospermun. - Berk. In. p. 291. f. 65. d.

(Spor.). - Dsin. III. 262.

melanosporum. - Vitt. t. 2, 3. - u. t. 3, 20. - Tul. F. hyp. t. 3. f. 2 ; t. 7. f. 1 ; t. 1 . f. 2 ; t. 19. f. 12. - Berg, Charakteristik. 1stol. t. 1.f. ว. - Kil. 1965.

_- Vitt. : ef. Oogaster m. Cd. - Ital. 45. - mesentericun. - Vitt. t. 3, 19. - Tul. F. hyp. t. 5. f. 5 ; t. 7. f. 4 ; t. 17. f. 1.

- Vitt. : zu culinare Zob. Cd. Ic. VI.

p. $\$ 2\rangle$.

- microsporum. - Vitt. t. 2, 6. - Willkomm,

Wund. d. Mikr. 1556 . p. 95. b. (Spor.).

-_ Montagnei Lesp. - cf. Vittadinion Mont. Z. moschatum Sow. - cf. Melanogast. Broom. Bull. : ef. Melanogast. varieg.

nigrum Bull. - ef. Oogaster melanosp. Cd. nitidum Vitt. - Vitt. t. 2, 10. — ef. Oogast.

nit. C. - [S. $437^{*+*}$.] - Brit. f. 303.

__ niveum Dsf. (non Krbh.). - cf. Tulasneinia

Leon. Z.

- nuciforme Cd. - Cd. Ic. VI. t. 20. f. 143.

- obtextum Spr. - Wstd. 39.

- occidentale Cd. - Cd. Ic. VI. t. 20. f. 144.

(= nuciforme Zob. p. 80).

- oleraceum Web. - Wstd. 152.

- oligosporum. - Vitt. t. 3, 1 .

- panniferum. - Tul. F. hyp. t. 3. f. 3; t. 17.

f. 4. - Willkomm, Wund. d. Mikrosk. 1856. p. 95. f. a. (Fruet.).

- puberulum. - Tul. F. hyp. t. 19. f. 11.

Rabenhorstii Cd. - Cd. Ic. VI. t. 18. f. 131.

(zu culinare Zob., p. S3). - Kl. 934. 
Tuber rapaeodormm. - 'lul. $F$. hyр. t. 5. f. . ; t. 1S. f. 1. Willkomm, Wund. d. Mikrosk. 15.j6i. p. 95 . c.

Requienii. - Tul. 19. hyp. t. 19. f. 10. rufum. - Vitt. t. 1, 1. - 'Tul. l' hyp. t. 1 s.

f. 우 t. i. f. 2.. - KI. 1961 .

—- Pico: ef. Oogaster r. Cd.

- suillum Bormh. : ef. Oogast. r. C'd.

— tesselatum \%oh. - C (d. Ic. VI. t. 1!) f. 135? zu culinare \%ob., p. 52).

Tubere in ia sealies. - Journ. hort. soc. Is 16. I. t. 4. f. 30, 31. Berk.).

Tubercularia Acaciae Fr. - Wstd. :39!.

- Aceris. - Act. 'Turin. 1s41. III. Dec. 2. f. 5. Notar.

Aesculi. - Voges. 1.17s.

albida. - [S. $341 \div$.

Cerasi Schum. = Cenangium Cer., Fl. dan. - ciliata. - [S. 361.$]$ ef. Volutella cil. Fr., u.

Fusar. ciliat. 1.k. - Sturm h. 1. t. 11.

- Ditm.: Bisch. f. 3529 . sar, cil. Kiops.

- confluens. - [S. 361.] - K1. 1261. - Voges. 576 .

P.: Fuck. s. 7\%.

— v. Acaciae P.). - Kl. II. 595.

—- v. minor. - Letell. 691.3.

— v. Sophorae. - Kl. II. Ti.

discoidea. - 's. 361 .

- expallens. - [S. 361.] - Voges. 1091. -

Wstel. 396 .

- fascicularis Td. - [S. $3(i 2.3$.$] cf. Derma-$

tea carpinea Fr.

- fasciculata Td. - Wstd. 12T4.

- Filicis. - Kl. 1\$15.

- Hoccipes Cd. - Bisch. f. 3530. - Stum

h. T. t. 24 .

fungiformis Wigg. - Wstd. 109.

- granulata. - [S. 361.] - Bon. t. 11. f. 222.

— I.f. 279: forma concara. — Bisch.f. 3×31. -

Kil. 99. - Dsm. III. 452. - Voges. 1090. -

Carol. III. 40. - Letell. 691. 4. - Wstd. 395.

—- herbarum. - [S. 36i.] 1392.

- hirsuta Schum. - Fl. dan. XIII. 2337. 1.

___ liceoides. - [S. 361 .]

- longipes. - Lotos 1557. VII. t. 2.

Menispermi. - [S. 361.]

— minor Lk. - Wstd. 394. - Dsm. \$2 .

— minuta Schum. - Fl. dan. XIII. 2339. 2

— mutabilis, - [S. 36L.] - Wstd. 1191.

- mycophila. - [S. 172.] - Ann. sc. 11. 1436.

V. t. 13. Mont.). - S. 4i2.] , Pactil.

_ nigra sichum. - Fl. dan. XIII. 22\%3. 3.

— persicina Ditm. - [S. 361.] - Bisch. f. 3433.

- Dsm. 1042. - Kl.1163. - Carol. I11. 41.

- Sturm h. 4. t. 49.

- pezizoidea. - [S. 361.]

- Pinastri. - [S. 361.] - Lib. 296.

- P'ini Schum. = Ditiola rad., Fl. dan.

- Populi Schum. - Fl. dan. XIII. 2337. 2.

- Pruni Schum. - Fl. dan. XIII. 2336. 2.

- reticularioides. - Letell. 702 .

- Rubi. - [S. 361.9.]

- sarmentorum. - [S. 361.]

- sulcata Schum. - Fl. dan. XIII. 233〉. 2.

- vulgaris. - [S. 361.] - Fl. dan. XiII.

$2: 3: 9.1 .-$ Cd. Anl. t. G. f. 65: $36-11 \%-$

Fuck. s. 7 it. - Bisch. f. 3५32. - Dsm. 42:-

Schnzl. t. 12. f. 22.23. - Kützing, Philos. Bot.
15\%2. t. 19. f. 12. - Kl. 15.5. - (Aeseuli) Kil. II. $77 \%$ - (Corchori, Coluteae) Kl. 1556.forma conidifera Nectriae cinnabarinae: $\mathrm{l}$. f. 321.3. - Voges. 8.1. - Carol. V. 15. - Schwahe. l'l. anhalt. II. t. 6. f. 4. - Wstd. 90. - R. f. 545 (sarmentor.). - Krypt. Bad. 152. Fuck. rh. 141.

'Tubulina. - [S. 45?.]

— fragiformis. - C'd. Anl. t. C. f. 27: 17-19. Tuburcinia. - [S. 197.]

Tulasneinia J.conis. - cf. Oogast. algericus. Zob.: C'rl. Ie. VI. t. 16. f. 12\%.

'Tu lasn i a elcgans Lcsp. - ef. Tulasneinia Lcon. Kob."

'Tulasnodea. - S. 1.10.] ef. 'Tulostoma.

Tulostoma. - [S. 4111.] (Tulasnodea).

- brumale. - Cd. Ic. VI. f. 39. - Fl. dan.

X. 17 10. 1. Cd. Anl. t. C. f. $36: 12.13 .-$

I.ond. f. 16502 - ef. T. mammos. - Voges. 35:.

- Wstd. 122.

- exasperatum Mont. - Cd. Ic. VI. f. 12. -

Sagra, Cuba. t. 11 . f. 1.

- fimbriatum Fr. - Kl. II. 31. -- Cd. Ic. VI.

f. 10. - Carol. V. S1). - Wstd. 122.

granulosum Lév. - Démid. Russ. t. 1. f. 1. Leveilleanum Gaud. - Cd. Ic. VI. f. 41. -

Bonite, t. 140 . f. 4.

- mammosum Fr. - [S. 440.] - Kl. II. 30. -

Fuck. s. 315. - Krypt. Had. 331. - Kops Fl.

bat. X. 750. 2. - Pay. b. cr. p.9.f. 40-12.Bisch. f. 3609. - Dsm. 1121. - Kil. 17\%. cf. T. brumale. - Wstd. 122. - Ital. (39.) 59.

- - v. b.: Bisch. f. 3610 .

Meyenianum. - Nov. Act. XIX. Suppl. I.

t. 5. Ḱlotzsch).

- pusillum. - Hook. Lond. J. 19\$2. I. t. 7.

Tympanis. - [S. 399.] - Bail S. t. 19. - Berk.

In. p. 214. f. 60. c. (Spor.)

- alnea Fr. - \$s. 399.] - K1. 341. - Norweg. 191.

- Ariae. - [S. 400.]

- conspersa. - S. 399.] - Cd. Anl. t. G.

f. 1) : 31-39. - Ann. sc. n. 15.53. XX. t. 16.

f. 1.). 16. (Tul.) - Pay. b. cr. p. 62. f. 272 . Brit. f. 160. - Lib. 129.

— Cotoneastri. - [S. 400.]

_- Crataegi Lasch. - R. f. 353.

— Frangulae Fr. - [S. 400.] - Kl. 313. Kl. II. 661. - Fuck. s. 509. - Cenang. Fr. T'ul. (Wstd.). - Wstd. $12 ; 6$.

- Fraxini, - [S. 100.]

- incon'stans. - [S. 400.]

- Lonicerae. - Ann. sc. 11. 1536. V. t. 13. Mont.)

- nigra Horn. = Tubercularia 11., Fl. dan.

obtexta Wilr. - Kl. 1134.

ziza Rosae $P$.).

Patella. - [S. 365.] cf. Phacidium P. -

Wstd. 324 v. sphaeriaef. Wlir.). - Fuck. s. 764

Heterosphaer.).

picastra. - Carol. III. 63.

plinthis. - [S. 365.]

Prunastri. - [S. 400.]

Ravenelii. - Carol. IV. 66.

rigida. - [S. 400.]

Rubi Wllr. - Wstd. $127 \%$.

saligna. - [S. 4110.] - Bisch. f. 3155.

Berk. Ont. t. 1. f. 10. (Fruet.) - Hook. J.

1s51. III. t. 9.f. $1:-$ IR. f. 229 . - Fuck. s. 510 . turgida W7lr. - Wstd. 1279.

viticola Schw. - Kl. 1139. 



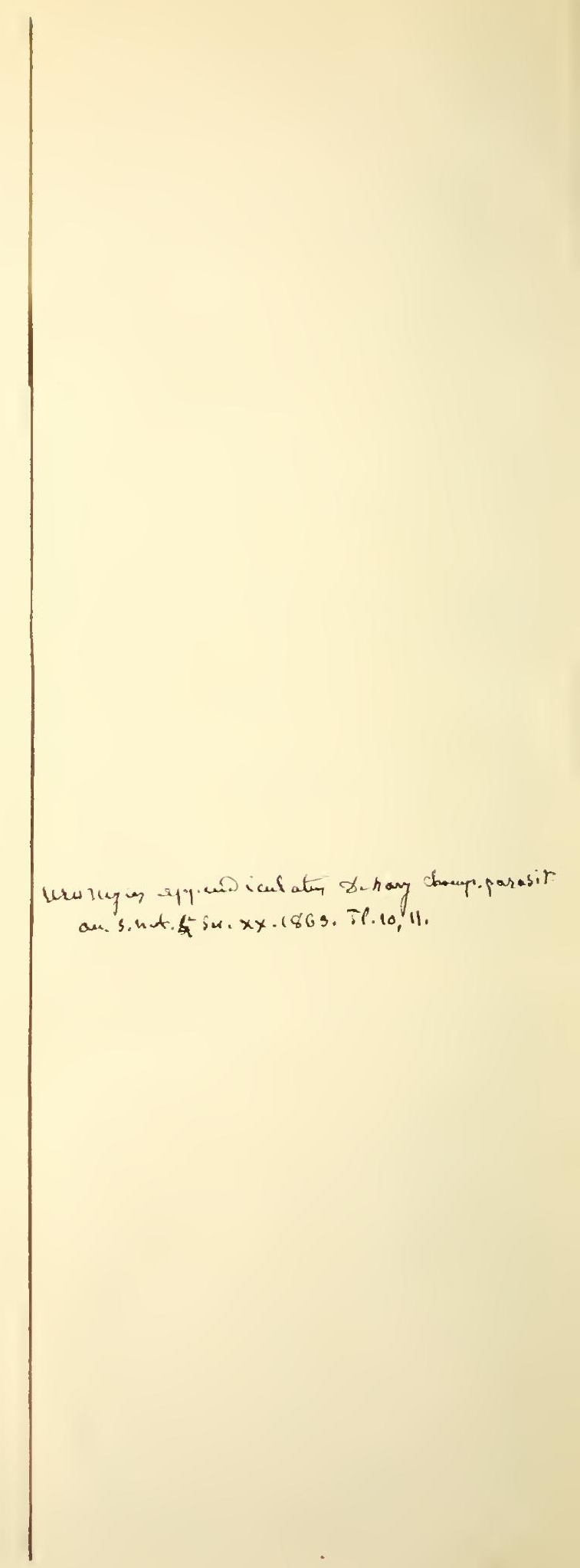


'T y ploodi um Graminis Lk. - Wstd. 522. - R. c.5 H. a. b.

Typhula. - S. 339.]

- crythropus. - [S. 339.] - Dsm. 101. - Fl. dan. XII. 2030. 1. - Fuck. s, s!33. - Cd. Aul. t. G. f. $72: 9 .-$ Stum h. 31-.t. 12, (Szl.).Bail S. t, 2\%. - Loud. f. 16193 . - Isl. $\$ 30$. zu Sclerot. erustuliforme D. Tul. . - III. II. 506. - Brit. f. 21.

- Euphorbiae Fuck. en. n. \96: ('laviceps. Fuck. in lit.).

— filiformis, - Cd. Anl. t. G. f. 72: 10. -

Loud. f. 16195. - Dsin. III. 310.

- gilva Lasch. - R. f. 419.

- ? gracilis. - [S. 339.] - Ann. Mg. n. h.

1 3 S. 1. t. i. f. 1. Berk.

- Grevillei Fr. - Bisch. f. 335\%. - Carol.

T. 35 .

- gyrans. - [S. 339.] - Fuck. s. $\$ 92$. - Dsm.

1019. - Brit. f. 66. - v. foliorum: ib. 151.

(Clavar. setip. Grev.).

- incarnata. - Kl. 1313.

— Laschii Rbh. - Kl. 1221.

- muscicola. - [S. 339. - Bisch. f. 3355. -

R. f. 417 . - Carol. III. 36.

- penicillata Horn. = Clavaria pen., Fl. dan.

- Persoonii Bail $=$ Phacorhiza selerotioides P.

- phacorhiza. - [\$. 339.] - Loud. f. 16192.

- Kl. 926. - R. f. 239 u. 115. huc sclerot. complan.). - Dsm. III. 659. - Hrit. f. 23.

_ ramentacea. - [S. 339.]

— sclerotioides. - Cd. Anl. t. G. f. $72: 11$.

- tenuis Fr. - Loud. f. 16191.

- Todei Fr. - [s. 339.] - Bisch. f. 33>1.

1'istillaria quisquil. Fr. (Tul.). - Dsm. III. 311.

— variabilis. - IIdw. I. t. 3. f. 2. a-c. - líl. 1725 .

- Riess. c. Sclerot. Scm. cult. Krl. II, 51) 7 . - Fuck. s. 595.

_ villosa. - [S. 339.] - Fl. dan. XI. 1967. 2.

Cloclarlium Botrytis. - Sturm l., 29-. t. 42. (Preuss.). - ?cf. Septosporium bifure. Fres.

Ulvina. - Mycelium.

- Aceti. - Kützing, Philos. Bot. 15.51. t. 2. f. 2. - IV std. 1319 .

( Sambuci. - Kützing, Philos. Bot. 1451. t. 2. f. 1 .

Un e inula. - ef. Erysiphe.

- adunca. - Ann. sc. n. $1 \$ 51$. XV. t. 7. f. 15.

(Lév.) $=$ Erys. Salicis Tul. - Berk. In. p. 27. f. $64 . \mathrm{d}$,

II. 164 : Erys. Salic. Tul.

— f. Populorum. - Fuck. s. 4ร5. b. - R. f. 560 .

- bicornis. - Ann. sc. n. I551.XT.t. 7. f. 17.

(Lév.) = Erys. Aceris Tul. - R. f. 559.

- Bivonac. - Ann. sc. n. 14.51. XV. t. 7. f. 14.

(Lév.) - Erys. Biv. Tul.; adunca f. Ulmorum.

polychaeta. - Carol. IV. 6 s.

spiralis B. C. - Berk. In. p. 2 is. f. 61. a.

Wallrothii. - Ann. sc. n. 1551. XV. t. 7 .

f. 16. Lév. = Erys. Prunastri Tul. Sel.

Uredo. - [S. 515.] - cf. Caeoma, Cystopus, Coleosporium, Fpitea, Melampsora, Podocystis,

Polycystis, Rubigo, 'Thecaphora, Tilletia, Tricho-

basis, Urocystis, Uromyces [S. 514.], Ustilago.

- acetosa Sehum. - Wstd. 25.5.

- Acherois Spr. - Wstd. 127.

Actaeae S-l. - Kl. 259.

Adoxae Awd. - Kl. 119 \%.

aecidioides DC. - Wstd. 127.

Hoffmann, Indix Fungorum.
Uredo acrigina Chev. - Wstd. 12\%.

Agrimoniae Schw. - ef. Potentillae, Carol.

Agropyri. - Sturm h. 25-.t. 1. (Preuss.).

- Kl. 1696.

Albertiniana Spr. - Wstd. 229.

Alchemillae P. - [S. 515.] - Ki]. 2צi. -

Til. II. 343. - hue: Trachyspora Alchem.

Fuck. Voges. 91. - Wstd. 1165. (Uromye.).

- Alliorum. - [S. 514.] - Bisch. f. 3574.

Dsm. 573. - Lib. S6. - Wstd. 567. alnea. - S. 520.]

Alsineacearum. - [S. 515.$]$

- Amaranthi. - Joum. hort. Soc. 1919. III.

p. 270. f. 4. (Berk.).

anbigua. - Kil. 1797. - Fuck. s. 92. -

Fuck. rh. 400. (limbata et ambig.).

- Andropogonis Podocystis). - Kl. 1997. -

Fuck. Nass. f. 9. - Fuck. s. $76 .-$ R. f. 595.

- Ital. 663. - Fuck, rh. 407.

Androsaemi DC. - Wstd. 565.

Anemones (Polycystis) P. - [S. 516.] -

Wstcl.675. - Krypt. Bad. 511. - Dsm. 721.

- Kil. 1297.

— annularis Strss. - Wstd. 75.3.

antherarum. - [S. 516.] - cf. Ur. violacea. apiculata Strss. - [S. 511.] - Til. 27. -

IVstd. 257. - Schweiz. 1. - Fuck. rh. 353-356.

- - v. Astragali Lasch. - Kl. 595.

f. Cytisi. - R. f. 93.

f. Plraseoli. - Kl. II. 364 : appendic.).

- Fuck. s. 59. b.

— f. Pisi sativi. - Kl. II. 363.

— v. 'Trifolii arv. - Kil, 169\%. - Kl. II.

362.

- v. Viciae. - Kil. II. 760.

apiculosa. - Wstd. 357.

— Berk: Dsm. 572. - Brit. f. 116. 117. v. Laburni. - Dsm. 233. - Wstd. 1172.

- v. P'raseoli. - Letell. 619. 1.

v. Trifolii. - Dsm. 956 .

Apii graveolentis. - Wstd. \$11.

appendiculata P. - [S. 514.] - Kl. 93. -

Krypt. Bad. 12. - (Uromyces) Kílaczek, Lehrb.

d. Bot. 1456. 9. (1). - Fuck. s. \$s. b. - de

Jary, Br. t. 3. f. 6. - Kühn, Krkh. t. 5. f. 47

-52. - Fuck. s. S5. a-c. - Voges. 191. I'.

Th.). - Wstd. 125. 1174. (Lathyri). - Fuck.

rh. $340-352$.

- v. Fabae. - An1. sc. n. 1554. 11. t. 9.

f. 12-14. ('Tul.).

v. Phaseol. P. - Dsm. 360 .

- v. Pisi P. - Letell. 619, 2. - Dsm.

361. - Wstd. S39.

- Armeriae. - Kl. 493. - Dsm.356. - Wstd. 1166. (Uromyc.).

Artemisiac. - Kl. 456 ; - it. Cent. XVI.

Vistd. T4S.

- arundinacea N. - Wstd. 390. - Fuck. s.

40. - Dsm. 107\%. - cf. Puccin. ar. : Fuck, rh. 320.

Asparagi Iasch. - Kl. 1150.

Avenae. - Oekon. Neuigk,v. Hlubeck. 1846.

t. 1. f. 23-26. (cf. Ur. segetum).

Balsamitae Strss. - Kl. Cent. XIII.

Behenis. - [S. 514.]

Berberidearum Lk. - Kl. 95.

Betae P. - [S. 515.] - Kl. 5\$2. - K1. II.

795. - Fuck. s. 99. - Brit. f. 60. - Wstd.

2\$6. - Ital. 249. - Fuck. rh. 398.

betaecola Bellk. - Wstd. 1170.

Betonicae Strss. - Wstd. 6Sf. 
Uredo Betulae Schum. - Wste. 129.

betulina Vahl. - Wstcl. 12!!. - Kl. 192.

bifrons DC. - W'stc. 255.

- Bistortac. - lib. Si.

- Bistortarum D( - Fuck. s. 46. - Lib. \&s.

- Wstel. 116\%. ustilaginea'.

- Iorraginearum I.k. - Kil. 91.

- bulbosa Strss. - Wstd. I†

- bullata 1'. - Wstd. S.j.

- Cacaliae DC. - Kil. 29\%. - IR. f. 395. Uro-

myces, .

- Calthac Reg. - Wstd. 1171.

Calystegiae. - Dsmu. 1363.

- Campanulae P. - S. 5J5.] - Kl. 2-9, it.

Cent. VII, Suppl. - Kl. II. 99. - Brit. f. 331 .

- W'std. 3>5.

- Campanularum, - Pringsh. Jahrb. II. t. 2 i.

f. 5. (Keimg., IIoffin.) - Fuck, s. 31 .

cancellata Sipr. - Wistd. 123.

candida. - [S. 512.] - Cystopus]. - Lng.

Ex. t. 3. f. 15;-t. 6. f. 32. - Bisch. f. 3673.

—Kl. 199. 792. 596. 497. 594. 699. 1097. 1094.

1194. 1299. - Dsm. 451. - Kiitzing, Phil. Bot.

1५51. t. t. f. 3. I. p. 236 . - Loud. f. 16656 . -

Pringsls. Jahrb. II. t. 27. f. 14. Keing., Hoffm.!.

- it.: Bot. Zt. 15.,9. t. 11. f. 2. - Letell. 621.

1. - Wstd. 125. 399. v. Cichorac.; ; 667. Pyrethri . - Krypt. Bad. 43.

- var. a. ef. Cystopus cubicus,

v. Scorzoncrae. - Voges. 1 !).

f. Cynarocephal. - Dsm. I079.

Capracarum DC: - ef. Melampsora salicin.

— bisch. f. 3904. - Fuck. s. 24. d. - Kl. 4!1.

- I'ringsh. Jalırb. II. t. 2-7. f. 7. (lieing.,

IIuffm. . - Wstd. 67̈. — Fuck. rh. 209.

- Carbo DC: - Wstd. 334.

- caricina. - S. 515.] - Kl. 1794. Dsm.

$47.5 .-W$ std. 4 is.

- Caricis P. - Kl. 195. - Fuck. s. 1119. -

Pringsh. Jahrb. II. t. 26. f. 1. IIoffin.) - cf.

Ustilago urceolor. - Wstd. 750. 1163. Polygoni!).

- Caries DC. - Loud. f. 16659. - Bisch. f. 3५५ . - ef. 'Tilletia C. - Brit. f. 113. - Jolln.

hort. Soc. 1417. II. p. 113. Keing., Berk.). -

Wistd. $\$ 333$.

__ Carpini. - lil. 1595. - Dsm. 131.

carpoplita Schum. - Wstd. 750.

Caryophyllacearum. - Wistd. 6it.

- v. Lychnidis. - Kil. 1295.

- v. Silenes. - Kl. 995.

- v. Stellariae. - Dsm. III. 337.

Castagnei. - Carol. V. 99.

Centumnodii Schum. - Wstd. 293.

Cerastii Mart. - Iil. 546.

chrysoides Orchidis Mart. - Kil. 196.

Cichoracearum. - [S. 51\%] - Ung. Fx.

t. †. f. 40. - Bisch. f. 3497. -. Wstd. $24 \%$.

Cicutae DC. - Wstd. 359.

cincta Strss. - Wstd. $2 \varsigma 6$.

- r. Fabae Strss. - Wstd. 2St.

cinnamomea. - Bon. t. 2. f. 42 .

Circacae A. S. - $[$ S. 515.$]-$ Kl. $175 .-$

iil. 1I. 296. - R. f. 494 . Fuck. s. 15. -

Wstcl. $1 \mid<0$.

- Cirsii Iasch. - R. f. 90. - Fuck. s, 52.

- Cissi DC. = Puccinia incarcerata Lér.

- clavigera Lasch. - Kil. 1295.

Colchici Ik. - R. f. 396. Polycrstis. -

Brit. f. 309. Sporisorium). - I.ib. 194. -

Wistl. H41. - Fuek. rh, 251. 25i Ścillae.
Uredo Compransor. - Kl. \5. - Dsm. 179. IVistd. 126.

_ - v. Tussilaginis. - Dsm. $35 \varsigma$. concentrica. - Dsm. 1115. - Wstd. 673. confluens A. S. - Wistd. 3S J. 665.

Convallariae Spr. - Wstd. Si.i.

- Convolvuli, - Bivona t. 3. f. 1. A. B.

cornuta Spr. - Wstel. 742 .

- Cruciferarum. - \$.514.] - Voges. 290. -

IVitd. 125.

- cubica Strss. - W'std. 329.

- Cucubali. - Dsin. III. 33〕.

- enlmorum Schum. - Wstd. 222.

- Cyani DC. - Wstd. 247.675.

cylindrica. - Brit. f. $33 \%$ - Kl. 191.

1) sm. 35. 364. - Wstd. 12!).

— Cylindrites (Roestelia) Lk. - Kl. !6.

Cynapii DC. - Wstcl. 349.

Decaisneana. - Kil. 1996. - Démid. IRuss.

t. 6. f. 2. p. 129 .

- Lév. : cf. Pileolaria Tereb.

decipiens Strss. - Wstd. Toll.

destruens S-l. - Kl. \2. - Fuck. s. 10 \%

I'ringsl. Jahrb. II. t. 26. f. 3. Hoftin.). - Oe-

konom. Neuigk. v. Hlubeck. 1 \$6. t. 3. f. 3-7.

v. Panici glauci Lasch. - Kl. 1296.

Dianthi P. - [S. 515. 9.] - K1. 59.

Digitariae Ustilago) Rbh. - Kl. 1199.

effusa. - [S. 5l?.] - Lond. f. 16639. -

lirit. f. 234. - Wstd. 563.

- Elepliantopodis. - Carol. II. 92.

Elymi. - Wstd. 391.

Eimpetri. - [S. 515.] - Voges. 391.

ephialtes Spr. - Wstd. $25 \%$.

Epilobii. - S. 515.] - Kl. 479. - Fuck.

s. 51. - R. f. 459 - ef. Melampsora Ep.:

Fuck, rh. 3100.

- epitea Kz. - [S. 512.] - Fuck. s. 25. b. -

I $\mathrm{sm}$. 36. - ef. Epitea vulgaris Fr. - Carol.

ソ. 94. - Wstd. 670. Capracar.) \$43. ef. Melampsora Sal.: Fuck. rh. 297.

Ervi Wstd. - Bull. ac. belg. 1. Sér. XXI.

2. p. 246 . f. 3. - Wstd. \$19.

Ervi hirsuti. - Kl. Si.

Erythronii. - Kl. II. 35 S.

Euphorbiae. - Pringsh. Jahrb. II. t. 27. f. (i.

(Keimg., IIoffm.) - Fuck. s. 27. - Dsm. I0s0.

- Wsta. 2๖:. - (f. Melampsora E.

- Euploorbiarum L.k. - Kl. 197.

_- Euphrasiae Schunı. - Wstd. 394.

- Evonymi Mart. - [S. 515.] - Kl. 1994. 79:3.

— Kil. II. $3 \triangleleft 0$. - Dsn. 723 .

- excavata. - Brit. f. 119.

Fabae Grev. - Bisch. f. 3906. - Lng. Ex.

t. ‡. f. 39. a. - Loud. f. 16626. - Ann. se. $n$.

14 47. VII. t. 7. f. 35. (Tul.).

- - I. ef. Uromyc. append. Fabae. - Trst

244. - Ital. 250. Uredo Leguminum v. Fabise I) sm.

_-_ Falcarine. - Kl. II. 390.

falcariata Ik. - Kl. I9ii.

fallens. - Dsin. 725.

farinosa. - 11 std. 230.670. - v. Salicis

Capreac. - Vuges. 150.

— ferruginea Sclium. - Wstd. 232.

- Ficariae ('olycystis) A. S. - Brit. f. 23\%. -

Kil. 499. - Fuck. s. 94. - Kil. II. i95. (Uromyces. - Wstd. is. - Fuck, rh. 3!13.

Fici ('ist. - D:m. i362.

filicina cher. - W'st. jtil.

Filicum. - [S. 515.] - Isn. 132. - Kil. 


293. - Kl. II. 797. - Fuck. s. 53. - Brit. f. 339. - Carol. IV. 97. - Ital. \99. — Fuck. rh. 106 .

Ure d o Filipendulae Iasch, - Iil. 5S0.

- Hosculorum. - [S. 5]6i.]

Flosculosarum DC. - Ioud. f. 16 titjl.

- - A. S.: R. f. 91. - Fuck. s. 5\%. - Iil.

II, 360. Uromyces. - Wstd. 2\T.

- - v. Arctii tom. - Ii. f. 499.

foetida. - Aun. sc. n. 1<21. II. t. 7 .

formosa. - [S. 515.] - Kl. 2- - - Wstd. 15 .

— v. Cichoracearum. - Kil. 1695.

fraterua (Uromyces). - Iil. II. (j9)3.

frumenti. - Usta. y1. 3:31.

fulva P. - Pringsl. Jahrb. II. t. 27. f. 11.

Kcing., IIoffin.. - Wstch. 12(i. 1 i (jj. Senec.).

- v. Sonchorum Sch. - Fuck. s. 33. b. fusca Bon. - R. f. 192. - cf. globoso-fusca. fusco-virens 'Lstilago' - KI. $1 \mathrm{i} 9 \mathrm{i}$.

Galii. - Kl. 545. - K. f. 506). Dsm. $i 20$. Wstd. S14.

Geranii Uromyces). - [S. 51.1.] - Kil. in.

- DC.: Loud. f. 166i17. - Fuck. s. i1.

- Dsm. 722. - Kl. II. 295. - Wstd. 1971.

glohoso-fusca. - Bon. Con. p. 1!1i. t. 2.

f. $21=$ fusca Bon. ap. 1R. f. 19ㄹ.

- globoso-rufa. - Bon. C'on. p. 197; lue

C'aeoma porplyrogeneta L.k.

— glumarum Rob. - Dsm. 1076. - Wstd.564.

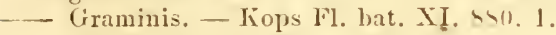

- gryrusa Rbt. — Wstd. 346. - Bisch. f. $35 \% 2$.

- Epitea) Kl. 177. - Fuck. s. 36, b. - ?R.

f. 159 .

- Ireliantlii. - Carol. II. 93.

IIelioscopiate. - Kl. 2!); it. C't. V, Suppl.

- Wistd. 25 .

-_- IIydrocotyles. — Dsm. 17:33.

- Hydropiperis Schum. - of. Ustilago Can-

dollei Tul. - Wstd. 1163.

- IIppericorum DC. - [S. 515.] - Kl. 153.

- Fuck. s. 77. - Dsm. 660. - Wistd. 56.j. -

Fuck, rh. 40)?.

hypodytes Ustilago. - [S. 5l6.] - Kl. \$3.

- Wstd. 572. 1161. Tritici.

- f. Tritici. - Dsm. 473.

Idaei Mart. - Wstd. 3<6.

Impatientis Schultz. - Kl. $2 \succ 5$.

inaequialta (Uromyces) Lasch. - R. f. 91.

inconstans. - Wstd. 331. 'gram.), 333 (se-

min.), 334 (spiculaecola'.

- intrusa Grer. - Wstd. 1169.

- Inulae Kz. - Kl. 5\$9. - Fuck. Nass. f. 10.

Fuck. s. 92.

- Ipomaeae. - Carol. IV. 99.

- Iridis Dub. - Brit. f. 59. - Iil. 5\$1.

Junci. - Fuck. rh. 379.

Labiatarum Lk. - S. 515.] - Wstd. $3 \varsigma 5$.

- f. Calaminthae. - Brit. f. 233.

f. Clinopodii. - Kil. 95. 1195.

f. Menthae. - Kl. 95. $1195 ;$ Kl. II.

?97. - Brit. f. 232. - Krypt. Bad. 142.

- Laburni DC. - Anu. sc. n. 1547. VII. t. 7.

f. 32-34. Tul.). - Wstd. 1172 .

- Lapsanae Rbh. - Kil. 475. - Fuck. Nass.

f. 6. p. 13 .

_ Lathyri Bellk. - Wstd. 117.

Ledi A. S. - [S. 515.] - Kl. 195.

__ Leguminosarum. - Kolaczek, Lehrb. d. Bot.

is.56.9: 2. — K1. 92. cf. Uromyces, - Letell.

619. 3. - Wstd. 254. S16. - Fuck. rh. 357

$-391$.
Uredo I.eguminosarum v. Fabae. - Dsm. 362. - Kil. 440. - Kl. II. 361. - Fuck. s. 90. b. d. - cf. Uredo Fabae.

- v. Fabarum. - Krypt. Bad. 405.

— - v. Glycyrhizac. - Kl. 799.

— v. Lathyri. - Kl. 11\$9.

- v. Medicaginis. - Kl. II. 356.

ง. Viciae. - lil. 1159.

Leguminum. - Dsm. 23jt.

leucosperma Spr. - Wstd. 662.

I.igustri Kblı. - Kil. 579.

lilacina Rob. - Dsm. I0\2.

linbata. - ef. Lr. ambigua.

— v. Alliorum. - Kl.1193. - Fuck. s. 75.

Limonii Dub. - Démid. Russ. t. 6. f. 1.

lincaris P. - [S. 515.] cf. Trichobasis.

Wistcl. $333 \mathrm{I}$

__ - Strs: : Wstd. 91. - 561: r. Polypo-

dii. - Krypt. Bad. 315. - Kl. 794. - Fuck s.

3\%. - I'ringsh. Jahrb. II. t. 27. f. 13. (Keing.,

Iloffn. . - zu Puccin. Gramin. : Kühn, Krankh.

t. 5. f. 16; - 1 nn. sc, n. 1551. II. t. 9. f. $1-$ 4. - "lul.

- _ frumenti: Joges. 2̧s.

— - r. Polypodii : Voges. 259. - ef. I'uc-

cin. Gramin.: Fuck. rh. 319.

- Iini. - S. 512.$\}$ - Dsm. 133. - Loud. f.

lfilit3. - Fuck. s. 31); zu MIclampsora, Tul. -

Podocystis Kl. 142. - Voges. !10. - Brit. t.

114. - Wstd. 11\%\% - ef. Melanps. Lin.:

Fuck. rh. 311 .

Lobeliarum. - Kil. 159 i.

longicapsula. - Wstd. 129. (v. Betulac)

cf. Melampsora pop., Carpin.

- — f. betulina IC. - ef. Melampsora bet.

- f. Carpini. - Fuck. s. 2ij.

- f. populina DC. - ef. Melamps. pop.

- Fuck. s. 25. c.

- longipes. - Kl. 1995. 1295. - Epitea

kil. 593 .

\section{— f. Legumin. - Kl. 1799.}

longissima Ustilago) Sow. - [S. 516.] -

Wstd. 232. - Kl. >1. - Fuck. s. 103. - cf.

Caeoma 1. - Pringsh. Jahrb. II. t. 26. f. 5.

keimg., Hoffm.). - Brit. f. 230.

- v. Holci. - Kl. 149 .

— v. megalospora. - Kl. 1597.

Luzulae. - Dsm. 1360 ; III. 651. - Wstd.

๖)

— Lychnidearum. - [S. 515.] - Dsm. 662. -

Kil. ن. 699 .

- - macrospora. - Dsm. III. 401. maculosa Strss. - Wstd. \$.15.

Majanthae. - Norweg. S5.

marginalis. - Kl. 1499. - Kl. II. 35 s.

- Maydis (Ustilago;. - Carol. IV. 100.

Bisch. f. 3912. - Fuck. S. 105. - Dsm. 10 S1.

- Kil. 193. - Pringsh. Jahrb. II. t. 21 . f. 4.

Keimg., Hoffm.). - cf. U. Zeae. - Oekonom.

Neuigk. v. Hlubeck. 15.6. t. 3. f. 1-2.

Mei Kz. - Kl. 5ss.

Melampyri Rbt. - Kl. 5S1. - cf. Coleo-

spor. Rhinanthac. - Wstd. 669.

— Menthae P. - S. 515. 5.] - Fuck. s. 51).

- Wstd. 355.

Mercurialis. - [S. 515.] - Kl. 1190. - Kl.

II. $35 \%$. Fuck. s. $7 .-D s m .955 .-$ Wstd. $66 \%$.

- miniata Epitea) P. - Voges. 15\% - Kl.

1100. - Bon. t. 2. f. 41. - Fuck. s. 5. -

Pringsh. Jahrb. II. t. 27. f. 9. (Keimg., Hoffm.).

- Letell. 619, 1. - Wstd. 1179. Lini .

minina. - Bon. Con. p. 196. t. 2. f. 23. 
Uredo mixta Fpitea) Kz. - Kil. 119. - Fuck. s. 2S. a. - 13rit. f, 120. - Wstd. S12. - cf. Melimpsora sal.: Fuck. rh. 296.

_ mucronita Strss. - Wstd. 131.

_ muricella Wilr. (Angélicae, Saniculae). - Kil.

1196 ; it. Cent. XV; 1164 , Suppl. - Fuck, s. 61. - f. Oreoselini. - Kl. II. 366.

Muscari. - Dsm. 1724.

mycophila $\mathrm{P}$. - Wstd. 136.

- nivalis Baner. - Wstd. 790 .

- obconica bon. - R. f. 1!11. - Bon. Con.

t. 2. f. 22. 'p. 196.)

oblonga. - kil. 169 f.

oblongata. - [S. 515.] - Kil. II. 341.

Loud. f. 16629.

— obtegens I/k. - Wstd. S7.

- obtusa Strss. - Wstd. S61). obtusata Lk. - W'std. 329.

— occulta. - Kühn, lirankhı. t. 2. f. 13-3.1; t. 4. f. 11-13. - Kl. 11. 393. - Ki. 1 \9. (1'olyeystis). - Dsm. 1725. - Wstd. 335. Fuck. rh. 256.

- olida - Kl. 1695. - Cacoma ol. Bon. Con.

17.1 .

1192.

_ orbicularis Mart. - Wstd. $\$ 43$.

—_ Orchidis. - [S. 515.]

- Oreoselini. - cf. Puccin. Umbellif.

__ Ornithogali s. K. - Kil. 5si.

ovata Strss. - Dsm. 661. - Fuck. s. 25. a.

- Wstd. 129. 1173.1175. Uromyc : - R.f.

596. (Semperv.).

— Oxalidis. - [S. 511.] - Ann. se. n. 1541.

XVI. t. 14. u. 15. f. S. Lév.) - Caeoma canum Bon. Con. 174.

Padi. - [S. 515.] - Kil. 1699.

Paeoniarum. - Dsm. 1364. - Wstd. 1172.

parallela. - [S. 5 I6.]

Parnassiae. - Wstd. 676.

penicillata Spr. - Wstd. 556.

- Phaseoli Strss. - Bisch. f. 3\$91. - Carol.

11. 94. - Wistd. 357 .

- Phaseolorum? ef. Puccinia Phaseol. - Wistd.

124. 835. (Pisi).

Phragmitis Schum. - Wistd. 1 66.

- Phyteumatum. - [S. 5/..] - Ung. Ex.

t. T. f. 35 .

piluliformis. - Hook. Lond. Journ. 15.13.

II. t. 22. f. 6. (Berk.).

- Pimpinellae Bellk. Wstd. S11.

Pisi DC. - TVstd. \$3s.

Poae sudeticae. - Wstd. 1265.

Polygoni Fuck. (Uromyces). - Fuck. rh. 399.

Convolvuli: Wstd. 749.

- Polygonornm (Uromyces) DC. - [S. 514.]

- Dsm. 355. 476; III. 751. - Kl. 292. -

Fuck. s. 46 . - Ital. 665. - Kl. II. 294. -

Loud. f. 16620. - R. f. 457 . - Brit. f. 231.-

Carol. IV. 95. - Wstd. 253.

- polymorpha Strss. - Wstd. 2ss. 672. 1179.

Polypodii DC. - Wstd. 561.

- pompholygodes (Polycystis) S-l. - Kl. S6.

- Brit. f. 236. - Wstd. 1151.

_- Populi Mart. - Kil. 455. 795. - cf. Caeoma

p. B. - Purton. I. t. 27.

populina. - Wstd. 129.

- - f, betulina. - Fuck. s. 29. - ef. Me-

lampsora bet. : Fuck. rh. 299.

— porphyrogeneta. - [S. 515.]

-_ Portulacae Dec. - Wstd. 330.
Uredo Portulacae DC.: Dsm. 363.

- Potentillac. - ef. Pliragmid. Pot. - Carol.

1I. 97. (Agrimoniae Schw.).

Potentillarun. - Kil. 91. - Dsm. 130.

- DC : zu l'hragmid. obtus. : Ann. sc.n.

14.5. II. t. 9. f. $15-23$ (Tul.). - Fuek. s. 31.

3.). a. - Brit. f. 115. — Wstd. 561. (i72.

— f. Agrimoniae. - Kl. $796,1<0)$.

- Poterii Spr. - Wstd. 561. - ef. J'hragmid

apicul.: Fuck, rh. 312.

— — S-l. - Kil. 252. - Fuck. s. 35. b.

I'runastri. - Carol. II. 95.

- Pruni Cast. - Dsm. 1051. - Fuck. Nass.

f. 12. - Fuck. s. 96. - Wstd 5ti9.

- Prunorum 1)C. - ? Wstd. 5ti!.

— Pseudo-Cyperi, - [S.5]6. 1.] - Wstd. 415. puccinioides. - Kl. 1596 .

Pulsatillae DC. - Kil. 999.

—- pustulata. - [S. 515:] - Fuck. s. S.5. Podocystis). - Dsm. i24. - v. Epilobiil': Wstd. 570.674 . (c. v. Cerast., Caryoph.). 1267 (Salic.).

— Fuck. rh. 409 .

Prrolae. - [S. 515.] — Bisch. f. 3905. -

Wstd. 106\%. - Kl. 1194. - Krypt. Bad. 401.

Ung. Ex. t. 6. f. 30. - Fuck. rh. 101.

— Quercus. - Dsm. 1721. - Brit. f. 239. -

Wstd. 562. - Ital. 600.

Quineunx Str - Wstd. 550.

Ranunculacearum (Uromyces). - [S. 516.

514.] - Brit. f. $23 \%$ - (Ilellebori) Lib. 291...

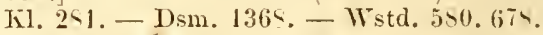

- - f. Ficariae. - Krypt. Bad. 322.

Ranunculi. - Lib. 19\%. (Sporisorium).

Receptaculorum DC. - [S.516.] - ef. Usti-

lago. - Dsm. 124.

- Rhinanthacearum Coleosporium! - Dsm.

951. - Kil. 2-3. - Fuck. s. 32. - Wstd. 35i

(v. Euphras.) ; 669.

— Rhododendri. - Kl. 1900. - R. f. 92.

- ribicola Lasch. - Ki. 190. - Ki. II. 367.

— Ribis. - [S. 515.] - Kl. 459.

_- Ringentium Mart. - Woestelites Lk, - cf. Rocstelia cancellata.

- Rosae. - Letell. 619.4. $\longrightarrow$ Ann. sc. n. 1st7.

YI1. t. 6. f. 19. (Tul.). - Epitea) Kl. 90.--

Dsin. 359. - Pringsh. Jahrb. II. t. 27. f. 10 .

lieing., Hoffm.). - Wstd. 229. — Fuck. rh.

313 c. Phragmid. incrass.).

- Rubi. - Ung. Ex. t. 7. f. 36. d. - Fuck. s. 36. a. - Wstd. 563 . Idaei P. - Wistd. $3 \varsigma 6$.

— Rubigo Uromyces'. - Kl. II. 365.

- Rubigo vera. - [S. 515.] - Bon. t. 2. f. 511.

- Fuck. s. H1. - Kl. 1599. - Bot. Zt. 1559.

t. 11, f. 1. Keimg., Hoffm.). - zu Puccin. coron. : Kühn, Kirkht. t. 5. f. 43-15. - Carol. I. 100. - Wstcl. 231. - ef. Puccin Straminis: Fuck. rh. 321.

- Ruborum DC. - Voges. 92. - ? Fuck. s. 37. - Ann. sc. n. 1547. VII. t. 6. f. $11-15$. (Tul.) - Fuck. s. 36. b. - Pay. b. cr. p. 75. f. 343 u. $347-350$ - Epitea) DC. : Kl. 797. - zu Phragmid. bulbos. : Ann. se. n. 14.54. II. t. 9. f. 15-17. (Tul.). - Wstd. 563. - Ital. 351. - Fuck, rh. 314. (c. Phragmid. incrass.).

- Rumicis Lasch. - Kl. $\$ 76$.

Rumicum DC. - K1. 49\%. - Fuck. s. 9 i.

(cf. Uromyces R.). - Krypt. Bad. 103. - Kil. II. 359. - Wstd. 2\5. - Ital. $\$ 95$. - Fuck. rl. 397.

Sagittariae. - Wstd. 117\%.

Saliceti. - Kl. 455. - Wstd. 56. 


Uredo Salicis. - Ung. Fx. t. 5. f. 25 ; t. 6. f. 31 . - Bisch. f. 3907. - Kl. 191.

- Saxifragarum. - [S.5 I5.] - K1. 1096. I191. - Iil. II. 3:5.

- Sehlechtendalii DC. - Kl. \$7.

_. Scillae (Uromyc.). - Fuck, rh. 401.

- Scirpinii. - Wstal. 1266 - R. f. 591.

- Serophulariae Laseh. - I1. 194. - Ital. 3.33.

Uromyc., Aecil, . Fuck, rlı, 39..

- scutellata P. - Voges. 93. - Kl. 5\$3; it. Cent. IX. Suppl. - Uromyces. - Kl. II. 29!3.

- Fuck. s. 91. - Fuck. rh. 396.

- Secalis. - K1.1399. - André, Ökon. Neuigkeiten. 1 19. t. 1. (Cd.)

- Sedi DC: - Wstd. $1173 .-$ R. f. 597.

segetum ]'. (Ustilago Carbo.). - [S. 516.] ef. Avenae. - Fl. dan. XII. 2150. 1. - Kil. 1. - Iringsl. Jahrb. II. t. 26. f. 2. Kieimg., IIoffu. - Voges. 291. - Carol. II.99. - Öko110n. Neuigk. v. Illubeck. 14 16. t. 2. f. 1--与.Wotd. 331. IIord. Aven.).

- Sempervivi. - Ung. Ex. t. 3. f. 16; t. 5. f. 24 . - IBisel. f. 3904. - Kil. 1599. - Kl. II. 699. - I.etell. 621. 4. Sedi . - Wstcl.1173.IR. f. 597 . - Ital, 6ifil.

- Senecionis. - Wstcl. 230. - Kops. Fl. bat. X1. \40. 2. - (oleosporium Kl. $2 \checkmark 6$. - Fuck, s. 33. d. - Pringsh. Jahrb. II. t. 27. f. 12. lieimg., IIoffin.

- Schum. = U. globoso-flava Bon. Con. Silenes. - Fuck, rh, 3!12.

- sitopliila Ustilago Ditm. - [S.,jli. Caries ]

Oekonom. Neuigk. v. Hlubeck. Is l6, t. t. f, 1

22. - Ki. 13!9. Tilletia Caries'1ul - Fuek. s. 112. - Wstd. 3333. - Sturm l. 3. t. 31.

- Smilicis. - Carol. I1. 96.

solicla Uromyc. :- Carol. IV. 92.

- Solidaginis. - Fuck. Nass. f. 11. - Fuck.

s. 95. - Carol. III. 96.

Sonchi P. - W'std. 126.

sororia Laseh (Uromye.). - Kil. 1291.

sparsa. - [S. 514.

Spermaeoces. - Carol. II. 91.

- Spiraeae Sow. - Wstd. 1167. - zu Tri-

phragm. Ulmar.: Amu. se, n. IS51. II. t. 11.

f. 17. 'Tul.) .

Stellariae. - Fuck. s. S1.

striacformis, - Wstd. 6\%\%. Ustilago macrospora Dsin.).

Striola Strss, - W'std. 186.

suaveolens. - [S.515.]-Ann. sc. n. 1477.

VII. t. 6. f. $1-10$. (Tul.). - Fuck. s. 59. -

Pay. b. er. p. 75. f. 345.346 . de Bary Br.

t. 3. f. $1-4 .-D \sin .129$ - Kl. 2S0. - Kl. II.

359. - Voges. 159. - Wstd. 57. - Ital. 664.

subeorticalis. - [S. 520.]

Sympleyti DC. - [S. 515.$]$ - Dsm. 35\%. -

Kl. 25ว. - Fuck. s. 75. - Kil. II. 200. - Brit.

f. 320. - Wstcl. 332. - Ital. 50. - Fuek. rh. 103.

- Syntherismae. - Carol. II. 94.

- 'Tanaceti. - Kl. 457. - Iil. II. 195. -

W'ste. $\$ 17$.

- Taraxaci P. - Kl. 474.

tecta. - Fuek. Nass. f. 3. p. 10.

- Terebinthi DC. - Kl. 1095. - Kl. II. 199.

$\mathrm{D}$-m. 1365. - ef, Pileolaria T.

Toxicodendri. - Carol. III. 97.

Tragopogonum. - Kl. $4 \$ 1$.

- prat. P. - ef. Ustilago recept. f. Tragopog. - Wstd. 329.
Uredo tremellosa. - [S. 512.] cf. Erannium aureum Bon. - Wstd. 355.

- $\checkmark$ Sonchi Strss. - Kil. 294. - ef. Cileospor. Sonchi.

1165. v. Senecionis. - Kil. 295. - Wistd.

Trifolii DC. - IVstd. 397.

Tropaeoli, - Dsm. 37. - Wstd. \$39.

tuberculata. - Fuck. s. S6. - Fuck. rl. $10 \mathrm{~s}$. 'Tussilaginis 1'. - Jiseh. f. 3911. - Ung.

Ex. t. 5. f. 26. - Fuck. s. 33. a. - ef. Eram. aurantiae, u. Caeoma Tuss. - Wstcl. 126.

—_ Farfarae P. - ef. Coleospor. 'Tuss. Ulmariae Grev. - Wstcl, 116i. (Uronyc.). Umbellatarum Lk. - Kl. 2\$ 1. - Dsm. 31.

Fuck. s. (61. = Ur. murieella f. Conii ete. l.ctell 621. 3. Cicutae). - Wstd. 3\9. 411. Ii. f. $5 ! 96$.

Unubelliferarum. - [S. 511.515, 7.]

- ureeolorum ('aricis I'.). - Ism. 10\$3. -

Brit. f. 111. - Wstd. 750. 1162.

- utriculosa. - [S. 516.] - Cd. Anl. t. 13.

f. 3: 3. 4. - Fuek. s. 110. (ef. Ustil. Candoll.). I)sm. 32. - Ki. 1099. - Wstd. 1163.

- Vaccinii A. S. - Kil. 4\$4. 795. - Fuck. s. $>0$.

- Vaceiniorum. - -S. 515.] - Wstd. 1066.

I) sm. 17y. - Fuek. rl. 105 .

Valerianae. - Kl. 594. - Kl. II. 342. -

Fuek. s. \$1. - Dsm. 152 . - Brit. f. 319.

Wstd. 571. - Fuek, rh. 39..

- Vepris. - Nom. 1722.

Veratri I) - Kl. 296. - Kl. II. 94. -

I) $=11$. 1361. - Ital. s 180.

- Viciae liellk. - Mstl. S16. \$19 Ervi) 117,

(r. erace.)

Vincae DC. - Brit. f. 231. - Dsin. 177.

Vineetoxici DC. - Fuck. s. 100.

violacea $P^{\prime}$. - Kl. S 7 ; it. Cent. VI. Suppl.; -

5.b. (ent. XIV.) ; † Cent. XVIII.). — Fuck.

s. [11.;. - I.etell. (i24). 2 (antherar.).

Violae. - Kl. 1796.

Fiolarum DC: - Fuck. s. 71. - 1)sm. 150. Wrstd. S 10 .

Vitellinae DC. - Fuck. s. 25. c. - Wstd. 66. Zeae. - cf. Ur. Maydis. - Dsm. 47.

U r n u la. - [S. 361.]

Craterium. - Carol. I. 39 (ef. Peziza (r.).

minor. - Afzel. t. 12. f. 25.

Uroeystis. - ef. Uredo.

Uromyees. - [S. 364.] cf. Uredo.

Ustilago. - [S. 5]6.] ef. Uredo, Tuburcinia. antherarum. - [S. 516.$]$ - Ann. se. n. IS17.

VII. t. 1. f. $12-19 .-$ t. 5. f. 23 (Tul.) - - de I3ary Br. t. 1. f. $6 \rightarrow$ - - Kl. II. 100. - I sm. III. 103.

- axicola. - Ann. Mg. n. h. 1552. IX. t. `. f. 2, p. 200. (Berk.).

- Candollei. - Anu. se. n. 18t7. VII. t. 3. f. 20. 21. (Tul.) - Fuck. s. 110 (Uredo utriculosa Cd. - - Kíl. II. 700. - R. f. 399 (Uredo

IIydropip. S.). - Fuek. rlı. 250.

eapsularum. - S. $516:$ Th. h.]

Carbo (cf. U. segetum). - (hordei) Pay. b. cr.p. 120). f. 565 ; p.55.f. 244. - Kühn, Krankh. t. 4. f. T-9. - (aven.) Kühn, Krankh. t. 3. f. 10-21. - (evolutio) Ann. se. n. 1530. XX. t. 2. A. Brongniart). - Ann. se. n. 1547. VII. t. 3. f. 1-12 (T'ul.). - Kolaczek, Lehrb. d. Bot. 1556. 6: a. - (aven.) Pay. b. er. p. 120. f. $56 \mathrm{l}$. Caricis. - Fuck. s. 109. - Fuek. rh. 219. Cissi DC. = Puccinia incarc. Lév. ('Tul.). 
Ustilago destruens Dub. - Sturm h. 24) - t. 2l'reuss.). - Külın, Krankh. t. 3. f. 1-9;t. f. f. 10. - Fuck. s. 107 . - Iil. II ! 100. Fuck, rh. 24i.

- Dregeana. - Ann se. n. 1417. Vil. t. 3.

f. 13. (T)

- Duriacana. - Dsm. III. 4112 .

Duriaei. - Amn. se. n. in 17. VII. t. 5. f. 32. Hosculormm. - [S. 516.] - Dsm. III. 151. foetens. - Carol. V. 100.

- hypodytes. - S. 516.] - Ann. se. n. IS17.

III. t. 3. f. 14. (Tul.). - de lary 13r. t. 1.

1. 2-5. - Iil. 43; Cent. XV. - Kl. II. 391. -

11std, 572. 1161 ('Tritic.) - Fuck, rh. 216.

- hypogzea. - Tul. F. hyp. t. 2l. f. 17.

- Ischaemi. - Fuck. Nas`. f. 13. - Fuck.

s. 111. - R. f. 394. - Fuck rh 251.

- lenecderma. - Ann. IIg. n. h. 1452. IX.

t. S. f. 3. p. 200 Spor., lierk.)

- longissima. - S. 516.] - Fuck. s. 103. lirypt. Bad. 112. - O $\mathrm{sm}$. 1366. - Fuck. rh. 242.

- macrospora. - I sm. 1727. - cf. Uredo striacformis.

_- Maydis. - Amn. sc. n. 1547. VII, t. 2. "Tul.

- de Bary Br. t. 1. f. 1. - Pay. b. er. p. 120. f. 563..-Fuck. s. 104. - Kühn, Krankh. t. 3. f. $22.23 ;-$ t. 1. f. $11-17 . \ldots$ Tiolaczek, Lehrl).

d. Bot. 15.56. (j: b.) - Fuck. rh. 21.

Montagnei. - Dim. 1720. - Ann. sc. n.

1517. V11. t. i. f. 31. (Yul.) - R. f. 243.-

Ann. Mg. n. h. IS52. IX. t. \&. f t. Spor, lierk.)

- oeculta. - Kl. 1 ৎ9ৎ. - Kl. I1. 393. - Dsm. III. 653 .

- olivacea. - [S. 516.] - Ann. se. n. IS17.

TII. t. -1. f. 11. 'Tul.) - Bon. Con. t. 2. f. 24.

- Dsm. 1526. - R. f. 599.

- Orobanches. - [S. 19i.]

- Persicariae Mentz. - Wstd. 1163.

-_- Phoenicis Cd. - ? Ust. destruens Bon. Con.

p. 192.

- pilulaeformis. - Amn. sc, n. 1 \$17. VII. t. $s$.

f. $27-311$. Tul.).

purpurea = Erysibe baccata Wlir.

- receptaculorum. - [S. 516.] - Ann. se. n.

1s 17. VII. t. 1. f. 1. Tul.). - Fuek. s. $104-$

ki. II. SO()-Fuck. rh. 214.

- - f. Scorzonerae Fr. - Ann. sc, n. 1554.

II. t. 12. f. $31-40$. Tul.).

- f. Tragopogonis Fr. - Ann. se. n. 15.91.

II. t. 12. f. $27-33$. "Tul.).

Rhynchosporae. - Iil. 1596.

Rudolphii. - Ann. sc. n. infi. VII. t. 5.

f. $21-26$. (Tul.)

Salveii B. B. - Dsm. III. 1. - Wstd. 1161.

-_ Salviae B. B. = Uredo globoso-fusea. Bon.

- Secalis. - Kühn, Krankh. t. 2. f. 35. segetum. - [S.516.] ef. U. Carbo. - Bisch.

f. 1. 3५s5. - Fuek. s. 102. - Cd. Anl. t. 13.

f. $3: 1$. 2. - Schnzl. t. 12. f. $1-3$. - Ital. 59?.

- Iiryt. Jad. 241. Avenae) +1. - Fuck. rh.

213. - Sturm h. 3. t. 33 .

f. Arrhenatheri, - Kil. II. 395 .

- f. Avenae pubescentis. - Kl. II. 399.

- f. IIordei. - K1. II. 397 .

- sitophila Bon. - Uredo s. Ditm. bei Sturm

h. 3.t. 3 :.

- Tragopog. prat. - cf. Ust. receptac. Trag.

- Tritici. - Wste. 333.

- urceolorum. - [S.516.] - Ann. sc.n. 1517.

Fil. t. 1. f. T-10. (Tul.). - Kl. II. 396.
Ustilago urceolorum DC. - ef. Uredo Caricis I'. -. Wstd. 7.50. 1161 .

- utriculosa. - [S. il6.] - Ann. se. n. 1417 . VII. t. 1. f. 2-6. (Tul.). - Kil. II. 395. Wistd. 1163.

- Vaillantii. - Amn. se. n. 1\$17. VII. t. 3. f. 15-19. (Tul.). - I) sm. III. 652 .

- violacea. - Fuck. s. 105. - (Microbotryum)

Ii. f. $3 !$ i. - cf. Caeoma Schlechtendalii líl. Yuck. rh. 245.

\section{Valsa. -- c.f. Sphaeria.}

Abictis Fr. - [S. 112.] - Fuek. s. 4.j.

abnormis. - [S. 112.55.]

acelinis. - [S. 11․]

aglaostoma (Circinatae). - Ann. Mg. n. h

[\:). III. t. III. f. IT. (J). Ii.).

alnea. - S. 112.:3\%.]

f. $($.

alnifraga. - [S. 111.] - Hedw. I. t. I1.

- ambiens. - [s, 112.] - Fuck. s. 1 [2.

angulata. - [S. 411 .

anomia Fr. - Sturm h. 31. t. 1. (Strauss, aurea. - Fuck. Nass. f, 20. - Fuck. s. 111 i. bitorulosa Circinat. - Ann. Mg. n. l.

1459. I11. t. 10. f. 15. (13. B.).

- Carpini Fr. - [s. 111.] - Fuck. s. 153.-

R. f. 322 .

- ccratina. - [S. 112.$]$

- cerviculata. - [S. \$11.1 - Ital. 615.

- chrysostroma. - [S. H12!] - Ann. Mg. M. h.

1459. III. t. 10. f. 16. (B. B.)

- ciliata. - [S. 111.] - Fuck. s. $41^{4}$.

ciliatula. - S. 112.]

cincta. - [S. 411.]

- clopima. - [S. 112.]

conferta. - [S. 412. 11..]

conjuncta. - S. 412 .]

convergens. - [S. 412 .]

- comiculata Fr. - Fuck. s. 461 .

coronata. - [S. 112.] - K1. II. 250. - Fuck.

s. 156 .

- decorticans Fr. - [S. 112.] - R. f. 1415. -

Fuck. s, 11\%. - R, f. 146. b. (Cent. IV. Suppl.). deformis. - [S. 112 .

- deplanata Fr. - Fuck. s, 457.

- cetrusa Fr. - [S. 411.] - Fuck. s. 462.

- diatrypa. - [\$. 111.$]$

- digitata Scop. - Wstd. 167.

dissepta. - [S. 411.]

- enteroleuca. - [S. 111 .]

- extensa. - [S. 111.]

faginea. - [S. 112 . $]$

- fenestrata Obvallatae!. - Ann. MIg. n. h.

I-5\%. III. t. 10. f. 14. (B. B.).

fibrosa Fr. - [S. 411.] - R. f. 41.

Frit. - [S. $411 * *$ *]

furfuracea. - [S. 112.]

- hapalocystis. - Berk. Out. t. 1. f. 12. d. (Spor.

- hypodermia (Circinatae). - [S. 112.] - An11. Mg. n. h. 1459. III. t. 10. f. Is. (J3. B.). Berk. Out. t. 1. f. 13. d. (Spor.). - 1. f. 320.

- Iiunzei. - [S. 111.] - R. f. 526. leiphaemia. - [S. 41 \%.]

leucopis. - [S. $\$ 12$. $]$

leucostoma. - [S. 411.] - Hdw. I. t. 15.

f. ว. - Kil. 145 (). - Carol. III. (i7. - Wistl. 904 .

lixivia. - [S. 411.]

melastoma Fr. - [S. 111.] - Fuck. s. 450. 


$$
\mid
$$


1 
Vals a melastroma Fr. - [S. 412.$]$ - R. f. 251. I V. stellulata Fr.!

- - microcarpa. - [S. 112.]

- microstoma. - S. J11.] ef. Microstoma rulg.

Awd. - Fuck, s. 449.

monadelpha Fr. - S. 111.$]$ - Fuck. s. 16.5.

nivea. - [S. $\left.411.410^{* * *}.\right]$ KI. II. 1i35. -

Fuck. s. 452.

- Pini A. S. - [S. 412.] - R. f. 117.

- platanigera (Obvallatae). -- Ann. Mg.n. l.

14.5\%. III. t. 9. f. 12. (B. B.).

profusa. - S. 111.]

l'rumastri. - [S. 411.] - Fuck. s. J(i).

- pulchella. - S. 412.] - Fuck. s. 411. -

li. f. 525 .

pusilla. - [S. 112 .

quaternata. - [S. 412.] - R. f. 25. -

Fuck. s, 13!. - Krypt. liad. 331.

__ salicina Fr. - S. 112.$]$ - Fuck. s. 44.

- scutellata Fr. - [S. 111.] - Fuck.S. 151. -

li. f. 321 .

Sorbi. - [S. 111.$]$

spectabilis. - [S. +12 .

sphinetrina. - S. 1]2.

stellulata Fr. - [S. H11.] - Fuck. s. 111 !

- R. f. 25 \%. (mel.)

stilbostoma - [S. 112.] Fuck. s. 413. -

Carol. III. 6భ. - Wstd. 906.

suffulta. - Kl. II. 5ti.

suffusa. - [S, 112 .]

syngenesia Fr. - [S. 411.] - Fuck. s. 4i].

Taleola Incusae), - S. 411.? - Amn. Mg.

11. h. 15.5!\% III. t. 9. f. 11. (13, B.) - Carol. III. 6!).

_- tessella. - [S. 411.]

- tetratrupha (Obvallatac). - Ann. Mg. n. li.

1 .59. III. t. 10. f. 13. 13. 13.

thelebola. - [S. 412.]

turgida (faginea). - [S. 112.$]-$ R.f.114. -

Fuck. s. 445 . - Wstd. 907 .

umbilicata. - S. 412. 40.

vasculosa Fr. - S. 412.$]$ - Fuck. s. 134. vestita. - [S. 112.$]$

Valsaria ef. Sphaeria.

Variolaria melogramma Bull. - (ff. Melogr. vagans Not.

Venturia. - [S. 405.] ef. Sphaeria.

circinata. - [S. 1115.

Vermicularia. - [S. 419.] cf. Sphacria, Depazea, Excipula. - Bail S. t. 16.

- Chenopodii. - Wstd. 1235.

clavuligera Schw. - ef. Sporidesmium con-

cinnum, Carol.

— culmifraga. - [S. 419***420.] — Wstd !)

culmigena. - [S. 120.] Dsm. 930.

—- Dematium Fr. - [5. 120.] - Fuck. s. 397. -

I) $\mathrm{sm} .1242 .-\mathrm{Wstd}$. $57 \mathrm{~s}$.

- Dianthi. - W'std. 393.

ditricha Fr. - [S. 420.] - Fuck. s. 395.

epixyla. - S. 120.]

Geranii. - Wstd. 123!.

graminicola. - Wistd. 1241.

Graminum. - Lib. 315. - Dsm. 929. (Pe-

ziza strigosa Fr.). - Wstd. 14t. - Excipula

Gram. Cd. - Dinemaspor. gramineum Lév. herbarum. -

- - f. Sedi. - Bull. ac. belg. 1. Sér. XXI.

2. p. 246. f. 5. (Wstd.). - Wstd. 1065.

hispida. - Lib. 3-17.

hispidula, - [S. 120.]

hypoderma. - [S. 420.]
Verm i cularia Liliacearum. - Wstd. $1235^{\circ} \mathrm{Or}^{-}$ nithogali, Scillae .

—_- Luzulac. - Bull, ac, belg. 2. Sér. 14.5. II. p. 5i!. f. 12. Wstd.). - Wstd. 1212.

- macrochaeta Dsm. - R. f. 345. - Dsm. lis2. - Wstd. 1240. - Ital. is

- maculans. - S. 120.]

- Mercurialis. - Bull, ac, belg. 2. Sér. 1557.

II. p. 579. f. 13. (Wstd.)

- metallorum. - [S. $\left.119^{* * *}.\right]$

— minuta. - Lib. 350 . (Exospor.).

oblonga. - I) sm. (131.

protracta. - [S. 420.]

- Pseudo-Sphaeria. - [S. 11! $9^{*+} ; 451$. Carc.;

$470 * *$.

- pubencens, - [S. 45]. Carc. ; $4: t^{\star t}$ ]

- relicina. - [S. 420.]

- Schoenoprasi Awd. - R. f. 316.

- strigosa. - Lib. 349. ['ez. strig. v. hyst.!

— trichella. - [S. 420.]- J)sm. I2s1. - Fil.

1615. - Fuck. s. 396 . Lindl. v. K. p. 29.

f'. (i. - Voges. 973. - Wstd. 133.

- velutina. - Carol. V. 7 i;

Terpa. - [S. 347.] — (Cidaris) - Bails. $t$ ?I.

- agaricoides. - Sturm h. 6. t. 3.

- atro-alba. - [S. 317.] - Jisch. f. 331.1i, -

sturm h. 6. t. 5 .

- conica. - S. 317.] - Lourl. f. 16205.Sturm h. i. t. t.

_- digitaliformis. - [\$. $310^{*+* *}$.] - Pay. I. (r. p. Si. f. Bno. - Kl. 1629. - Berk. Out.

t. 2l. f. 6. - Sturm h. li. t. 2.

- grisea. - Sturm h. 6. t. 6.

Krombliolzii. - Cd. Anl. t. G. f. 67: 1-3.

Sturm h. 6. t. 1.

Verticicladium trificlum Preuss. - Stum h. 3.). t.

Verticillium. - [S. $\left.196^{* *} \cdot\right]$ - Terlultnist zu Trichothec, ros. : Bot. Zt. 1 554 t. 4. A.

Ioffm.

- allochroum. - sturm h. 11. t. G.

t. 7. f. 1 i. 1 . B. B.).

- Candelabrum. - Bon. t. 5. f. 121

eapitatum. - Bon. t. j. f. 1]

corymbosum. - Zeitschr. f. wiss. Zool 14.55.

IX. t. 16. f. 1-5. (Lebert).

crassum. - Bon. t. T. f. 119.

crustosum. - Kl. $197 \%$

eylindrosporum. - Cd, Anl, t. I3, f. 17: 1 .

2. - Pay. b. cr. p. 72. f. 316.

distans. - Ann. Mg. n. h, 1551. VII. t. .

f. 16. p. 102. (B. B.).

epimyces. - Ann. Mg. n. h. 1551. VII. t. 7.

f. 15. p. 102 . B. B. .

-- glaueum, - Bon. t. 5. f. 120.

hamatum. - Lon. t. 5, f. $11 \%$.

lateritium Htg. - Verhandl. Amsterd. Akarl.

XII. -- Amn. sc. n. 1516. VI. = Acrostalagm, cinnab. $\mathrm{Cd}$.

- minutulum P. - Lotos, 1557. VII. t. 1. f. 2.

- nanum. - Ann. Mg. n. h. $155 \%$. VII. t. 7 .

f. 1 S. p. 102 . (B. B.).

- ochro-rubrum. - Ann. sc. n. 1834. II. t. 2.

— pyranidale. - Bon. t. S. f. 179.

- ruberrimum. - Bon. t. i. f. 166. tenerum. - [S. 491.]

Vibrissea. - [S. 360 .]

- flavipes. - KI. $162 \%$.

Guernisaci. - Ann. se, n. IS5 T. VII. t. 4.

f. F. (Crouan).

Persoonii, - Cd. Anl. t. G. f. 6ii: 3. 1. 
Vibrissea rimarmm. - s. $36 ; 1$.

_- truncorum. - [S. 36il.] - Bisch. f. 33i4. -

Schnel. t. 16. f. 16. 17. - Bail S. t. 21. -

Berk. In. p. 2s4. f. 66. d. - Isin. 30. - Ca.

Anl. t. G. f. 66: 1. 2.

- vermieularis. - [S. $36,1$.

Virgaria. - [S. 492 . $]$

$V$ it t a dinion fuseum. - cf. luber $f$.

- Montagnei \%ob. - Cd. Ic. VI. t. 20. f. 112 ;

Tuber excavitum Tul.

Volutella. - [S. 363.] Fusar., Peziz., 'Tubercul.

- carmea. - [S. 363.

— ciliata. - [S. 363.] - Bisch. f. 352T; Fusarium ciliat. $\mathrm{Lk}$. $\mathrm{Fr}$.).

- melaloma. - Am. Mg. n. h. 1550. V. t. 11.

f. 3. p. 466. I3. B.!

— pallens. - \$s. $34 i 3$.$] - Bisch. f. 3525$.

- volvata. - [S. 3ti3.] - Cd. Anl. t. G. f. 6t:

9-11. - Bail S. t. 20. - Bisch. f. 3526.

W e in mannodora. - [S. 409.]

X eilaria Agrostidis. - Lib. 63.

— Urticae Lib. - ef. Rhytisma Urt. Fr.

Xenodochus. - [S. 505.]

- brevis S-l, - Bisch. f. 3466. - Fuck, s. 161.

- carbonarius. - Lib. 249. - Bisch, f. 3sbij.

- Fuck. s. 111. - Microse. Journ. 1557. V.

t. ร. f. 31. Currey, - Fuck. rh. 410 .

- paradoxus. - Brit. f. 324. - Berk. In. p.

325. f. $73 . \mathrm{b}$.

Ierotus afer. - Afzel. t. 11. f. 24.

- degener. - [S. 315.]

_- discolor. — C'hile no. 7. f. 3.

—- lateritius. - Carol. IV. 3.

Xylaria. - [S. 3S1.] - ef. Sphaeria, Hrpoxylon.

- alutacea P. - Loud. f. 16357.

- aristata. - Ann. se. n. 1-55. III. t. 5. f. 6 . Mont.

- axifera. - Ann. se. n. 1S55. III. t. 5. f. 7. Mont.

- _ bulbosa P. - R. f. 133. - Berk. Out. t. 21.

f. 2 .

- capitata. - Loud. f. 16356. - Wstd. 21.

- carpophila, - [S. 352.] - Kl. 1915. -

Fuck. s. 701. - Wstd. 111.

_- clarata Math. - Wstd. 902.

- collabens. - [S. 351.]

— comosa. - S. 381.]

- corniformis. - [S. 3 1.] - Sturm h. 3.1-.

t. 1. Strauss . C Carol. IV. 30?

cornuta Schrk. - Wstd. J6T.

eupressiformis. - [S. 352.] - Vistd. 365. digitata. - [S. 351.] - Loud. f. 16353

,, 163556 - Fuek. s. 699: polymorpha, - Kl. 252. - Krypt. Bad. 432. - Kil. II. 46. - Carol. V. 50.

— entomorhiza Dicks. - Loud. f. 16355. links).

cf. Claviceps.

- eupeliaca $=\mathbf{X}$. Guepini v. eup. C'es.).

- filiformis A. S. - [S. 352.] - R. f. 57. -

Dsm. III. 37 .

- flabelliformis. - [S. 351 .

— flexuosa Schrk. - Wstd. 114.

- gracilis Grev. - Loud. f. 16354.

-.. Guepini.

- v. eupeliaea. - Comm. soc. erit. it. 1〉61. 2. t. 5. Ces.

- Hypoxylon. - [S. 3)1.] - Loud. f. 16351, 163.54 - Berk. Ont. t. 21. f. 1. - Iil. 251. Iil. II. 429. - Fuck. s. 69ะ. - Krypt. Bad. 336.
Xylaria mieroeephala. - Chile no, 9. f. 2.

Oedipus. - Carol. III. 47.

ophioglossoides. - Wstd. 69.

pedata. - [S. 352.]

- pedunculata. - [S. 352.]

- polymorpha. -- $[\mathrm{S} .351]-.\mathrm{Cd} . \mathrm{Ic} . \mathrm{V} . \mathrm{t} . \mathrm{s}$

f. 52. - Fuck. s. (i99. 700. - Loud. f. 16353.

- (d. Anl. t. F. f. 56: 15-20. - Kl. II. 129.

- Dsm. III. 376. - Ital. 291.

- portentosa. - Chile no. 9. f. 1.

- punila. - [S. 351.]

— punctata Sow. - Loud. f. 16359. (, 1635y".)

- subularis. -. [S. 352.]

Xyloehoeras. - S. 476.$]$

Xylographa. - S. 372.

attenuata. - Alger. t. 2!, 7 .

_ flexella. - [S. 372 .]

-... Hederae Lib. - Kl. 105.5.

- longa. - [S. 372.]

- parallela. - [S. 3:2.] - K1. 14:1.

- stietica. - [S. 372.$]$

$\mathrm{Xylographuin.} \mathrm{-} \mathrm{cf.} \mathrm{Aylographum.}$

A yloma. - [S. 521.] ef. Melampsora, Sclerotium, Leptostroma, Rhytisma.

- acerinum P. - Loud.f. 16190. - Voges. 77.

- Wstd. 31.

__ alneum. - S. 123.] - Voges. 75. - Wstd.

26it. - cf. Dothidea aln.

- Andromedae. - Virges. 176.

Aquifolii DC. - Wstd. 273.

- betulinum. - Voges. 370. - cf. Dotlid. bet. Lib.

- Bistortac, - Voges. 652. - Wstd. 1245.

Carpini Fr. - Wstd. 275.

circinans. - Voges. 450.

- concarum Grer. - Loud. f. 16496. - Wstr. 36 .

- coneentricum. - Voges. 369. - ef. Leptothyrium Tremulae.

- Empetri. - Voges. 4\$1. - Ital. S\$1.

- fagineum Schum. = Sphacria Artuereas, Fl.

dan. - Voges. 4is. - Wstd. 27.

- foliicolum. - Voges. 761. Hysterium f. Fr.:.

- herbarum Dub. - Melampsora h. Dsm.

hysterioides P. - Lib. 72. Hysterium fo-

liicolum; - Lophodermium xylomoid. C'her.

Dub. . - Wstd. 92.).

- Ilicis, - [S. 367. 3.] - Wstd. 273.

- Juglandis. - ef. Leptothyrium

- leucocreas. - Toges. 175. - Wstcl. 33. u.

1244.

- lichemoides DC: - Wstd. 17\%.

Liriodendri Wllr. - Wstd. 45().

- multivalve. - ef. Ascochyta Aq. - Wstd. $52 \pi$.

pezizoides $\mathrm{P}$ '. - Phacidium coronat., Fl, dan. pezizoideum I'. - Loud. f. I6197. - ef. 1'hacid. coronat. - Wistd. 919.

- Pini. - Wstd. 1117.

- populinum. - Toges. 269. - Wstd. 7 .

- punctatum. - Voges. 47\%. Rhytisma . -

Wistd. 659.

- punctulatum DC: - Wstd. 535 .

—_ pyrigenum D. L. - Labrella Pomi.

Rosae DC. - Wstd. 476.

rubrum. - ef. Polystigma, Ascochyta Iib.

- Wstd. 637.

salicinum P. - Loud. f. 16191. - Wstd.

736. 1244 .

- salignum. - Toges. 26ৎ. - Wstd. 521.

Spiraeae. - Voges, i60.

Stcllariae S. — Fuck. s. 222. 


Xyloma sticticum Mart. - Wstd. 111.

_ ?striaeforme. - Voges. 476.

ulmeum schw. - cf. Sphaeria u

umbonatum. - Schnzl. t. 15. f. 1-3.

varium. - Voges. 653. Leptostroma juncinum Fr.).

- Virgaureae. - Voges. 479. - ef. Puccinia V., Lib. - Wstd. 6S1.

- Vitis Lév. - Wstd. 725.

Aylomites Aceris. - Heer, Fl. tert. Helv. t. 1. f. 111 .

— Daphnogones. - Heer, Fl. tert. Helv, t. 1. f. 11 .

- maculifer. - Heer, Fl. tert. Helv. t. 1. f. S. - protogaeus. - Heer, Fl. tert. Helret. t. 1 . f. 12 .

- varius. - Heer, Fl, tert. Helv. t. 1. f. 9.

Xylopodium. - [S. 413.]

- Delastrii. - Pay. b. er. p. 112. f. 513. Alger. t. 20, 1.

Xylosphaera Dmtr. - ef. Sphaeria.

Xylostroma. - [S.521.] - Kl. II. 700. (Suppl.). Corium P. - Wstd. 100. - Kil. 1200. Fuck. s. 201.

giganteum Td. - Wstd, 100. - R. f. 100. - Voges. 6i9. Mycelium Polypori albi cujus- dam fagicoli in sylya Compendiensi frequentis, Tul.). - Carol. III. 9s.

Ypsilonia. - [S. 424. 377.]

Z a s midium. - [S. 407.] - ef. Racodium.

Tela. - [S. 407.]

vulgare. - [S. 407.]

Zygodesmus. - [S. 494. 337.]

- ferrugineus. - Sturm h. 29-- t. 39. (Preuss.). fuscus. - Cd. Anl, t. B. f. S: 1.2. - R. f.

379. - Microse. Journ. 1S57. V. t. S. f. Hl.

(Curr.) - Pay. b. cr. p. 72. f. 322. - Berk.

In. p. 294. f. 65. g. - Fuck. rh. 156.

- tristis. - Kl. II. 274.

Zygosporium. - [S. 501.]

oscheoides. - Cd. Anl, t. H. f. TS: 3-9. -

Cd. Ic. VI. f. 2S. - Sagra, Cuba. t. 11. f. 2. Zythia. - S. 107.$]$

- Dentariae. - Bull. ac. belg. 2. Sér. 1857.

II. p. 579. f. 11. (Wstd.). - R. f. 452. -

Wstd. 1236.

- elegans. - [S. 408.]

- epimyces. - [S. 40S.]

- ferox: - [S. 10૫.]

- Georginae. - S. 105.

- Rhinanthi. - [S. HoS.]

Zythinia. - [S. 400.]

\section{Corrigenda et addenda.}

l'ag. 1. Ac antloostigma cf. Sphaeria.

," , Acremonium alternatum. - Sturm l. 1. t. 2.

,2. A etinonema Crataegi. - Fuck. rh. 15̃f.

,, , - Populorum. - Fuck. rh. 15s,

,, , Rosae. - Fuck. rh. 457.

,, Agarieus collariatus, - Sturm h. 11. t. 52.

- coprinoides. - Sturm h. 14. t. 50.

, 10. - fragilis Russula, - sturm h. 14. t. 53.

,, 21. — virescens. - Sturm h. 3. t. 17.

, 23. Amylocarpus encephaloides. - Proceed. roy. Soc. Lond. 1\$59. IX. p. 123. c. ic. Anat. Curr. .

, 21. Ascobolus vinosus. - Berk. Out. t. 23. f. 1.

," , Ascochyta Cheilaria Aceris. - Fuck. rh. 17t.

", "- Armoraciae. - Fuck, rlı. 4 bi.

,, - Cannabis. - Fuck. rh. 14t.

,, 2̌s. - Polygoni. - Kl. II. 442.

,, 29. Boletus picrodes Rostk. = impolitus? Fr. in lit.).

, 30. Botrytis Jonesii B. B. = Chaetocladium J., Fres.

, 36. Chaetoeladi um Jonesii Fres. = Botrytis Jonesii B. B.

", 10. Comiothecium Evonymi. - Fres. p. 103. t. 13. f. 25-28.

,, 55. Fumago vagans. - cf. Coniothecium Tiliae Fres.

,, 61. He li com a Mülleri Fuck. = Helicosporium Fuckelii Fres.

,, \$2. Oidi um fusisporioides cf. et Ramularia Urticae.

,, 95. Podospora fimicola Ces, - cf. Sordaria fimiseda Not.

,, , Polyangium vitellinum. - Sturm h. 2. t. 27. 
Typis Breitkopfii et Harteli, Lipsiae. 

1 

1 





$$
\mid
$$



1 


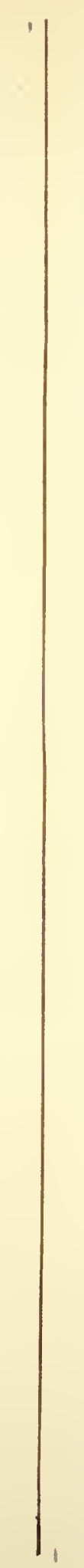




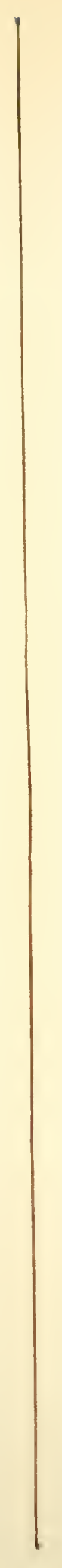









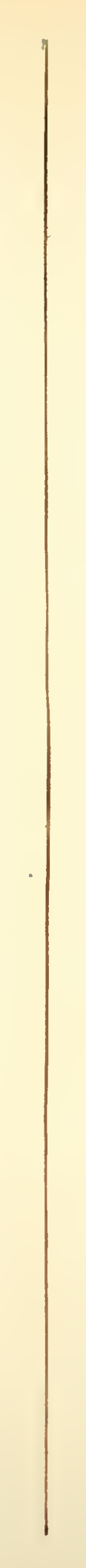



1 

1 


$$
\mid
$$




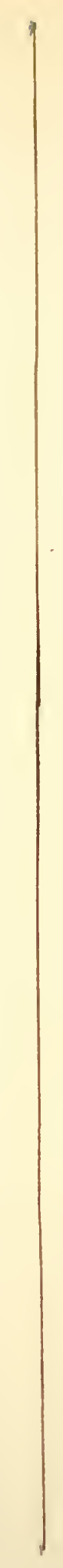




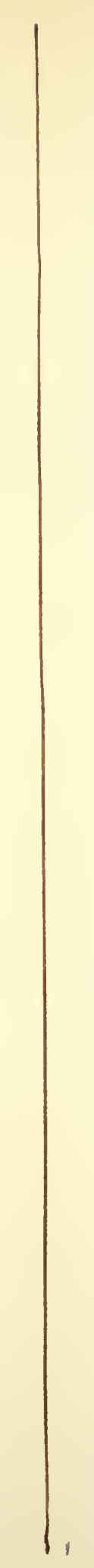




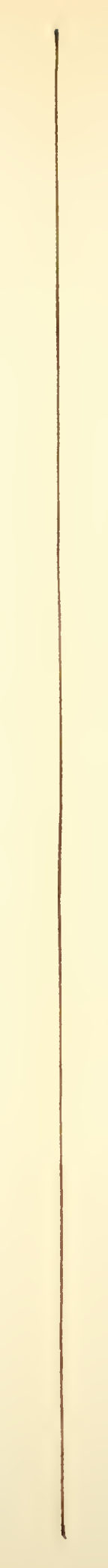




$$
1 .
$$




$$
\text { | }
$$




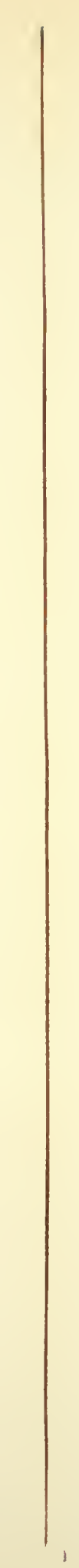





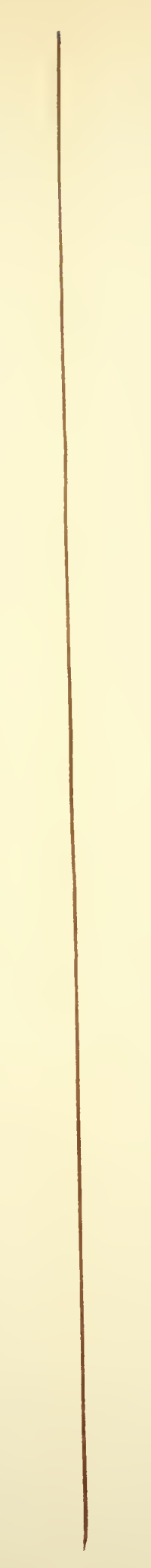








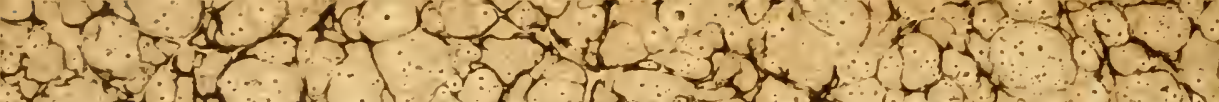

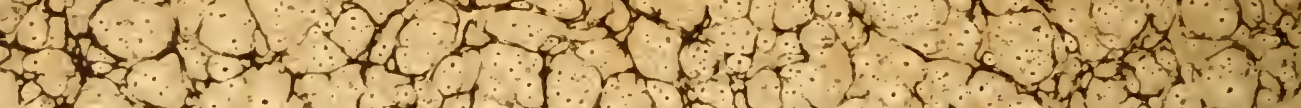

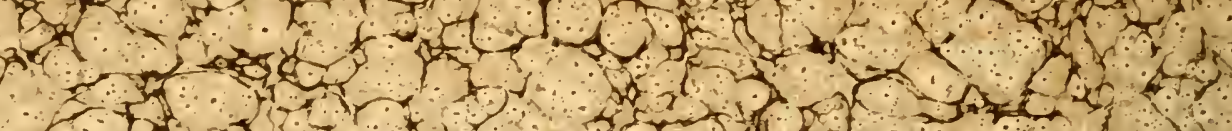

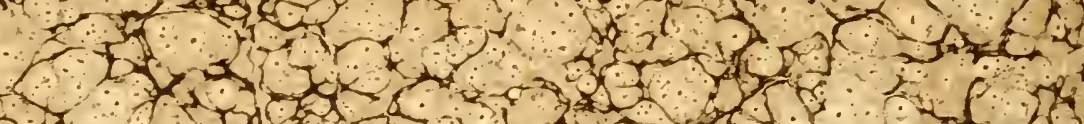

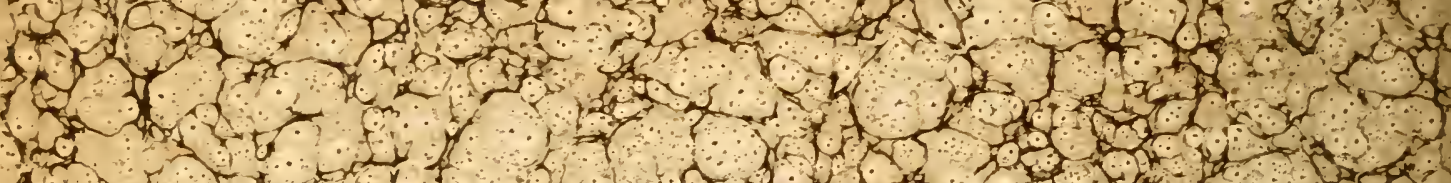

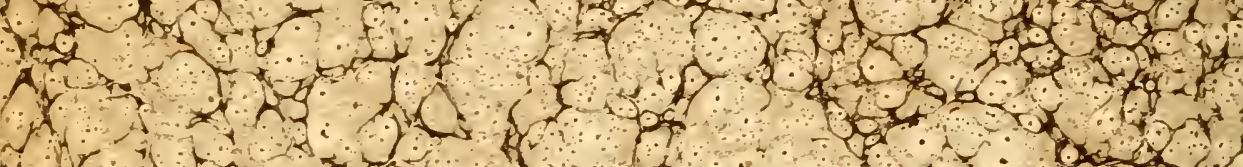

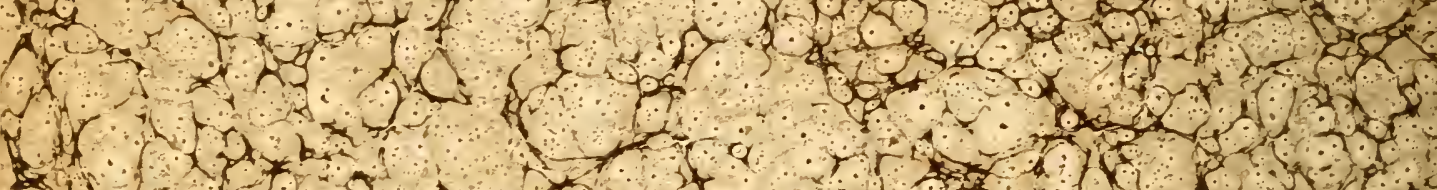

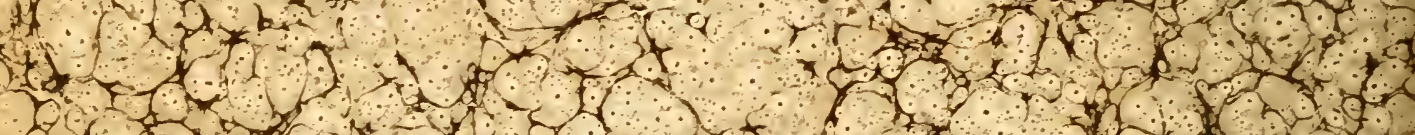

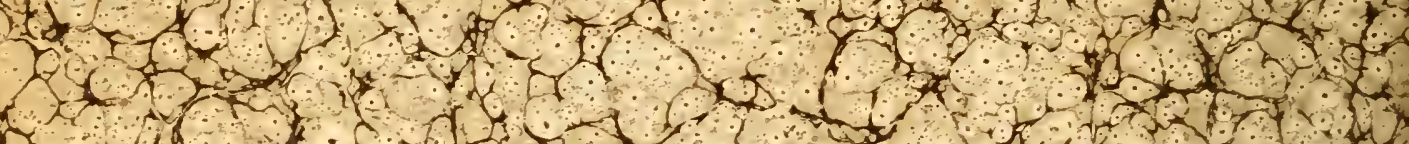

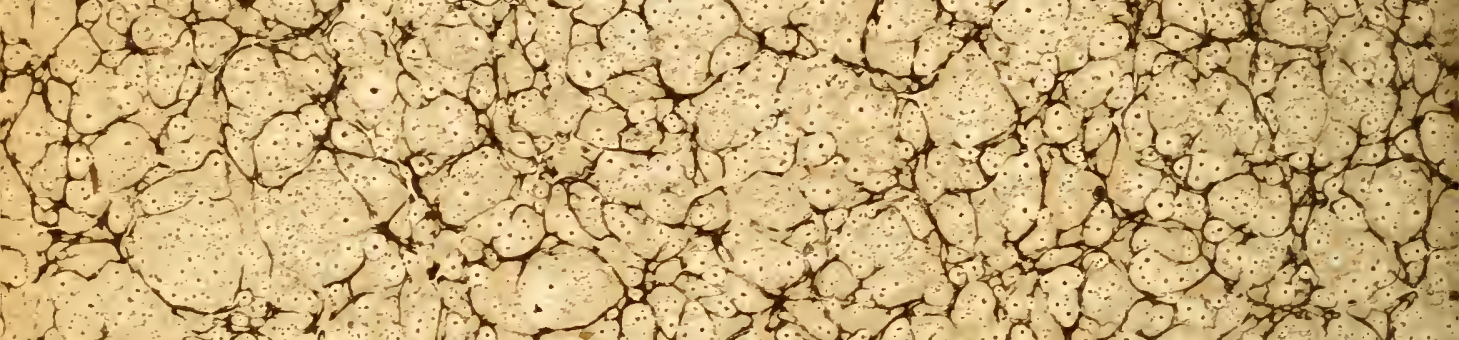

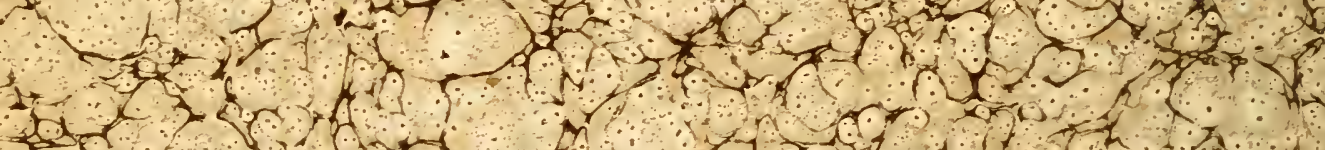

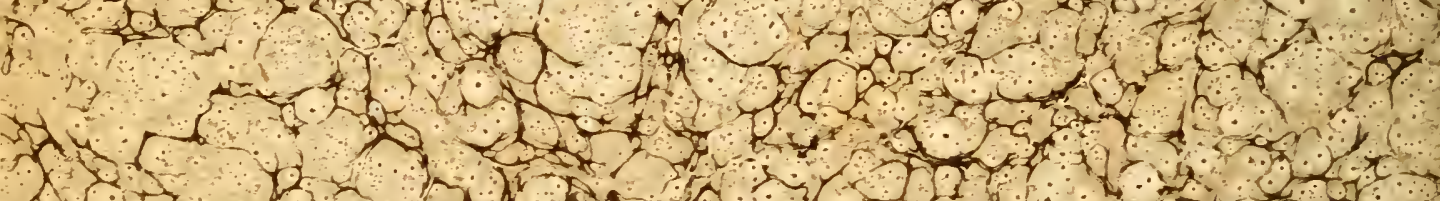

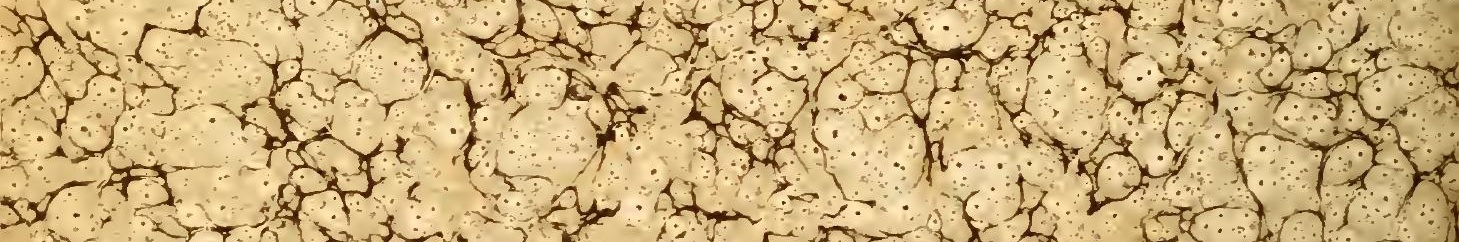

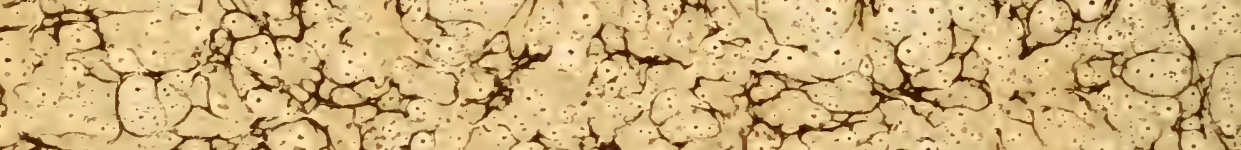

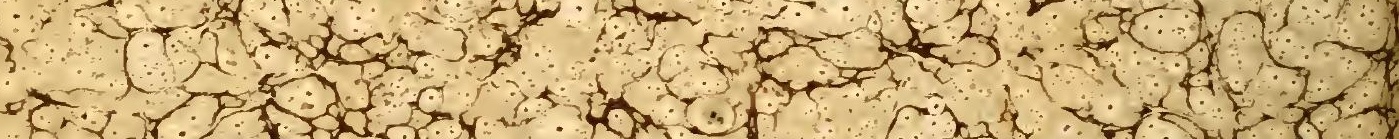

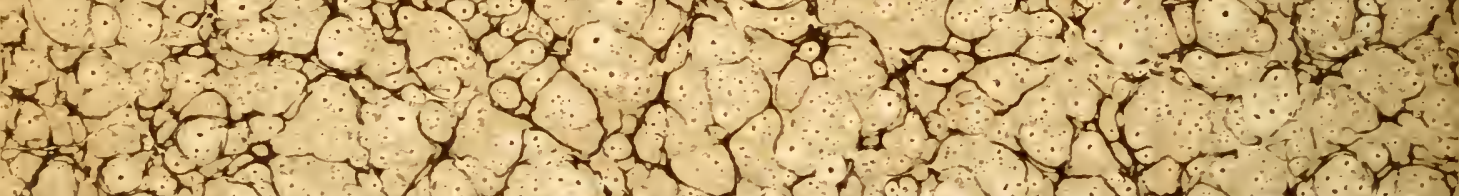
at.

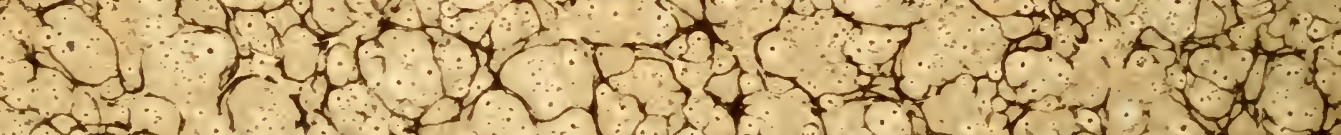

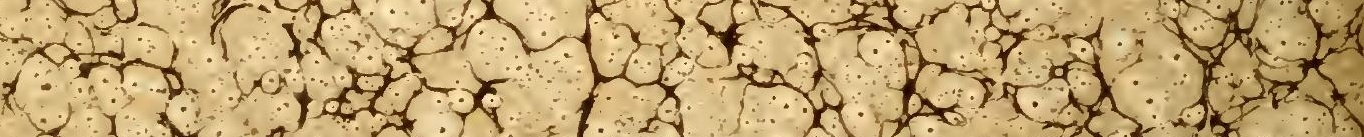
- 10 a

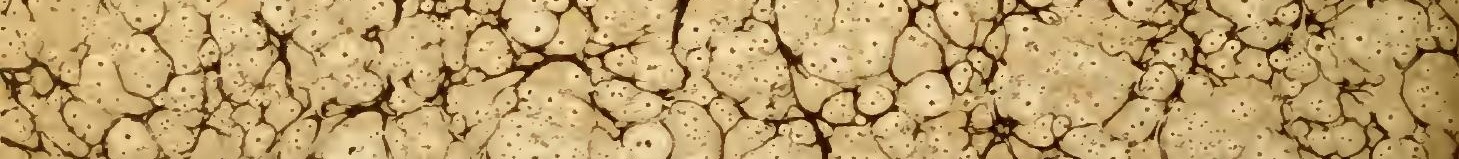
(1) 


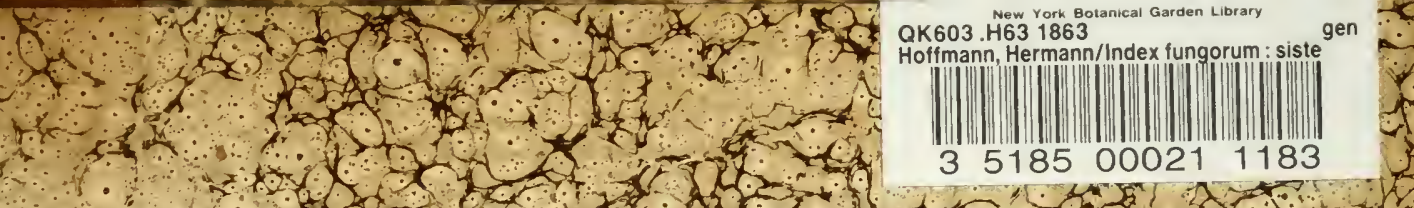

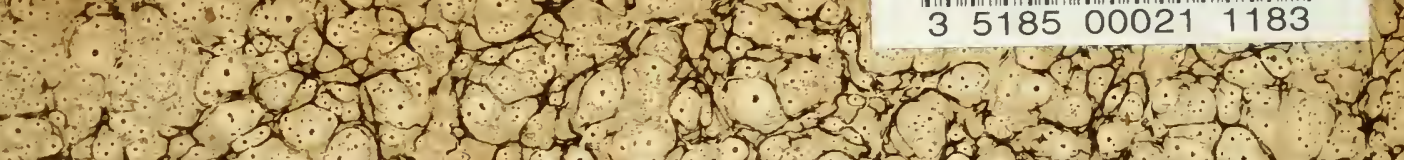

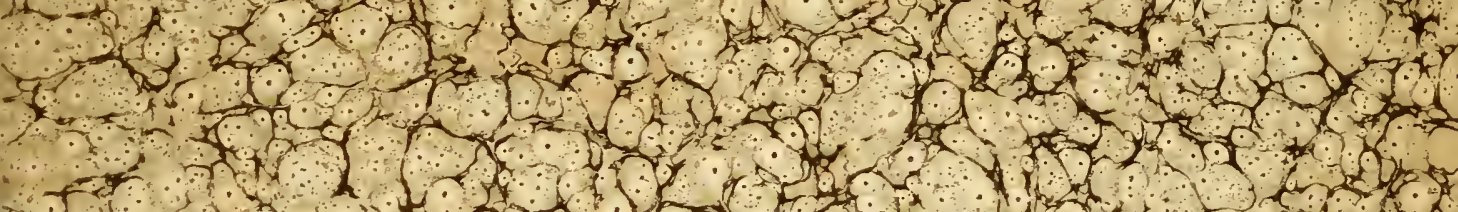

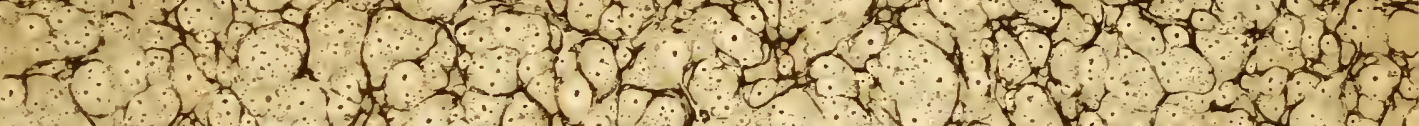

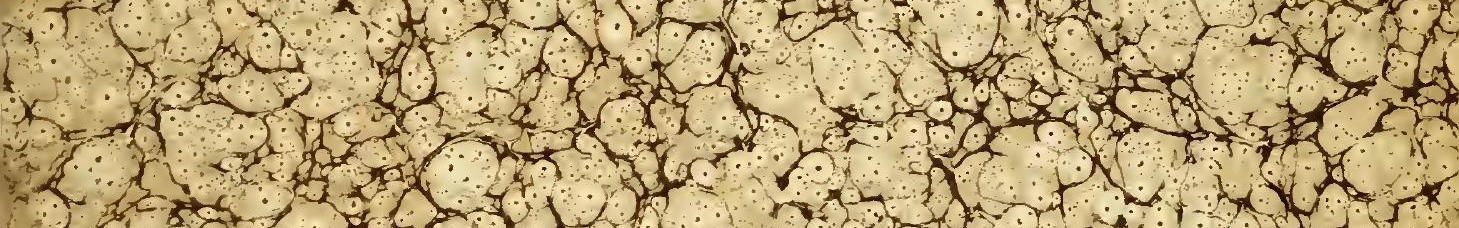

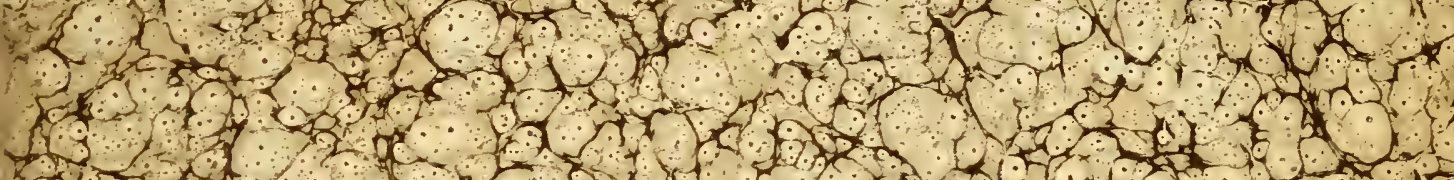

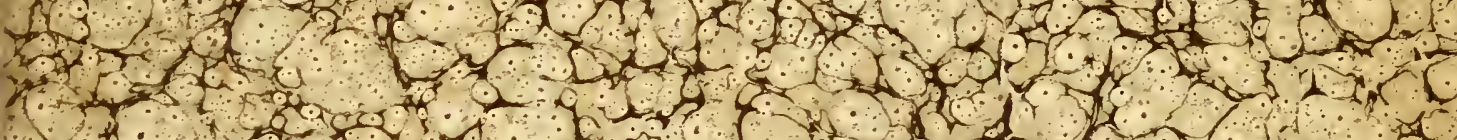

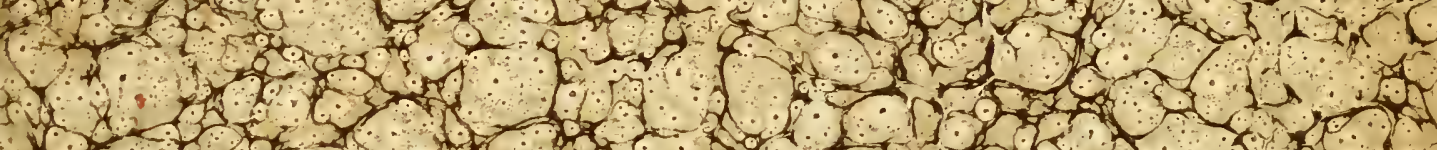
$x+6 \times 2 \times 2$ In 10,

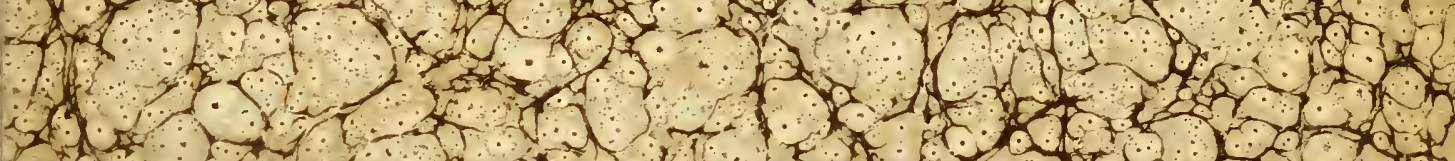
$1+26$.

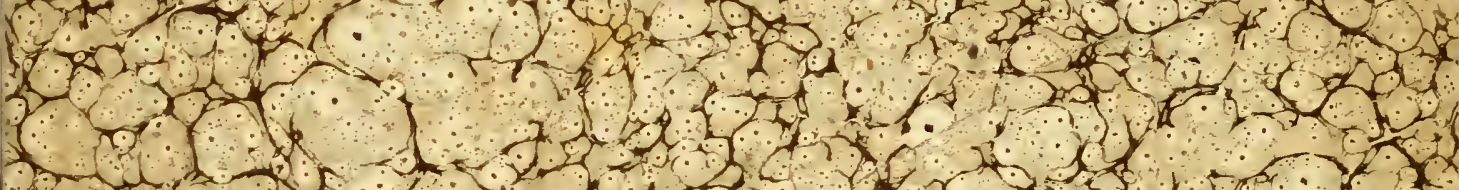

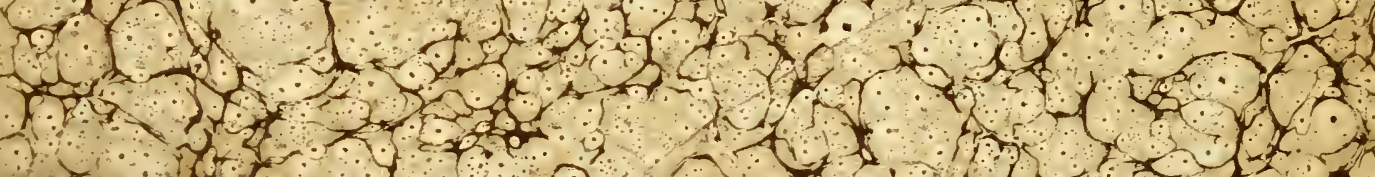

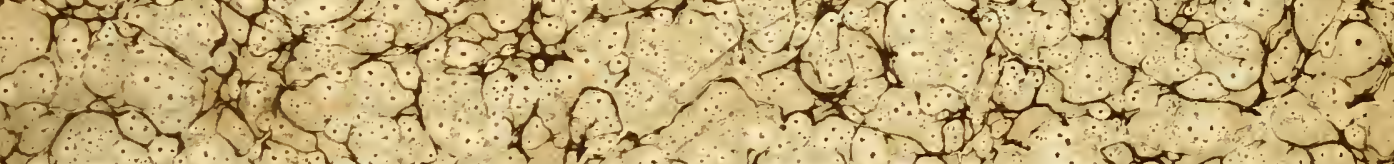

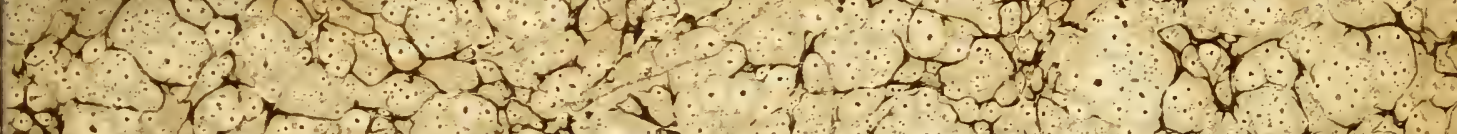

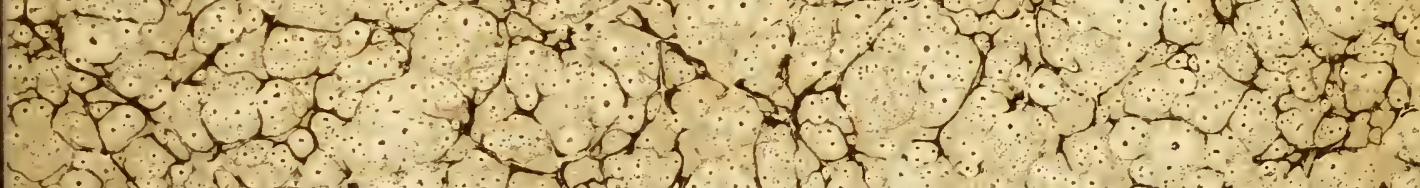

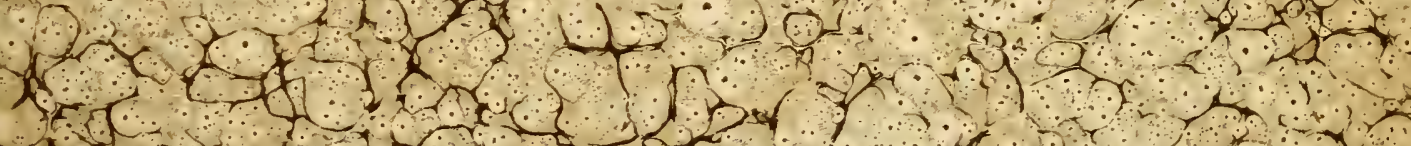

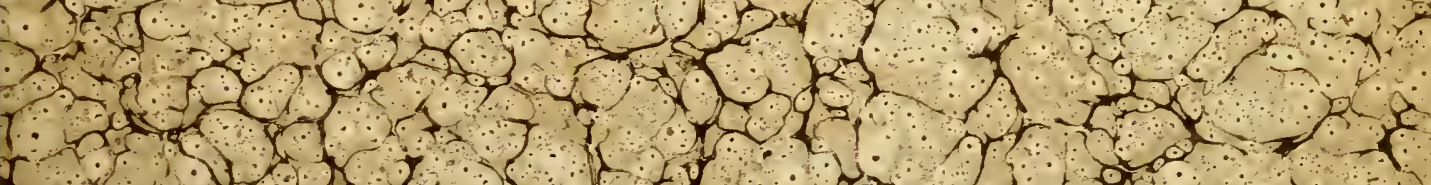

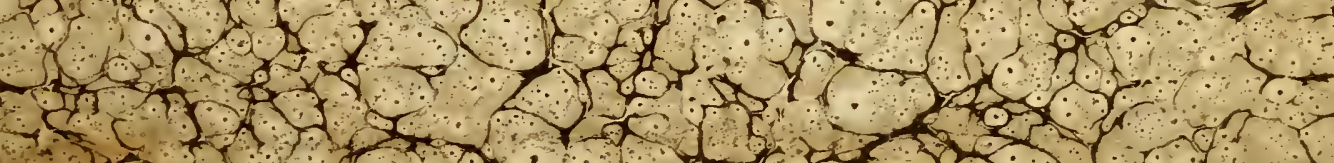

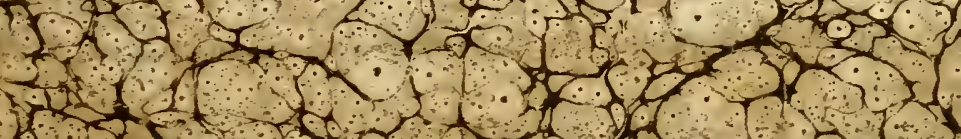




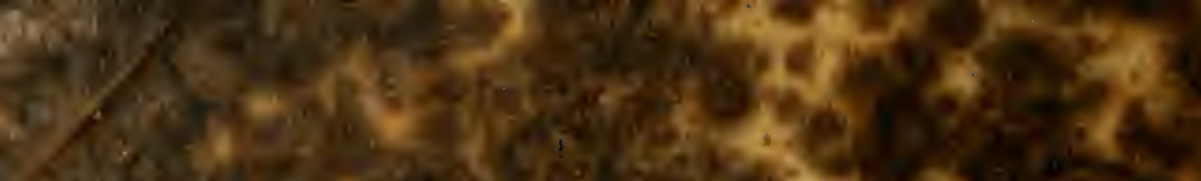

the

.

$x$ sit.

- ow

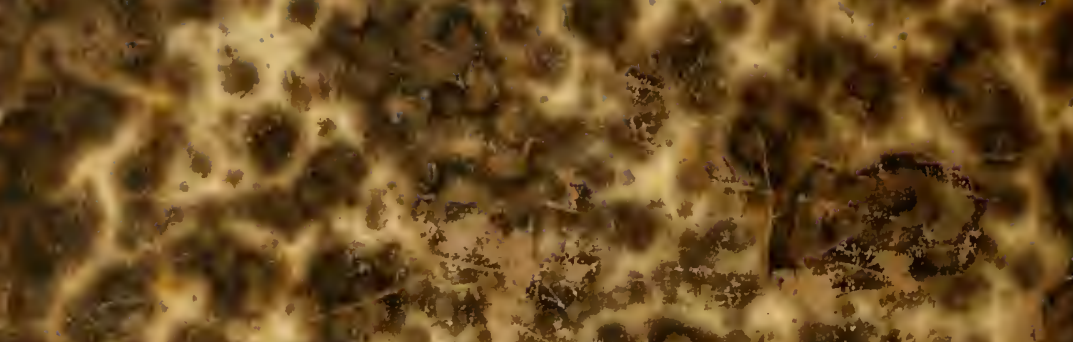

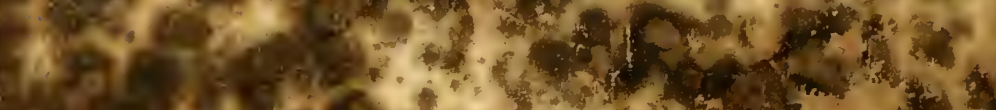

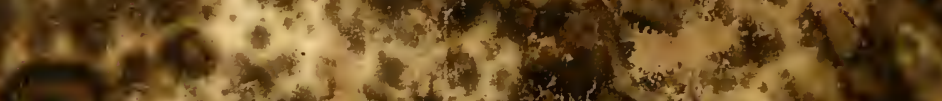

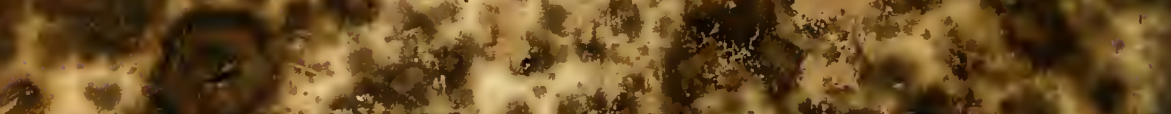

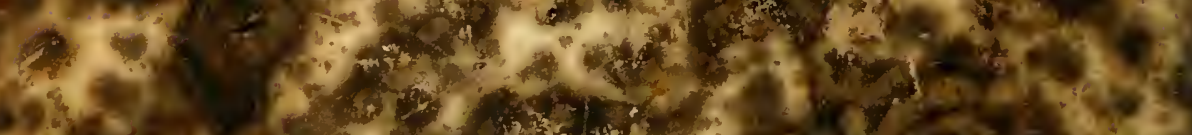
2.8.

(25)

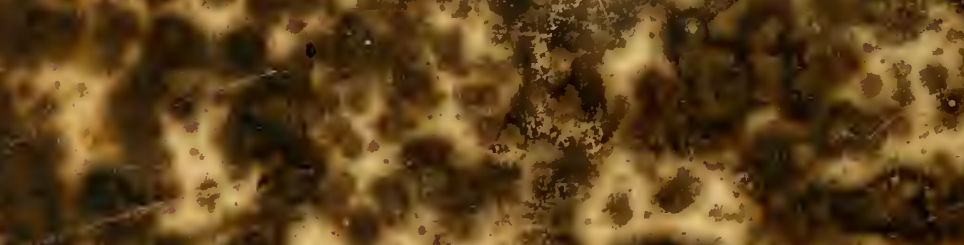

(2)

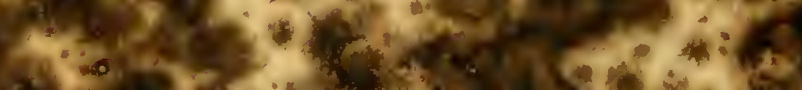

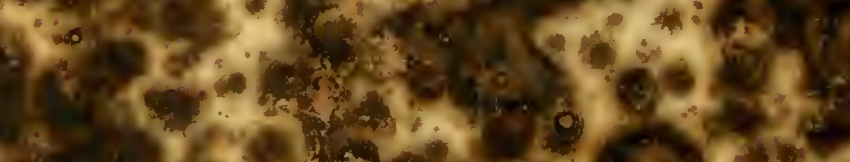

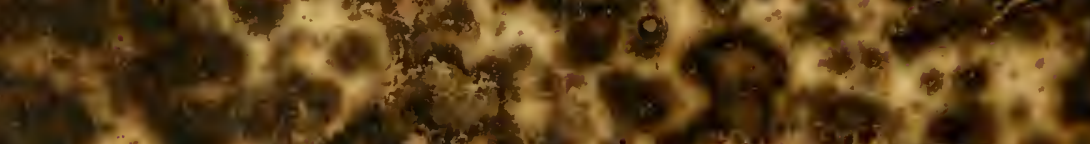

H."

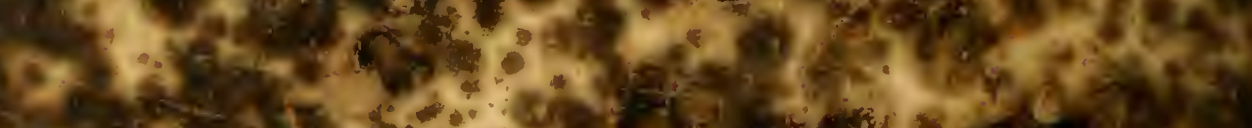

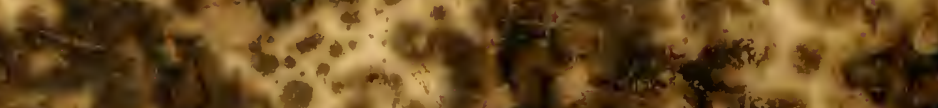

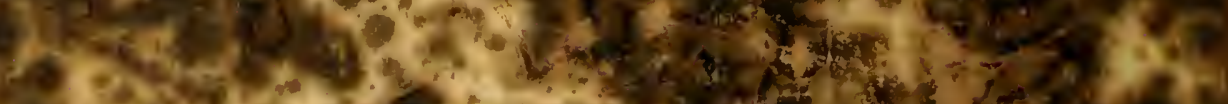

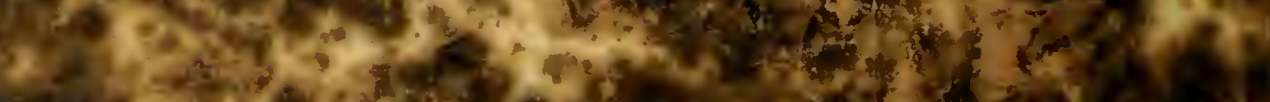

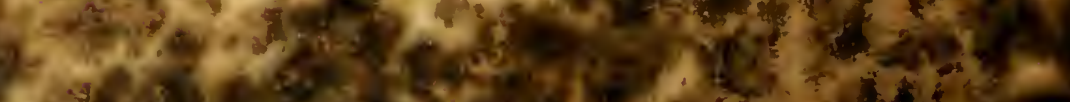

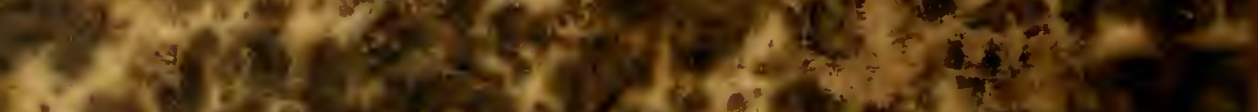

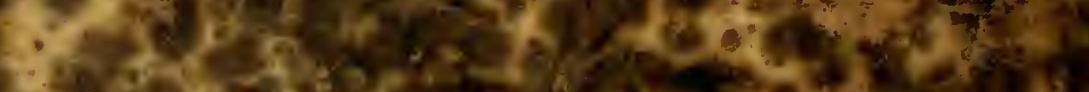

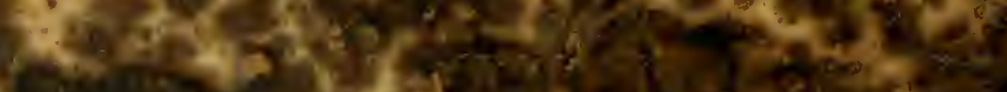

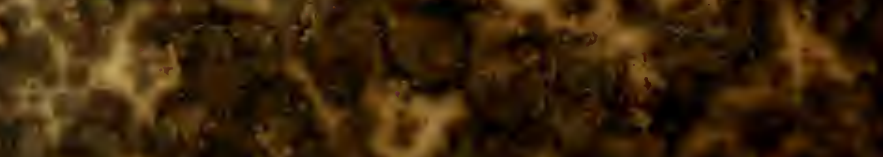

W. 